Final Report

FHWA/IN/JTRP-2004/38

\title{
STABILIZATION AND IMPROVEMENT OF ORGANIC SOILS
}

\author{
by \\ Joonho Hwang \\ Graduate Research Assistant \\ Aaron Humphrey \\ Graduate Research Assistant \\ Antonio Bobet \\ Associate Professor \\ and \\ Maria Caterina Santaga \\ Assistant Professor \\ School of Civil Engineering \\ Purdue University \\ Joint Transportation Research Program \\ Project No. C-36-50U \\ File No. 6-19-20 \\ SPR-2460 \\ Conducted in Cooperation with the \\ Indiana Department of Transportation \\ and the U.S. Department of Transportation \\ Federal Highway Administration
}

The contents of this report reflect the views of the authors who are responsible for the facts and the accuracy of the data presented herein. The contents do not necessarily reflect the official views or policies of the Indiana Department of Transportation or the Federal Highway Administration at the time of publication. This report does not constitute a standard, specification, or regulation.

School of Civil Engineering

Purdue University

September 2005 
TECHNICAL REPORT STANDARD TITLE PAGE

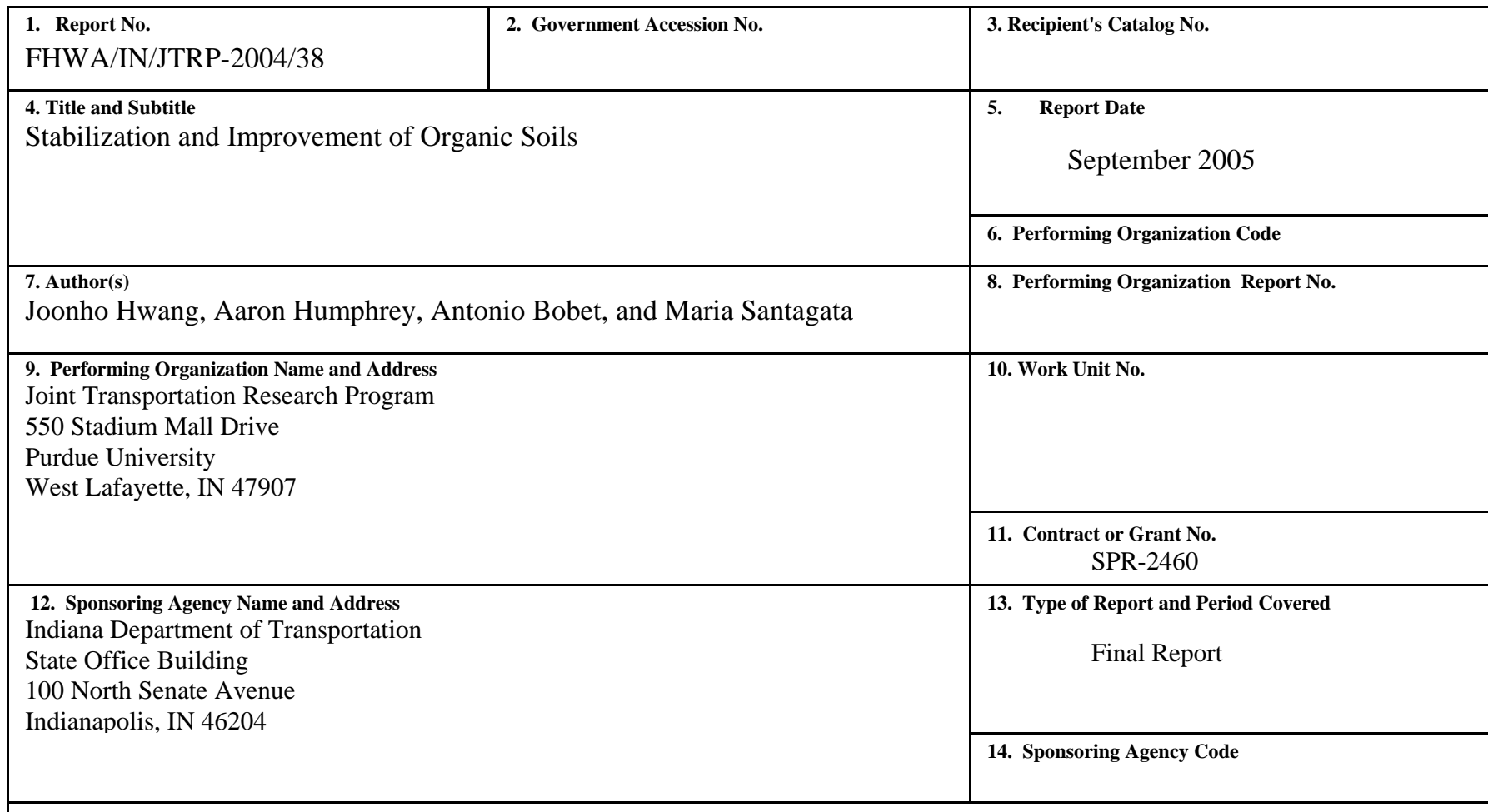

\section{Supplementary Notes}

Prepared in cooperation with the Indiana Department of Transportation and Federal Highway Administration.

\section{Abstract}

Peats and organic soils in general pose significant problems to geotechnical engineers due to their low strength, high compressibility and elevated creep. The research performed addressed one soil improving technique, deep soil mixing, that has been widely used for treating soft clays, but that especially in the US has found limited use in presence of organic soils. The work performed made use primarily of one soil sampled on Lindberg Road (LR) in West Lafayette, IN characterized by LOI= 45-52\%, LL= 327\%, PL= $162 \%, L_{\text {oven dried }} / L_{\text {non-dried }}$ $=0.31$, Gs $=2.05-2.12$, fiber content $\sim 2.29 \%$, clay fraction $=40.6 \%$. In addition, a limited number of tests were performed making use of soils with LOI of 10-20\%, manufactured in the lab from LR soil and an illitic clay.

A procedure was developed for preparing samples of reconstituted LR soil both untreated and mixed with a binder and which included a "curing" stage under a surcharge to simulate treatment at depth. Specimens obtained from these samples were used for the engineering tests which included constant rate of strain (CRS) consolidation tests, end-of-primary incremental loading (EOP-IL) consolidation tests with one long term creep stage, and unconfined compression tests. A battery of characterization tests and an in depth review of the literature complemented this work.

Unconfined compression tests provided a preliminary evaluation of the effects of treatment on the strength of the soil; highlighted the effects of curing under a surcharge; and allowed to identify in Portland cement (PC) the most promising binder, which was subsequently used for all other engineering tests, at dosages ranging from $8 \%\left(\sim 25 \mathrm{~kg} / \mathrm{m}^{3}\right)$ to $100 \%\left(\sim 320 \mathrm{~kg} / \mathrm{m}^{3}\right)$ by dry mass of the soil. The results of the consolidation tests highlighted how the accurate characterization of the primary consolidation behavior of soils characterized by high tendency to creep must rely on either CRS or EOP-IL loading tests and demonstrated the effects of treatment with cement on the stiffness, the hydraulic conductivity, the rate of consolidation and the rate of creep of the soil. Specifically, the tests showed how the addition of cement is associated with the development of a preconsolidation pressure and the shift of the compression curve towards higher effective stresses. Once this yield stress is exceeded the compressibility in the virgin compression range is found not to vary significantly with cement content. Also associated with the addition of cement is an increase in the hydraulic conductivity, an increase in the coefficient of consolidation, and a reduction in the creep coefficient at any given stress level. Moreover, the $\mathrm{C}_{\alpha} / \mathrm{C}_{\mathrm{c}}$ ratio decreases markedly with cement addition indicating a decreased susceptibility of the soil to creep. All these effects are more marked with increasing cement content and the treatment appear especially effective once the PC\% exceeds $50 \%\left(\sim 160 \mathrm{~kg} / \mathrm{m}^{3}\right)$.

\section{Key Words}

organic soils, LOI, compressibility, consolidation, constant-rate-of-strain (CRS), secondary compression, strength, ground improvement, cement.

\section{Distribution Statement}

No restrictions. This document is available to the public through the National Technical Information Service, Springfield, VA 22161

\section{Security Classif. (of this report) \\ Unclassified}

\author{
20. Security Classif. (of this page) \\ Unclassified
}

21. No. of Pages
268

22. Price 


\section{TECHNICAL Summary}

INDOT Research

Technology Transfer and Project Implementation Information

TRB Subject Code: 61-6 Soil Properties

Publication No.: FHWA/IN/JTRP-2004/38, SPR-2460

September 2005

Final Report

\section{STABILIZATION AND IMPROVEMENT OF ORGANIC SOILS}

\section{Introduction}

Peats and organic soils in general pose significant problems to geotechnical engineers due to their low strength, high compressibility and elevated creep. The research performed addressed one soil improving technique, deep soil mixing, which has been widely used for treating soft clays, but that especially in the one-dimensional compression behavior of organic soils following treatment, a topic which has been so far for the most part unexplored.

The work performed made use primarily of one soil sampled on Lindberg Road (LR) in West Lafayette, IN characterized by LOI $=45-52 \%$, LL= $327 \%, \mathrm{PL}=162 \%, \mathrm{LL}_{\text {oven dried }} / \mathrm{LL}_{\text {non-dried }}=0.31$, Gs $=2.05-2.12$, fiber content $\sim 2.29 \%$, clay fraction $=$ $40.6 \%$. In addition, a limited number of tests were performed making use of soils with LOI of 10$20 \%$, manufactured in the lab from LR soil and an illitic clay.

A procedure was developed for preparing samples of reconstituted LR soil both untreated and mixed with a binder and which included a "curing" stage under a surcharge to simulate treatment at depth. Specimens obtained from these samples were used for engineering tests which included constant rate of strain (CRS) consolidation tests, end-of-primary incremental loading (EOP-IL) consolidation tests with one long term creep stage, and unconfined compression tests. A battery of characterization tests and an in depth review of the literature complemented this work.

\section{Findings}

Unconfined compression tests provided a preliminary evaluation of the effects of treatment on the strength of the soil and allowed to identify in Portland cement (PC) the most promising binder, which was subsequently used for all other engineering tests, at dosages ranging from $8 \%$ $\left(\sim 25 \mathrm{~kg} / \mathrm{m}^{3}\right)$ to $100 \%\left(\sim 320 \mathrm{~kg} / \mathrm{m}^{3}\right)$ by dry mass of the soil, reflecting dosages typically used in practice. The unconfined compression tests also highlighted that treatment with PC is more effective for soils with lower organic content, and showed that the surcharge applied during curing has a significant effect on the strength and stiffness of the soil mixture. In particular, the unconfined compressive strength was found to be directly proportional to the surcharge applied.

The results of the consolidation tests, which comprised the most significant portion of the experimental work, highlighted how an accurate characterization of the primary consolidation behavior of soils characterized by high tendency to creep must rely on either CRS or EOP-IL loading tests and demonstrated the effects of treatment with cement on the stiffness, the hydraulic conductivity, the rate of consolidation and the rate of creep of the soil.

Specifically the tests showed how the addition of cement is associated the development of a preconsolidation pressure and the shift of the compression curve towards higher effective stresses. In the recompression range, the slope of the compression curve decreases with \% of PC indicating that the soil becomes stiffer with treatment. Once the yield stress is exceeded the compressibility in the virgin compression range is found not to vary significantly with cement content. Also associated with the addition of cement is an increase in the hydraulic conductivity (e.g. with 50\% PC, the hydraulic conductivity at a given stress level increased by more than two orders of magnitude with respect to the reconstituted soil). The coefficient of 
consolidation of PC treated LR soil was also found to increase with addition of Portland cement (e.g. for 50\% PC, the results indicate that consolidation takes place 10 times faster than in the reconstituted LR soil). Phase relation calculations for both untreated and treated soil indicate that the addition of cement leads to an increase in the void ratio of the material at a given stress level.

\section{Implementation}

The research performed has highlighted the importance of carefully characterizing/classifying organic soils; has suggested methods for evaluating the consolidation and creep behavior of highly organic soils both in their untreated state and following treatment with a cementitious binder; has provided insight into the effects of deep mixing on the engineering properties of a highly organic soil; and has provided recommendations for field implementation of the findings based on depth of the organic soil and the percentage of organic matter in the soil. While the work has focused primarily on one soil, the findings have broader application. As demonstrated by a sample calculation included in the report, in which deep mixing was compared to construction using preloading and/or sand drains, with PC\% equal to $50 \%$ not only was the
Finally, the creep tests showed that the addition of cement causes a reduction in the creep coefficient of secondary compression (creep) at any given stress level. Moreover, the $\mathrm{C}_{\alpha} / \mathrm{C}_{\mathrm{c}}$ ratio decreases markedly with cement addition indicating a decreased susceptibility of the soil to creep. All the effects described are more marked with increasing cement content and the treatment appear especially effective once the PC \% is 50\% $\left(\sim 160 \mathrm{~kg} / \mathrm{m}^{3}\right)$ or greater.

\section{Contacts}

For more information:

Prof. Antonio Bobet

Principal Investigator

School of Civil Engineering

Purdue University

West Lafayette IN 47907

Phone: (765) 494-5033

Fax: (765) 496-1364

E-mail: bobet@ecn.purdue.edu

\section{Prof. Maria Caterina Santagata}

Co-Principal Investigator

School of Civil Engineering

Purdue University

West Lafayette IN 47907

Phone: (765) 494-0697

Fax: (765) 496-1364

E-mail: $\underline{\text { mks@ecn.purdue.edu }}$ predicted final consolidation settlement reduced significantly due to the high preconsolidation pressure developed, but it occurred at a much faster rate, therefore expediting the construction process. In addition creep deformation were also greatly reduced. As a result, the study suggests that deep mixing may be an attractive solution for construction on soft organic soils when the soil deposit is deeper than about $10 \mathrm{ft}$. An Implementation Project is recommended to investigate actual field execution of the method and behavior of the treated soil. Such Implementation Project will be useful to develop recommendations, specifications, and guidelines for design, construction, and quality control of deep organic soil deposits treated with Portland cement.

\author{
Indiana Department of Transportation \\ Division of Research \\ 1205 Montgomery Street \\ P.O. Box 2279 \\ West Lafayette, IN 47906 \\ Phone: (765) 463-1521 \\ Fax: (765) 497-1665 \\ Purdue University \\ Joint Transportation Research Program \\ School of Civil Engineering \\ West Lafayette, IN 47907-1284 \\ Phone: (765) 494-9310 \\ Fax: (765) 496-7996 \\ E-mail: jtrp@ecn.purdue.edu \\ http://www.purdue.edu/jtrp
}


TABLE OF CONTENTS

page

TABLE OF CONTENTS.......................................................................................... ii

LIST OF TABLES............................................................................................ vi

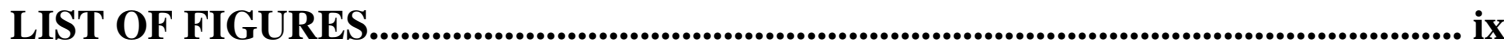

IMPLEMENTATION REPORT ..................................................................................... xvii

CHAPTER 1: INTRODUCTION................................................................. 1

CHAPTER 2: BACKGROUND................................................................................ 4

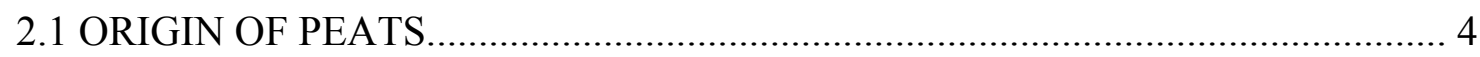

2.2 CLASSIFICATION AND INDEX PROPERTIES............................................ 5

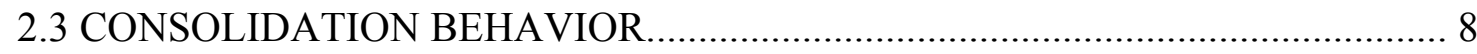

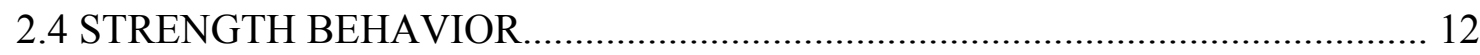

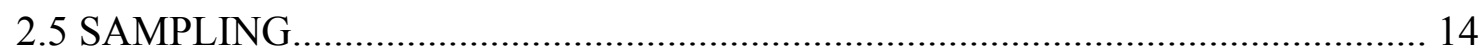

2.6 IMPROVEMENT OF ORGANIC SOILS................................................... 15

CHAPTER 3: LINDBERG ROAD SOIL_......................................................... 46

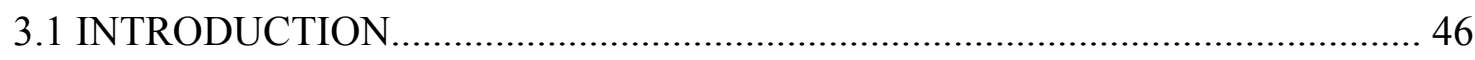

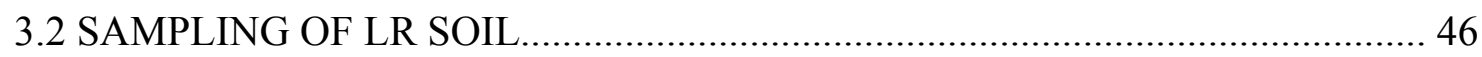

3.3 CHARACTERIZATION AND CLASSIFICATION OF LR SOIL...................... 49

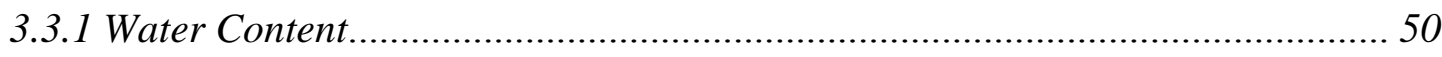

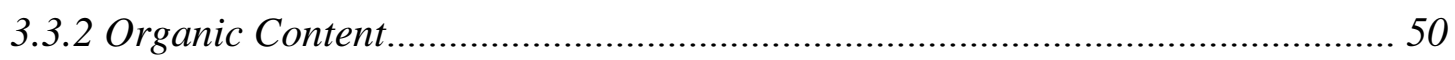

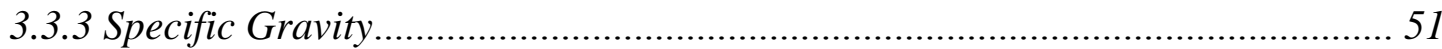

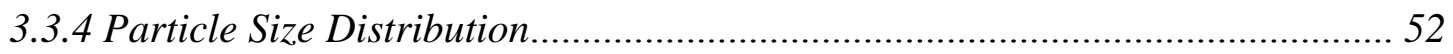

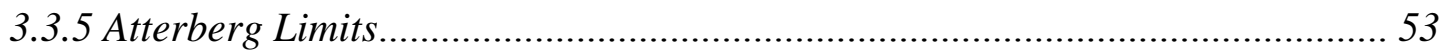

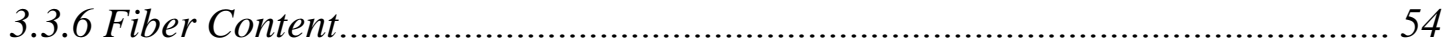

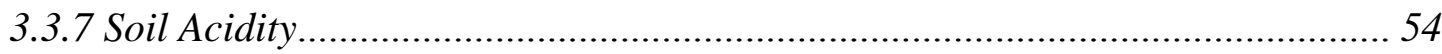

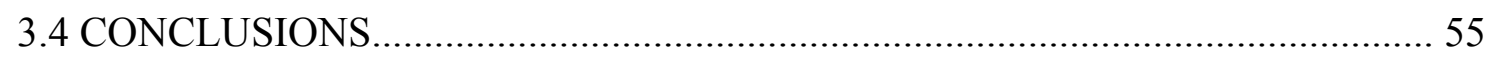

CHAPTER 4: EXPERIMENTAL METHODS...................................................... 69

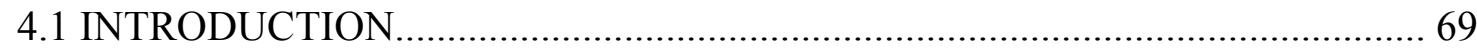

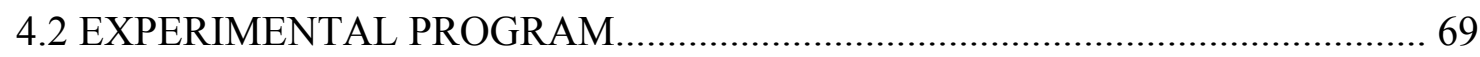

4.3 LABORATORY SAMPLE PREPARATION PROCEDURE.............................. 71 


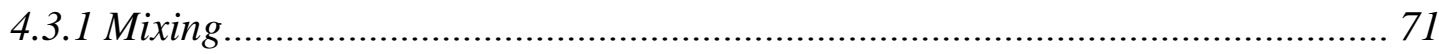

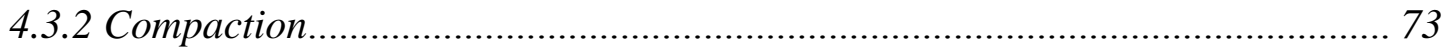

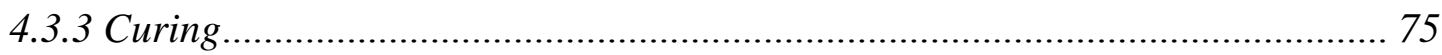

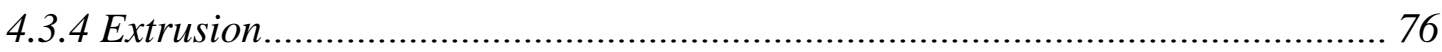

4.4 UNCONFINED COMPRESSION TEST........................................................ 76

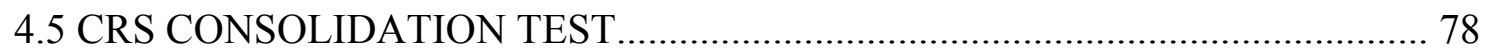

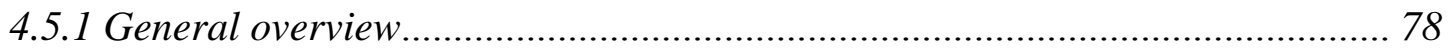

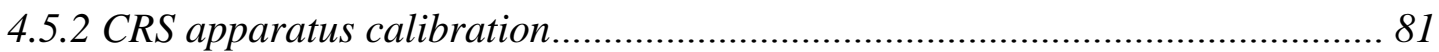

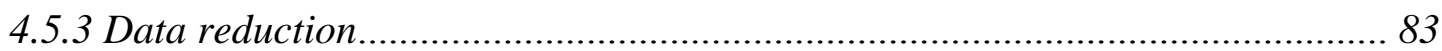

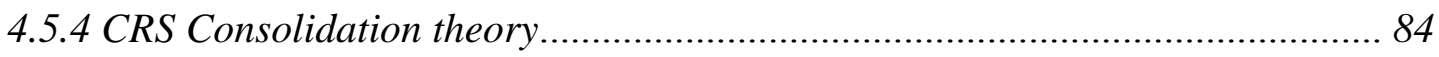

CHAPTER 5: STRENGTH: UNCONFINED COMPRESSION TESTS.................. 114

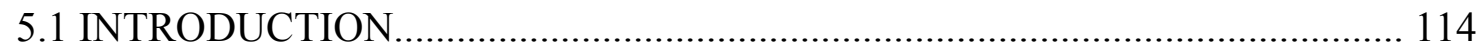

5.2 UNCONFINED COMPRESSIVE STRENGTH OF TREATED SOILS WITH

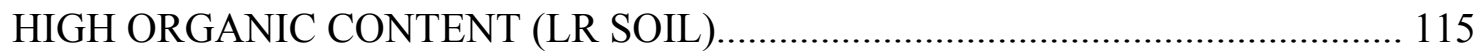

5.2.1 Untreated and Reconstituted LR Soil........................................................ 115

5.2.2 Effect of curing time and surcharge on LR soil treated with 8\% PC............. 117

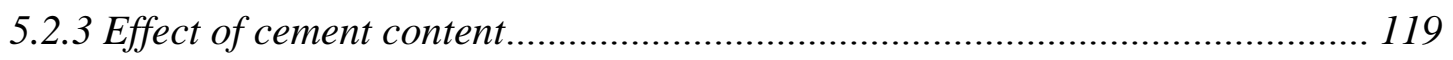

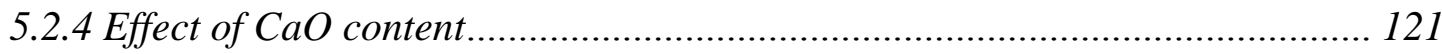

5.2.5 Effect of $\mathrm{CaO}$ content on cement treated soil................................................ 122

5.2.6 Effect of High Calcium Flue Dust (HC), Marblehead Buffington Dust (MB)

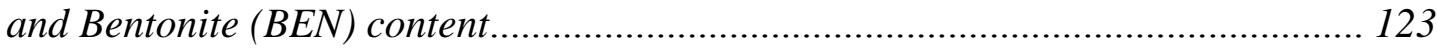

5.3 UNCONFINED COMPRESSIVE STRENGTH OF CEMENT TREATED SOILS

WITH LOW ORGANIC CONTENTS............................................................... 124

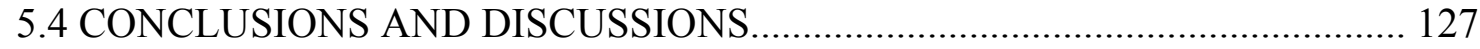

CHAPTER 6: STIFFNESS: CRS CONSOLIDATION TESTS................................... 165

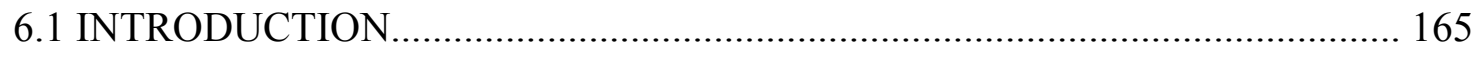

6.2 1-D CONSOLIDATION BEHAVIOR OF RECONSTITUTED LR SOIL........... 166

6.2.1 Preconsolidation Pressure and Compressibility of Reconstituted LR soil...... 166

6.2.2 Constrained Modulus $(D=1 / \mathrm{mv})$................................................................. 169

6.2.3 Generation of Excess Pore Pressure............................................................. 170 


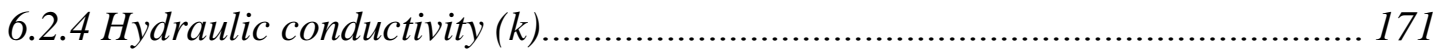

6.2.5 Coefficient of Consolidation (Cv).............................................................. 172

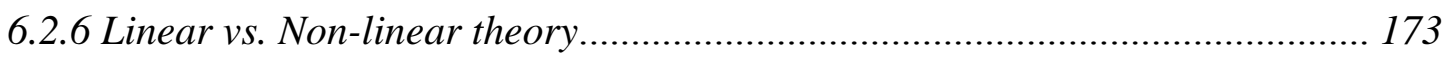

6.3 STRAIN RATE SELECTION AND RATE EFFECTS...................................... 175

6.3.1 Strain rate effect on the preconsolidation pressure and the compressibility... 176

6.3.2 Strain rate effect on the generation of the excess pore pressure...................... 177

6.3.3 Strain rate effect on the hydraulic conductivity and the coefficient of

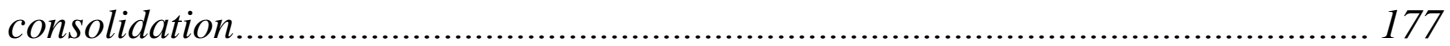

6.4 EOP-IL CONSOLIDATION TEST ON RECONSTITUTED LR SOIL.............. 178

6.4.1 Determination of end-of-primary (EOP) point............................................. 179

6.4.2 Results of EOP-IL consolidation tests performed on reconstituted LR soil.... 180

6.4.3 Comparison with CRS consolidation tests data............................................... 181

6.5 EFFECTS OF TREATMENT WITH PORTLAND CEMENT............................ 183

6.5.1 Effects of treatment with 8.0\% PC.............................................................. 183

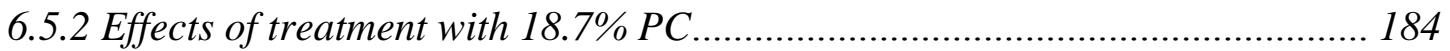

6.5.3 Effects of treatment with 51.4\% PC.......................................................... 185

6.5.4 Effects of treatment with 103.4\% PC............................................................... 186

6.6 SUMMARY OF THE EFFECTS OF TREATMENT ON 1-D

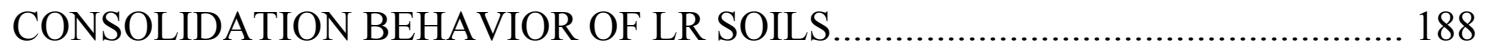

6.6.1 Effects of treatment with PC on the preconsolidation pressure and the

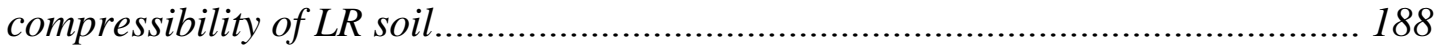

6.6.2 Effects of treatment with PC on the hydraulic conductivity and the

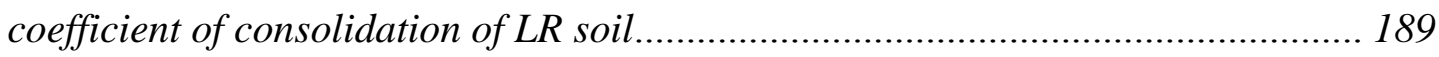

CHAPTER 7: SECONDARY COMPRESSION: CREEP TESTS............................. 223

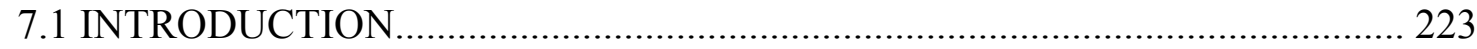

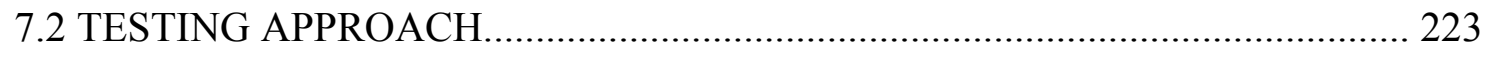

7.3 CREEP BEHAVIOR OF RECONSTIUTUED LR SOIL.................................... 224

7.4 EFFECTS OF TREATMENT WITH PORTLAND CEMENT........................... 228

7.4.1 Effects of treatment with 8.0\% PC................................................................ 228

7.4.2 Effects of treatment with 18.7\% PC............................................................... 229 
7.4.3 Effects of treatment with 51.4\% PC............................................................. 230

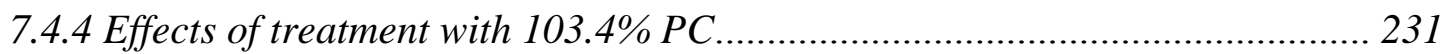

7.5 SUMMARY OF THE EFFECTS OF TREATMENT ON CREEP BEHAVIOR

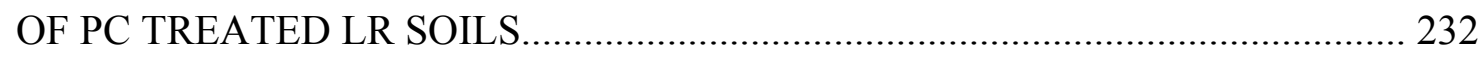

CHAPTER 8: CONCLUSIONS AND RECOMMENDATIONS............................... 247

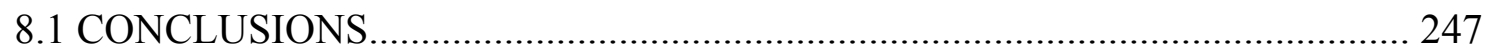

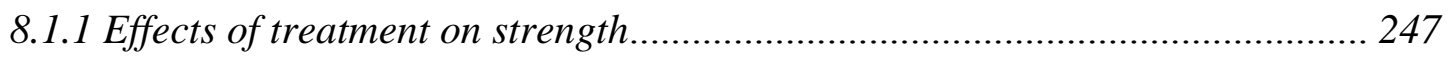

8.1.2 Effects of treatment on stiffness and creep behavior...................................... 249

8.2 COMPARISON TO OTHER IMPROVEMENT METHODS AND

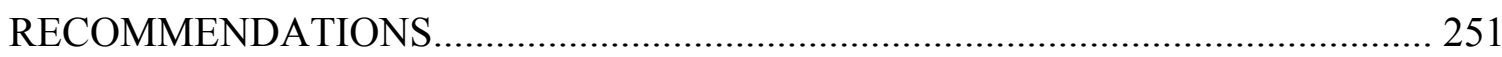

8.2.1 Construction of embankment in 1 stage (similar results apply for

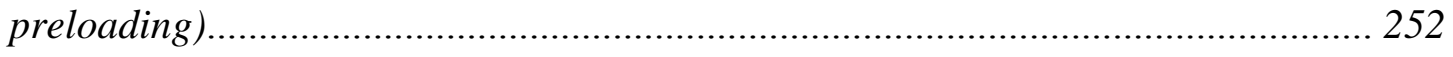

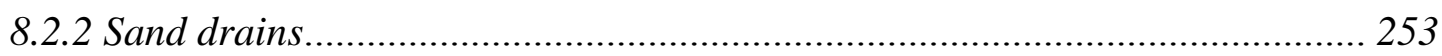

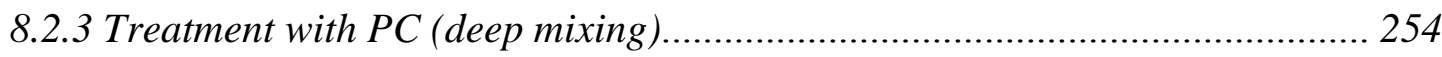

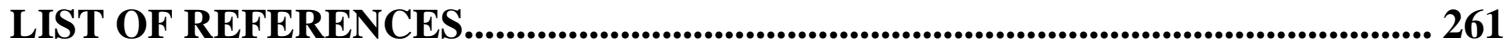




\section{LIST OF TABLES}

page

Table 2.1: Classification of peat according to Radforth System (Radforth, 1969).......... 21

Table 2.2: Classification of peat according to Von Post System (After Landva and

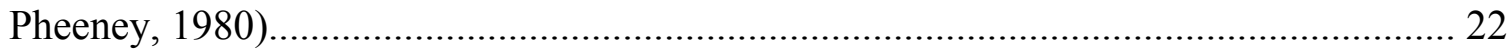

Table 2.3: Classification of peat according to ASTM System....................................... 24

Table 2.4: Range of values encountered from term "peat".......................................... 24

Table 2.5: Complete database of typical values for soils described as "peat"................. 25

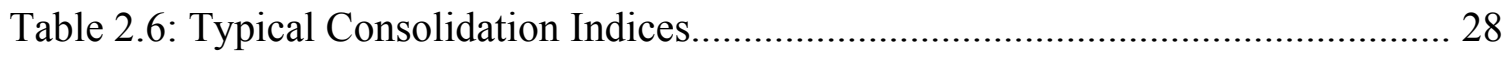

Table 3.1: Summary of index properties and unit weights of peats and organic soils (Edil

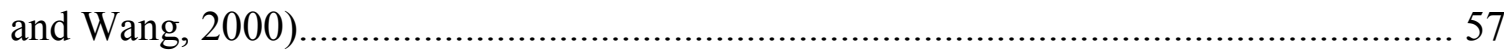

Table 3.2: Average water content of LR soil taken from three sampling trips................ 57

Table 3.3: Average water content (Wn) and organic content (OC) of LR soil................ 58

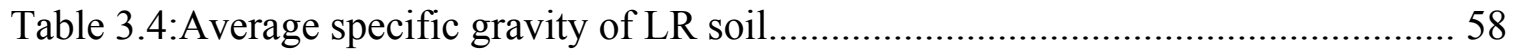

Table 3.5: The Atterberg Limits of LR soil (from II-14).......................................... 59

Table 3.6: Results from $\mathrm{pH}$ testing of LR soil......................................................... 59

Table 4.1: Summary of the experimental program carried out in the research................ 91

Table 4.2: Weights of cements per unit volume of treated and untreated soil................ 92

Table 4.3: Information of the Sensors used in CRS1 system....................................... 93

Table 4.4: Information of the Sensors used in CRS2 system................................... 93

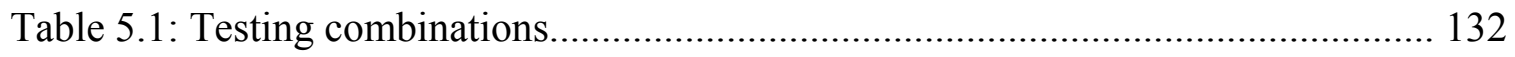

Table 5.2: Summary table of UC Tests on PC and $\mathrm{CaO}$ treated LR soil...................... 133

Table 5.3: Summary table of UC tests on PC treated Mixture I and II, and LR soil..... 136

Table 6.1: CRS tests performed on reconstituted LR soil........................................ 191

Table 6.2: Summary of the results from CRS tests performed on reconstituted LR

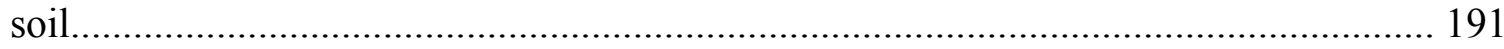

Table 6.3: CRS tests performed to evaluate the strain rate effect............................... 191

Table 6.4: Summary of the results from CRS tests performed to evaluate the strain rate effect. 
Table 6.5: Summary of reconstituted LR soil specimen data used for CRS and IL consolidation tests. 192

Table 6.6: Summary of data from EOP-IL consolidation test performed on reconstituted LR soil (CRS037)...... 193

Table 6.7: Summary of results from CRS and IL consolidation tests performed on reconstituted LR soil.

Table 6.8: Summary of PC treated LR soil specimen data used for CRS and IL

Consolidation tests..

Table 6.9: CRS tests performed to evaluate the effects of treatment with PC.

Table 6.10: Summary of data from EOP-IL consolidation test performed on $8 \%$ PC

treated LR soil (CRS048)

Table 6.11: Summary of data from EOP-IL consolidation test performed on $18.7 \%$ PC treated LR soil (CRS042)

Table 6.12: Summary of data from EOP-IL consolidation test performed on $51.4 \%$ PC treated LR soil (CRS050) 196

Table 6.13: Summary of data from EOP-IL consolidation test performed on $103.4 \%$ PC treated LR soil (CRS054). 196

Table 6.14: Summary of the results from CRS and IL consolidation tests performed to evaluate the effects of treatment with PC

Table 7.1: Summary of soil specimen data used in EOP-IL consolidation tests. 234 Table 7.2: Summary of data from EOP-IL consolidation test performed on reconstituted LR soil (CRS037)...... 234

Table 7.3: Values of $\mathrm{C} \alpha / \mathrm{Cc}$ for various geotechnical materials (Terzaghi et al, 1996).. 235 Table 7.4: Values of natural water content (wo), and $\mathrm{C} \alpha / \mathrm{Cc}$ for peat deposits (Mesri et al., 1997). 235

Table 7.5: Summary of data from EOP-IL consolidation test performed on $8 \%$ PC treated LR soil (CRS048)...... 236

Table 7.6: Summary of data from EOP-IL consolidation test performed on $18.7 \%$ PC treated LR soil (CRS042). 236 
Table 7.7: Summary of data from EOP-IL consolidation test performed on 51.4\% PC

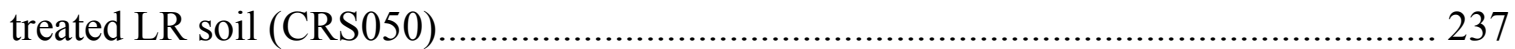

Table 7.8: Summary of data from EOP-IL consolidation test performed on 103.4\% PC

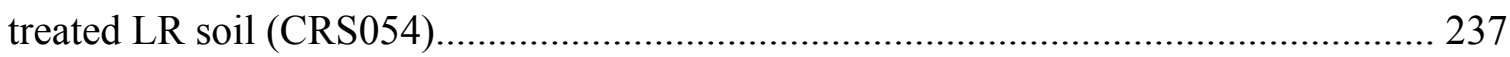

Table 7.9: Summary of the secondary compression index and the ratio of $\mathrm{C} \alpha / \mathrm{Cc}$ for untreated (reconstituted) and PC treated LR soils.................................................. 238

Table 8.1: Comparisons of settlement due to primary consolidation, time for $95 \%$

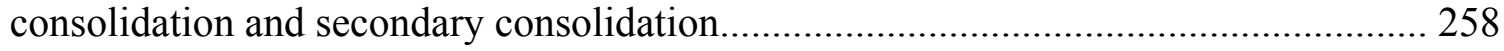




\section{LIST OF FIGURES}

page

Figure 2.1: Strain versus time plot for consolidation of Portage Peat at $\sigma=25 \mathrm{kPa}$

(Dhowian and Edil, 1981). 29

Figure 2.2: Strain versus time plot for consolidation of Portage Peat at $\sigma=200 \mathrm{kPa}$ (Dhowian and Edil, 1981)..... 30

Figure 2.3: Compression curves for consolidation tests on multiple peats (Dhowian and Edil, 1981) 31

Figure 2.4: Effective friction angle ( $\left.\varphi^{\prime}\right)$ versus Loss of Ignition (LOI) (Edil and Wang, 2000) 32

Figure 2.5: Ko versus Loss of Ignition (LOI) (Edil and Wang, 2000).......................... 33

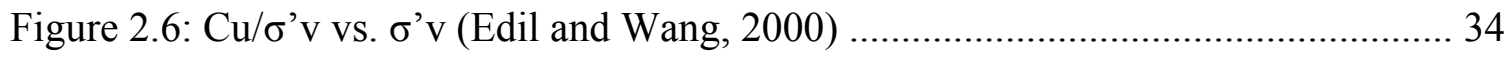

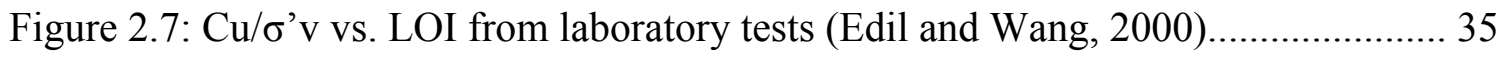

Figure 2.8: Cu/ $\sigma^{\prime} v$ vs. LOI from field vane tests (Edil and Wang, 2000)...................... 36

Figure 2.9: Schematic of $100 \mathrm{~mm}$ piston sampler (Landva et al., 1983)........................ 37

Figure 2.10: Photograph of $100 \mathrm{~mm}$ piston sampler............................................... 38

Figure 2.11: Diagram of 250-mm-square block sampler (Landva et al. 1983)............... 39

Figure 2.12: Photograph of 250-mm-square block sampler (Landva et al. 1983)........... 40

Figure 2.13: Lougheed Highway at Maillardville (Saski, 1982)................................. 41

Figure 2.14: Experimental curves of the change of water content and shear strength of

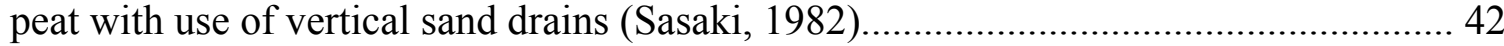

Figure 2.15: Effects of humic acid on the strength of clay samples stabilized with $\mathrm{CaO}$ $(8 \%)$ and ordinary Portland cement (8\%) as binding agents (Kujala et al. 1996).....

Figure 2.16: Effects of humic acid on the strength of samples of coarse-grained materials stabilized with ordinary Portland cement (8\%) (Kujala et al. 1996) 44 Figure 2.17: Shear strength of stabilized peat as determined by column drilling and vane shear tests after intervals of 30 days and one year. 45 Figure 3.1: Location of Lindberg Road in West Lafayette, Tippecanoe County, Indiana (After Earth Exploration, Inc., 1996). 
Figure 3.2: Soil boring location plan and profile along Lindberg Road (Earth Exploration,

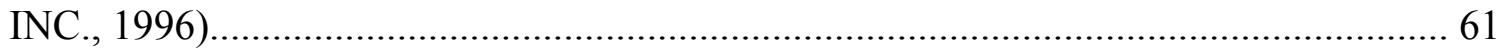

Figure 3.3: Sampling activities during April 2001................................................ 62

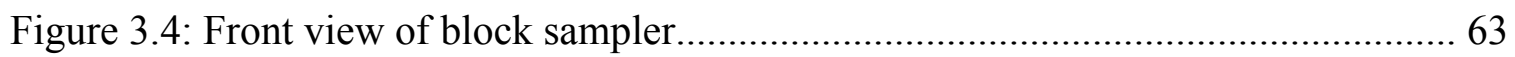

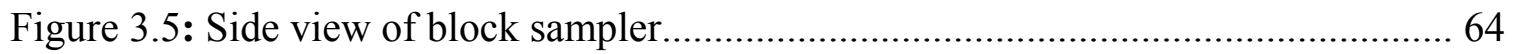

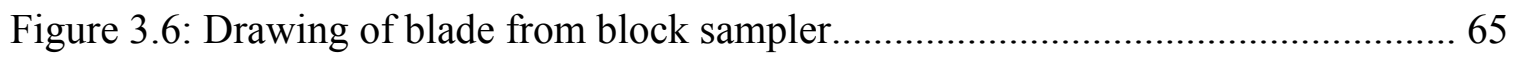

Figure 3.7: A $30 \mathrm{~cm}$ x $30 \mathrm{~cm}$ x $30 \mathrm{~cm}$ block sampler made out of Lexan plates............. 66

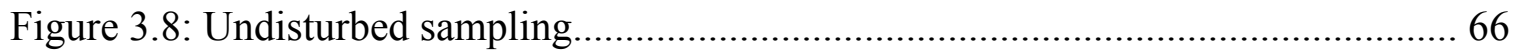

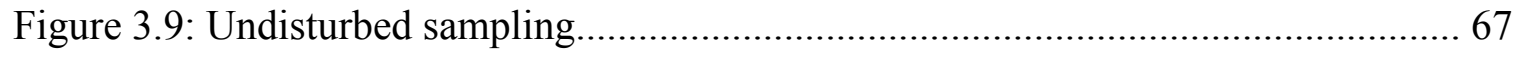

Figure 3.10: Undisturbed block sample sealed with plastic wrap and wax.................... 67

Figure 3.11: Particle size distribution of LR soil obtained from combined analysis........ 68

Figure 4.1: Mixer with two different mixing tools employed to prepare samples for CRS

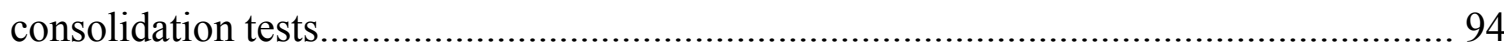

Figure 4.2: Modified mechanical proctor compactor employed for treated LR soil

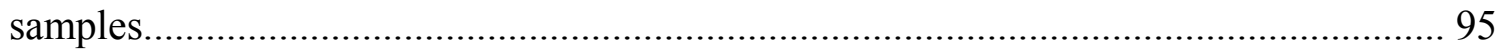

Figure 4.3: Original $(\mathrm{D}=5.08 \mathrm{~cm}$, above $)$ and modified compaction hammer $(\mathrm{D}=2.54$

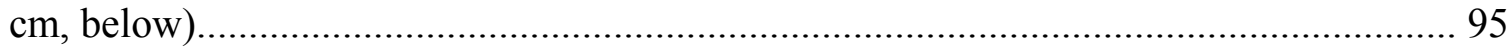

Figure 4.4: A plastic cylinder and modified compaction mold employed for compaction.

Figure 4.5: Stress control loading frame employed for preparation of treated MI and MII samples. 97

Figure 4.6: Cutting of plastic cylinder with Wells metal band saw for preparation of soil

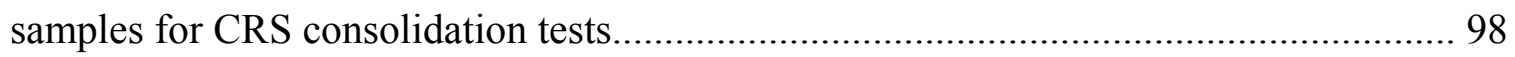

Figure 4.7: Curing of soil specimen in water bath under a surcharge of $48 \mathrm{kPa}$............. 99

Figure 4.8: Specimen curing in consolidation frame under a surcharge of $96 \mathrm{kPa}$.......... 99

Figure 4.9: Unconfined compression test apparatus............................................ 100

Figure 4.10: Schematic of four components of CRS consolidation test apparatus; CRS Cell, Load Frame, Air-Water Interface Pressure Regulator and Data Acquisition System 
Figure 4.11: Specimen trimming apparatus: trimming frame, a thin spatula, cutting blade and a wire saw. 102

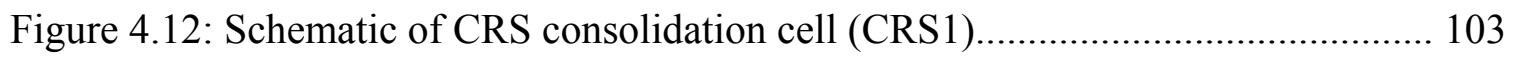

Figure 4.13: Resolution of the Data Acquisition System and sensors......................... 104

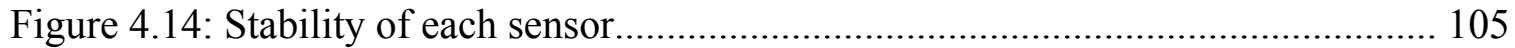

Figure 4.15: Uplift calibration for CRS cell (The area subject to cell or backpressure is

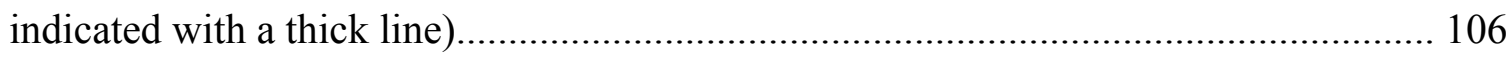

Figure 4.16: Uplift calibration curves for (a) CRS1 and (b) CRS2 cells (The piston areas

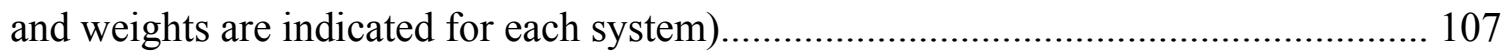

Figure 4.17: Machine Deflection (Compliance) of (a) CRS1 and (b) CRS2 ................ 108

Figure 4.18: Flow chart for data reduction............................................................... 109

Figure 4.19: Deviation of strain as function of death for different time factor (From Wissa

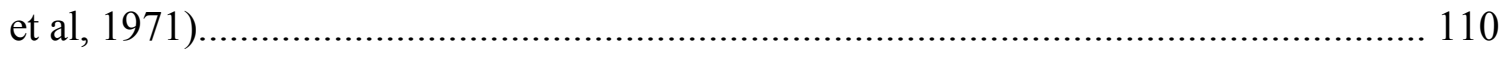

Figure 4.20: Plot of the function F3 as a function of Tv ...................................... 111

Figure 4.21: Comparison of vertical effective stress computed using linear and non-linear

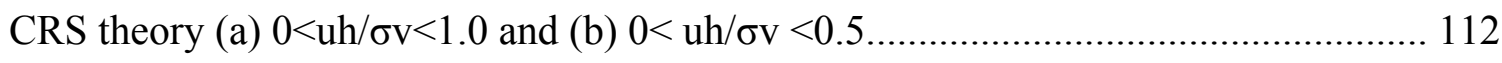

Figure 4.22: Comparison of coefficient of consolidation computed using linear and

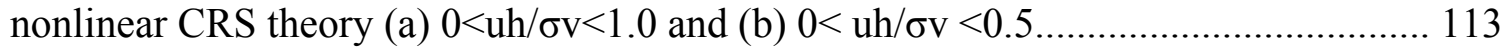

Figure 5.1: Typical uncorrected Unconfined Compression test results....................... 139

Figure 5.2: Typical corrected Unconfined Compression test results............................ 139

Figure 5.3: Stress vs. strain curve showing peak and non-peak behavior, and parameter selection

Figure 5.4: Stress-strain plot from UC test on $0 \%$ PC mixture surcharged at $14.5 \mathrm{kPa} .140$ Figure 5.5: Stress-strain plot from UC test on 0\% PC mixture surcharged at $48 \mathrm{kPa} . . .141$ Figure 5.6: Stress-strain plot from UC test on $0 \% \mathrm{PC}$ mixture surcharged at $96 \mathrm{kPa}$.... 141 Figure 5.7: Effect of surcharge on strength of LR soil without binder......................... 142 Figure 5.8: Effect of surcharge on stiffness of LR soil without binder........................ 142 Figure 5.9: Effect of surcharge on strain at failure of LR soil without binder.............. 143 Figure 5.10: Stress-strain plot from UC test on 8\% PC mixture surcharged at $14.5 \mathrm{kPa} 143$ Figure 5.11: Stress-strain plot from UC test on 8\% PC mixture surcharged at $48 \mathrm{kPa} . .144$ 
Figure 5.12: Stress-strain plot from UC test on 8\% PC mixture surcharged at $96 \mathrm{kPa} . .144$

Figure 5.13: Effect of surcharge on strength of LR soil with 8\% PC.......................... 145

Figure 5.14: Effect of surcharge on stiffness of LR soil with 8\% PC........................ 145

Figure 5.15: Effect of surcharge on strain at failure of LR soil with 8\% PC................ 146

Figure 5.16: Compressive strength of $8 \%$ PC LR soil normalized by compressive strength of $0 \%$ PC LR soil for same surcharge and curing period........................................ 146

Figure 5.17: Various \%

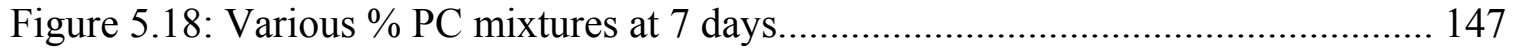

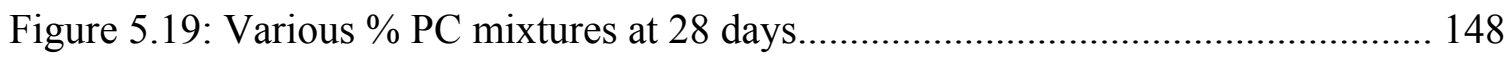

Figure 5.20: Effect of \% $\mathrm{PC}$ on compressive strength............................................. 148

Figure 5.21: Effect of \% PC on LR soil stiffness...................................................... 149

Figure 5.22: Effect of \% $\%$ PC on strain at failure...................................................... 149

Figure 5.23: Stress-strain plot from UC tests with $\mathrm{CaO}$ mixture surcharged at $48 \mathrm{kPa} .150$

Figure 5.24: Effect of $\mathrm{CaO}$ on strength of LR soil surcharged at $48 \mathrm{kPa}$..................... 150

Figure 5.25: Effect of $\mathrm{CaO}$ on stiffness of LR soil surcharged at $48 \mathrm{kPa}$.................... 151

Figure 5.26: Effect of $\mathrm{CaO}$ on stiffness of LR soil surcharged at $48 \mathrm{kPa}$..................... 151

Figure 5.27: Comparison of $\mathrm{CaO}$ at 7 days against $\mathrm{PC}$ at 7 and 28 days.................... 152

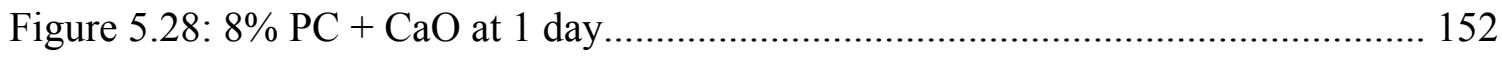

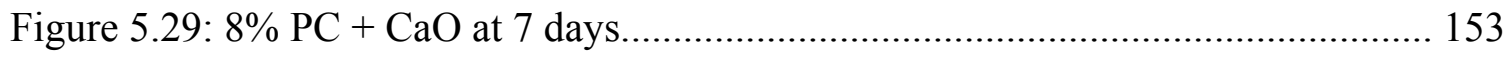

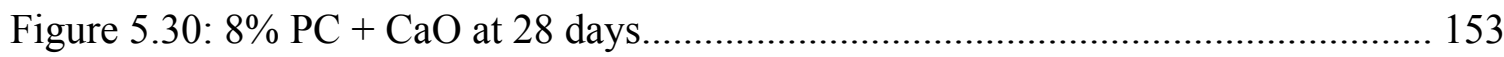

Figure 5.31: Effect of $\mathrm{CaO}$ on compressive strength of cement treated LR soil............ 154

Figure 5.32: Effect of $\mathrm{CaO}$ on stiffness of cement treated LR soil.............................. 154

Figure 5.33: Effect of $\mathrm{CaO}$ on failure strain of cement treated LR soil........................ 155

Figure 5.34: Stress-strain plot from UC tests on HC treated LR soil......................... 155

Figure 5.35: Effect of \% $\mathrm{HC}$ on compressive strength............................................ 156

Figure 5.36: Partially hydrated High Calcium Flue Dust employed in the UC tests...... 156

Figure 5.37: Stress-strain plot from UC tests on MB treated LR soil.......................... 157

Figure 5.38: Effect of \%

Figure 5.39: Comparison of effects of $\mathrm{CaO}, \mathrm{HC}$ and $\mathrm{MB}$ on compressive strength...... 158

Figure 5.40: Stress-strain plot from UC tests on bentonite treated LR soil.................. 158 
Figure 5.41: Effect of \%BEN on compressive strength

Figure 5.42:Effects of curing time on the compressive strength of PC treated Low organic soil (Mixture I)

Figure 5.43: Effects of \% PC on the compressive strength of Low organic soil (Mixture I) 160

Figure 5.44: Effect of curing surcharge on compressive strength of untreated Mixture I

Figure 5.45: Effect of curing time on compressive strength of untreated Mixture I...... 161

Figure 5.46: Effect of curing surcharge on compressive strength of $8 \%$ PC treated Mixture I.

Figure 5.47: Effect of curing time on compressive strength of 8\% PC treated Mixture I

Figure 5.48: Effect of curing time on compressive strength of untreated and $8 \%$ treated

Mixture I and II. 162

Figure 5.49: Comparison of compressive strength of 50\% PC LR soil and 20\% PC Mixture II 163

Figure 5.50: Comparison of normalized undrained shear strengths for $8 \% \mathrm{PC}$ and $0 \% \mathrm{PC}$

LR soil at 28 days. 163

Figure 5.51: Comparison of strength for cement and/or lime treated LR soil..... 164

Figure 6.1: Compression curves from three CRS consolidation tests performed on reconstituted LR soil. 198

Figure 6.2: Determination of preconsolidation pressure with the strain energy method (CRS006) 198

Figure 6.3: Change of the constrained modulus $(\mathrm{D}=1 / \mathrm{mv})$ during loading. 199

Figure 6.4: Generation of excess pore pressure $(\Delta \mathrm{uh})$ during loading and reloading..... 199

Figure 6.5: Plot of the pore pressure ratio vs. vertical effective stress........................ 200

Figure 6.6: Plot of the hydraulic conductivity (k) vs. axial strain.............................. 200

Figure 6.7: Plot of the hydraulic conductivity (k) vs. vertical effective stress.............. 201

Figure 6.8: Coefficient of consolidation $(\mathrm{Cv})$ as a function of the vertical effective stress:

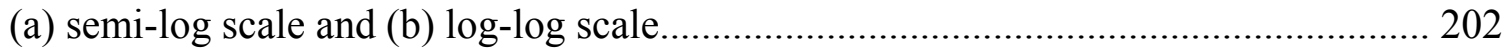


Figure 6.9: Comparison of linear vs. non-linear theory (CRS006): (a) compression curve, (b) the constrained modulus, (c) the hydraulic conductivity and (d) the coefficient of consolidation. 204

Figure 6.10: Increase of the actual strain rate during CRS consolidation test (CRS006)205

Figure 6.11: Strain rate effect on the compression curve........................................ 205

Figure 6.12: Strain rate effect on generation of excess pore pressure ......................... 206

Figure 6.13: Normalization of excess pore pressure with actual strain rate................. 206

Figure 6.14: Strain rate effect on pore pressure ratio................................................. 207

Figure 6.15: Strain rate effect of the hydraulic conductivity..................................... 207

Figure 6.16: Strain rate effect of the coefficient of consolidation................................ 208

Figure 6.17: Determination of End-of-primary point based on measurements of excess

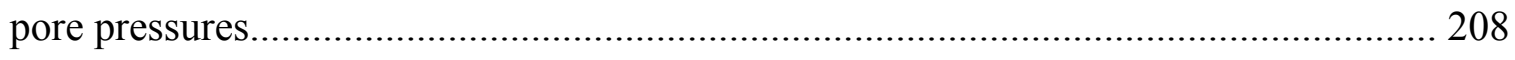

Figure 6.18: EOP compression curve of reconstituted LR soil (CRS037)................... 209

Figure 6.19: Determination of preconsolidation pressure of reconstituted LR soil

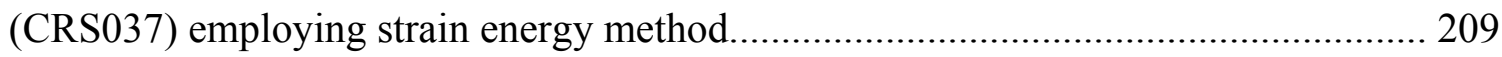

Figure 6.20: Coefficient of consolidation of reconstituted LR soil (CRS037).............. 210

Figure 6.21: Hydraulic conductivity of reconstituted LR soil (CRS037)..................... 210

Figure 6.22: Comparison of EOP compression curves of reconstituted LR soil obtained

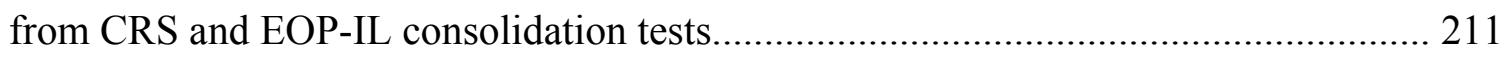

Figure 6.23: Comparison of hydraulic conductivity of reconstituted LR soil obtained from

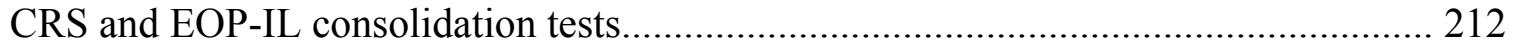

Figure 6.24: Comparison of coefficient of consolidation of reconstituted LR soil obtained from CRS and EOP-IL consolidation tests.......................................................... 212

Figure 6.25: EOP compression curves of 8\% PC treated LR soil obtained from CRS and

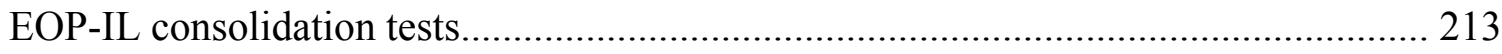

Figure 6.26: Hydraulic conductivity of $8 \%$ PC treated LR soil obtained from CRS and

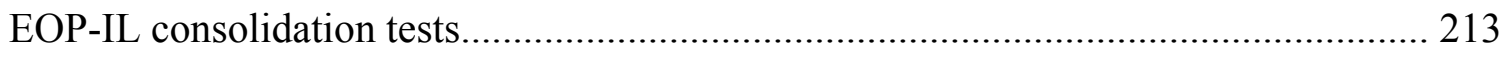

Figure 6.27: Coefficient of consolidation of 8\% PC treated LR soil obtained from CRS

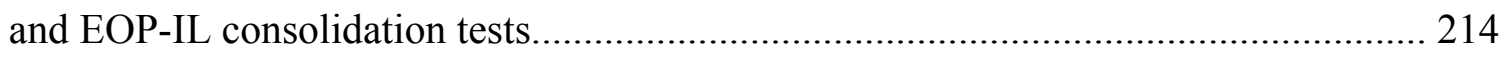

Figure 6.28: EOP compression curve of $18.7 \%$ PC treated LR soil obtained from CRS 
and EOP-IL consolidation tests......

Figure 6.29: Hydraulic conductivity of $18.7 \%$ PC treated LR soil from CRS and EOP-IL consolidation tests.

Figure 6.30: Coefficient of consolidation of 18.7\% PC treated LR soil from CRS and EOP-IL consolidation tests

Figure 6.31: EOP compression curve of 51.4\% PC treated LR soil obtained from CRS and EOP-IL consolidation tests. 216

Figure 6.32: Hydraulic conductivity of 51.4\% PC treated LR soil obtained from CRS and EOP-IL consolidation tests 216

Figure 6.33: Coefficient of consolidation of 51.4\% PC treated LR soil obtained from CRS and EOP-IL consolidation tests. 217

Figure 6.34: EOP compression curve of $103.4 \%$ PC treated LR soil obtained from CRS and EOP-IL consolidation tests.

Figure 6.35: Hydraulic conductivity of $103.4 \%$ PC treated LR soil obtained from CRS and EOP-IL consolidation tests. 218

Figure 6.36: Coefficient of consolidation of 103.4\% PC treated LR soil obtained from CRS. 218

Figure 6.37: Comparison of the compression curves of LR soil treated with different amounts of PC

Figure 6.38: Increase of the preconsolidation pressure with increase in the amount of PC 219

Figure 6.39: Effect of treatment with PC on the compression index of LR soil............ 220 Figure 6.40: Effect of treatment with PC on the recompression index of LR soil........ 220 Figure 6.41: Comparison of the hydraulic conductivity of LR soil treated with different amounts of PC. 221

Figure 6.42: Comparison of the coefficient of consolidation of untreated and PC treated LR soil. 221

Figure 6.43: Normalized vertical effective stress vs. coefficient of consolidation........ 222 Figure 7.1: EOP compression curve of reconstituted LR soil obtained form two CRS and one EOP-IL consolidation tests. 
Figure 7.2: Long term creep test for determination of secondary compression index $(\mathrm{C} \alpha)$ of reconstituted LR soil... 239

Figure 7.3: Comparison of the secondary compression index of reconstituted LR soil at different vertical effective stress levels 240

Figure 7.4: Calculation of $\mathrm{C} \alpha / \mathrm{Cc}$ for reconstituted LR soil (CRS037)..... 241

Figure 7.5: EOP compression curves of 8\% PC treated LR soil obtained from CRS and EOP-IL consolidation tests...

Figure 7.6: Long term creep test for determination of secondary compression index $(\mathrm{C} \alpha)$ of $8 \%$ PC treated LR soil

Figure 7.7: EOP compression curve of $18.7 \%$ PC treated LR soil obtained from CRS and

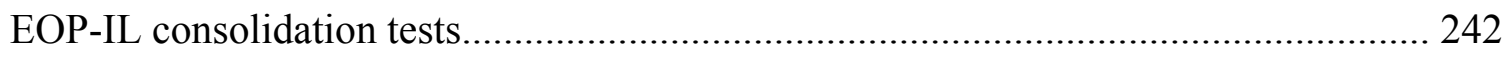
Figure 7.8: Long term creep test for determination of secondary compression index $(\mathrm{C} \alpha)$ of $18.7 \%$ PC treated LR soil. 243

Figure 7.9: EOP compression curve of 51.4\% PC treated LR soil obtained from CRS and EOP-IL consolidation tests 243

Figure 7.10: Long term creep test for determination of secondary compression index $(\mathrm{C} \alpha)$

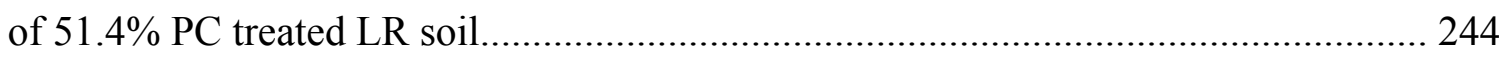
Figure 7.11: Determination of $\mathrm{C} \alpha / \mathrm{Cc}$ for $51.4 \%$ PC treated LR soil (CRS050)........... 244 Figure 7.12: EOP compression curve of $103.4 \%$ PC treated LR soil obtained from CRS and EOP-IL consolidation tests. 245

Figure 7.13: Long term creep test for determination of secondary compression index $(\mathrm{C} \alpha)$

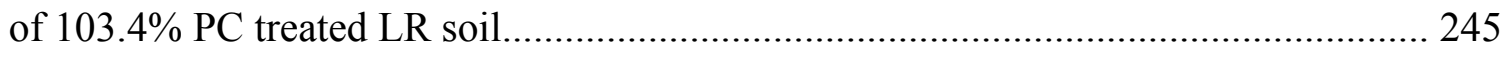

Figure 7.14: Comparison of time settlement curves from long term creep tests........... 246 Figure 7.15: Change of the ratio of $\mathrm{Ca} / \mathrm{Cc}$ for PC treated LR soil............................... 246

Figure 8.1: Soil profile under the embankment...................................................... 259

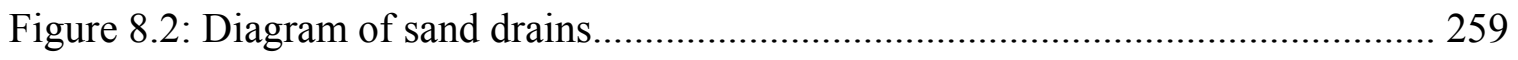

Figure 8.3: Long term behavior of a road embankment stabilized with deep mixing

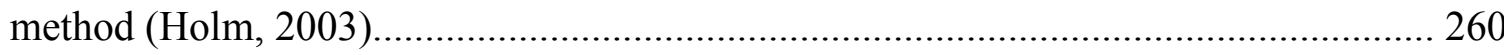

Figure 8.4: Comparison of the time-settlement curves.............................................. 260 


\section{IMPLEMENTATION REPORT}

Numerous projects have been designed and developed in areas in Indiana, and in the Mid-west in general, where poor soils such as peats or highly organic soils are encountered. The presence of peats and highly organic soils, which are characterized by low strength, high compressibility and very significant creep, increases the risk of foundation failure or inadmissible settlements. As a result, foundations, embankments, excavations, and other ground works become very difficult.

One of the most widely used methods for improving the engineering properties of soft problem soils is the Deep Mixing Method (DMM). The principle of this method is to mix in situ hardening agents (generally lime or cement) with soil. The mixing requires controlled proportions of hardening agents to produce columns of soil with higher strength and stiffness.

There have been many studies on the physical and mechanical properties of soils treated with hardening agents. Most of the research has focused on the improvement of the strength of soft inorganic clays, while, with only a few exceptions (e.g. some work performed in Sweden), there has been very limited experience with organic soils. In particular, the consolidation behavior of these soils following treatment remains for the most part unexplored.

In this research an extensive experimental program comprising characterization tests, constant rate of strain and incremental loading consolidation tests, long term creep tests, as well as unconfined compression tests was carried out. The work focused

primarily on testing one soil sampled in proximity to the Purdue campus and referred to throughout this report as Lindberg Road (LR) soil (LOI =45-52\%; LL=327\%; PI=162\%; $\mathrm{G}_{\mathrm{s}}=2.05-2.12 ; 16.1 \%$ sand, $43.3 \%$ silt, $40.6 \%$ clay; fiber content $2.29 \%$ ). On a secondary basis additional tests were carried out on soils with LOI in the 10-20\% range, manufactured in the laboratory by mixing LR soil with an illitic clay. This experimental program was complemented by an in depth literature review on the properties of organic soils. 
The research performed has highlighted the importance of carefully characterizing/classifying organic soils; has suggested methods for evaluating the consolidation and creep behavior of highly organic soils both in their untreated state and following treatment with a cementitious binder; and has provided insight into the effects of deep mixing on the engineering properties of a highly organic soil. While the work has focused primarily on one soil, the findings have broader application. The following paragraphs highlight the main conclusions derived from the study in these three areas, which have the potential to find immediate application in practice.

\section{(1) Characterization of organic soils}

An extensive review of the literature in this area has highlighted the great variability in the nature and properties of organic soils, as well as the lack of established methods and an accepted system for classifying these soils.

While characterization of organic soils beyond what is currently routinely done in practice (Atterberg limits and loss on ignition) can be quite complex, it appears that additional valuable insights may be gained from a more fundamental, soil chemistry based approach to this problem.

Testing performed as part of this research work, as well as data from the literature, highlight for example how expressing the organic content using the Loss of Ignition (LOI) can be misleading since other minerals such as calcite, kaolinite, etc. can be lost at the high temperatures employed to determine the LOI. Instead, the organic content should be measured by extracting organic matter from the soil following chemical treatment. Even more insight could be obtained by performing chemical analyses to establish the specific nature of the organic material present.

Fiber content is another very important parameter for characterization of organic soil since the shear behavior of organic soil changes dramatically depending on whether the soil is fibrous or amorphous.

For organic soils difficulties may be also encountered in evaluating properties such as specific gravity and particle size distribution. The specific gravity was found in this research to vary quite significantly $(1.93-2.12)$ as a result of fairly modest changes 
in the LOI (45-52\%). The presence of fibers is likely to further complicate evaluation of this property, possibly requiring the use of a fluid different from water. These issues all require careful consideration as a reliable evaluation of Gs is necessary for accurately calculating void ratios and compressibility parameters.

The particle size distribution of LR was obtained in this research employing wet sieving in combination with hydrometer testing. This approach is recommended for organic soils with no or very limited fibers provided that special care is exercised to keep the organic soil from drying. In the presence of fibers the hydrometer test should not be considered due to both the shape of the "particles" and their inability to settle.

\section{(2) Testing methods}

Extensive work was performed in this research to identify the optimal testing method and approach for characterizing the 1-D consolidation behavior of organic soils. In particular the limitations of the conventional 24 hour incremental loading consolidation test were highlighted. In presence of highly organic soils characterized by significant creep, separation of primary and secondary consolidation can be problematic, and as a result, conventional IL test may underestimate the preconsolidation pressure and overestimate the compressibility parameters.

Constant rate of strain (CRS) and end-of-primary incremental loading (EOP-IL) consolidation tests are proposed as reliable alternatives for evaluating the primary consolidation of organic soils.

The CRS test in particular provides a continuous compression curve and $\mathrm{C}_{\mathrm{v}}$ and $\mathrm{k}$ as a continuous function of the vertical effective stress. A well established theory is available for evaluating these properties eliminating the need for subjective constructions. In performing this test special consideration should be placed in selection of the strain rate and in evaluating the strain rate sensitivity of the soil under consideration. Back pressure saturation (to $300-600 \mathrm{kPa}$ ) is a necessary step, so that the excess pore pressures can be accurately measured to calculate the coefficient of consolidation and the hydraulic conductivity. 
The results of CRS tests were found to be consistent with those of incremental loading tests provided that the application of load increments occurred allowing negligible secondary compression (EOP-IL tests). In these tests the end of primary was best estimated based on measurements of the excess pore pressure developed at the base of the specimen. As a result these tests too require back pressure saturation.

The creep behavior of organic soil is best investigated with IL tests. Particularly in the case of organic soils it appears important to evaluate creep deformations beyond the standard 24-72 hour period (some researchers have documented an increase in the creep rate with time, e.g. Fox et al, 1992). In this research each IL test included a creep stage lasting a minimum of 30 days. In the case of treated soils such a long creep stage may also highlight changes to the soil properties associated with reactions of the binder. Note however, that in this research no change in creep behavior with time was observed.

The $C_{\alpha} / C_{c}$ concept developed by Mesri (e.g. see Mesri et al. 1997) was found to be very useful in quantifying the susceptibility to creep of the soils investigated as well as the changes produced by cement treatment. Consolidation to the pre-creep effective stress following the EOP compression curve facilitated selection of the compressibility index to be used in calculating the ratio.

\section{(3) Evaluation of the effects of treatment}

The following flowchart summarizes the testing methods employed in this testing program to evaluate the effects of treatment on the strength, stiffness and creep behavior of a highly organic soil, and the variables and results obtained from each testing method.

Among the binders employed in this testing program, based on the results of unconfined compression tests, PC was judged to be the most effective binding agent. As a result all subsequent testing focused on use of this binder. The dosages of Portland cement employed in the testing program reflect values typically used in the field and ranged between $8 \%$ (corresponding to approximately $25 \mathrm{~kg}$ per $\mathrm{m}^{3}$ of treated soil) to $100 \%\left(\sim 320 \mathrm{~kg} / \mathrm{m}^{3}\right)$. 


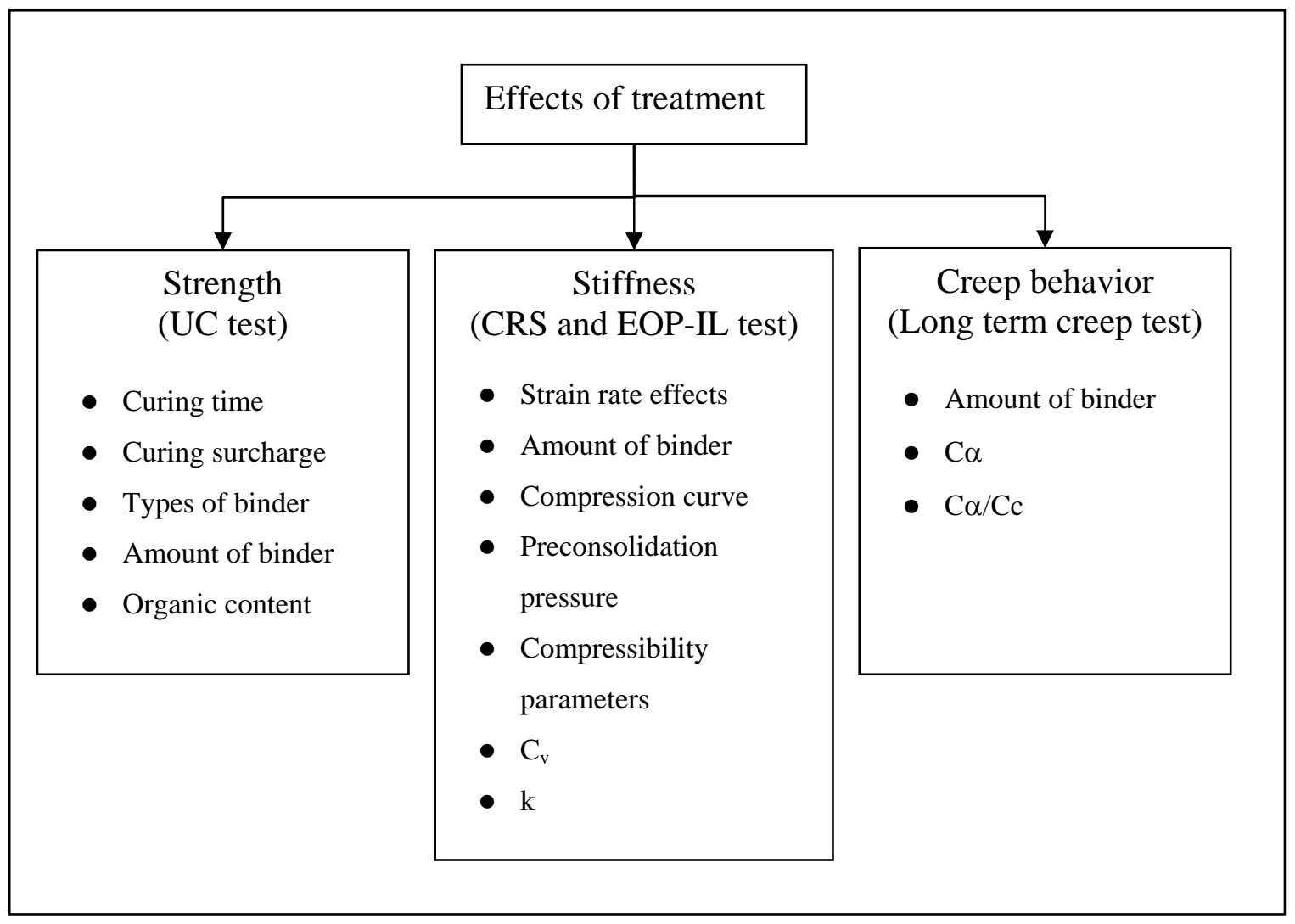

In the consolidation tests the most evident effect of treatment with PC was the increase in the preconsolidation pressure. The increase in preconsolidation pressure was observed to be fairly modest for low dosages of PC (8 - 20\%). Above 20\% PC the increase in $\sigma^{\prime}{ }_{p}$ was instead very significant: 8 times increase with 51.4\% PC and 25 times increase with $103.4 \%$ PC. Given that the compressibility of the soil in the virgin compression range is not found to be affected by the presence of cement, the development of this preconsolidation pressure is the mechanism responsible for the reduction in deformations associated with any increase in effective stresses. As a result design of a treatment should be finalized at obtaining a preconsolidation pressure that exceeds the maximum effective stress resulting from the application of the loads at the surface.

The hydraulic conductivity and coefficient of consolidation of the treated soil increase with increasing cement content. The increase in hydraulic conductivity with treatment is most likely a result of the change of the fabric of soil (flocculation and 
aggregation), which leads to an increase in the size of the macropores. The increase in coefficient of consolidation is caused by the increases in hydraulic conductivity and the stiffness. The increase in coefficient of consolidation implies that the consolidation process takes place faster in LR soil when treated. Compared to the untreated reconstituted LR soil the coefficient of consolidation increases by 1.4 times with $8.0 \%$ PC, 8.1 times with $18.7 \%$ PC, and about 38 times with $51.4 \%$ and $103.4 \%$ PC. Note that also in this case the most marked change in properties occurs when the PC\% exceeds $50 \%$.

Treatment with PC also significantly reduced creep deformations. For reconstituted LR soil the $C_{\alpha} / C_{c}$ was found to be equal to 0.103 , at the upper range of values reported for peats. With $8.0 \%$ and $18.7 \% \mathrm{PC}, \mathrm{C}_{\alpha} / \mathrm{C}_{\mathrm{c}}$ decreased to 0.085 and 0.063 , still remaining higher than the average value for peats and muskeg $(0.06 \pm 0.01)$. With $51.4 \% \mathrm{PC}, \mathrm{C}_{\alpha} / \mathrm{C}_{\mathrm{c}}$ decreased to 0.04 , falling in the range of values reported for inorganic clays and silts $(0.04 \pm 0.01)$. With $103.4 \% \mathrm{PC}, \mathrm{C}_{\alpha} / \mathrm{C}_{\mathrm{c}}$ further decreased to 0.024 falling in the range of values reported for granular soils $(0.02 \pm 0.01)$.

\section{Recommendations for field implementation}

Even though most of the effort in the research concentrated on the treatment of high organic soils with Portland cement, an extensive literature review complemented with additional tests on soils with smaller organic content and treatment with other binding agents, has led to the following recommendations for implementation of these research to sites containing organic soils:

Shallow organic soil. Organic soils that extend from the surface to about 6-10 ft deep. The solution recommended is, if possible, excavation of the problem soil and replacement with an engineered fill. For soils with small organic content, less than 15\% as a first approximation, in situ mixing of the soil with lime or cement will reduce the water content of the soil, increase its strength and decrease its deformability.

Deep organic soils. Organic soils that extend further than 6-10 ft deep. The solution recommended is deep mixing with cement. For soils with low organic content, preloading with vertical drains could also be considered. 
It must be pointed out that these recommendations are general and need to be evaluated for each case. Field and laboratory testing should be conducted to determine if a particular solution is appropriate for the case. In particular soil improvement with lime or cement requires laboratory testing with the soil at the site with different percentages of the desired binding agent. Laboratory results from this research indicate that percentages of binding agent that are too small or too large may not provide the results desired.

It is recommended that for each project a series of tests be conducted, which should include at a minimum, the following:

$$
\text { Identification tests: density, granulometry, LOI, Atterberg limits, }
$$
water content, fiber content.

CRS or IL tests with pore pressure measurement of natural soil. The tests should produce the stress-strain compression behavior of the soil, preconsolidation pressure, compressibility parameters, permeability, $\mathrm{C}_{\mathrm{v}}$, and $\mathrm{C}_{\alpha}$.

CRS or IL tests with pore pressure measurement of treated soil. The tests are conduced on soils treated with different percentages of Portland cement and consolidated at different depths. In this test series, the full stress-strain behavior of the treated soil should be obtained and should include, at a minimum, preconsolidation pressure, compressibility parameters, permeability, $\mathrm{C}_{\mathrm{v}}$, and $\mathrm{C}_{\alpha}$.

Unconfined compression tests on treated soils. The tests should yield a correlation between strength of the treated soil in uniaxial compression and percentage of added cement. The tests can then be used for quality control in the field to ascertain the amount of cement added in specimens retrieved at different depths after treatment completion.

It is recommended to conduct an Implementation Project where field conditions can be assessed and field results can be used to complement laboratory results. It is suggested to select a location where deep organic deposits exist and conduct a treatment of the soils using the Deep Mixing Method with Portland cement. The objectives of the 
study will be to develop recommendations for field implementation of the treatment. The work would include laboratory characterization of both initial and treated soils, field monitoring and testing and assessment of treatment success. The establishment of correlations between UCS (unconfined compression tests) and percentage of binder has the potential for a reliable quality control indicator. Results from the field test could then be used for the development of guidelines, specifications, and construction documents necessary for an appropriate use of the technology in Indiana. 


\section{CHAPTER 1: INTRODUCTION}

Numerous projects have been designed and developed in Indiana in area where poor soils such peats or highly organic soils are encountered. The presence of peats and highly organic soils increase the risk of foundation failure or inadmissible settlements, which derived from their low strengths, high compressibility and the significant impact of secondary consolidation. As a result, foundations, embankments, excavations, and other ground works become very difficult and often require costly treatments.

Several options are generally available to modify and improve the ground conditions: (1) strengthening of the foundation; (2) elimination of the problem soils; (3) treatment of the problem soils; (4) relocation of the project. In many cases, the only option is strengthening the foundation (e.g. deep foundation) or elimination of part of the problem soils, since the other options are impractical or too expensive. Considerable benefits can be obtained with new creative application that may achieve the needed results without the necessity of massive ground treatments. Use of materials that will change strength or stiffness when needed in only a portion of the soil volume may have the desired effects. This approach may be more cost-effective than traditional solution such as extensive soil modification.

One of the most widely used methods for the improvement of the engineering properties of problem soils is the Deep Mixing Method (DMM). The deep mixing method was developed in Sweden and Japan as a method for treatment of soft soils in late 1960's. It was introduced to the United States in late 1980's and has been widely used over the past 10 years for many applications: the works at Jackson Lake Dam, WY, Logan Airport, Boston, MA and Fort Point Channel, Boston, MA (Bruce et al, 2003). Although still very limited compared to the Europe, there has been a few cases in which the deep mixing method was successfully applied to improve the engineering properties of organic soils in the United States: stabilization of a 3.5 to $5 \mathrm{~m}$ thick organic silty clay deposit for construction of railroad embankment in a section of the Hudson-Bergen Light Rail Transit System, New Jersey (Esrig et al, 2003), and stabilization of a $2.5-7.5 \mathrm{~m}$ thick 
organic clay layer (with organic content of 4 to over 30\%) for I-95 widening, Alexandria, VA (Lambrechts et al, 2003).

The principle of the deep mixing method is to mix hardening agents (generally lime or cement) with soil in situ in controlled proportion to produce columns of hardened soil, which display higher strength and stiffness and lower hydraulic conductivity. Depending on the configuration of the deep mixing system used, the binder agents can be introduced in slurry (wet method) or dry form (dry method). The binding agents are mixed with soil at the distal end of the shaft or along the drill shaft by pure rotation of the mixing augers or by a combination of rotation of mixing tool and injection of binder in slurry form at high pressure (Bruce et al, 2003).

There have been many studies on the physical and mechanical properties of treated soil. It was found that the properties of deep mixed soils are controlled by a variety of factors: (1) physical and chemical properties of the soil and groundwater, (2) types and amounts of binders, (3) curing period and condition, and (4) mixing effectiveness. However, most of the researches were focused on the improvement of the strength of inorganic clays, and there has been very limited experience with organic soils.

The overall scope of the work presented in this report is to investigate the treatment of peats or highly organic soils through the use of binding agents including Portland cement and lime simulating the deep mixing method. The specific goals of the work are to:

- Acquire a basic understanding of the behavior of highly organic soils from the available literature.

- Investigate methods for improvement of highly organic soils from the available literature.

- Conduct laboratory experiments to characterize the behavior of a highly organic soil.

- Develop experimental procedures to simulate treatment through soil mixing in the laboratory. 
- Assess the effectiveness of treatment of a highly organic soil with two binding agents (Portland cement and calcium oxide) in improving the strength and compressibility.

This report consists of eight chapters, including this introduction

Chapter 2 presents background information on the characteristics and behaviors of highly organic soils, and an overview of the methods used in practice for improvement of the engineering properties of highly organic soils obtained from the available literature.

Chapter 3 presents the characteristics and index properties of Lindberg Road (LR) soil, which is the soil used in the entire experimental program.

Chapter 4 describes the experimental methods employed in the research to investigate the effects of treatment on the engineering properties of LR soil. The variables investigated in the experimental program are presented. The sample preparation procedure developed to produce laboratory soil samples and the experimental equipments used to perform the tests presented in the report are discussed.

In this report, the results are presented and analyzed in two separate chapters. Chapter 5 presents the results obtained from unconfined compression tests performed on reconstituted and treated soils to investigate the effects of treatment on the strength.

Chapter 6 presents the results obtained from Constant Rate of Strain (CRS) and End-of primary (EOP) Incremental Loading (IL) consolidation tests performed on reconstituted and PC treated soils to investigate the effects of treatment on the stiffness.

Chapter 7 presents the effects of treatment with PC on creep behavior of LR soil evaluated based on the results from EOP-IL and long term creep tests.

Chapter 8 presents the conclusions drawn from the analysis of the results and the recommendations. 


\section{CHAPTER 2: BACKGROUND}

The broad topic of "organic" soils provides a substantial margin of variation in the types of soils to be considered. The most important single characteristic of the soils that are studied is the organic content. Although a soil with substantial organic content has been studied, the primary focus of the literature research effort is on soil with very high organic content. These highly organic soils, also referred to as "peats" ("muskeg” in Canada and "histosols" in soil science literature), possess unique characteristics. Study of these soils helps isolate and demonstrate the influence of organic content and the engineering difficulties that it poses.

\subsection{Origin of Peats}

Peats consist primarily of decomposing plant materials with a much smaller amount of mineral deposits. Approximately ninety-five percent of all peat deposits have been formed from plants decomposing under aerobic conditions. The rate of decomposition is several thousand times faster under aerobic as opposed to anaerobic conditions, which are characterized by cool climate and surplus of water. The aerobic conditions are conducive to growth and decomposition, whereas anaerobic conditions stop surface growth and slow down subsurface decomposition. These conditions lead to complex growth and decomposition patterns of moss and fibrous sedge plants. Sedge plants are much more resistant to decomposition than moss, which leads to natural reinforcement of the weaker moss plants. This reinforcement effect is even more pronounced and the soil variability is much more significant if trees and shrubs grow in the area (Landva and Pheeney, 1980). Although this description of peat formation is quite simplistic, it provides a basic conceptual building block for the understanding of peats and organic soils. 


\subsection{Classification and Index properties}

The Unified Soil Classification System (USCS) is the most widely used standard for the classification of soils. This system consists of applying group symbols and group names for soils depending on their grain size distribution, composition, and Atterberg limits. This system takes into account organic soils through three different classifications: organic clay, organic silt, and peat. Organic clay and organic silt satisfy the requirements to be classified as a clay or silt, except that the liquid limit after oven drying is less than $75 \%$ of its liquid limit before oven drying. Peat is defined by the USCS system as: “a soil composed primarily of vegetable tissue in various stages of decomposition usually with an organic odor, a dark brown to black color, a spongy consistency, and a texture ranging from fibrous to amorphous (ASTM D2488-93).” While the classification of organic clays and silts becomes more involved depending on the grain size distribution, the classification of peat by this system is based solely on the previous definition. This large margin of uncertainty necessitates that another system be used to more specifically identify a particular peat.

Three classification systems are most often used for the description and classification of peats: Radforth, Von Post, and ASTM. The Radforth classification system is relevant only for soils with extremely high organic content (i.e. $>80 \%$ ). This system is based on the visual identification of texture and botanical composition, although no specific reference is made to moss type plant materials. It is assumed that the peat is entirely organic, although wind or water transported colloids may be present. This system provides seventeen different categories depending upon the size of the fibers present and the botanical composition (see Table 2.1). These categories are based upon general descriptions of basic form and texture information. These descriptions do not

include precise nor quantitative information on the specific orientation or strength properties of the fibers. The primary tools used in this classification system are visual identification from hand samples from the field, or microscopic photographs and x-rays of undisturbed samples. The creators note that although a peat category may include several types of peat, no specific type can belong to multiple categories (Radforth, 1969). 
Unlike the Radforth classification system which provides discreet categories, the classification of peats using the Von Post system is done through the assignment of a series of code letters depending on the type of botanical matter, degree of decomposition, water content, abundance of designated fiber sizes, and abundance of wood and shrub remnants (Table 2.2). This system provides some numerically specific indices for use in classification, specifically for the evaluation of water content and separation of fiber sizes (Landva and Pheeney, 1980). Unlike the previous classification system, the Von Post system consists of a much larger number of possibilities for classification. For example, the complete Von Post designation for a peat consisting of lightly decomposed, extremely wet sphagnum moss peat, with some sedge fibers smaller than $1 \mathrm{~mm}$ may be designated by: $\mathrm{SCH}_{3} \mathrm{~B}_{5} \mathrm{~F}_{2} \mathrm{R}_{0} \mathrm{~W}_{0}$. This same soil using the Radforth system would only require a designation of category 2 or 3.

Despite the introduction of some quantifiable properties compared to the Radforth classification, the Von Post system also utilizes visual-manual identification methods. These methods are used particularly for evaluating the degree of decomposition. The degree of decomposition, also known as degree of humification, is the most significant contribution from the Von Post system. This characteristic is coupled with fiber content in the ASTM standard for peat classification, and is also the origin for the terms "amorphous" and "fibrous" peat. An "amorphous" peat is that which is in advanced stages of decomposition and has an essentially granular texture, while a fibrous peat corresponds to relatively early stages of decomposition in which stems and plant components are still intact. These two consistencies may be compared to jelly and fiberglass matting, respectively. The more granular types of peat usually correspond to those containing large amounts of moss plants, whereas the fibrous peats would contain more sedge and "woodier" type plant materials (Landva and Pheeney, 1980).

Despite the relative merits of the previously mentioned classification systems, the most common and easiest system to use for classification of peats is that used by the American Society for Testing and Materials (ASTM). This system provides a much more standardized framework and its quantitative nature reduces the uncertainty associated with assigning a classification to a soil. The definition for peat as stated by ASTM is: "a 
naturally-occurring highly organic substance derived primarily from plant materials. Peat is distinguished from other organic soil materials by its lower ash content (less than 25\% ash by dry weight), and from other phytogenic material of higher rank (that is, lignite coal) by its lower calorific value on a water saturated basis (ASTM D 4427-92).” Further classification of peat is then approached through the quantitative characterization of fiber content (related to degree of humification), ash/organic content, acidity, absorbency, and botanical content (see Table 2.3). Although this system is not particularly detailed with respect to botanical origin, the designation of plant types is allowed for provided that a significant percentage of the peat originates from a particular plant. Despite this, the ASTM classification system seems to provide a much more concise and quantitative approach than the previously mentioned systems given the well-defined and quantitative categories.

In addition to the classification systems described above and documented in the literature, the Indiana Department of Transportation (INDOT) offers its criterion to distinguish between peats and organic soils in Section 903.05 of the INDOT Standards Specifications. This states that an "organic soil" contains between 19-30\% organic material, while a "peat" contains an amount of organic material greater than $30 \%$. This classification is noteworthy since it is derived from the AASHTO standard T 267.

A small database has been created for this project to summarize different properties of soils described as peat from the available literature. Table 2.4 summarizes the ranges encountered for certain properties, and Table 2.5 presents the entire database. From these data the following is observed: the organic content, measured by the Loss of Ignition (LOI) ranges from twenty seven percent to ninety five percent, the unit weight ranges from 8.8-12.0 $\mathrm{kN} / \mathrm{m}^{3}$, and is considerably lower than the values of $16-18 \mathrm{kN} / \mathrm{m}^{3}$ for most other soils (Lambe and Whitman, 1969). These numbers are consistent with the low specific gravity $\left(\mathrm{G}_{\mathrm{s}}\right)$ of these soils, which ranges from 1.40 to 2.68 , the upper bound similar to other soils (Lambe and Whitman, 1969). The water content ranges from 100$1400 \%$ and is not surprising given the nature of these soils. The low density and highly organic nature, coupled with the deposition below the ground water table indicates that large quantities of water are present. Similarly, the high organic content indicates that 
fibrous plant materials may be present, as illustrated by the fiber contents ranging from twenty to ninety-two percent.

Other properties such as particle size distribution and void ratio depend strongly on the field conditions. The particle size distribution depends on the fiber size and abundance as well as the surrounding geology. It is expected that a high percentage of fines may be present due to the ability of these particle sizes to be carried by water into peat deposits (Radforth, 1969). The particle size distribution coupled with the overburden stress will determine the void ratio, and thus the hydraulic conductivity. It is expected that peat found at greater depths will exhibit lower void ratios due to the consolidation process resulting from the deposition of new materials on the ground surface. All of these factors, combined with the large potential variability, make it difficult to attempt to characterize "common" particle size distribution and void ratio that may be encountered in a peat sample.

\subsection{Consolidation Behavior}

The characteristically low mineral composition, the abundance of water arising from the origin and formation of peats, and the fact that peats are generally found very near the surface causes these soils to have low density and a rather porous fabric, characterized by high in-situ void ratio and subsequently high permeability (Fox and Edil, 1996). Thus, understanding the consolidation behavior is extremely important for construction and design on these soils.

Consolidation is the process through which soil is compressed through the forced displacement of water from the pore spaces. The simplest form of the consolidation test, the oedometer test, involves a soil specimen restrained laterally and loaded axially through the application of a static force. Measurements of the elapsed time, and the deformation of the specimen constitute the primary data from the test. The data from this test can be used to estimate both the magnitude and the rate of settlement that can be expected in a field condition, and is thus a crucial piece of information for almost any geotechnical application (ASTM D2435). Despite this simplistic description, the actual test and subsequent interpretation may become quite involved and complex. 
Figure 2.1 (Dhowian and Edil, 1981) shows an example of the strain vs. log t $(\varepsilon$ vs. $\log \mathrm{t})$ curve obtained from the first load increment $(\sigma=25 \mathrm{kPa})$ of an incremental oedometer test performed on Portage Peat $\left(\mathrm{LOI}=80.5 \%, \mathrm{G}_{\mathrm{s}}=1.72, \mathrm{w}=600 \%\right)$. Dhowian and Edil identify four components of strain:

(1) Instantaneous strain $\left(\varepsilon_{\mathrm{i}}\right)$ that occurs immediately and is the result of the elastic response of the peat and the removal of air from voids.

(2) Primary strain $\left(\varepsilon_{\mathrm{p}}\right)$ that corresponds to the removal of excess pore pressure and normally lasts only a few minutes in peat. This component occurs at a relatively rapid rate and continues for several minutes to a time $t_{\mathrm{a}}$.

(3) Secondary strain $\left(\varepsilon_{s}\right)$ that is the result of creep of the soil and is time dependent. This results from a linear increase of strain with the logarithm of time for additional log cycles of time until a time $t_{k}$, after which the rate of compression increases substantially giving rise to further deformation.

(4) Tertiary strain $\left(\varepsilon_{t}\right)$ that continues indefinitely.

Figure 2.2 (Dhowian and Edil, 1981) shows a strain versus time curve for the same test at a higher load increment $(\sigma=200 \mathrm{kPa})$. In this figure, the four different strain components are not as distinguishable as is the case for the lower stress. This indicates that the primary and secondary strain components occur simultaneously.

The complete dissipation of excess pore pressure marks the end of primary (EOP) consolidation and the point at which any compression is primarily due to "creep", which is the increase of strain under constant stress. While creep occurs also during primary consolidation, the high magnitude of primary consolidation masks this behavior during the early stages of the test. The two most commonly used methods for determining the end of primary consolidation, namely the Taylor and Casagrande graphical constructions from a void ratio (e) or axial strain ( $(\varepsilon)$ versus time curve. In the case of peats and organic soils, the usual primary consolidation curve is not apparent. Subsequently, the conventional curve fitting methods described by the ASTM standards are not useful (Dhowian and Edil, 1981). As a result, the end of primary is best identified from the monitoring of pore pressure measurements during the consolidation test (Fox et al 1992). 
An example of how the excess pore pressure data is used to determine the EOP is shown in the inset of Figures 2.1 and 2.2. These graphs show the measurements of excess pore pressure with respect to time. The EOP is taken at the point where the excess pore pressure has dissipated nearly completely. Fox and Edil (1992) recommend that the dissipation of $95 \%$ of the excess pore pressure be selected as the EOP for highly organic soils.

The complications involved with the evaluation of the end of primary consolidation for peats appear to be due to the structure of the soil (Dhowian and Edil, 1981). Fibrous peats have a two-level microstructure, consisting of interconnected macropores and micro-pores. Macro-pores are the void spaces between soil particles, while micro-pores are voids within the plant fibers (Dhowian and Edil 1981). Primary consolidation for these soils is complete once the flow from the macropores has ceased. The reduction of the size of the micro and macro-pores results in decreased soil permeability. While the permeability for most peats starts out being near common values for sand (i.e. $10^{-1}-10^{-3} \mathrm{~cm} / \mathrm{s}$ (Lambe and Whitman, 1969)), it decreases nearly 10,000 fold with loading to be comparable to the permeability of clay (i.e. $10^{-6}-10^{-9} \mathrm{~cm} / \mathrm{s}$ (Lambe and Whitman, 1969). This results in increasing time until the complete generation and dissipation of excess pore pressure, and subsequently longer times until the end of primary consolidation for increased stress increments (Dhowian and Edil, 1981).

Plotting of the void ratios corresponding to the end of primary consolidation against the stress for each increment yields the compression curve. This plot illustrates the relationship between stress and deformation for a particular soil. Figure 2.3 gives the results for consolidation tests performed by Dhowian and Edil (1981), including that for the test on Portage peat, discussed earlier. The plot also shows the values of $C_{c}$ and $C_{s}$, which represent the slope of the compression curve $\left(\Delta \mathrm{e} / \Delta \log \sigma^{\prime}{ }_{\mathrm{v}}\right)$ in the normally consolidated $\left(\mathrm{C}_{\mathrm{c}}\right)$ overconsolidated $\left(\mathrm{C}_{\mathrm{s}}\right)$ range, respectively. Typical values of these indices for peats are given in Table 2.6 and compared against those for soft clays, which are some of the most highly compressible of natural soils. The high values for $C_{c}$ and $C_{s}$ 
as compared to the smaller values for soft clays indicate that both normally and overconsolidated peats undergo very large deformations as stress increases.

The large deformations experienced in highly organic soils are also investigated in a discussion by Mesri et al. (1997) through the parameter $C_{k}$. $C_{k}$ indicates the change in the void ratio of a soil with respect to a change in the permeability $\left(C_{k}=\Delta e / \Delta \log k\right)$. For soft clays and silts, the value of $C_{k}$ is close to one half of the initial void ratio $\left(\mathrm{e}_{0} / 2\right)$. For peats, this value is usually closer to one quarter of the initial void ratio $\left(\mathrm{e}_{0} / 4\right)$. The low $\mathrm{C}_{\mathrm{k}} / \mathrm{e}_{0}$ and high $\mathrm{C}_{\mathrm{k}}$ values as compared to soft clays and silts suggest that only the macro-pores are serving as flow channels. Similarly, $\mathrm{C}_{\mathrm{k}} / \mathrm{C}_{\mathrm{c}}\left(\Delta \log \sigma^{\prime} / \Delta \log \mathrm{k}\right)$ is found to be between one-third and one-half for peats as compared to values near one for many clays and silts. This suggests that only macro-pores serve as flow channels, while macro and micro pores contribute to the overall compression (Mesri et al. 1997).

Following the completion of primary consolidation, secondary compression becomes much more pronounced. Secondary compression is defined by Fox and Edil (1996) as "one-dimensional creep settlement under constant effective stress and zero lateral strain $\left(\mathrm{K}_{0}\right)$ conditions.” The most significant parameter in quantifying this time dependent portion of the test is $C_{\alpha}(\Delta \mathrm{e} / \Delta \log \mathrm{t})$, which is expressed as the slope of the tangential portion of the deformation versus time curve during which excess pore pressure is negligible (Fox and Edil, 1996). Mesri et al. (1997) list three reasons why the secondary compression of peat is often more important in peats than in other soils:

(1) Peat deposits exist at high natural water content and void ratio. They accumulate high void ratio because plant matter that constitutes peat particles are of relatively low density and hold a considerable amount of water. Because of high in-situ void ratios, compression indices $\left(\mathrm{C}_{\mathrm{c}}, \mathrm{C}_{\mathrm{s}}\right)$ are high. Because secondary compression index is related to $C_{c}$, peat deposits tend to display high values of $C_{\alpha}$.

(2) Peats have the highest $C_{\alpha} / C_{c}$ values of natural soil deposits with values of 0.06 +/- 0.01. It appears that the magnitude $\mathrm{C}_{\alpha} / \mathrm{C}_{\mathrm{c}}$ depends on the deformability and compressibility of the soil particles. The lowest values of $\mathrm{C}_{\alpha} / \mathrm{C}_{\mathrm{c}}$ for natural soil deposits are observed in granular soils with relatively undeformable particles and $C_{\alpha} / C_{c}$ are 0.02 $+/-0.01$. 
(3) The duration of the primary consolidation is quite short. Primary consolidation for field conditions is usually completed within a few weeks, since the permeability is generally 100-1000 times larger than that of soft clays and the coefficient of consolidation $\left(C_{v}\right)$ is generally $10-100$ times larger.

A consequence of these findings is that the secondary compression behavior of peats is quite significant. In practical applications, the primary consolidation of these soils would be nearly completed by the end of construction activities. The effect of this is that most of the settlements that a structure founded on peat would encounter during its lifetime would be the result of secondary compression. Failure to recognize this fact may lead to substantial underestimation of field settlements (Mesri et al., 1997).

In addition to the settlement misevaluation possible from the neglection of secondary compression, similar errors are possible from the assumption of a constant $\mathrm{C}_{\alpha}$. Fox and Edil (1996) find that $\mathrm{C}_{\alpha}$ increases strongly with time. This behavior, which is also observed in some soft clays, is often missed by researchers who do not allow consolidation tests to proceed for a sufficiently lengthy time period. Fox et al. (1999) investigated the possible mechanism of this $\mathrm{C}_{\alpha}$ increase through the comparison of consolidation tests from $\gamma$-irradiated and untreated soil. It was found that the irradiated sample displayed a decrease in the rate and magnitude of secondary compression. The destruction of microorganisms decreased the rate of secondary compression. They hypothesized that the secondary compression of these soils is due to the biodegradation of plant material.

A detailed study of the consolidation behavior of these soils is a complicated topic. However, the literature provides several recommendations of values to be expected from the consolidation behavior of peats. Table 2.6 lists some key indices found from available literature, and a comparison to typical values found for soft clays.

\subsection{Strength Behavior}

Since peats tend to have extremely high water contents, they are generally weak in their natural state. However, fibrous materials that are often present in peats provide a 
mode of internal reinforcement as well as some anisotropy (Edil and Wang, 2000). It has also been found that the undrained shear strength and effective strength parameters (cohesion and effective friction angle) of peats and organic soils increase with increasing organic content and the corresponding increase in moisture content (Figure 2.4). This counter-intuitive behavior is again due to the presence of fibers and the fact that fiber content tends to increase with increasing water content and decreasing unit weight (Edil and Wang 2000). For other soils, water tends to reduce mineral-to-mineral contact and shearing resistance is lowered as water content increases (Lambe and Whitman, 1969).

Shear strength data for peats and organic soils available in the literature is most often derived from consolidated undrained (CU) triaxial compression tests. In addition, data for drained direct, ring, and simple shear tests have also been reported. Drained triaxial tests are rarely used due to the large changes in specimen size and shape during consolidation and shearing. Edil and Wang (2000) present an extensive review of the strength behavior of peats based on data available in the literature as well as tests performed at the University of Wisconsin in Madison. Their conclusions can be summarized as follows:

1) The effective friction angle ( $\left.\phi^{\prime}\right)$ is influenced by organic content (see Figure 2.4). Organic soils tested in a CU triaxial test posses an average friction angle of $41^{\circ}$ for organic contents up to approximately thirty percent. An increase to an average of $53^{\circ}$ is noted for soils with an organic content beyond thirty percent, with these soils being called "peat" in this study.

2) Ring shear tests give much lower result with friction angles of $32^{\circ}-33^{\circ}$. These values seem more reasonable when compared to typical friction angles of well-graded sand which exhibit friction angles of $38^{\circ}-46^{\circ}$, and is one of the most frictional of natural soils (Lambe and Whitman, 1969).

3) The cohesion intercept is generally found to be between 0-6 kPa.

4) $K_{o}$ values of 0.34 are found for fibrous peats (Figure 2.5), as compared to 0.53 for amorphous peats, regardless of the organic content (Edil and Wang, 2000). These values indicate a large range of variability when compared with $k_{0}$ values of $0.40-0.50$ for most other NC soils (Lambe and Whitman, 1969) 
5) The normalized undrained shear strength ratio $\left(\mathrm{s}_{\mathrm{u}} / \sigma_{\mathrm{v}}{ }_{\mathrm{v}}\right)$ is not constant for peats and organic soils as it is with inorganic clays (Figure 2.6). For normally consolidated peats tested in isotropically and anisotropically consolidated undrained triaxial compression tests, the normalized undrained strength ratio $\left(\mathrm{s}_{\mathrm{u}} / \sigma^{\prime}{ }_{\mathrm{v}}\right)$ varied between 0.5-0.7 with an average value of 0.59 (Figure 2.7). These values are significantly higher than the values of $0.25-0.35$ observed for most other soils (Edil and Wang, 2000). It was also noted that these values cannot be related to organic content, type of peat, level of consolidation, and type of consolidation (isotropic or anisotropic). Field vane shear tests from two highway sites in Wisconsin yield greater dispersion than the laboratory results, but similar results with respect to organic content and soil type (Figure 2.8).

The effect of fiber reinforcement and orientation provide a substantial complication in the testing and description of fibrous peats. Scale effects may artificially increase the strengthening mechanism of the fibers (Edil and Wang, 2000). For example, while in the field a two-centimeter long fiber may be essentially negligible, the presence of a similar fiber in a triaxial specimen may give artificially high strength. While the focus on use and testing of non-ideal soils has recently become of increased interest in the geotechnical engineering community, it is apparent that much more research needs to be done to establish testing and sampling methods that account for fiber presence in these highly organic soils.

\subsection{Sampling}

Due to the effect of fiber presence and orientation on the engineering properties measured in the laboratory, maintaining the soil's natural fabric during sampling is critical. Soil obtained through disturbed sampling may be used in classification and index tests. This soil may be gathered by use of conventional tube samplers or simply with a shovel and wheelbarrow type system.

Compared to other soft soils, the acquisition of undisturbed samples of peat is further complicated by the presence of fibers and the highly compressible nature of the soil. Different methods for undisturbed sampling of peats, primarily used for research 
purposes, are documented in the literature. Landva et al. (1983) described a $100 \mathrm{~mm}$ diameter piston sampler which features a plexiglass tube insert to hold the sample inside a piece of bronze tubing with a stainless steel cutting edge (Figure 2.9 and 2.10). A double leather suction cup provides a mechanism to hold the soil in place without applying unnecessary pressure and disturbance to the sample. Landva et al. (1983) note that no discernible disturbance of the peat horizons is present using this sampler.

Another solution for undisturbed sampling of peats is block sampling. This involves placing an open cube on top of the soil surface, forcing it into the soil and then removing it. While traditional techniques for block sampling can be used to obtain samples only from very shallow depths, more complicated variations such as that proposed by Landva et al. (1983) (Figure 2.11 and 2.12) have been developed to obtain samples from a greater depth.

Although these methods can provide large undisturbed samples, the dilemma of sampling recurs when the sample is divided into manageable blocks for laboratory testing. For fibrous peats with little or no mineral content and relatively low decomposition, most of the trimming can be done with an electric knife (Landva et al., 1983). A guide should be used during the trimming of more highly decomposed materials that may be unable to support their own weight. The guide also serves to insure that the sample is never touched by hand, which may cause disturbance and moisture loss. Samples should then be stored in humid conditions to insure minimal moisture loss during storage (Landva et al., 1983).

\subsection{Improvement of Organic soils}

With undeveloped land becoming increasingly rare and correspondingly expensive, engineers are being forced to design structures for construction on less than desirable soil conditions. It is becoming increasingly necessary to construct embankments and foundations either adjacent to or across highly organic soil deposits, whereas these deposits may have simply been circumvented in the past. The following discussion investigates some of the more common methods that are employed in practice when highly compressible organic soils are present. 
The oldest and most obvious method for dealing with highly compressible soils is to remove and replace them with quality fills. This is an extremely effective and simple method. This method proves to be practical if quality fill is available and if the problem soil is a relatively thin surface layer. If the peat layer is not completely removed, the compaction of the fill material may be difficult and unwanted displacements may occur. Incomplete removal of the organic soil would lead to further compression during conventional field compaction (Sasaki, 1982). Venema et al. (1989) describe a case where a fourteen-story building was to be constructed over an organic soil deposit in Minneapolis, Minnesota. The top layer of soil was an organic silt (OL) that stretched to three feet below the surface, underlain by an organic silt and peat deposit reaching approximately eleven feet below the surface. In this particular case, it was determined to be more economically feasible to remove the organic layers and replace them with a vibro-compacted sand backfill. This provided sufficient bearing capacity to allow the use of a shallow foundation, as opposed to the alternative of using a pile foundation to reach the fifty-five foot deep limestone bedrock layer. The application of this method is very clear: provide suitable foundation soil based upon economic constraints.

The application of a surcharge is another common method that may ensure that settlements due to subsequent construction are limited. In this method, a layer of surcharge fill is placed on top of the organic soil, and then removed after the appropriate preloading period. The soil is then in the overconsolidated state, resulting in smaller settlements and increased shear strength. The hindrance of this method is that it requires an extremely flexible and lengthy construction time (Sasaki, 1982).

Techniques to increase the drainage are often used in conjunction with preloading to accelerate the primary consolidation process under the effect of surcharge and/or the permanent fill. Many different methods have been used throughout the world depending on ground conditions and the materials that are readily available. The most common form of drainage, which has been used and investigated extensively (e.g. Sasaki, 1982), is constituted by vertical sand drains. These are constructed by digging a hole in the peat layer and then filling it with compacted sand. Other drainage systems that have been employed in peat deposits include: compacted stone drains (Sasaki, 1982), wick (plastic 
tube) drains (Ng and Rudd, 1984), bamboo drains (Aziz, 1984), strip (geotextile) drains (Koda and Wolski, 1994), and sand drains wrapped with a geotextile to add strength and prevent clogging (Koda and Wolski, 1994). Data available in the literature indicates that in peats, drains are often not very effective in promoting settlements. This is demonstrated in Figure 2.13, which compares settlements with time under an embankment in British Columbia, Canada in the presence and absence of sand drains.

In addition, problems often arise due to the large settlements that soft peat layers undergo which may cause buckling of the drains. There is some evidence that sand drains may be somewhat effective in promoting strength gain, as shown for example in Figure 2.14. Any strength gains in these cases will be small (depending on drain spacing) and are the result of arching between individual drains (Koda and Wolski, 1994). This strengthening effect resulting from arching increases with increasing compaction of the sand or stone. Although this method reduces the drainage capabilities, it provides an increase in the overall shear strength of the foundation, and may increase bearing strength in the center of the drains by up to twenty times (Sasaki, 1982). Despite the limitations, this low-cost improvement technique makes it a complementary addition to other improvement methods.

Lightweight fills are often used alone or in conjunction with the methods described previously (Ng and Rudd, 1984). In some instances it may prove feasible to substitute a soil embankment with an array of polystyrene blocks. The low weight of these blocks result in smaller settlements of the foundation soil. Provided that the blocks possess adequate strength properties to support a road, the use of these blocks may result in much less costly improvement methods. Lightweight fills may also be used in conditions where the foundation soil is too soft to even support construction activities. One such application is documented by Ng and Rudd (1984). In this case, a retention basin was to be constructed at the edge of a lake, over an existing swamp. This involved the construction of a fifteen-foot high, 500-foot long earth dike with three to one side slopes, over a fifty-five feet thick peat deposit. The problem in this case was that the swamp soil was too soft to allow the necessary equipment to move about as needed. The solution involved layering a two hundred by five hundred foot geotextile layer on the 
surface of the swamp. A three-foot layer of wood chips was then placed on top, followed by another geotextile and three-foot wood chip layer. It was then possible for construction activities to be conducted over the entire area after a one-foot gravel layer was placed. While the selection of materials for this working platform was governed by local economics, the principles can be extrapolated to many different scenarios (Ng and Rudd, 1984).

The previous discussion of improvement methods has focused on mechanical improvement methods. Although these methods are the basis for most improvement activities, a seemingly infinite number of variations on these methods are possible. The sequencing and alternation of embankment strips to prevent shear failure and to meet certain settlement requirements is a variation on the principles of preloading and surcharging (Ng and Rudd, 1984). Drainage systems may be varied to accommodate horizontal drainage, or may be replaced with compacted stone columns to enhance the arching effect between columns (Sasaki, 1982). Lightweight fills may also be used in conjunction with other methods to lower the foundation load or to simply provide tolerable working conditions (Ng and Rudd, 1984).

A second class of methods involves chemical treatment of the problem soil. Chemical improvement methods consist of the addition of a binding compound into the soil. The application of these methods for deep or mass stabilization of any soil involves the injection of a binding material into the soil to form a stabilized column or block (Åhnberg and Holm, 1999). Deep stabilization creates a stabilized column in the soil while mass stabilization creates a stabilized slab under the ground surface. The most common binding materials used are: Portland cement, quick lime, gypsum, flyash, coal slag, and other pozzolanic materials (Esrig, 1999) These binding compounds all work in a manner to increase the $\mathrm{pH}$ of the soil environment above a value of 12.4, which allows the silica and alumina of the soil to become available for the pozzolanic reaction to take place (Esrig, 1999). The strengthening is also contributed to by the reduction of water content in the soil that takes place upon hydration of the binding agent. This is most pronounced with the usage of cement and/or quicklime, but becomes essentially negligible when considering soils with extremely high moisture contents (Esrig, 1999). 
The strengthening mechanism for organic soils depends on several properties. The most significant characteristics that determine the effectiveness of the treatment are the type of peat, size and abundance of fibers, type of binder, concentration of binder, elapsed time after stabilization, and degree of decomposition (Huttunen and Kujala, 1996). Of particular relevance concerning the use of binding agents with organic soils is the role of the degree of decomposition (Huttunen and Kujala, 1996).

The chemical composition of an organic soil consists primarily of polymers, humic substances, and polysaccharides (Kujala et al. 1996). The humic substances are most interesting in this context due to their relation to the decomposition of the organic matter in a soil. Decomposing organic matter results in increased levels of humic and fulvic acids in the soil that combine with metal ions to form complexes that hinder the binding reaction (Kujala et al. 1996). Thus, a binder will introduce less strength gain in a highly decomposed soil as compared to an equivalent binder introduced in a relatively undecomposed soil. Kujala et al. (1996) ran a series of experiments on a number of soils, ranging from organic clays to gravels, with a number of different binders. For these tests, a synthetic humic acid was added to the soils. The results indicate that the humus and humic acid had a significant effect on the strength gain of treated soils, reducing the shear strength up to $77 \%$ in lime and cement treated clays, and up to $95 \%$ in cement treated granular soils (Figure 2.15 and 2.16). The study then went on to reveal that although humus content is not the only property of a soil that determines the effectiveness of a binder, little strength gain could be achieved if the soil has humus content above two percent (Kujala et al. 1996).

Equipment used for in-situ mixing also play a significant role on the improvement of the soil properties that can be achieved in the field. A field test was performed by the Finnish Roads Administration's Research and Development Unit of Oulu to test the quality of mass and deep stabilized peat and clay (Huttunen et al. 1996). Two adjacent plots measuring $13 \times 18 \mathrm{~m}^{2}$ were selected for the test, at a site characterized by three meters of surface peat underlain by 15 meters of clay. In one plot, the peat layer was stabilized with either a mixture of Finnstabi (a gypsum based by-product) and cement, or a mixture of cement and blast furnace slag creating $3 \mathrm{~m}$ x 3m x 3m blocks. At the second 
plot, $700 \mathrm{~mm}$ diameter columns extending to a depth of 18 were formed with a mixture of the Finnstabi and cement or cement and blast furnace slag (Huttunen et al. 1996). After thirty days and again after one year, various evaluations were performed at the site. These evaluations included column drilling, vane shear tests, dynamic cone penetrometer, $\mathrm{pH}$ measurement, thermal conductivity, and sampling for laboratory tests. The results of the vane measurements shown in Figure 2.17 suggest that a more uniform and effective stabilization was accomplished with the mass treatment equipment. The results also show the greater effectiveness in terms of the strength increase of the cement-slag binder and demonstrate that the strength gain may be significant even after thirty days (Huttunen et al., 1996). 
Table 2.1: Classification of peat according to Radforth System (Radforth, 1969)

\begin{tabular}{|c|c|c|}
\hline $\begin{array}{l}\text { Predominant } \\
\text { Characteristic }\end{array}$ & Category & Name \\
\hline \multirow{7}{*}{$\begin{array}{l}\text { Amorphous- } \\
\text { granular }\end{array}$} & 1 & Amorphous-granular peat \\
\hline & 2 & Non-woody, fine-fibrous peat \\
\hline & 3 & $\begin{array}{l}\text { Amorphous-granular peat containing non-woody fine } \\
\text { fibres }\end{array}$ \\
\hline & 4 & Amorphous-granular peat containing woody fine fibres \\
\hline & 5 & $\begin{array}{l}\text { Peat, predominantly amorphous-granular, containing } \\
\text { non-woody fine fibres, held in a woody, fine-fibrous } \\
\text { framework }\end{array}$ \\
\hline & 6 & $\begin{array}{l}\text { Peat, predominantly amorphous-granular containing } \\
\text { woody fine fibres, held in a woody, coarse-fibrous } \\
\text { framework }\end{array}$ \\
\hline & 7 & $\begin{array}{l}\text { Alternate layering of non-woody, fine-fibrous peat and } \\
\text { amorphous-granular peat containing non-woody fine } \\
\text { fibres }\end{array}$ \\
\hline \multirow[t]{4}{*}{ Fine-fibrous } & 8 & $\begin{array}{l}\text { Non-woody, fine fibrous peat containing a mound of } \\
\text { coarse fibres }\end{array}$ \\
\hline & 9 & $\begin{array}{l}\text { Woody, fine fibrous peat held in a woody, coarse-fibrous } \\
\text { framework }\end{array}$ \\
\hline & 10 & Woody particles held in a non-woody, fine fibrous peat \\
\hline & 11 & Woody and non-woody particles held in fine-fibrous peat \\
\hline \multirow[t]{6}{*}{ Coarse-fibrous } & 12 & Woody, coarse-fibrous peat \\
\hline & 13 & Coarse fibres criss-crossing fine fibrous peat \\
\hline & 14 & $\begin{array}{l}\text { Non-woody and woody fine fibrous peat held in a coarse } \\
\text { fibrous framework }\end{array}$ \\
\hline & 15 & $\begin{array}{l}\text { Woody mesh of fibres and particles enclosing } \\
\text { amorphous-granular peat containing fine fibres }\end{array}$ \\
\hline & 16 & $\begin{array}{l}\text { Woody, coarse-fibrous peat containing scattered woody } \\
\text { chunks }\end{array}$ \\
\hline & 17 & $\begin{array}{l}\text { Mesh of closely applied logs and roots enclosing woody } \\
\text { coarse-fibrous peat with woody chunks. }\end{array}$ \\
\hline
\end{tabular}


Table 2.2: Classification of peat according to Von Post System (After Landva and Pheeney, 1980)

\begin{tabular}{|c|c|c|c|c|c|}
\hline \multicolumn{2}{|c|}{ Property } & \multicolumn{2}{|c|}{ Description } & \multicolumn{2}{|l|}{ Code } \\
\hline \multicolumn{2}{|l|}{ Genera } & \multicolumn{4}{|c|}{ Type of plant material present } \\
\hline & & \multicolumn{2}{|c|}{ Bryales (Moss) } & \multicolumn{2}{|l|}{$\mathrm{B}$} \\
\hline & & \multicolumn{2}{|c|}{ Carex (Sedge) } & \multicolumn{2}{|l|}{$\mathrm{C}$} \\
\hline & & \multicolumn{2}{|c|}{ Equisetum (horse tail) } & \multicolumn{2}{|l|}{ Eq } \\
\hline & & \multicolumn{2}{|c|}{ Eriphorum (cottong grass) } & \multicolumn{2}{|l|}{ Er } \\
\hline & & \multicolumn{2}{|c|}{ Hypnum (moss) } & \multicolumn{2}{|l|}{$\mathrm{H}$} \\
\hline & & \multicolumn{2}{|c|}{ Lignidi (wood) } & \multicolumn{2}{|l|}{$\mathrm{W}$} \\
\hline & & \multicolumn{2}{|c|}{ Nanolignidi (shrubs) } & \multicolumn{2}{|l|}{$\mathrm{N}$} \\
\hline & & \multicolumn{2}{|c|}{ Phragmites } & \multicolumn{2}{|l|}{$\mathrm{Ph}$} \\
\hline & & \multicolumn{2}{|c|}{ Scheuchzeria(aquatic herbs) } & \multicolumn{2}{|l|}{ Sch } \\
\hline & & \multicolumn{2}{|c|}{ Sphagnum (moss) } & $\mathrm{S}$ & \\
\hline \multirow{2}{*}{\multicolumn{2}{|c|}{ Designation }} & \multicolumn{4}{|c|}{ Genera is listed in decreasing order of content } \\
\hline & & \multicolumn{4}{|c|}{$\begin{array}{l}\text { For example, a peat classified as ErCS would consist mainly of } \\
\text { Eriphorum remnants, some Carex remnants, and even less Sphagnum } \\
\text { remnants }\end{array}$} \\
\hline \multicolumn{2}{|l|}{ Humification } & \multicolumn{4}{|c|}{ Extent of decomposition } \\
\hline Decomposition & $\begin{array}{l}\text { Plant } \\
\text { Structure }\end{array}$ & $\begin{array}{l}\text { Content of } \\
\text { amorphous } \\
\text { material }\end{array}$ & $\begin{array}{l}\text { Material Extruded on } \\
\text { squeezing }\end{array}$ & \begin{tabular}{|l} 
Nature of \\
residue
\end{tabular} & \begin{tabular}{|l} 
Degree of \\
Humification
\end{tabular} \\
\hline None & $\begin{array}{l}\text { Easily } \\
\text { Identified }\end{array}$ & None & Clear, colorless water & & $\mathrm{H}_{1}$ \\
\hline Insignificant & $\begin{array}{l}\text { Easily } \\
\text { Identified }\end{array}$ & None & Yellowish water & & $\mathrm{H}_{2}$ \\
\hline Very Slight & $\begin{array}{l}\text { Still } \\
\text { Identifiable }\end{array}$ & Slight & \begin{tabular}{|l} 
Brown, Muddy \\
water; no peat
\end{tabular} & Not pasty & $\mathrm{H}_{3}$ \\
\hline Slight & $\begin{array}{l}\text { Not easily } \\
\text { Identified }\end{array}$ & Some & $\begin{array}{l}\text { Dark brown, muddy } \\
\text { water; no peat }\end{array}$ & $\begin{array}{l}\text { Somewhat } \\
\text { pasty }\end{array}$ & $\mathrm{H}_{4}$ \\
\hline Moderate & $\begin{array}{l}\text { Recognizabl } \\
\text { e, but vague }\end{array}$ & Considerable & $\begin{array}{l}\text { Muddy water and } \\
\text { some peat }\end{array}$ & Strongly pasty & $\mathrm{H}_{5}$ \\
\hline $\begin{array}{l}\text { Moderately } \\
\text { Strong }\end{array}$ & $\begin{array}{l}\text { Indistinct } \\
\text { (more } \\
\text { distinct after } \\
\text { squeezing) } \\
\end{array}$ & Considerable & $\begin{array}{l}\text { About } 1 / 3 \text { of peat } \\
\text { squeezed out; water } \\
\text { dark brown }\end{array}$ & & $\mathrm{H}_{6}$ \\
\hline Strong & $\begin{array}{l}\text { Fairly } \\
\text { Recognizabl } \\
\text { e }\end{array}$ & High & $\begin{array}{l}\text { About } 1 / 2 \text { of peat } \\
\text { squeezed out; any } \\
\text { water very dark brown }\end{array}$ & & $\mathrm{H}_{7}$ \\
\hline Very Strong & $\begin{array}{l}\text { Very } \\
\text { Indistinct }\end{array}$ & High & $\begin{array}{l}\text { About } 2 / 3 \text { of peat } \\
\text { squeezed out; also } \\
\text { some pasty water }\end{array}$ & $\begin{array}{l}\text { Plant tissue } \\
\text { capable of } \\
\text { resisting } \\
\text { decomposition } \\
\text { (roots, fibres) }\end{array}$ & $\mathrm{H}_{8}$ \\
\hline $\begin{array}{l}\text { Nearly } \\
\text { Complete }\end{array}$ & $\begin{array}{l}\text { Almost not } \\
\text { recognizable }\end{array}$ & & $\begin{array}{l}\text { Nearly all the peat } \\
\text { squeezed out as a } \\
\text { fairly uniform paste }\end{array}$ & & $\mathrm{H}_{9}$ \\
\hline Complete & $\begin{array}{l}\text { Not } \\
\text { discernible }\end{array}$ & & $\begin{array}{l}\text { All the peat passes } \\
\text { between the fingers; no } \\
\text { free water visible }\end{array}$ & & $\mathrm{H}_{10}$ \\
\hline
\end{tabular}


Table 2.2 (continued)

\begin{tabular}{|c|c|c|}
\hline \multirow[t]{5}{*}{ Water Content } & \multicolumn{2}{|c|}{ Mass based water content } \\
\hline & $\mathrm{w}<500 \%$ & $\mathrm{~B}_{2}$ \\
\hline & $500 \%<\mathrm{W}<1000 \%$ & $\mathrm{~B}_{3}$ \\
\hline & $1000 \%<\mathrm{W}<2000 \%$ & $\mathrm{~B}_{4}$ \\
\hline & $\mathrm{w}>2000 \%$ & $\mathrm{~B}_{5}$ \\
\hline \multirow{6}{*}{ Fine Fibers } & \multicolumn{2}{|c|}{ Abundance of fibers and stems smaller than $1 \mathrm{~mm}$} \\
\hline & None & $\mathrm{F}_{0}$ \\
\hline & Low & $\mathrm{F}_{1}$ \\
\hline & Moderate & $\mathrm{F}_{2}$ \\
\hline & High & $\mathrm{F}_{3}$ \\
\hline & \multicolumn{2}{|c|}{$\begin{array}{l}\text { Note: The composition of fibres may be included if properly specified. } \\
\text { For example, } \mathrm{F}(\mathrm{S})_{3} \text { would indicate abundance of sphagnum fibres. }\end{array}$} \\
\hline \multirow[t]{6}{*}{ Coarse Fibers } & \multicolumn{2}{|c|}{ Abundance of fibers and stems larger than $1 \mathrm{~mm}$} \\
\hline & None & $\mathrm{R}_{0}$ \\
\hline & Low & $\mathrm{R}_{1}$ \\
\hline & Moderate & $\mathrm{R}_{2}$ \\
\hline & High & $\mathrm{R}_{3}$ \\
\hline & \multicolumn{2}{|c|}{$\begin{array}{l}\text { Note: The composition of fibres may be included if properly specified. } \\
\text { For example, } \mathrm{R}(\mathrm{S})_{3} \text { would indicate abundance of sphagnum fibres. }\end{array}$} \\
\hline \multirow[t]{5}{*}{ Wood Remnants } & \multicolumn{2}{|c|}{ Abundance of wood remnants } \\
\hline & None & $\mathrm{W}_{0}$ \\
\hline & Low & $\mathrm{W}_{1}$ \\
\hline & Moderate & $\mathrm{W}_{2}$ \\
\hline & High & $\mathrm{W}_{3}$ \\
\hline \multirow[t]{5}{*}{ Strub Remnants } & \multicolumn{2}{|c|}{ Abundance of wood remnants } \\
\hline & None & $\mathrm{N}_{0}$ \\
\hline & Low & $\mathrm{N}_{1}$ \\
\hline & Moderate & $\mathrm{N}_{2}$ \\
\hline & High & $\mathrm{N}_{3}$ \\
\hline
\end{tabular}


Table 2.3: Classification of peat according to ASTM System

\begin{tabular}{|c|c|c|}
\hline ASTM Standard & Criteria & Designation \\
\hline \multirow[t]{3}{*}{ Fiber Content (D 1997) } & $>67 \%$ fibers & Fibric $\left(\mathrm{H}_{1}-\mathrm{H}_{3}\right)$ \\
\hline & $33 \%-67 \%$ fibers & Hemic $\left(\mathrm{H}_{4}-\mathrm{H}_{10}\right)$ \\
\hline & $<33 \%$ fibers & Sapric $\left(\mathrm{H}_{7}-\mathrm{H}_{10}\right)$ \\
\hline \multirow[t]{3}{*}{ Ash Content (D 2974) } & $<5 \%$ ash & Low Ash \\
\hline & 5\%-15\% ash & Medium Ash \\
\hline & $15-25 \%$ ash & High Ash \\
\hline \multirow[t]{4}{*}{ Acidity (D 2976) } & $\mathrm{pH}<4.5$ & Highly Acidic \\
\hline & $4.5<\mathrm{pH}<5.5$ & Moderately Acidic \\
\hline & $5.5<\mathrm{pH}<7$ & Slightly Acidic \\
\hline & $\mathrm{pH}>7$ & Basic \\
\hline \multirow[t]{4}{*}{ Absorbency (D 2980) } & $\mathrm{w}>1500 \%$ & Extremely Absorbent \\
\hline & $800 \%<w<1500 \%$ & Highly Absorbent \\
\hline & $300 \%<\mathrm{w}<800 \%$ & Moderately Absorbent \\
\hline & $\mathrm{w}<300 \%$ & Slightly Absorbent \\
\hline Botanical Composition & \multicolumn{2}{|c|}{$\begin{array}{l}\text { If a single botanical name is to be used (sphagnum peat, } \\
\text { etc.), it is required that at least } 75 \% \text { of the fiber content of } \\
\text { that peat be derived from the designated type of plant } \\
\text { material. }\end{array}$} \\
\hline
\end{tabular}

Table 2.4: Range of values encountered from term "peat"

\begin{tabular}{|c|c|}
\hline Property & Values \\
\hline Organic Content $(\%)$ & $27-95$ \\
\hline Unit Weight $\left(\mathrm{kN} / \mathrm{m}^{3}\right)$ & $8.8-12.9$ \\
\hline Specific Gravity $\left(\mathrm{G}_{\mathrm{s}}\right)$ & $1.40-2.68$ \\
\hline Water Content $(\%)$ & $100-1400$ \\
\hline Fiber Content $(\%)$ & $20-92$ \\
\hline
\end{tabular}


Table 2.5: Complete database of typical values for soils described as "peat"

\begin{tabular}{|c|c|c|c|c|c|c|c|}
\hline Source & Description & W (\%) & LOI (\%) & $\gamma\left(\mathrm{kN} / \mathrm{m}^{3}\right)$ & Fiber (\%) & $\mathbf{G}_{\mathrm{s}}$ & Ref. \\
\hline Middleton, WI & Fibrous & $500-600$ & 83-95 & $9.1-10.1$ & 64 & $\begin{array}{l}1.40- \\
1.70\end{array}$ & 1 \\
\hline Middleton, WI & Amorphous & $430-520$ & $83-95$ & 10.3 & $20-30$ & $\begin{array}{l}1.60- \\
1.90\end{array}$ & 1 \\
\hline Portage, WI & Fibrous & 600 & 81 & 9.6 & 31 & 1.72 & 1 \\
\hline $\begin{array}{l}\text { Fond du Lac, } \\
\text { WI }\end{array}$ & Amorphous & 240 & 60 & 10.2 & 20 & 1.94 & 1 \\
\hline $\begin{array}{l}\text { Nine Spring, } \\
\text { WI }\end{array}$ & Fibrous & $450-655$ & $74-84$ & 9.8 & $75-92$ & $\begin{array}{l}1.62- \\
1.85 \\
\end{array}$ & 1 \\
\hline USH12 & Fibrous & $157-165$ & - & 12.9 & - & - & 1 \\
\hline USH12/18 & Fibrous & 361 & 66 & - & - & 1.82 & 1 \\
\hline STH29 & Fibrous & $250-516$ & $35-66$ & $9.6-12.1$ & - & $\begin{array}{l}1.82- \\
2.23 \\
\end{array}$ & 1 \\
\hline $\begin{array}{l}\text { Hoyt Lakes, } \\
\text { MN } \\
\end{array}$ & Fibrous & $270-470$ & $50-85$ & 10.4 & $36-76$ & $\begin{array}{l}1.59- \\
1.70 \\
\end{array}$ & 1 \\
\hline Richfield, MN & Amorphous & $175-300$ & $31-37$ & 11.6 & $37-45$ & 2.02 & 1 \\
\hline Middleton, WI & Fibrous & 510 & 88 & - & - & 1.41 & 1 \\
\hline Portage, WI & Fibrous & 600 & 81 & - & - & 1.72 & 1 \\
\hline $\begin{array}{l}\text { Fond du Lac, } \\
\text { WI }\end{array}$ & Amorphous & 240 & 60 & - & - & 1.94 & 1 \\
\hline R2-7 & Fibrous & 624 & 91 & - & - & 1.48 & 1 \\
\hline B2-6 & Fibrous & 446 & 83 & - & - & 1.48 & 1 \\
\hline B3-2 & Amorphous & 321 & 50 & - & - & 1.58 & 1 \\
\hline Hoyt Lakes \#1 & Fibrous & 304 & 50 & - & - & 1.75 & 1 \\
\hline $\begin{array}{l}\text { Hoyt Lakes } \\
\# 11\end{array}$ & Fibrous & 244 & 78 & - & - & 1.52 & 1 \\
\hline Netherlands & Fibrous & 500 & 67 & - & - & - & 1 \\
\hline Netherlands \#1 & Fibrous & 669 & 66 & - & - & 1.52 & 1 \\
\hline Netherlands \#2 & Fibrous & - & 74 & - & - & - & 1 \\
\hline Netherlands \#3 & Fibrous & - & 84 & - & - & - & 1 \\
\hline Netherlands \#4 & Fibrous & - & 66 & - & - & - & 1 \\
\hline Netherlands \#5 & Fibrous & - & 72 & - & - & - & 1 \\
\hline Indiana \#1 & Amorphous & - & 27 & - & - & - & 1 \\
\hline Indiana \#2 & Amorphous & - & 38 & - & - & - & 1 \\
\hline Indiana \#3 & Amorphous & - & 60 & - & - & - & 1 \\
\hline Japan & Fibrous & 507 & 58 & - & - & - & 1 \\
\hline Nine Spring \#1 & Amorphous & 562 & 74 & - & - & - & 1 \\
\hline $\begin{array}{ll}\text { Nine } & \text { Springs } \\
\# 2 & \\
\end{array}$ & Amorphous & 589 & 76 & - & - & - & 1 \\
\hline $\begin{array}{l}\text { Nine Springs } \\
\# 3\end{array}$ & Amorphous & 626 & 81 & - & - & - & 1 \\
\hline $\begin{array}{ll}\text { Nine } & \text { Springs } \\
\# 4 & \\
\end{array}$ & Amorphous & 616 & 84 & - & - & - & 1 \\
\hline $\begin{array}{ll}\text { Nine } & \text { Springs } \\
\# 5 & \\
\end{array}$ & Amorphous & 655 & 83 & - & - & - & 1 \\
\hline $\begin{array}{ll}\text { Nine } & \text { Springs } \\
\# 6 & \\
\end{array}$ & Amorphous & 562 & 74 & - & - & - & 1 \\
\hline $\begin{array}{l}\text { Nine } \text { Springs } \\
\# 7\end{array}$ & Amorphous & 589 & 76 & - & - & - & 1 \\
\hline
\end{tabular}




\begin{tabular}{|c|c|c|c|c|c|c|c|}
\hline Source & Description & W (\%) & LOI (\%) & $\gamma\left(\mathrm{kN} / \mathrm{m}^{3}\right)$ & Fiber (\%) & $\mathbf{G}_{\mathbf{s}}$ & Ref. \\
\hline $\begin{array}{l}\text { Nine Springs } \\
\# 8\end{array}$ & Amorphous & 626 & 81 & - & - & - & 1 \\
\hline $\begin{array}{ll}\text { Nine } & \text { Springs } \\
\# 9 & \end{array}$ & Amorphous & 616 & 84 & - & - & - & 1 \\
\hline Richfield \#1 & Amorphous & 181 & 31 & - & - & - & 1 \\
\hline Richfield \#2 & Amorphous & 175 & 37 & - & - & - & 1 \\
\hline $\begin{array}{ll}\text { Hoyt } & \text { Lakes } \\
\# 1-1 & \end{array}$ & Amorphous & - & 70 & - & - & - & 1 \\
\hline $\begin{array}{ll}\text { Hoyt } & \text { Lakes } \\
\# 1-2 & \\
\end{array}$ & Amorphous & & 58 & - & - & - & 1 \\
\hline $\begin{array}{ll}\text { Hoyt } & \text { Lakes } \\
\# 1-3 & \end{array}$ & Amorphous & - & 88 & - & - & - & 1 \\
\hline $\begin{array}{l}\text { Hoyt } \quad \text { Lakes } \\
\# 2-1\end{array}$ & Amorphous & - & 83 & - & - & - & 1 \\
\hline $\begin{array}{l}\text { Hoyt } \quad \text { Lakes } \\
\# 2-2\end{array}$ & Amorphous & - & - & - & - & - & 1 \\
\hline $\begin{array}{l}\text { Hoyt Lakes } \\
\# 2-3\end{array}$ & Amorphous & - & - & - & - & - & 1 \\
\hline USH 12 & Fibrous & 165 & - & - & - & - & 1 \\
\hline USH 12/18 & Fibrous & 361 & - & - & - & - & 1 \\
\hline STH 29 & Fibrous & 374 & - & - & - & - & 1 \\
\hline Middleton, WI & Fibrous & - & - & - & - & - & 1 \\
\hline Portage, WI & Fibrous & - & - & - & - & - & 1 \\
\hline $\begin{array}{l}\text { Fond du Lac, } \\
\text { WI }\end{array}$ & Amorphous & - & - & - & - & - & 1 \\
\hline Ohmiya \#1 & Fibrous & - & - & - & - & - & 1 \\
\hline Ohmiya \#2 & Fibrous & - & - & - & - & - & 1 \\
\hline Ohmiya \#3 & Fibrous & - & - & - & - & - & 1 \\
\hline Ohmiya \#4 & Fibrous & - & - & - & - & - & 1 \\
\hline Ohmiya \#5 & Fibrous & - & - & - & - & - & 1 \\
\hline Ohmiya \#6 & Fibrous & - & - & - & - & - & 1 \\
\hline Ohmiya \#7 & Fibrous & - & - & - & - & - & 1 \\
\hline Ohmiya \#8 & Fibrous & - & - & - & - & - & 1 \\
\hline $\begin{array}{l}\text { Sacramento, } \\
\text { CA }\end{array}$ & Fibrous & $146-783$ & - & - & - & - & 1 \\
\hline Japan \#1 & Amorphous & - & - & - & - & - & 1 \\
\hline Japan \#2 & Amorphous & - & - & - & - & - & 1 \\
\hline Japan \#3 & Amorphous & - & - & - & - & - & 1 \\
\hline $\begin{array}{l}\text { Raheenmore, } \\
\text { IR }\end{array}$ & Amorphous & $800-900$ & - & - & - & - & 1 \\
\hline Akita, Japan & Amorphous & $560-680$ & - & - & - & - & 1 \\
\hline France & - & - & $>30$ & - & - & - & 1 \\
\hline $\begin{array}{l}\text { Kamedago, } \\
\text { Japan }\end{array}$ & Fibrous & - & 58 & - & - & - & 1 \\
\hline Kamedago \#2 & Fibrous & - & 58 & - & - & - & 1 \\
\hline Kamedago \#3 & Fibrous & - & 8 & - & - & - & 1 \\
\hline Canada \#1 & Fibrous & $375-400$ & $78-88$ & - & - & - & 1 \\
\hline Canada \#2 & Fibrous & $375-401$ & 78-89 & - & - & - & 1 \\
\hline Canada \#3 & Fibrous & $200-600$ & - & - & - & - & 1 \\
\hline Japan \#1 & Amorphous & - & $57-67$ & - & - & - & 1 \\
\hline Japan \#2 & Amorphous & - & $57-68$ & - & - & - & 1 \\
\hline
\end{tabular}




\begin{tabular}{|c|c|c|c|c|c|c|c|}
\hline Source & Description & W (\%) & LOI (\%) & $\gamma\left(\mathrm{kN} / \mathrm{m}^{3}\right)$ & Fiber (\%) & $\mathbf{G}_{\mathbf{s}}$ & Ref. \\
\hline Japan \#3 & Amorphous & - & 56 & - & - & - & 1 \\
\hline Japan \#4 & Amorphous & - & 56 & - & - & - & 1 \\
\hline $\begin{array}{l}\text { Antoniny, } \\
\text { POL }\end{array}$ & Amorphous & 250 & - & - & - & - & 1 \\
\hline Antoniny \#2 & Amorphous & 100 & - & - & - & - & 1 \\
\hline $\begin{array}{l}\text { Raheenmore, } \\
\text { IR }\end{array}$ & Amorphous & $800-900$ & 80 & - & - & - & 1 \\
\hline Adria-1 & - & $330-421$ & $68-75$ & $10.3-10.7$ & - & $\begin{array}{l}1.55- \\
1.58 \\
\end{array}$ & 2 \\
\hline Adria-2 & - & $226-427$ & $65-84$ & 8.8-11.7 & - & $\begin{array}{l}1.42- \\
1.52 \\
\end{array}$ & 2 \\
\hline Correzzola & - & $606-790$ & $70-72$ & $10.3-10.8$ & - & $\begin{array}{l}1.46- \\
1.60 \\
\end{array}$ & 2 \\
\hline P11 & - & 572 & 92.5 & - & - & - & 3 \\
\hline P12 & - & 572 & 91.3 & - & - & - & 3 \\
\hline $\mathrm{P} 13$ & - & 522 & 89.6 & - & - & - & 3 \\
\hline $\mathrm{P} 14$ & - & 609 & 92.8 & - & - & - & 3 \\
\hline P16 & - & 546 & 89.8 & - & - & - & 3 \\
\hline $\mathrm{P} 17$ & - & 622 & 93.2 & - & - & - & 3 \\
\hline $\mathrm{P} 18$ & - & 605 & 92.9 & - & - & - & 3 \\
\hline $\mathrm{P} 19$ & - & 521 & 89.2 & - & - & - & 3 \\
\hline $\mathrm{P} 21$ & - & 604 & 93.3 & - & - & - & 3 \\
\hline $\mathrm{P} 24$ & - & 491 & 91.4 & - & - & - & 3 \\
\hline $\mathrm{P} 25$ & - & 568 & 92.9 & - & - & - & 3 \\
\hline P27 & - & 525 & 91.1 & - & - & - & 3 \\
\hline P28 & - & 624 & 93.9 & - & - & - & 3 \\
\hline $\begin{array}{l}\text { Fond du Lac, } \\
\text { WI }\end{array}$ & sapric & 240 & 60.2 & 10.2 & 20 & 1.94 & 4 \\
\hline Portage, WI & hemic & 600 & 80.5 & 9.6 & 31 & 1.72 & 4 \\
\hline Waupaca & fibric & 460 & 85 & 9.6 & 50 & 1.68 & 4 \\
\hline Middleton & fibric & 510 & 88 & 9.1 & 64 & 1.41 & 4 \\
\hline Adams \#1 & - & $200-600$ & 77.5-87.8 & - & 1.62 & - & 4 \\
\hline Adams \#2 & - & $355-425$ & 84.1 & - & 1.73 & - & 5 \\
\hline Adams \#3 & - & $330-375$ & 87.7 & - & 1.65 & - & 5 \\
\hline $\begin{array}{l}\text { Anderson \& } \\
\text { Haas }\end{array}$ & - & $105-470$ & $15-53$ & - & 2.3 & - & 5 \\
\hline$\# 2$ & - & $100-470$ & $5-53$ & - & - & - & 5 \\
\hline Brochu \& Pare & - & $300-650$ & $60-85$ & - & $1.3-1.7$ & - & 5 \\
\hline Cassagrande & - & $250-800$ & & - & - & - & 5 \\
\hline Colley & - & $485-910$ & 83 & - & - & - & 5 \\
\hline $\begin{array}{l}\text { Goodman \& } \\
\text { Lee }\end{array}$ & - & $280-320$ & $40.5-75.2$ & - & $1.51-1.62$ & - & 5 \\
\hline$\#$ & - & $240-575$ & $42-75$ & - & 1.53 & - & 5 \\
\hline Hanrahan & - & $\begin{array}{l}340- \\
1465\end{array}$ & & - & $1.1-1.8$ & - & 5 \\
\hline Tessier \#1 & - & $200-800$ & - & - & $1.9-2.7$ & - & 5 \\
\hline Tessier \#2 & - & $300-650$ & - & - & $1.9-2.68$ & - & 5 \\
\hline
\end{tabular}




\section{Reference:}

1) Edil and Wang (2000)

2) Colleselli et. Al. (2000)

3)Fox and Edil (1996)

4)Dhowian and Edil (1980)

5)Radforth (1969)

Table 2.6: Typical Consolidation Indices

\begin{tabular}{|c|l|l|l|l|}
\hline Index & Peat & Reference & Soft Clay & Reference \\
\hline $\mathrm{C}_{\mathrm{c}}$ & $2.9-4.7$ & $\begin{array}{l}\text { Dhowian and Edil } \\
(1981)\end{array}$ & $0.2-0.9$ & $\begin{array}{l}\text { Lambe and } \\
\text { Whitman (1968) }\end{array}$ \\
\hline $\mathrm{C}_{\mathrm{s}}$ & $\mathrm{C}_{\mathrm{c}} / 10$ & $\begin{array}{l}\text { Dhowian and Edil } \\
(1981)\end{array}$ & $\mathrm{C}_{\mathrm{c}} / 10-\mathrm{C}_{\mathrm{c}} / 8$ & $\begin{array}{l}\text { Lambe and } \\
\text { Whitman (1968) }\end{array}$ \\
\hline $\mathrm{C}_{\mathrm{k}}$ & $\mathrm{e}_{\mathrm{o}} / 4$ & Mesri et. Al. (1997) & $\mathrm{e}_{0} / 2$ & $\begin{array}{l}\text { Mesri et. Al. } \\
(1997)\end{array}$ \\
\hline $\mathrm{C}_{\alpha} / \mathrm{C}_{\mathrm{c}}$ & $0.06+/-0.01$ & Mesri et. Al (1997) & $0.04+/-0.01$ & $\begin{array}{l}\text { Mesri et. Al. } \\
(1992)\end{array}$ \\
\hline $\mathrm{C}_{\mathrm{v}}$ & $10-100 * \mathrm{C}_{\mathrm{v} \text { soft clay }}$ & Mesri et. Al. (1997) & - & - \\
\hline
\end{tabular}




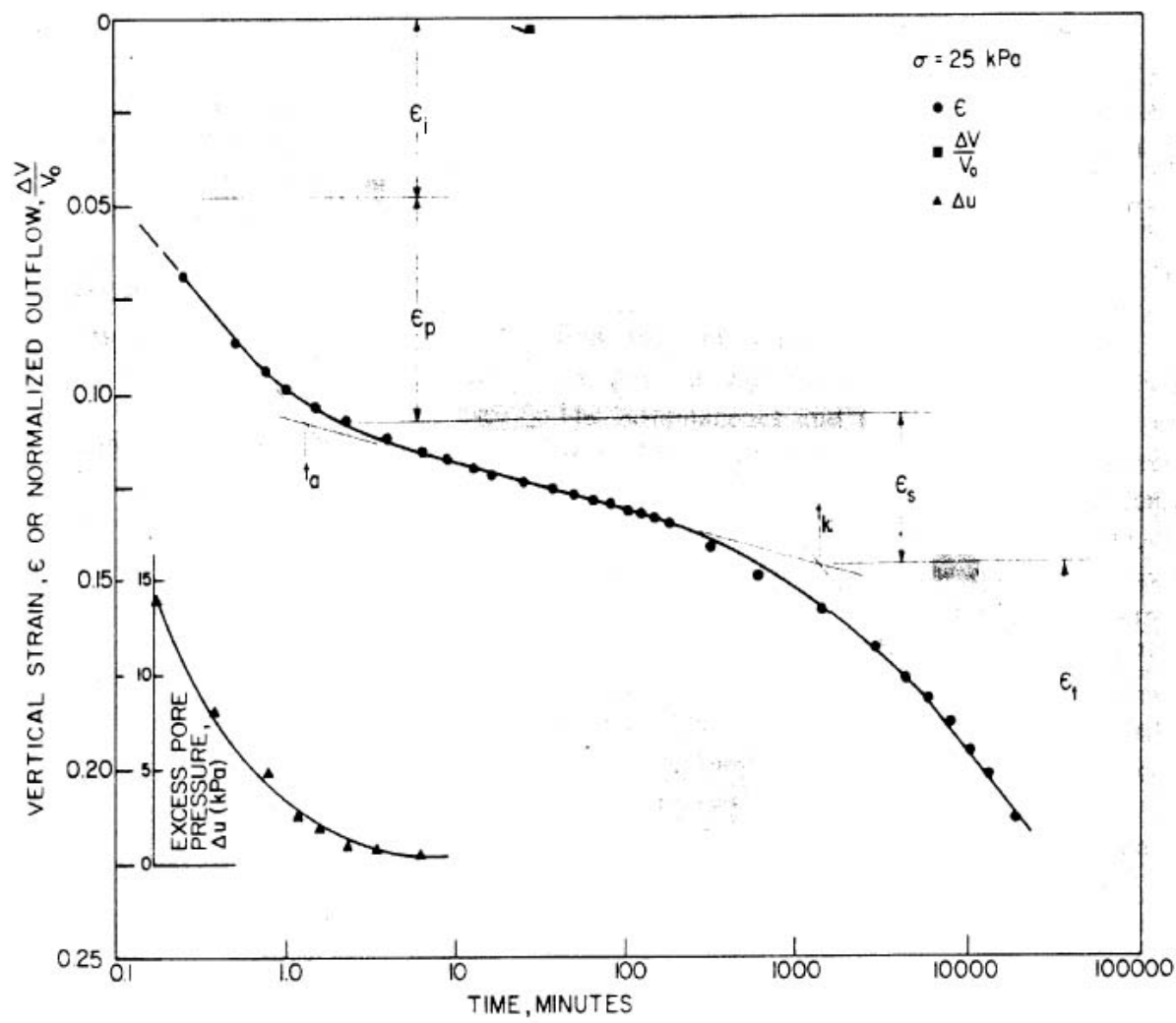

Figure 2.1: Strain versus time plot for consolidation of Portage Peat at $\sigma=25 \mathrm{kPa}$ (Dhowian and Edil, 1981) 


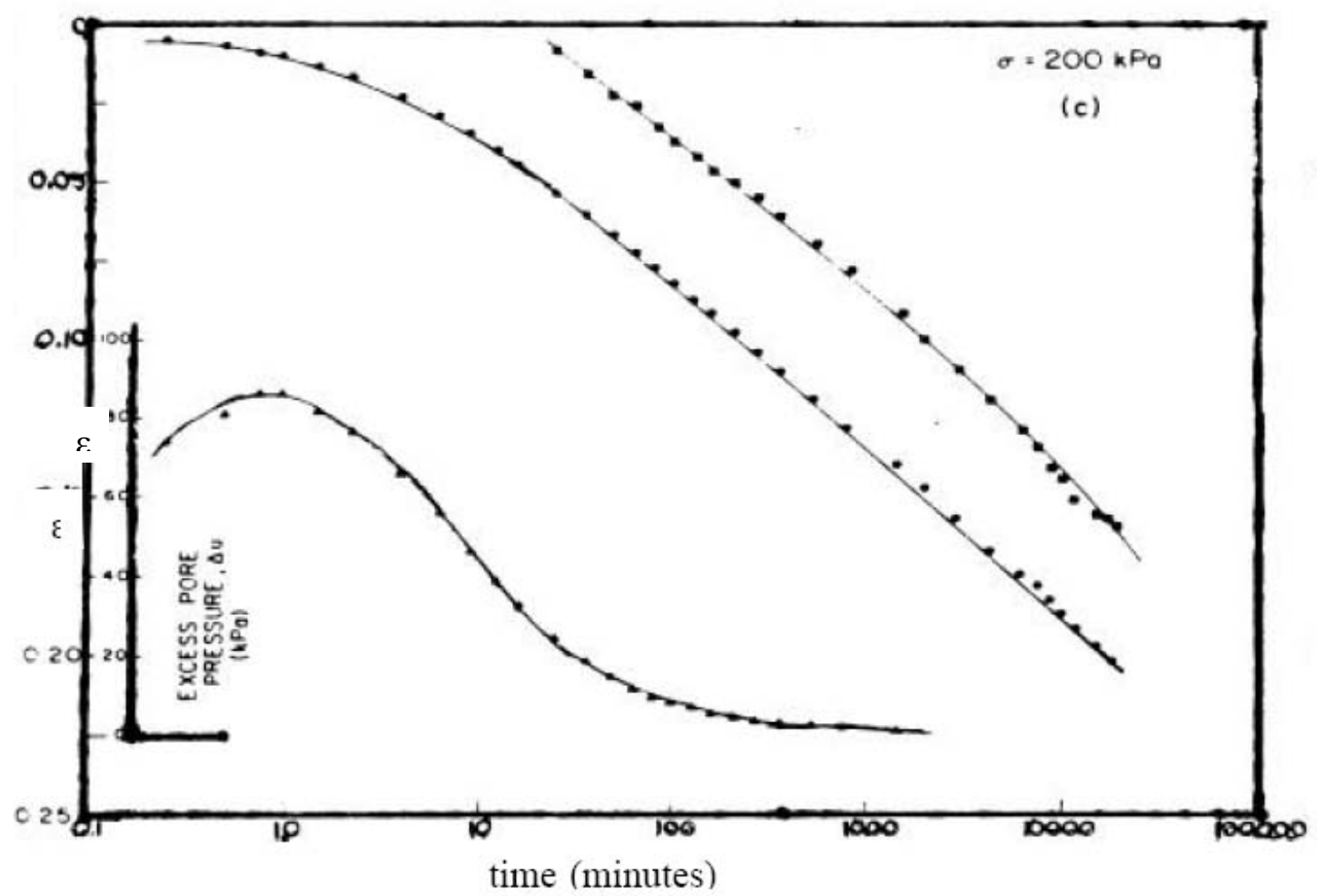

Figure 2.2: Strain versus time plot for consolidation of Portage Peat at $\sigma=200 \mathrm{kPa}$ (Dhowian and Edil, 1981) 


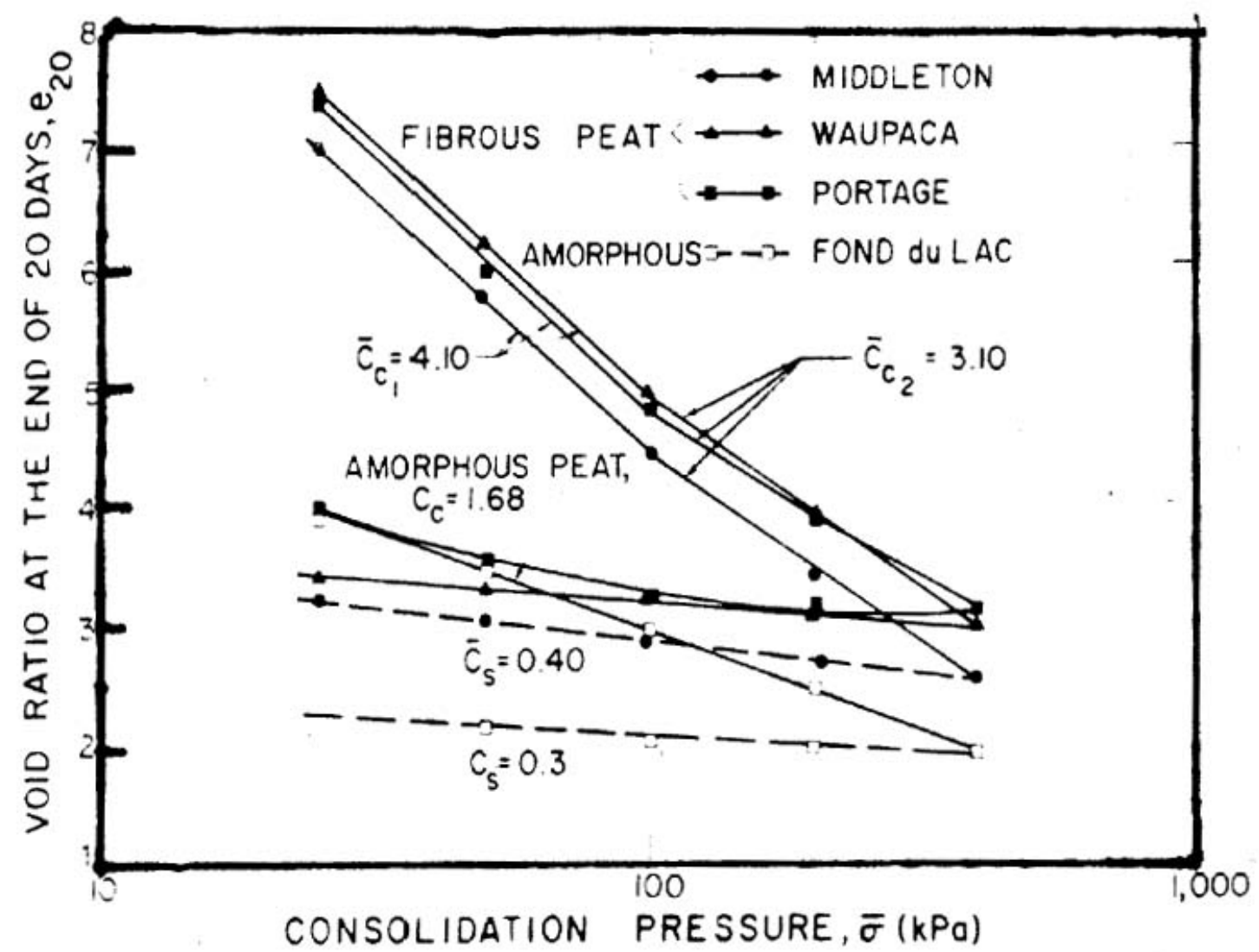

Figure 2.3: Compression curves for consolidation tests on multiple peats (Dhowian and Edil, 1981) 


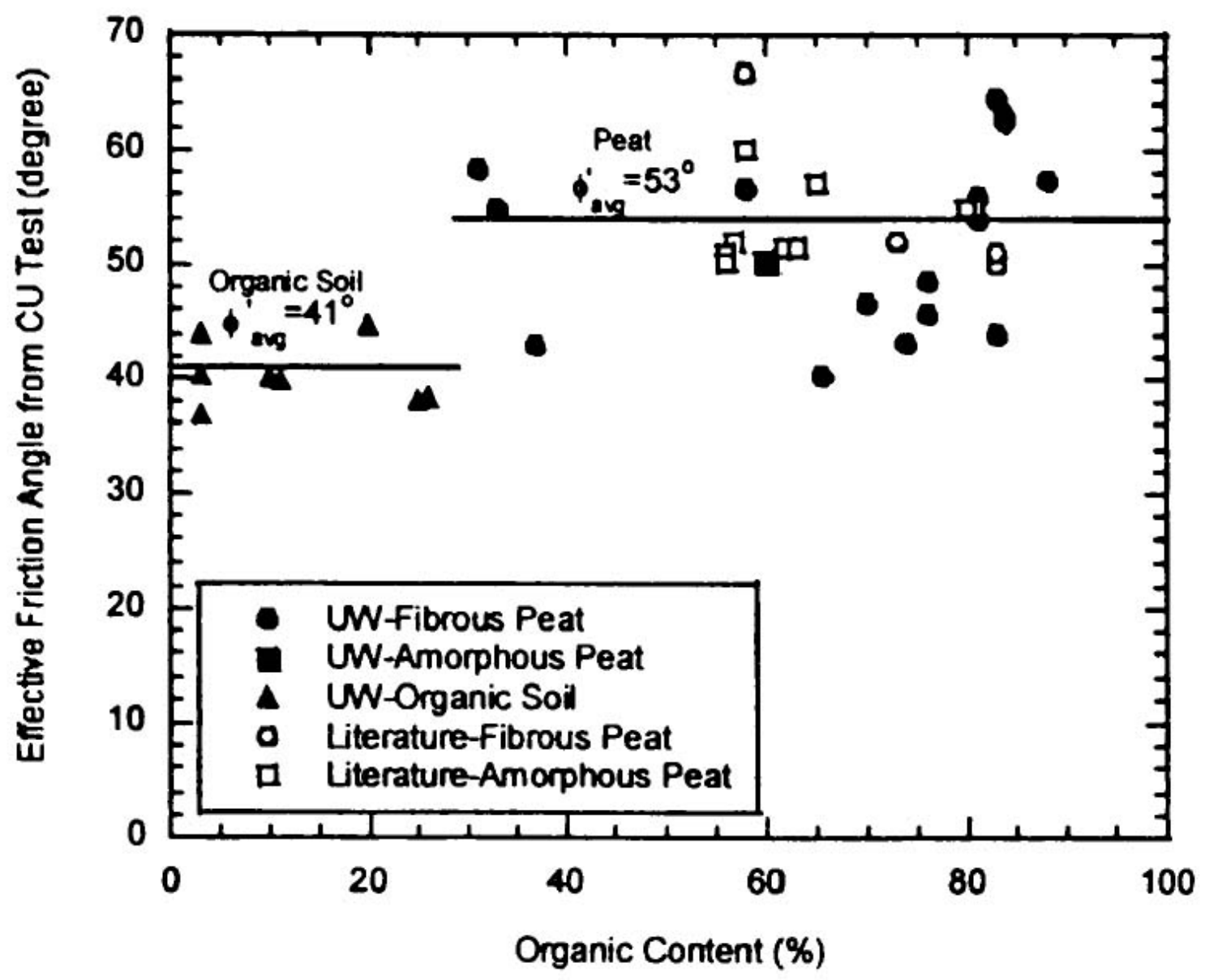

Figure 2.4: Effective friction angle ( $\phi$ ') versus Loss of Ignition (LOI) (Edil and Wang, 2000) 


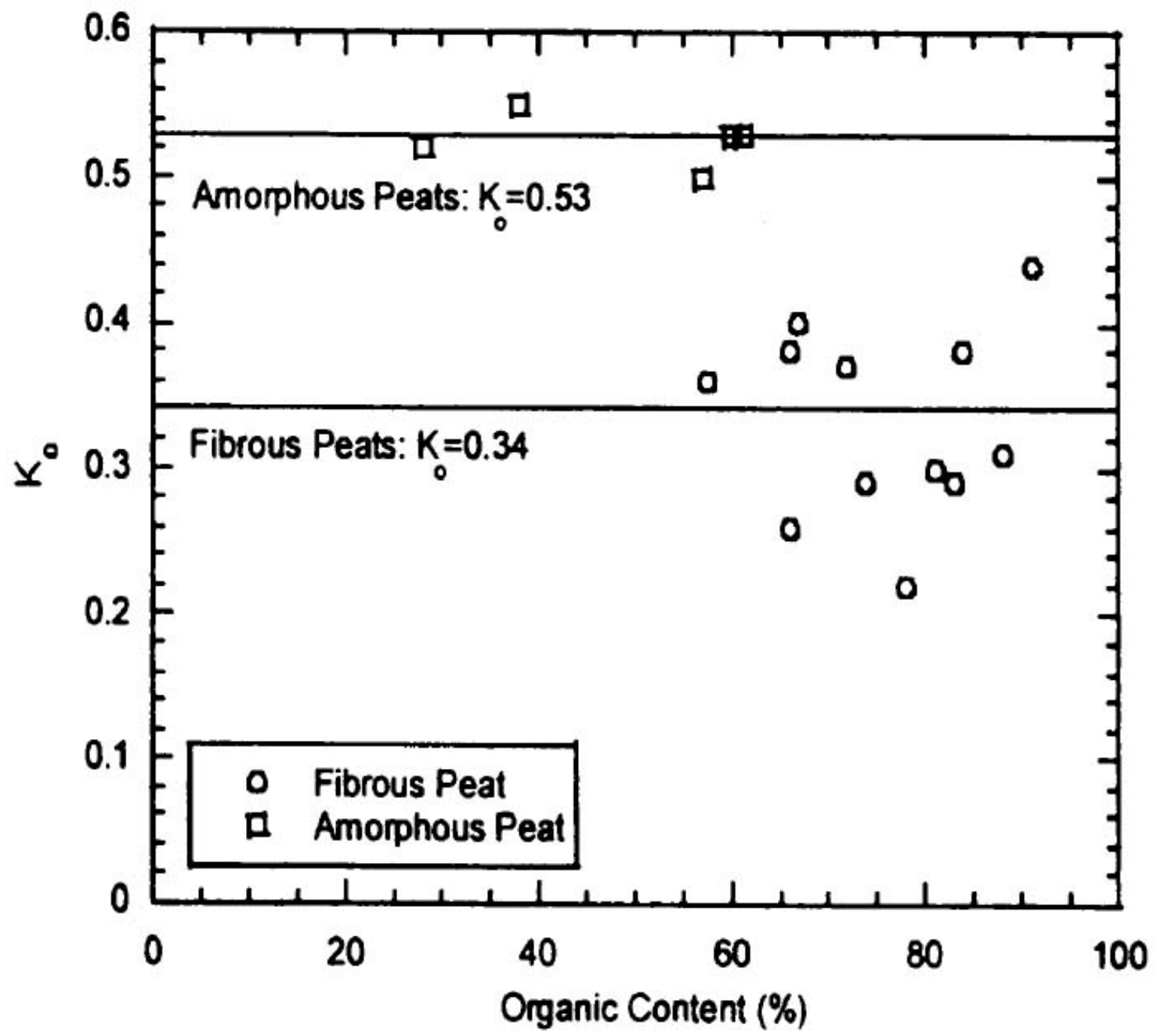

Figure 2.5: $\mathrm{K}_{\mathrm{o}}$ versus Loss of Ignition (LOI) (Edil and Wang, 2000) 


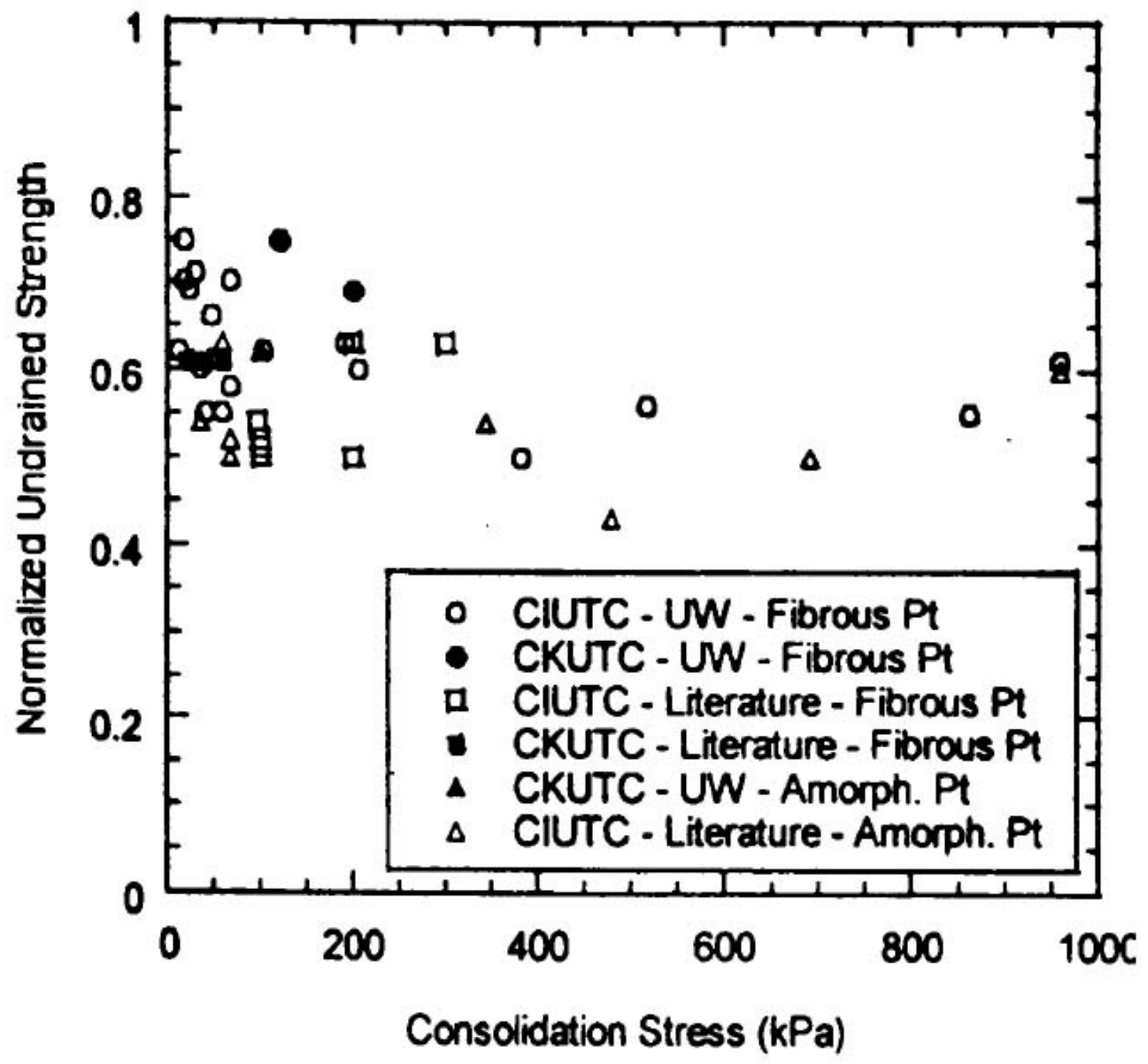

Figure 2.6: $\mathrm{C}_{\mathrm{u}} / \sigma^{\prime}{ }_{\mathrm{v}}$ vs. $\sigma^{\prime}{ }_{\mathrm{v}}$ (Edil and Wang, 2000) 


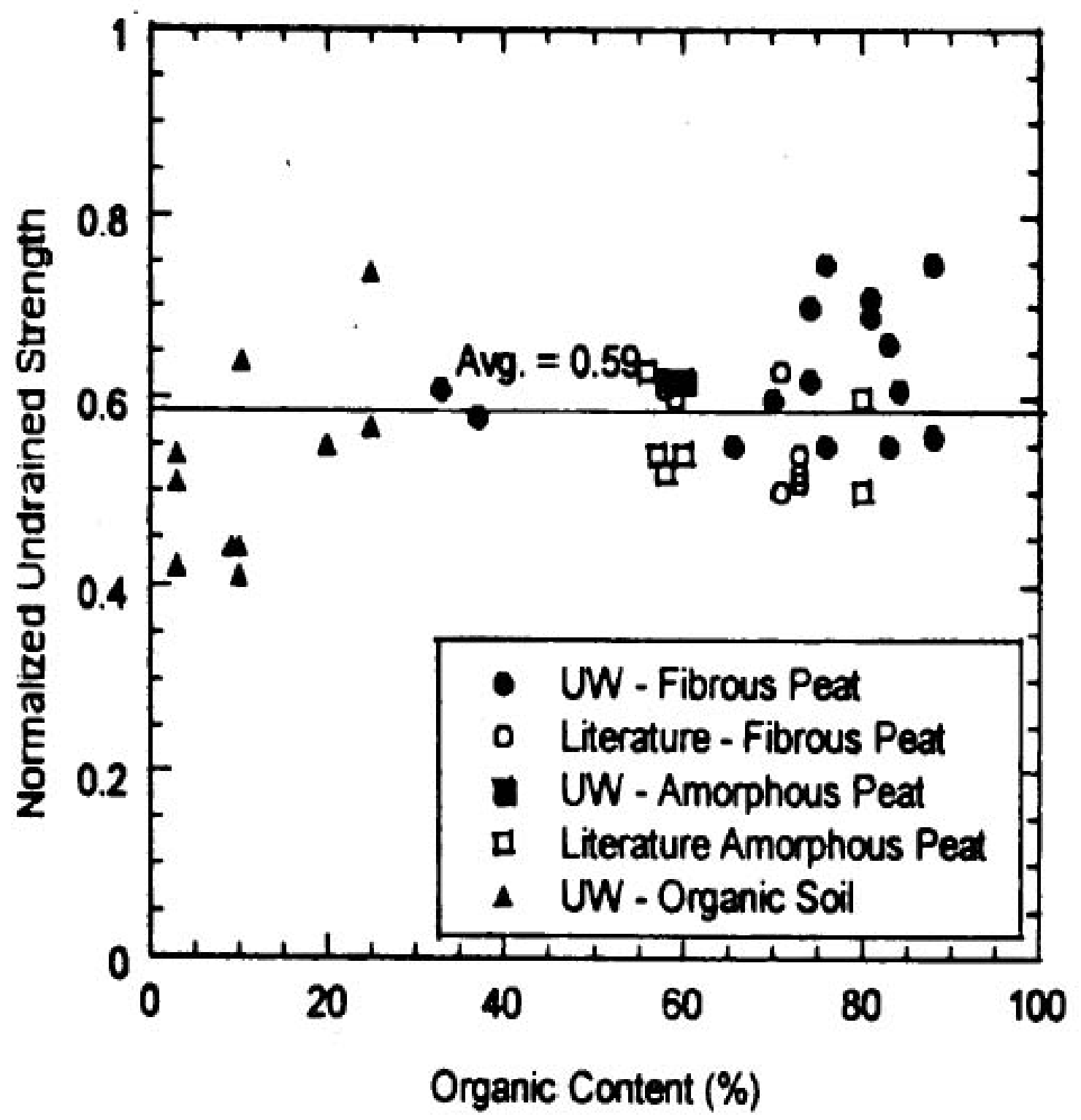

Figure 2.7: $\mathrm{C}_{\mathrm{u}} / \sigma^{\prime}{ }_{\mathrm{v}}$ vs. LOI from laboratory tests (Edil and Wang, 2000) 


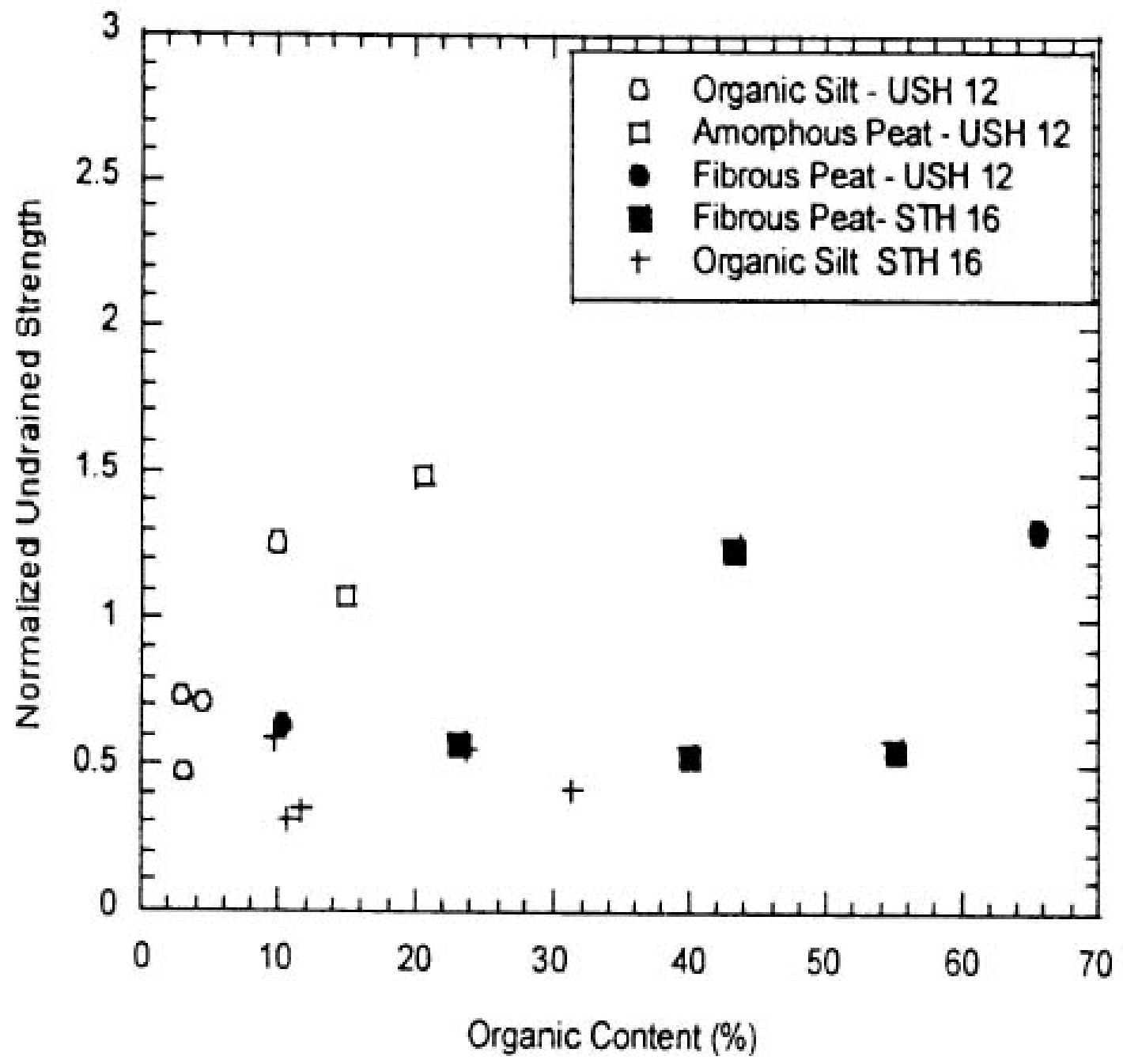

Figure 2.8: $\mathrm{C}_{\mathrm{u}} / \sigma^{\prime}{ }_{\mathrm{v}}$ vs. LOI from field vane tests (Edil and Wang, 2000) 


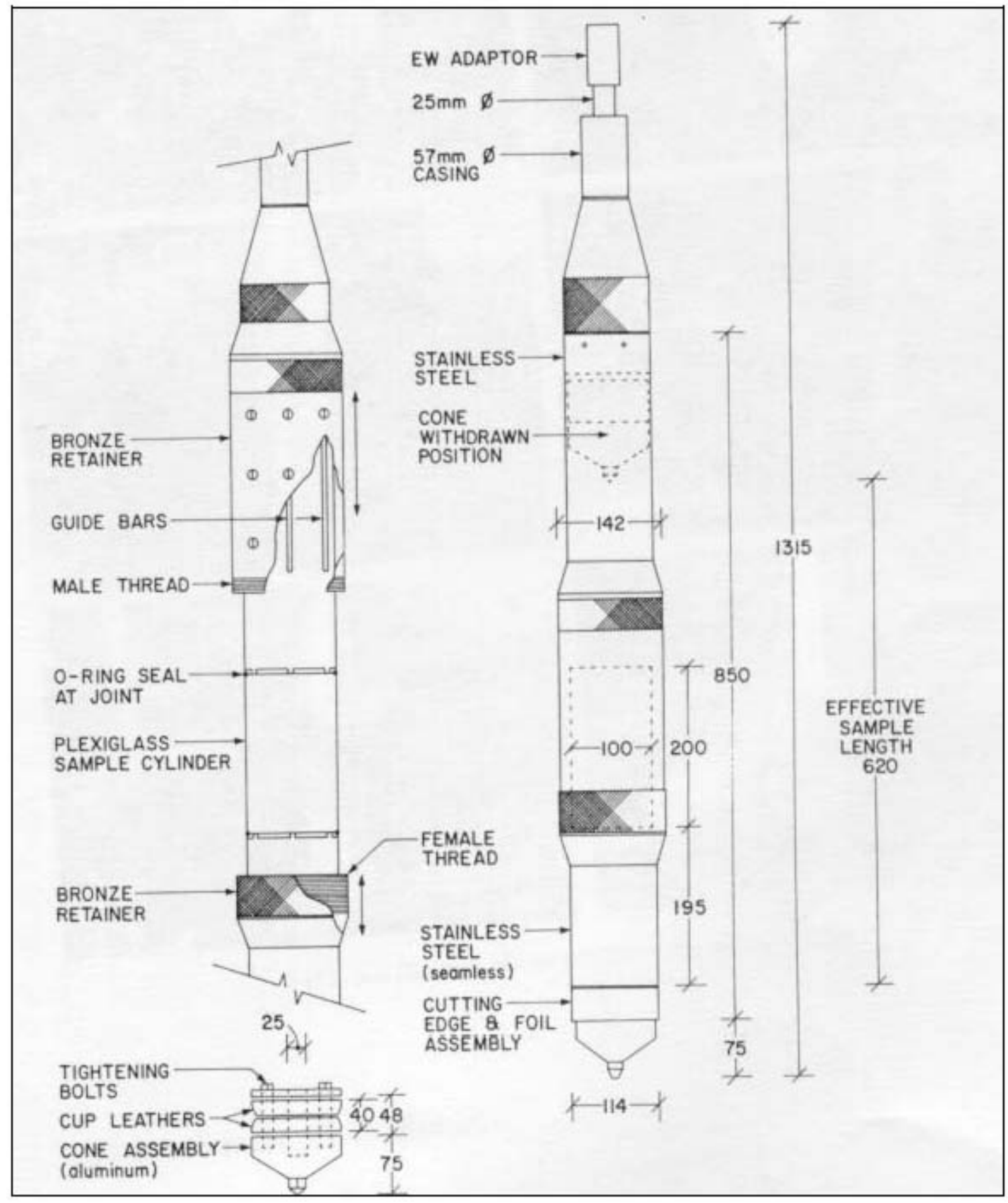

Figure 2.9: Schematic of 100 mm piston sampler (Landva et al., 1983) 


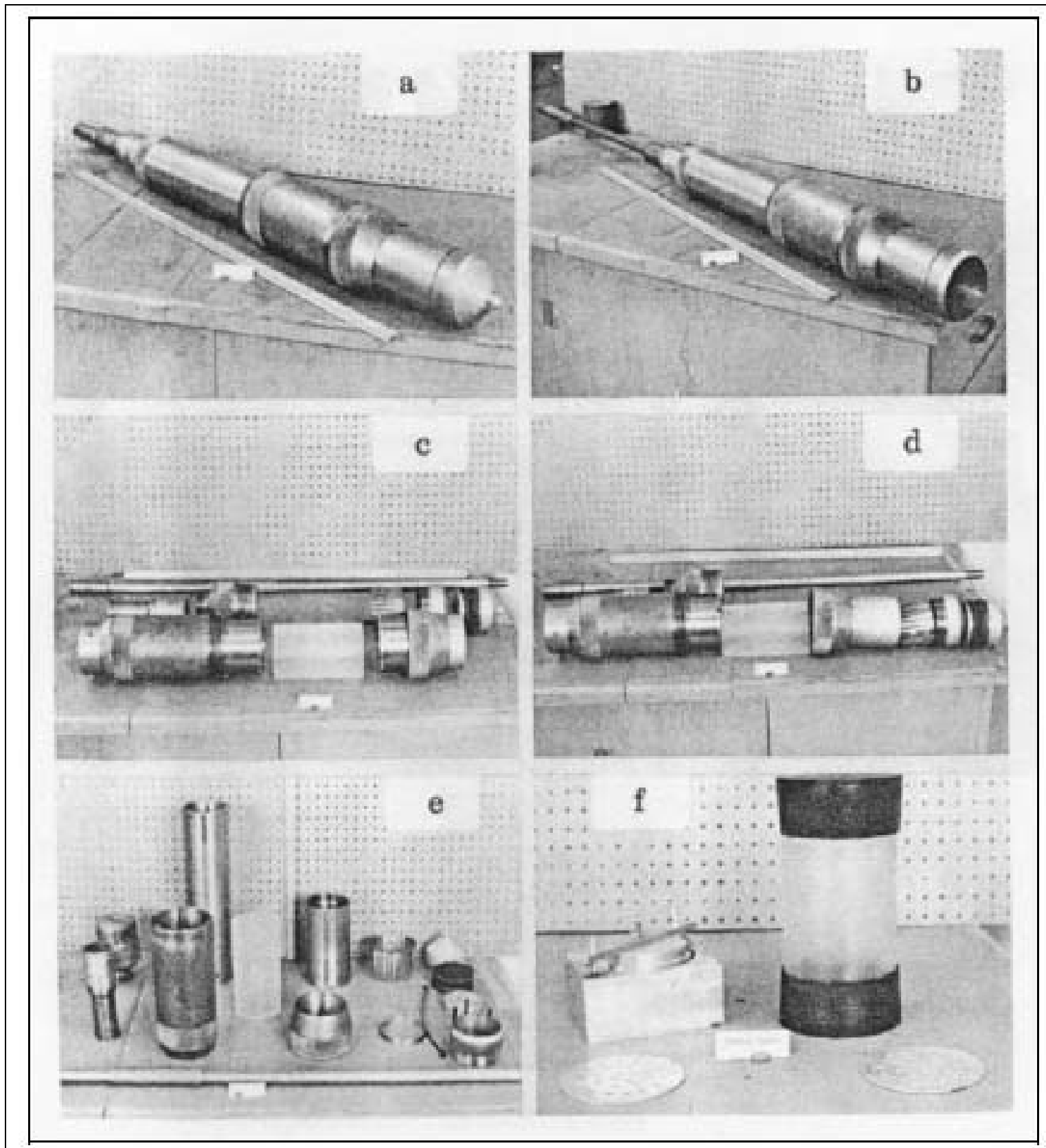

(a) to (b): Fully assembled piston sampler

(c) to (f): Plexiglas insert

(d): optional foil retainer

(f): mold for making rubber caps

Figure 2.10: Photograph of $100 \mathrm{~mm}$ piston sampler 


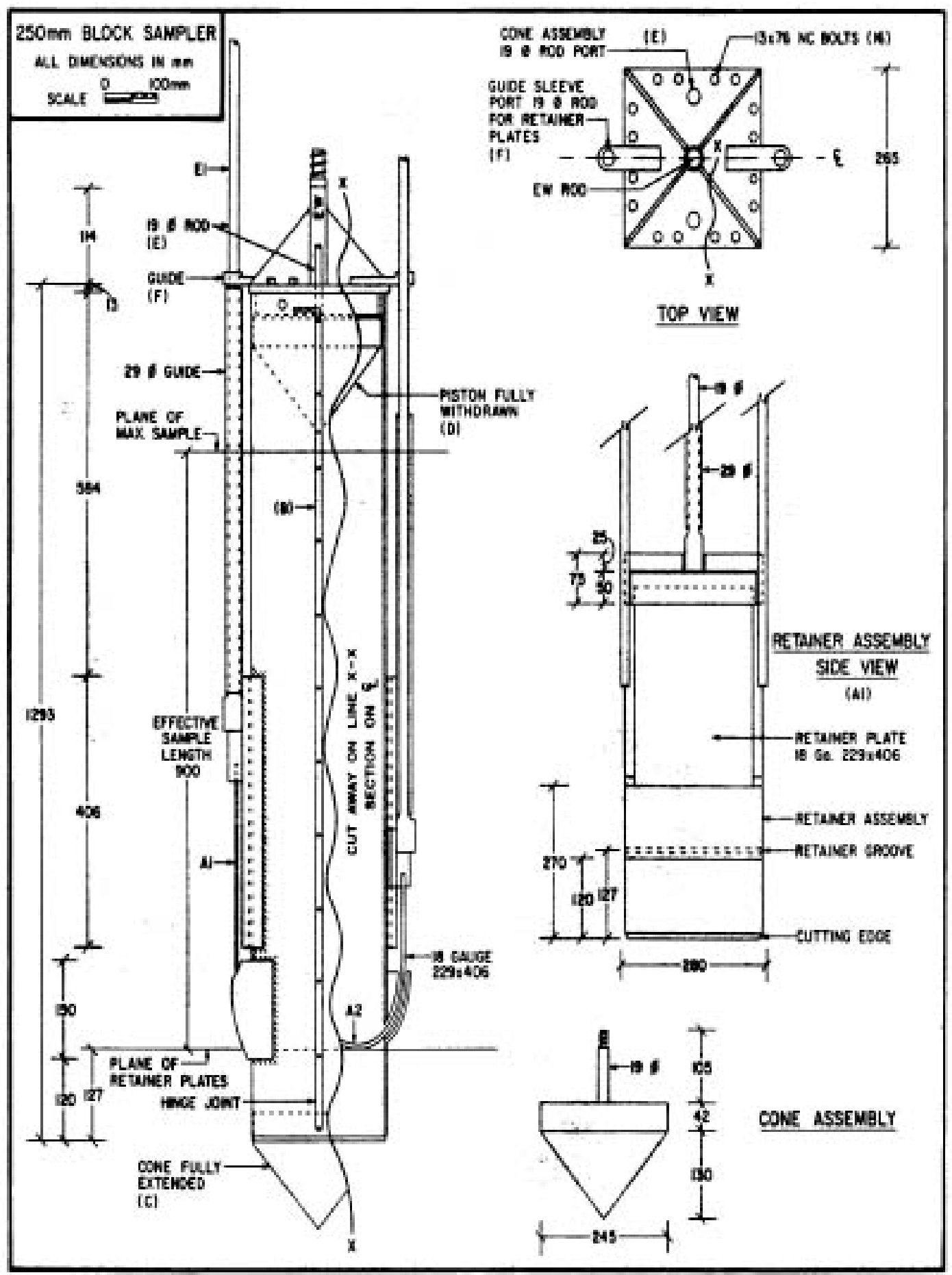

Figure 2.11: Diagram of 250-mm-square block sampler (Landva et al. 1983) 


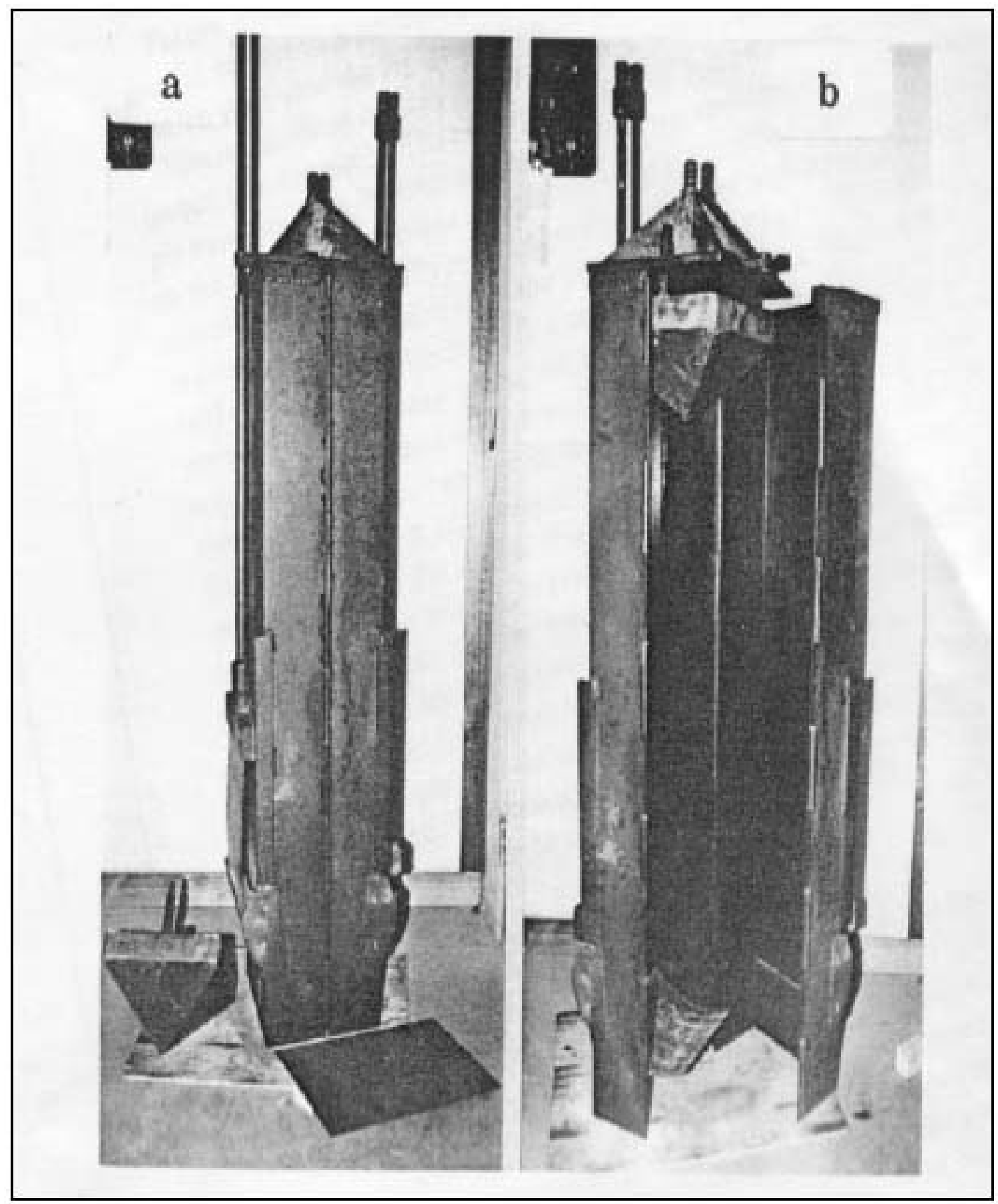

(a): sampler in sampling position

(b): sampler open with retainer plates in fully closed position

Figure 2.12: Photograph of 250-mm-square block sampler (Landva et al. 1983) 

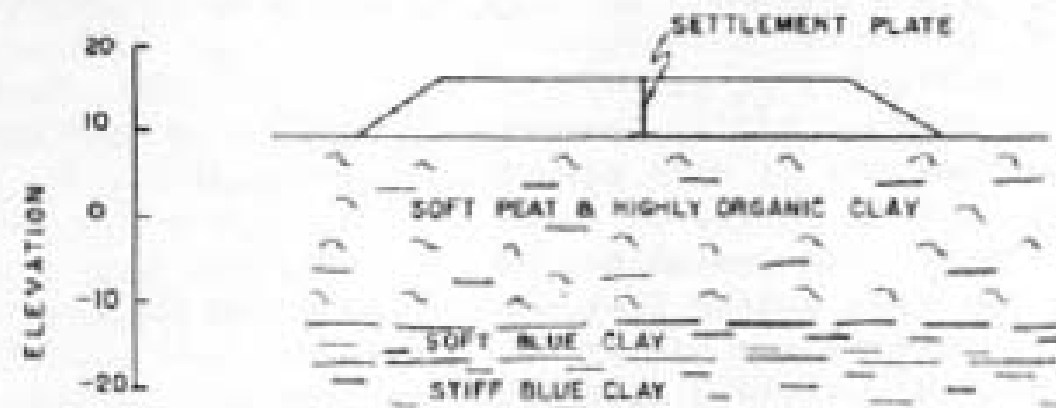

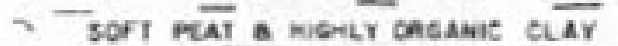

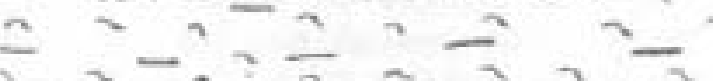

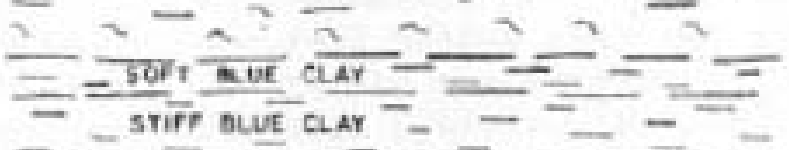

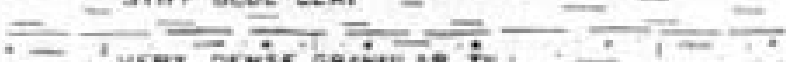

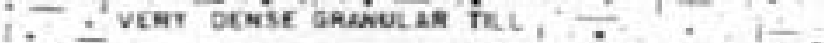
nOTE SET TLEUINT NOT ILUNTAATE *

[a] SECTICN SHOWNS STRATIGAMMr a INSTRUNENTATION

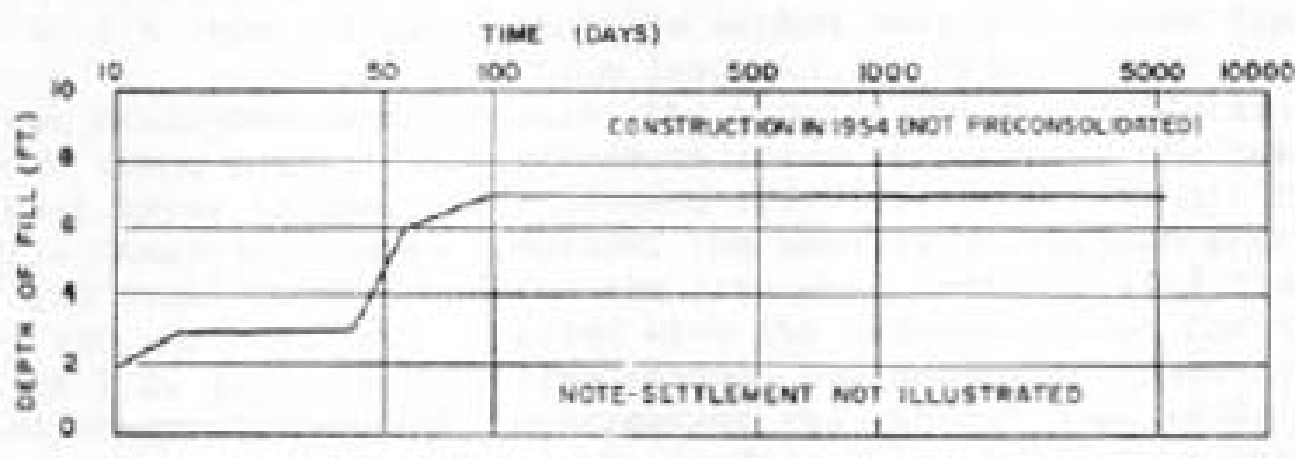

(b) LOAD Diaghau

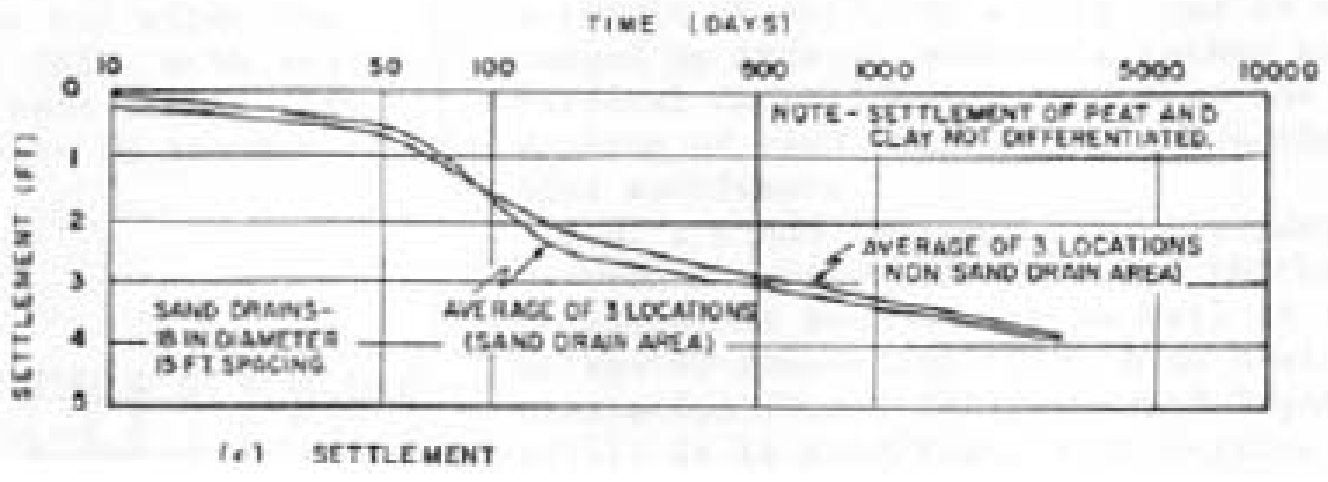

Figure 2.13: Lougheed Highway at Maillardville (Saski, 1982) 


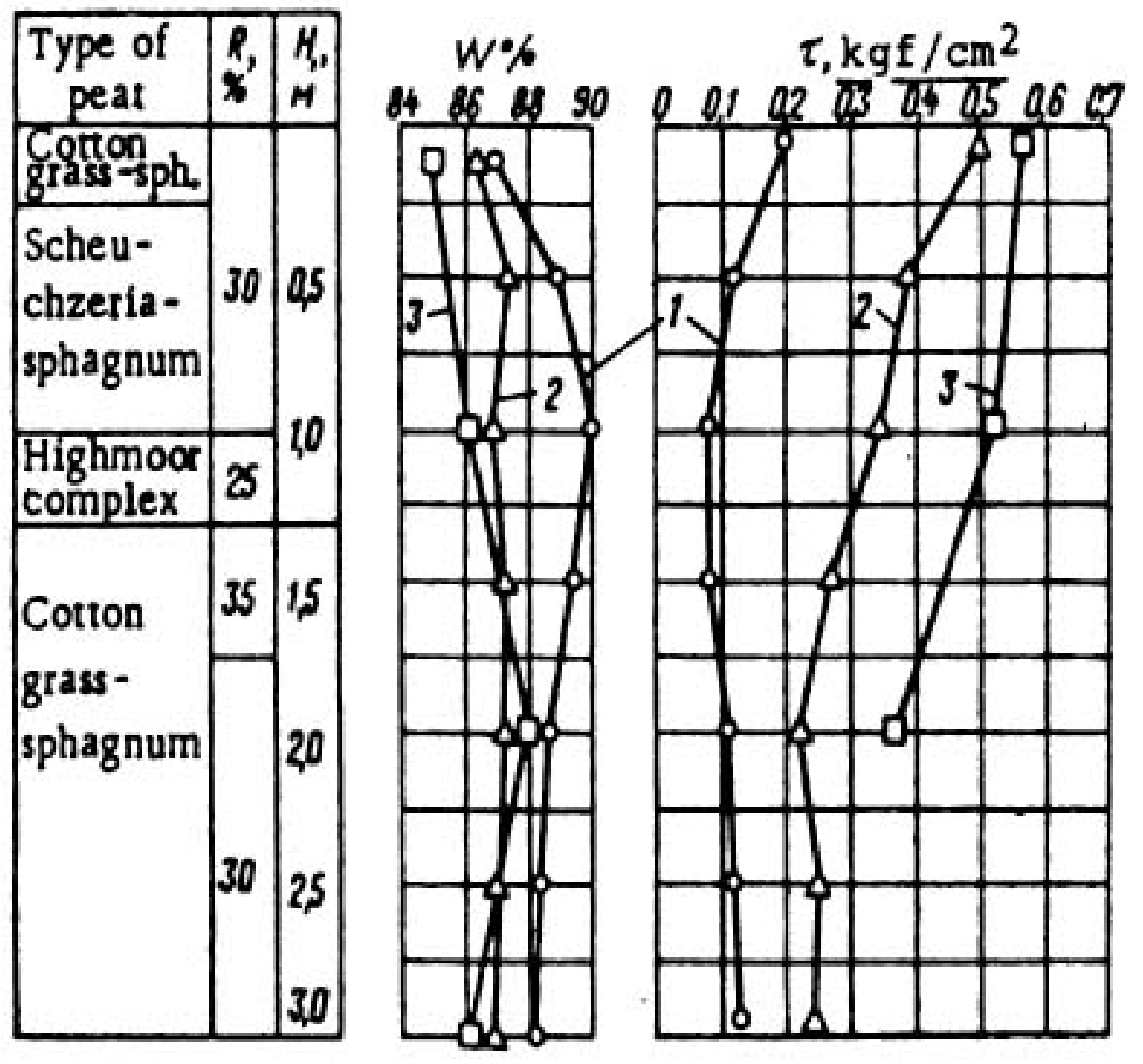

1) Before fill placement on the embankment

2) 30 days after fill placement on undrained peat

3) 30 days after fill placement on drained peat

Figure 2.14: Experimental curves of the change of water content and shear strength of peat with use of vertical sand drains (Sasaki, 1982). 


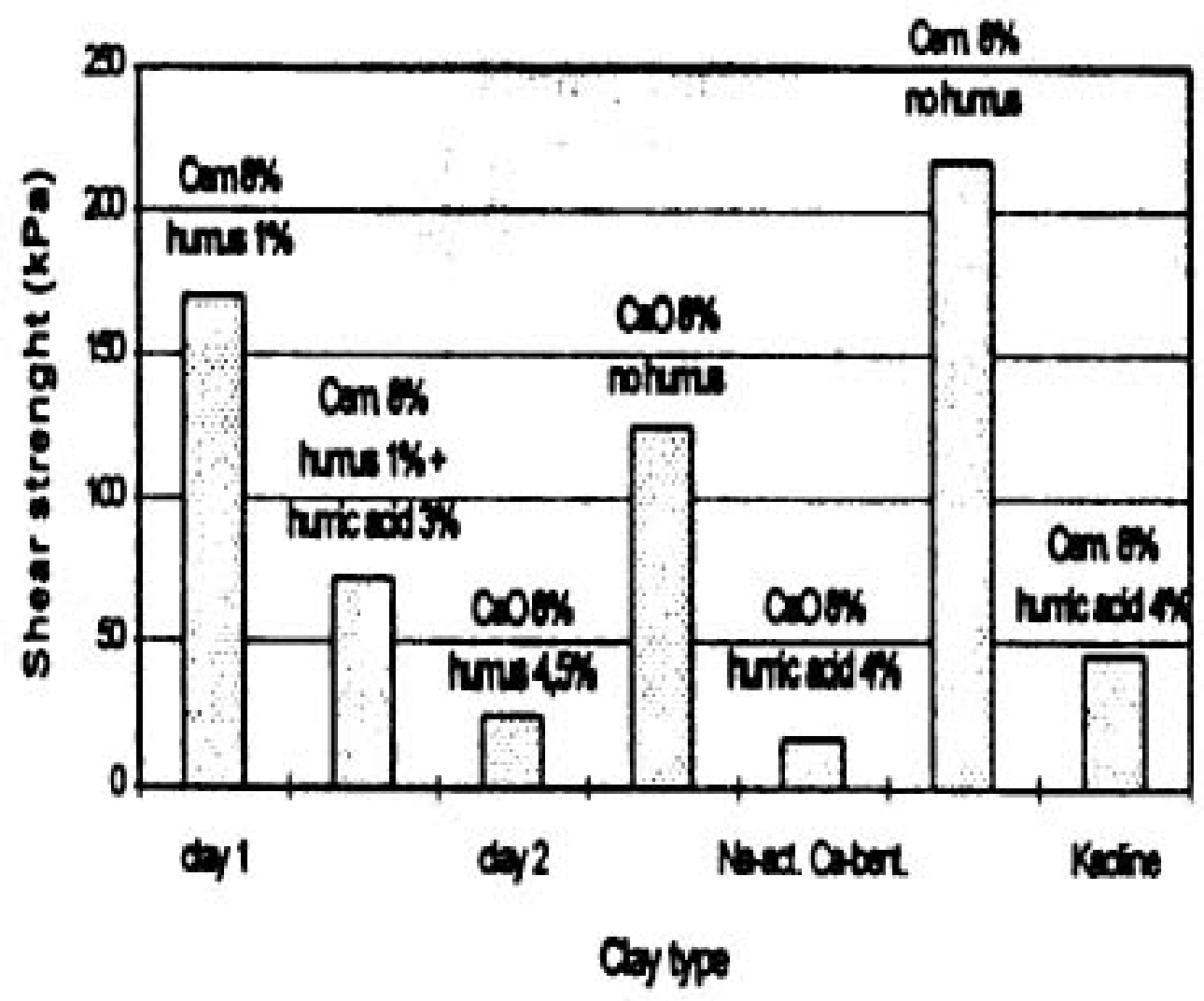

Figure 2.15: Effects of humic acid on the strength of clay samples stabilized with $\mathrm{CaO}$ (8\%) and ordinary Portland cement (8\%) as binding agents (Kujala et al. 1996). 


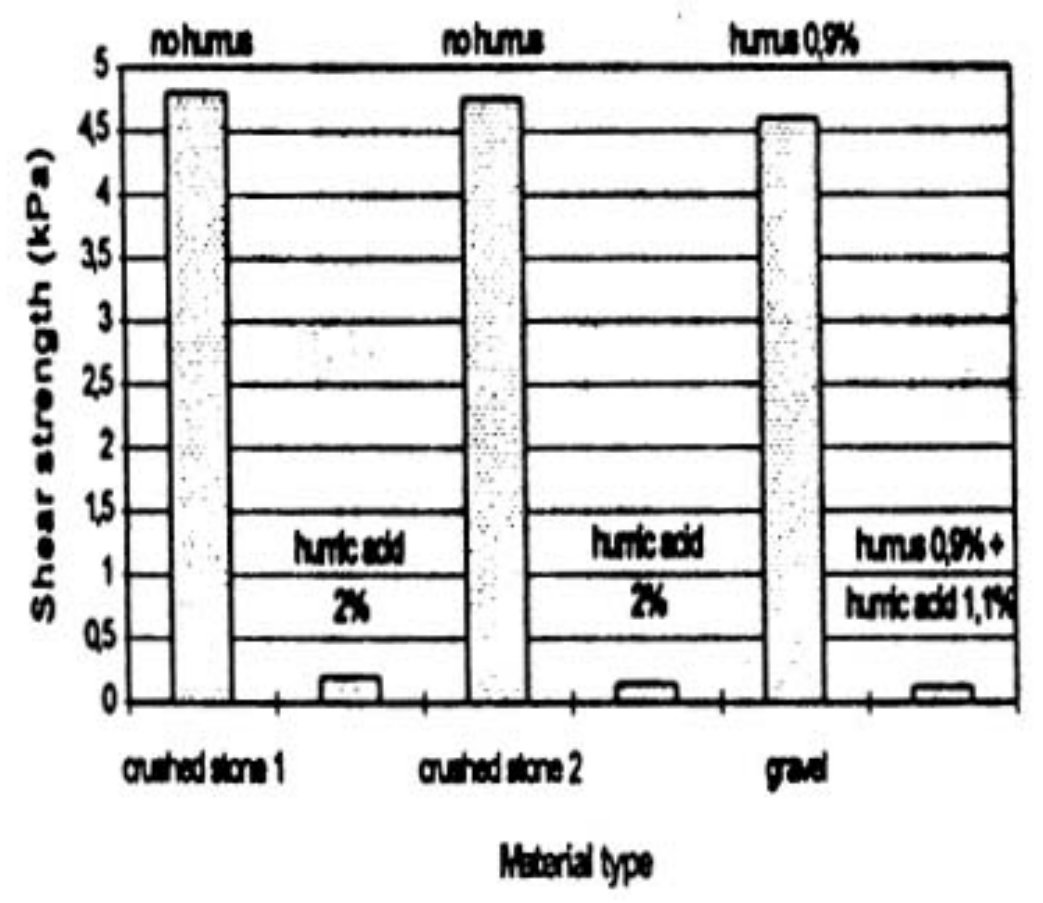

Figure 2.16: Effects of humic acid on the strength of samples of coarse-grained materials stabilized with ordinary Portland cement (8\%) (Kujala et al. 1996) 


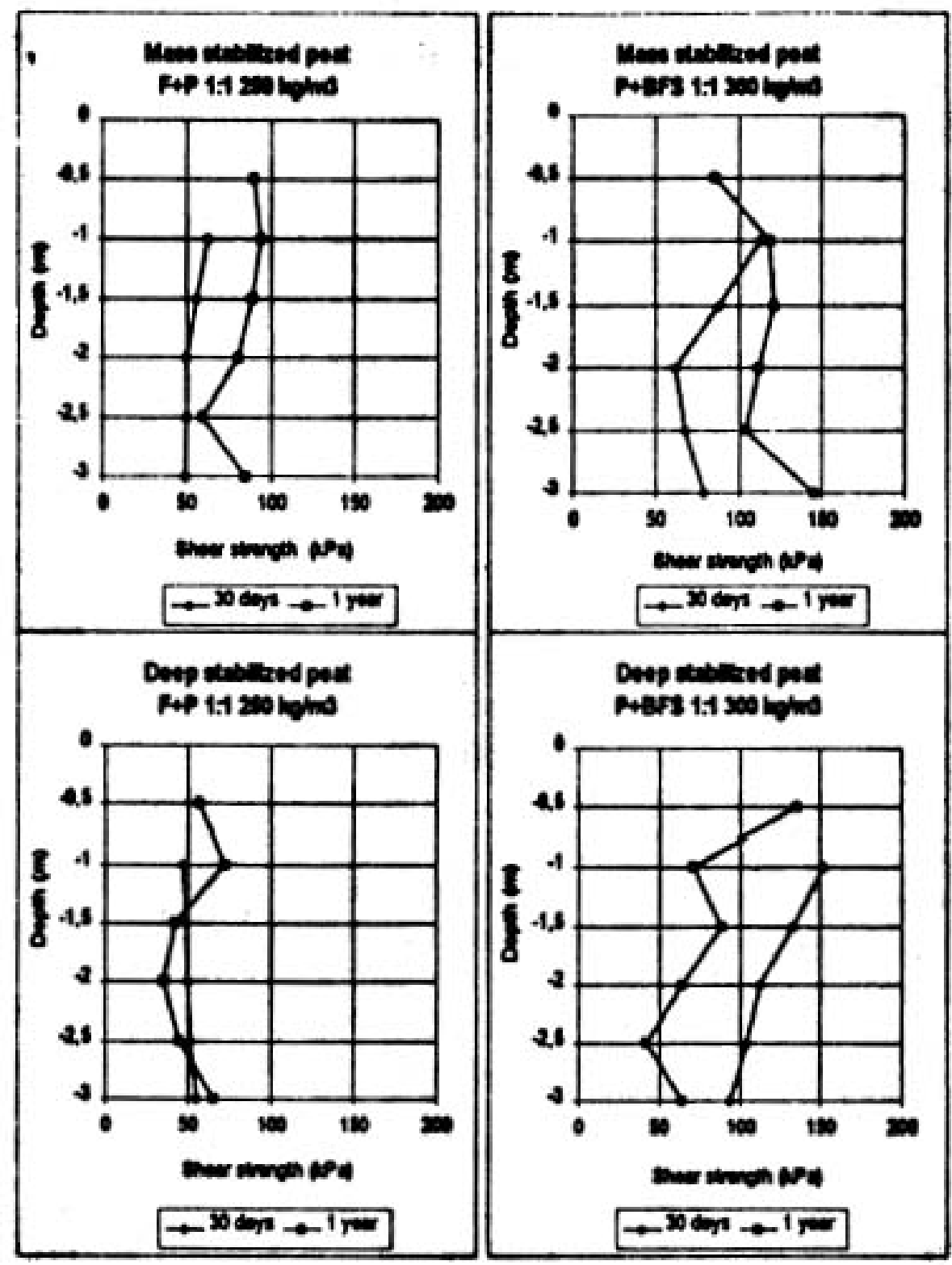

Figure 2.17: Shear strength of stabilized peat as determined by column drilling and vane shear tests after intervals of 30 days and one year. 
CHAPTER 3: LINDBERG ROAD SOIL

CHAPTER 4: EXPERIMENTAL METHODS

CHAPTER 5: STRENGTH: UNCONFINED COMPRESSION TESTS

CHAPTER 6: STIFFNESS: CRS CONSOLIDATION TESTS

CHAPTER 7: CONCLUSIONS AND RECOMMENDATIONS 


\section{CHAPTER 3: LINDBERG ROAD SOIL}

\subsection{INTRODUCTION}

To assess the effects of treatment on the compressive strength and the stiffness of highly organic soils, the soil sampled in the vicinity of Lindberg Road was employed in the experimental program. Section 3.2 describes the procedure to obtain disturbed and undisturbed LR soil samples. Section 3.3 presents the natural water content and the results of index tests of LR soil.

\subsection{SAMPLING OF LR SOIL}

The soil that is studied in this report and referred to as Lindberg Road soil was obtained from the edge of a bog near Purdue University. The Indiana Department of Transportation in conjunction with the City of West Lafayette were involved with the identification of this particular site as being likely to contain highly organic soils. This site is at the edge of the "Celery Bog" park, and can be found to the North side of Lindberg Road in West Lafayette, approximately $0.8 \mathrm{~km}$ West of the intersection with Northwestern Avenue (Figure 3.1).

Following visual reconnaissance from Lindberg Road, this site could easily be identified as being likely to contain highly organic soil. The high water table, abundant vegetation such as cattails and reed plants, and other swamp-like characteristics were strongly indicative of organic soils. Further conference with the city engineer of West Lafayette yielded boring logs from 1996. As shown in Figure 3.2, these boring logs indicated that the site possessed highly organic soils and provided a satisfactory source from which to begin an investigation on the treatment of these soils.

According to the four boreholes taken from the bog area, a $1-1.5$ thick crushed limestone fill layer was encountered beneath the pavement of the roadway. The crushed limestone was underlain by a second fill layer described as silty clay or clay loam with occasional sand seams, down to a depth between 1.37 and $2.74 \mathrm{~m}$. Below the second fill layer, a peat layer of about 3.0 - 3.7 m thick was encountered, which was underlain by a 
$1.4-1.7 \mathrm{~m}$ thick marl layer. The groundwater table observed after the completion of drilling was above (RB-1 and 2) or in the upper part (RB-3 and 6) of the peat layer. The marl layer was underlain by silty clay, clay loam and loam soils to the maximum depth explored in the borings. Two borehole data taken from the outside of the bog area (RB-4 and 5) showed discontinuity of some of the layers in that neither the peat nor the marl layers were encountered up to the depth of $4.6 \mathrm{~m}$

Following the identification of this site as a source for relevant and abundant testing material, efforts were made to obtain soil from this site. These efforts were divided into obtaining two different types of samples: disturbed and undisturbed. As mentioned in the previous chapter, disturbed samples are useful for classification and evaluation of index properties. These samples were also used in the treatment testing and evaluation, as is discussed in the following chapter. Undisturbed samples were also collected and are crucial for the evaluation of in-situ fabric and stress states.

Disturbed samples were collected during three different sampling trips made in February, April, and July of 2001. The trip that was made in February of 2001 involved extensive manual labor. A test pit was dug by hand measuring approximately two meters in diameter to a depth of approximately $1.5 \mathrm{~m}$ below the ground surface. A second trip was made in April of 2001 and the acquisition of disturbed samples coincided with the acquisition of undisturbed samples as will be discussed in greater detail later. In this case, a backhoe was used to open a $4.5 \mathrm{~m}$ diameter hole to a depth of approximately two meters (Figure 3.3). Due to the high groundwater table and lessons learned from the February sampling trip, a pump was used to dewater the excavation. The third trip was made during July of 2001 and involved only disturbed sampling. Since this trip coincided with the beginning of a two-year closing of Lindberg Road for construction activities, a large backhoe was present at the site and was used to excavate a six-meter diameter hole to a depth of approximately two meters. In all three cases, large plastic bins were used to collect and transport the soil to the laboratory. In addition, groundwater was collected during each sampling trip for use in the testing phase of the project.

In addition to disturbed samples, undisturbed samples were collected during the April sampling trip. The primary tool used for this was a box sampler that was created 
specifically for this project. This box sampler was created out of $1.3 \mathrm{~cm}$ thick Lexan plates to allow for a $30 \mathrm{~cm}$ x $30 \mathrm{~cm}$ x $30 \mathrm{~cm}$ block of soil to be collected. Diagrams of the box sampler are shown in Figure 3.4 - 3.6. Some of the important features are listed below in no particular order.

1. Lexan plates were used due to low weight and low permeability

2. Air holes on top allow for air to escape during driving

3. Eye-bolts on top allow box to be carried with cables

4. Sliding metal plate allows bottom of soil block to be cut

5. Beveled bottom edges allow for easier driving

6. Bolted top and back allow for easy disassembly for sample removal

The sampling box (Figure 3.7) provided a relatively efficient method for producing undisturbed samples. The following description details the steps involved with this process.

1. The location for sampling was identified.

2. The backhoe removed surface vegetation to prepare a clean surface.

3. The backhoe proceeded to open an excavation approximately $1.7 \mathrm{~m}$ deep and $4.6 \mathrm{~m}$ in diameter.

4. The dewatering pump was inserted into the excavation as the walls and bottom of the excavation were straightened by hand.

5. The backhoe excavated $0.5 \mathrm{~m}$ below the existing excavation floor, leaving a $0.5 \mathrm{~m}$ wide by $0.5 \mathrm{~m}$ tall bench in the middle of the excavation (Figure 3.8).

6. The bottom metal blade of the block sampler was removed and the box was placed on the top of the bench.

7. The block sampler was systematically pushed down by hand while the soil was trimmed away from the leading edges.

8. Once the soil had filled the entire block sampler, the bottom-cutting blade was inserted into the box (Figure 3.9).

9. Another block sample was obtained from the same bench as the first sample. 
10. The block samples were removed from the excavation and the hole was backfilled with the original soil.

Additional samples were collected into glass jars during the sampling trips to maintain the soil at its natural water content. The disturbed samples were stored in large plastic containers that were sealed with duct-tape and plastic wrap. Following sampling, the undisturbed samples were immediately transported to a humid room with approximately 93\% relative humidity adjacent to the geotechnical engineering laboratory at Purdue University. These samples were stored inside the block sampler after sealing all openings on the sampler with a combination of duct-tape and plastic wrap.

Approximately one month after sampling, the boxes were opened and the soil block is divided into smaller blocks for testing and for better preservation during storage. The bolts on the sides of the box allowed it to be taken apart to access the soil inside. Once the large soil block was removed from the sampling box, it was divided into smaller cubes through the use of a wire saw and a sharp knife blade. The smaller blocks were then placed on a sheet of Plexiglas and sealed with two layers of plastic wrap and wax to preserve the moisture content as completely as possible (Figure 3.10). All of the blocks were then placed back in the humid room where they were stored until testing.

\subsection{CHARACTERIZATION AND CLASSIFICATION OF LR SOIL}

Once the soil is obtained, it is necessary to begin the process of characterizing the material. The classification and evaluation of index properties provide a starting point from which to begin to understand the behavior of the soil. The analysis of these characteristics gives a framework from which other soils from available literature or future sampled soils may be compared. Estimations can then be made as to the behavioral characteristics that are likely to be encountered.

The index properties and the unit weights of different types of peats and organic soils collected in Wisconsin and Minnesota are summarized in Table 3.1 for comparison with LR soil. In this table, soils with organic content greater than $20 \%$ are classified as organic soils and peats with more than $30 \%$ fiber content are classified as fibrous peats. 
Peats have the natural water content ranging from 150 to $655 \%$, and the specific gravity ranging from 1.40 to 2.23. Organic soils have the natural water content ranging from 50 to $367 \%$, and the specific gravity ranging from 2.29 to 2.63 .

This section focuses on the characterization tests that were performed on the disturbed soil samples. The following sections are organized according to the tests performed.

\subsubsection{Water Content}

The characterization process begins with the evaluation of the water content of the soil. British Standard (1377, Test 1(A)) recommends a drying temperature of $60^{\circ} \mathrm{C}$ for peats and soils containing organic matter to prevent oxidation of the organic content. However, this test was performed in accordance with ASTM D2974, in which the change in mass of a soil sample was measured following a drying period under a temperature of $105+/-5^{\circ} \mathrm{C}$. This test was performed immediately following sampling on soil collected in sealed glass jars. Expediency was necessary between the sampling and testing phase to insure that the measured water contents reflect the field conditions as accurately as possible.

Table 3.2 lists the average results from these tests organized by the month in which the samples were taken. The average water content of the samples collected in February is significantly lower than the average values of the other two samples. This is because the soil was collected above the ground water table in February. However, since the undisturbed block samples were taken during the April sampling trip, and since this was the first trip where large quantities of disturbed samples were taken for testing, the water content of $289 \%$ is taken as the in-situ water content.

\subsubsection{Organic Content}

Following the immediate assessment of the water content, the next piece of vital information is the organic content. Organic content, also referred to as Loss On Ignition (LOI), is simply a mass measurement of the abundance of organic material present in the 
soil. This test was performed in accordance with ASTM D2974 during or prior to the sample preparation procedure (see section 4.3 for detail). In this test, an oven dried soil specimen was placed in a muffle furnace and subjected to a temperature of $440^{\circ} \mathrm{C}$ until the mass of the sample did not change upon further heating. The remaining "ash" is the mineral content of the soil, with the lost mass constituting the organic material. The organic content may then be expressed as a percentage of the original oven-dried mass.

The average water content and organic content of LR soil from each soil bin employed in the experimental program are summarized in Table 3.3. The source number indicates the trip number during which the soil was sampled: for instance, source II indicates the soil sampled during the second trip, April of 2001. The April sample exhibits slightly higher water content and organic content: $261.2 \%+/-32.1$ and $51.8+/-$ 4.8, respectively, than the July sample: $195.3+/-20.7$ and $44.5+/-4.0$, respectively. It should be noted that although failing to satisfy the ASTM requirement (D4427) to be classified as a peat (higher than 75\%), LR soil satisfies the INDOT criteria (higher than $25 \%$ ) for being labeled as peat.

\subsubsection{Specific Gravity}

The specific gravity is essential for phase relationship and calculations of unit weights and stress. The specific gravity of most soils generally lies between 2.65 and 2.85 , and thus is not very useful in classification. For soils consist of various minerals or contain substantial quantities of organic matters, the specific gravity may be considerably lower values, sometimes below 2.0 (Table 3.1). In that case, the specific gravity is the average of all the grains constitute the soil.

To obtain accurate value of specific gravity of LR soil, tests were performed in accordance with ASTM D854. It is recommended to use Gas pycnometers (ASTM D5550) or kerosene as the fluid for soils having solids that can dissolve or float in water. However, those materials were not observed in LR soil during testing, and the test was performed using deionized water. In the test, combination of heat and vacuum was applied to remove the entrapped air in the soil. Using only vacuum was not sufficient to remove entrapped water and yielded values significantly different from the values 
measured using heat and vacuum combination. During the deairing process, the slurry was agitated often to make sure that small air bubbles could move up easily and the soil would not stick to the glass.

The average values of specific gravity of LR soil are summarized in Table 3.4. The specific gravity of LR soil ranges from 1.929 to 1.934 . For calculation of initial void ratio of each soil sample, the specific gravity of LR soil is assumed as 1.93 in this research.

\subsubsection{Particle Size Distribution}

In addition to quantifying the density of the solid particles contained within the soil, the size of soil particles is extremely important. The classification of size, in combination with the determination of the Atterberg limits, provides sufficient information to assign a USCS classification.

The analysis of the particle sizes was done through a combination of wet sieving and hydrometer test, in accordance with ASTM D422. Organic matters can shrink or break when dried. Therefore, it is very difficult to use the mechanical sieving method for soils containing organic matters. For these soils, the soil particles and organic matters should be separated by washing them with water. An independent hydrometer test was performed to obtain the particle size distribution of the soil particles smaller than $2 \mathrm{~mm}$ in diameter (\#10 sieve). The specific gravity of LR soil, as the mean value of all particles, was applied to calculate the diameter of fine particles and organic matters.

The particle size distribution of LR soil is obtained by combining the results from the wet sieve and the hydrometer analysis. The particle size distribution curve is shown in Figure 3.11. The result indicates that the Lindberg Road soil contains approximately 16.1 $\%$ of sand, $43.3 \%$ of silt and $40.6 \%$ of clay as defined by USCS. Therefore, LR soil can be classified as fine-grained soils according to the USCS. 


\subsubsection{Atterberg Limits}

The Atterberg Limits provide a useful method for identifying and classifying the fine-grained cohesive soils. The liquid limit (LL) is defined as the moisture content at which the soil changes from a liquid to plastic state. The plastic limit (PL) is the moisture content at which the soil changes from a plastic to a semi-solid state. The difference between the liquid limit and the plastic limit is defined as the plasticity index (PI). The liquidity index (LI) is the ratio of the difference between liquid limit and plastic limit to the plasticity index. The liquidity index describes the relative consistency of fine-grained soils on the basis of water content in its natural state. The shear strength and the compressibility of fine-grained soils also depend on the relative consistency, or Liquidity Index (LI), of the soil.

The determination of the Atterberg limits of LR soil was performed in accordance with ASTM D4318. The soil was treated with water to 15 drop consistency prior to testing (wet method). Two types of water: deionized and tap water, were used for pretreatment of the soil. When tap water is used, the Atterberg limits, especially the liquid limit, can be affected due to the possibility of ion exchange between the soil and impurities in the water. In general, the liquid limit obtained from the soil treated with tap water is lower than the value obtained using deionized water. The liquid limit increases if excessive amount of $\mathrm{NaCl}$ presents in the water. Therefore, it is recommended to use deionized or distilled water to determine the Atterberg limits. After allowing the soil to hydrate for 16 hours, the test was preceded while drying the soil with a fan.

The Atterberg limits of LR soil from the soil bin \# II-14 are summarized in Table 3.5. The liquid limit obtained using deionized water was higher than the value obtained using tap water: $327 \%$ and $302 \%$, respectively. The plastic limit is $162 \%$, which yields the plastic index of $165 \%$. In the natural state ( $\mathrm{Wn}=289 \%$ ), the liquidity index (LI) of LR soil is 0.77 .

Another liquid limit was determined with soil from the same bin, but after the soil was dried out in an oven at $105+/-5^{\circ} \mathrm{C}$. When dried, soils can alter in many ways: chemical of the soil can change, soil particles can subdivide or agglomerate, and some of the absorbed water can evaporate, which can not be reversed by rewetting. These changes 
can affect the index properties, especially for organic clays. LR soil particles were agglomerated after oven drying, and thus the soil was grinded into finer size before the determination of the liquid limit. The liquid limit of LR soil decreased to $102 \%$ after oven drying, which yields the ratio of the liquid limits without drying and after drying about 0.312 . This value satisfies the USCS criteria to be classified as an organic soil (< $0.75)$

Based on the results from particle size distribution analysis in conjunction with the Atterberg limits, LR soil can be classified as $\mathrm{OH}$ according to the USCS (ASTM D2488).

\subsubsection{Fiber Content}

In addition to the evaluation of the size of mineral particles in the soil, the size of the organic fibrous materials was also determined. Although not a peat according to the ASTM standard, this soil was subjected to a fiber content test in accordance with ASTM D1997. This test is used to measure the amount of plant materials that are larger than 0.15 $\mathrm{mm}$ and smaller than $20 \mathrm{~mm}$. Based on this test, the average fiber content for this soil was found to be $2.29 \%$. This would categorize the soil as being "sapric" according to the ASTM definition.

\subsubsection{Soil Acidity}

As explained in the previous chapter, the acidity of a soil has a significant effect on the effectiveness of binding agents in the soil. In order to complete the characterization for this soil, a pH test was performed in accordance with ASTM D4972. In this test, an air-dried soil passed through the \#10 sieve was soaked in water and in a $0.01 \mathrm{M} \mathrm{CaCl}_{2}$ solution for one hour before testing. The results, given in Table 4.3, indicate that the soil is essentially neutral in both situations. This is in agreement with the moderate organic content, as a highly decomposed and highly organic peat would likely be very acidic. 


\subsection{CONCLUSIONS}

The soil employed in the experimental program was sampled in the vicinity of Lindberg road in West Lafayette, Indiana, and is referred to as LR (Lindberg road) soil. The subsurface profile and groundwater information were obtained from six boreholes drilled along the center of Lindberg road. A 3.0 - $3.7 \mathrm{~m}$ thick peat layer was encountered at a depth between $1.4-2.8 \mathrm{~m}$. The groundwater table was above or at the upper part of the peat layer.

Two undisturbed block samples (30 cm x $30 \mathrm{~cm}$ x $30 \mathrm{~cm}$ ), disturbed samples and site water were collected from three trips were made in February, April, and July of 2001. The undisturbed samples were collected during the April trip. A block sampler specially designed to reduce the effect of disturbance during sampling was used to collect undisturbed samples. Block samples were sealed with two layers of plastic wrap and wax to preserve the natural water content. The disturbed samples were stored in plastic bins and sealed with plastic wrap. All the samples were stored in a humid room until used for testing.

The natural water content and the index properties of LR soil were determined using disturbed samples to classify the soil. The natural water content was measured from disturbed samples collected from each of the three trips immediately after sampling. The natural water content of LR soil shows variation with time. However, since the block samples and the majority of disturbed samples were collected in April, the water content measured in April (289\%) is assumed as the natural water content of LR soil. The organic content of LR soil also shows variation with time. The organic content of the April and July samples are $51.8+/-4.8$ and $44.5+/-4.0$, respectively. The specific gravity of LR soil was determined using pycnometers and water as fluid. The average value of the specific gravity of LR soil is 1.93. This value was used in phase relationship to determine the initial void ratio of the soil specimen employed in the experimental program. The particle size distribution of LR soil was determined using a combined analysis: wet sieving and hydrometer test. According to the results, LR soil consists of $16.1 \%$ of sand, 43.3\% of silt and $40.6 \%$ of clay. The Atterberg Limits of LR soil were determined with both disturbed and oven dried soil samples. The liquid and plastic limits of non-dried LR 
soil are $327 \%$ and $162 \%$, respectively. In the natural state, LR soil exhibits liquidity index of 0.77 . The liquid limit of the oven dried LR soil is about $102 \%$. The ratio of the liquid limits of non-dried and oven dried LR soil is 0.31. Based on the results from particle size distribution and Atterberg limits, LR soil can be classified as $\mathrm{OH}$ according to the USCS (ASTM D2488). The fiber content of LR soil is about 2.29\%, and thus LR soil can be classified as "sapric" according to the ASTM definition. 
Table 3.1: Summary of index properties and unit weights of peats and organic soils (Edil and Wang, 2000)

\begin{tabular}{|c|c|c|c|c|c|c|}
\hline Soil & Description & Wn $(\%)$ & $\gamma\left(\mathrm{kN} / \mathrm{m}^{3}\right)$ & OC (\%) & $\begin{array}{c}\text { Fiber } \\
\text { content (\%) }\end{array}$ & $\mathrm{G}_{\mathrm{s}}$ \\
\hline Middleton, WI & Fibrous Peat & $500-600$ & $9.1-10.1$ & $83-95$ & 64 & $1.40-1.70$ \\
\hline Middleton, WI & Amorph. Peat & $430-520$ & 10.3 & $58-65$ & $20-30$ & $1.60-1.90$ \\
\hline Portage, WI & Fibrous Peat & 600 & 9.6 & 81 & 31 & 1.72 \\
\hline Fond du Lac, WI & Amorph. Peat & 240 & 10.2 & 60 & 20 & 1.94 \\
\hline Nine Springs, WI & Fibrous Peat & $150-655$ & 9.8 & $74-84$ & $75-92$ & $1.62-1.85$ \\
\hline USH 12, Middleton, WI & Fibrous Peat & $157-165$ & 12.9 & - & - & - \\
\hline USH 12/18, Cambridge, WI & Fibrous Peat & 361 & - & 66 & - & 1.82 \\
\hline USH 12/18, Cambridge, WI & Organic Soil & 321 & - & 10 & - & 2.56 \\
\hline STH 29, Shawano, WI & Fibrous Peat & $250-516$ & $9.6-12.1$ & $35-66$ & - & $1.82-2.23$ \\
\hline STH 29, Shawano, WI & Organic Soil & $125-367$ & 18.4 & $6-10$ & - & 2.55 \\
\hline Hoyt Lakes, MN & Fibrous Peat & $270-470$ & 10.4 & $50-85$ & $36-76$ & $1.59-1.70$ \\
\hline Hoyt Lakes, MN & Organic Soil & $50-100$ & 12.5 & 3 & - & $2.47-2.63$ \\
\hline Richfield, MN & Amorph. Peat & $175-300$ & 11.6 & $31-37$ & $37-45$ & 2.02 \\
\hline Richfield, MN & Organic Soil & $150-160$ & - & 25 & - & 2.29 \\
\hline
\end{tabular}

Table 3.2: Average water content of LR soil taken from three sampling trips

\begin{tabular}{|c|c|c|c|}
\hline Sampling Time & Sampling Depth (m) & Average Water Content (\%) & Number of tests \\
\hline February, 2001 & $1.5 \mathrm{~m}$ & $58 \%+/-9.1 \%$ & 6 \\
\hline April, 2001 & $2.1 \mathrm{~m}$ & $289 \%+/-25 \%$ & 7 \\
\hline July, 2001 & $2.1 \mathrm{~m}$ & $188 \%+/-31 \%$ & 5 \\
\hline
\end{tabular}


Table 3.3: Average water content (Wn) and organic content (OC) of LR soil

\begin{tabular}{|c|c|c|c|c|}
\hline Series & Bin number & Wn (\%) & OC (\%) & Test Performed \\
\hline \multirow{15}{*}{$\begin{array}{c}\text { II } \\
\text { (April, 2001) }\end{array}$} & 01 & 249.0 & 51.7 & UC \\
\hline & 02 & 278.4 & 48.7 & UC \\
\hline & 03 & 293.0 & 58.6 & UC \\
\hline & 04 & 231.0 & 50.4 & UC \\
\hline & 05 & 230.0 & 53.9 & UC \\
\hline & 06 & 245.0 & 57.6 & UC \\
\hline & 07 & 318.8 & 41.8 & UC \\
\hline & 08 & 238.0 & 52.3 & UC \\
\hline & 09 & 230.3 & 54.5 & UC \\
\hline & 10 & 323.4 & 54.7 & Mixture I and II, UC \\
\hline & 11 & 275.2 & 51.5 & Mixture I and II, UC \\
\hline & 12 & 238.9 & 51.7 & Mixture I and II, UC \\
\hline & 13 & 264.5 & $42.6+/-0.5$ & CRS consolidation \\
\hline & 14 & 240.6 & $54.6+/-1.5$ & CRS consolidation \\
\hline & Average & $261.2+/-32.1$ & $51.8+/-4.8$ & \\
\hline \multirow{8}{*}{$\begin{array}{c}\text { III } \\
\text { (July, 2001) }\end{array}$} & 01 & - & 46.4 & UC \\
\hline & 02 & 215.5 & 47.6 & UC \\
\hline & 03 & 210.0 & 50.8 & UC \\
\hline & 04 & 163.7 & 42.6 & UC \\
\hline & 05 & 210.0 & 43.8 & Mixture I and II, UC \\
\hline & 06 & 194.7 & $40.9+/-7.6$ & CRS consolidation \\
\hline & 07 & 177.9 & 39.4 & CRS consolidation \\
\hline & Average & $195.3+/-20.7$ & $44.5+/-4.0$ & \\
\hline
\end{tabular}

Table 3.4:Average specific gravity of LR soil

\begin{tabular}{|c|c|}
\hline Bin Number & Average Specific Gravity \\
\hline II -14 & $1.934+/-0.005$ \\
\hline III - 07 & $1.929+/-0.017$ \\
\hline
\end{tabular}


Table 3.5: The Atterberg Limits of LR soil (from II-14)

\begin{tabular}{|c|c|}
\hline Liquid Limit with deionized water & $327 \%$ \\
\hline Liquid Limit with tap water & $302 \%$ \\
\hline Plastic Limit (PL) & $162 \%$ \\
\hline Plasticity Index (PI) & $165 \%$ \\
\hline Natural water content & $289 \%$ \\
\hline Liquidity Index (LI) & 0.77 \\
\hline Liquid Limit after oven drying & $102 \%$ \\
\hline LL $_{\text {oven-dried }}$ & 0.312 \\
\hline
\end{tabular}

Table 3.6: Results from $\mathrm{pH}$ testing of LR soil

\begin{tabular}{|c|c|}
\hline Solution & $\mathrm{pH}$ \\
\hline $0.01 \mathrm{M} \mathrm{CaCl}_{2}$ & 7.26 \\
\hline $\mathrm{H}_{2} \mathrm{O}$ & 6.96 \\
\hline
\end{tabular}




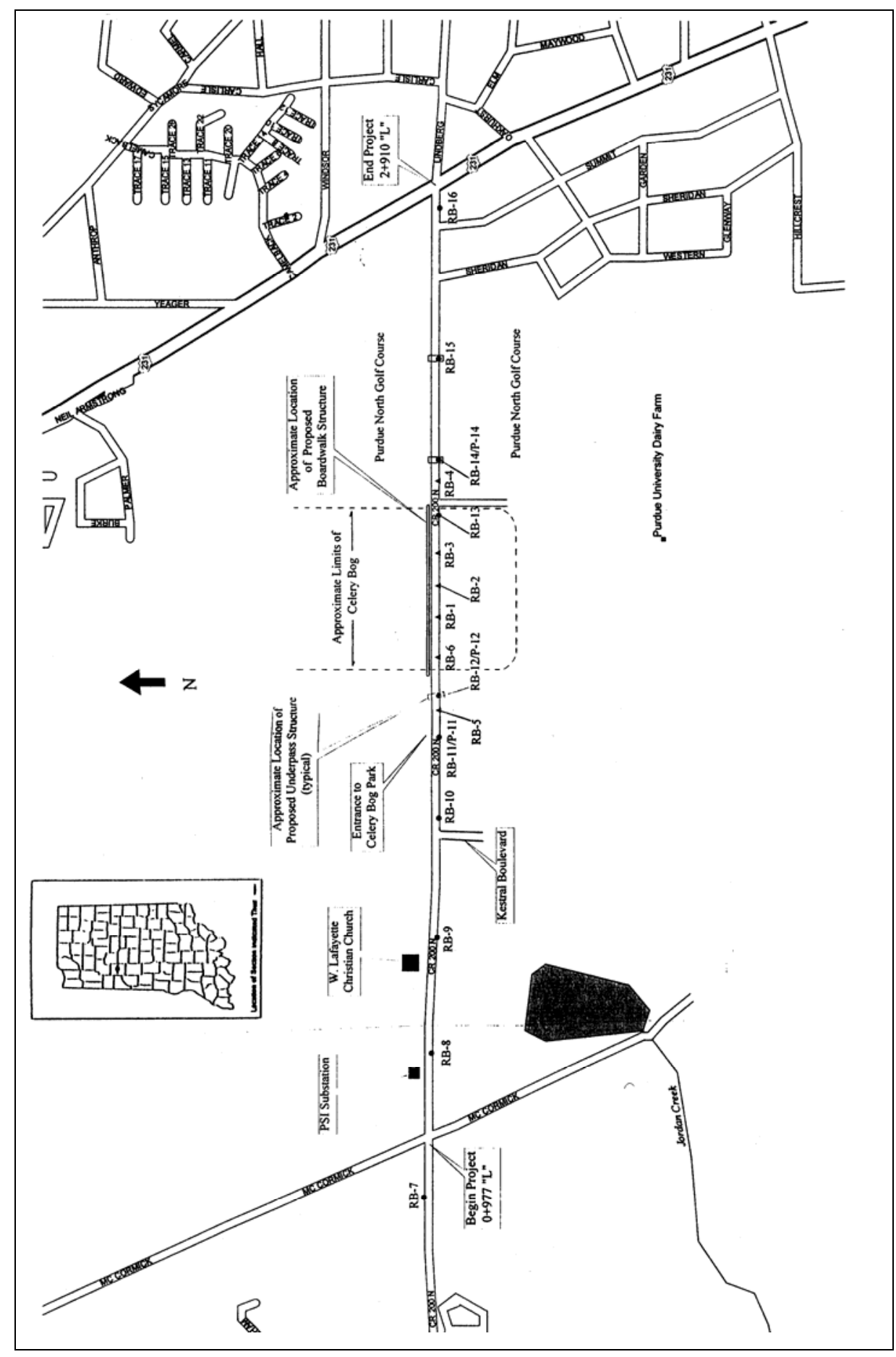

Figure 3.1: Location of Lindberg Road in West Lafayette, Tippecanoe County, Indiana (After Earth Exploration, Inc., 1996) 


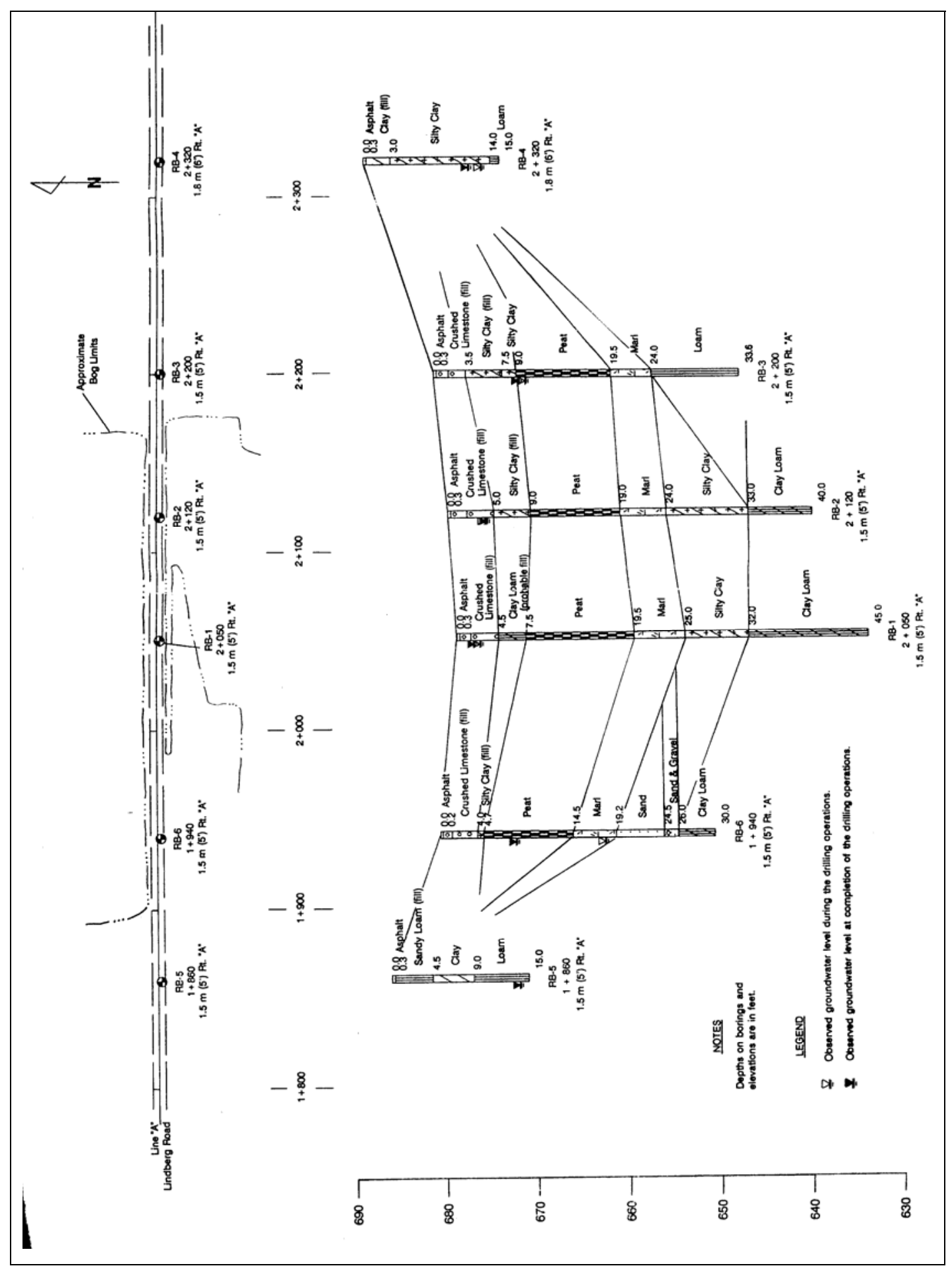

Figure 3.2: Soil boring location plan and profile along Lindberg Road (Earth Exploration, INC., 1996) 


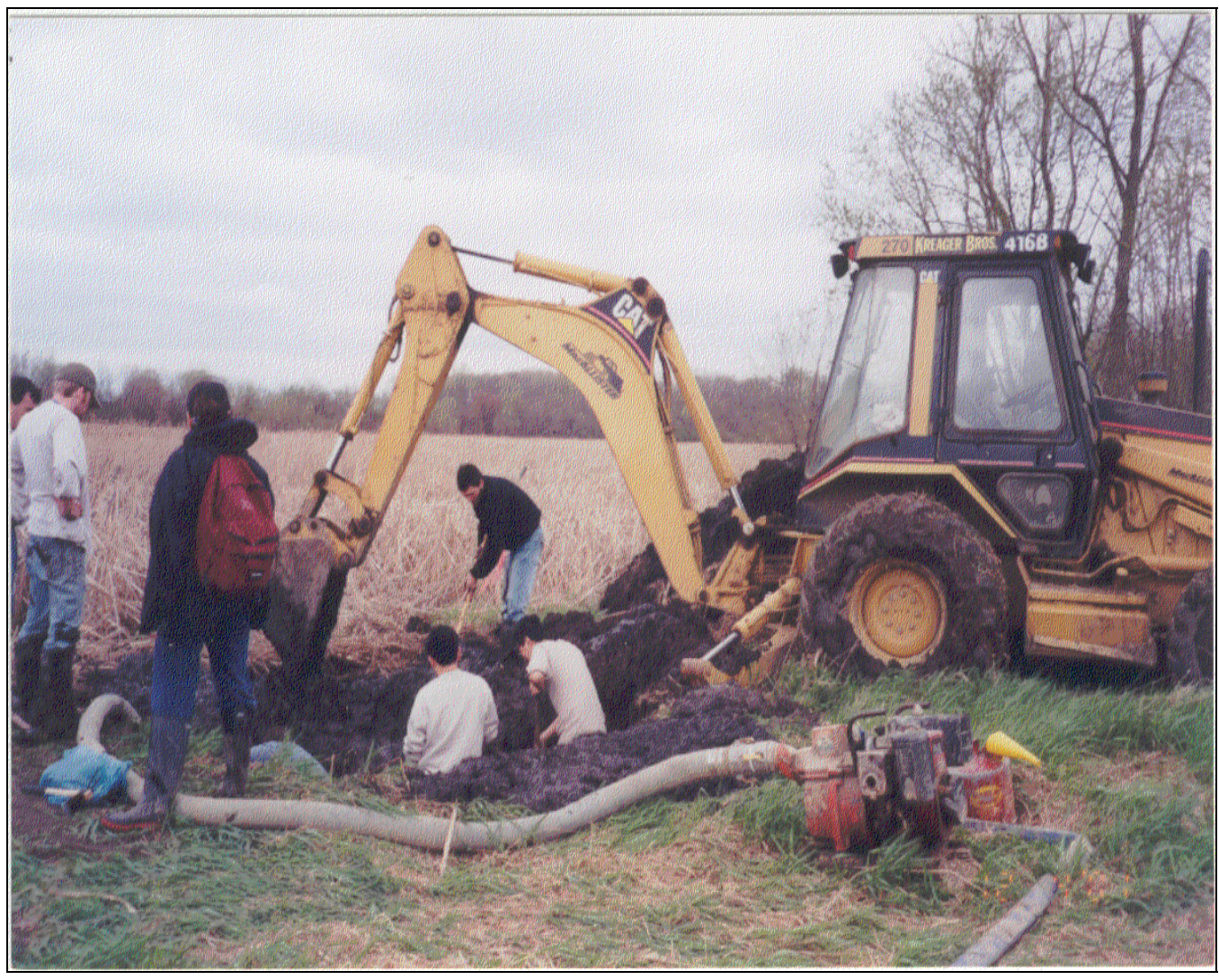

Figure 3.3: Sampling activities during April 2001 


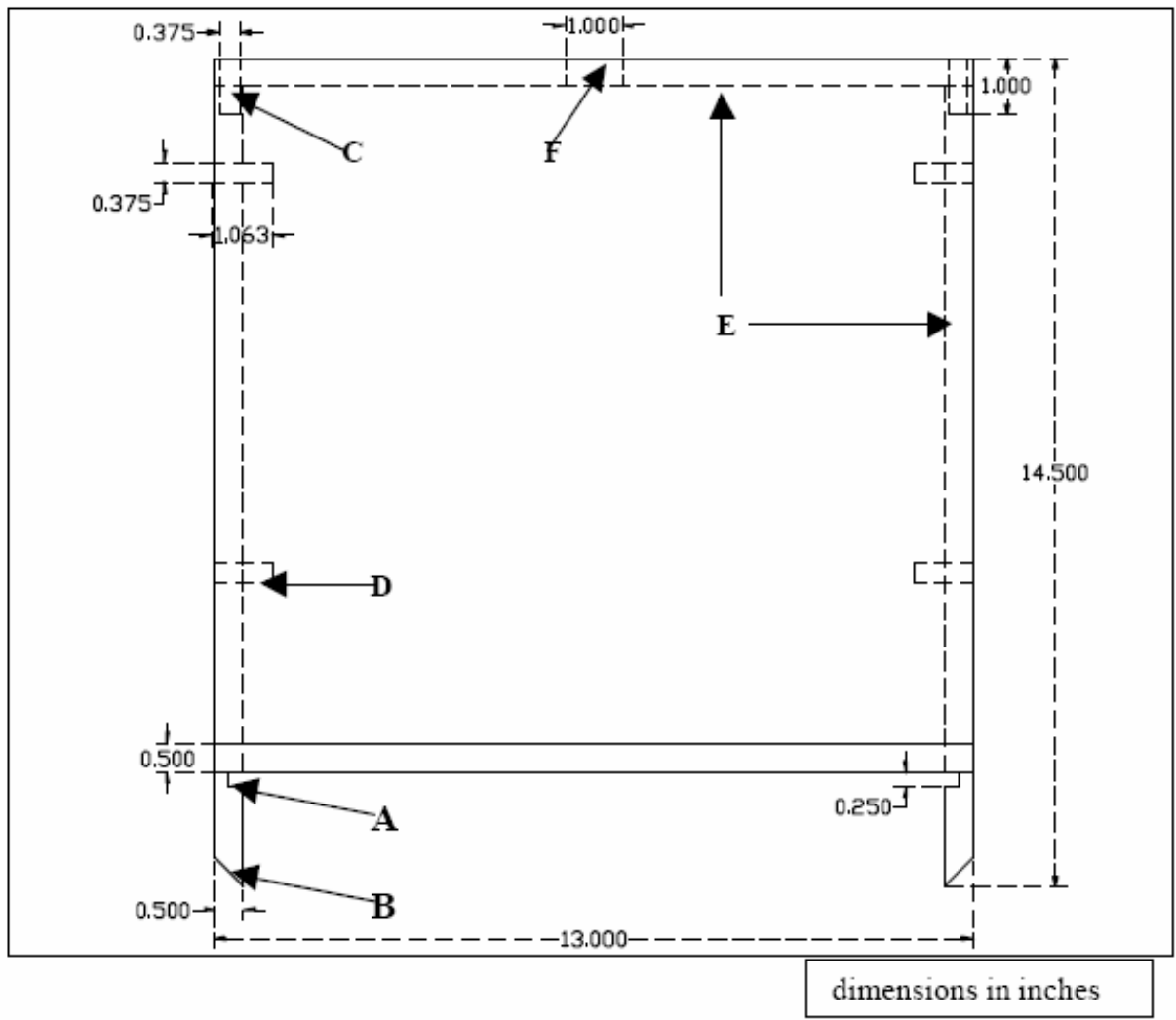

A: Groove for bottom cutting blade

$B$ : Beveled edge for cutting

C: Top fastener thread

D: Side fastener thread

E: $1 / 2$ inch thick Lexan side and top

F: Air release hole

Figure 3.4: Front view of block sampler 


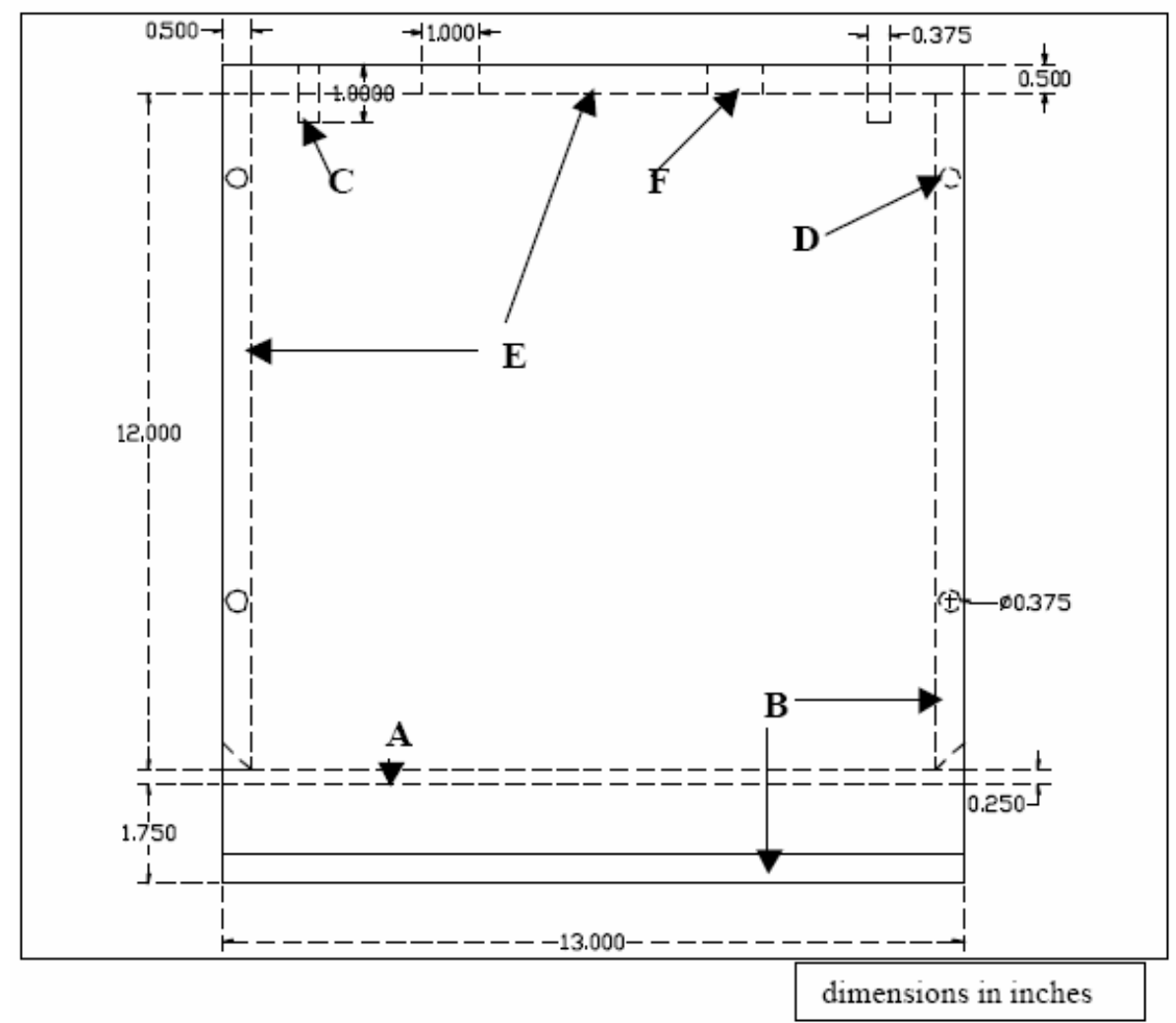
A: Groove for bottom cutting blade
$\mathrm{B}$ : Beveled edge for cutting
C: Top fastener thread
D: Side fastener thread
E: $1 / 2$ inch thick Lexan side and top
F: Air release hole

Figure 3.5: Side view of block sampler 


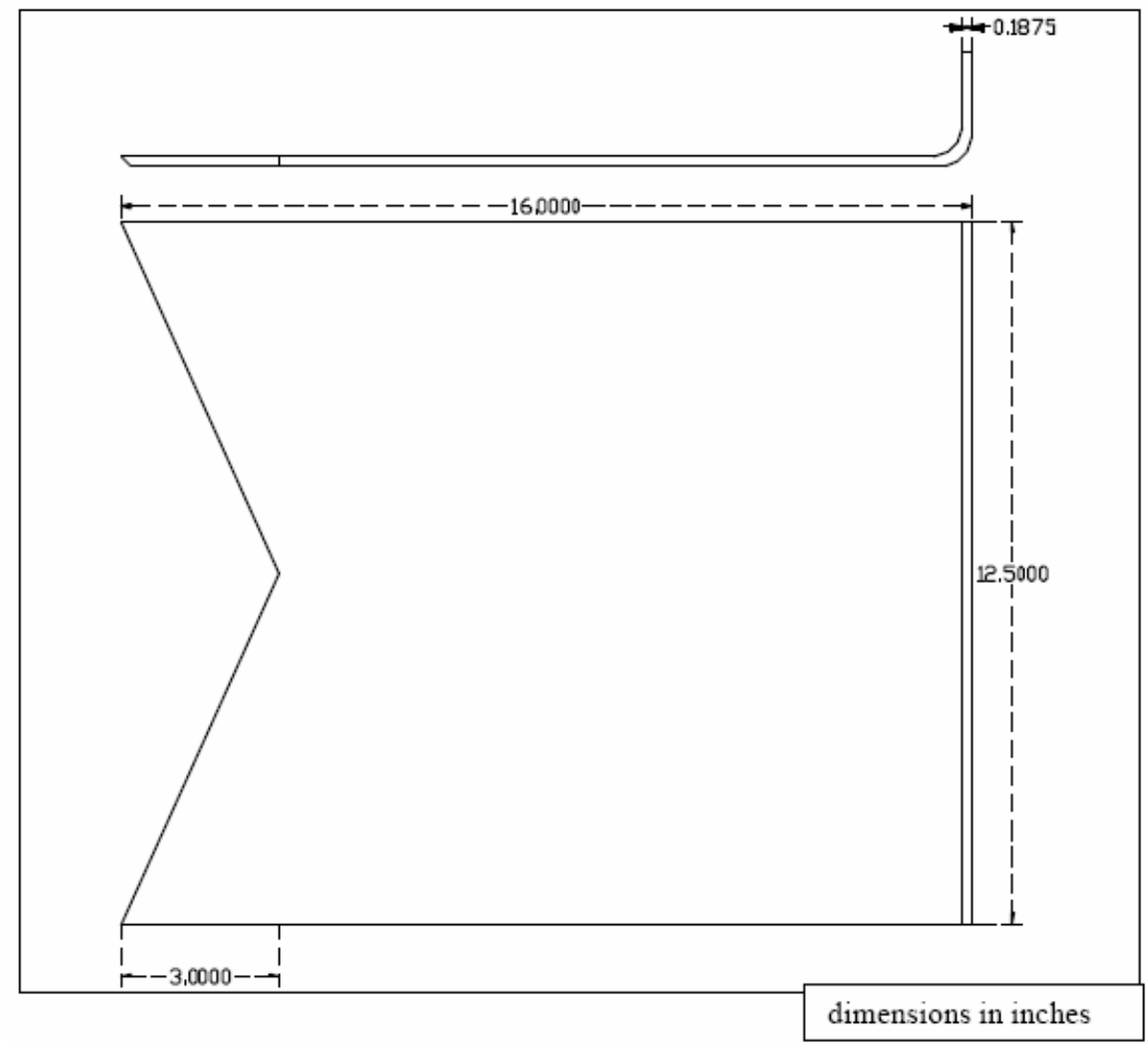

Figure 3.6: Drawing of blade from block sampler 


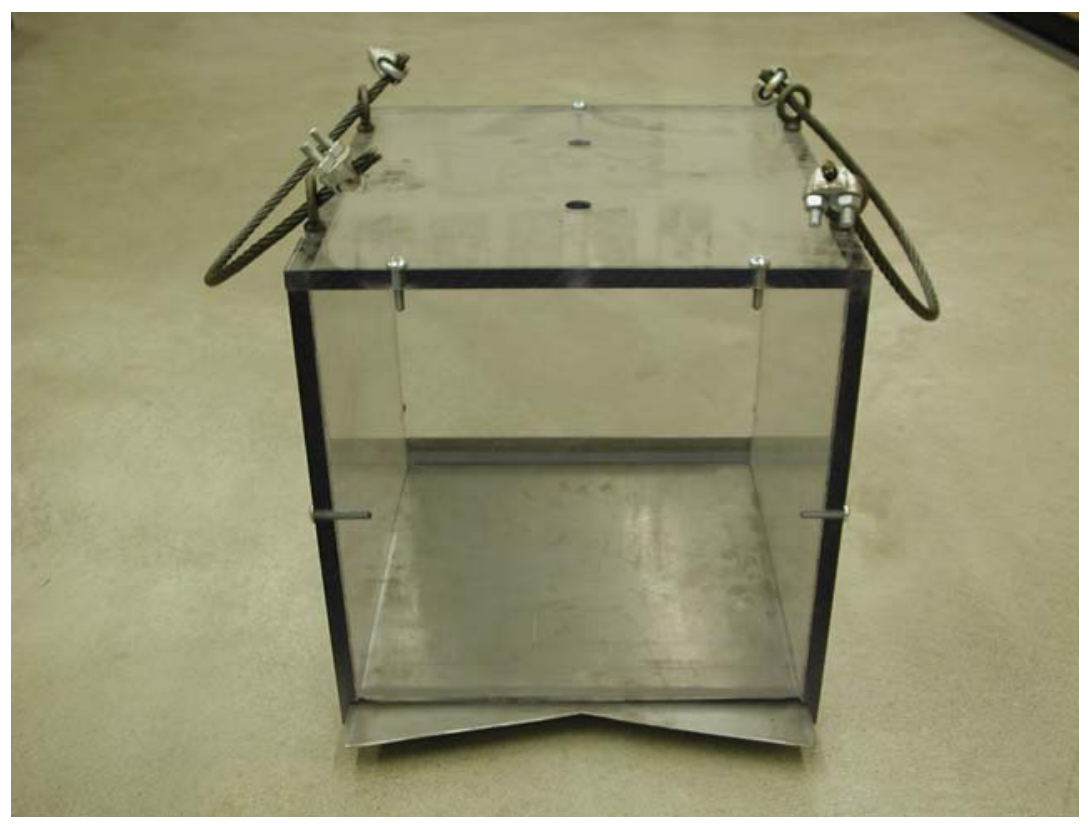

Figure 3.7: A $30 \mathrm{~cm}$ x $30 \mathrm{~cm}$ x $30 \mathrm{~cm}$ block sampler made out of Lexan plates

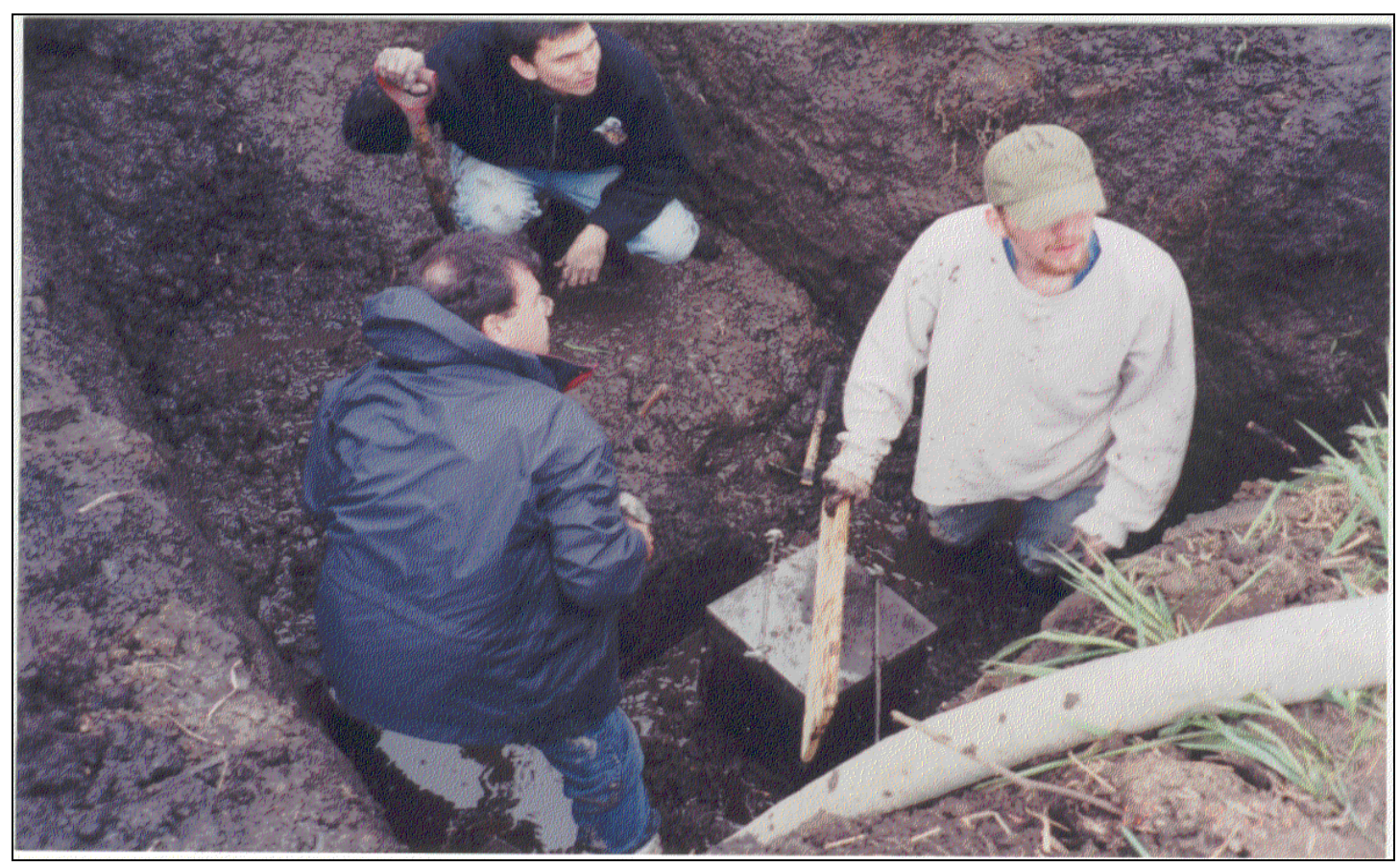

Figure 3.8: Undisturbed sampling 


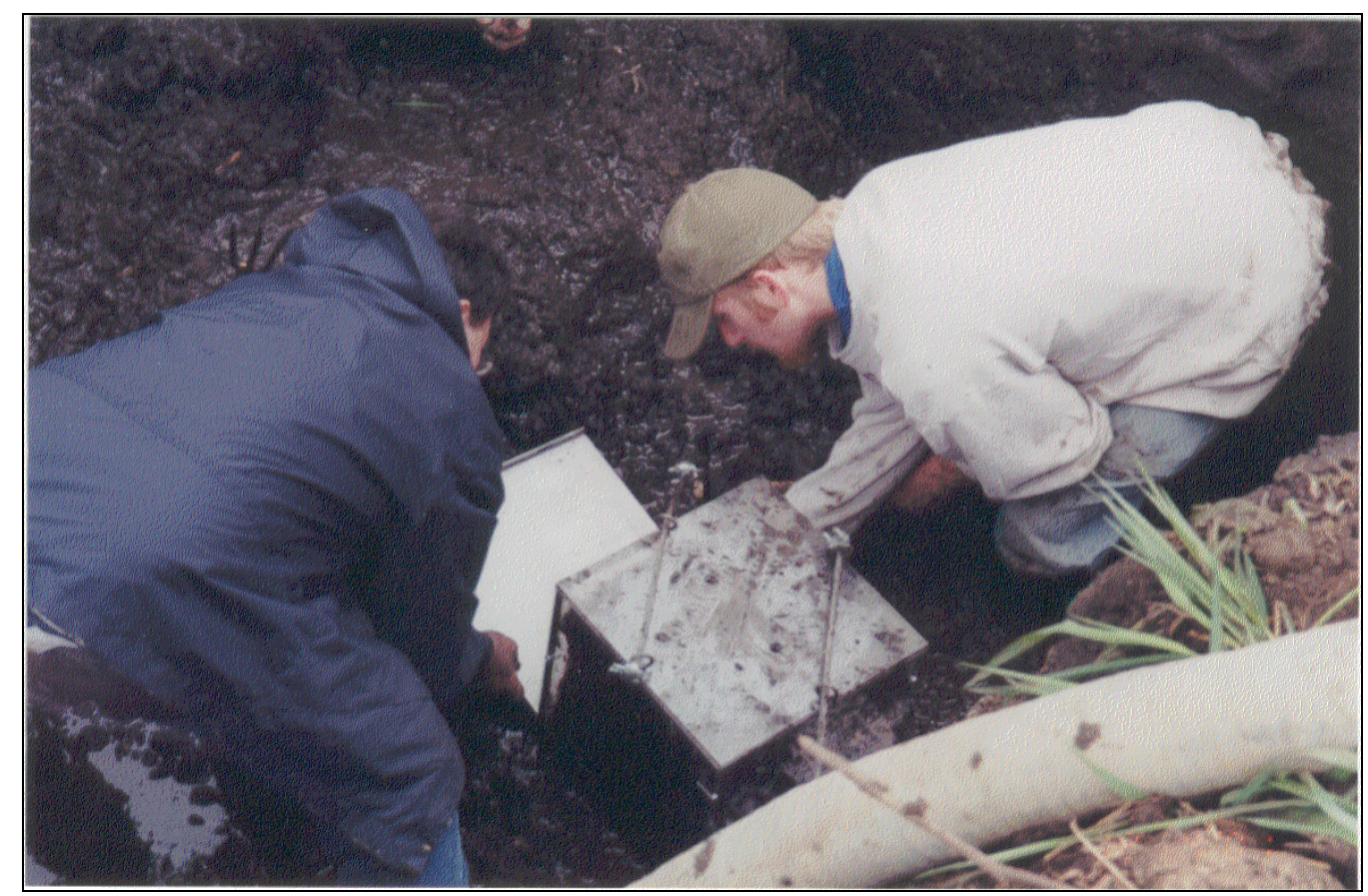

Figure 3.9: Undisturbed sampling

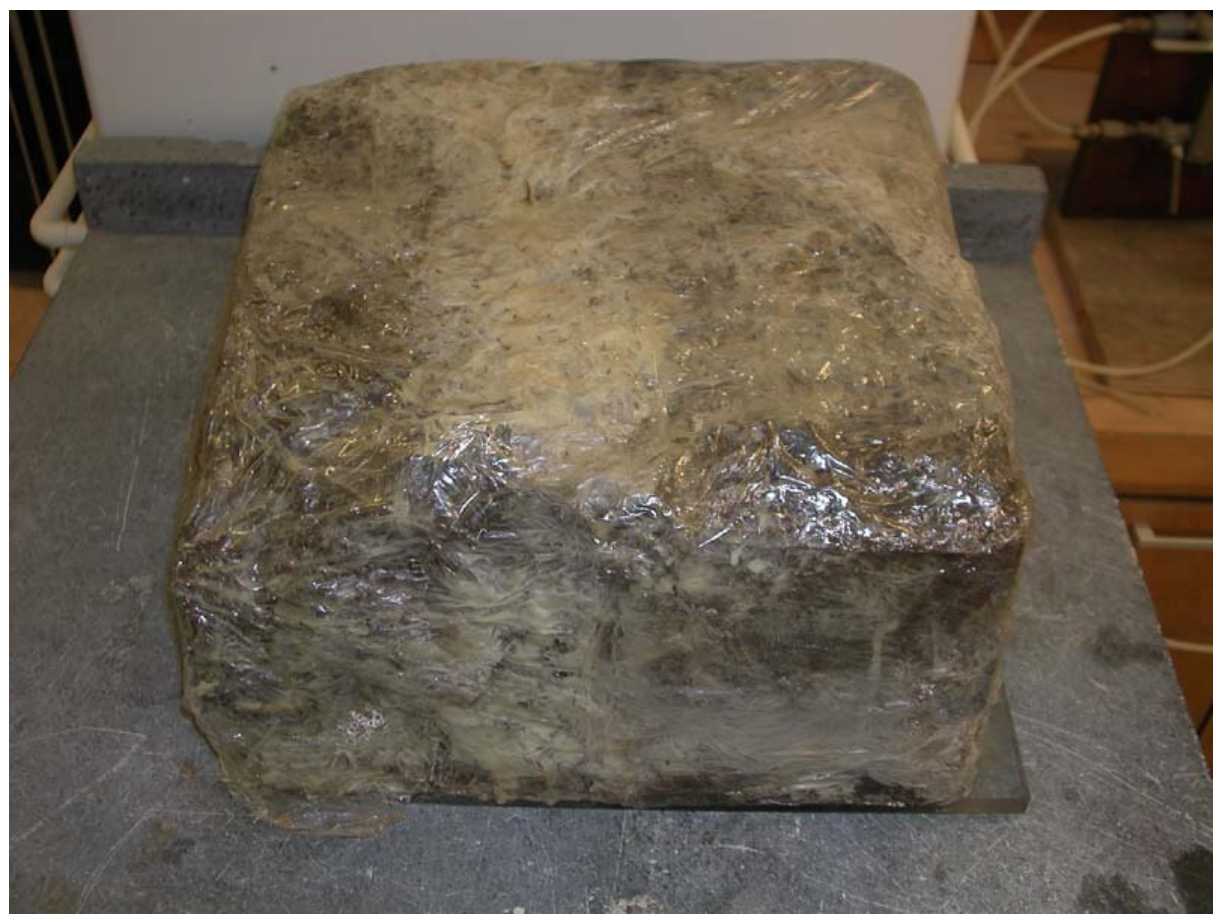

Figure 3.10: Undisturbed block sample sealed with plastic wrap and wax 


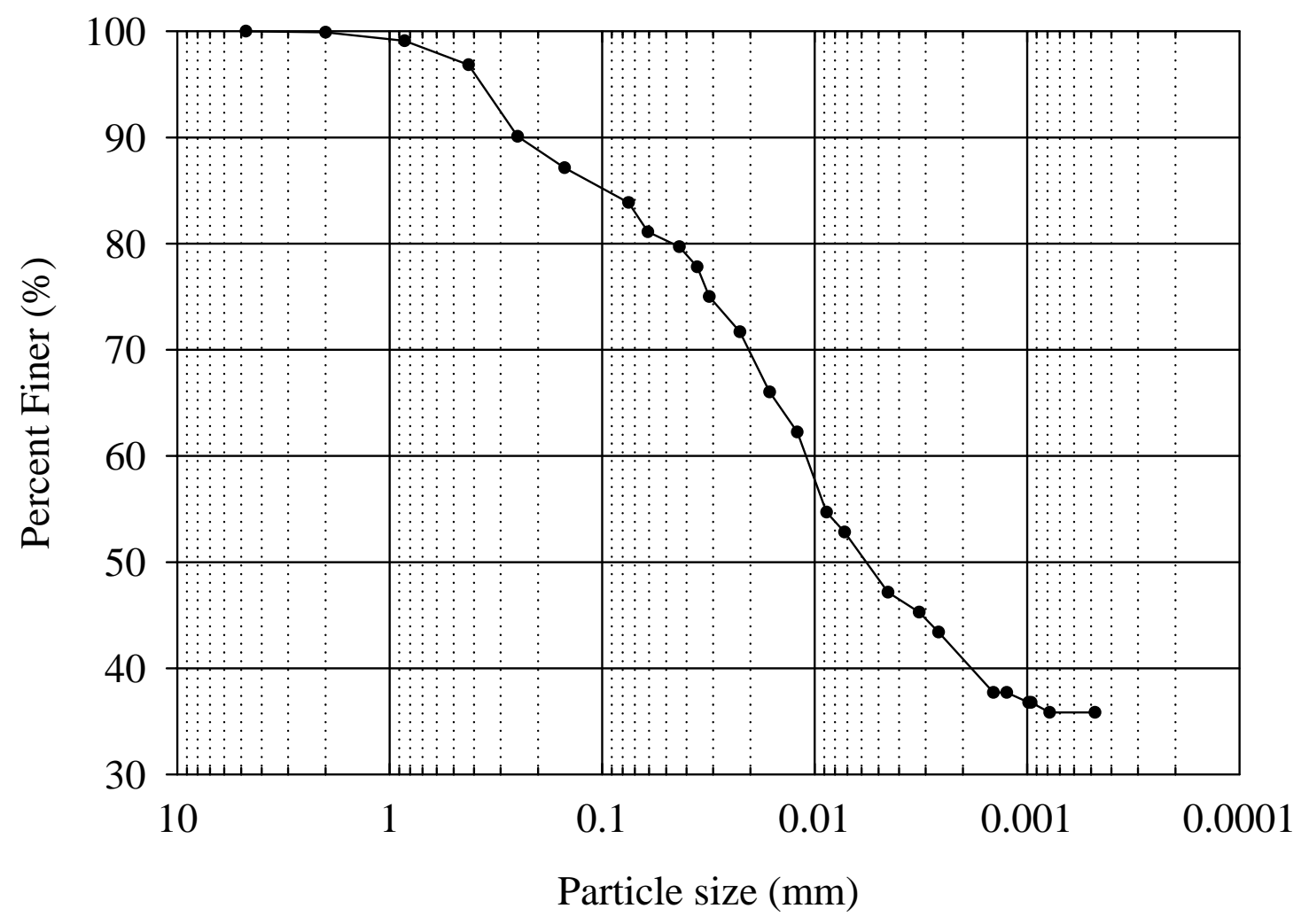

Figure 3.11: Particle size distribution of LR soil obtained from combined analysis 
CHAPTER 4: EXPERIMENTAL METHODS

CHAPTER 5: STRENGTH: UNCONFINED COMPRESSION TESTS

CHAPTER 6: STIFFNESS: CRS CONSOLIDATION TESTS

CHAPTER 7: CONCLUSIONS AND RECOMMENDATIONS 


\section{CHAPTER 4: EXPERIMENTAL METHODS}

\subsection{INTRODUCTION}

In the first part of this chapter, the procedure employed to prepare reconstituted and treated soil samples is described. In the second part, the overview of the Constant Rate of Strain (CRS) consolidation tests is described.

\subsection{Experimental Program}

Following the completion of the literature review (Chapter 2) and the characterization of LR soil (Chapter 3), the next step in the research project was to identify the testing variables and the plan of the experimental program.

The effects of treatment on the strength of soils of high and low organic contents were investigated by performing unconfined compression tests. To evaluate the effects of treatment on the compressive strength of a highly organic soil, ordinary Portland cement (PC), lime (CaO), High Calcium Flue Dust (HC), Marblehead Buffington Dust (MB), and Bentonite (BEN) were selected as binding agents to treat LR soil. The effects of these binding agents were tested alone and in combination. The Portland cement that was tested is given as percentage of dry mass as: $0 \%, 4 \%, 8 \%, 12 \%, 20 \%$ and $50 \%$. CaO, $\mathrm{HC}$ and MB as well as bentonite were mixed with LR soil at $4 \%, 8 \%, 12 \%$, and $20 \%$ by dry weight of the soil. A combination of $8 \%$ Portland cement and $4 \%, 8 \%$, and $12 \% \mathrm{CaO}$ was also investigated. The 8\% Portland cement mixture was selected as the "base-case", against which the effects of $\mathrm{CaO}$ were investigated. This value was selected since $8 \%$ binder addition is a commonly investigated binder content encountered in the literature.

Soils with low organic contents: Mixture I (LOI = 9.5\%) and Mixture II (LOI = 20\%) were manufactured in the laboratory by mixing Grundite, an illitic clay, with appropriated amount of LR soil. Water contents of $60 \%$ and $102.2 \%$ respectively were targeted to meet a constant LI of 0.65 for both. The MI and MII were mixed with 4\%, $8 \%, 12 \%, 20 \%$ and $30 \%$ of PC by dry mass of untreated soil. 
In addition to the effects of each particular binding agent, the elapsed time after the preparation of the mixed soil was an important variable. Considering that the hydration of Portland cement continues with time, it was deemed important that the improvement with cement should be evaluated after an extensive curing time. The selection of 28 days as the maximum curing time was based on time constraints that make a longer curing time impractical, and because it is commonly accepted that cement achieves most of its strength after approximately 28 days. In order to evaluate the progression of strength gain, the mixtures containing Portland cement were tested after one day, seven days, and twenty-eight days.

As the hydration of lime is complete soon after the lime comes into contact with water, the effect of the addition of lime into the soil mixture is an almost instantaneous process. For this reason, the mixtures containing lime, $\mathrm{HC}$ and $\mathrm{MB}$ were tested exclusively after seven days. The seven day period was chosen in order to guarantee that the lime absorbs its full capacity of water, without sacrificing valuable laboratory time and space.

In addition to the mixture composition and curing time characteristics, the surcharge applied to the soil was the third variable considered. The surcharge refers to the stress that was applied to the treated soil cylinders during the appropriate curing period. This load was used to simulate various overburden stresses that the soil experiences at different depths. For this study, stresses of $14.5 \mathrm{kPa}, 48 \mathrm{kPa}$, and $96 \mathrm{kPa}$ are chosen. These values correspond to approximate depths below the ground surface of approximately one, three, and six meters respectively, assuming an average unit weight of the soil of $16 \mathrm{kN} / \mathrm{m}^{3}$.

The effects of treatment on the stiffness of a highly organic soil were investigated by performing Constant Rate of Strain (CRS) consolidation tests on treated LR soil samples with $0,8,20,50,100$, and $150 \%$ of PC by dry mass. The soil samples were cured for 14 days while applying a surcharge of $48 \mathrm{kPa}$. The curing time was selected to provide sufficient time for hydration of Portland cement to take place but to prevent the soil samples to gain excessive strength due to secondary compression. 
The range of the testing variables investigated employed in the experimental program: soil type, type and amounts of binder, surcharge and curing time is summarized in Table 4.1.

\subsection{LABORATORY SAMPLE PREPARATION PROCEDURE}

The procedure employed in the experimental program to prepare reconstituted and treated soil samples was developed originally to produce homogeneous and reproducible samples for unconfined compression tests on LR soil (Humphrey, 2001). The sample preparation procedure consists of four steps: mixing, compaction, curing and extrusion. The details of each step of sample preparation procedure are described in this section

\subsubsection{Mixing}

The mixing procedure was specially designed to simulate the kneading action applied to soil in one of the most widely used soil stabilization methods, the Deep Mixing Method (DMM). The basic concept of the DMM is to improve the deformation properties and the strength of the soil in situ by mixing it with binding agents, usually lime or Portland cement. The binding agent can be introduced to the soil in slurry or grout form (wet method) or in dry form (dry method). In both methods, the binder can be mixed with the soil either by pure rotation of the mixing tool at relatively low stress or by the combination of the rotation of the mixing tool and injection of the binding slurry or grout into the soil at high stress (Bruce and Bruce 2003).

To prepare treated soil samples for unconfined compression tests, a moisture content for the soil to be treated was obtained at least twenty-four hours prior to the mixing procedure, along with a test of the organic content. The appropriate quantity of soil was placed in a large metal mixing bowl. Water from the source of the soil was added to bring the soil water content up to a value that is representative of the field conditions $(\mathrm{w}=289 \%$ for LR soil, and $\mathrm{w}=60 \%$ for Mixture I and $102.2 \%$ for Mixture II). Binder was added to soil in appropriate quantity. Portland cement was added in form of slurry with water to cement ratio of 0.5 , while $\mathrm{CaO}, \mathrm{HC}, \mathrm{MB}$ and bentonite were added as dry powder. Mixture was mixed on medium setting in large Hobart mixture for two 
minutes. Mixture was mixed with metal spoon by hand for one minute to remove lumps. A moisture content for the soil mixture was obtained.

The devices employed to prepare soil samples for CRS consolidation tests in the mixing step include the KitchenAid ${ }^{\circledR}$ stand mixer and the two mixing tools (Figure 4.1). Two different mixing tools, a flat beater and a hook-shaped tool, were used to apply kneading action to the soil during mixing. Before samples were prepared, the water content of each soil bin was determined. About 1500 grams of disturbed soil was placed in a mixing bowl, and deionized water was added to bring the water content of the soil up to the natural water content (289\%). The mixture of soil and water was mixed thoroughly for two minutes using flat beater in the mixer, and then was mixed for additional five minutes with a hook-shaped mixing tool. Two water content measurements were made after the mixing of soil with water was completed.

Portland cement was added to the soil mixture as slurry form to prepare treated soil samples. A water/cement ratio of 0.5 was used to prepare cement slurry. After cement slurry was poured into the soil, the mixture was mixed following the same procedure described above: with a flat beater for two minutes and with a hook-shaped mixing tool for five minutes. Additional two water contents of the soil-cement mixture were determined after the mixing is completed.

Reconstituted soil sample were prepared following the same procedures but without introducing binding agents for both unconfined compression and CRS consolidation tests.

In the experimental program, the amount of binder added to prepare treated soil samples are expressed as percentage by the dry mass of soil. However, since the water content of each soil: LR soil, MI and MII is different, the actual amount of binder in each soil samples can be significantly different. To be able to directly compare the effects of treatment on each soil type, the actual amounts of PC in soil samples are expresses as the cement factor, which is defined as the mass of PC per unit volume of soil, and are summarized in Table 4.2. The typical value of the cement factor used in the practice for wet method ranges from 100 to $500 \mathrm{~kg} / \mathrm{m}^{3}$, which is equivalent to $32 \%$ and $160 \%$ for LR soil, $10.5 \%$ and 58\% for MI, and 13\% and 74\% for MII. 


\subsubsection{Compaction}

For unconfined compression tests, the shape of the specimen needs to be cylindrical to avoid edge effects and the height/diameter ratio needs to be greater than two to avoid end effects (Farmer, 1983). In this regards, plastic cylinders of $7.62 \mathrm{~cm}$ in diameter and $15.24 \mathrm{~cm}$ were employed to prepare soil samples for unconfined compression tests. These cylinders featured one open end and a $0.3175 \mathrm{~cm}$ hole drilled in the bottom of the cylinder for drainage during the curing phase.

A very important consideration for the manufacture of these soil cylinders was the method with which the mixed soil is placed in the cylinder. In an effort to ensure production of a homogeneous soil mass, various methods were investigated, including: static compaction, vibro-compaction, and dynamic compaction.

After performing tests with each of these methods, it was decided that the dynamic compaction method produced the most homogenous and repeatable results for LR soil samples. This method involved the use of a modified mechanical compactor (Figure 4.2). A modification was made to the drop hammer that was used in the machine. This machine, which was intended to be used for both modified and standard proctor compaction using $10.16 \mathrm{~cm}$ and $15.24 \mathrm{~cm}$ diameter compaction molds, was equipped with a $5.08 \mathrm{~cm}$ in diameter hammer. Considering the smaller size of the cylinders that were used for the specimen manufacture, the diameter of this hammer was reduced to $2.54 \mathrm{~cm}$ (Figure 4.3). The use of the original proctor mold allowed for the use of the revolving turntable of the mechanical compactor, which was designed to be used with these molds. A $2.54 \mathrm{~cm}$ thick aluminum spacer was machined for insertion into the molds in order to accommodate the smaller plastic cylinders. In addition, a $6.35 \mathrm{~cm}$ extension collar was also manufactured so that the cylinder could be filled above its $15.24 \mathrm{~cm}$ height to allow for surface leveling after the filling (Figure 4.4). The compaction procedure was performed as follows:

1. Cylinder is coated with a thin film of concrete form oil to allow for easy extrusion prior to testing.

2. A piece of filter paper is cut to $7.62 \mathrm{~cm}$ (3 in) in diameter

3. Cylinder and moist filter paper mass are obtained and recorded 
4. Cylinder is placed inside compaction mold and bolted to compaction machine

5. Cylinder is filled approximately $3 / 4$ full with soil mixture

6. Mechanical compactor is set to apply 40 hammer blows for this layer with the revolving turntable set on medium

7. Cylinder is filled with soil mixture

8. Mechanical compactor is set to apply 40 hammer blows for this layer with the revolving turntable adjusted to a medium-low setting.

9. Cylinder is filled approximately $2.54 \mathrm{~cm}$ above the top of the $15.24 \mathrm{~cm}$ mold.

10. Mechanical compactor is set to apply 40 hammer blows for this layer with the revolving turntable adjusted to a medium-low setting.

11. Steps 9-10 are repeated

12. Cylinder is removed from mold.

13. Top of surface is leveled and patching is done as necessary.

14. Filter paper is placed on top of the cylinder and the total mass is recorded

On the other hand, due to the more cohesive nature of the MI and MII, the static compaction method, recommended by EuroSOILSTAB, was selected for preparation of treated MI and MII samples. In this method, a stress control loading frame (Figure 4.5) was used to apply static pressure on soil specimens and satisfying results were obtained. The detailed procedure of this method is described as follows:

1. Apply a thin layer of oil to the inner side of cylinder for easy extrusion of specimen after curing.

2. Place a plastic glass plate in cylinder.

3. Put about $1 / 4$ volume of prepared mixture in cylinder.

4. Fit the cylinder with soil in compaction mold and bolt tightly.

5. Cover the soil in cylinder with a plastic wrap sheet. This sheet work as an interface to separate soil from the loading cap.

6. Apply pressure on specimen through flat metal cap. Give specimen three static strikes with $120^{\circ}$ rotation of cylinder after each strike. The pressure on specimen was controlled at $100 \mathrm{kPa}$ for 3 seconds. 
7. Repeat the previous step using a metal cap with $15^{\circ}$ inclination instead.

8. Remove the cylinder from mold and scarify the soil surface with a metal fork for quality binding between soil layers.

9. Repeat step 3 to 8 until cylinder was filled. When compact the last layer soil should be filled over the cylinder top to allow for the settlement due to pressure.

10. Remove cylinder from mold and level the top of specimen with a trimmer.

The soil samples for CRS consolidation test were prepared following the same compaction method employed for preparation of treated LR soil samples for unconfined compression tests (dynamic compaction method). In the beginning of the investigation of the effects of treatment on stiffness, five preliminary CRS consolidation tests were performed and it was found that the preconsolidation pressures of the test specimens were significantly lower (about $40 \%$ ) than the loads applied to the samples during curing phase. This was attributed to the effects of friction along the wall of the plastic cylinder which were especially enhanced by the high H/D ratio. Therefore to reduce the H/D ratio of the specimen, the compacted soil cylinder was cut with a bench in half immediately after compaction was completed (Figure 4.6).

\subsubsection{Curing}

The application of the surcharge was performed using two different methods. In the first method, concrete caps were cast with a $1.27 \mathrm{~cm}$ diameter epoxy coated steel rebar in the center. These caps were cast inside the plastic cylinders using Portland cement at a water/cement ratio of 0.5 and sandy gravel that was readily available in the testing laboratory. The epoxy coated rebar was placed inside the mold before the concrete has set. These caps were then extruded after one week of hydration and filed down slightly so that they fit snugly inside the cylinder. Once the caps were placed on the soil cylinders, appropriate weights were added to the top of the cylinder, centered around the rebar, and balanced using duct tape (Figure 4.7). The change of samples height during curing phase 
was monitored for samples used in CRS consolidation tests to check the degree of consolidation of samples.

The other method involved the manufacture of concrete caps in an identical method as described before, but without the steel re-bar. These caps were then placed on the soil cylinders and placed in the consolidation frame in the Geotechnical laboratory (Figure 4.8). The consolidation frame was utilized for the $96 \mathrm{kPa}$ surcharge cases in order to avoid the instability caused by piling large amounts of weight on top of the cylinders. In both cases, the cylinders were immersed in tap water and kept moist during the entire curing phase by replenishing the water on a continuous basis. This was done in order to guarantee that the soil remained saturated as would be the case in the field. Under these conditions, ample water would be available for the hydration of the cement.

\subsubsection{Extrusion}

After curing process is completed, the sample cylinder is removed from the water bath. Hot water is run over the sides of the plastic cylinder for easier extrusion of soil sample. The cylinder is then inverted on a piece of wax paper so that the soil sample can be moved for testing later without losing its water content. The soil sample is extruded from the cylinder by carefully applying air pressure thorough the opening on the bottom.

\subsection{Unconfined Compression Test}

Following the curing and extrusion, an unconfined compression test was performed on each of the soil cylinders to evaluate the change in compressive strength and stiffness associated with the treatment.

The test was performed using a constant displacement load frame with a $10 \mathrm{kN}$ capacity. This frame was instrumented with a $2.2 \mathrm{kN}$ capacity load cell and an LVDT to monitor displacements (Figure 4.9). The data was recorded using the TestNet-GP version 4.06 data acquisition system manufactured by GeoTac Inc. 
In this test, the extruded specimen was placed with the longitudinal axis orientation identical to the orientation during the curing period. A strain rate of one percent per minute was chosen for the displacement rate of the frame, and the load cell and LVDT data was recorded every second. This setup was used for all of the unconfined compression testing that was conducted.

Upon completion of tests, the collected data were reduced to obtain two important values: axial stress and axial strain.

The strain $(\varepsilon)$ is given as a percentage and is calculated using the displacement measurements as follows:

$\varepsilon=\Delta \mathrm{H} / \mathrm{H}_{\mathrm{o}} \times 100 \%$

where:

$\varepsilon$ : vertical strain (\%)

$\Delta \mathrm{H}$ : change in height of the specimen

$\mathrm{H}_{0}$ : height of specimen at beginning of the test.

The stress is calculated from the value of the force measured by the load cell, divided by the area of the specimen to yield the normal stress $\sigma$ as follows:

$\sigma=\mathrm{F} / \mathrm{A}_{\mathrm{C}}$

where:

$\sigma:$ normal stress

F: force measured by load cell

$\mathrm{A}_{c}$ : corrected area

The corrected area $\left(A_{c}\right)$ is used in place of the original area $\left(A_{0}\right)$ as calculated from the measured dimensions following extrusion in order to correct for the change in cross-sectional area that is incurred during compression. This "barreling” effect yields a cross sectional area that is larger than the original area $\left(\mathrm{A}_{0}\right)$ from pre-test dimensions, leading to artificially high stresses if the original area is used. This corrected area $\left(A_{c}\right)$ is calculated as follows:

$$
A_{c}=A_{o}\left(-1 / 4+\left(25-20 \varepsilon-5 \varepsilon^{2}\right)^{1 / 2} / 4(1-\varepsilon)\right)^{2} \quad(\text { Germaine and Ladd, 1988) }
$$




\subsection{CRS consolidation test}

\subsubsection{General overview}

The CRS consolidation test was first introduced by Smith and Wahls in 1969 to overcome some of the disadvantages of the conventional incremental loading (IL) oedometer test. Wissa et al. (1971) published the solutions for the coefficient of consolidation $\left(\mathrm{C}_{\mathrm{v}}\right)$ and the hydraulic conductivity $(\mathrm{k})$ for CRS consolidation test based on both linear and non-linear theory, and since then this method has been widely used among the researchers. In 1989, the CRS test was adopted by ASTM as one of the standard method for 1-D consolidation (ASTM D4186).

Compared to the IL test, which is a stress-controlled test, CRS test is a straincontrolled test, in which the stress-strain relationship of the soil specimen is obtained by imposing a constant rate of displacement on the soil specimen. The dimension of soil specimen is equivalent to oedometer specimen, $2.54 \mathrm{~cm}$ in height and $6.35 \mathrm{~cm}$ in diameter. The specimen can be saturated at constant volume by applying back pressure from the bottom of the specimen prior to loading. During consolidation, the drainage is allowed only at the top and the pore pressure is measured at the bottom of the specimen with pore pressure transducer. One of the advantages of CRS test is that the test provides continuous data. The compression curve can be defined as a continuous function of the vertical effective stress, and thus, the preconsolidation pressure can be easily defined with Casagrande's graphical construction. The coefficient of consolidation $\left(\mathrm{C}_{\mathrm{v}}\right)$ and the hydraulic conductivity $(\mathrm{k})$ can be directly calculated from the excess pore pressure as a continuous function of the vertical effective stress or axial strain. While an IL oedometer test may take several weeks to complete, since each load increment is left on for 24 hours, CRS consolidation test can be completed in much shorter period of time, usually within one to two weeks. However, the creep information may be limited. In this regard, the creep behavior of LR soil will be investigated with IL oedometer tests.

In the experimental program, one of the two computer controlled CRS apparatuses: CRS1 and CRS2, available at the Purdue's Bechtel Geotechnical Engineering Laboratory has been employed. The main difference of the two systems is 
the type of sealing installed on the loading piston to prevent leakage of cell pressure: Oring for CRS1 and rolling diaphragm for CRS2. Figure 4.10 shows a schematic of a CRS apparatus includes the following basic components: 1) the CRS cell and sensors to measure axial load, axial displacement, cell pressure and pore pressure; 2) the load frame used to impose a constant displacement rate, 3) the air-water interface system through which the cell pressure is applied; and 4) the data acquisition system and personal computer.

The soil specimens for CRS testing was prepared employing a procedure and devices designed for trimming very soft soils with minimal disturbance. The trimming devices consist of a specimen ring characterized by a sharp cutting edge, a specimen trimming frame, a thin metal spatula, a cutting blade and a wire saw (Figure 4.11). The specimen ring was attached to the vertical rod of trimming frame through a collar. After the curing was completed and the sample was extruded, the soil sample was placed on the rotating plate of trimming frame aligned along the centerline of the frame. Trimming was performed by advancing the specimen ring in small increments. A thin metal spatula was used to trim the soil ahead of the ring, so that no load could be exerted on the soil as the ring advances. After trimming was completed, excess soil above and below the specimen was cut roughly with a wire saw. The top and bottom sides of the specimen were carefully trimmed with a sharp metal knife blade. Two water contents were determined with soils roughly cut above and below the portion used for specimen. From the average of these two water contents, the initial void ratio of specimen was estimated.

The prepared soil specimen was placed in the base of the CRS cell. The CRS cell incorporates a loading piston, low friction ball bearings, a top cap, and two porous stones within a Plexiglas chamber that can withstand up to $700 \mathrm{kPa}$ of cell pressure. The schematic of one of the CRS cells (CRS1) is shown in Figure 4.12. A $8900 \mathrm{~N}$ capacity load cell is bolted to the cross head of the loading frame and measures the axial load transmitted though the loading piston. A DCDT is connected to the loading piston and measures the displacement of the piston. The cell and the pore pressures are monitored with $1400 \mathrm{kPa}$ capacity pressure transducers. 
Once assembled, the CRS cell was mounted on the platen of the load frame. A constant rate of displacement can be imposed on the specimen by moving the platen both in the upward and downward direction. The platen is driven by a high-precision double gear type motor which can be controlled as precisely as $1.175 \times 10^{-6} \mathrm{~cm}$ with a PC and a control program.

During the test, signals from sensors were recorded by the data acquisition system and stored into data file in the personal computer. The analog signal (voltage) from each of the sensor is converted to digital signal in the ADIO (Analog Digital Input Output) module of the 22-bit data acquisition system. The ADIO module also provides excitations $(10 \mathrm{~V})$ to the sensors connected to it. With full utilization of its 22-bit resolution, the data acquisition system is able to discriminate up to $4.77 \times 10^{-3} \mathrm{mV}$ for $+/-10 \mathrm{~V}$ input range. To get the best resolution, the input range was selected to $+/-10.0 \mathrm{~V}$ for the DCDT and +/- 0.1V for the Load Cell and two pressure transducers. To evaluate the resolution of the data acquisition system, the output voltage from each sensor was monitored over 3 hours. As shown in Figure 4.13, the Load Cell and the pressure transducers have resolutions of $0.001 \mathrm{mV}$ (corresponds to $0.287 \mathrm{~N}, 0.003 \% \mathrm{FSO}$ and $0.0138 \mathrm{kPa}, 0.001 \% \mathrm{FSO}$, respectively). The resolution of the DCDT is $0.1 \mathrm{mV}(.232 \mu \mathrm{m}, 0.001 \% \mathrm{FSO})$. The output signal from each sensor can be integrated over a time span of $12.5 \mathrm{~ms}(80 \mathrm{~Hz})$ to $3.125 \mathrm{~ms}$ (320 HZ). For the highest stability, the sampling rate was set to $80 \mathrm{~Hz}$. The stability of each sensor was checked by monitoring the outputs for 24 hours (Figure 4.14). The range, resolution and stability of each sensor used in the research are summarized in Table 4.3 for CRS1 and in Table 4.4 for CRS2.

Each of the tests presented in this report was performed following the same procedure. First the soil was saturated for 24 hours by applying back pressure through the bottom of the specimen. The back pressure line is hydraulically connected to cell pressure line, and thus the same pressure is applied to the top and bottom of the specimen. The cell and back pressure was increased in small increments of $7 \mathrm{kPa} /$ minute by manually controlling the air-water interface pressure regulator. For reconstituted specimens and treated specimens with less than 50\% PC, about $300 \mathrm{kPa}$ of back pressure was applied, while the magnitude of back pressure was increased to $450 \mathrm{kPa}$ for treated specimens with 
more than 50\% PC. The volume of specimen was kept constant during back pressure saturation after $0.3 \%$ of seating strain was applied to ensure the contact between the loading piston and the specimen. Following backpressure saturation, the specimen was loaded one dimensionally at a constant displacement rate until the desired target stress was reached (200 - $1600 \mathrm{kPa})$. At the beginning of the consolidation phase, the back pressure line was closed to monitor the pore pressure generated at the base of the specimen during loading with pore pressure transducer. The excess pore pressure was calculated as the difference of the pore pressure and the cell pressure. The maximum load was maintained for two to three days until at least $95 \%$ of excess pore pressure was dissipated. Then, the specimen was unloaded to $10 \%$ of the maximum load at $10 \%$ of the displacement rate employed during loading. The reduction of the rate was required to prevent generation of excessive negative pore pressure during unloading. The load was again maintained for two to three days until $95 \%$ of negative pore pressure was dissipated. Finally, the specimen was reloaded with the same displacement rate employed for the consolidation phase until maximum target stress was reached (2000 kPa).

\subsubsection{CRS apparatus calibration}

During back pressure saturation, the cell and back pressure of the same magnitude is applied to the top and the bottom of the specimen, respectively. However, since the cell pressure cannot be applied to the area occupied by the loading piston at the top of the specimen, higher force is applied to the bottom of the specimen (Figure 4.15). The additional force acting on the bottom of the specimen, called the uplift force, is transmitted through the loading piston to the load cell. Therefore, the load measured with the load cell is not the actual load applied to the specimen, and needs to be corrected to account for the uplift force. In addition, since the loading piston and the top cap are imposing loads, which can not be measured with the load cell, their weights should be added to the axial load to obtain the actual load.

To evaluate the uplift force, the CRS cell was set up but without a specimen. The load platen was adjusted so that about $5.0 \mathrm{~N}$ of load was applied before applying the cell and the back pressure. The cell pressure was increased in small increments to about 500 $\mathrm{kPa}$, and the axial load and the cell pressure were monitored. 
As shown in Figure 4.16, the increase of axial load shows linear relationship with cell pressure. The slope of the linear best fitting curve of these data points represents the piston area and the y-intercept represents the piston weight. With these two parameters, the actual load can be calculated as follows:

Actual load $=$ Axial load - Piston area $*$ Cell pressure + Piston weight

When compressed, the load frame, the loading piston, the filter papers and the porous stones deflect as a function of the actual load. In the current configuration of the apparatus, the deformations of the specimen are measured at the top of the CRS cell as the relative change in the position of the DCDT clamped onto the loading piston. Therefore, the DCDT measurements include the displacement of the specimen as well as the deflection of the whole system due to compression. To obtain the actual deformation of the specimen, the compliance (machine deflection) must be quantified and the data from the DCDT should be corrected.

To evaluate the compliance of the system, the cell was set up with a steel dummy specimen with the same dimensions as specimen. The cell was filled with de-aired and de-ionized water but no cell pressure was applied. In this way, the load measured with the load cell corresponds to the actual load applied to the specimen. Once the cell was filled, a seating load of about $0.5 \mathrm{~kg}$ was applied. The CRS cell was loaded and unloaded at a constant rate while measuring the load and the displacement. The steel specimen deforms by about $3.39 \times 10^{-4} \mathrm{~mm}(0.001 \%$ axial strain) under the maximum applied load (8900 $\mathrm{N})$, thus it can be assumed as incompressible. Therefore, the displacement measured during calibration is the displacements of the system.

Figure 4.17 shows the compliance of the system during loading and unloading as a function of the actual load, and the best fitting curves obtained using power function. Under the maximum load, the system deflects about $0.30 \mathrm{~mm}$ (1.2\% axial strain) for CRS1 and $0.16 \mathrm{~mm}(0.63 \%$ axial strain) for CRS2. The maximum difference of the displacement at the same load during loading and unloading is about $0.025 \mathrm{~mm}(0.1 \%$ axial strain) for CRS1 and $0.007 \mathrm{~mm}$ (0.03\% axial strain) for CRS2. Although the best fitting curve for unloading has a higher $r^{2}$ value for CRS2, the best fitting curve for 
loading better captures the machine deflection below the actual load of $200 \mathrm{~N}$, which corresponds to the preconsolidation pressure of reconstituted specimen (70 kPa), where the strain of specimen is small. Therefore, the best fitting curve for loading was used to account for the compliance of both systems during loading and unloading.

\subsubsection{Data reduction}

As described in the previous section, the readings (voltages) from each sensor and the corresponding reading time were recorded by the data acquisition system and stored into data file during CRS test. After completion of test, the test data was reduced for analysis using a data reduction program written in Visual Basic and is embedded in a Microsoft Excel file. Two options are available for reduction of the CRS data: the linear and the non-linear solution (e.g. Wissa et al., 1971). The solutions for CRS test are described in the following section.

The sequence of data reduction is shown as a flow chart in Figure 4.18. First, the actual load was calculated by correcting the axial load for uplift force as a function of the cell pressure using the theoretical values of piston area and piston weight. The actual displacements of the specimen $(\Delta \mathrm{H})$ were calculated by correcting the displacements for the compliance as a function of the actual load. The axial strain, total stress and vertical effective stress were computed from the actual load, actual displacement and excess pore pressure. These calculations are based on the initial specimen height and the specimen area.

During consolidation, three strain rates were calculated: the axial strain rate based on the initial specimen height, the current strain rate based on the current specimen height, and the encoder strain rate based on the encoder that monitors the relative movement of the load platen. The starting and the ending points of each phase of CRS consolidation test were determined from the encoder strain rate. 


\subsubsection{CRS Consolidation theory}

Since the boundary conditions for the CRS test are different from those for conventional IL test, the Terzaghi's1-D consolidation theory cannot be applied to interpret the test data from CRS consolidation test. In 1941, Wissa et al. developed solutions for CRS tests based on both linear and non-linear stress-strain relationship including the initial transient and steady states. The basic assumption of the solutions for CRS tests is that infinitesimal strains exist and that the coefficient of consolidation $\left(\mathrm{C}_{\mathrm{v}}\right)$ is constant and is independent of the depth at any time.

Based on the assumptions, the basic equation of consolidation is expressed as

$$
C_{v} \frac{\partial^{2} \varepsilon}{\partial z^{2}}=\frac{\partial \varepsilon}{\partial t}
$$

where $C_{v}=k / m_{v} \gamma_{w} ; \mathrm{t}=$ time; $\mathrm{z}=$ the vertical coordinate of a point from the top of the specimen.

The solution of equation (4.3) for the strain at any point, $\mathrm{z}$ and time, $\mathrm{t}$ is:

$$
\varepsilon(z, t)=\gamma t[1+F(z, t)]
$$

in which $F(z, t)=\frac{1}{6 T_{v}}\left[2-6 \frac{z}{H}+3\left(\frac{z}{H}\right)^{2}\right]-\frac{2}{\pi^{2} T_{v}} \sum_{n=1}^{\infty} \frac{\cos n \pi z}{n^{2} H} \exp \left(-n^{2} \pi^{2} T v\right)$

and, $T_{v}=\frac{C_{v} t}{H^{2}}$

The first part of function F represents the deviation from the average strain in the steady state, and the second part describes the decay of the initial discontinuities setup in the transient state. As shown in Figure 4.19, By the time $T_{v}$ is 0.5 , the transient component becomes insignificant

The data obtained from CRS tests include, as described in the previous section, the total stress, $\sigma_{\mathrm{v}}$, measured at the top of the specimen $(\mathrm{z}=0)$, which is constant throughout the specimen, the excess pore pressure, $u_{h}$, measured at the bottom of the specimen $(\mathrm{z}=\mathrm{H})$, and the average strain rate, $\gamma$. 
In order to interpret the CRS test data, it is necessary to assume the relationship between the stress and the strain, and the following two possibilities are considered: linear and non-linear stress-strain relationship.

\subsubsection{Linear theory}

The basic assumption of the linear theory is that the coefficient of volume compressibility, $\mathrm{m}_{\mathrm{v}}$, is constant. In other words, the change of effective stress is linearly proportional to the change of strain, and the relation can be expressed as:

$$
\Delta \sigma_{v}^{\prime}=\frac{\Delta \varepsilon}{m_{v}}
$$

The transient state occurs at the start of loading or throughout the duration of tests performed at higher strain rates in which large excess pore pressure is generated. The transient conditions can be interpreted from equation (4.4). At any time t, the ratio of the strain at the bottom $(\mathrm{z}=\mathrm{H})$ and the top $(\mathrm{z}=0)$ of the specimen can be expresses as:

$$
\frac{\varepsilon(H, t)}{\varepsilon(0, t)}=\frac{1+F(H, t)}{1+F(0, t)}=F_{3}\left(T_{v}\right)
$$

For a linear material, since the change of strain is proportional to the change of effective stress from time 0 and t, equation (4.4) can be expressed as

$$
F_{3}=\frac{\sigma^{\prime}{ }_{v}(H, t)-\sigma^{\prime}{ }_{v}(H, 0)}{\sigma^{\prime}{ }_{v}(0, t)-\sigma^{\prime}{ }_{v}(0,0)}=\frac{\left(\sigma_{v}-u_{h}\right)-\sigma_{v, t=0}}{\left(\sigma_{v}\right)-\sigma_{v, t=0}}
$$

The function $F_{3}$ is dependent only on $T_{v}$. and $F_{3}$ can be plotted as a function of $T_{v}$ (Figure 4.20). Sheahan et al (1997) proposed an equation for $\mathrm{T}_{\mathrm{v}}$ after performing a regression analysis on the plot of $\mathrm{T}_{\mathrm{v}}$ versus $\mathrm{F}_{3}$ as follows:

$$
T_{v}=4.78\left(F_{3}\right)^{3}-3.21\left(F_{3}\right)^{2}+1.65 F_{3}+0.0356
$$

If $\mathrm{T}_{\mathrm{V}}$ is smaller than 0.5 at any time t, the specimen is assumed to be in the transient state. Once the value of $F_{3}$ is obtained from CRS tests data and equation (4.7), $\mathrm{T}_{\mathrm{v}}$ can be calculated from equation (4.8), and the coefficient of consolidation and the 
hydraulic conductivity in the transient state can be calculated using Terzaghi's theory as follows:

$$
\begin{aligned}
& C_{v}=\frac{T_{v} H^{2}}{t} \\
& k=C_{v} m_{v} \gamma_{w}
\end{aligned}
$$

If $T_{v}$ becomes greater than 0.5 , the soil is assumed to be in the steady state, and the second term of function $\mathrm{F}(\mathrm{z}, \mathrm{t})$ in equation (4.4) can be neglected. In the steady state, the strain is parabolically distributed through the depth of the specimen. Therefore, the average strain can be obtained as follows:

$$
\varepsilon_{\text {ave }}=\frac{1}{3}[2 \varepsilon(H, t)+\varepsilon(0, t)]=\gamma t
$$

and the average effective stress corresponding to the average strain is

$$
\sigma_{\text {vave }}^{\prime}=\frac{1}{3}\left[2 \sigma_{v}^{\prime}(H, t)+\sigma_{v}^{\prime}(0, t)\right]=\sigma_{v}-\frac{2}{3} u_{h}
$$

The hydraulic conductivity of a linear soil in the steady state can be directly calculated from the difference of the stress and strain at the top and bottom of the specimen. The strain and the effective stress at the top and bottom of the specimen at any time $t$ are:

At top: $\varepsilon(0, t)=\gamma t+\frac{\gamma H^{2}}{3 C_{v}} \quad \sigma_{v}^{\prime}=\sigma_{v}$

At bottom: $\varepsilon(H, t)=\gamma t-\frac{\gamma H^{2}}{6 C_{v}} \quad \sigma_{v}^{\prime}=\sigma_{v}-u_{h}$

Using equations (4.5) and (4.10), the differences in strain stress between top and bottom is expressed as:

$$
\begin{aligned}
& \left(\sigma_{v}-u_{h}\right)-\sigma_{v}=\frac{\varepsilon(0, t)-\varepsilon(H, t)}{m_{v}}, \\
& u_{h}=\frac{\gamma H^{2}}{2 C_{v} m_{v}}=\frac{\gamma H^{2} \gamma_{w}}{2 k}
\end{aligned}
$$

Rearranging equation (4.11) for the hydraulic conductivity, k: 


$$
k=\frac{\gamma H^{2} \gamma_{w}}{2 u_{h}}
$$

Also from equation (4.13), the coefficient of consolidation is:

$$
C_{v}=\frac{\gamma H^{2}}{2 m_{v} u_{h}}
$$

\subsubsection{Non-linear theory}

The assumption for non-linear theory is that the difference of strain is related to the difference of the logarithm of effective stress by a constant $\mathrm{C}_{\varepsilon}$ called the strain index as:

$$
-\frac{d \varepsilon}{d\left(\log \sigma_{v}^{\prime}\right)}=C \varepsilon
$$

Since the change in strain is proportional to the change in logarithms of the effective stress, the function $\mathrm{F}_{3}$ is expressed as:

$$
F_{3}=\frac{\log \left[\sigma_{v}^{\prime}(H, t)\right]-\log \left[\sigma_{v}^{\prime}(H, 0)\right]}{\log \left[\sigma_{v}{ }_{v}(0, t)\right]-\log \left[\sigma_{v}^{\prime}(0,0)\right]}=\frac{\log \left(\sigma_{v}-u_{h}\right)-\log \left(\sigma_{v, t=0}\right)}{\log \left(\sigma_{v}\right)-\log \left(\sigma_{v, t=0}\right)}
$$

From the value $F_{3}, T_{v}$ can be estimated using equation (4.8). If the transient condition exists $\left(T_{\mathrm{v}}<0.5\right)$ at any time $t, k$ and $C_{v}$ can be calculated from the value $T_{v}$ using equations (4.9) and (4.10), respectively.

In the steady state, using the effective stresses and the strains at the top and bottom of specimen at any time t, equation (4.16) can be approximated as:

$$
\frac{\varepsilon(0, t)-\varepsilon(H, t)}{\log \left(\sigma_{v}\right)-\log \left(\sigma_{v}-u_{h}\right)}=\frac{-\gamma H^{2}}{2 C_{v} \log \left(\frac{\sigma_{v}-u_{h}}{\sigma_{v}}\right)}=C_{\varepsilon}
$$

Similarly, by comparing conditions at two different times, $t_{1}$ and $t_{2}$, at the top of the specimen yields: 


$$
\frac{\varepsilon\left(0, t_{1}\right)-\varepsilon\left(0, t_{2}\right)}{\log \left(\sigma_{v 1}\right)-\log \left(\sigma_{v 2}\right)}=\frac{\gamma \Delta t}{\log \left(\frac{\sigma_{v 2}}{\sigma_{v 1}}\right)}=C_{\varepsilon}
$$

Combining equations (4.18) and (4.19), the coefficient of consolidation is:

$$
C_{v}=\frac{-H^{2} \log \left(\frac{\sigma_{v 2}}{\sigma_{v 1}}\right)}{2 \Delta t \log \left(1-\frac{u_{h}}{\sigma_{v}}\right)}
$$

The coefficient of volume compressibility, $\mathrm{m}_{\mathrm{v}}$, can be computed from equation (4.19) as:

$$
m_{v}=\frac{0.434 C_{\varepsilon}}{\sigma_{v}^{\prime}}
$$

in which $\sigma^{\prime}{ }_{v}$ is the average effective stress between time $t_{1}$ and $t_{2}$.

From equations (4.20) and (4.21), the hydraulic conductivity can be calculated as follows:

$$
k=C_{v} m_{v} \gamma_{w}=\frac{-0.434 \gamma H^{2} \gamma_{w}}{2 \sigma^{\prime}{ }_{v} \log \left(1-\frac{u_{h}}{\sigma_{v}}\right)}
$$

For a non-linear soil, the distribution of strain in steady state is parabolic and the average strain can be calculated using equation (4.11). Then, the average effective stress corresponding to the average strain is calculated from the following equation:

$$
\sigma_{\text {vave }}^{\prime}=\left(\sigma_{v}^{3}-2 \sigma_{v}^{2} u_{h}+\sigma_{v} u_{h}^{2}\right)^{1 / 3}
$$

\subsubsection{Linear vs. Non-linear theory}

The difference of the results obtained from the linear and non-linear theories can be evaluated by comparing the equations for the vertical effective stress, the hydraulic conductivity and the coefficient of consolidation.

From equations (4.12) and (4.23), the ratio of the vertical effective stresses from the linear and non-linear theories is 


$$
\frac{\sigma_{\text {vlinear }}^{\prime}}{\sigma_{\text {vnon-linear }}^{\prime}}=\frac{\sigma_{v}-\frac{2}{3} u_{h}}{\left(\sigma_{v}^{3}-2 \sigma_{v}{ }^{2} u_{h}+\sigma_{v} u_{h}^{2}\right)^{1 / 3}}=\frac{1-\frac{2}{3} \frac{u_{h}}{\sigma_{v}}}{\left[1-2 \frac{u_{h}}{\sigma_{v}}+\left(\frac{u_{h}}{\sigma_{v}}\right)^{2}\right]^{1 / 3}}
$$

The ratio of the vertical effective stresses from the linear and non-linear theories is plotted against $\mathrm{u}_{\mathrm{h}} / \sigma_{\mathrm{v}}$ in Figure 4.21. The plot shows that for positive excess pore pressure, the linear theory yields greater vertical effective stress than the non-linear theory, and that the divergence between the two theories increases with the ratio of $\mathrm{u}_{\mathrm{h}} / \sigma_{\mathrm{v}}$.

Similarly, the ratios of the hydraulic conductivity and the coefficient of consolidation can be derived as, using $\sigma^{\prime}{ }_{v}=\sigma_{v}$ for small $u_{\mathrm{h}}$ :

$$
\frac{k_{\text {linear }}}{k_{\text {non-linear }}}=\frac{\frac{\gamma H^{2} \gamma_{w}}{2 u_{h}}}{-\frac{0.434 \gamma H^{2} \gamma_{w}}{2 \sigma_{v}^{\prime} \log \left(1-\frac{u_{h}}{\sigma_{v}}\right)}}=-\frac{\log \left(1-\frac{u_{h}}{\sigma_{v}}\right)}{0.434 \frac{u_{h}}{\sigma_{v}}}
$$

and,

$$
\frac{C_{\text {vlinear }}}{C_{\text {v non-linear }}}=\frac{\frac{\gamma H^{2}}{2 m_{v} u_{h}}}{-\frac{0.434 \gamma H^{2}}{2 \sigma_{v}{ }_{v} m_{v} \log \left(1-\frac{u_{h}}{\sigma_{v}}\right)}}=-\frac{\log \left(1-\frac{u_{h}}{\sigma_{v}}\right)}{0.434 \frac{u_{h}}{\sigma_{v}}}
$$

Since the hydraulic conductivity and the coefficient of consolidation are related to each other through equation (4.10), the ratios of these are the same. The ratio of the coefficient of consolidations obtained from using the linear and non-linear theories is plotted against $u_{h} / \sigma_{v}$ in Figure 4.22. The ratio of the coefficient of consolidation is similar to the ratio of the vertical effective stress that for positive excess pore pressure, the linear theory produced the greater value of the coefficient of consolidation than the non-linear 
theory. The difference between the two theories diverges as the ratio $\mathrm{u}_{\mathrm{h}} / \sigma_{\mathrm{v}}$ increases, but the divergence of the coefficient of consolidation is greater than that of vertical effective stress. It should be noted that this comparison is valid only for small values of $\mathrm{u}_{\mathrm{h}} / \sigma_{\mathrm{v}}$ so that the constrained modulus from the linear and non-linear theories can be cancelled out and the vertical effective stress can be replaced with the total stress is equations (4. 25) and (4.26). 
Table 4.1: Summary of the experimental program carried out in the research

\begin{tabular}{|c|c|c|c|c|}
\hline Test Type & Soil & Binding Agent & Surcharge $(\mathrm{kPa})$ & Curing Time (days) \\
\hline \multirow[t]{8}{*}{ UC tests: (Strength) } & \multirow[t]{6}{*}{ LR Soil } & PC: 4,8,12,20,50 \% & $14.5,48,96$ & $1,7,28$ \\
\hline & & CaO: 4,8,12,20\% & 48 & 7 \\
\hline & & $\begin{array}{l}\mathrm{PC}+\mathrm{CaO}: \\
8 \% \mathrm{PC}+4 \% \mathrm{CaO} \\
8 \% \mathrm{PC}+8 \% \mathrm{CaO} \\
8 \% \mathrm{PC}+12 \% \mathrm{CaO}\end{array}$ & 48 & $1,7,28$ \\
\hline & & HC: 4,8,12,20\% & 48 & 7 \\
\hline & & MB: 4,8,12,20\% & 48 & 7 \\
\hline & & BEN: 4,8,12,20\% & 48 & 7 \\
\hline & MI & PC: 4,8,12,20,30 & $14.5,48,96$ & $1,7,28$ \\
\hline & MII & PC: $8 \%$ & 48 & $1,7,28$ \\
\hline $\begin{array}{l}\text { CRS consolidation } \\
\text { tests: (Stiffness) }\end{array}$ & LR Soil & PC: $8,20,50,100,150 \%$ & 48 & 14 \\
\hline
\end{tabular}


Table 4.2: Weights of cements per unit volume of treated and untreated soil

\begin{tabular}{|c|c|c|c|}
\hline Soil Type & $\begin{array}{l}\text { Cement content } \\
\text { By dry mass (\%) }\end{array}$ & $\begin{array}{l}\text { Cement in treated } \\
\text { soil }\left(\mathrm{kg} / \mathrm{m}^{3}\right)\end{array}$ & $\begin{array}{c}\text { Cement in untreated } \\
\text { soil }\left(\mathrm{kg} / \mathrm{m}^{3}\right)\end{array}$ \\
\hline \multirow{5}{*}{$\mathrm{MI}(\mathrm{w}=60 \%)$} & 4 & 40.66 & 41.75 \\
\hline & 8 & 79.23 & 83.51 \\
\hline & 12 & 115.88 & 125.26 \\
\hline & 20 & 183.96 & 208.77 \\
\hline & 30 & 260.46 & 313.15 \\
\hline MII (w= $102.2 \%)$ & 8 & 63.28 & 65.97 \\
\hline \multirow{7}{*}{ LR soil $(w=289 \%)$} & 4 & 12.70 & 12.80 \\
\hline & 8 & 25.19 & 25.61 \\
\hline & 12 & 37.48 & 38.41 \\
\hline & 20 & 61.47 & 64.02 \\
\hline & 50 & 145.05 & 160.04 \\
\hline & 100 & 290.09 & 320.08 \\
\hline & 150 & 435.13 & 480.12 \\
\hline
\end{tabular}


Table 4.3: Information of the Sensors used in CRS1 system.

\begin{tabular}{|l|l|l|l|l|}
\hline Sensor & I.D. & Range & Resolution & Stability \\
\hline DCDT & $0243-0141$ & $2.54 \mathrm{~cm}$ & $\begin{array}{l}0.232 \mu \mathrm{m} \\
(0.1 \mathrm{mV})\end{array}$ & $\begin{array}{l}3.016 \mu \mathrm{m} \\
(1.3 \mathrm{mV})\end{array}$ \\
\hline Load Cell & IC18824 & $8900 \mathrm{~N}$ & $\begin{array}{l}0.287 \mathrm{~N} \\
(0.001 \mathrm{mV})\end{array}$ & $\begin{array}{l}2.296 \mathrm{~N} \\
(0.008 \mathrm{mV})\end{array}$ \\
\hline Pore Pressure & $8162-014$ & $1379 \mathrm{kPa}$ & $\begin{array}{l}0.0138 \mathrm{kPa} \\
(0.001 \mathrm{mV})\end{array}$ & $\begin{array}{l}0.1104 \mathrm{kPa} \\
(0.008 \mathrm{mV})\end{array}$ \\
Transducer & & & $0.0138 \mathrm{kPa}$ & $0.114 \mathrm{kPa}$ \\
Cell Pressure & $8162-024$ & $1379 \mathrm{kPa}$ & $(0.001 \mathrm{mV})$ & $(0.008 \mathrm{mV})$ \\
\hline
\end{tabular}

Table 4.4: Information of the Sensors used in CRS2 system.

\begin{tabular}{|l|l|l|l|l|}
\hline Sensor & I.D. & Range & Resolution & Stability \\
\hline DCDT & $0244-0177$ & $5.08 \mathrm{~cm}$ & $\begin{array}{l}0.308 \mu \mathrm{m} \\
(0.1 \mathrm{mV})\end{array}$ & $\begin{array}{l}4.004 \mu \mathrm{m} \\
(1.3 \mathrm{mV})\end{array}$ \\
\hline Load Cell & 102116 & $8900 \mathrm{~N}$ & $\begin{array}{l}0.479 \mathrm{~N} \\
(0.001 \mathrm{mV})\end{array}$ & $\begin{array}{l}3.382 \mathrm{~N} \\
(0.008 \mathrm{mV})\end{array}$ \\
\hline Pore Pressure & PS2036 & $1379 \mathrm{kPa}$ & $0.0138 \mathrm{kPa}$ & $0.1104 \mathrm{kPa}$ \\
Transducer & & & $(0.001 \mathrm{mV})$ & $(0.008 \mathrm{mV})$ \\
\hline Cell Pressure \\
Transducer
\end{tabular}




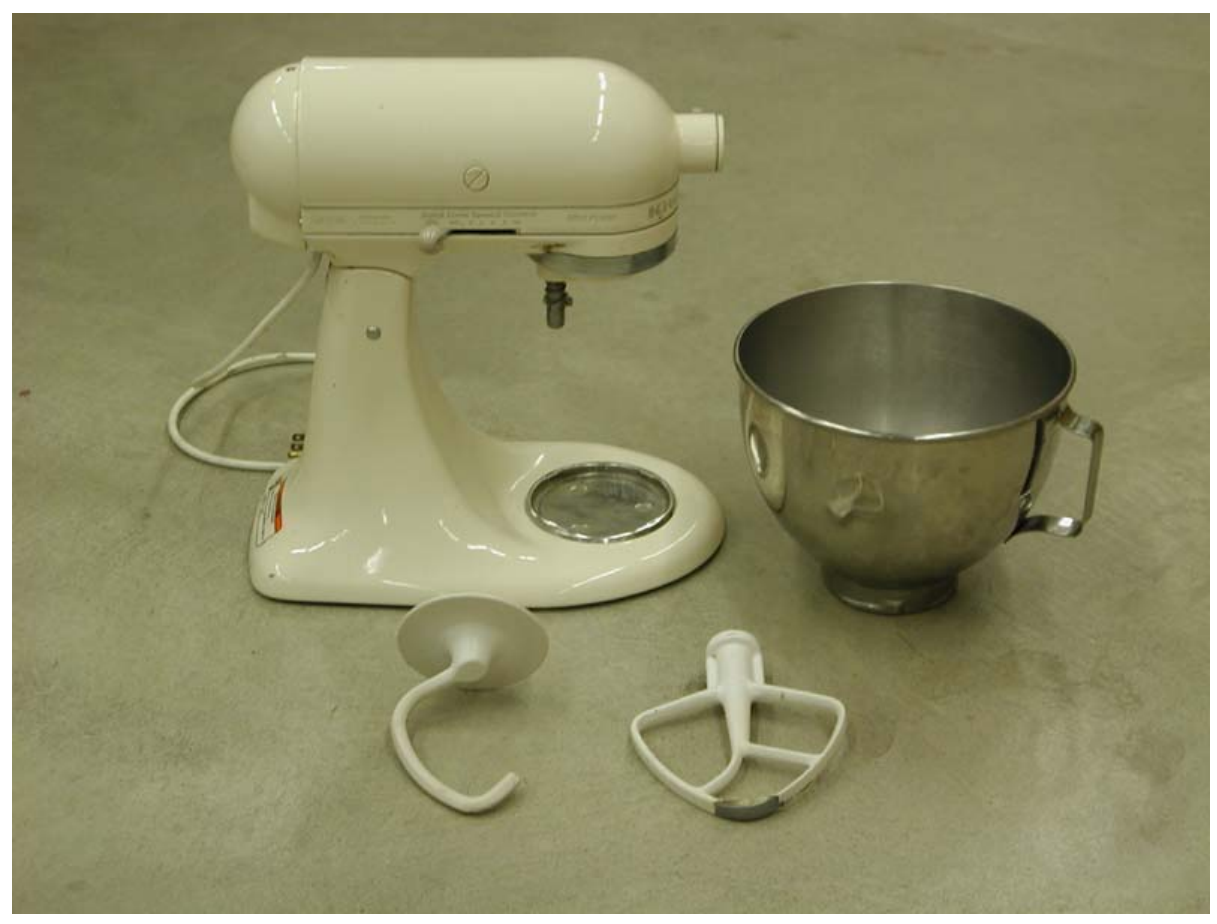

Figure 4.1: Mixer with two different mixing tools employed to prepare samples for CRS consolidation tests. 


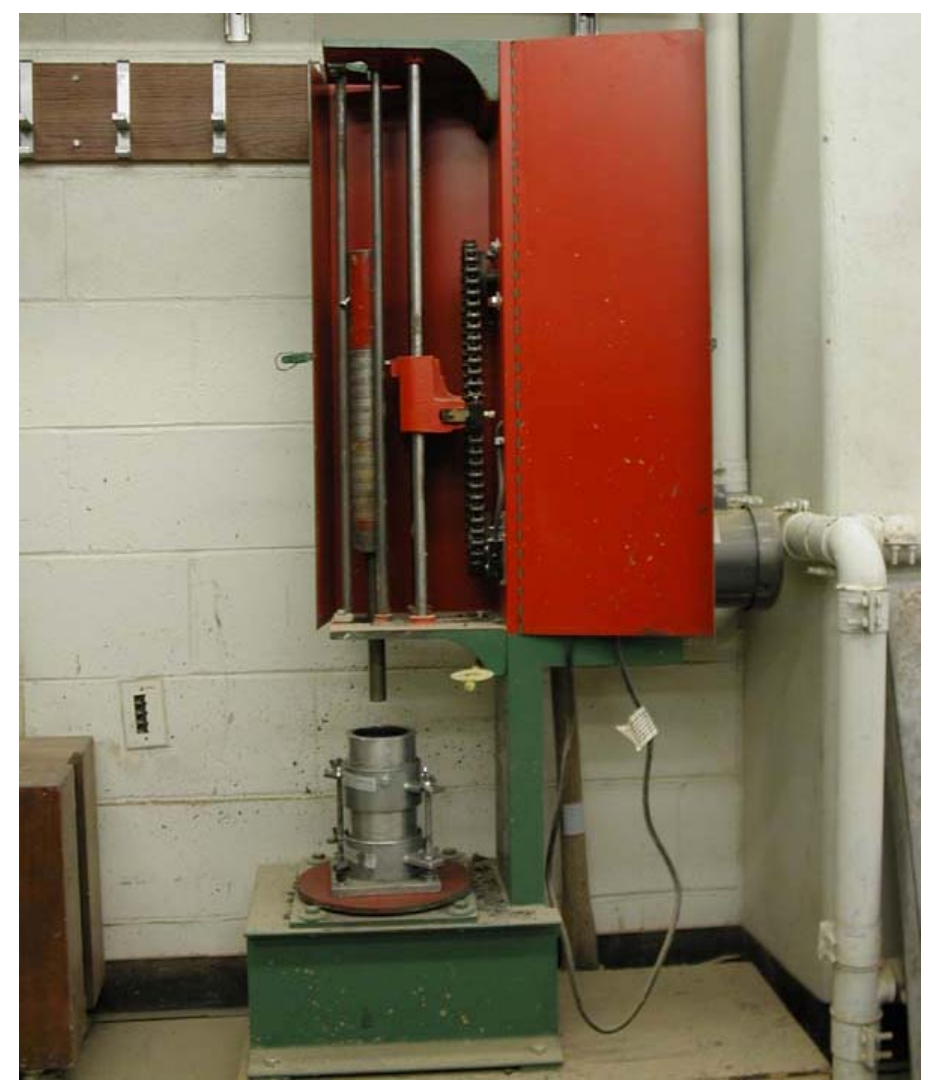

Figure 4.2: Modified mechanical proctor compactor employed for treated LR soil samples

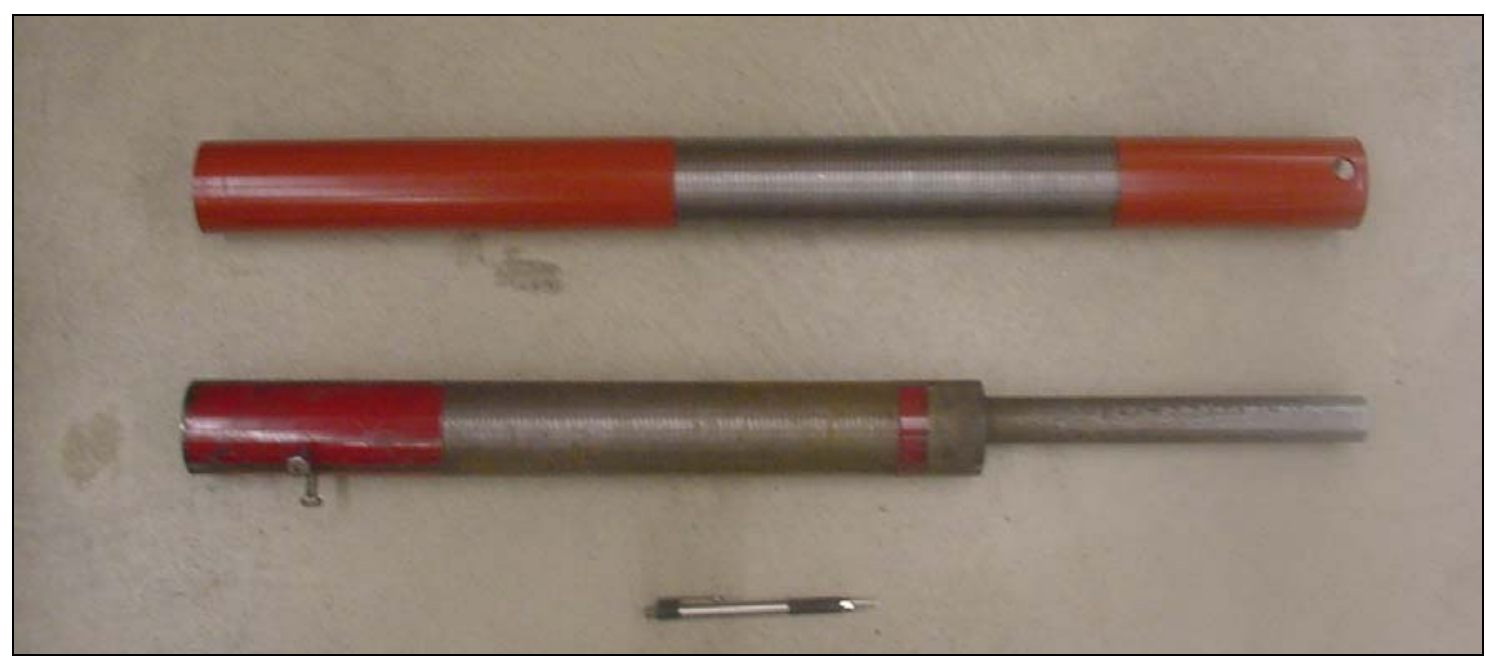

Figure 4.3: Original $(\mathrm{D}=5.08 \mathrm{~cm}$, above) and modified compaction hammer $(\mathrm{D}=2.54$ cm, below) 


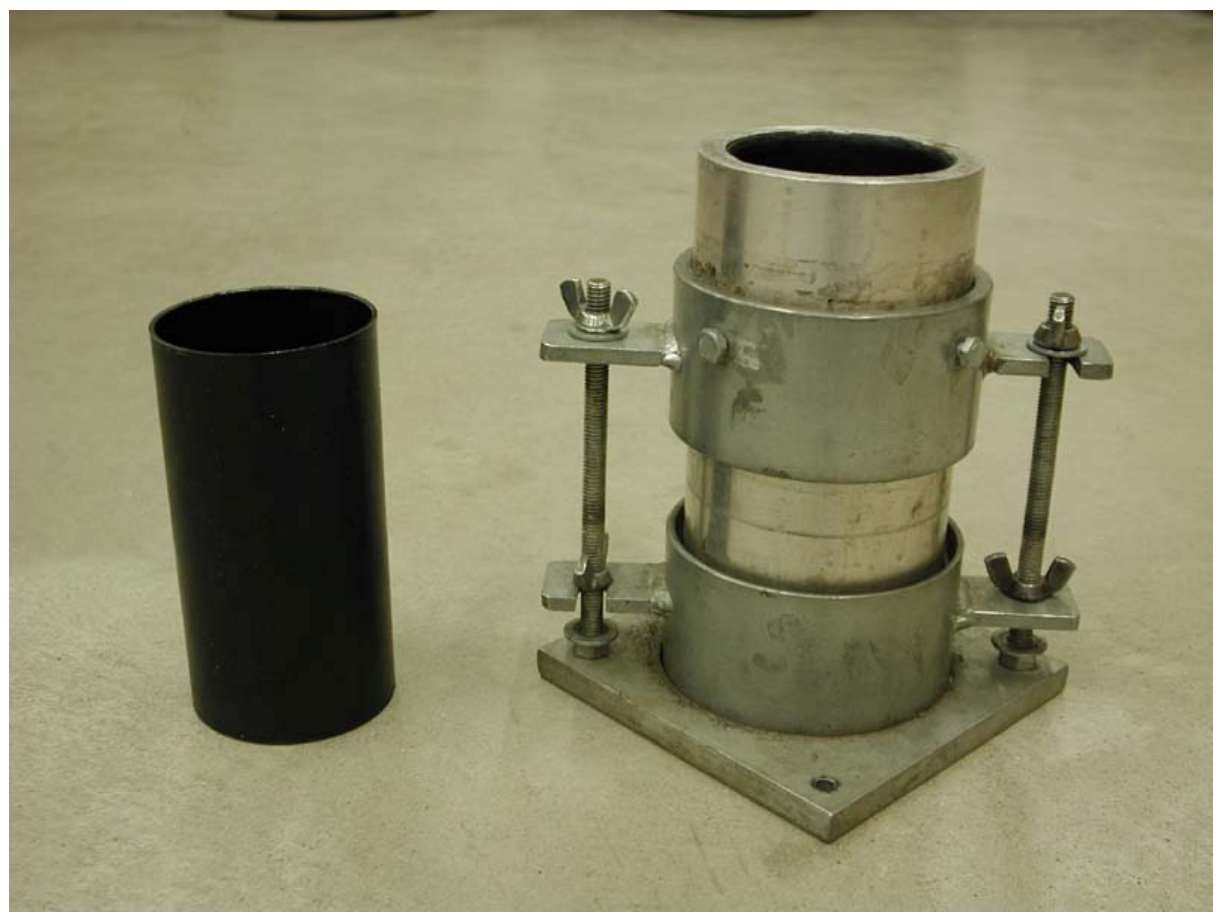

Figure 4.4: A plastic cylinder and modified compaction mold employed for compaction. 


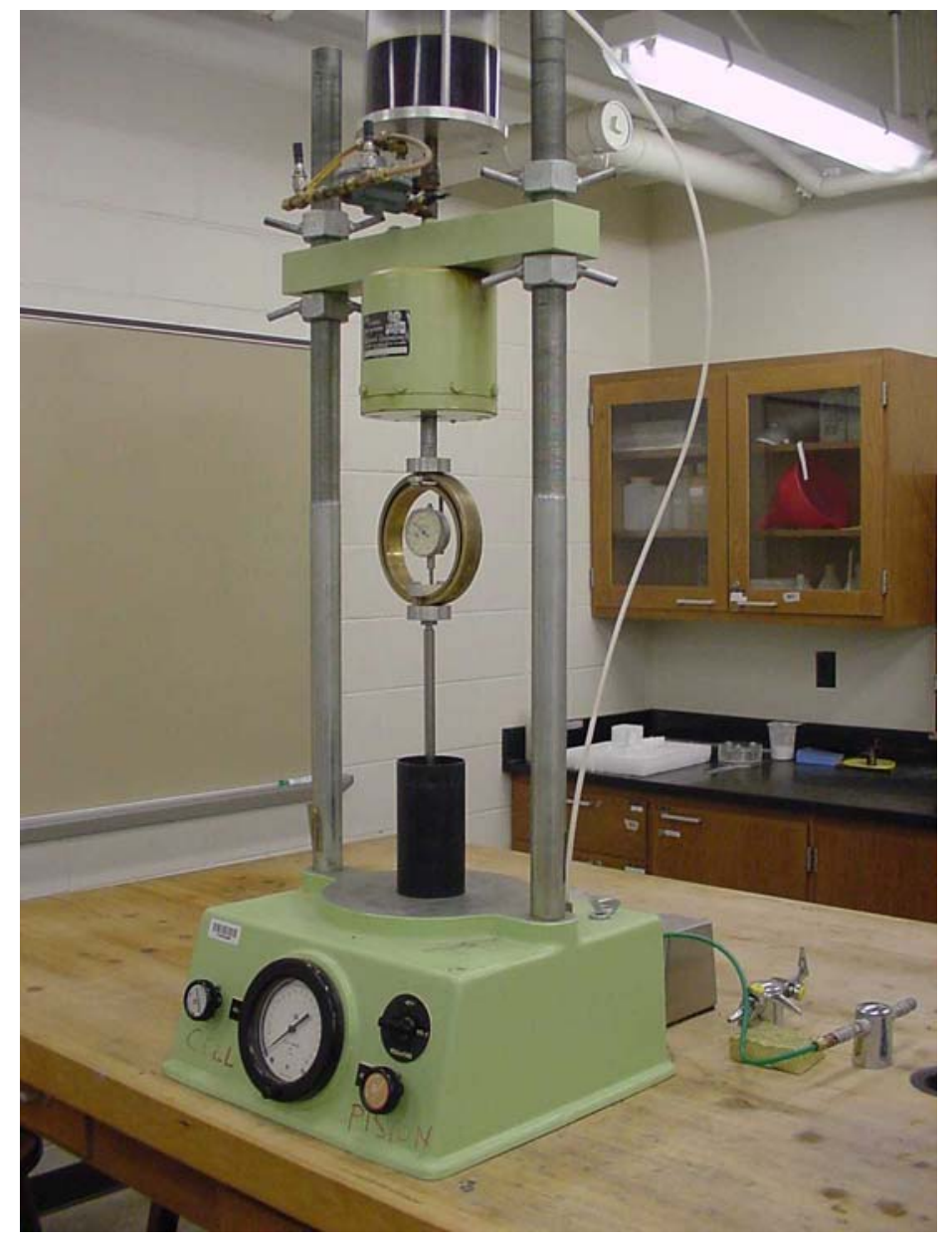

Figure 4.5: Stress control loading frame employed for preparation of treated MI and MII samples 


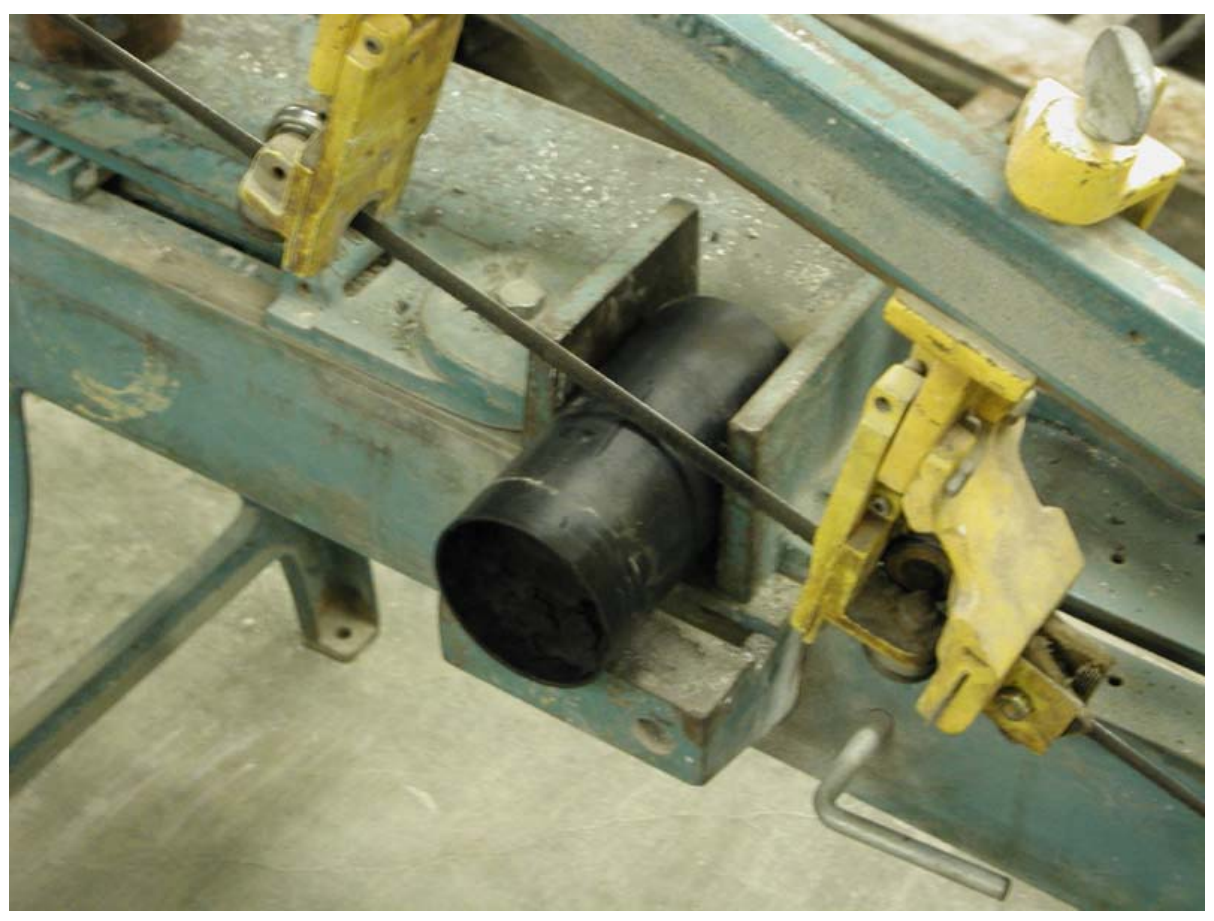

Figure 4.6: Cutting of plastic cylinder with Wells metal band saw for preparation of soil samples for CRS consolidation tests 


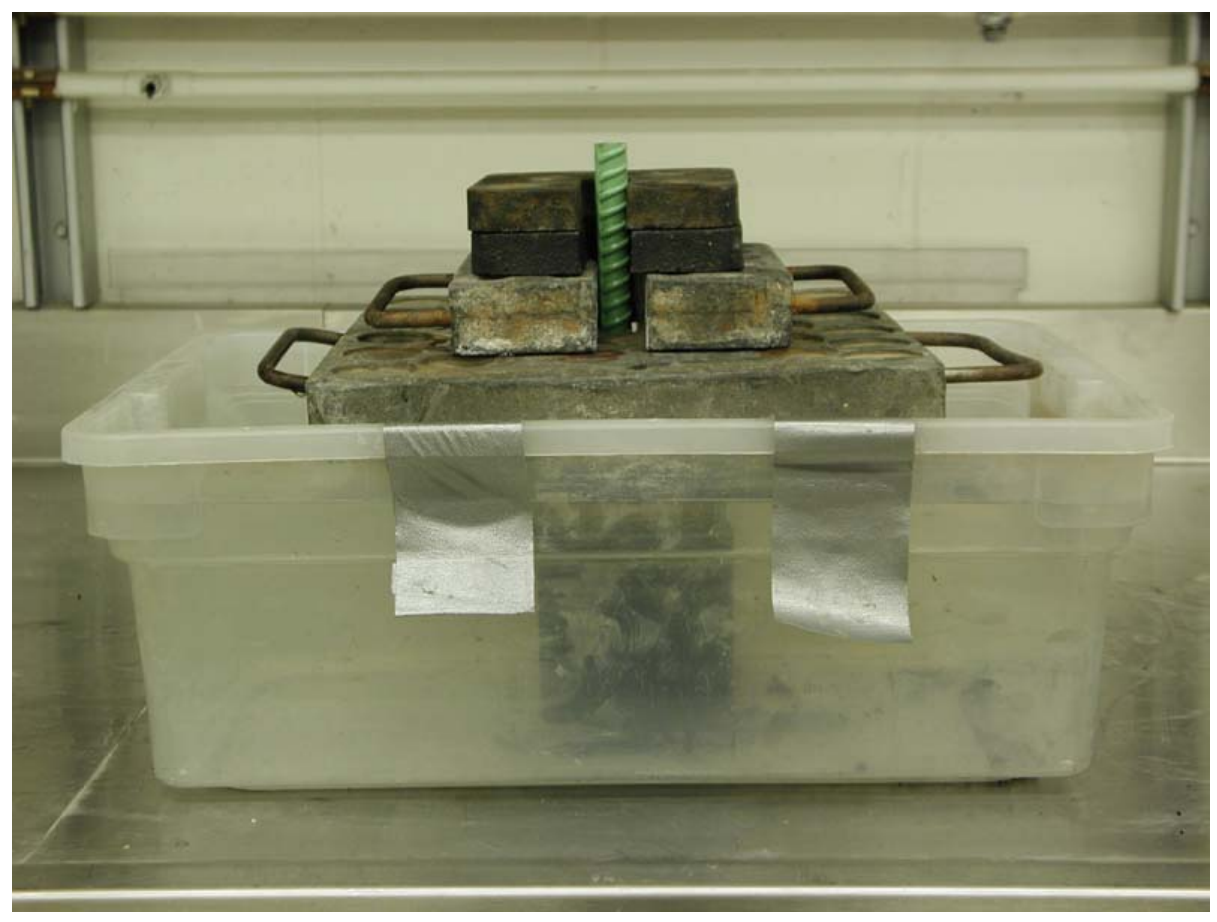

Figure 4.7: Curing of soil specimen in water bath under a surcharge of $48 \mathrm{kPa}$

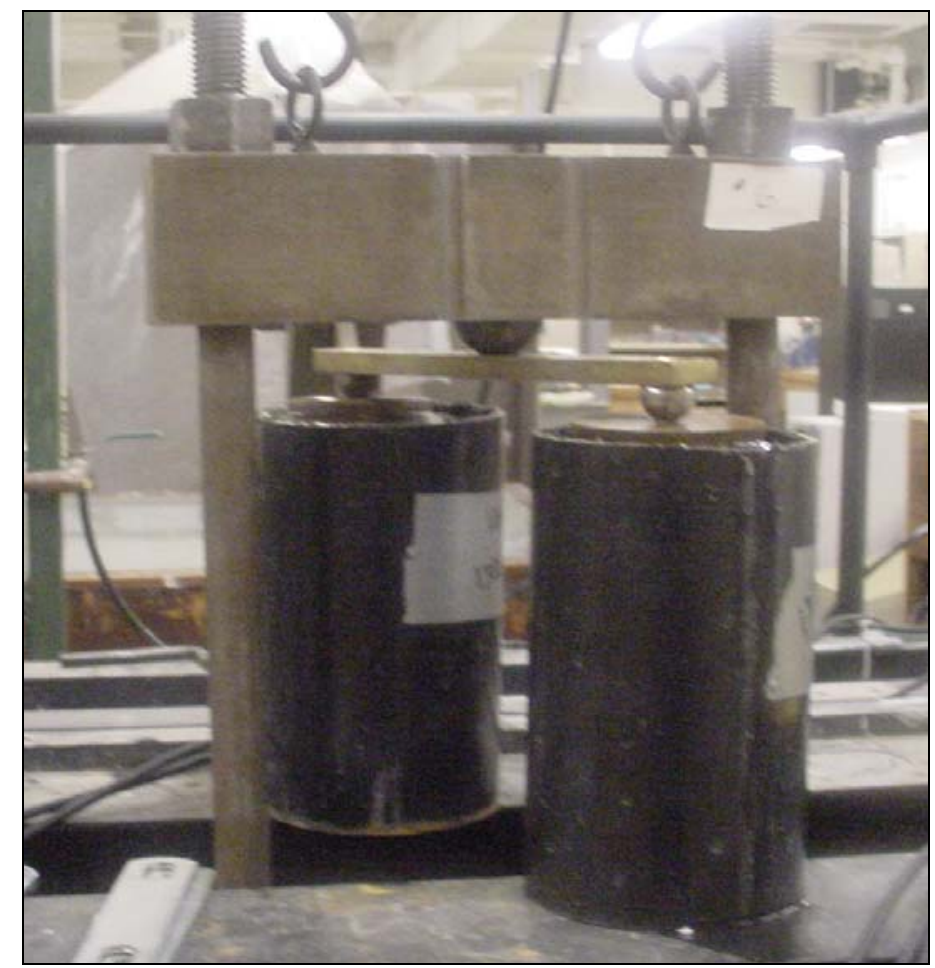

Figure 4.8: Specimen curing in consolidation frame under a surcharge of $96 \mathrm{kPa}$ 


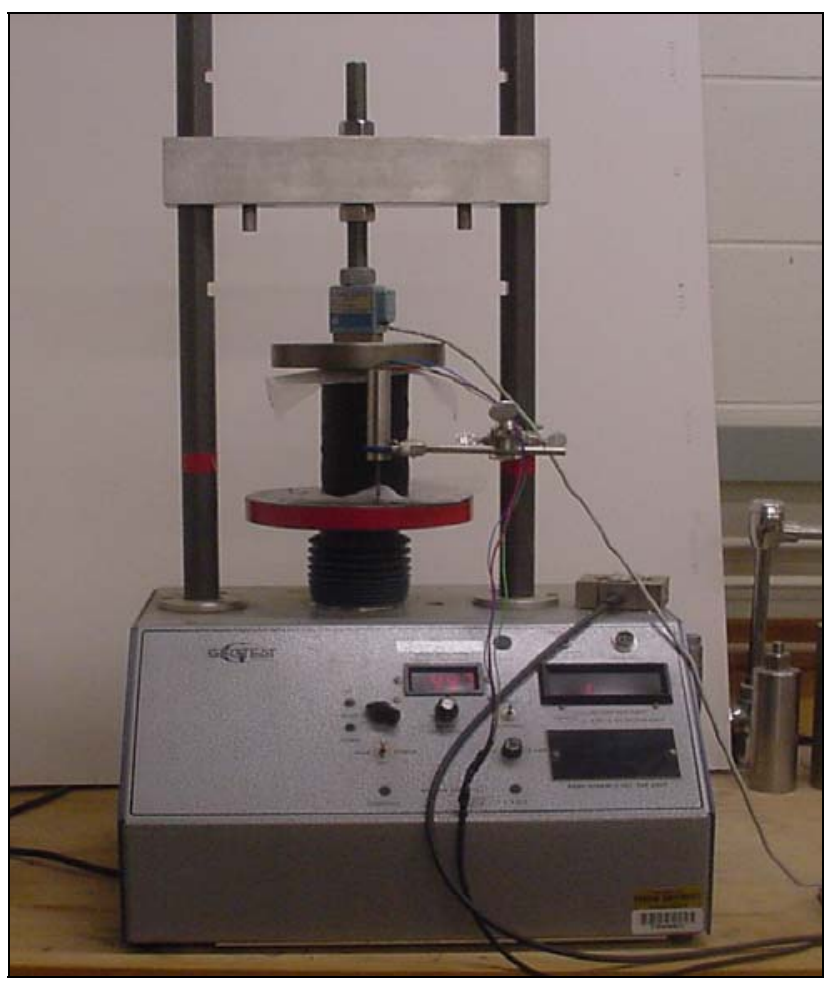

Figure 4.9: Unconfined compression test apparatus 


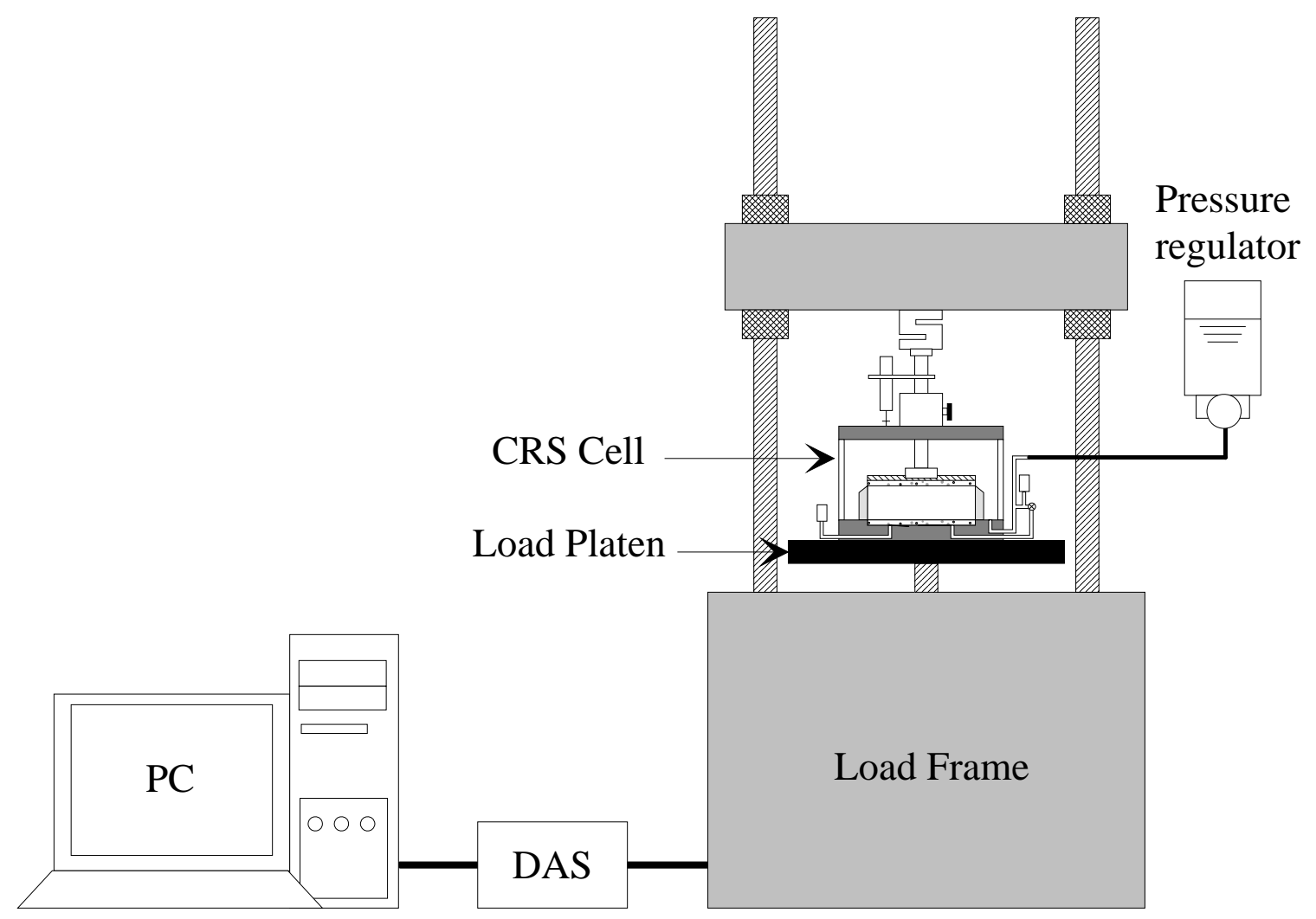

Figure 4.10: Schematic of four components of CRS consolidation test apparatus; CRS Cell, Load Frame, Air-Water Interface Pressure Regulator and Data Acquisition System 


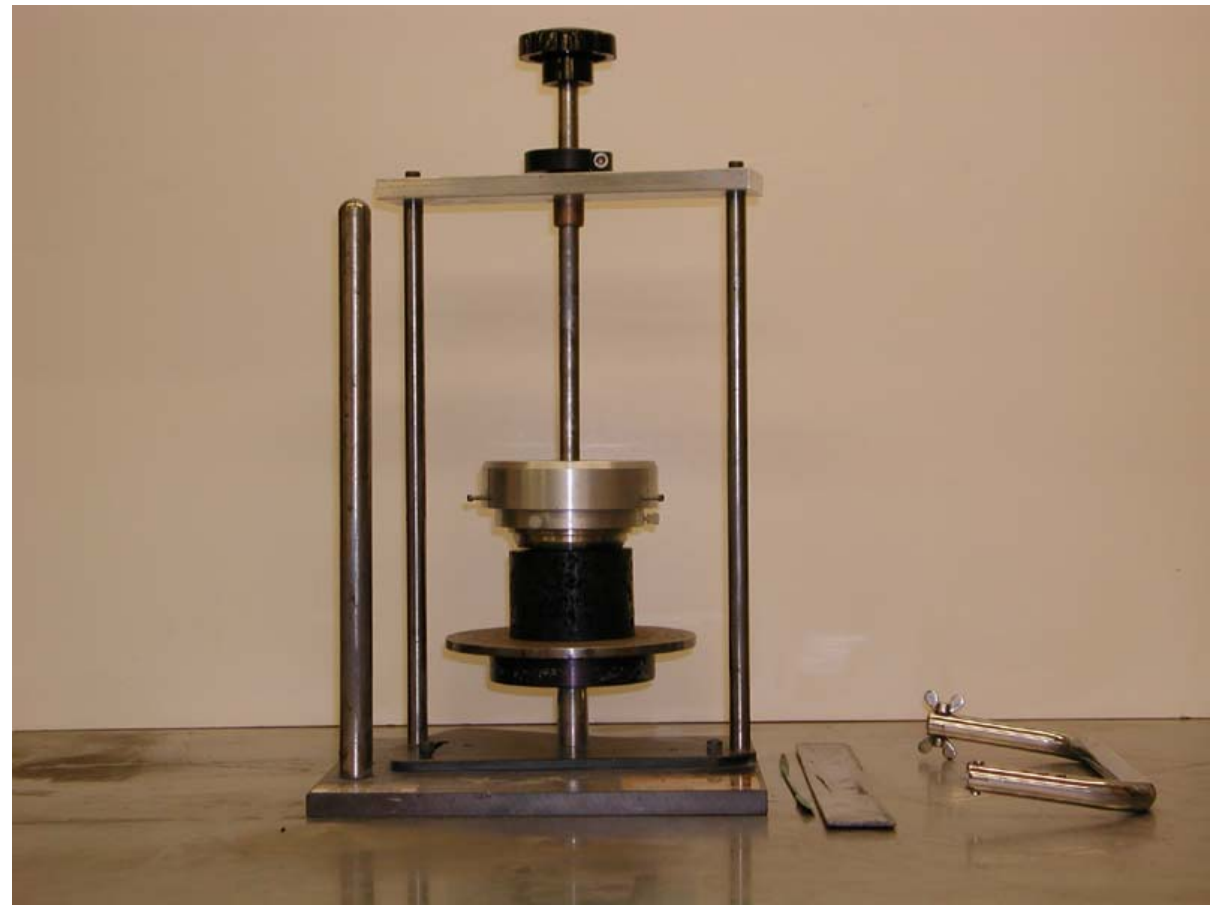

Figure 4.11: Specimen trimming apparatus: trimming frame, a thin spatula, cutting blade and a wire saw. 


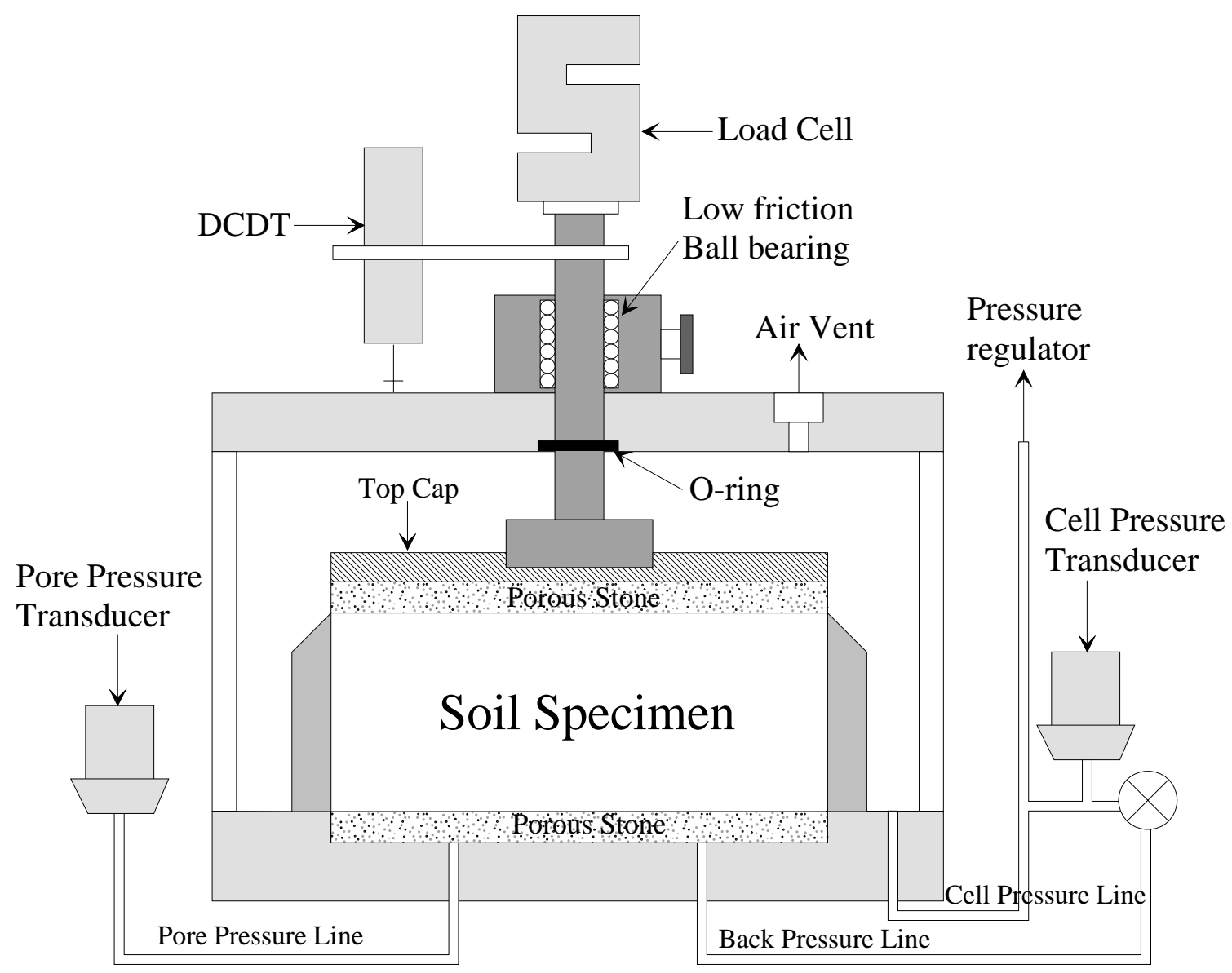

Figure 4.12: Schematic of CRS consolidation cell (CRS1) 

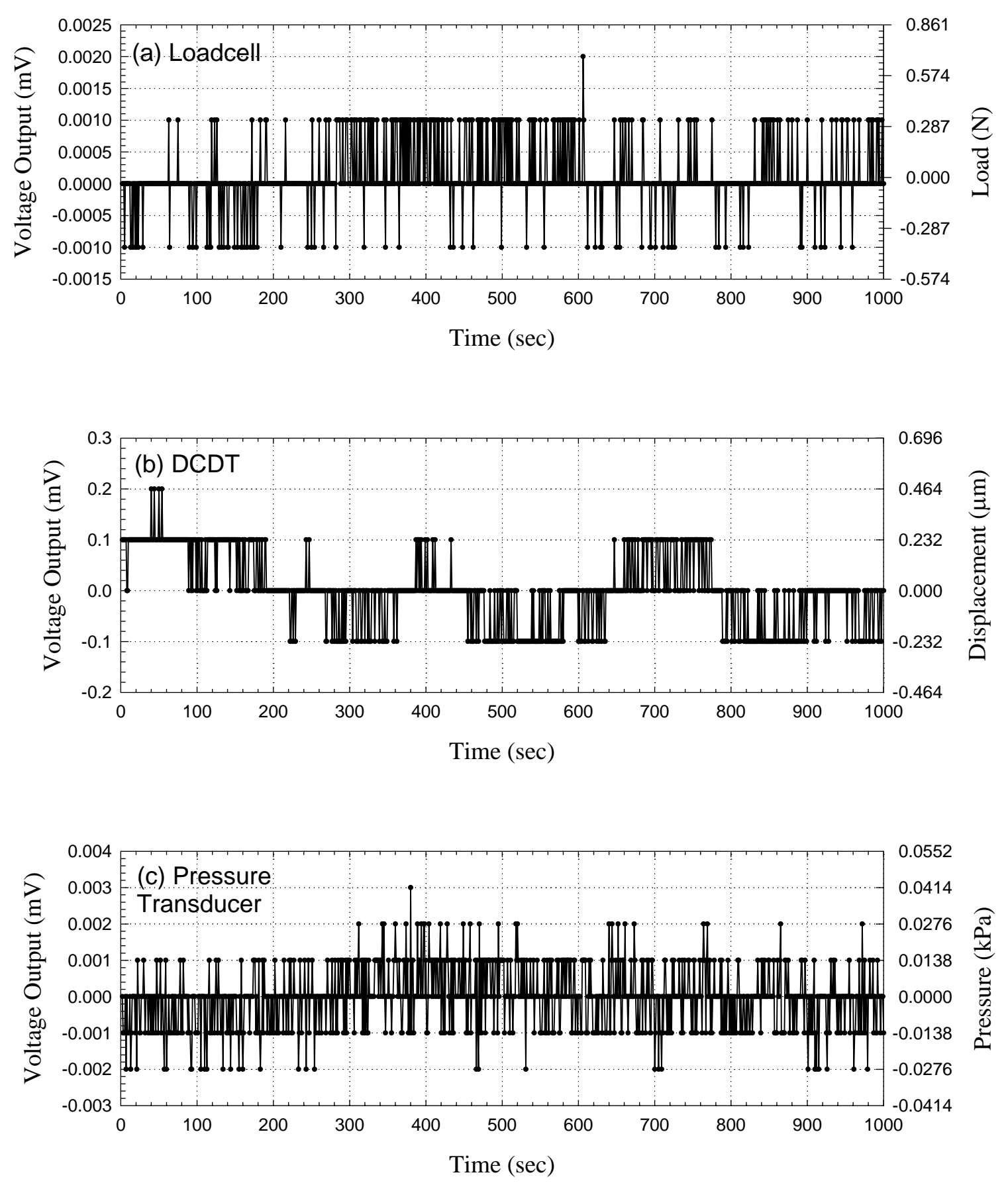

Figure 4.13: Resolution of the Data Acquisition System and sensors 

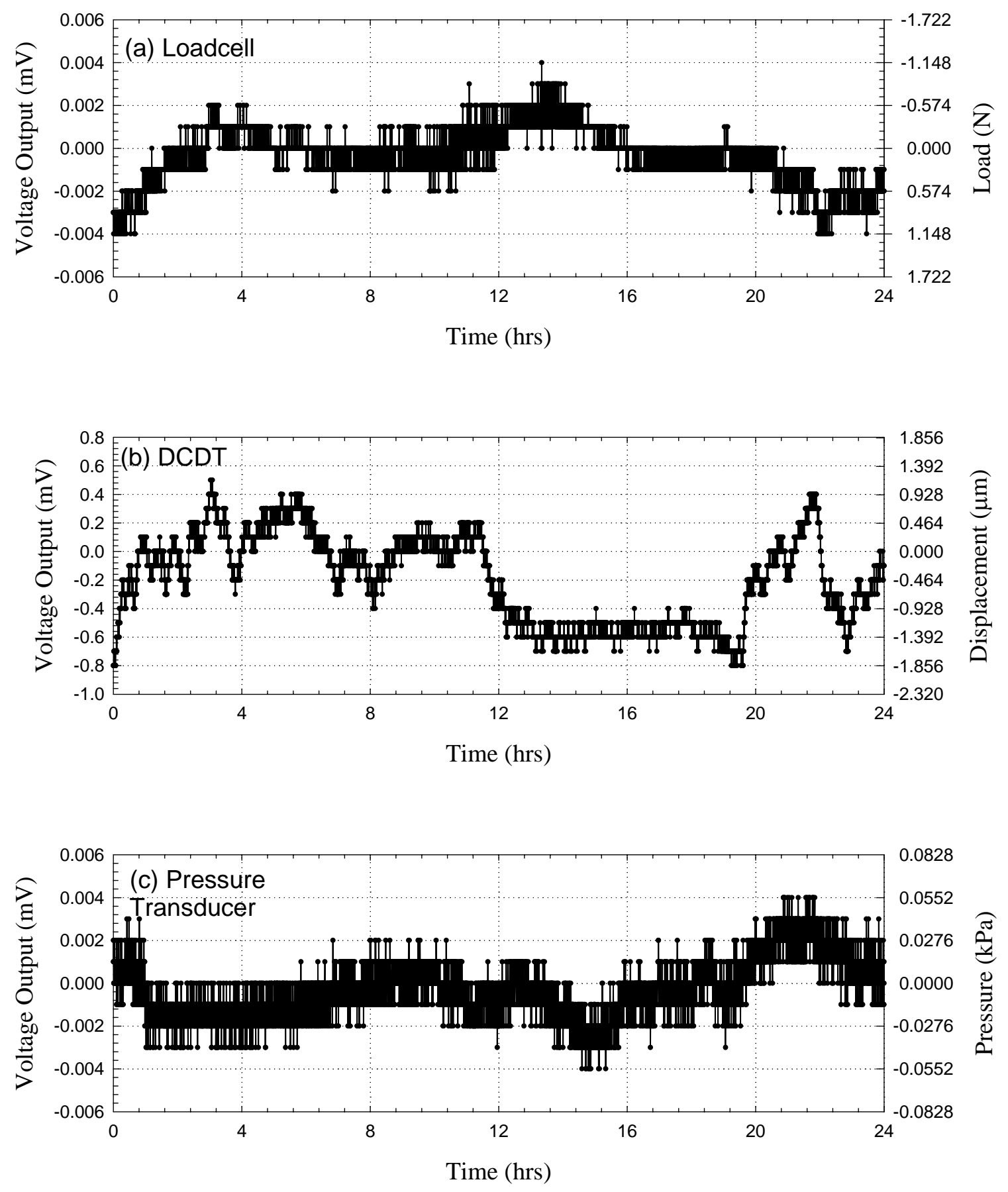

Figure 4.14: Stability of each sensor 


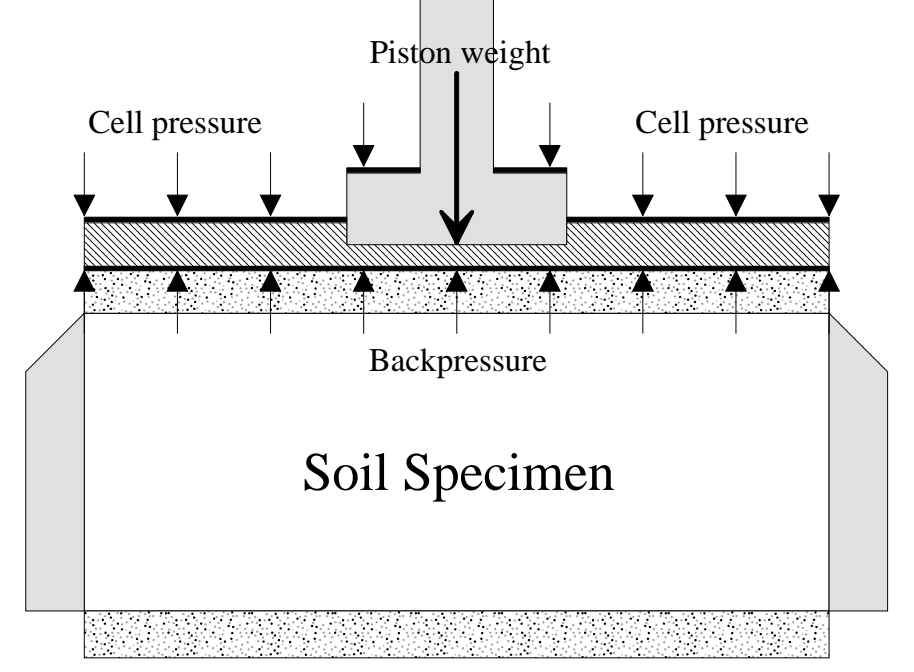

Figure 4.15: Uplift calibration for CRS cell (The area subject to cell or backpressure is indicated with a thick line) 

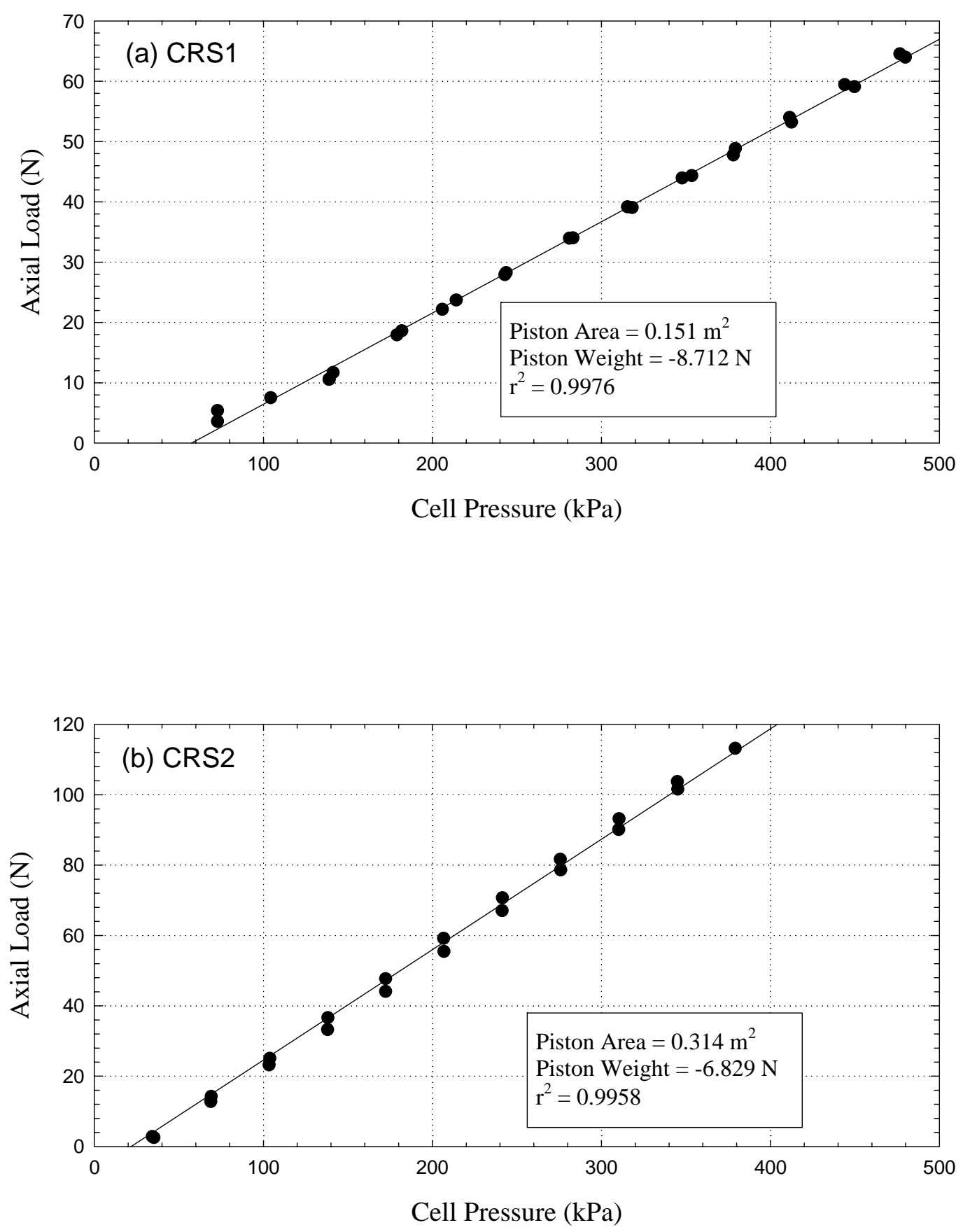

Figure 4.16: Uplift calibration curves for (a) CRS1 and (b) CRS2 cells (The piston areas and weights are indicated for each system). 

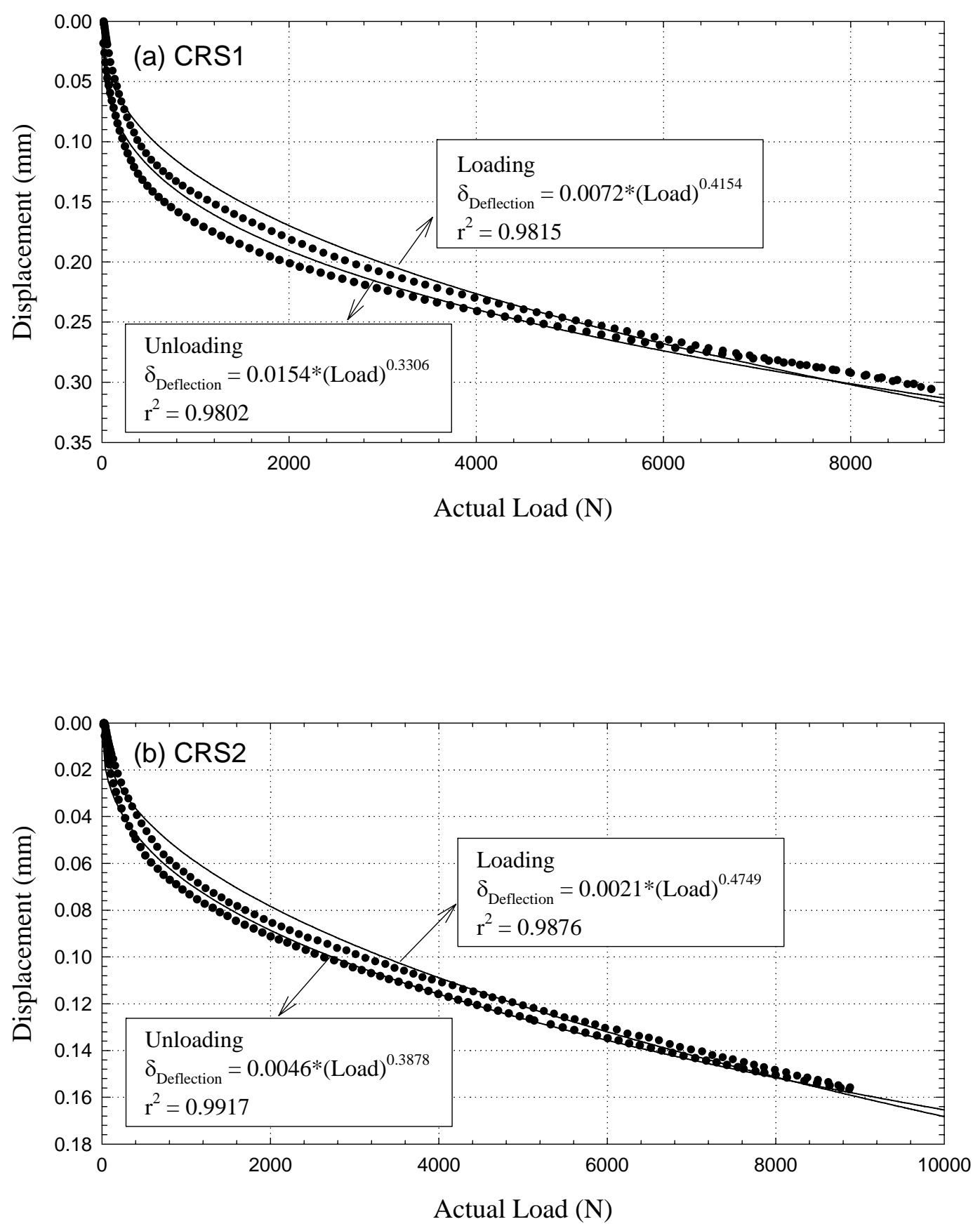

Figure 4.17: Machine Deflection (Compliance) of (a) CRS1 and (b) CRS2. 


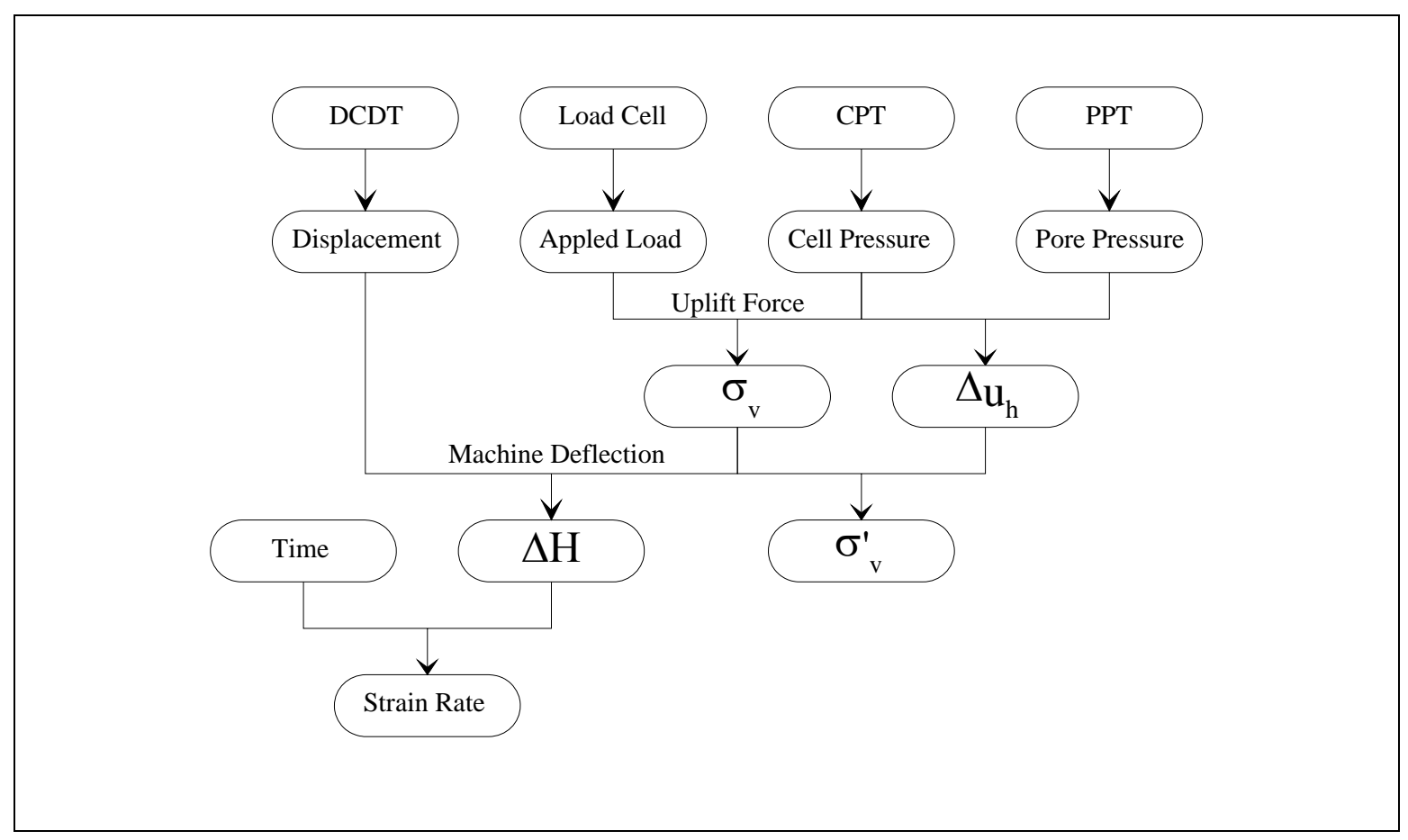

Figure 4.18: Flow chart for data reduction 


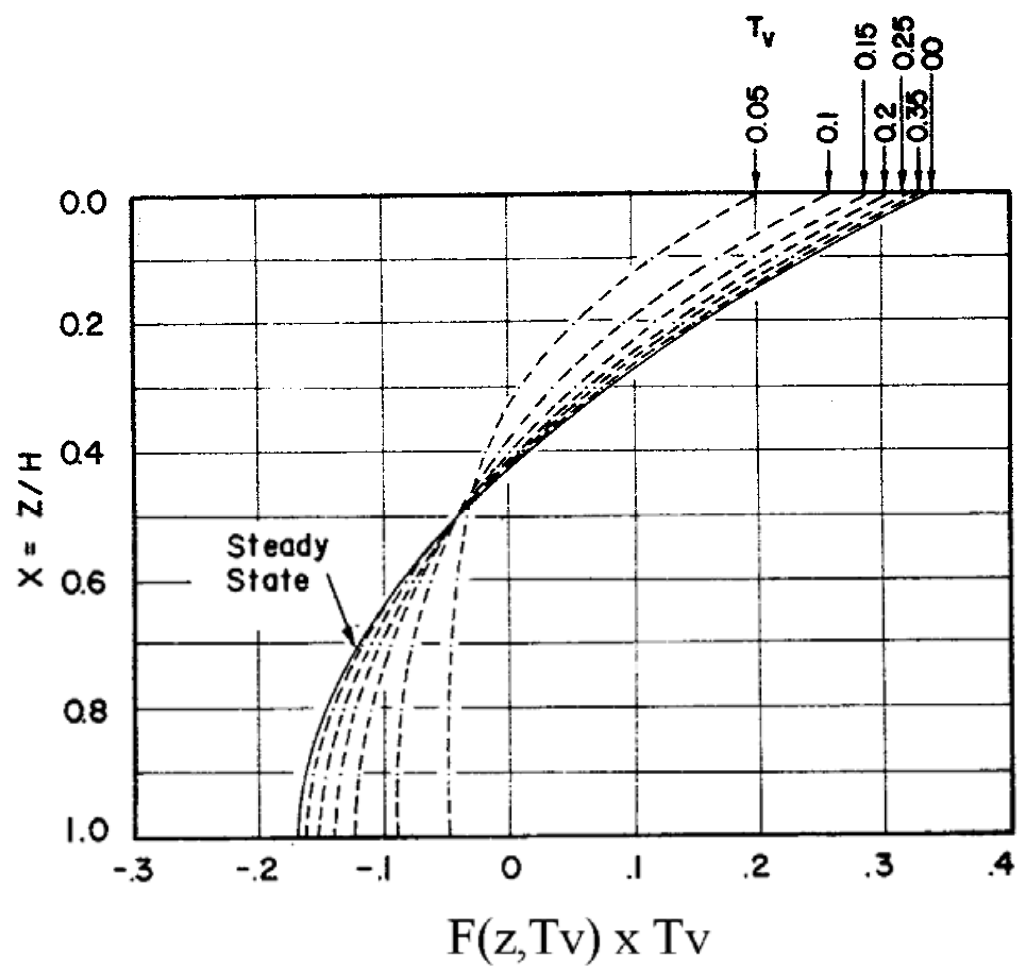

Figure 4.19: Deviation of strain as function of death for different time factor (From Wissa et al, 1971) 


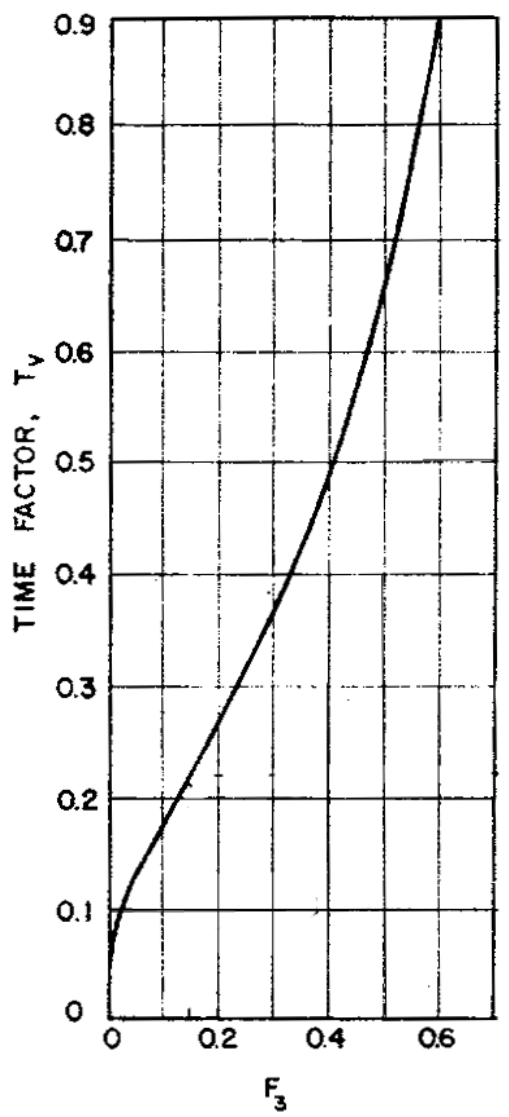

Figure 4.20: Plot of the function F3 as a function of Tv 

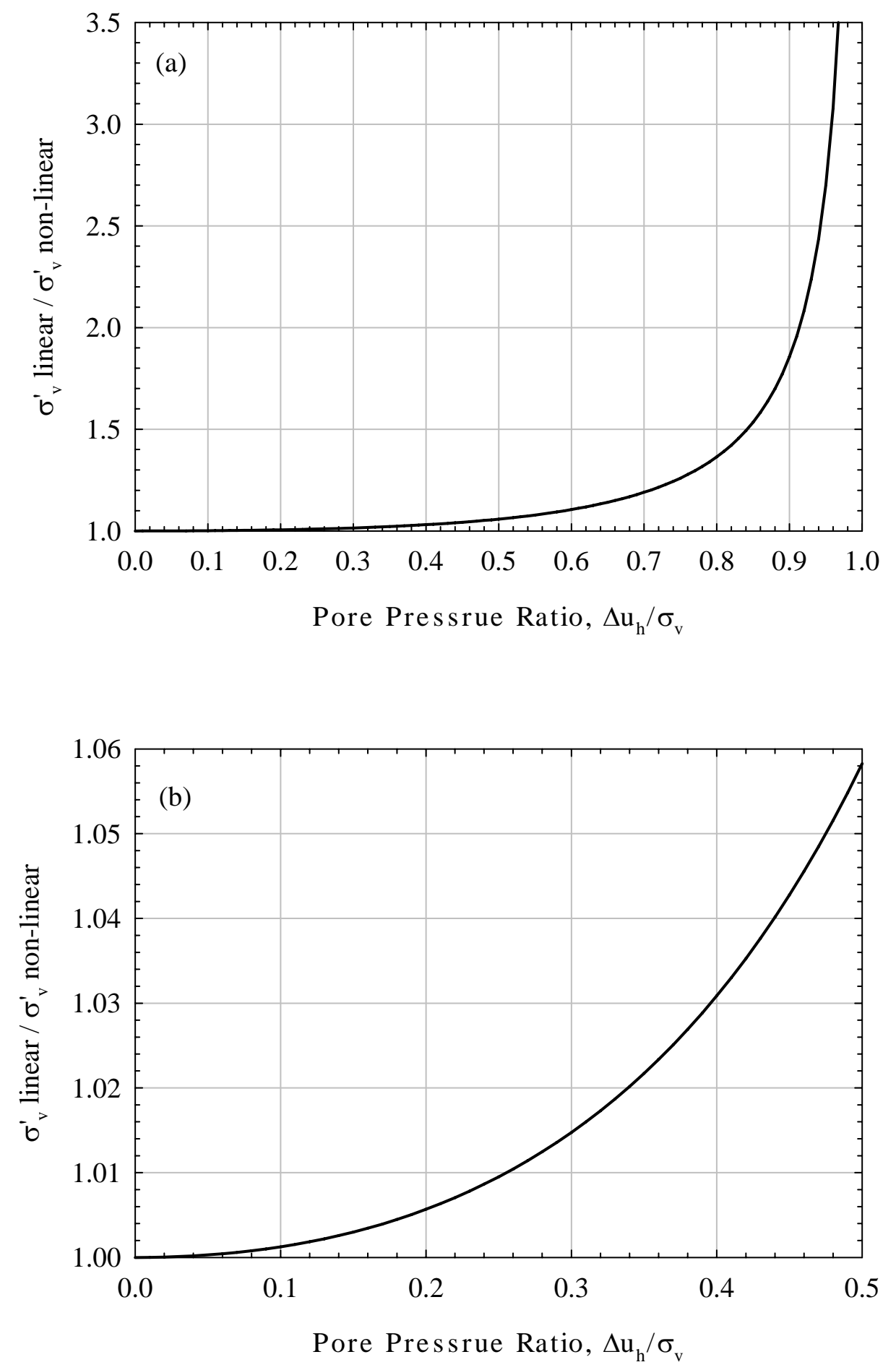

Figure 4.21: Comparison of vertical effective stress computed using linear and non-linear CRS theory (a) $0<\mathrm{u}_{\mathrm{h}} / \sigma_{\mathrm{v}}<1.0$ and (b) $0<\mathrm{u}_{\mathrm{h}} / \sigma_{\mathrm{v}}<0.5$ 

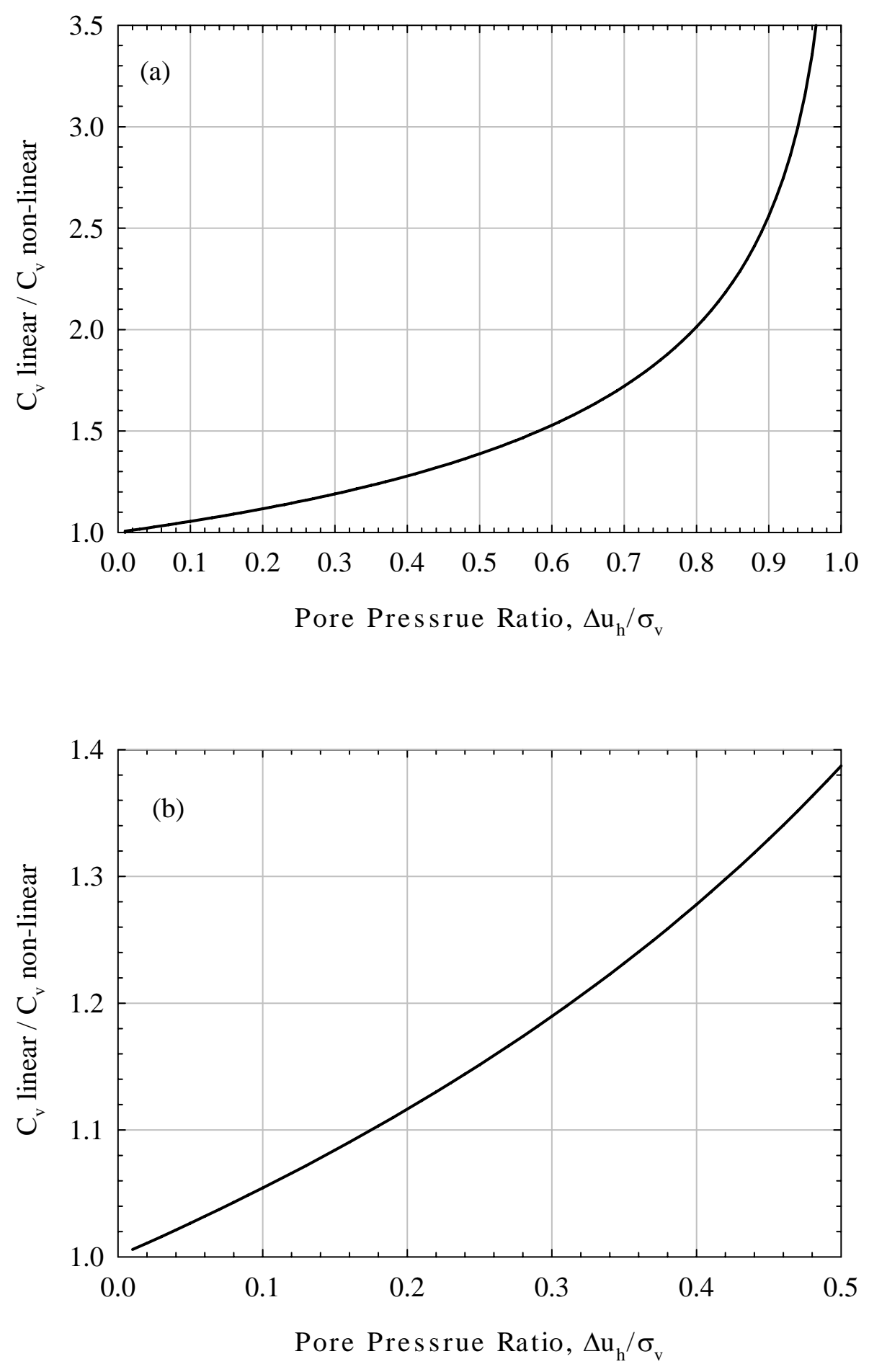

Figure 4.22: Comparison of coefficient of consolidation computed using linear and nonlinear CRS theory (a) $0<\mathrm{u}_{\mathrm{h}} / \sigma_{\mathrm{v}}<1.0$ and (b) $0<\mathrm{u}_{\mathrm{h}} / \sigma_{\mathrm{v}}<0.5$ 
CHAPTER 5: STRENGTH: UNCONFINED COMPRESSION TESTS

CHAPTER 6: STIFFNESS: CRS CONSOLIDATION TESTS

CHAPTER 7: CONCLUSIONS AND RECOMMENDATIONS 


\section{CHAPTER 5: STRENGTH: UNCONFINED COMPRESSION TESTS}

\subsection{INTRODUCTION}

This chapter evaluates the effectiveness of the soil treatment methods identified in Chapter 3, as measured through unconfined compression tests. As indicated in Chapter 3, the following variables were investigated: amount of binder, curing time, and applied surcharge. The investigated binder includes Portland cement (PC), Calcium Oxide (CaO), combination of PC and CaO, two commercial limes, High Calcium Flue Dust (HC) and Marblehead Buffington Dust (MB), and Bentonite (BEN). Table 5.1 summarizes the combinations of the variables investigated in the experimental program.

Three important pieces of information are contained within the stress-strain plots, particularly the compressive strength $\left(\sigma_{\mathrm{c}}\right)$, the strain at failure $\left(\varepsilon_{\mathrm{f}}\right)$, and the initial stiffness (E). The compressive strength is the peak axial stress that is reached during the test. This peak stress occurs at the strain at failure $\left(\varepsilon_{\mathrm{f}}\right)$. The slope of the initial linear portion of the stress-strain curve up to an axial strain of one percent is taken as the initial stiffness of the soil. Table 5.2 summarizes the testing conditions (type and quantity of binder, amount and duration of surcharge) and the main data for all the tests performed on PC and $\mathrm{CaO}$ treated specimens. These include:

Specimen identification number

Binder type and content

The compressive strength $\left(\sigma_{\mathrm{c}}\right)$

The strain at failure $\left(\varepsilon_{\mathrm{f}}\right)$

The initial Young's modulus (E)

The change in height during curing $\left(\Delta \mathrm{H}_{\text {curing }}\right)$

The initial $\left(\mathrm{e}_{0}\right)$ and final $\left(\mathrm{e}_{\mathrm{f}}\right)$ void ratio determined from the measured volumes and final dry mass.

Due to the fact that the measurement of the strain is not performed on-specimen, the curve displays an initial concave portion caused by equipment deformation with 
minor pickup of the load. This is due to bedding effects while the top platen of the loading frame establishes contact with the soil specimen. In order to correct for this behavior, the linear portion of the curve is extrapolated backwards to zero strain. Figures 5.1 and 5.2 show the difference between uncorrected and corrected stress-strain curves, respectively.

The stress-strain curves obtained are basically of two types: one in which a peak stress can clearly be identified (strain softening behavior), and a second type in which no decrease in the stress is observed even at very large strain (strain hardening behavior). In these latter cases, an arbitrary strain at failure of $13 \%$ is chosen and the corresponding stress is designated as the compressive strength. This criterion is selected since all of the tests that do not contain a clearly defined peak were allowed to progress to at least $13 \%$ axial strain. Figure 5.3 illustrates these two types of behavior, as well as the identification of the compressive strength, the axial strain at failure, and the initial Young's modulus. In this figure, the labels "114A" and "113B" correspond to the identification number that is assigned to the soil specimens. The labels " $28 \mathrm{~d}$ " and " $7 \mathrm{~d}$ " correspond to curing times of 28 days and 7 days for the specimens, respectively.

\subsection{UNCONFINED COMPRESSIVE STRENGTH OF TREATED SOILS WITH HIGH ORGANIC CONTENT (LR SOIL)}

\subsubsection{Untreated and Reconstituted LR Soil}

Figures 5.4 through 5.6 show the stress-strain curves for the soil that is prepared following the procedures described in Chapter 3, but with no binder introduced into the mixture. These tests were conducted to investigate the effects of both time and surcharge. The results pertain to the unconfined compression tests performed following one, seven, and twenty-eight day curing periods under a surcharge of $14.5 \mathrm{kPa}$ (Figure 5.4), $48 \mathrm{kPa}$ (Figure 5.5), and $96 \mathrm{kPa}$ (Figure 5.6), respectively.

The stress-strain plot of Figure 5.4 shows the UC test results for the untreated LR soil surcharged at $14.5 \mathrm{kPa}$. From this plot, it can be seen that the strength and stiffness of the soil increase with curing time. The lack of a clear peak in the curves and the high 
failure strains as shown in Table 5.2 indicate that this soil does not exhibit strainsoftening behavior. Figure 5.5 and Figure 5.6 show that the strength of the pure soil increases with curing time, similarly to Figure 5.4. Also, the increasing surcharge results in higher strength, stiffness, and strain softening behavior.

From each of these figures, additional observations may be made regarding the behavior of the LR soil. From Figure 5.4, it can be seen that the strength difference between the one and seven day tests is less than the difference between the seven and twenty-eight day tests. In all of these cases, the difference between the two tests is quite small as can be observed from the basically "overlapping” behavior of the curves. All of these tests also exhibit strain-hardening behavior, in which a peak compressive stress is not reached.

Figure 5.5 shows similar results in that the difference in the curves between the one and seven day tests is much less than that between the seven and twenty-eight day tests. However, the difference in the initial stiffness between the one and seven day tests is more pronounced than that shown in Figure 5.4. Figure 5.5 shows that the differences between two tests for the same curing period are much larger as the curing time increases. In addition, the twenty-eight day test exhibits strain-softening behavior while the one and seven day tests both exhibit strain hardening behavior.

Figure 5.6 shows that the strength and stiffness differences between the one, seven, and twenty-eight day tests is not as significant as those found in Figure 5.4 and 5.5. However, these differences may be shrouded by the large difference between the two twenty-eight day tests. This plot shows that similarly to the tests shown in Figure 5.5, the twenty-eight day tests exhibit strain softening behavior, while the one and seven day tests both exhibit strain hardening. However, the peak strength is not as easily recognizable as that shown for the twenty-eight day test of Figure 5.5.

Figure 5.7-5.9 plot the average results of strength, stiffness, and strain at failure, for tests conducted to evaluate the effects of curing time and surcharge on the unconfined compressive strength of the soil. The error bars indicate the range in which the results vary. It can be seen that the strength and stiffness of the soil exhibit an increase with surcharge, for the one, seven, and twenty-eight day cured specimens. However, the effect 
of the surcharge appears to be rather insignificant for the one-day cured specimens, and very substantial for the twenty-eight day cured specimens. The difference between the results for the strength appears to be much less than the differences associated with the stiffness measurements, as evidenced by the smaller error bars for the strength values. These errors are quite large for the values of the strain at failure. In addition, it can also be seen from Figure 5.9 that the strain at failure decreases for increasing curing time and surcharge. However, the reduction in failure strain appears to only be significant at a surcharge of $48 \mathrm{kPa}$, and appears to increase from this case with the $96 \mathrm{kPa}$ surcharge. However, the very large errors associate with this data limits the identification of possible trends.

The strength and stiffness increase with increased curing time and surcharge may be dependent on the change in the height of specimens during curing $(\Delta \mathrm{H})$ or the initial void ratio $\left(\mathrm{e}_{\mathrm{o}}\right)$ of specimens as shown in Table 5.2. It can be noticed that $\Delta \mathrm{H}$ decreases with increasing curing time and surcharge. This time and stress dependent effect is due to the increased consolidation that occurs with increased surcharge and curing time. The reduction of the void ratio results in a more compact and consequently stronger and stiffer soil structure.

Although this set of tests provides a base point from which to analyze the effects of the addition of binding agent, it does not provide information on the strength of the insitu soil. This set of tests using "pure soil” only gives the strength of the reconstituted soil after the mixing process. From this starting point, the relative strength increase caused by the addition of binding agents may be evaluated.

\subsubsection{Effect of curing time and surcharge on LR soil treated with 8\% PC}

Stress strain curves relevant to soil treated with 8\% PC are shown in Figures 5.10 through 5.12 These tests were conducted to investigate the effects of time and surcharge on a soil treated with $8 \%$ PC. The results pertain to the unconfined compression tests performed on mixed soil specimens following one, seven, and twenty-eight day curing periods under a surcharge of $14.5 \mathrm{kPa}$ (Figure 5.10), $48 \mathrm{kPa}$ (Figure 5.11), and $96 \mathrm{kPa}$ 
(Figure 5.12), respectively. The results from these tests are plotted in Figures 5.13-5.15, in which the average values of compressive strength, initial Young's modulus, and failure strain for each set of testing variables are plotted against the surcharge.

Figure 5.10 shows that the strength and stiffness increase with increasing curing time. Although the results from the two tests conducted after one day of curing are quite similar, the results for the seven and twenty-eight days of curing exhibit much greater differences. Unlike the tests performed on the mixture with $0 \%$ PC, the increase in strength and stiffness appear to be approximately equal between the one and seven day tests and the seven and twenty-eight day tests. In all of the cases, the soil exhibits strainsoftening behavior, however the peak stress is not clearly defined for the one-day test labeled “103B.”

Figure 5.11 also shows that the strength and stiffness increase with increasing curing time. However, these results appear to exhibit large differences in the results, especially for the seven and twenty-eight day tests. In this figure, the results from four different tests for the seven-day curing period are presented. This case is one of those where the errors between the first two tests (101A and 101B) were so large that they were supplemented with the manufacture of two additional specimens (101C and 101D). From these results, it can be seen that the one-day tests exhibit strain-hardening behavior while the seven and twenty-eight day tests exhibit strain-softening behavior.

Similarly to Figure 5.10 and 5.11, Figure 5.12 exhibits an increase in strength and stiffness with curing time. Also, the differences between the one and seven day tests and the seven and twenty-eight day test do not appear to be dramatically different. In all of the cases shown in this plot, the results between the two tests are quite similar. Each of these tests exhibit strain softening behavior, although the peak is much more pronounced with increasing curing time.

The results indicate that the curing time and surcharge have a significant effect on the behavior of the treated soil, similarly to untreated and reconstituted soil. Figures 5.13 and 5.14 show that the strength and stiffness of the treated soil increase with curing time. Similarly to the results shown for the $0 \%$ PC soil, the effect of the surcharge does not appear to be significant for the test conducted on the specimen cured for one day, despite 
the seemingly anomalous results for the strength of the specimen cured for one day at 48 $\mathrm{kPa}$. However, there appears to be an essentially linear increase in strength and stiffness for both the seven and twenty-eight day tests. Again, the error associated with the stiffness measurements are generally much larger than that associated with the strength.

Figure 5.15 also shows very high errors associated with the strain at failure measurements. However, these results seem to show that the strain at failure decreases with increasing time and surcharge, indicating more pronounced strain softening behavior with time and surcharge. Subsequently, the soil exhibits strain-softening behavior with increased curing time as evidenced by the decrease in the failure strains.

Curing time and surcharge have similar effects on both the treated and untreated soil. In both cases, the compressive strength and stiffness increase with curing time and surcharge accompanied by strain softening behavior. However, the addition of cement results in higher compressive strength and stiffness. Figure 5.16 shows the strength of the 8\% PC mixture $\left(\sigma_{c}\right)$ compared to that of the $0 \%$ PC mixture for the same surcharge and curing period $\left(\sigma_{0}\right)$. These results indicate that the surcharge has a greater strengthening effect on the cement treated soil as opposed to the pure soil, as evidenced by the basically linear increase of the normalized strength with surcharge. Comparison of the failure strains for the cement treated and pure soil (Figure 5.15 and 5.9) reveals that the addition of cement causes failure at smaller strains, as evidenced by the peaks in the stress-strain curves and the lower failure strains as compared to the $0 \%$ PC mixture for the one, seven, and twenty-eight day specimens. This behavior coincides with the increase of strength and stiffness that are observed.

\subsubsection{Effect of cement content}

A number of tests were conducted to investigate the effects of the cement percentage. They were performed on mixed soil that was cured under a surcharge of 48 $\mathrm{kPa}$ and treated with 4, 8, 12, and 20\% Portland cement. The stress-strain plots given pertain to the unconfined compression tests performed following one (Figure 5.17), seven (Figure 5.18), and twenty-eight days curing period (Figure 5.19). The results as shown in 
Figures 5.20-5.22 that plot the average values of compressive strength, Young's modulus, and strain at failure for each set of testing variables are plotted against the percentage of cement.

Figure 5.17 shows the stress-strain plots obtained for specimens containing different percentages of cement after one day of curing. It can be seen from this graph that the strength and stiffness increase with increasing cement percentage, up to a value of approximately 8\% Portland cement. Specimens with Portland cement content above this value tend to exhibit lower values for strength and stiffness. It can also be seen that a change in the behavior of the soil occurs between cement contents of $4 \%$ and $8 \%$ Portland cement. The mixture containing 4\% PC exhibits strain-hardening behavior, as opposed to strain softening behavior exhibited for mixtures possessing a Portland cement content of $8 \%$ or more. These plots also show large errors between two similar tests (i.e. mixtures containing $4 \%$ and $12 \%$ PC) as may be seen by the divergence of the curves beyond axial strain values of $0.5 \%$.

Figure 5.18 shows similar results in that the strength and stiffness of the soil mixtures increase with cement content, up to a value of approximately 8\% PC. Similarly to the previously described observations, the strength and stiffness decrease with cement concentrations greater than $8 \%$. The plot also shows similar behavior with respect to the strain hardening behavior for specimens with cement content of $4 \%$, and strain softening behavior for the specimens with cement content of $8 \%$ or more.

Figure 5.19 shows the UC test results for the same cement contents as in Figure 5.17 and 5.18 after 28 days of curing. Again, the behavior with respect to the cement content results in the greatest strength gain for the mixtures containing $8 \%$ PC, with a reduction at higher values. However, unlike the behavior for the one and seven day tests, these tests exhibit strain softening behavior for all of the cement contents tested.

It should be noted that the reproducibility of the tests (i.e. difference between tests using identical parameters) does not appear to be related to the cement content or curing time. The discrepancies between the tests in these cases, and in all subsequent cases, are not likely to be dependent on curing time, surcharge, or binder. These differences are 
likely due to variability within the mixed soil fabric that are a consequence of the mixing and compaction procedures.

The most important trend that is noticeable from Figures 5.20-5.22 is that the compressive strength and stiffness of the soil mixture increases with an increasing cement content up to amounts between $8 \%$ and $12 \%$ (Figure 5.20 and 5.21), beyond which the strength and stiffness are reduced. It can be seen that compressive strength and stiffness both increase with curing time and surcharge similarly to the test performed on the untreated soil. Also, the cement content has an effect on strength and stiffness regardless of curing time. Similarly, the strain at failure (Figure 5.22) decreases as the cement concentration is increased from $0 \%$ to $8 \%$, and then remains essentially constant at twenty-eight days for increasing cement contents. This indicates that although decreasing the strength, that high Portland cement content does not significantly alter the stiffness or failure mode. However, this interpretation is limited by the large errors associated with the stiffness and failure strain values.

\subsubsection{Effect of $\mathrm{CaO}$ content}

Figure 5.23 shows the stress-strain plots from the soil mixtures containing $\mathrm{CaO}$ alone. These tests were conducted on mixed soil that was cured under a surcharge of 48 $\mathrm{kPa}$ for a curing time of seven days, with $\mathrm{CaO}$ contents of $4 \%, 8 \%, 12 \%$, and $20 \%$. Figures 5.24-5.26 plot the average values of compressive strength, Young's modulus, and failure strains for these tests versus percentage of $\mathrm{CaO}$.

Despite some scatter in the data, particularly for the soil prepared with $4 \% \mathrm{CaO}$, these figures along with the stress-strain curves show that the soil becomes stiffer and stronger with increasing $\mathrm{CaO}$ concentration. The plots also show a transition from strain hardening to strain softening associated with the addition of $\mathrm{CaO}$. In this case, the strengthening provided by the addition of $4 \% \mathrm{CaO}$ is difficult to evaluate due to the large variation between the two curves for the mixtures containing $4 \% \mathrm{CaO}$.

It is found that increasing $\mathrm{CaO}$ content results in a marked increase in strength and stiffness up to a content of $8 \%$. This is followed by a smaller increase between $8 \%$ and $12 \%$ after which they remain essentially constant to $20 \%$. Similarly to the behavior 
observed for the cement treated soil, the strength and stiffness increase are associated with more pronounced strain softening behavior as shown in Figure 5.26.

Although the $\mathrm{CaO}$ does act to increase the strength of the soil, the strength increase is not as significant as that associated with Portland cement. Figure 5.27 compares the average seven-day strength associated with different $\mathrm{CaO}$ percentages, as opposed to the seven and twenty-eight day results for soil treated with Portland cement. From this plot it can be seen that although the $\mathrm{CaO}$ treated soil exhibits higher strength at 7 days than the Portland cement, the fully hydrated Portland cement (28 days) is much stronger. However, the previously described strength reduction associated with the increase in PC content from $12 \%$ to $20 \%$ results in the 28 day strength for $20 \%$ PC being slightly lower than the 7 day results for $20 \% \mathrm{CaO}$. These trends for the strength are very similar to those for the stiffness, and the reduction of the strain at failure with increasing binder content occurs similarly for both Portland cement and CaO.

\subsubsection{Effect of $\mathrm{CaO}$ content on cement treated soil}

Figures 5.28-5.30 show the results of the UC tests performed to evaluate the effect of the addition of $\mathrm{CaO}$ to a soil treated with cement. These tests were conducted on soil that was cured for one (Figure 5.28), seven (Figure 5.29), and twenty-eight days (Figure 5.30) with a surcharge of $48 \mathrm{kPa}$. The soil was treated with $8 \%$ Portland cement plus 4,8 , and $12 \% \mathrm{CaO}$. The average values for strength, stiffness, and strain at failure versus percent of $\mathrm{CaO}$ added are plotted in Figures 5.31-5.33. These plots indicate that the soil becomes stronger and stiffer with curing time.

Figure 5.28 shows the stress-strain plots for the soil treated with $8 \%$ PC and different percentages of $\mathrm{CaO}$ after one day of curing. This plot shows that the strength and stiffness of the soil increase with increasing $\mathrm{CaO}$ content, and each of the tests exhibit strain-hardening behavior. Figure 5.29 shows the stress strain plots for the same binder contents after seven days of curing. Again, the results show that the strength and stiffness increase with curing time. However, a transition from strain hardening to strain softening occurs between the one and seven day tests. Following with the patterns established between the one day and seven day tests, the twenty-eight day tests (Figure 
5.30) exhibit an increase in strength and stiffness with increasing $\mathrm{CaO}$ content and strain softening behavior for all of the specimens more pronounced than that exhibited for the testing conducted after one and seven days.

However, the most interesting behavior regarding these tests is apparent when comparing these results to those obtained for the soil treated with only $8 \%$ cement, as shown in Figures 5.31-5.33. It can be seen that the strength and stiffness of the cement treated soil decreases dramatically upon the addition of $4 \% \mathrm{CaO}$. The strength increases a little beyond $4 \% \mathrm{CaO}$, although it remains less than that for the cement only treated soil. The stiffness of the soil is reduced upon the addition of $4 \% \mathrm{CaO}$, however it increases substantially above the cement treated soil upon the addition of $8 \% \mathrm{CaO}$. It may be seen that the effect of the addition $\mathrm{CaO}$ is much less significant for the one and seven day strength and stiffness than that of the twenty-eight day strength and stiffness. It can be seen that the addition of $\mathrm{CaO}$ has little effect on the strain at failure of the twenty-eight day tests, but a substantial change on the failure strain of the one-day tests. This change in failure strains indicates that the addition of $\mathrm{CaO}$ to a cement treated soil changes the behavior from strain softening to strain hardening for the one-day tests, with little change for the twenty-eight day specimens.

\subsubsection{Effect of High Calcium Flue Dust (HC), Marblehead Buffington Dust (MB)} and Bentonite (BEN) content

In addition to Portland cement and $\mathrm{CaO}$, two commercial limes, High Calcium Flue dust (HC) and Marblehead Buffington Dust (MB), as well as bentonite (BEN) were used to evaluate the effect of treatment on the unconfined compressive strength of LR soil. All the specimens were cured under a surcharge of $48 \mathrm{kPa}$ for 7 days with $4 \%, 8 \%$, $12 \%$ and $20 \%$ of binder.

Figure 5.34 shows the stress-strain curves for the High Calcium Flue Dust (HC) samples. These tests exhibit strain hardening behavior for all of the HC contents tested. Although the specimen treated with $20 \%$ of HC shows the highest stiffness and compressive strength, the increase of compressive strength is not apparently related to the amount of HC added. The average compressive strength of specimen treated with HC is 
shown in Figure 5.35. Compared to the compressive strength of the untreated specimen cured under a surcharge of $48 \mathrm{kPa}$ for 7 days, there is a slight increase in strength from 8 $\mathrm{kPa}$ to $12 \mathrm{kPa}$ at $4 \%, 10 \mathrm{kPa}$ at $8 \%$, and $11 \mathrm{kPa}$ at $20 \% \mathrm{HC}$ but no increase in strength at $12 \%$ HC. This may be due to inconsistencies in the material.

Upon arrival at Purdue University, the HC appeared to be partially hydrated as shown in Figure 5.36. If the material is partially hydrated, adding HC to organic soil will only strengthen the soil by decreasing the water content.

The stress-strain curves from the soil specimens treated with Marblehead Buffington Dust (MB) are shown in Figure 5.37. Similar to the HC treated specimens, the test results show strain-hardening behavior for all the MB contents tested. The stiffness does not show increase with treatment. The experiments using Marblehead Buffington Dust as the additive indicate an increasing trend in compressive strength from 4\% to $20 \%$ as shown in Figure 5.38. However, the compressive strengths of specimen treated with $4 \%$ and $8 \%$ of MB are lower than that of the untreated soil.

The average compressive strengths of specimens treated with $\mathrm{CaO}, \mathrm{HC}$ and $\mathrm{MB}$ are compared in Figure 5.39. Among the three lime-based binders, the $\mathrm{CaO}$ has the most significant effect on the compressive strength of LR soil.

The results of the unconfined compression tests of the soil treated with bentonite are shown in Figure 5.40 and Figure 5.41. The bentonite samples were much softer after curing than the samples using either High Calcium Flue dust or Marblehead Buffington dust. The samples using $20 \%$ bentonite by dry weight were so soft after curing that they could not be extracted from the molds and, therefore, could not undergo the unconfined compression test. The compressive strengths of the bentonite treated specimens decreased to $4 \mathrm{kPa}$, to about $50 \%$ of the compressive strength of untreated specimen, for all the bentonite contents.

\subsection{UNCONFINED COMPRESSIVE STRENGTH OF CEMENT TREATED SOILS WITH LOW ORGANIC CONTENTS}

The experimental program was carried out further to assess the effect of treatment on the compressive strength of soils with low organic content. As described in chapter 4, 
soils with low organic content were manufactured in the laboratory by mixing grundite, an illitic clay, with appropriate amounts of LR soil. Two types of mixtures were produced: Mixture I with Loss Of Ignition (LOI) $=9.5 \%$ and Mixture II with LOI $=20 \%$. The liquidity index (LI) of Mixture I and Mixture II were designed to be identical so that the results would be comparable.

Similar to the UC tests performed on LR soil, the following variables were investigated for Mixture I: cement content, curing time and curing surcharge. The cement content was selected as 4\%, 8\%, 12\%, 20\% and 30\% of untreated soil by dry mass. 1 day, 7 days and 28 days were chosen as the curing time. $14.5 \mathrm{kPa}, 48 \mathrm{kPa}$ and $96 \mathrm{kPa}$ were applied on $0 \%$ and $8 \%$ cement content specimens to investigate the effect of various curing surcharges on strength. All the other specimens were cured under a surcharge of $48 \mathrm{kPa}$.

A number of tests on Mixture II were performed to provide knowledge on the effect of organic content on unconfined compressive strength. The results of UC tests on PC treated Mixture I and II are summarized in Table 5.3 including the amount of PC, the compressive strength, the Young's modulus and the strain at failure.

Figure 5.42 shows the effect of curing time on the compressive strength of PC treated Mixture I. The UCS increases with curing time for all $4 \%$ to $30 \%$ cement content specimens. The strength of the specimens increased much faster during the first week than in the later three weeks, especially for specimens with cement content larger than 8\%. The compressive strength of seven-day and twenty-eight day curing specimens, except specimens with $0 \%$ and $4 \%$ cement content, increased by a factor of about 2 to 2.6 over the strength of one-day specimens.

The increase in the compressive strength of PC treated Mixture I with cement content is shown in Figure 5.43. Compared to the results from PC treated LR soil, where the compressive strength did not increase beyond 8\% PC, the compressive strength of Mixture I continuously increases with PC. The increase in compressive strength is negligible with $4 \%$ of PC. With more than 4\% PC, the compressive strength shows a quasi-linear increase with \% PC. The compressive strengths of $30 \%$ PC treated 
specimens increased by factors of 45, 56 and 68 after one, seven and twenty-eight days of curing, respectively, over the strength of the untreated specimen.

The effects of surcharge and curing time on the compressive strength of untreated Mixture I are shown in Figure 5.44 and Figure 5.45, respectively. Similar to the results from UC tests on LR soil, the effect of the surcharge appears to be negligible after one day of curing, but is substantial after seven days of curing. However, after seven days, the rate of increase in the compressive strength seems to decrease. With a surcharge of 14.5 $\mathrm{kPa}$, which is equivalent to the stress under about $1 \mathrm{~m}$ thick fill, the compressive strength does not increase with curing time. With a surcharge of $96 \mathrm{kPa}$, equivalent to 5-6m thick fill, the compressive strength increased from $18 \mathrm{kPa}$ to $56 \mathrm{kPa}$ after seven days of curing, and to $67 \mathrm{kPa}$ after twenty-eight days of curing.

The effects of curing surcharge and curing time on 8\% PC treated Mixture I are shown in Figure 5.46 and Figure 5.47, respectively. Similar to the result from untreated soil mixture I, the compressive strength increases with curing surcharge. However, the compressive strength also increases substantially with curing time, even after seven days. This is probably due to the continuous hydration of cement added after treatment.

The effect of curing time on the untreated and 8\% PC treated Mixture II (LOI = 9.5\%) are compared with the results from Mixture I $(\mathrm{LOI}=20 \%)$ in Figure 5.48. With treatment, the compressive strength increases for both Mixtures I and II compared to the untreated specimens. However, the increase of compressive strength is much significant for Mixture II, with lower LOI, than for Mixture I, although more cements are added to Mixture I. The amount of cement added for $8 \%$ PC Mixtures I and II are $84 \mathrm{~kg} / \mathrm{m}^{3}$ and $66 \mathrm{~kg} / \mathrm{m}^{3}$, respectively. Also the rate of increase in compressive strength for Mixture II is greater than for Mixture I after seven days of curing. There seems to be no further increase in compressive strength after seven day.

Additional UC tests were performed on PC treated LR soil to understand the effect of organic content on compressive strength. LR soil was treated with 50\% PC and was cured under a surcharge of $48 \mathrm{kPa}$ for one, seven and twenty eight days (see Table 5.3). 
To better understand the effect of organic contents on compressive strength, additional UC tests were performed on 50\% PC LR soil (LOI 50\%). The actual amount of PC added per unit volume of 50\% PC LR soil is about $160 \mathrm{~kg}$. This value is similar to the amount of cement added for $20 \%$ PC Mixture I, which is about $209 \mathrm{~kg} / \mathrm{m}^{3}$. The results from these two specimens are compared in Figure 5.49. The result indicate that 50\% PC LR soil has much lower UCS than 20\% PC Mixture I. This is similar to the observation made on the results from 8\% PC Mixture I and II that the effect of treatment with PC is smaller for soils containing higher organic content.

\subsection{CONCLUSIONS AND DISCUSSIONS}

To assess the effect of treatment on the compressive strength of soils with considerable organic contents, UC tests were performed on LR soil (LOI 50\%), and manufactured soil Mixture I (LOI 9.5\%) and Mixture II (LOI 20\%). The effects of the type and the amount of binder, curing time and curing surcharge were investigated. The employed binder includes Portland cement, Calcium Oxide $(\mathrm{CaO})$, combination of PC and $\mathrm{CaO}$, two commercial limes, High Calcium Flue dust (HC) and Marblehead Buffington Dust (MB), and Bentonite (BEN).

It is clear from all of the UC tests performed that increased curing time increases the compressive strength and stiffness, and decreases the strain at failure. This phenomenon is very likely due to the reduction of the void ratios in the soil. The degree of consolidation increases with curing time, leading to a more compact soil structure. This change in density results in the soil becoming stiffer and stronger. This increase in strength with curing time may also be attributed to the aging of the specimen, as new bonds may be created between particles.

The accepted soil mechanics concept applicable to clays that normalized shear strength $\left(s_{\mathrm{u}} / \sigma_{\mathrm{v}}{ }_{\mathrm{v}}\right.$, where $\left.s_{\mathrm{u}}=1 / 2 \sigma_{\mathrm{c}}\right)$ is constant explains the increase in strength with surcharge. The increase in strength with surcharge for twenty-eight day specimens of untreated and treated LR soil with 8\% PC is plotted in Figure 5.50. This shows a basically linear relationship between surcharge and strength. 
Although an increase in surcharge results in an increase in the strength of soil without binder, this effect is even more pronounced in a cement treated soil. As discussed earlier, Figure 5.16 plots the strength of an 8\% PC LR soil normalized by the strength of a $0 \%$ PC LR soil against surcharge. Due to the linear increase of the strength for both the $0 \%$ and $8 \%$ treated soil, it can be stated that the surcharge has a more pronounced strengthening effect on a cement treated soil. This may be due to the cement particles coming in contact with more soil particles due to the reduction in the void spaces, thus establishing better bonding and even more strengthening capability beyond that caused by the densification of the soil.

In addition to the increased strength caused by curing time and surcharge, it is shown that the introduction of Portland cement into the soil has a similar effect. The results indicate that the strength and stiffness of the soil increase with cement content up to $8-12 \%$. However, beyond this point strength decreases as shown by the dramatically lower strength and stiffness at a cement content of $20 \%$. Perhaps a limit state is reached near $12 \%$ where the cement achieves optimum bonding between soil particles. It is possible that at a cement content beyond $12 \%$ the excess cement particles bond together to form large cement grains as opposed to maximizing the cement-soil bonding. This could lead to the soil behaving in a more frictional rather than cohesive nature, thus reducing the strength and stiffness.

The introduction of $\mathrm{CaO}$ into a soil acts to increase the strength and stiffness of the soil. The strengthening accompanies an increase in $\mathrm{CaO}$ content to a concentration of $8 \%$, after which it remains essentially constant. Since treatment with $\mathrm{CaO}$ is thought to contribute to the reduction of the water content of the soil, its improvement capabilities appear to be limited. This may be due to the increase in size of $\mathrm{CaO}$ particles upon hydration. The improvement in the strength of the soil mixture is due to the ability of $\mathrm{CaO}$ to exhibit shear resistance, unlike water. The replacement of water with shearresistant hydrated $\mathrm{CaO}$ on the shearing plane may be responsible for the strength increase. However, as the amount of $\mathrm{CaO}$ contained within the soil increases, the strength does not increase. This may be because $8 \% \mathrm{CaO}$ is sufficiently high to fill nearly all voids with hydrated $\mathrm{CaO}$. 
The addition of $\mathrm{CaO}$ into the cement treated soil has an adverse effect on the strength of the soil mixture. Although a slight increase occurs between a CaO content of $4 \%$ to $8 \%$, the twenty-eight day strength in both cases is lower than that for the untreated soil. Despite this, the stiffness of the soil cured for twenty-eight days increases upon the addition of $8 \% \mathrm{CaO}$ to a value higher than that for the cement treatment only. However, the one and seven day strength and stiffness are not altered much with the addition of $\mathrm{CaO}$. The result pertaining to the strength reduction is surprising in that the use of limecement columns is well documented in the literature. Despite the widespread usage, no information was found to document the behavior of cement treated soil upon the addition of $\mathrm{CaO}$. From these results, it appears that the benefit of the addition of $\mathrm{CaO}$ to a cement treated soil is through the increase in stiffness. Studies were found in which researchers evaluated the strength of lime-cement mixes such as Hebib and Farrell (1999) and Cortellazzo and Cola (1999). However, these researchers do not offer results from a cement-lime treated soil with a comparison to cement only treated soil.

Huttunen and Kujala (1996) conducted a series of tests on various types of peats using cement alone and a 1:1 cement-CaO mixture. They indicate that in certain soils, cement alone has a greater strengthening effect than the combination of cement and $\mathrm{CaO}$. Their results show that cement alone is more effective in Sphagnum peats and cement and lime mixtures are more effective in Carex (fibrous) peats. These results seem to be independent of the degree of decomposition.

Hebib and Farrell (1999) provide an explanation of the role that $\mathrm{CaO}$ and the liberated $\mathrm{Ca}^{++}$ions play in the binding reaction. They state that organic matter interferes with the hardening of soil-cement mixtures by retaining calcium ions liberated during cement hydration. The addition of $\mathrm{CaO}$ results in free $\mathrm{Ca}^{++}$ions being used to satisfy the ion exchange capacity of the soil, thus creating sufficient calcium content for the strengthening of the cement.

These two findings indicate that the effectiveness of $\mathrm{CaO}$ and/or cement is controlled by the composition of the soil. For LR soil with an organic content of approximately fifty-percent, it is possible that little of the $\mathrm{CaO}$ is used to satisfy the ion exchange capacity of the soil. Due to the affinity of Portland cement for $\mathrm{Ca}^{++}$ions, the 
cement particles may bond to the $\mathrm{Ca}^{++}$ions rather than the soil particles. This may lead to the sharp reduction in strength upon the addition of $\mathrm{CaO}$, as evidenced by these tests. However, the strength increase with increasing $\mathrm{CaO}$ content would likely come from the absorption of pore water as evidenced for mixes containing only $\mathrm{CaO}$. It is possible that testing of lower $\mathrm{CaO}(<4 \%)$ content on cement treated soil could results in a slight strength increase of the cement treated soil, before being reduced at a certain $\mathrm{CaO}$ content. This may occur due to a small fraction of $\mathrm{CaO}$ being used to satisfy the ion exchange capacity of the organic material, leading to ready formation of the calcium silicates used in cement hydration. However, increasing the $\mathrm{CaO}$ content to $4 \%$ results in the cement being attracted directly to the $\mathrm{CaO}$ particles and not the soil, leading to poor soil-cement bonding. However, the increase in strength with increasing $\mathrm{CaO}$ content beyond $8 \%$ may be due to the same strengthening mechanism that is utilized for the $\mathrm{CaO}$ only soil.

After analysis of LR soil treated with High Calcium Flue dust, Marblehead Buffington dust, and bentonite it may be concluded that none of these three additives significantly increase the compressive strength of organic soil. The addition of High Calcium Flue dust and Marblehead Buffington Dust decreases the moisture content of the soil. Soil treated with these additives does not consistently increase in compressive strength as more additive is used. Furthermore, the addition of bentonite decreases the compressive strength of organic soil.

The compressive strength of PC treated soils with lower organic contents, Mixtures I and II, increases with increasing curing surcharge and curing time. Unlikely to the results from PC treated highly organic soil (LR soil), the compressive strength of Mixture I increases continuously in the \%PC range investigated, $0-30 \%$ PC. The increase of compressive strength with treatment with PC seems to be affected by the organic content of the soil treated. Comparing the results from UC tests on 50\% PC LR soil and 20\% PC Mixture I, the compressive strength of 20\% PC Mixture I is substantially higher than $50 \%$ LR soil although similar amounts of PC are added to treat the soils. 
Several implications are available from the results of these tests. Firstly, among the binders investigated, both cement and $\mathrm{CaO}$ increase the strength and stiffness of a soil mixture, while the effects of two commercial limes, $\mathrm{HC}$ and $\mathrm{MB}$, and bentonite are negligible. Secondly, cement provides a much more pronounced effect, especially when considering the increase in strength with curing time. Figure 5.51 compares the effectiveness of different percentages of cement, $\mathrm{CaO}$, and a combination of the two. It can be seen that cement alone provides much greater strengthening than $\mathrm{CaO}$ or $\mathrm{CaO}$ and cement, although the addition of at $\mathrm{CaO}$ to a cement treated soil appears to increase the stiffness of the treated soil. Thirdly, the surcharge has a significant effect on the strength and stiffness of the soil mixture. The compressive strength is directly proportional to the surcharge that is applied, as can be observed from the nearly linear increase in strength with surcharge. The surcharge provides a greater strengthening effect on cement treated soil than on a soil with no binder. Finally, the treatment with PC is more effective for soils with lower organic content.

Given the data and results previously presented, certain trends can be applied. In essence, LR soil treated with $8 \%$ cement and surcharged at $48 \mathrm{kPa}$ may be substituted for a 4\% cement treatment surcharged with $96 \mathrm{kPa}$ and vice versa. Many researchers that have conducted laboratory research on the effectiveness of binders have done so with no surcharge being applied during curing (e.g. Lahtinen et. al (1996)). This testing philosophy can lead to a significant underestimation of the strength that can be expected in the field.

Given the comparatively lower price of fill as compared with mass stabilization, the results indicate that binder may be substituted with surcharge for a more economically efficient ground improvement method. These findings may lead to a conclusion that there is an optimum cost-effective solution for combining preloading with mass stabilization. Although surcharging is time consuming and subsequently cost restrictive, the strength gain after 28 days may shorten the time that the surcharge needs to be in place. This may reduce the time necessary for preloading by several months. 
Table 5.1: Testing combinations

\begin{tabular}{|c|c|c|c|c|c|c|c|c|c|c|c|}
\hline \multirow{2}{*}{ Soil } & \multirow{2}{*}{ Binder } & Surcharge (kPa) & \multicolumn{3}{|c|}{14.5} & \multicolumn{3}{|c|}{48} & \multicolumn{3}{|c|}{96} \\
\hline & & Curing time (days) & 1 & 7 & 28 & 1 & 7 & 28 & 1 & 7 & 28 \\
\hline \multirow{25}{*}{ LR Soil } & \multirow{6}{*}{ PC } & $0 \%$ & $\mathrm{X}$ & $\mathrm{X}$ & $\mathrm{X}$ & $\mathrm{X}$ & $\mathrm{X}$ & $\mathrm{X}$ & $\mathrm{X}$ & $\mathrm{X}$ & $\mathrm{X}$ \\
\hline & & $4 \%$ & & & & $\mathrm{X}$ & $\mathrm{X}$ & $\mathrm{X}$ & & & \\
\hline & & $8 \%$ & $\mathrm{X}$ & $\mathrm{X}$ & $\mathrm{X}$ & $\mathrm{X}$ & $\mathrm{X}$ & $\mathrm{X}$ & $\mathrm{X}$ & $\mathrm{X}$ & $\mathrm{X}$ \\
\hline & & $12 \%$ & & & & $\mathrm{X}$ & $\mathrm{X}$ & $\mathrm{X}$ & & & \\
\hline & & $20 \%$ & & & & $\mathrm{X}$ & $\mathrm{X}$ & $\mathrm{X}$ & & & \\
\hline & & $50 \%$ & & & & $\mathrm{X}$ & $\mathrm{X}$ & $\mathrm{X}$ & & & \\
\hline & \multirow{4}{*}{$\mathrm{CaO}$} & $4 \%$ & & & & & $\mathrm{X}$ & & & & \\
\hline & & $8 \%$ & & & & & $\mathrm{X}$ & & & & \\
\hline & & $12 \%$ & & & & & $\mathrm{X}$ & & & & \\
\hline & & $20 \%$ & & & & & $\mathrm{X}$ & & & & \\
\hline & \multirow{3}{*}{$\mathrm{PC}+\mathrm{CaO}$} & $8 \% \mathrm{PC}+4 \% \mathrm{CaO}$ & & & & $\mathrm{X}$ & $\mathrm{X}$ & $\mathrm{X}$ & & & \\
\hline & & $8 \% \mathrm{PC}+8 \% \mathrm{CaO}$ & & & & $\mathrm{X}$ & $\mathrm{X}$ & $\mathrm{X}$ & & & \\
\hline & & $8 \% \mathrm{PC}+12 \% \mathrm{CaO}$ & & & & $\mathrm{X}$ & $\mathrm{X}$ & $\mathrm{X}$ & & & \\
\hline & \multirow{4}{*}{$\mathrm{HC}$} & $4 \%$ & & & & & $\mathrm{X}$ & & & & \\
\hline & & $8 \%$ & & & & & $\mathrm{X}$ & & & & \\
\hline & & $12 \%$ & & & & & $\mathrm{X}$ & & & & \\
\hline & & $20 \%$ & & & & & $\mathrm{X}$ & & & & \\
\hline & \multirow{4}{*}{ MB } & $4 \%$ & & & & & $\mathrm{X}$ & & & & \\
\hline & & $8 \%$ & & & & & $\mathrm{X}$ & & & & \\
\hline & & $12 \%$ & & & & & $\mathrm{X}$ & & & & \\
\hline & & $20 \%$ & & & & & $\mathrm{X}$ & & & & \\
\hline & \multirow{4}{*}{ BEN } & $4 \%$ & & & & & $\mathrm{X}$ & & & & \\
\hline & & $8 \%$ & & & & & $\mathrm{X}$ & & & & \\
\hline & & $12 \%$ & & & & & $\mathrm{X}$ & & & & \\
\hline & & $20 \%$ & & & & & $\mathrm{X}$ & & & & \\
\hline \multirow{6}{*}{ Mixture I } & \multirow{6}{*}{ PC } & $0 \%$ & $\mathrm{X}$ & $\mathrm{X}$ & $\mathrm{X}$ & $\mathrm{X}$ & $\mathrm{X}$ & $\mathrm{X}$ & $\mathrm{X}$ & $\mathrm{X}$ & $\mathrm{X}$ \\
\hline & & $4 \%$ & & & & $\mathrm{X}$ & $\mathrm{X}$ & $\mathrm{X}$ & & & \\
\hline & & $8 \%$ & $\mathrm{X}$ & $\mathrm{X}$ & $\mathrm{X}$ & $\mathrm{X}$ & $\mathrm{X}$ & $\mathrm{X}$ & $\mathrm{X}$ & $\mathrm{X}$ & $\mathrm{X}$ \\
\hline & & $12 \%$ & & & & $\mathrm{X}$ & $\mathrm{X}$ & $\mathrm{X}$ & & & \\
\hline & & $20 \%$ & & & & $\mathrm{X}$ & $\mathrm{X}$ & $\mathrm{X}$ & & & \\
\hline & & $30 \%$ & & & & $\mathrm{X}$ & $\mathrm{X}$ & $\mathrm{X}$ & & & \\
\hline \multirow{2}{*}{ Mixture II } & \multirow{2}{*}{ PC } & $0 \%$ & & & & $\mathrm{X}$ & $\mathrm{X}$ & $\mathrm{X}$ & & & \\
\hline & & $8 \%$ & & & & $\mathrm{X}$ & $\mathrm{X}$ & $\mathrm{X}$ & & & \\
\hline
\end{tabular}

$\mathrm{X}$ : investigated combination

HC: High Calcium Flue Dust

MB: Marblehead Buffington Dust

BEN: Bentonite 
Table 5.2: Summary table of UC Tests on PC and CaO treated LR soil

\begin{tabular}{|c|c|c|c|c|c|c|c|c|c|}
\hline I.D. & Binder & $\begin{array}{c}\text { Surcharge } \\
\text { (kPa) }\end{array}$ & $\begin{array}{c}\text { Curing Time } \\
\text { (days) }\end{array}$ & $\begin{array}{c}\sigma_{\mathrm{c}} \\
(\mathrm{kPa})\end{array}$ & $\begin{array}{c}E \\
(\mathrm{kPa})\end{array}$ & $\begin{array}{c}\varepsilon_{\mathrm{f}} \\
(\%)\end{array}$ & $\begin{array}{c}\Delta \mathbf{H}_{\text {curing }} \\
\text { (cm) }\end{array}$ & $\mathbf{e}_{0}$ & $\mathbf{e}_{f}$ \\
\hline 100A & $8 \%$ PC & 48 & 1 & 5.60 & 39 & 96 & .398 & 7.87 & 7.06 \\
\hline 100B & $8 \%$ PC & 48 & 1 & 14.28 & 253 & 9.07 & 1.441 & 7.86 & 7.03 \\
\hline 101A & $8 \%$ PC & 48 & 7 & 28.88 & 1242 & 5.18 & 2.218 & 7.80 & 6.52 \\
\hline 101B & $8 \%$ PC & 48 & 7 & 20.62 & 688 & 6.11 & 2.032 & 7.86 & 6.68 \\
\hline 101C & $8 \%$ PC & 48 & 7 & 17.10 & 898 & 6.37 & 2.172 & 6.52 & 5.45 \\
\hline 101D & $8 \%$ PC & 48 & 7 & 15.52 & 811 & 7.39 & 2.170 & 6.53 & 5.46 \\
\hline 102A & $8 \%$ PC & 48 & 28 & 36.32 & 1427 & 4.16 & 2.784 & 7.86 & 6.24 \\
\hline 102B & $8 \%$ PC & 48 & 28 & 41.60 & 1965 & 4.17 & 2.764 & 7.72 & 6.14 \\
\hline 103A & $8 \%$ PC & 14.5 & 1 & 6.26 & 234 & 8.46 & 1.570 & 8.47 & 7.49 \\
\hline 103B & $8 \%$ PC & 14.5 & 1 & 6.84 & 221 & 0.41 & 0.903 & 7.92 & 7.39 \\
\hline 104A & $8 \%$ PC & 14.5 & 7 & 11.50 & 699 & 6.83 & 1.466 & 7.78 & 6.94 \\
\hline 104B & $8 \%$ PC & 14.5 & 7 & 9.50 & 530 & 7.96 & 1.378 & 7.88 & 7.08 \\
\hline 105A & $8 \%$ PC & 14.5 & 28 & 13.06 & 728 & 5.50 & 1.668 & 7.93 & 6.96 \\
\hline 105B & $8 \%$ PC & 14.5 & 28 & 12.14 & 375 & 9.04 & 1.469 & 7.81 & 6.96 \\
\hline 106A & $8 \%$ PC & 96 & 1 & 10.86 & 383 & 8.98 & 2.051 & 7.86 & 6.67 \\
\hline 106B & $8 \%$ PC & 96 & 1 & 13.20 & 237 & 8.29 & 1.407 & 7.99 & 7.16 \\
\hline 107A & $8 \%$ PC & 96 & 7 & 40.84 & 1605 & 4.70 & 2.774 & 7.81 & 6.21 \\
\hline 107B & $8 \%$ PC & 96 & 7 & 41.22 & 1352 & 5.24 & 2.756 & 7.92 & 6.30 \\
\hline 108A & $8 \%$ PC & 96 & 28 & 73.76 & 2872 & 3.75 & 3.749 & 7.83 & 5.66 \\
\hline 108B & $8 \%$ PC & 96 & 28 & 70.60 & 3402 & 3.48 & 3.701 & 7.80 & 5.66 \\
\hline 109A & $12 \%$ PC & 48 & 1 & 15.12 & 525 & 6.34 & 1.052 & 7.47 & 6.89 \\
\hline 109B & $12 \%$ PC & 48 & 1 & 10.50 & 256 & 6.93 & 1.123 & 7.95 & 7.29 \\
\hline 110A & $12 \%$ PC & 48 & 7 & 19.20 & 587 & 6.32 & 1.151 & 7.37 & 6.74 \\
\hline $110 \mathrm{C}$ & $12 \%$ PC & 48 & 7 & 11.74 & 665 & 6.33 & 1.636 & 6.58 & 5.77 \\
\hline 110D & $12 \%$ PC & 48 & 7 & 14.30 & 777 & 7.49 & 1.596 & 6.36 & 5.59 \\
\hline 111A & $12 \%$ PC & 48 & 28 & 43.50 & 1656 & 5.42 & 2.256 & 7.33 & 6.09 \\
\hline 111B & $12 \%$ PC & 48 & 28 & 37.96 & 1422 & 4.64 & 2.256 & 7.35 & 6.11 \\
\hline 112A & $4 \%$ PC & 48 & 1 & 5.89 & 112 & 13.00 & 1.306 & 6.94 & 6.26 \\
\hline 112B & $4 \%$ PC & 48 & 1 & 2.76 & 90 & 13.00 & 1.232 & 7.38 & 6.71 \\
\hline 113A & $4 \%$ PC & 48 & 7 & 6.96 & 272 & 10.53 & 1.918 & 7.18 & 6.15 \\
\hline
\end{tabular}




\begin{tabular}{|c|c|c|c|c|c|c|c|c|c|}
\hline I.D. & Binder & $\begin{array}{c}\text { Surcharge } \\
\text { (kPa) }\end{array}$ & $\begin{array}{c}\text { Curing Time } \\
\text { (days) }\end{array}$ & $\begin{array}{c}\sigma_{\mathrm{c}} \\
(\mathrm{kPa})\end{array}$ & $\begin{array}{c}E \\
(\mathrm{kPa})\end{array}$ & $\begin{array}{c}\varepsilon_{\mathrm{f}} \\
(\%)\end{array}$ & $\begin{array}{c}\Delta \mathbf{H}_{\text {curing }} \\
\text { (cm) }\end{array}$ & $\mathbf{e}_{0}$ & $\mathbf{e}_{\mathrm{f}}$ \\
\hline 113B & $4 \% \mathrm{PC}$ & 48 & 7 & 12.26 & 255 & 12.80 & 2.404 & 7.26 & 5.96 \\
\hline 114A & 4\% PC & 48 & 28 & 23.44 & 1143 & 3.84 & 3.366 & 7.26 & 5.43 \\
\hline 114B & $4 \%$ PC & 48 & 28 & 21.44 & 661 & 6.79 & 3.576 & 7.43 & 5.45 \\
\hline 115A & $8 \% \mathrm{PC}+4 \% \mathrm{CaO}$ & 48 & 1 & 10.48 & 248 & 12.55 & 1.604 & 6.90 & 6.07 \\
\hline 115B & $8 \% \mathrm{PC}+4 \% \mathrm{CaO}$ & 48 & 1 & 9.72 & 151 & 13.00 & 1.751 & 7.02 & 6.10 \\
\hline 116A & $8 \% \mathrm{PC}+4 \% \mathrm{CaO}$ & 48 & 7 & 15.74 & 600 & 7.59 & 2.035 & 6.73 & 5.70 \\
\hline 116B & $8 \% \mathrm{PC}+4 \% \mathrm{CaO}$ & 48 & 7 & 18.46 & 645 & 5.41 & 2.109 & 6.68 & 5.62 \\
\hline 117A & $8 \% \mathrm{PC}+4 \% \mathrm{CaO}$ & 48 & 28 & 20.08 & 788 & 4.09 & 2.405 & 6.90 & 5.65 \\
\hline 117B & $8 \% \mathrm{PC}+4 \% \mathrm{CaO}$ & 48 & 28 & 20.60 & 1770 & 3.92 & 2.493 & 6.80 & 5.53 \\
\hline 118A & $0 \%$ & 48 & 1 & 3.08 & 49 & 13.00 & 1.288 & 8.01 & 7.25 \\
\hline 118B & $0 \%$ & 48 & 1 & 3.02 & 85 & 13.00 & 1.062 & 7.86 & 7.24 \\
\hline 119A & $0 \%$ & 48 & 7 & 8.18 & 145 & 13.00 & 2.110 & 8.09 & 6.83 \\
\hline 119B & $0 \%$ & 48 & 7 & 8.50 & 166 & 13.00 & 2.530 & 8.17 & 6.65 \\
\hline 120A & $0 \%$ & 48 & 28 & 17.90 & 808 & 4.21 & 3.064 & 8.21 & 6.36 \\
\hline 120B & $0 \%$ & 48 & 28 & 15.56 & 522 & 8.94 & 2.791 & 7.95 & 6.31 \\
\hline 121A & $0 \%$ & 14.5 & 1 & 1.18 & 16 & 11.23 & 0.974 & - & - \\
\hline 121B & $0 \%$ & 14.5 & 1 & 1.15 & 25 & 13.00 & 1.118 & - & - \\
\hline 122A & $0 \%$ & 14.5 & 7 & 2.44 & 75 & 13.00 & 1.560 & 8.41 & 7.45 \\
\hline 122B & $0 \%$ & 14.5 & 7 & 2.72 & 99 & 12.89 & 1.768 & 8.72 & 7.59 \\
\hline 123A & $0 \%$ & 14.5 & 28 & 6.56 & 205 & 13.00 & 2.508 & 8.55 & 6.98 \\
\hline 123B & $0 \%$ & 14.5 & 28 & 6.12 & 145 & 13.00 & 2.200 & 8.44 & 7.08 \\
\hline 124A & $20 \%$ PC & 48 & 1 & 10.90 & 223 & 8.93 & 1.132 & 6.55 & 5.99 \\
\hline 124B & $20 \%$ PC & 48 & 1 & 8.52 & 321 & 7.49 & 1.151 & 6.69 & 6.11 \\
\hline 125A & $20 \%$ PC & 48 & 7 & 21.54 & 1762 & 4.74 & 1.993 & 6.41 & 5.44 \\
\hline 125B & $20 \%$ PC & 48 & 7 & 18.94 & 1030 & 5.16 & 1.961 & 6.38 & 5.43 \\
\hline 126A & $20 \%$ PC & 48 & 28 & 19.70 & 896 & 5.10 & 1.896 & 6.50 & 5.57 \\
\hline 126B & $20 \%$ PC & 48 & 28 & 23.50 & 1490 & 4.53 & 2.343 & 6.49 & 5.34 \\
\hline $126 \mathrm{C}$ & $20 \%$ PC & 48 & 28 & 28.20 & 2910 & 4.27 & 2.451 & 5.10 & 4.12 \\
\hline 126D & $20 \%$ PC & 48 & 28 & 27.20 & 872 & 5.87 & 2.116 & 5.01 & 4.18 \\
\hline 127A & $8 \% \mathrm{PC}+8 \% \mathrm{CaO}$ & 48 & 1 & 12.03 & 381 & 11.53 & 2.068 & 6.04 & 5.09 \\
\hline 127B & $8 \% \mathrm{PC}+8 \% \mathrm{CaO}$ & 48 & 1 & 11.54 & 294 & 12.57 & 1.951 & 5.87 & 4.99 \\
\hline 128A & $8 \% \mathrm{PC}+8 \% \mathrm{CaO}$ & 48 & 7 & 21.28 & 946 & 6.75 & 2.643 & 5.97 & 4.76 \\
\hline
\end{tabular}




\begin{tabular}{|c|c|c|c|c|c|c|c|c|c|}
\hline I.D. & Binder & $\begin{array}{c}\text { Surcharge } \\
\text { (kPa) }\end{array}$ & $\begin{array}{c}\text { Curing Time } \\
\text { (days) }\end{array}$ & $\begin{array}{c}\sigma_{\mathrm{c}} \\
(\mathrm{kPa})\end{array}$ & $\begin{array}{c}E \\
(\mathrm{kPa})\end{array}$ & $\begin{array}{c}\varepsilon_{\mathrm{f}} \\
(\%)\end{array}$ & $\begin{array}{c}\Delta \mathbf{H}_{\text {curing }} \\
\text { (cm) }\end{array}$ & $\mathbf{e}_{0}$ & $\mathbf{e}_{\mathrm{f}}$ \\
\hline 128B & $8 \% \mathrm{PC}+8 \% \mathrm{CaO}$ & 48 & 7 & 19.46 & 1308 & 7.14 & 2.995 & 6.05 & 4.67 \\
\hline 129A & $8 \% \mathrm{PC}+8 \% \mathrm{CaO}$ & 48 & 28 & 27.30 & 1876 & 6.00 & 2.955 & 6.09 & 4.71 \\
\hline 129B & $8 \% \mathrm{PC}+8 \% \mathrm{CaO}$ & 48 & 28 & 25.64 & 2734 & 4.12 & 3.225 & 6.08 & 4.58 \\
\hline 130A & $8 \% \mathrm{PC}+12 \% \mathrm{CaO}$ & 48 & 1 & 12.68 & 336 & 12.33 & 1.822 & 5.93 & 5.10 \\
\hline 130B & $8 \% \mathrm{PC}+12 \% \mathrm{CaO}$ & 48 & 1 & 15.66 & 747 & 11.45 & 2.207 & 5.91 & 4.91 \\
\hline 131A & $8 \% \mathrm{PC}+12 \% \mathrm{CaO}$ & 48 & 7 & 22.90 & 1003 & 5.40 & 2.895 & 5.96 & 4.64 \\
\hline 131B & $8 \% \mathrm{PC}+12 \% \mathrm{CaO}$ & 48 & 7 & 19.90 & 1106 & 7.19 & 2.676 & 5.72 & 4.54 \\
\hline 132A & $8 \% \mathrm{PC}+12 \% \mathrm{CaO}$ & 48 & 28 & 27.94 & 1958 & 4.39 & 2.934 & 5.82 & 4.51 \\
\hline 132B & $8 \% \mathrm{PC}+12 \% \mathrm{CaO}$ & 48 & 28 & 27.94 & 2689 & 5.46 & 3.129 & 5.76 & 4.37 \\
\hline 133A & $0 \%$ & 48 & 7 & 5.78 & 296 & 12.97 & 3.325 & 7.06 & 5.30 \\
\hline 133B & $0 \%$ & 48 & 7 & 10.08 & 649 & 4.81 & 3.129 & 6.78 & 5.18 \\
\hline 134A & $4 \% \mathrm{CaO}$ & 48 & 7 & 19.82 & 1118 & 5.70 & 1.873 & 6.87 & 5.90 \\
\hline 134B & $4 \% \mathrm{CaO}$ & 48 & 7 & 6.18 & 222 & 7.00 & 3.284 & 7.02 & 5.29 \\
\hline 135A & $8 \% \mathrm{CaO}$ & 48 & 7 & 20.24 & 708 & 8.11 & 3.239 & 6.97 & 5.27 \\
\hline 135B & $8 \% \mathrm{CaO}$ & 48 & 7 & 26.52 & 1971 & 4.34 & 3.559 & 6.91 & 5.06 \\
\hline 136A & $12 \% \mathrm{CaO}$ & 48 & 7 & 26.42 & 1795 & 5.07 & 2.880 & 6.55 & 5.12 \\
\hline 136B & $12 \% \mathrm{CaO}$ & 14.5 & 7 & 24.74 & 1953 & 3.71 & 2.738 & 6.53 & 5.18 \\
\hline 137A & $20 \% \mathrm{CaO}$ & 14.5 & 7 & 28.58 & 2157 & 3.17 & 2.967 & 6.68 & 5.19 \\
\hline 137B & $20 \% \mathrm{CaO}$ & 14.5 & 7 & 24.06 & 1440 & 4.80 & 2.824 & 6.72 & 5.29 \\
\hline 138A & $0 \%$ & 96 & 28 & 22.51 & 1392 & 8.46 & 3.459 & 8.07 & 6.01 \\
\hline 138B & $0 \%$ & 96 & 28 & 2.9 & 615 & 8.74 & 3.350 & 8.33 & 6.28 \\
\hline 139A & $0 \%$ & 96 & 7 & 14.63 & 725 & 13.00 & 2.363 & 6.57 & 5.40 \\
\hline 139B & $0 \%$ & 96 & 7 & 14.84 & 453 & 13.00 & 2.637 & 6.57 & 5.26 \\
\hline 140A & $0 \%$ & 96 & 1 & 4.40 & 154 & 13.00 & 1.511 & 6.86 & 6.08 \\
\hline 140B & $0 \%$ & 96 & 1 & 5.29 & 153 & 13.00 & 1.725 & 7.10 & 6.18 \\
\hline
\end{tabular}


Table 5.3: Summary table of UC tests on PC treated Mixture I and II, and LR soil

\begin{tabular}{|c|c|c|c|c|c|c|c|c|}
\hline Specimen ID. & $\begin{array}{l}\% \\
\text { PC }\end{array}$ & $\begin{array}{c}\text { Surcharge } \\
\text { (kPa) }\end{array}$ & $\begin{array}{c}\text { Curing Time } \\
\text { (days) }\end{array}$ & $\begin{array}{c}\sigma_{\mathrm{c}} \\
(\mathrm{kPa})\end{array}$ & $\begin{array}{c}E \\
(\mathrm{kPa})\end{array}$ & $\begin{array}{c}\varepsilon_{\mathrm{f}} \\
(\%)\end{array}$ & $\begin{array}{c}\Delta \mathbf{H}_{\text {curing }} \\
\text { (cm) }\end{array}$ & $\begin{array}{l}\text { LOI } \\
(\%)\end{array}$ \\
\hline MI 001401A & 0 & 14.5 & 1 & 16.37 & 0.34 & 13 & 3.25 & 9.1 \\
\hline MI 001401B & 0 & 14.5 & 1 & 15.66 & 0.35 & 13 & 2.92 & 9.1 \\
\hline MI 001407A & 0 & 14.5 & 7 & 23.22 & 0.63 & 13 & 3.69 & 8.5 \\
\hline MI 001407B & 0 & 14.5 & 7 & 23.27 & 0.68 & 13 & 2.11 & 8.5 \\
\hline MI 001428A & 0 & 14.5 & 28 & 20.56 & 0.66 & 13 & 4.86 & 9.2 \\
\hline MI 001428B & 0 & 14.5 & 28 & 19.78 & 0.46 & 13 & 5.14 & 9.2 \\
\hline MI 004801A & 0 & 48 & 1 & 17.09 & 0.51 & 13 & 4.74 & 8.8 \\
\hline MI 004801B & 0 & 48 & 1 & 17.57 & 0.49 & 13 & 3.39 & 8.8 \\
\hline MI 004801C & 0 & 48 & 1 & 16.33 & 0.31 & 13 & 4.73 & 8.7 \\
\hline MI 004807A & 0 & 48 & 7 & 25.23 & 0.66 & 13 & 7.47 & 8.8 \\
\hline MI 004807B & 0 & 48 & 7 & 26.27 & 0.76 & 13 & 7.49 & 8.8 \\
\hline MI 004828A & 0 & 48 & 28 & 33.42 & 0.94 & 13 & 7.91 & 8.7 \\
\hline MI 004828B & 0 & 48 & 28 & 29.13 & 0.50 & 13 & 8.03 & 8.7 \\
\hline MI 009601A & 0 & 96 & 1 & 19.39 & 0.71 & 13 & 11.60 & 10.2 \\
\hline MI 009601B & 0 & 96 & 1 & 16.44 & 0.39 & 13 & 10.60 & 10.2 \\
\hline MI 009607A & 0 & 96 & 7 & 52.73 & 2.41 & 5.19 & 19.88 & 10.2 \\
\hline MI 009607B & 0 & 96 & 7 & 58.31 & 3.30 & 4.03 & 20.60 & 10.2 \\
\hline MI 009628A & 0 & 96 & 28 & 66.88 & 5.25 & 5.35 & 26.04 & 9.6 \\
\hline MI 044801A* & 4 & 48 & 1 & 82.65 & 7.79 & 3.25 & 1.35 & 8.7 \\
\hline MI 044801B* & 4 & 48 & 1 & 71.51 & 7.78 & 2.94 & 0.73 & 8.7 \\
\hline MI 044801C & 4 & 48 & 1 & 37.36 & 1.04 & 13 & 4.78 & 9.2 \\
\hline MI 044801D & 4 & 48 & 1 & 41.21 & 2.81 & 11.01 & 4.32 & 9.2 \\
\hline MI 044807A* & 4 & 48 & 7 & 114.50 & 7.57 & 3.35 & 2.28 & 8.7 \\
\hline MI 044807B* & 4 & 48 & 7 & 56.58 & 10.99 & 3.02 & 4.26 & 8.7 \\
\hline MI 044807C & 4 & 48 & 7 & 45.74 & 2.14 & 6.76 & 6.89 & 9.6 \\
\hline MI 044807D & 4 & 48 & 7 & 44.89 & 4.71 & 13 & 6.28 & 9.8 \\
\hline MI 044828B & 4 & 48 & 28 & 69.54 & 4.68 & 3.22 & 2.65 & 9.8 \\
\hline MI 081401A & 8 & 14.5 & 1 & 226.68 & 19.84 & 2.66 & 0.00 & 8.3 \\
\hline MI 081401B & 8 & 14.5 & 1 & 205.30 & 12.73 & 2.7 & 0.00 & 8.3 \\
\hline MI 081407A & 8 & 14.5 & 7 & 222.09 & 21.46 & 3.12 & 0.00 & 10.2 \\
\hline
\end{tabular}




\begin{tabular}{|c|c|c|c|c|c|c|c|c|}
\hline Specimen ID. & $\begin{array}{l}\% \\
\text { PC }\end{array}$ & $\begin{array}{c}\text { Surcharge } \\
\text { (kPa) }\end{array}$ & $\begin{array}{c}\text { Curing Time } \\
\text { (days) }\end{array}$ & $\begin{array}{c}\sigma_{\mathrm{c}} \\
(\mathrm{kPa})\end{array}$ & $\begin{array}{c}E \\
(\mathrm{kPa})\end{array}$ & $\begin{array}{c}\varepsilon_{\mathrm{f}} \\
(\%)\end{array}$ & $\begin{array}{c}\Delta \mathbf{H}_{\text {curing }} \\
\text { (cm) }\end{array}$ & $\begin{array}{l}\text { LOI } \\
\text { (\%) }\end{array}$ \\
\hline MI 081407B & 8 & 14.5 & 7 & 211.01 & 20.40 & 2.91 & 0.00 & 10.2 \\
\hline MI 081428A & 8 & 14.5 & 28 & 418.20 & 56.82 & 1.44 & 0.00 & 9.4 \\
\hline MI 081428B & 8 & 14.5 & 28 & 440.44 & 59.11 & 1.56 & 0.00 & 9.4 \\
\hline MI 084801A & 8 & 48 & 1 & 208.14 & 18.00 & 2.55 & 0.40 & 8.8 \\
\hline MI 084801B & 8 & 48 & 1 & 197.85 & 18.89 & 2.21 & 1.27 & 8.8 \\
\hline MI 084807A & 8 & 48 & 7 & 507.44 & 72.79 & 1.5 & 1.45 & 9.0 \\
\hline MI 084807B & 8 & 48 & 7 & 409.04 & 41.36 & 1.61 & 2.00 & 9.0 \\
\hline MI 084807C & 8 & 48 & 7 & 363.27 & 44.41 & 1.95 & 0.75 & 9.4 \\
\hline MI 084828A & 8 & 48 & 28 & 416.89 & 53.20 & 1.21 & 2.20 & 9.0 \\
\hline MI 084828B & 8 & 48 & 28 & 511.07 & 60.45 & 1.23 & 1.43 & 9.0 \\
\hline MI 089601A & 8 & 96 & 1 & 249.69 & 18.69 & 2.57 & 1.16 & 9.7 \\
\hline MI 089601B & 8 & 96 & 1 & 247.38 & 29.67 & 2.16 & 1.54 & 9.7 \\
\hline MI 089607A & 8 & 96 & 7 & 361.18 & 29.63 & 1.97 & 2.44 & 9.4 \\
\hline MI 089607B & 8 & 96 & 7 & 332.36 & 25.57 & 2.38 & 2.06 & 9.4 \\
\hline MI 089607C & 8 & 96 & 7 & 430.02 & 47.01 & 2.65 & 6.07 & 10.7 \\
\hline MI 089607D & 8 & 96 & 7 & 463.51 & 45.81 & 2.55 & 5.84 & 10.7 \\
\hline MI 089628A & 8 & 96 & 28 & 589.54 & 36.08 & 2.17 & 3.41 & 9.3 \\
\hline MI 089628B & 8 & 96 & 28 & 602.72 & 62.76 & 1.66 & 3.78 & 9.3 \\
\hline MI 124801A & 12 & 48 & 1 & 363.00 & 37.79 & 1.8 & 0.49 & 9.0 \\
\hline MI 124801B & 12 & 48 & 1 & 314.19 & 46.44 & 1.28 & 0.31 & 9.0 \\
\hline MI 124801C & 12 & 48 & 1 & 488.55 & 64.44 & 1.88 & 1.13 & 9.5 \\
\hline MI 124807A & 12 & 48 & 7 & 696.42 & 103.99 & 1.21 & 0.45 & 9.4 \\
\hline MI 124807B & 12 & 48 & 7 & 580.13 & 106.54 & 1.2 & 0.44 & 9.4 \\
\hline MI $124807 \mathrm{C}$ & 12 & 48 & 7 & 774.53 & 99.94 & 1.52 & 0.63 & 9.5 \\
\hline MI 124828A* & 12 & 48 & 28 & 857.75 & 93.81 & 1.14 & 0.22 & 9.4 \\
\hline MI 124828B* & 12 & 48 & 28 & 525.68 & 86.91 & 0.59 & -0.23 & 9.4 \\
\hline MI 124828C & 12 & 48 & 28 & 1041.08 & 143.19 & 1.42 & 1.51 & 10.1 \\
\hline MI 124828D & 12 & 48 & 28 & 975.40 & 138.33 & 1.46 & 1.34 & 10.1 \\
\hline MI 204801A & 20 & 48 & 1 & 530.53 & 55.35 & 1.65 & 0.47 & 8.8 \\
\hline MI 204801B & 20 & 48 & 1 & 455.70 & 61.78 & 1.04 & 0.49 & 8.8 \\
\hline MI 204807A & 20 & 48 & 7 & 1088.30 & 113.60 & 1.64 & 1.26 & 10.8 \\
\hline MI 204807B & 20 & 48 & 7 & 1213.45 & 152.74 & 1.45 & 0.01 & 10.8 \\
\hline
\end{tabular}




\begin{tabular}{|c|c|c|c|c|c|c|c|c|}
\hline Specimen ID. & $\begin{array}{l}\% \\
\text { PC }\end{array}$ & $\begin{array}{c}\text { Surcharge } \\
\text { (kPa) }\end{array}$ & $\begin{array}{c}\text { Curing Time } \\
\text { (days) }\end{array}$ & $\begin{array}{c}\sigma_{\mathrm{c}} \\
(\mathrm{kPa})\end{array}$ & $\begin{array}{c}E \\
(\mathrm{kPa})\end{array}$ & $\begin{array}{c}\varepsilon_{\mathrm{f}} \\
(\%)\end{array}$ & $\begin{array}{c}\Delta \mathbf{H}_{\text {curing }} \\
\text { (cm) }\end{array}$ & $\begin{array}{l}\text { LOI } \\
(\%)\end{array}$ \\
\hline MI 204828A & 20 & 48 & 28 & 1475.09 & 202.34 & 1.28 & 0.78 & 9.5 \\
\hline MI 204828B & 20 & 48 & 28 & 1282.68 & 131.10 & 1.07 & -0.24 & 9.5 \\
\hline MI 304801A & 30 & 48 & 1 & 770.67 & 72.13 & 1.88 & 1.46 & 8.3 \\
\hline MI 304801B & 30 & 48 & 1 & 766.21 & 58.13 & 1.71 & 1.09 & 8.3 \\
\hline MI 304807A & 30 & 48 & 7 & 1403.64 & 110.00 & 1.52 & 0.22 & 9.0 \\
\hline MI 304807B & 30 & 48 & 7 & 1466.84 & 151.26 & 1.39 & 0.74 & 9.0 \\
\hline MI 304828A & 30 & 48 & 28 & 2055.47 & 210.36 & 1.51 & 0.37 & 9.0 \\
\hline MI 304828B & 30 & 48 & 28 & 2198.65 & 295.85 & 1.22 & 1.02 & 9.0 \\
\hline MII 004801 & 0 & 48 & 1 & 20.69 & 0.60 & 13 & 5.78 & 19.6 \\
\hline MII 004807 & 0 & 48 & 7 & 31.35 & 1.58 & 7.95 & 9.51 & 19.6 \\
\hline MII 004828 & 0 & 48 & 28 & 47.60 & 2.26 & 5.81 & 13.19 & 19.6 \\
\hline MII 084801 & 8 & 48 & 1 & 51.09 & 4.23 & 6.31 & 5.26 & 21.2 \\
\hline MII 084807 & 8 & 48 & 7 & 66.63 & 6.65 & 3.95 & 4.92 & 21.2 \\
\hline MII 084828A & 8 & 48 & 28 & 89.02 & 5.32 & 4.42 & 7.34 & 21.2 \\
\hline MII 084828B & 8 & 48 & 28 & 87.30 & 12.50 & 3.28 & 8.53 & 21.2 \\
\hline LRP504801A* & 50 & 48 & 1 & 29.51 & 2.59 & 4.52 & 19.21 & 41.6 \\
\hline LRP504801B* & 50 & 48 & 1 & 37.44 & 2.71 & 3.97 & 21.36 & 41.6 \\
\hline LRP504801C & 50 & 48 & 1 & 65.33 & 4.39 & 4.03 & 10.44 & 53.9 \\
\hline LRP504801D & 50 & 48 & 1 & 70.41 & 3.98 & 4.07 & 10.50 & 53.9 \\
\hline LRP504807A* & 50 & 48 & 7 & 80.09 & 13.21 & 2.73 & 19.76 & 43.3 \\
\hline LRP504807B* & 50 & 48 & 7 & 79.52 & 10.38 & 3.22 & 19.20 & 43.3 \\
\hline LRP504807C* & 50 & 48 & 7 & 85.22 & 13.70 & 2.83 & 22.58 & 43.3 \\
\hline LRP504807D & 50 & 48 & 7 & 114.82 & 4.46 & 4.06 & 13.46 & 53.9 \\
\hline LRP504807E & 50 & 48 & 7 & 102.66 & 5.84 & 3.62 & 13.36 & 53.9 \\
\hline LRP504828A* & 50 & 48 & 28 & 57.63 & 7.99 & 2.41 & 21.91 & 41.6 \\
\hline LRP504828B* & 50 & 48 & 28 & 62.63 & 6.00 & 1.06 & 22.86 & 41.6 \\
\hline LRP504828C & 50 & 48 & 27 & 115.20 & 7.03 & 3.34 & 14.11 & 53.9 \\
\hline LRP504828D & 50 & 48 & 27 & 119.38 & 6.84 & 3.52 & 13.33 & 53.9 \\
\hline
\end{tabular}

*: Problems were encountered in performing these tests and thus the results should be disregarded. 


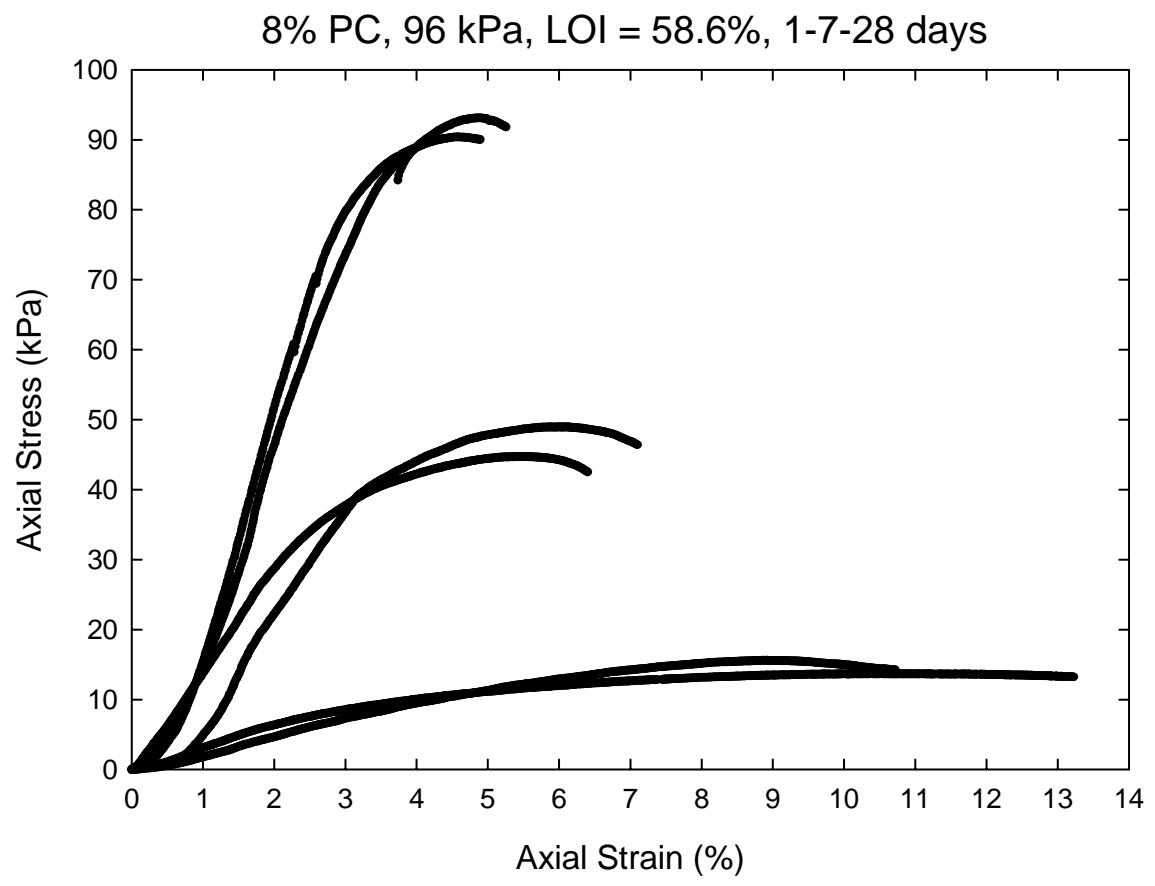

Figure 5.1: Typical uncorrected Unconfined Compression test results

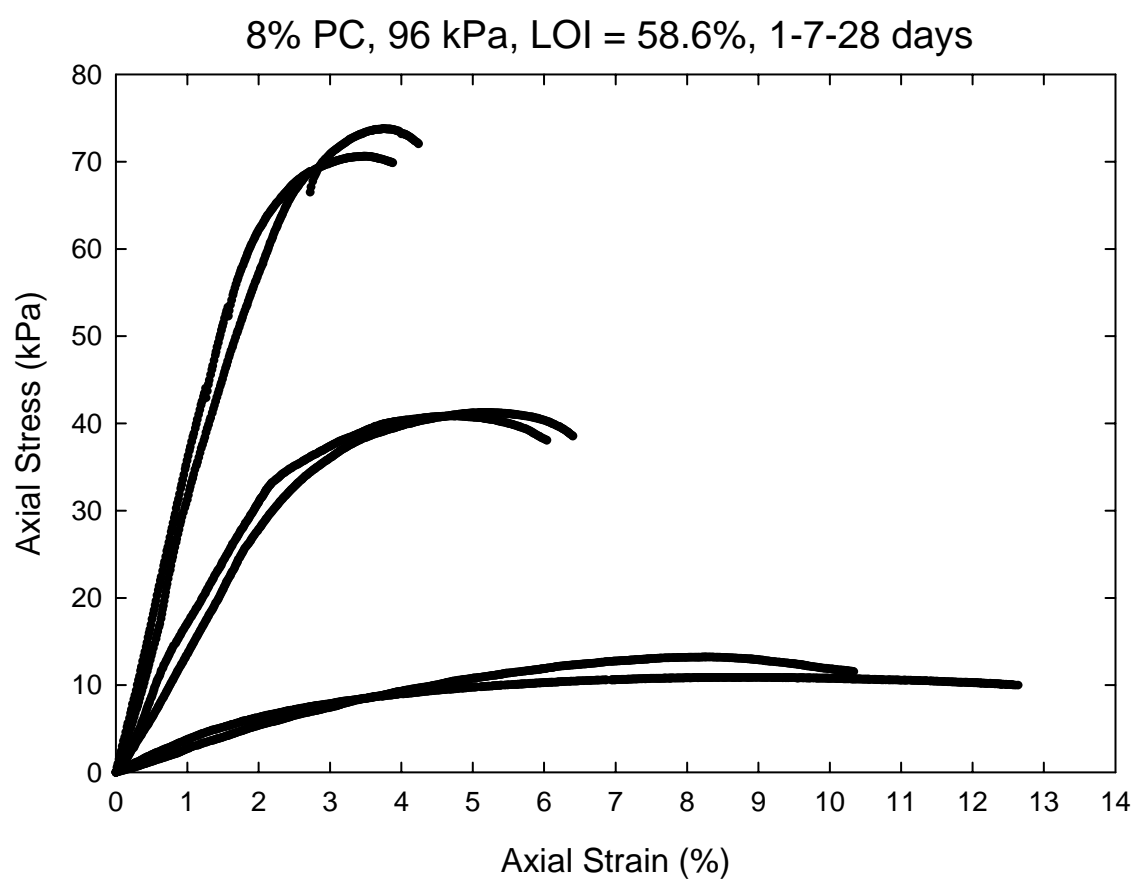

Figure 5.2: Typical corrected Unconfined Compression test results 


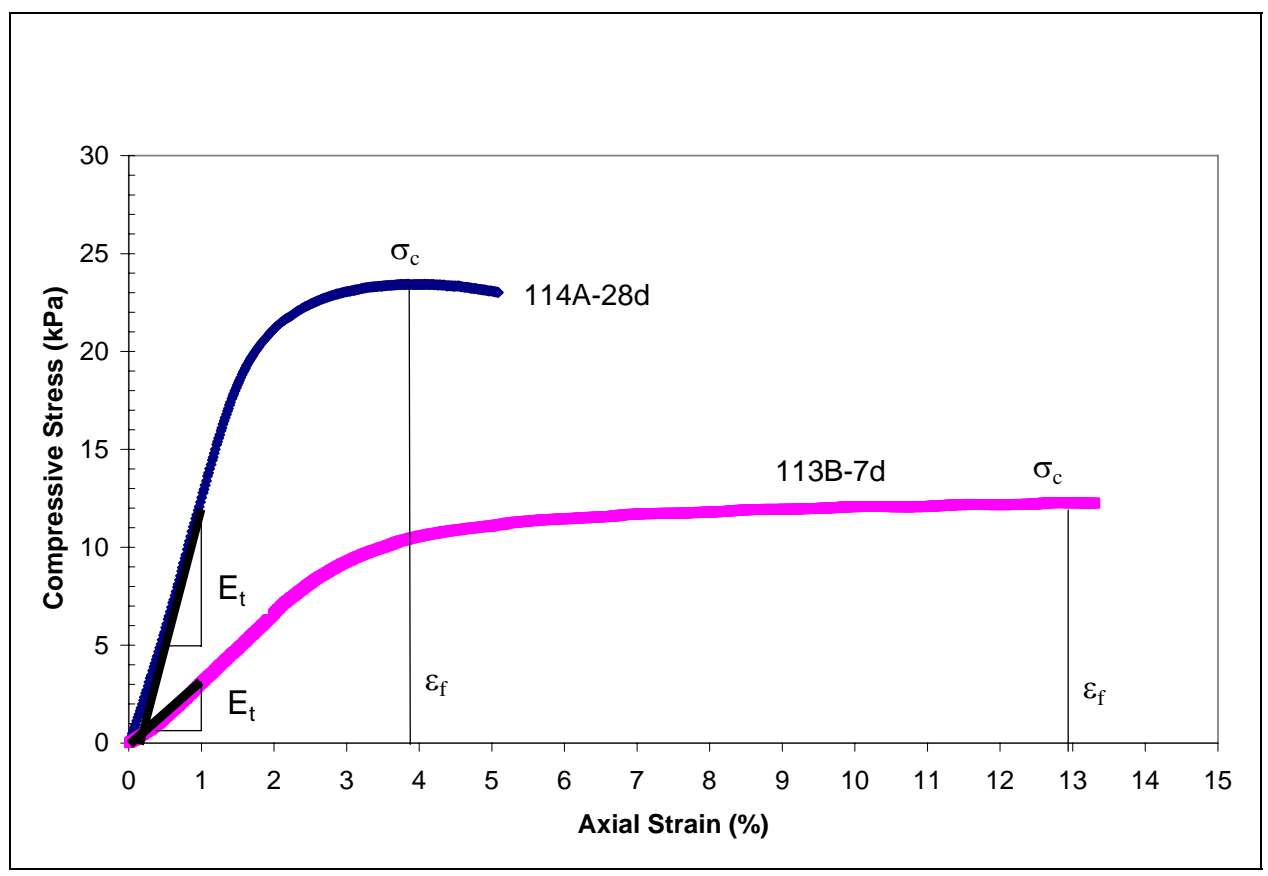

Figure 5.3: Stress vs. strain curve showing peak and non-peak behavior, and parameter selection.

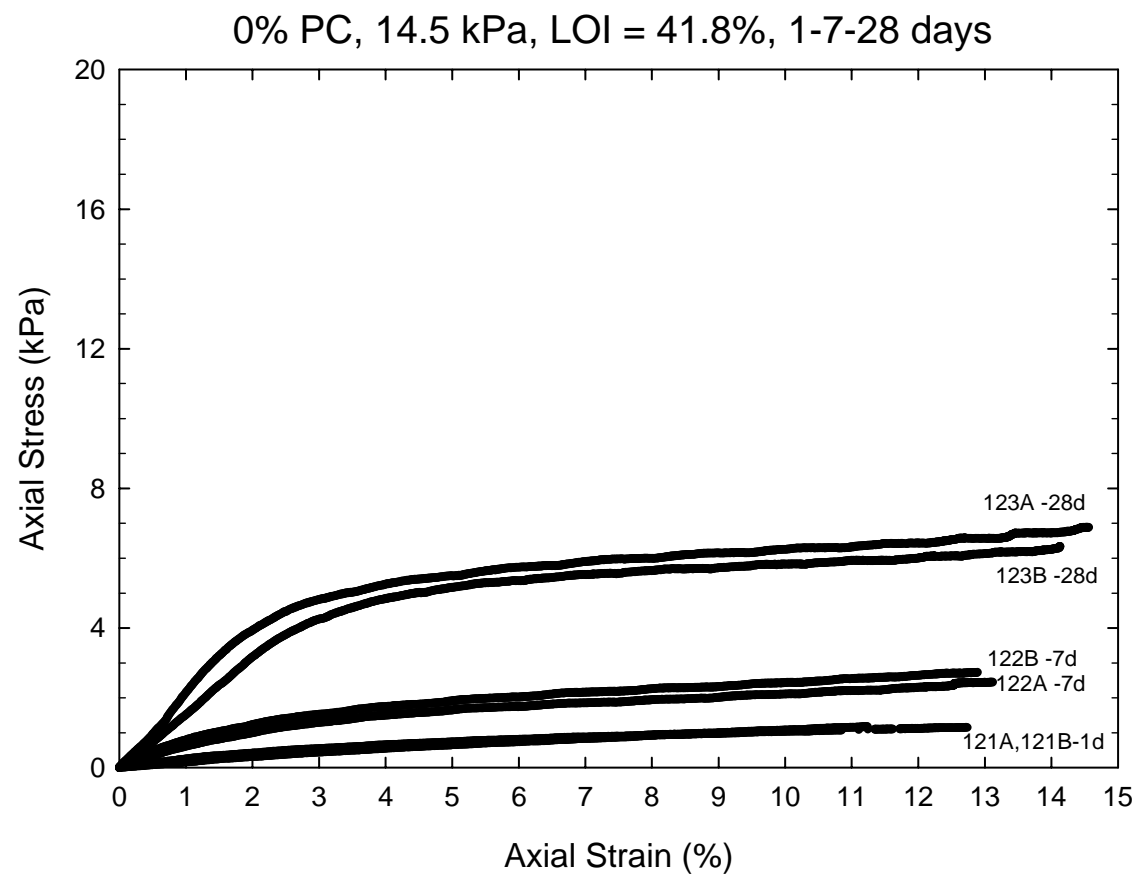

Figure 5.4: Stress-strain plot from UC test on 0\% PC mixture surcharged at $14.5 \mathrm{kPa}$ 


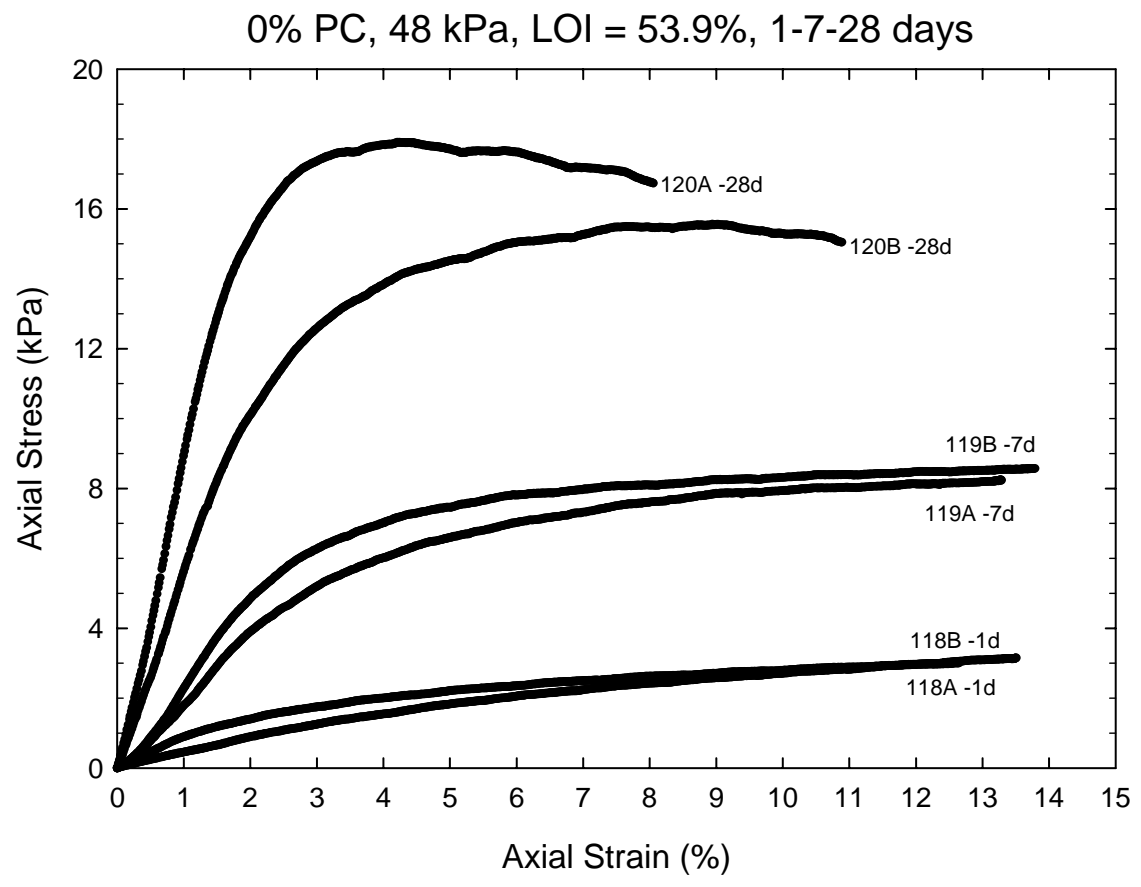

Figure 5.5: Stress-strain plot from UC test on $0 \%$ PC mixture surcharged at $48 \mathrm{kPa}$

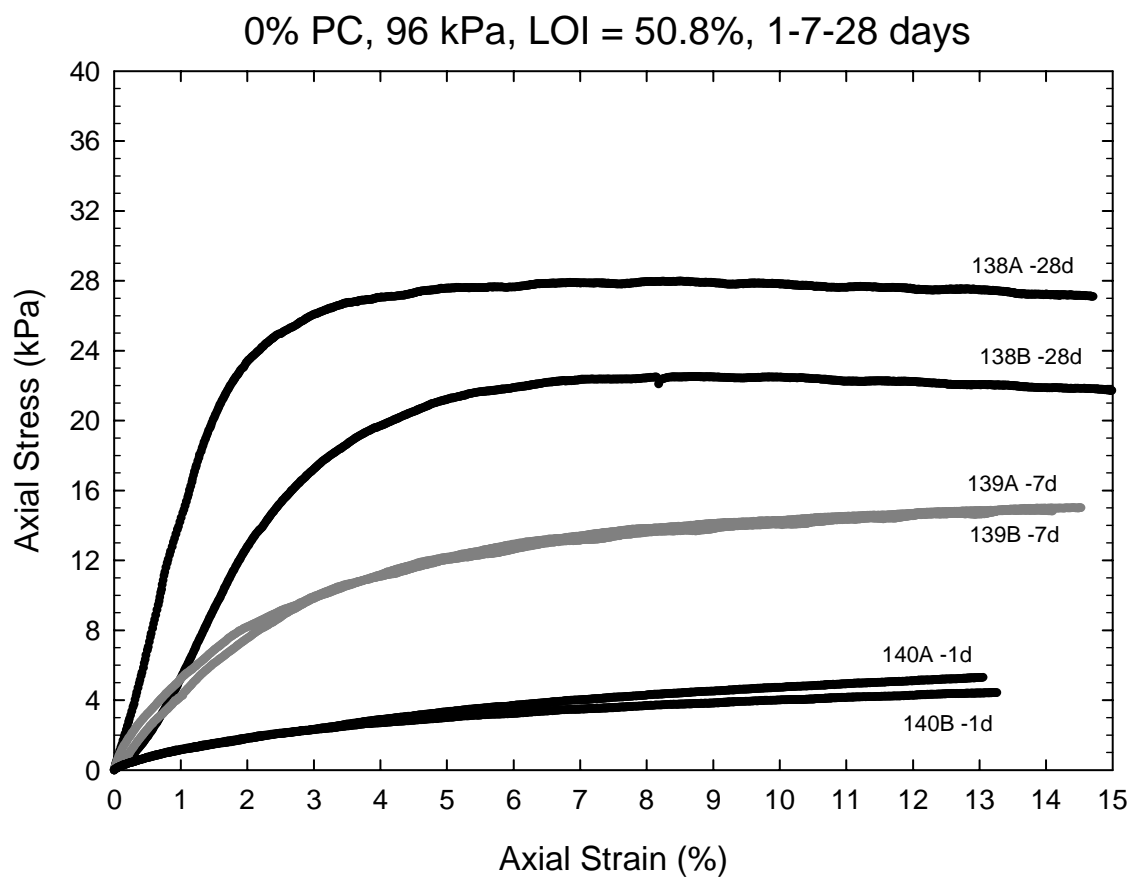

Figure 5.6: Stress-strain plot from UC test on 0\% PC mixture surcharged at $96 \mathrm{kPa}$ 


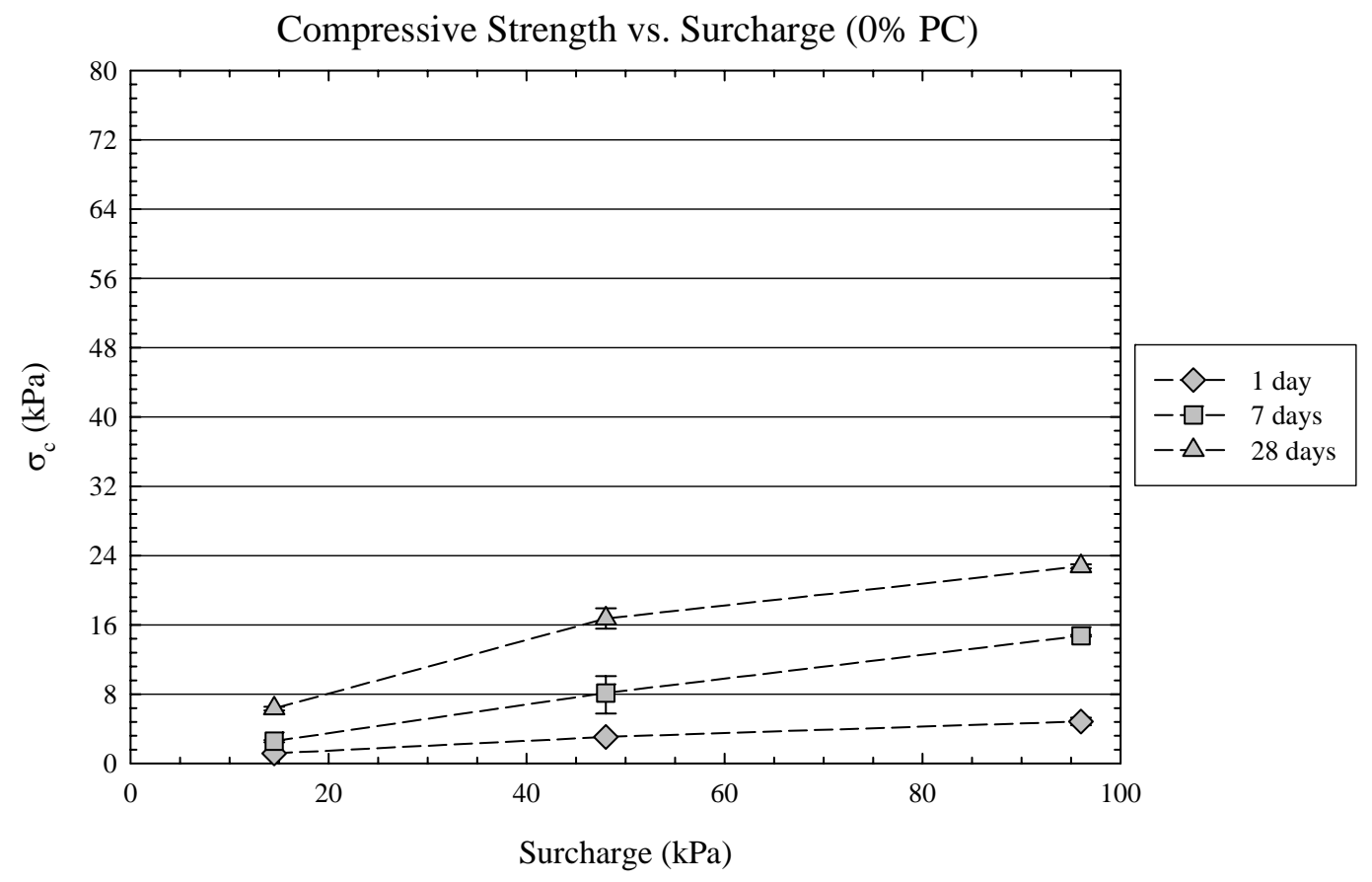

Figure 5.7: Effect of surcharge on strength of LR soil without binder

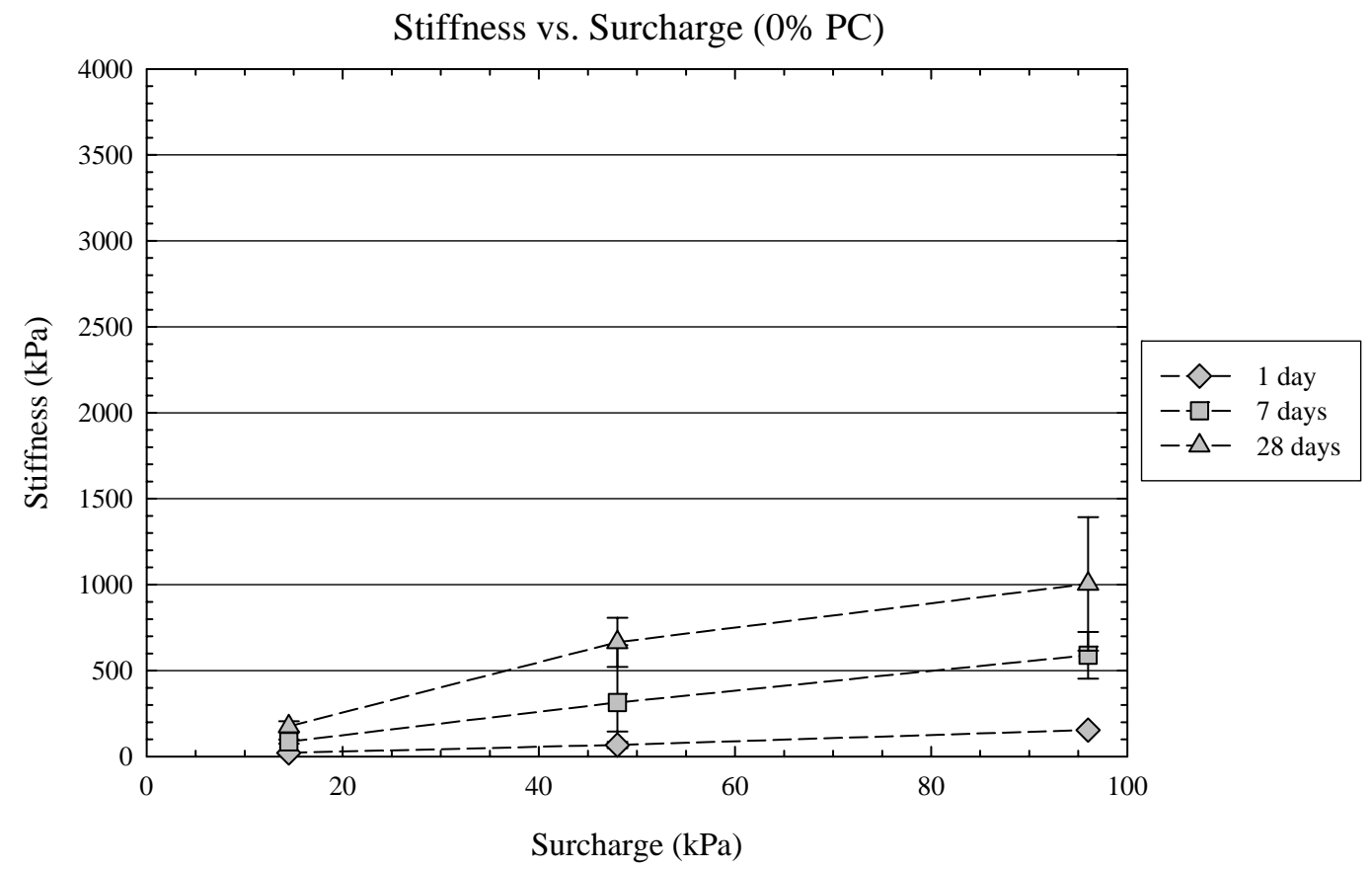

Figure 5.8: Effect of surcharge on stiffness of LR soil without binder 
Strain at Failure vs. Surcharge (0\% PC)

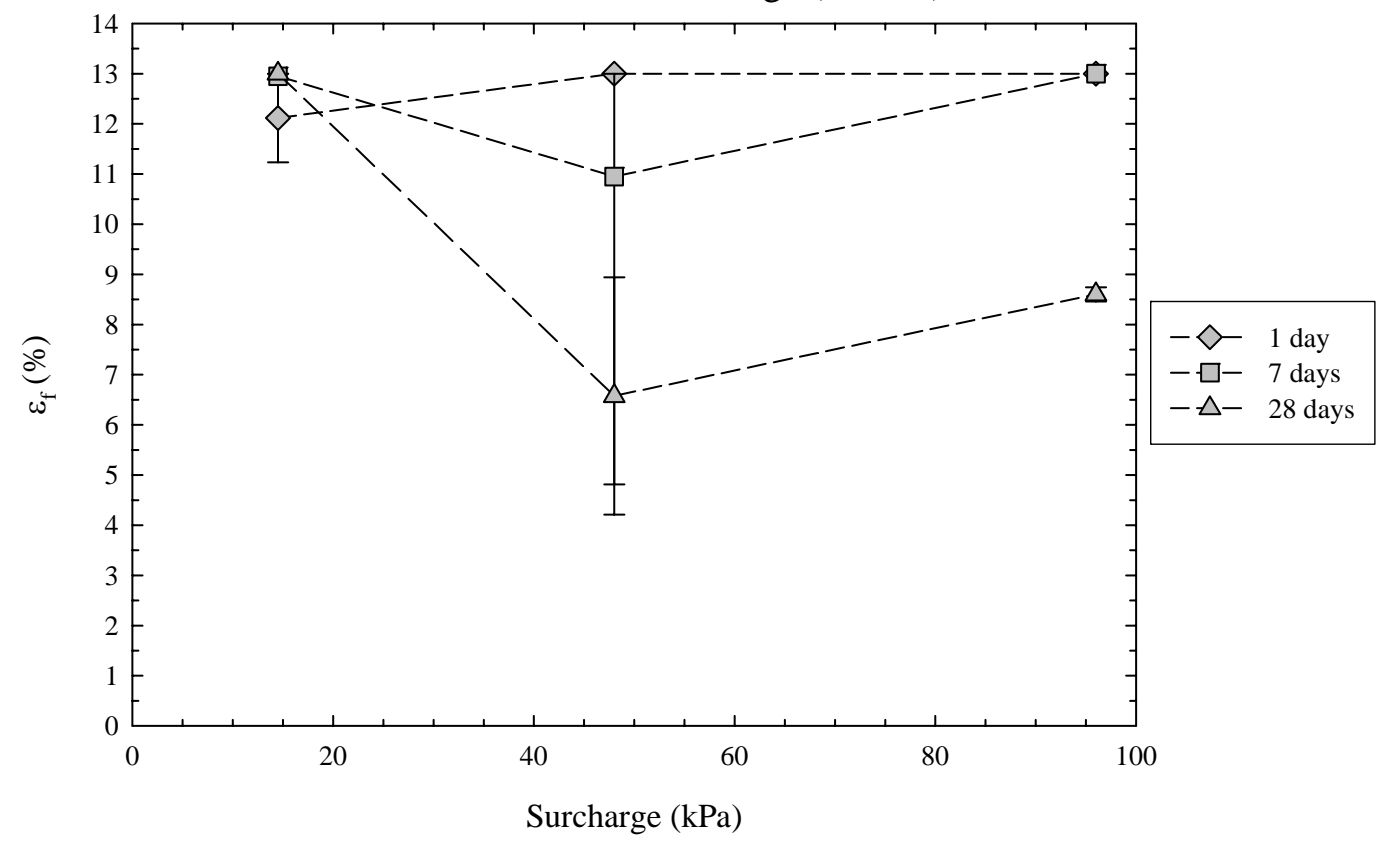

Figure 5.9: Effect of surcharge on strain at failure of LR soil without binder

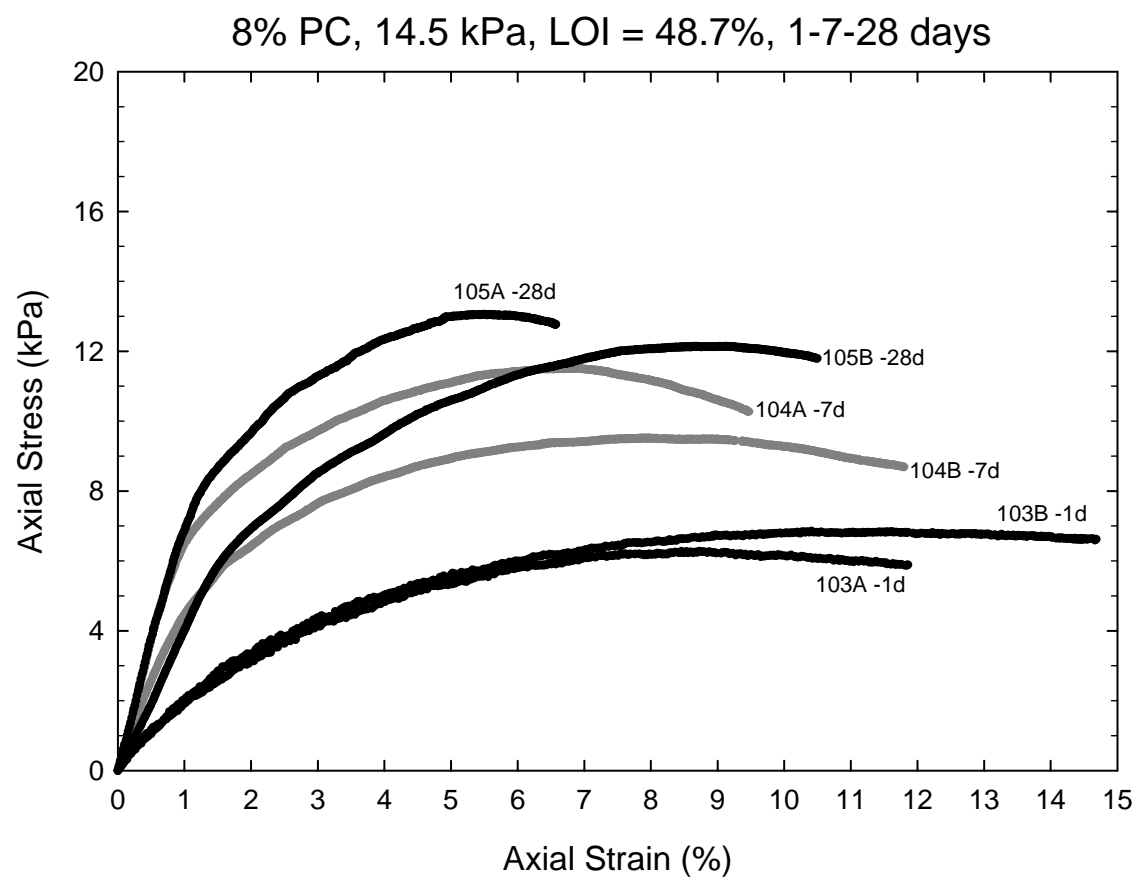

Figure 5.10: Stress-strain plot from UC test on 8\% PC mixture surcharged at $14.5 \mathrm{kPa}$ 


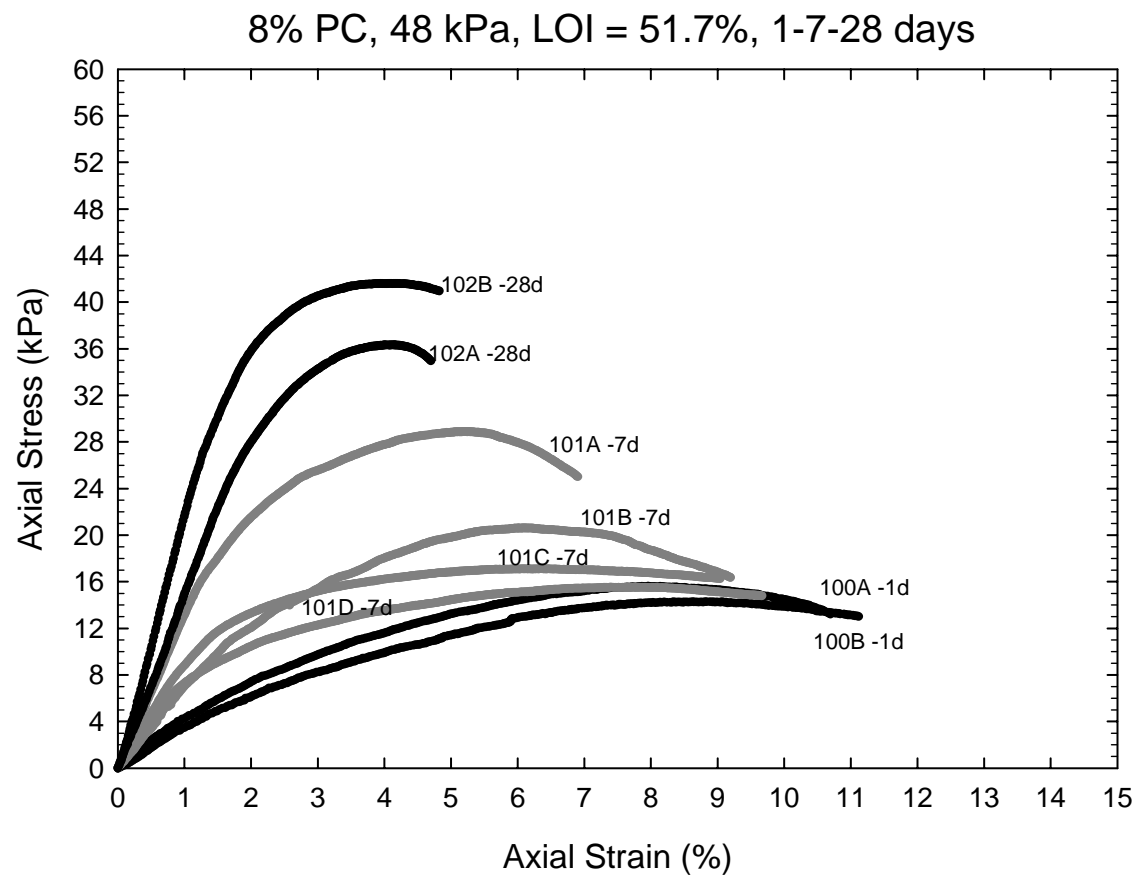

Figure 5.11: Stress-strain plot from UC test on 8\% PC mixture surcharged at $48 \mathrm{kPa}$

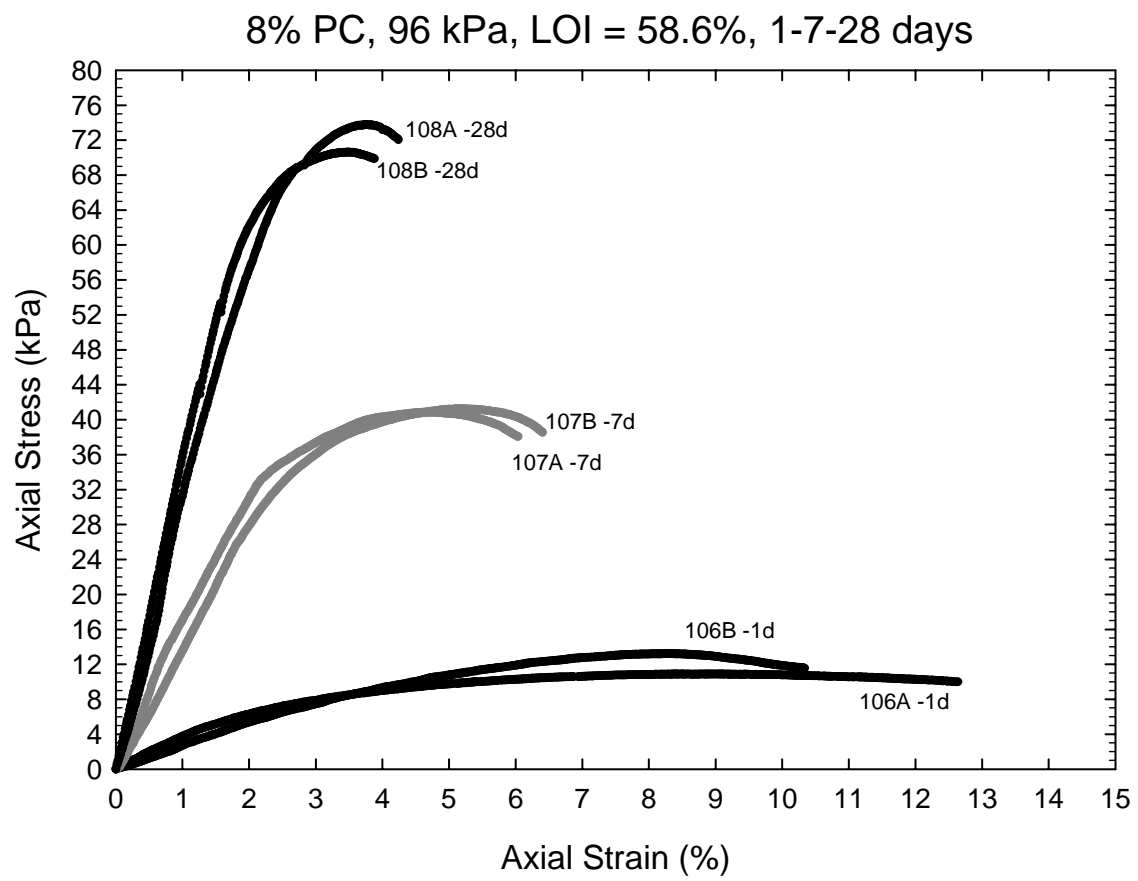

Figure 5.12: Stress-strain plot from UC test on 8\% PC mixture surcharged at $96 \mathrm{kPa}$ 


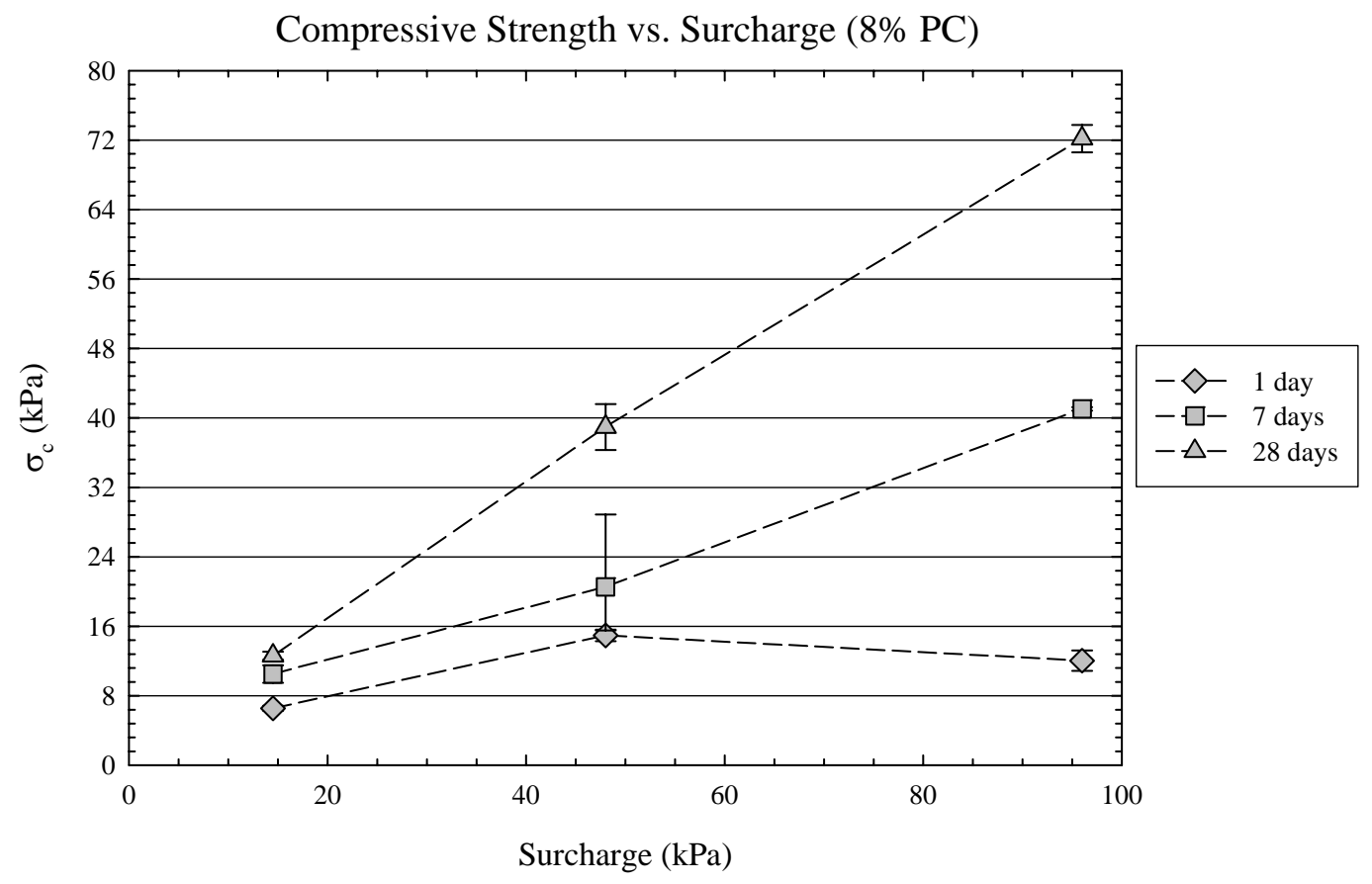

Figure 5.13: Effect of surcharge on strength of LR soil with 8\% PC

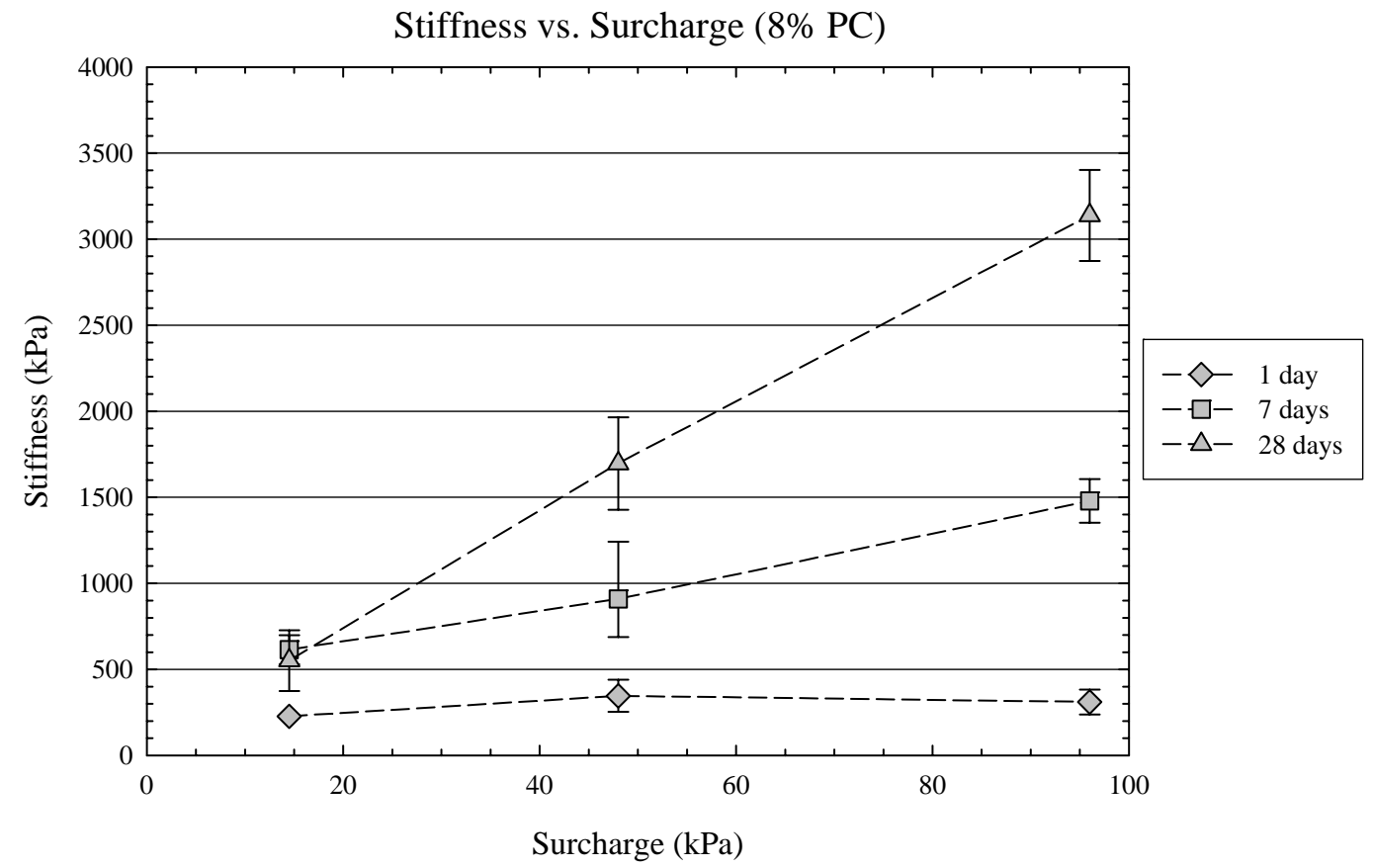

Figure 5.14: Effect of surcharge on stiffness of LR soil with 8\% PC 


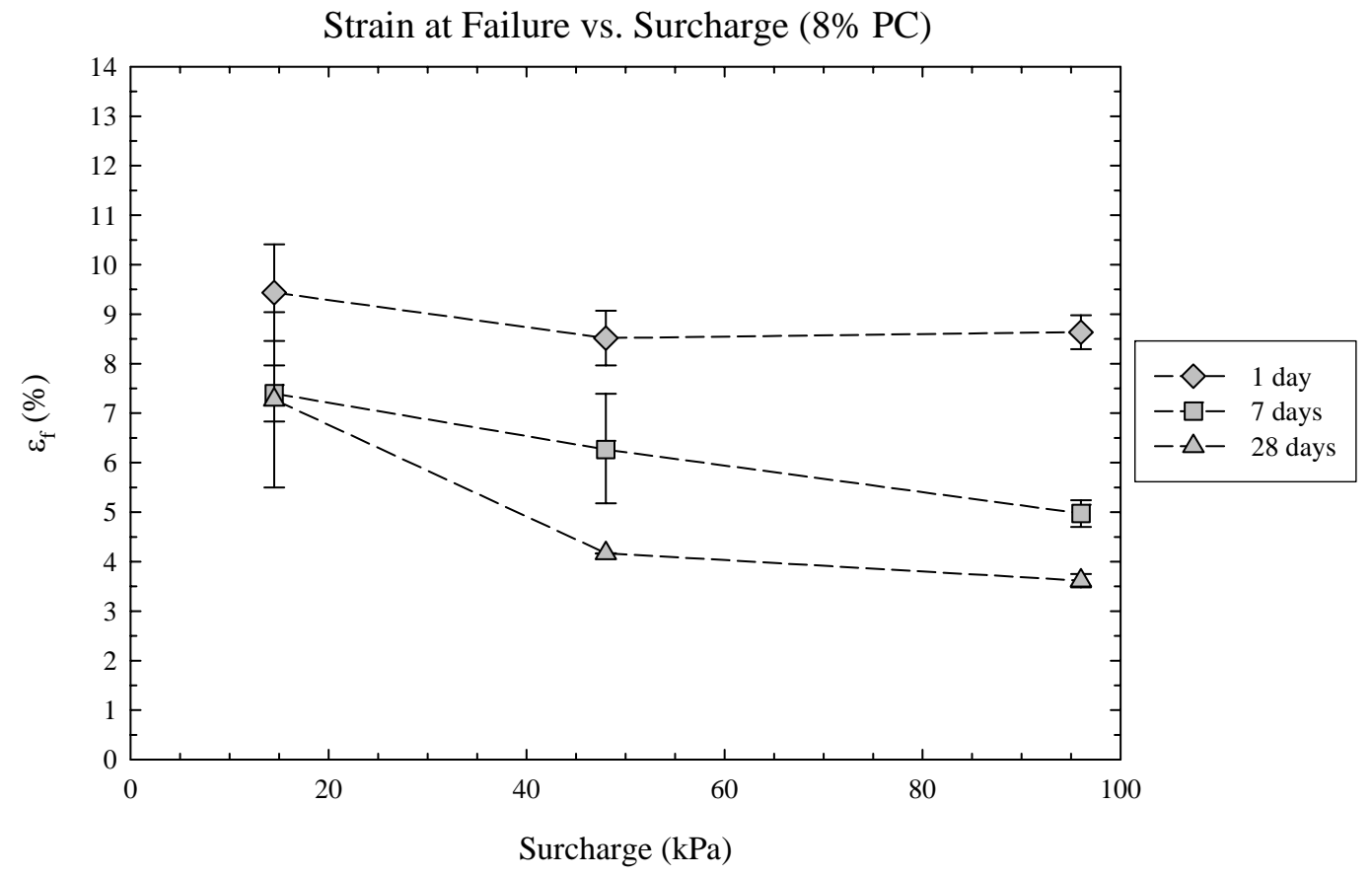

Figure 5.15: Effect of surcharge on strain at failure of LR soil with 8\% PC

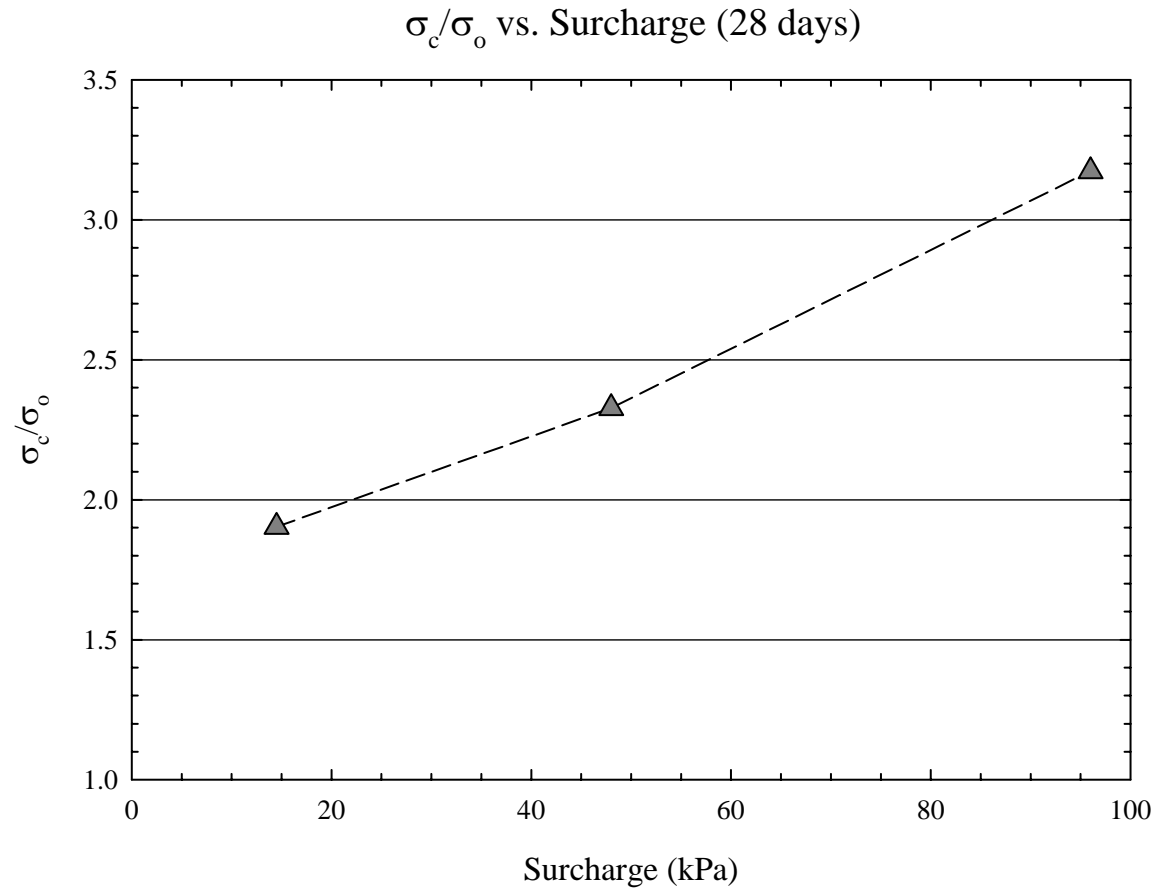

Figure 5.16: Compressive strength of 8\% PC LR soil normalized by compressive strength of $0 \%$ PC LR soil for same surcharge and curing period 


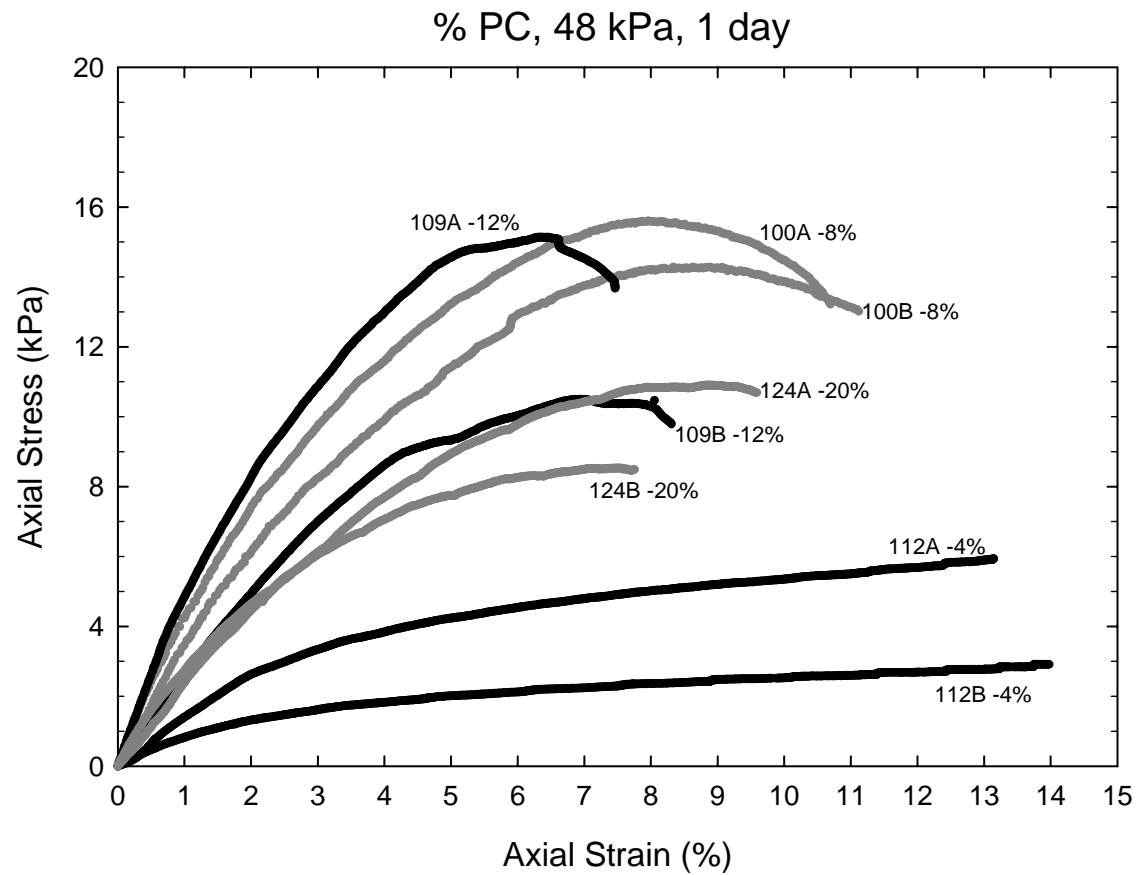

Figure 5.17: Various \%PC mixtures at one day

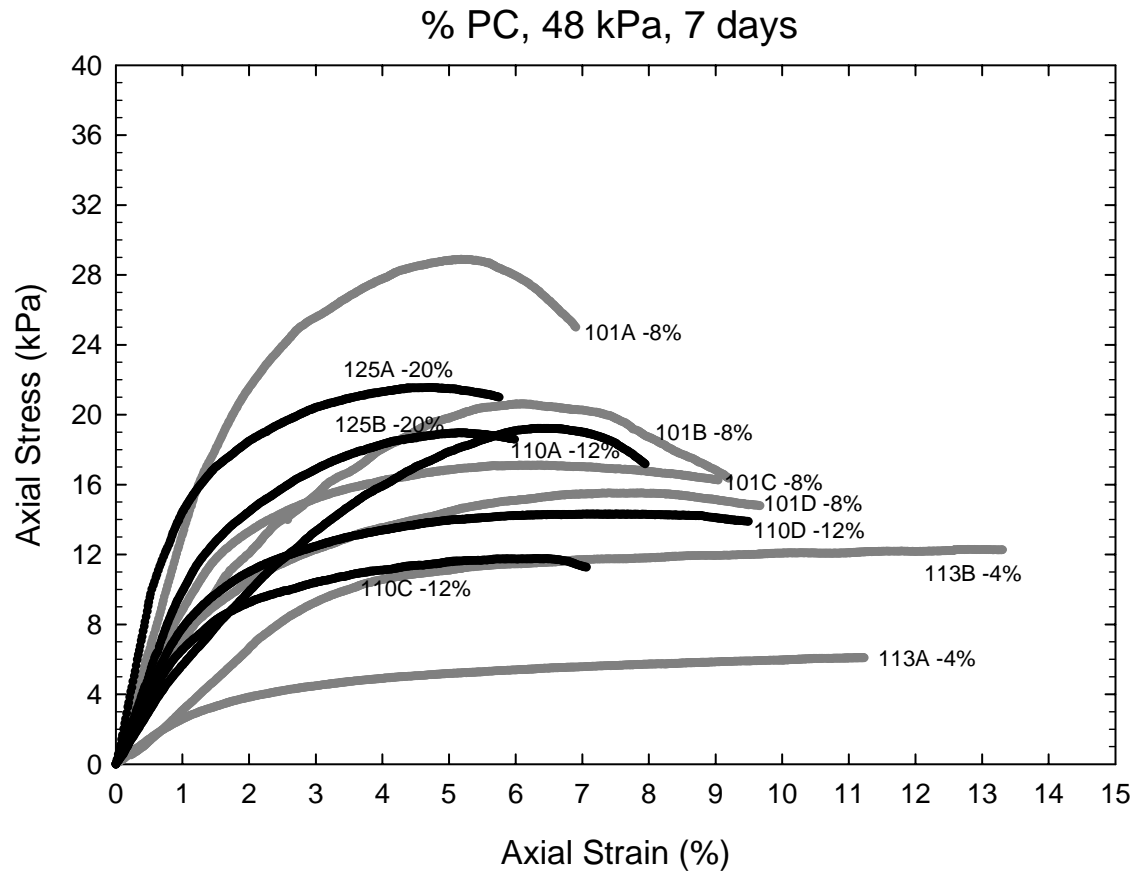

Figure 5.18: Various \% PC mixtures at 7 days 


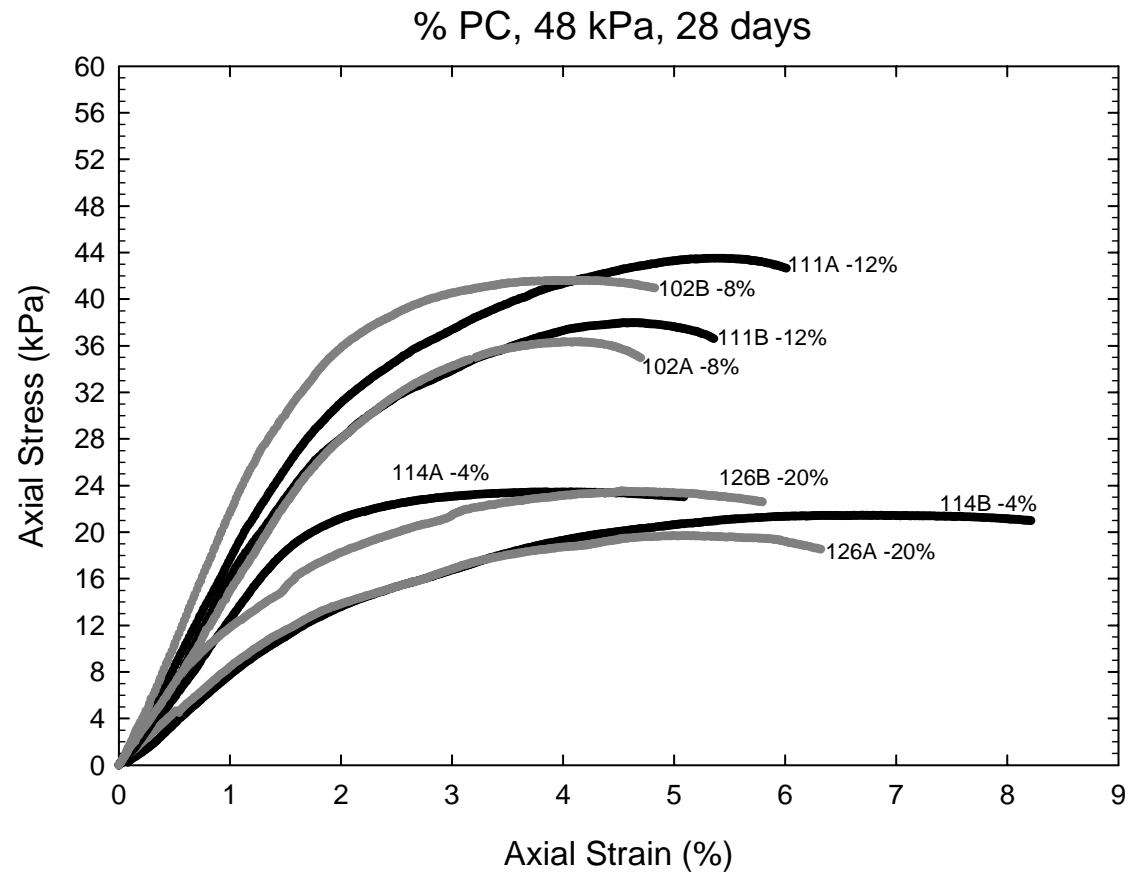

Figure 5.19: Various \% PC mixtures at 28 days

Compressive Strength vs. \% PC (48 kPa)

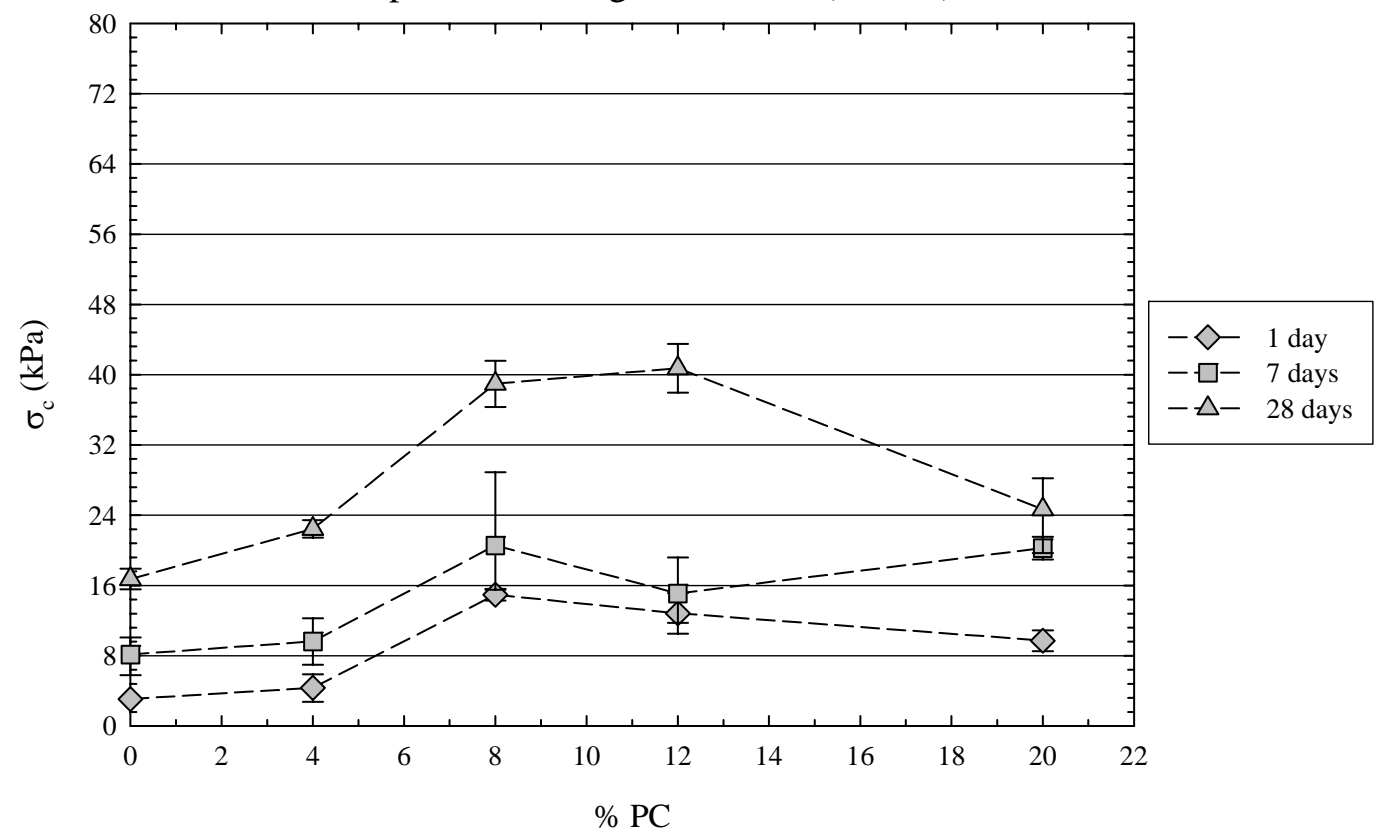

Figure 5.20: Effect of \%PC on compressive strength 
Stiffness vs. \% PC (48 kPa)

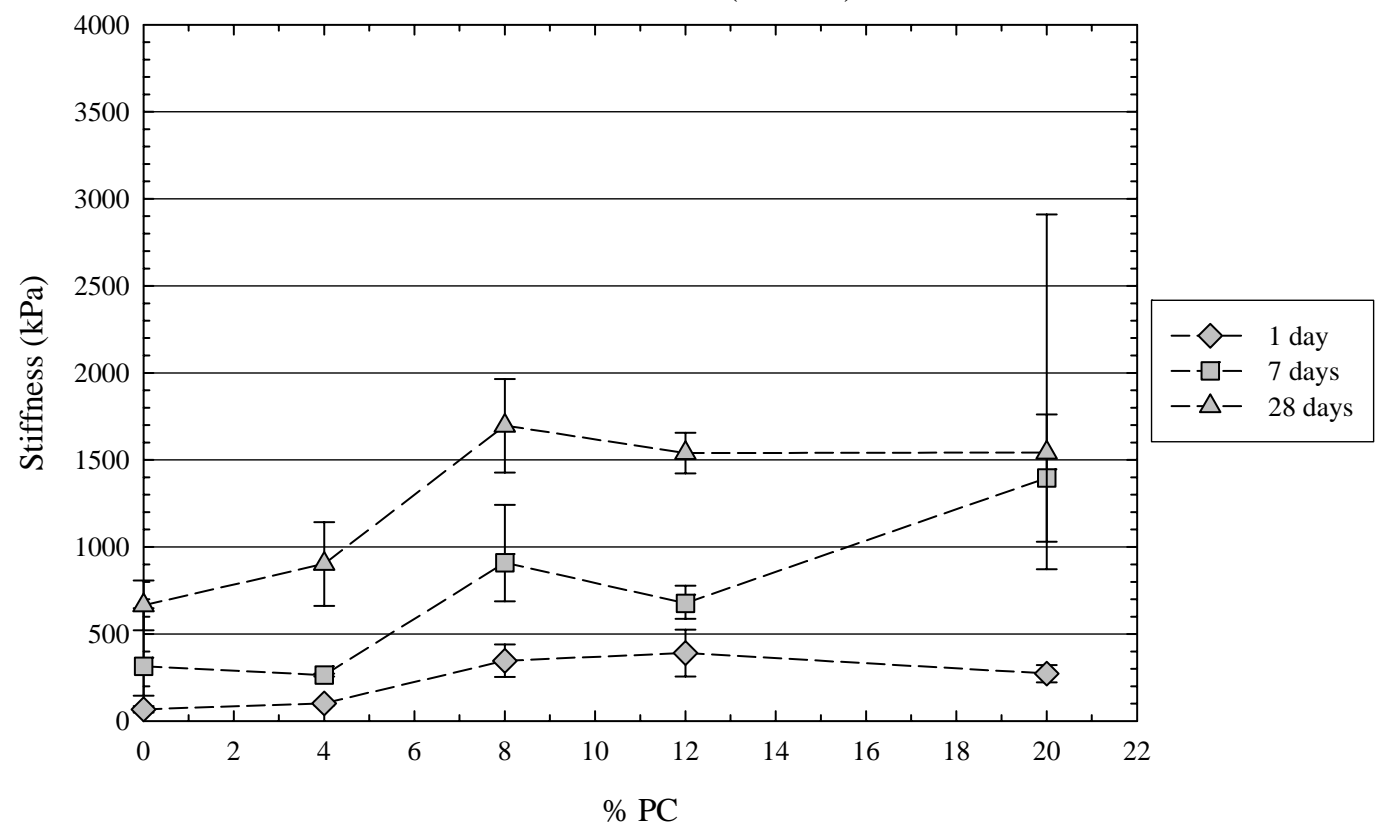

Figure 5.21: Effect of \% PC on LR soil stiffness

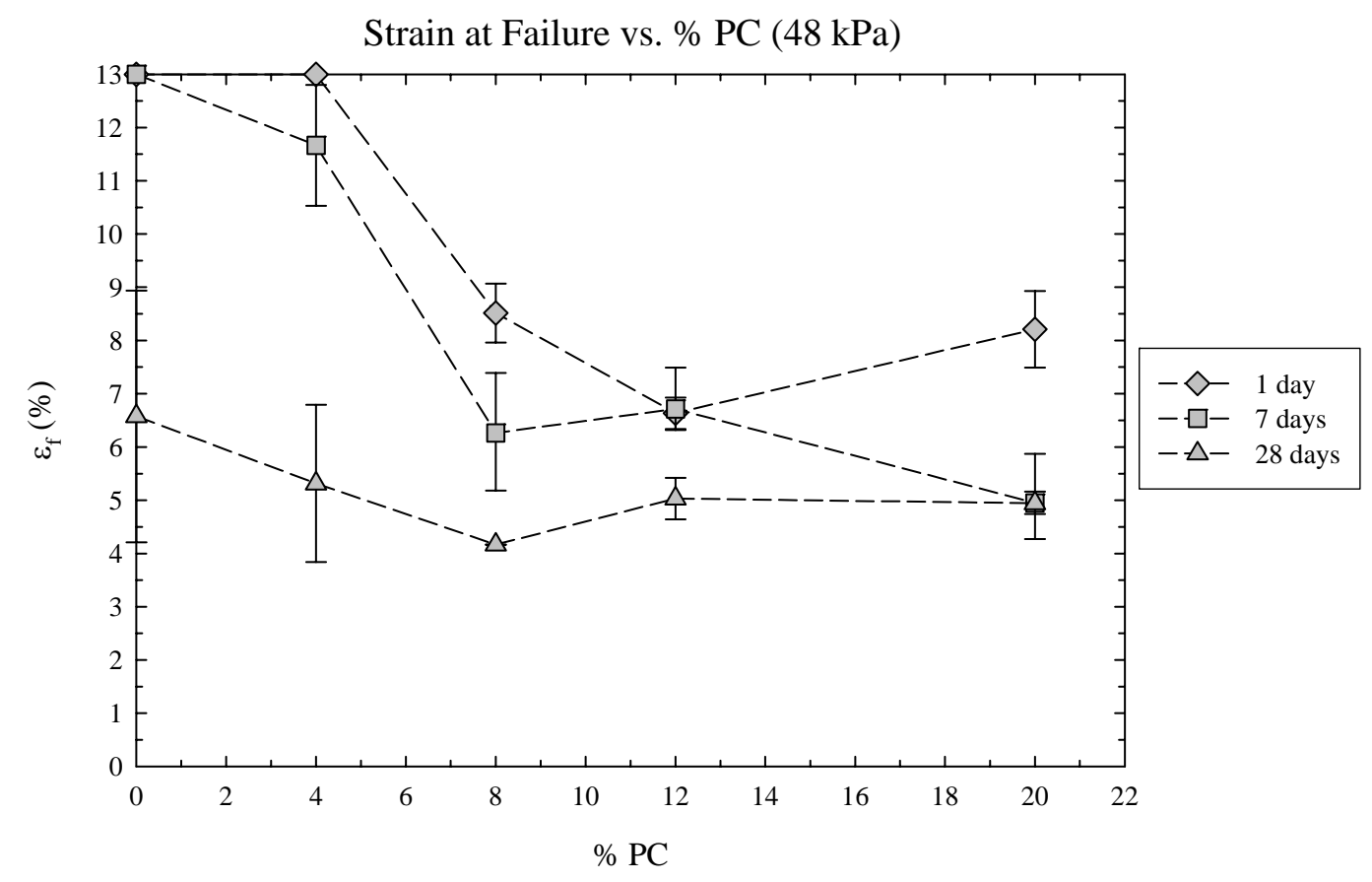

Figure 5.22: Effect of \%PC on strain at failure 


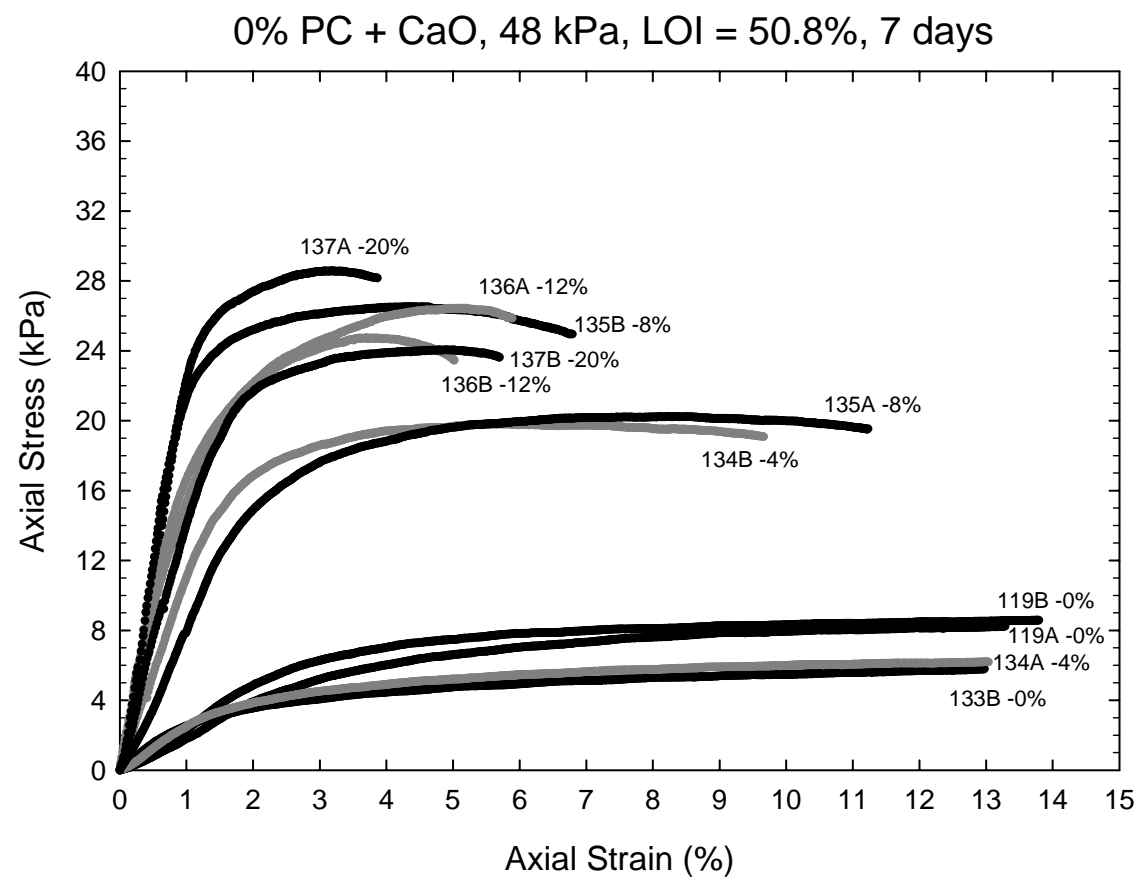

Figure 5.23: Stress-strain plot from UC tests with $\mathrm{CaO}$ mixture surcharged at $48 \mathrm{kPa}$

Compressive Strength vs. \% CaO (48 kPa, 7d)

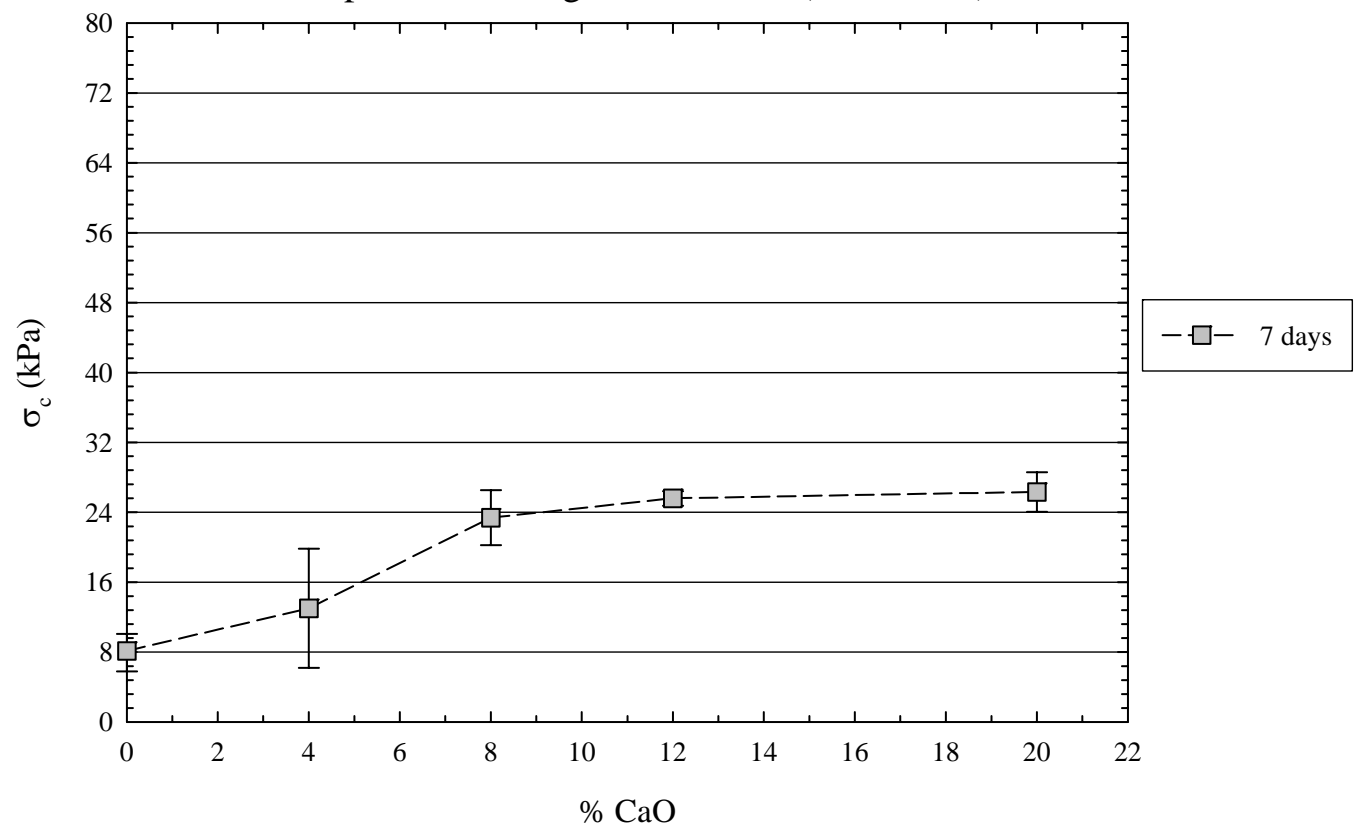

Figure 5.24: Effect of $\mathrm{CaO}$ on strength of LR soil surcharged at $48 \mathrm{kPa}$ 
Stiffness vs. \% CaO (48 kPa, 7d)

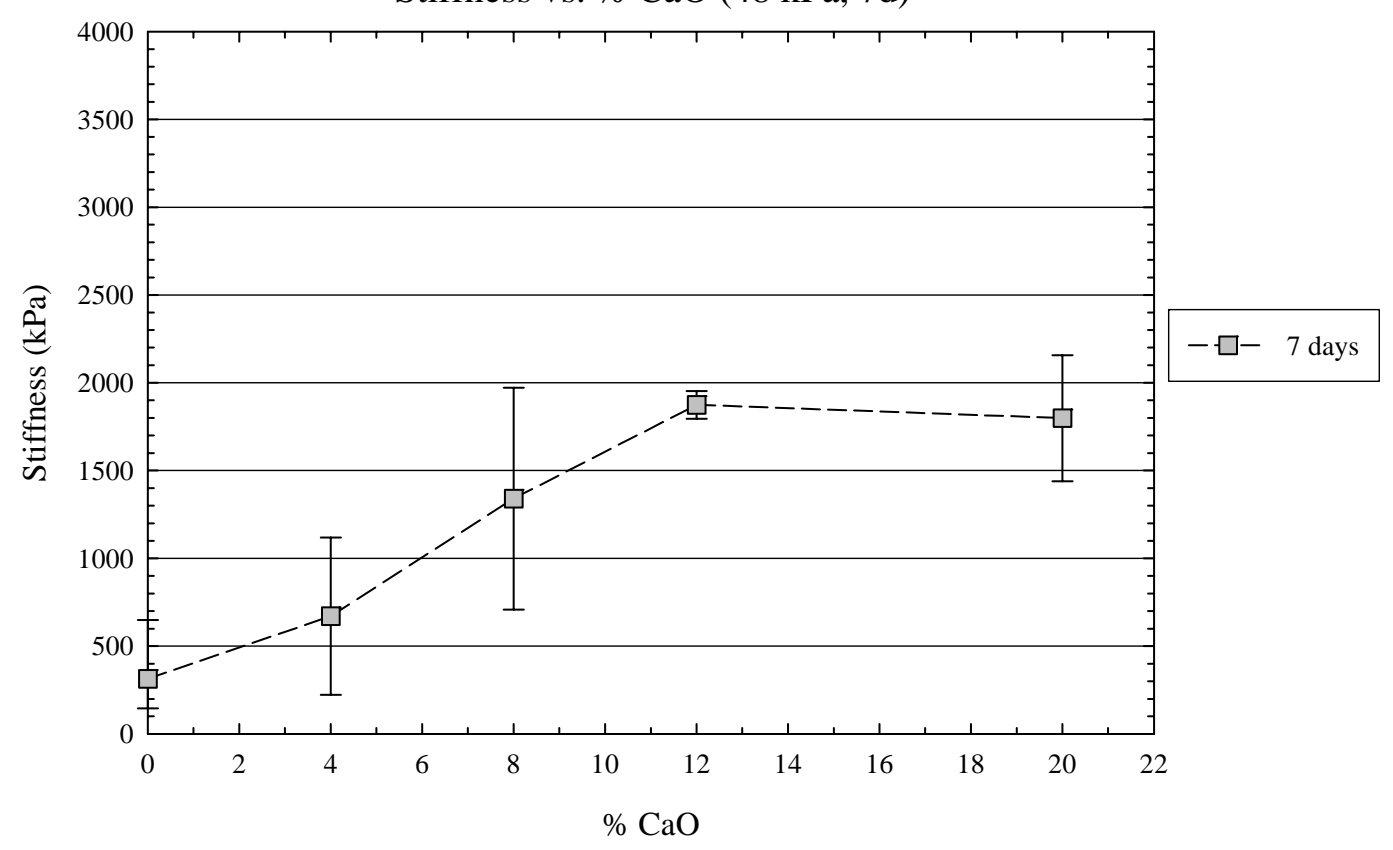

Figure 5.25: Effect of $\mathrm{CaO}$ on stiffness of LR soil surcharged at $48 \mathrm{kPa}$

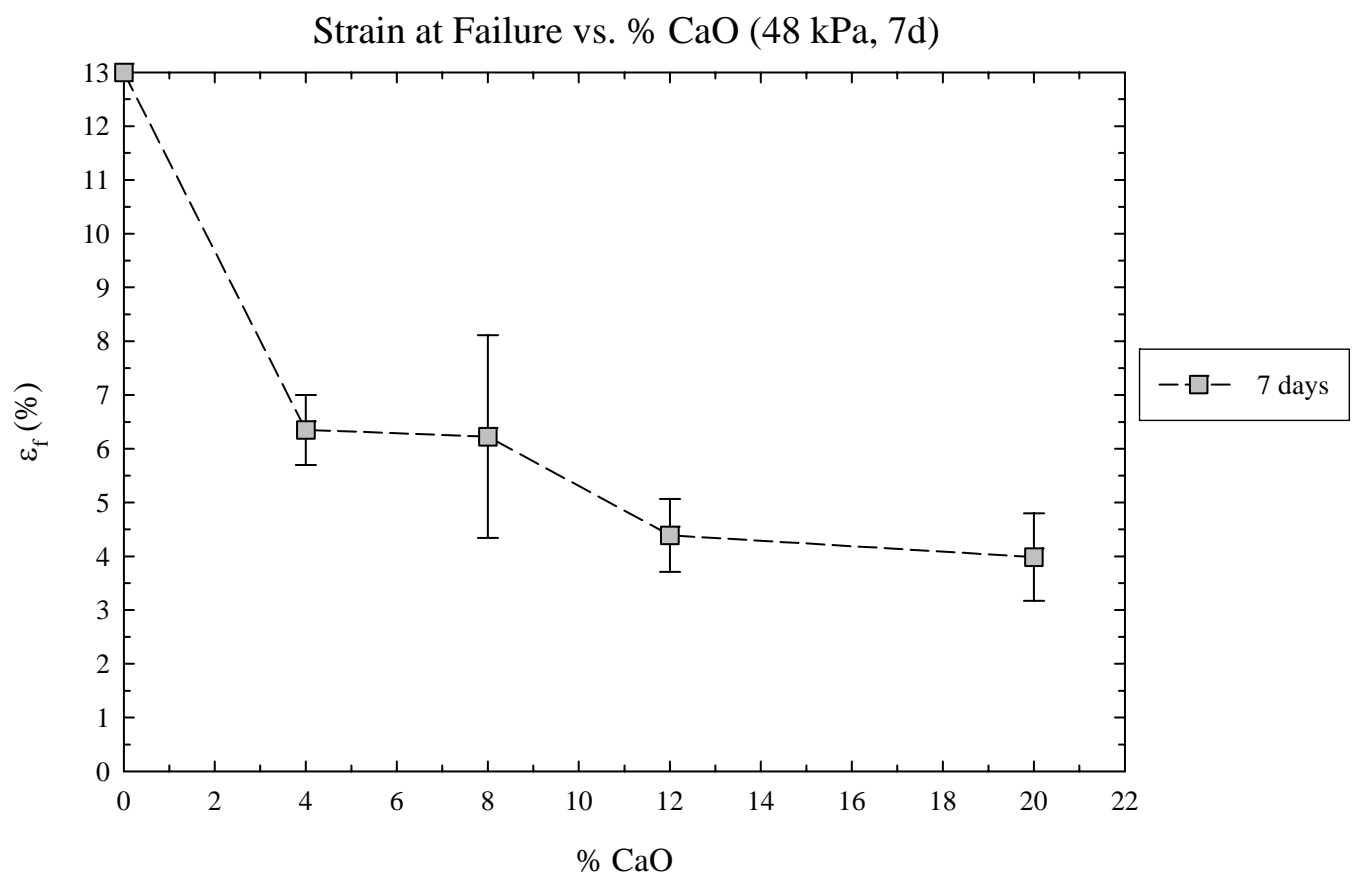

Figure 5.26: Effect of $\mathrm{CaO}$ on stiffness of LR soil surcharged at $48 \mathrm{kPa}$ 


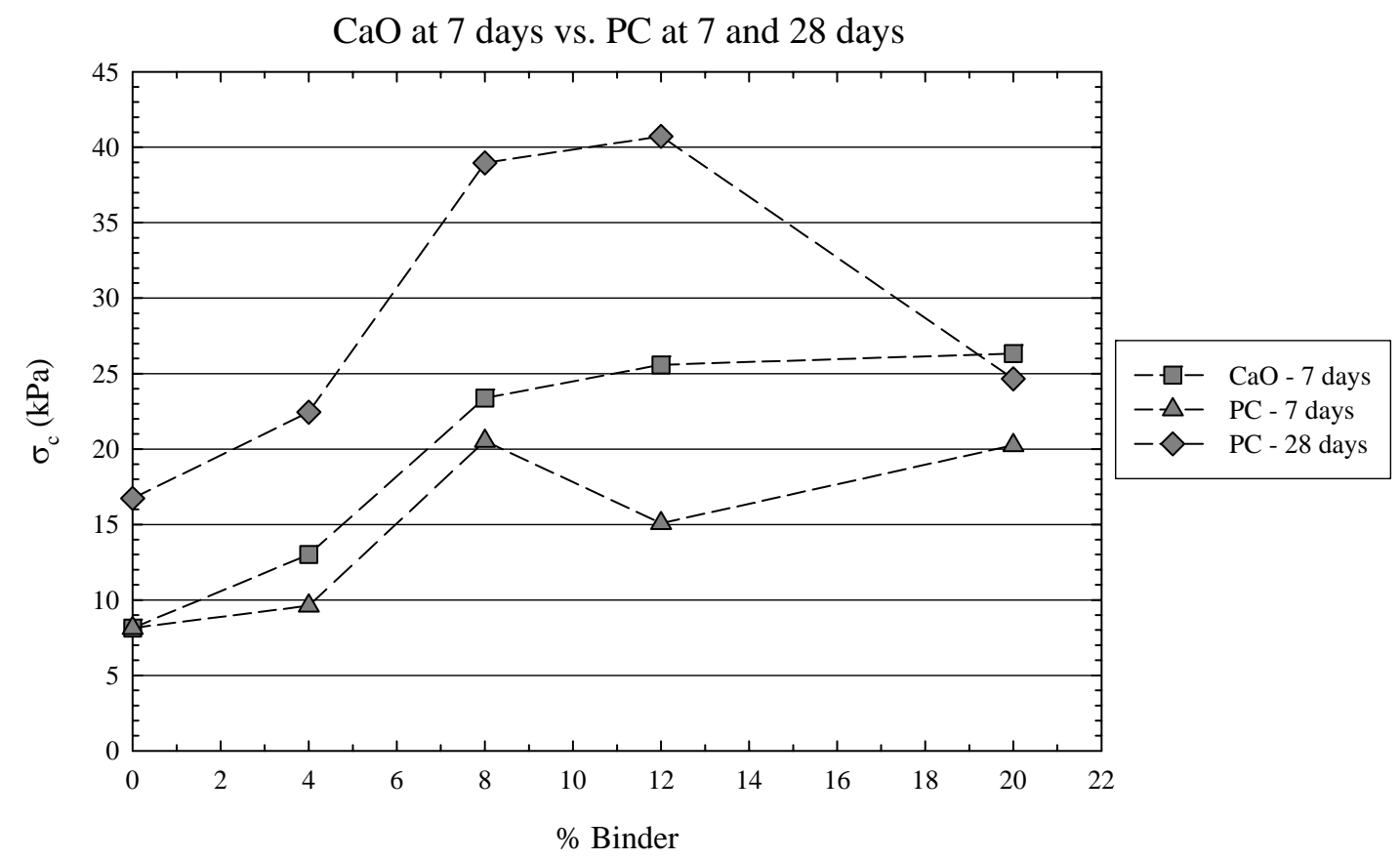

Figure 5.27: Comparison of $\mathrm{CaO}$ at 7 days against $\mathrm{PC}$ at 7 and 28 days

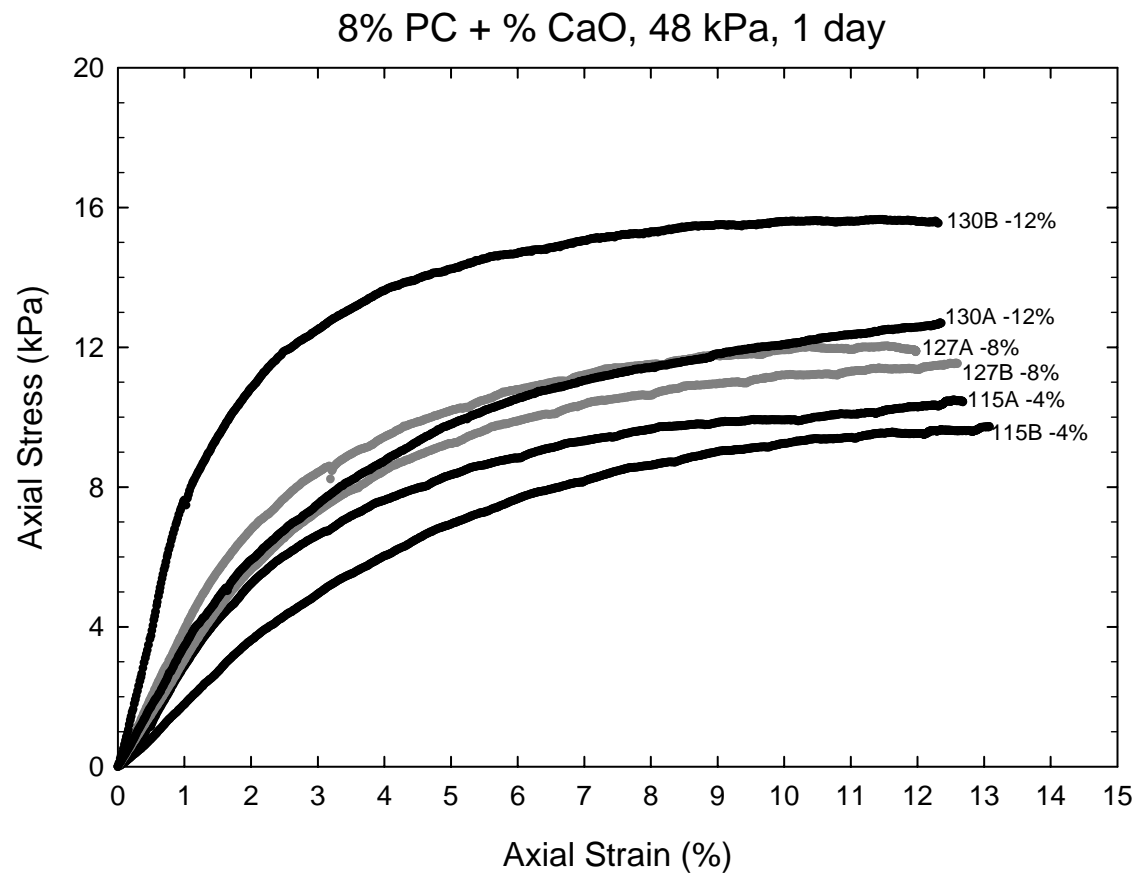

Figure 5.28: 8\% $\mathrm{PC}+\mathrm{CaO}$ at 1 day 


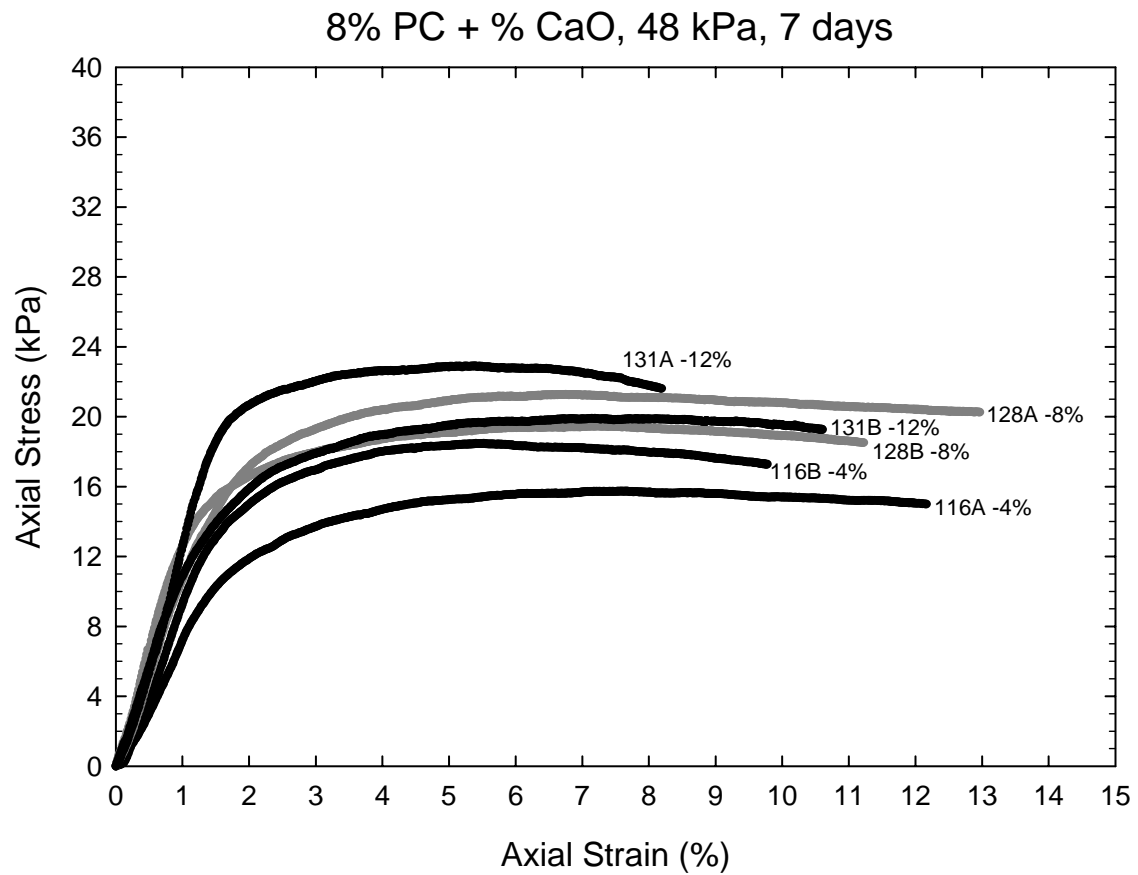

Figure 5.29: 8\% $\mathrm{PC}+\mathrm{CaO}$ at 7 days

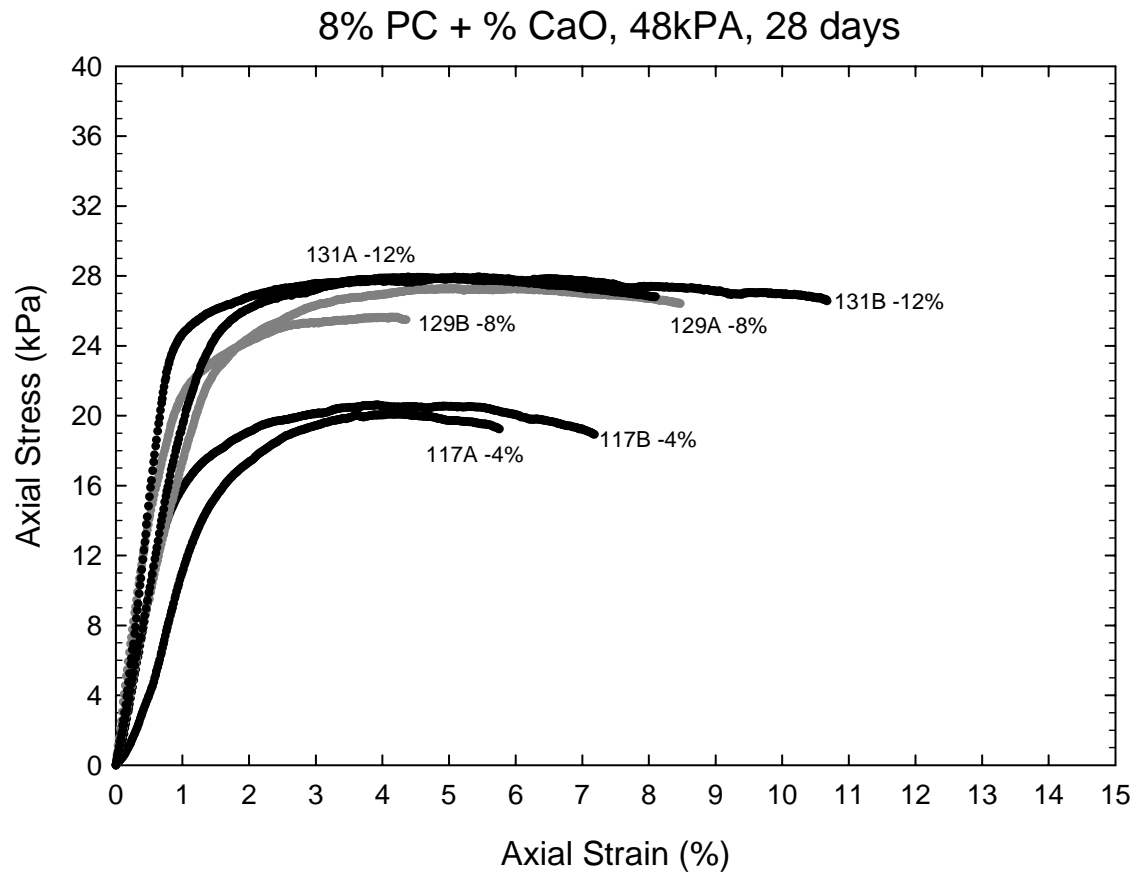

Figure 5.30: 8\% $\mathrm{PC}+\mathrm{CaO}$ at 28 days 


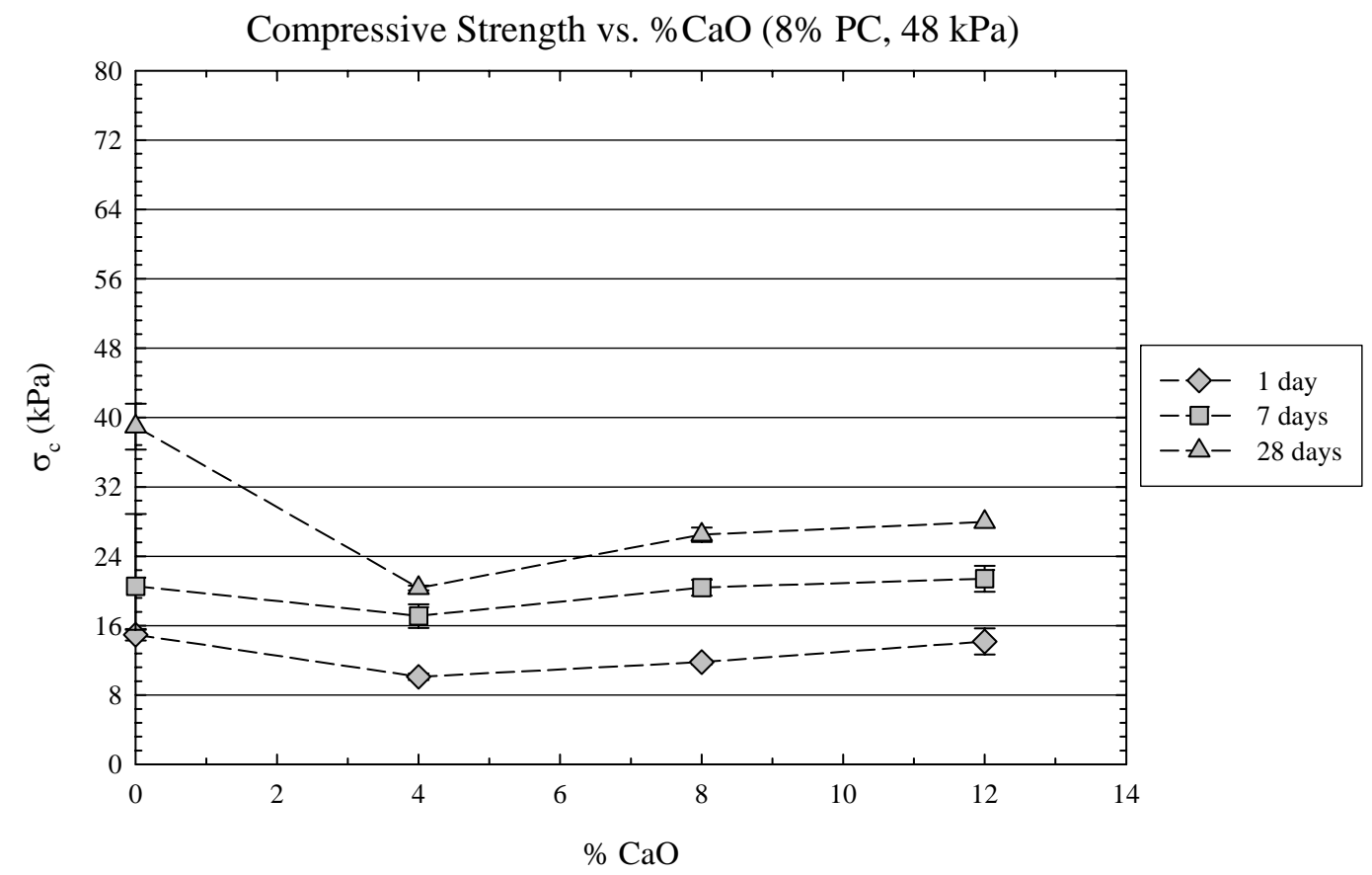

Figure 5.31: Effect of $\mathrm{CaO}$ on compressive strength of cement treated LR soil

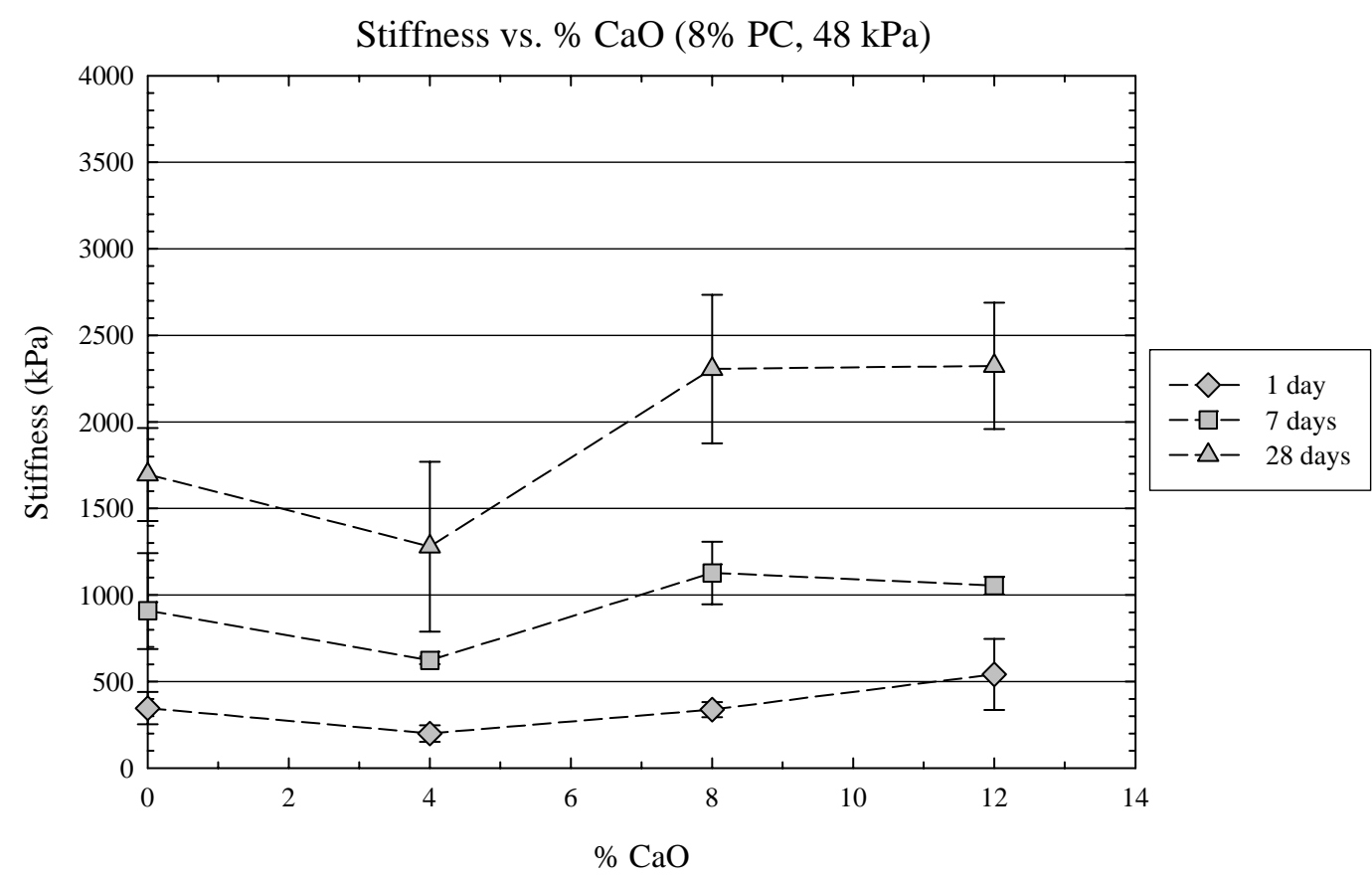

Figure 5.32: Effect of $\mathrm{CaO}$ on stiffness of cement treated LR soil 
Strain at Failure vs. \% CaO (8\% PC, $48 \mathrm{kPa})$

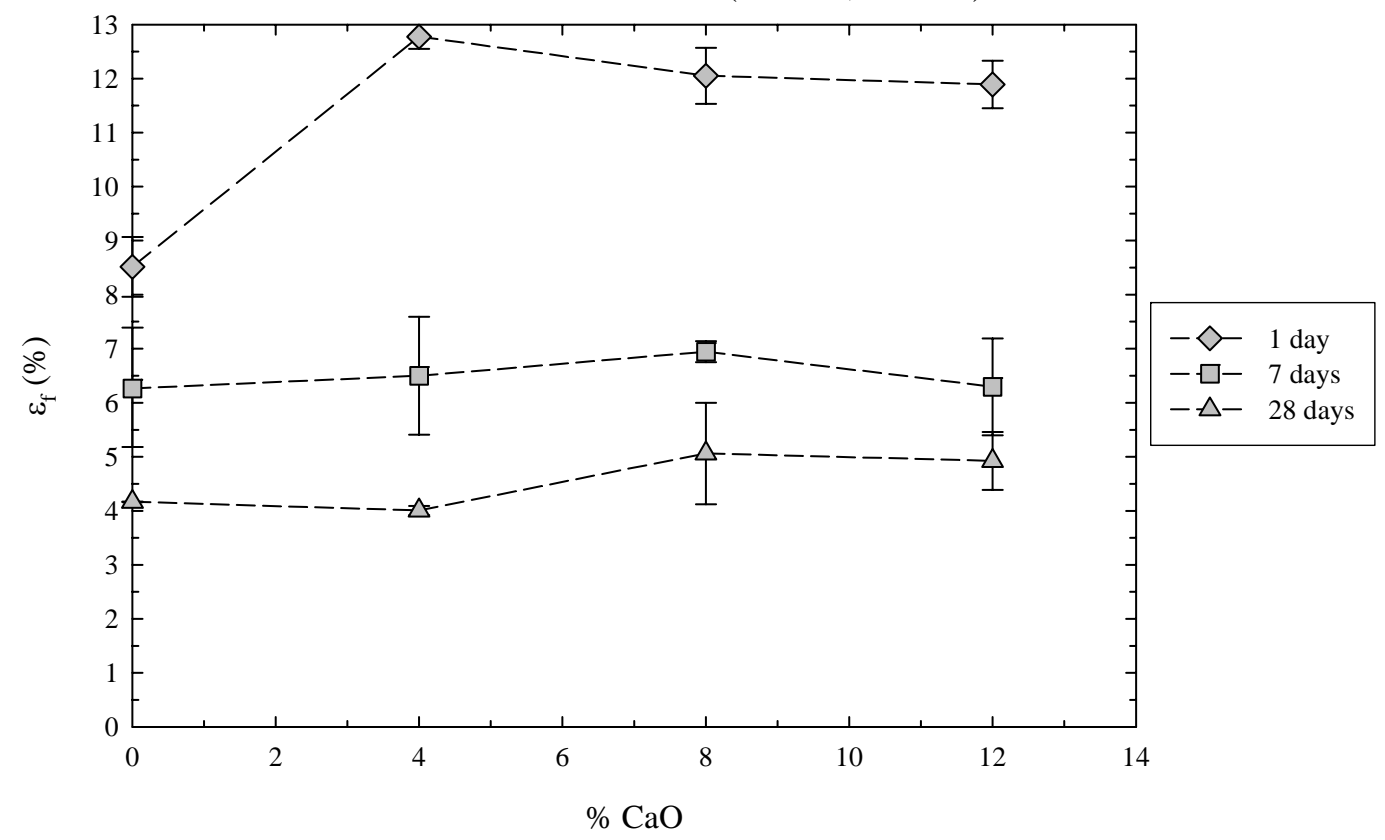

Figure 5.33: Effect of $\mathrm{CaO}$ on failure strain of cement treated LR soil

\% HC, $48 \mathrm{kPa}, 7$ daye

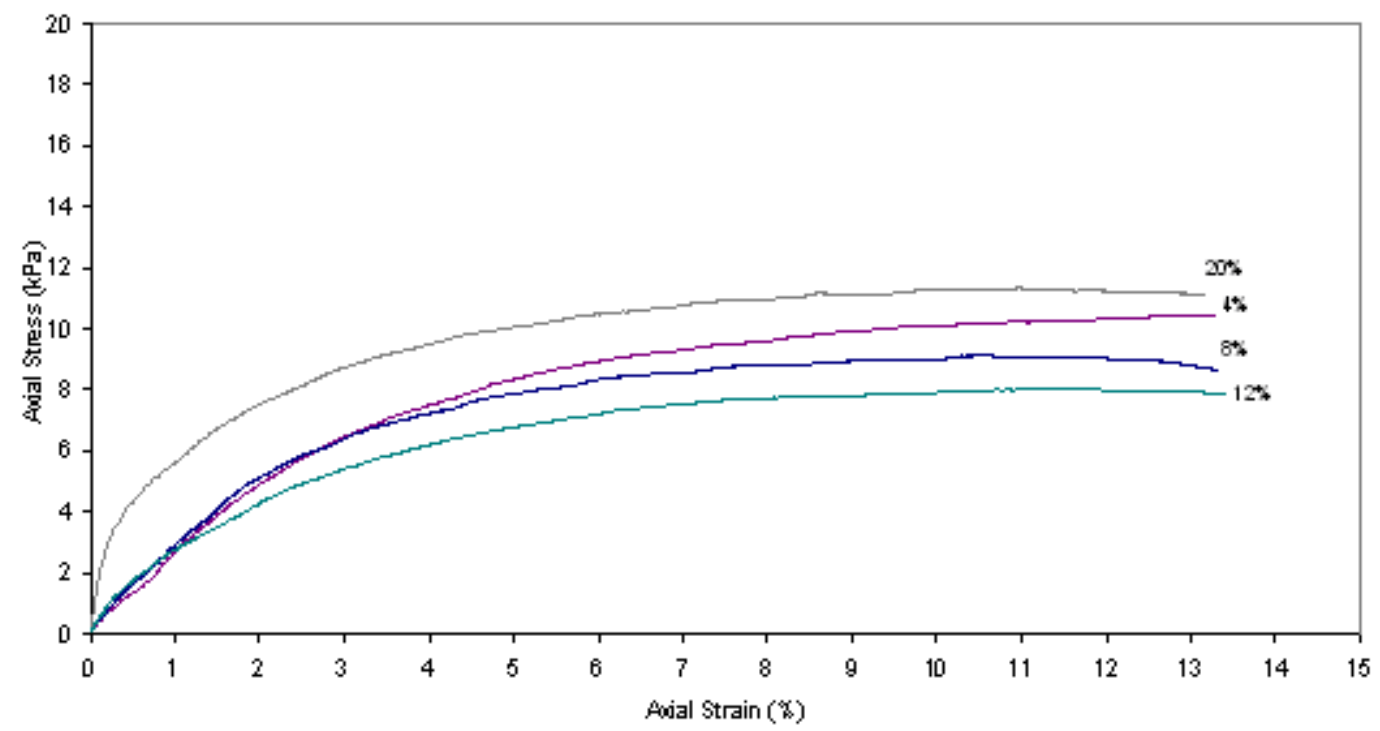

Figure 5.34: Stress-strain plot from UC tests on HC treated LR soil 


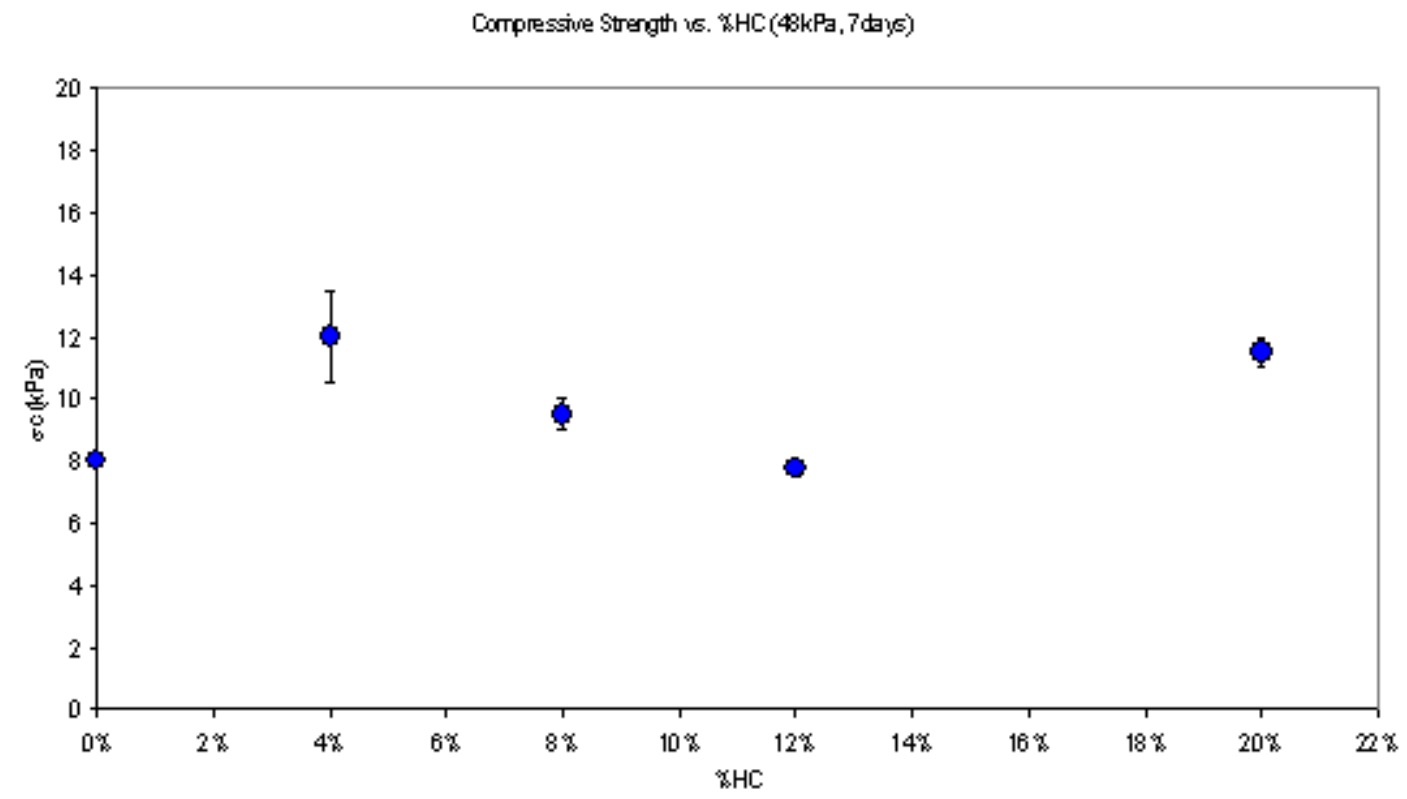

Figure 5.35: Effect of \%HC on compressive strength

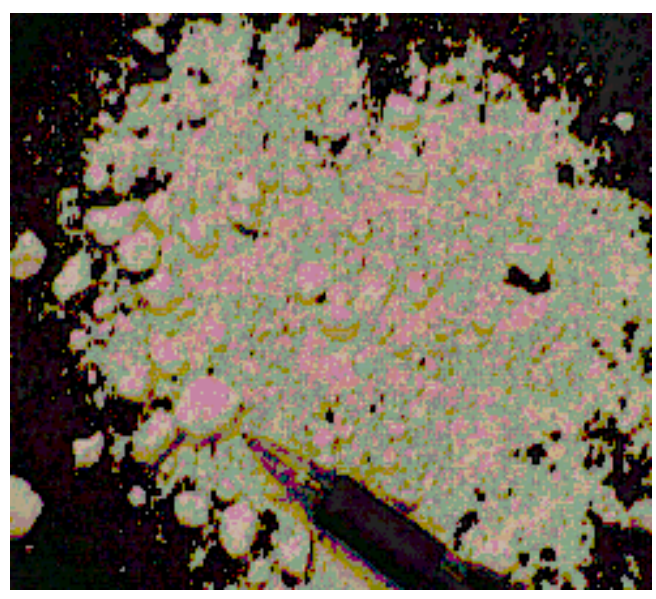

Figure 5.36: Partially hydrated High Calcium Flue Dust employed in the UC tests 


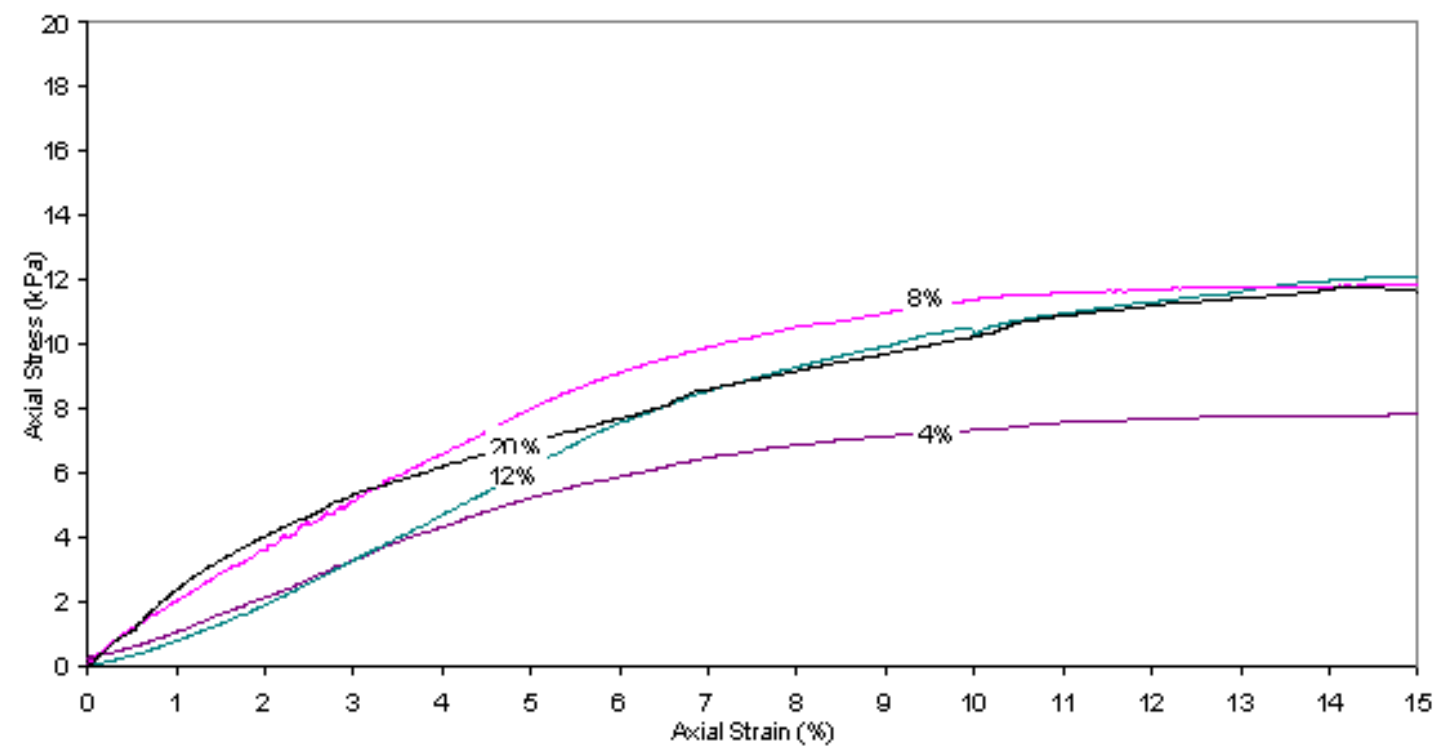

Figure 5.37: Stress-strain plot from UC tests on MB treated LR soil

Compressive Strength ws. \$MB (48kPa, 7days)

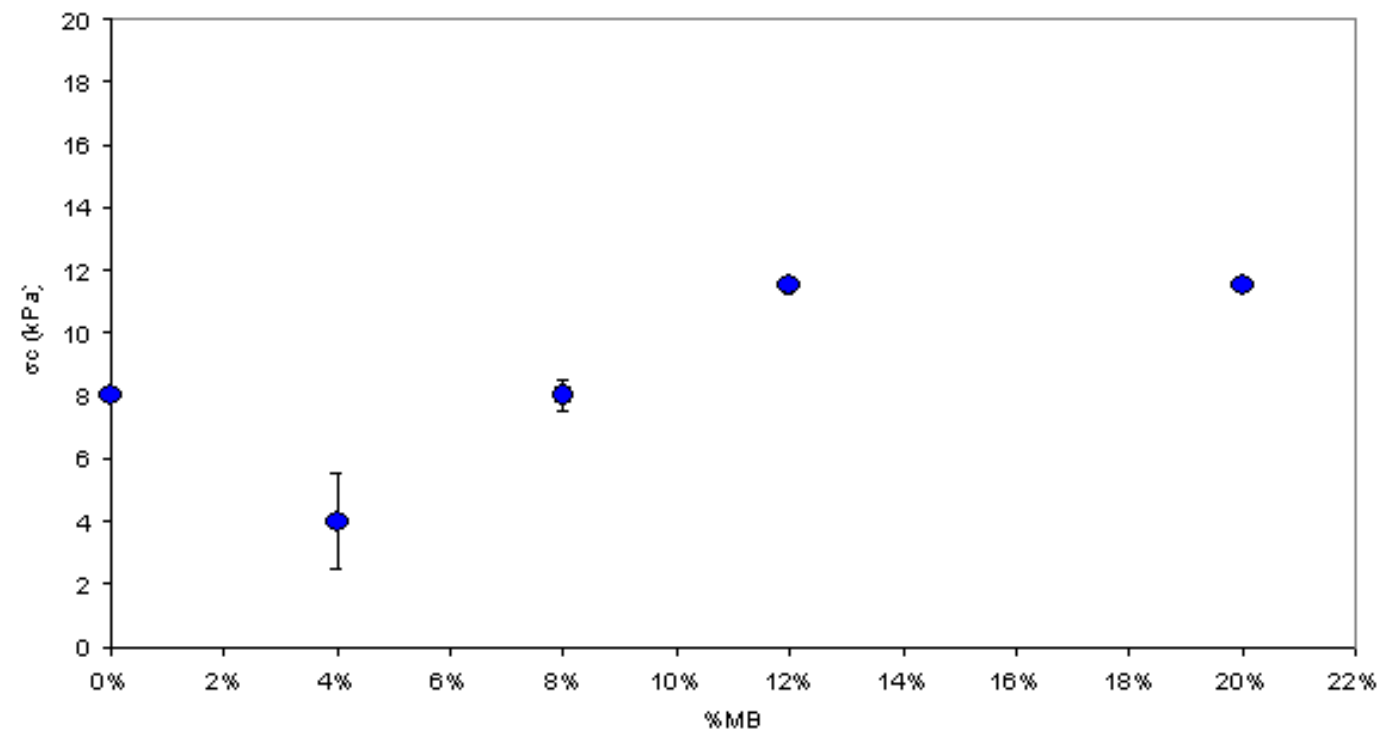

Figure 5.38: Effect of \%MB on compressive strength 
Compressive Strength vs. \%CaO (48 kPa, 7d)

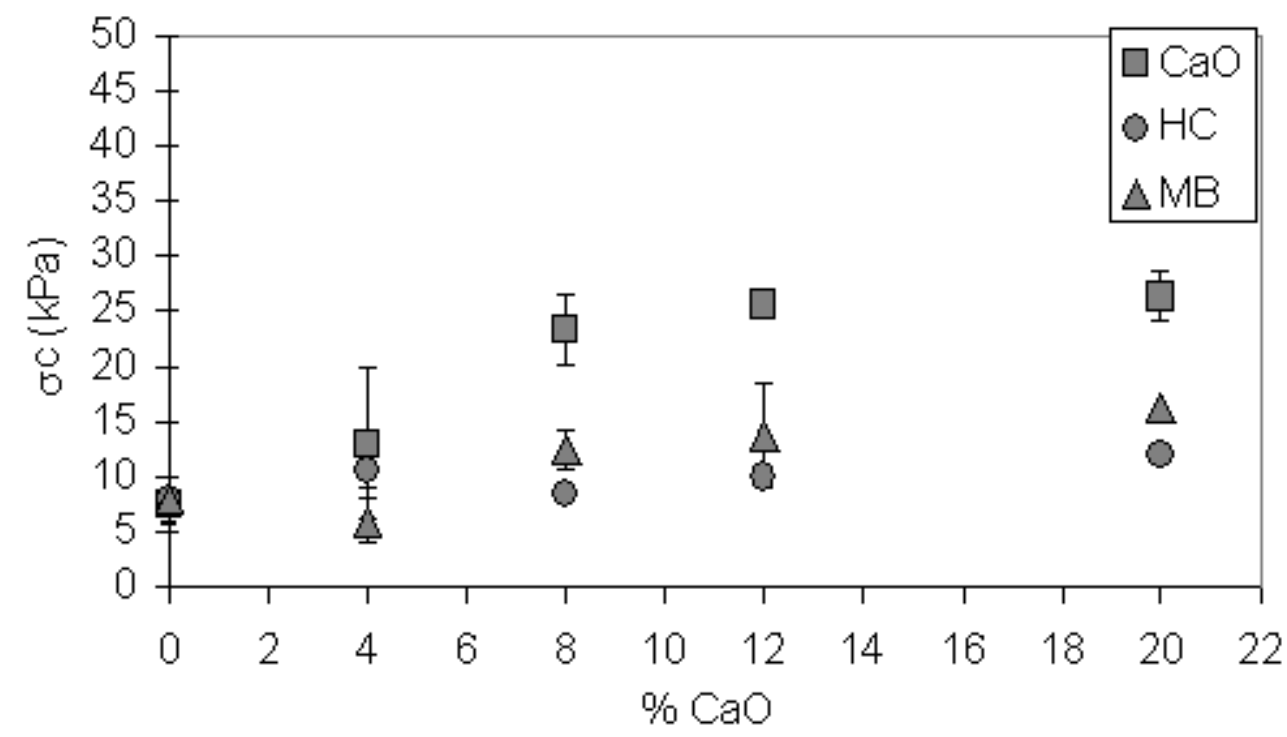

Figure 5.39: Comparison of effects of $\mathrm{CaO}, \mathrm{HC}$ and $\mathrm{MB}$ on compressive strength

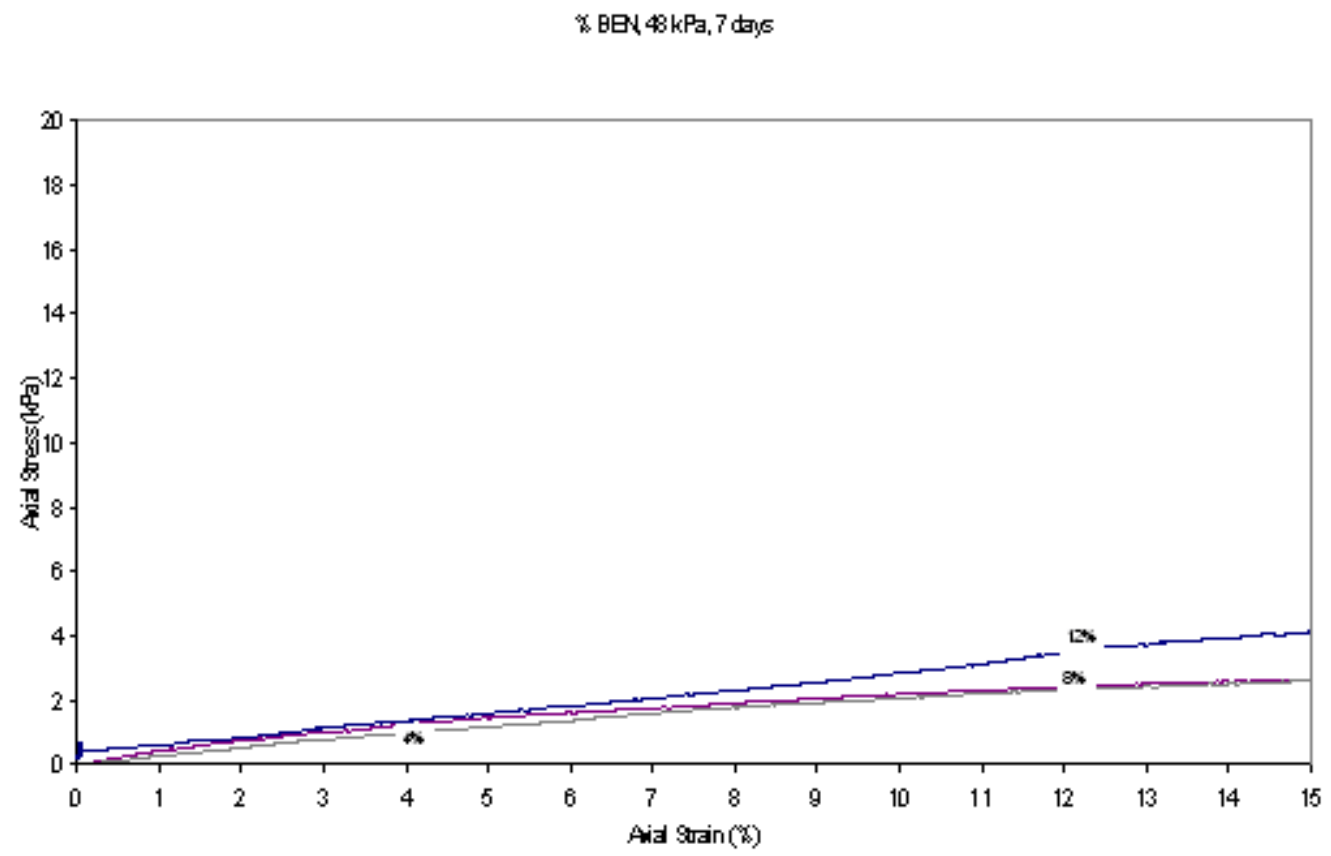

Figure 5.40: Stress-strain plot from UC tests on bentonite treated LR soil 
Compresstue steigth vs. * BEN (48KPa,7 days)

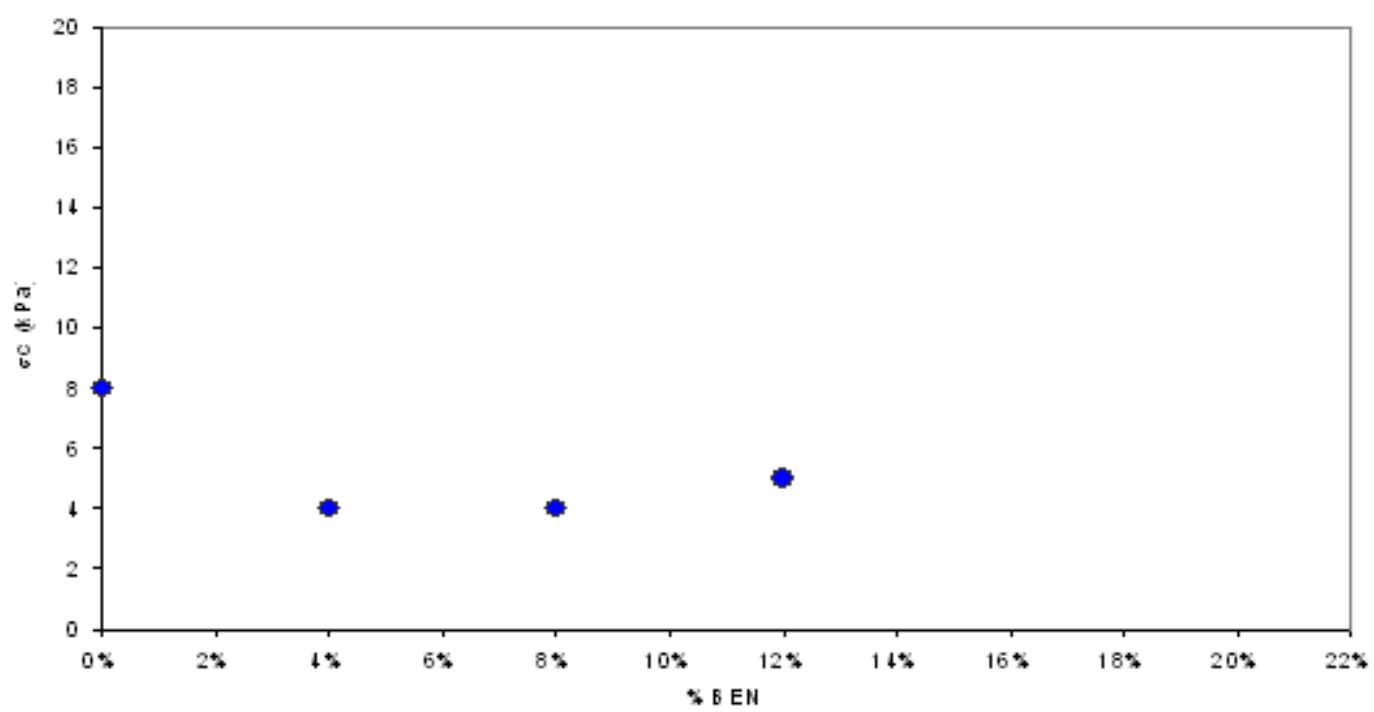

Figure 5.41: Effect of \%BEN on compressive strength

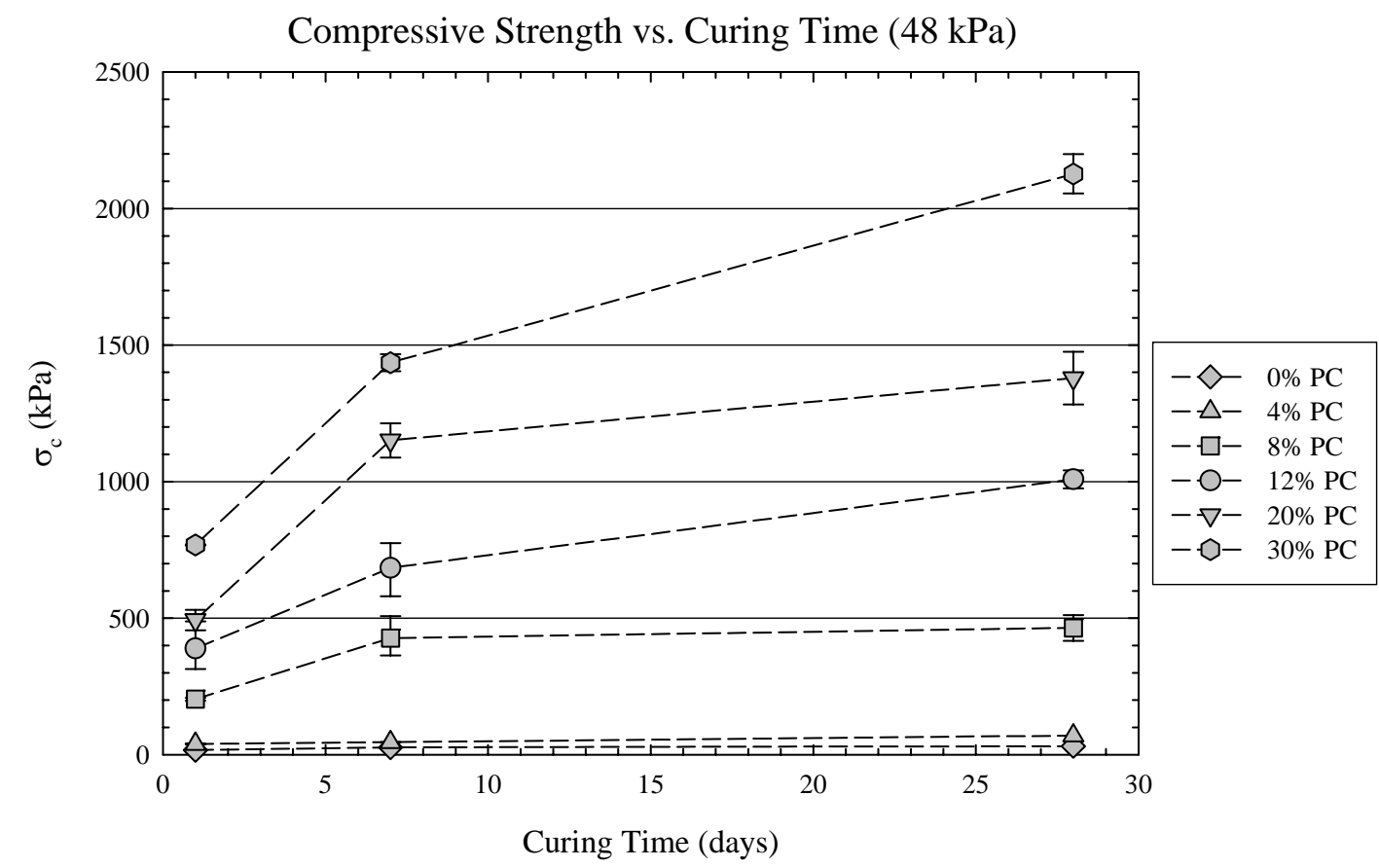

Figure 5.42:Effects of curing time on the compressive strength of PC treated Low organic soil (Mixture I) 


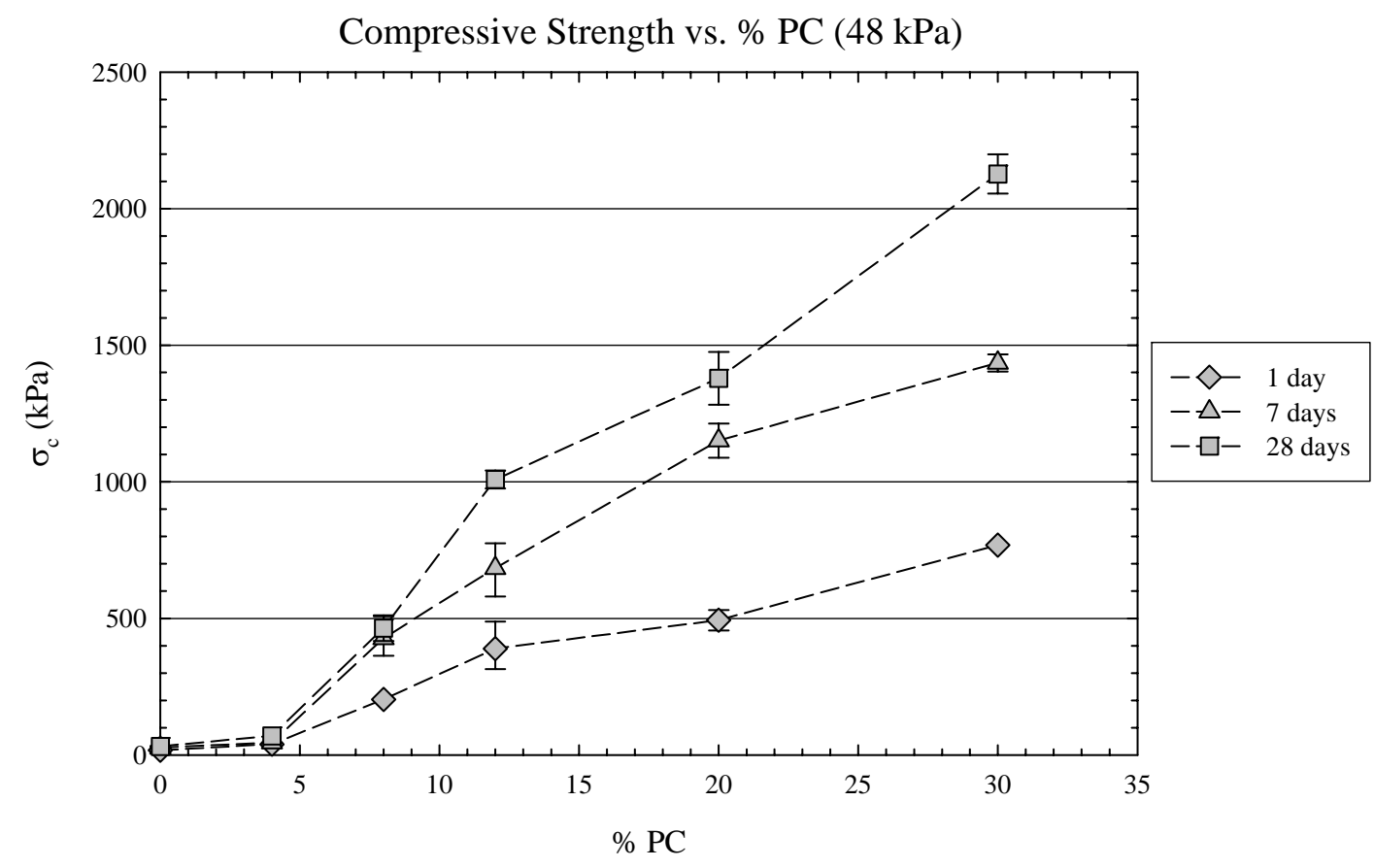

Figure 5.43: Effects of \% PC on the compressive strength of Low organic soil (Mixture I)

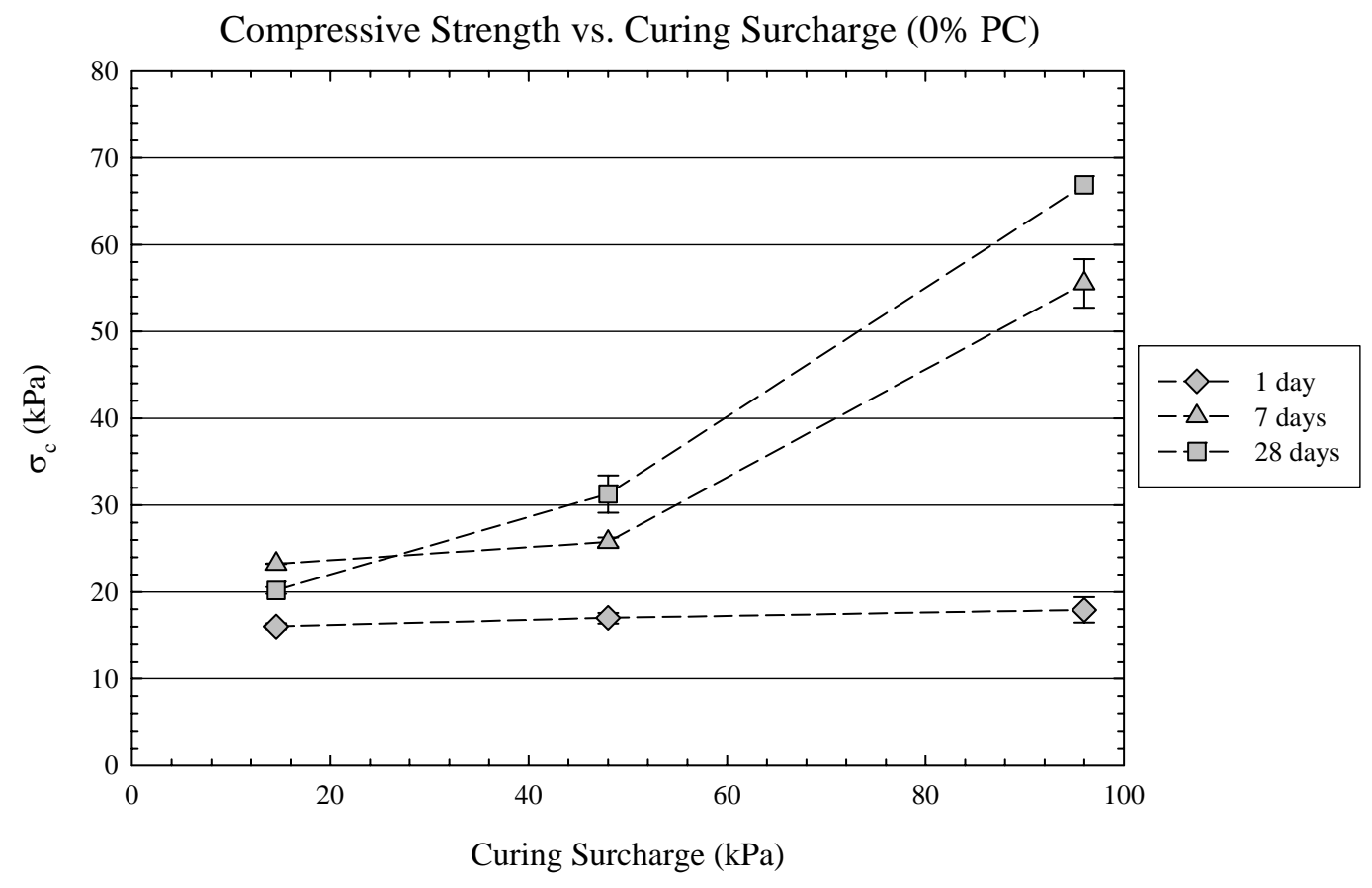

Figure 5.44: Effect of curing surcharge on compressive strength of untreated Mixture I 


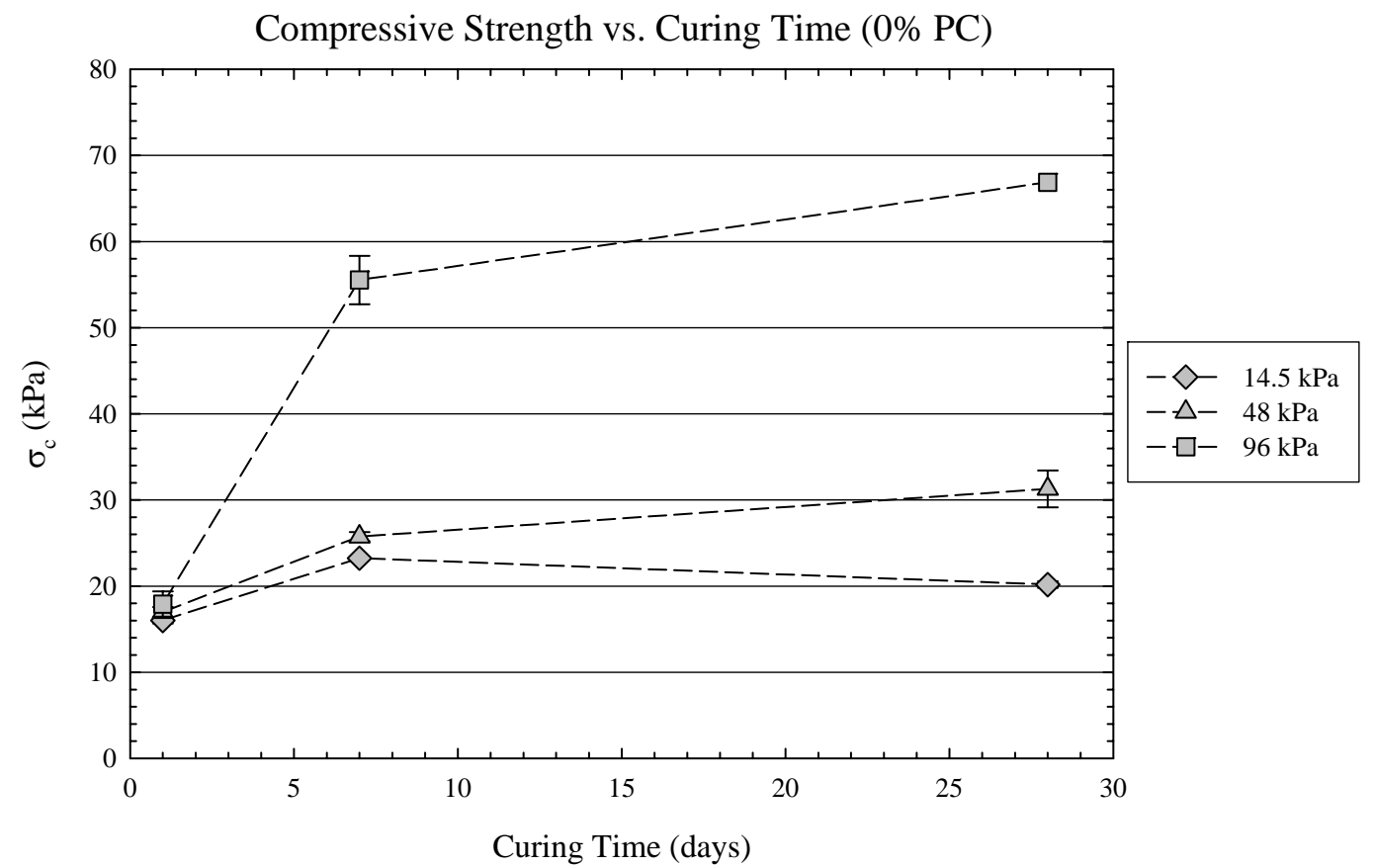

Figure 5.45: Effect of curing time on compressive strength of untreated Mixture I

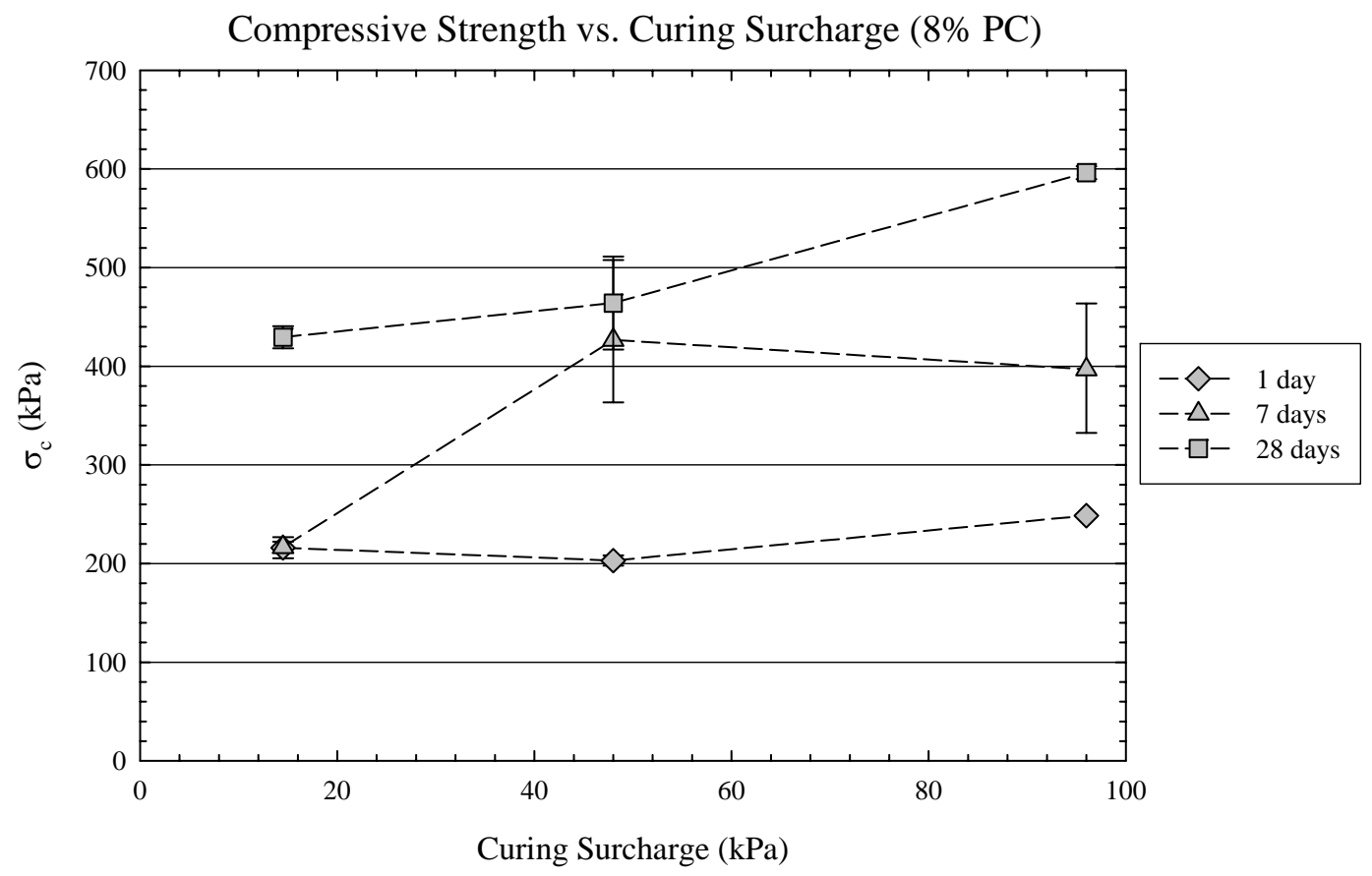

Figure 5.46: Effect of curing surcharge on compressive strength of $8 \%$ PC treated Mixture I 


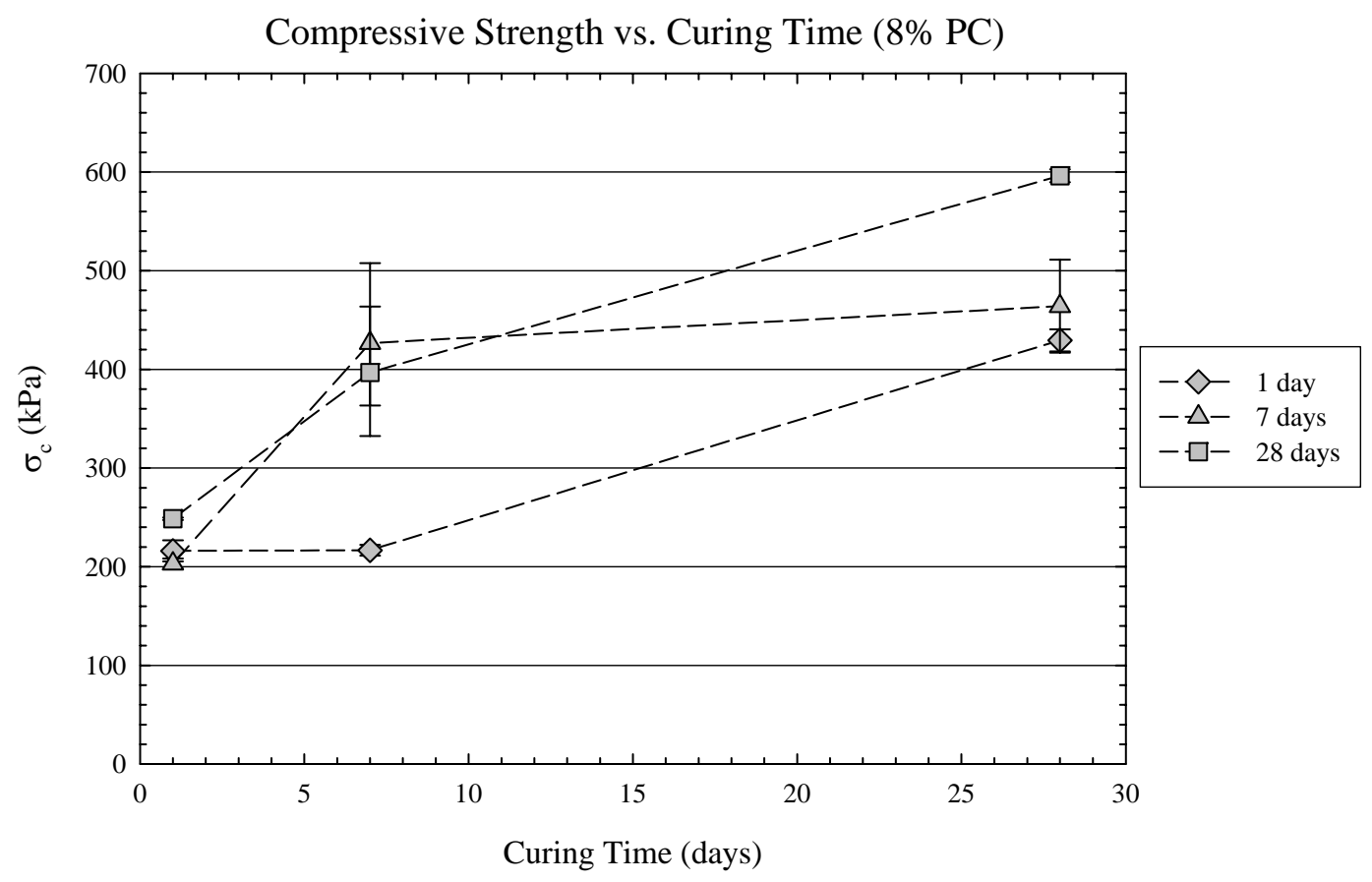

Figure 5.47: Effect of curing time on compressive strength of 8\% PC treated Mixture I

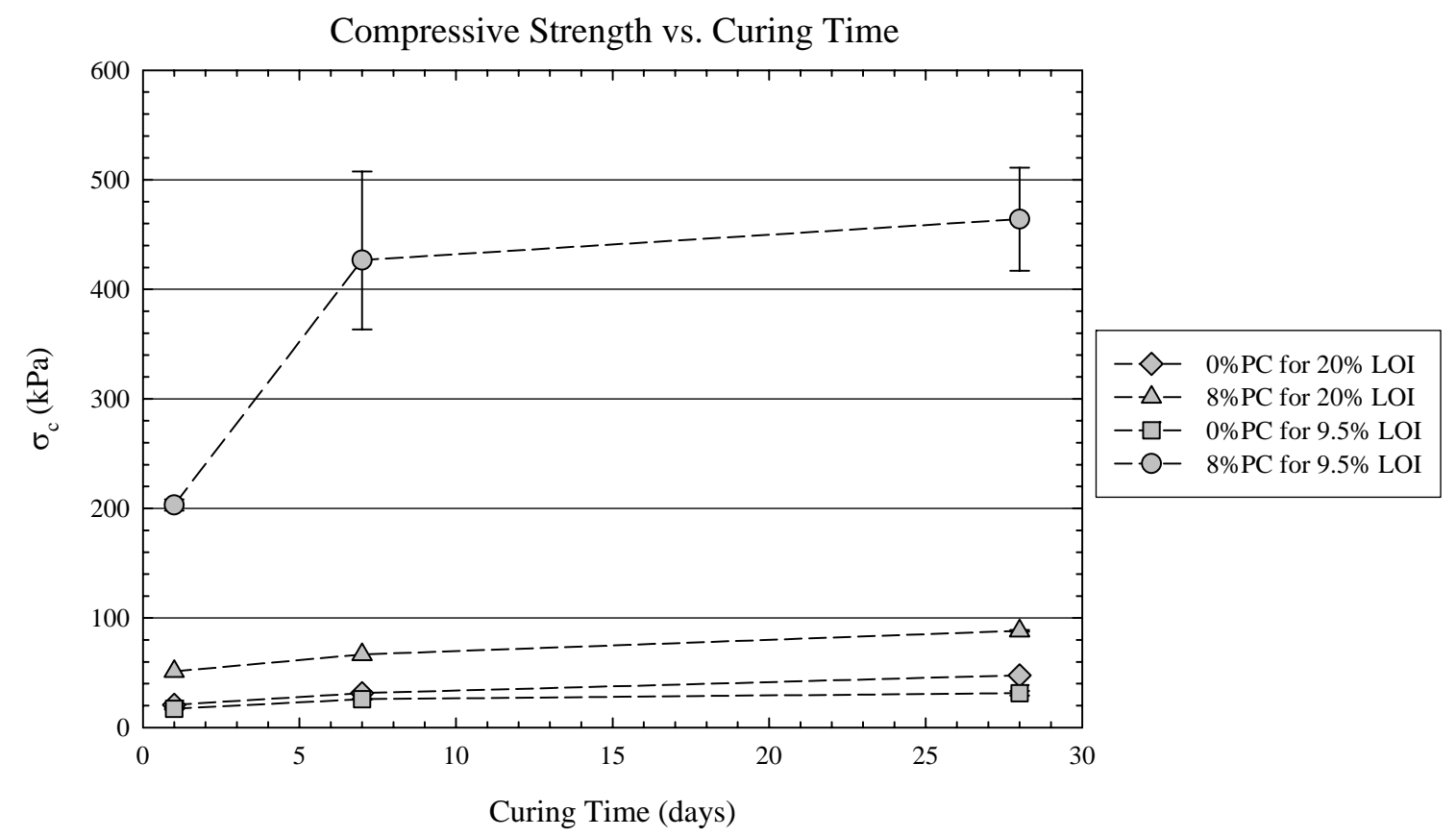

Figure 5.48: Effect of curing time on compressive strength of untreated and 8\% treated Mixture I and II 


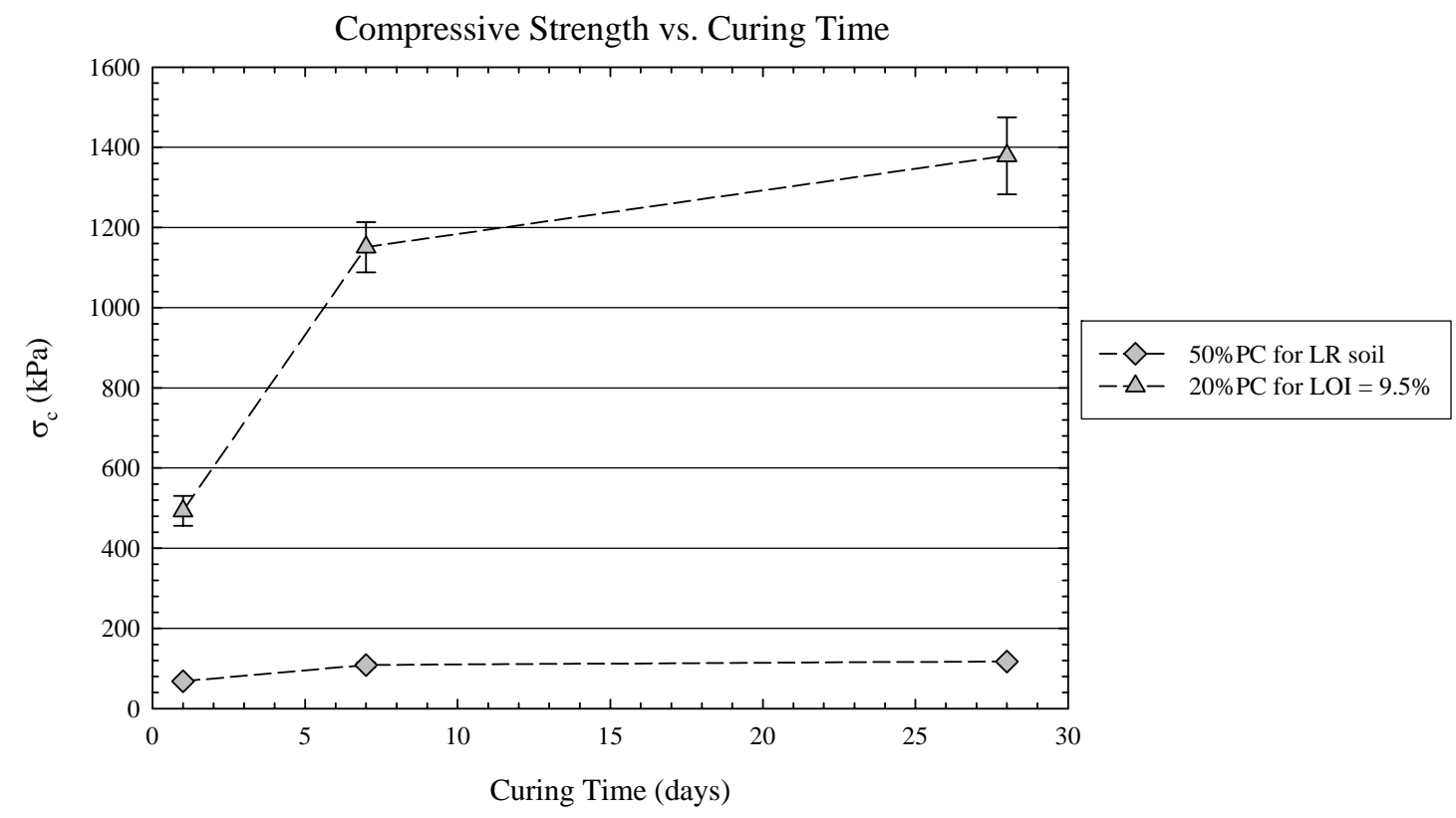

Figure 5.49: Comparison of compressive strength of 50\% PC LR soil and 20\% PC Mixture II

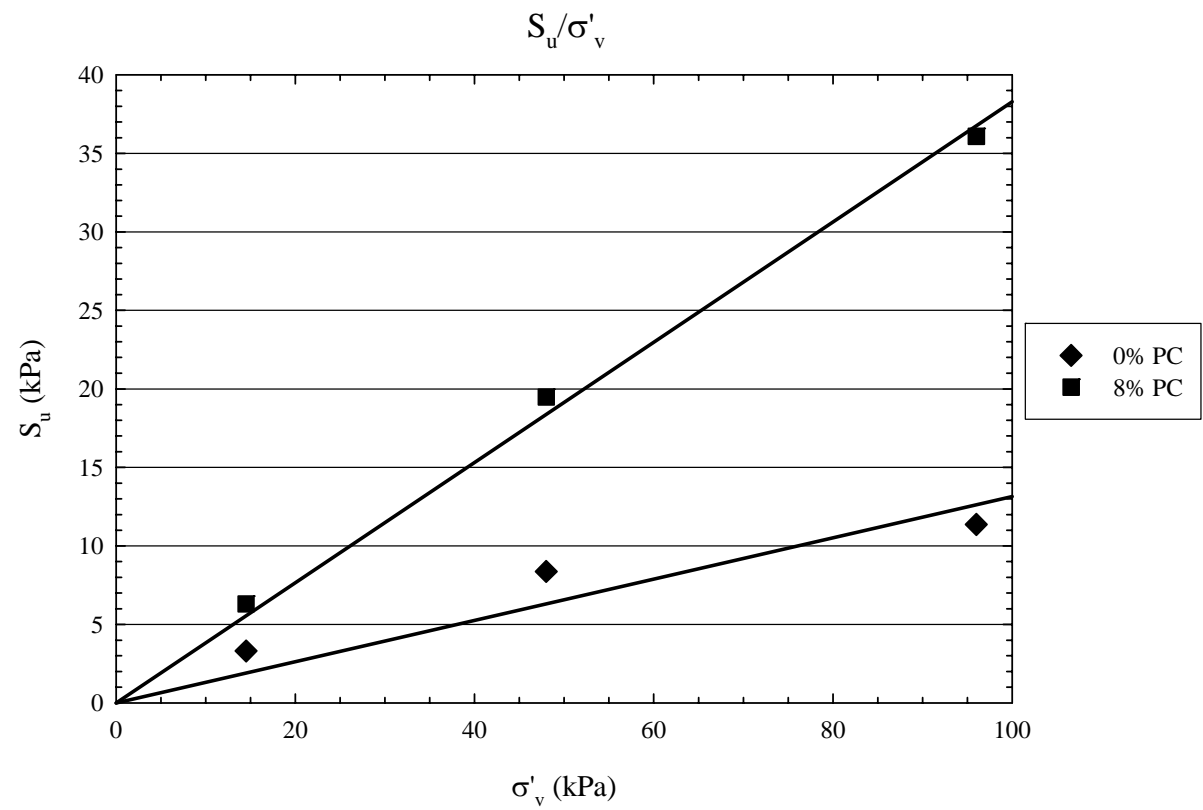

Figure 5.50: Comparison of normalized undrained shear strengths for $8 \%$ PC and 0\% PC LR soil at 28 days 


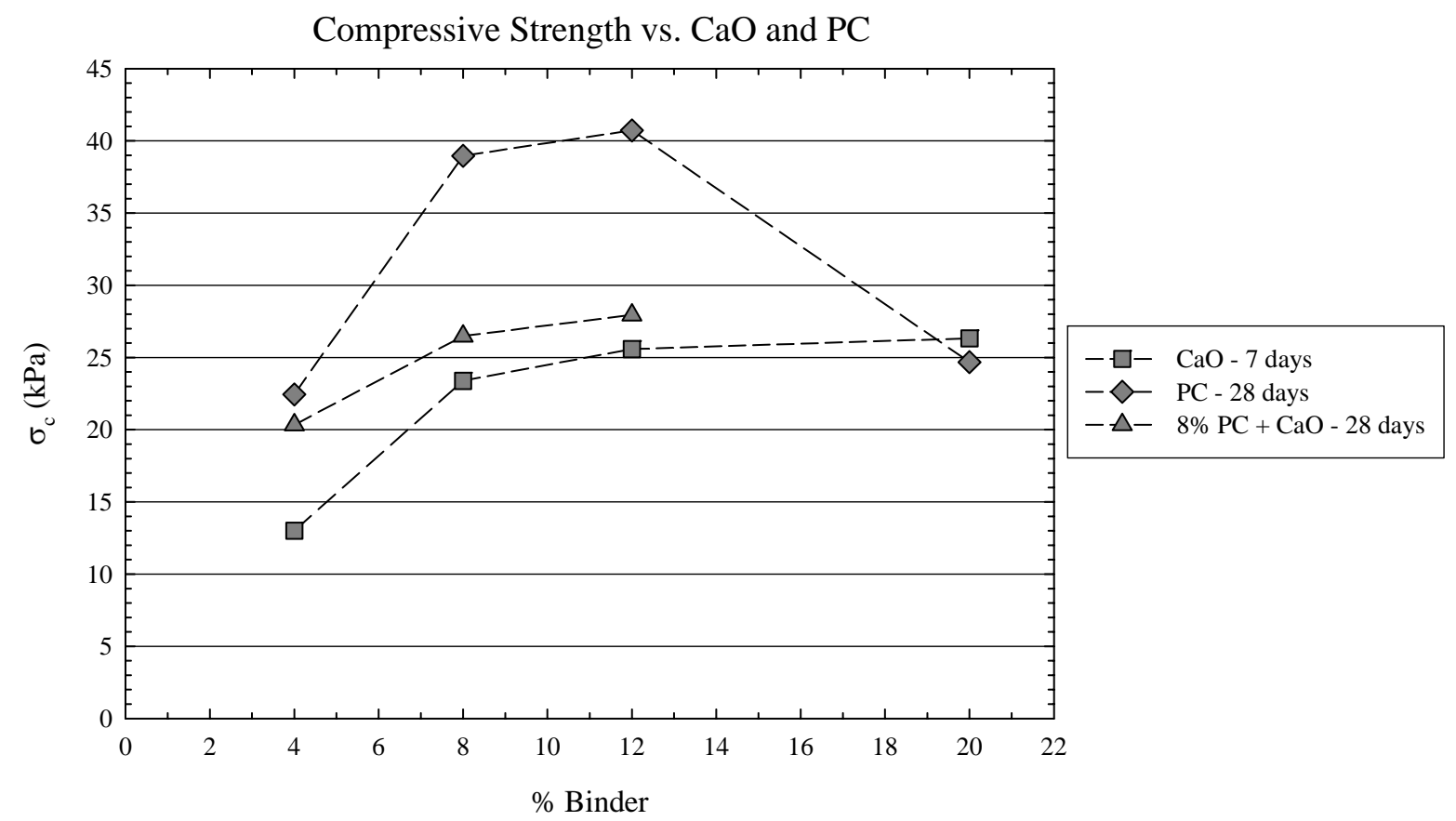

Figure 5.51: Comparison of strength for cement and/or lime treated LR soil 
CHAPTER 6: STIFFNESS: CRS CONSOLIDATION TESTS

CHAPTER 7: CONCLUSIONS AND RECOMMENDATIONS 


\section{CHAPTER 6: STIFFNESS: CRS CONSOLIDATION TESTS}

\subsection{INTRODUCTION}

The objective of this chapter is to present and discuss the results of constant rate of strain (CRS) and end-of-primary incremental loading (EOP-IL) consolidation tests performed on LR soil both untreated and treated with Portland cement.

Section 6.2 presents the results of consolidation tests on untreated and reconstituted LR soil. The test results for reconstituted LR soil serve as a base case for evaluating the effects of treatment with Portland cement (PC). When deep mixing is employed in the field, the existing soil structure is completely destroyed by the rotation of the mixing augers, which are used to introduce the binder(s) and to mix the soil to produce stabilized soil columns. The increases of the strength and stiffness, thus, come mainly from the chemical reaction of the binding agents. Therefore, evaluation of the improvement in properties associated with the presence of the binder is best performed using the behavior of the reconstituted as a reference.

Section 6.3 presents the results of CRS consolidation tests performed to evaluate the strain rate dependency of LR soil. The results of CRS consolidation tests have been shown to depend on the strain rate employed, especially for sensitive clays. The strain rate dependency of LR soil was evaluated by performing CRS consolidation tests with different strain rates ranging from 0.25 to $1.0 \% / \mathrm{hr}$.

Section 6.4 presents the results of CRS and EOP-IL consolidation tests on reconstituted LR soil. Comparisons are made for results from these tests to check the repeatability and the consistency of the results.

Section 6.5 presents the results of CRS and EOP-IL consolidation tests on PC treated LR soil. The effects of treatment are evaluated by comparing the results of PC treated LR soil with those of the reconstituted soil.

Section 6.6 summarizes the results presented in the chapter and compares the trends observed to those reported in the literature. 


\subsection{1-D CONSOLIDATION BEHAVIOR OF RECONSTITUTED LR SOIL}

The 1-D consolidation behavior of reconstituted LR soil specimens was investigated by performing CRS consolidation tests. After performing five pilot tests with strain rates equal to $0.5 \% / \mathrm{hr}$ and $1.0 \% / \mathrm{hr}$, the strain rate of $0.5 \% / \mathrm{hr}$ was selected to perform CRS consolidation tests in the experimental program. The information of each CRS consolidation test performed on reconstituted LR soil is summarized in Table 6.1. For example, during test CRS006, the soil specimen was consolidated up to the target stress of $1591.8 \mathrm{kPa}$ with a strain rate of $0.5 \% / \mathrm{hr}$. Once the target stress was reached, the specimen was allowed to creep for 48 hours until more than $95 \%$ of excess pore pressure was dissipated. After creep, the specimen was unloaded to the vertical effective stress of $72.7 \mathrm{kPa}$ with a strain rate of $0.5 \% / \mathrm{hr}$. The specimen was again allowed to creep for 72 hours to allow dissipation of the negative excess pore pressure. Finally, the specimen was reloaded up to the final stress of $2006.0 \mathrm{kPa}$ with a strain rate of $0.5 \% / \mathrm{hr}$.

\subsubsection{Preconsolidation Pressure and Compressibility of Reconstituted LR soil}

Figure 6.1 presents the compression curves obtained from the loading and reloading phases of the three CRS consolidation tests performed on reconstituted LR soil. The unloading curves are excluded due to the large negative excess pore pressure generated during this stage of the tests. As shown in Table 6.1, all the specimens were unloaded with the same strain rate $(0.5 \% / \mathrm{hr})$ employed for loading without reducing it by a factor of 0.1 as recommended by ASTM. As a result, significant negative excess pore pressure was developed at the end of unloading. The magnitude of negative excess pore pressure increased approximately linearly as the specimen was unloaded further. For instance, the magnitudes of the negative excess pore pressure for CRS006, 7 and 8 were $302.6 \mathrm{kPa},-199.4 \mathrm{kPa},-25.0 \mathrm{kPa}$, respectively, where the specimen was unloaded approximately by $1800 \mathrm{kPa}, 1100 \mathrm{kPa}$ and $200 \mathrm{kPa}$, respectively. The effect of the negative excess pore pressure on the unloading behavior is not well understood. However, it was observed that the slope of the unloading curve did not increase with increasing OCR but remained constant or decreased slightly with OCR. 
The compression curve of reconstituted LR soil exhibits the characteristics of the compression curve of non-structured soft soils: stiffer response in the recompression range, a well defined break corresponding to the preconsolidation pressure $\left(\sigma_{p}{ }_{p}\right)$, and the linear and steeper slope along the virgin compression line. The compression curve flattens gradually at the effective stress level higher than $1000 \mathrm{kPa}$ and yields more of an S-shaped curve. Compression curves of similar shape were observed from other highly organic soils and peats (Dhowian and Edil, 1981, Mesri et al, 1997). Although the compression curve from CRS007 shows slight sample disturbance effect near the preconsolidation pressure with modest change of the slope, all the compression curves coincide with each other in the compression range and show exceptional repeatability. The preconsolidation pressure and the compressibility parameters of reconstituted LR soil are summarized in Table 6.2.

The preconsolidation pressure of reconstituted LR soil was estimated employing the strain energy method (Becker et al, 1987). In this method, the work per unit volume is used as a yield criterion to define the preconsolidation pressure, where the change from small strain response to large strain response takes place. As shown in Figure 6.2, the relationship between the work per unit volume and the vertical effective stress is close to linear at low stress level and in the compression range. Therefore, linear best-fitting curves can be drawn through the initial portion of the curve (pre-yield line) and through the curve corresponding to the virgin compression line (post-yield line). The preconsolidation pressure can, then, be estimated as the intersection of the pre-yield and the post-yield lines.

As shown in Table 6.2, the preconsolidation pressure of reconstituted LR soil, which ranged from 61.5 to $68.0 \mathrm{kPa}$, is greater than the surcharge applied during soil sample preparation. According to Mesri and Castro (1986), soft soils develop preconsolidation pressure as a result of secondary compression. The overconsolidation resulting from secondary compression depends mainly on the duration of the aging (secondary compression stage) and on the ratio of $\mathrm{C}_{\alpha} / \mathrm{C}_{\mathrm{c}}$ of the soil and can be estimated using the following equation: 
$O C R=\frac{\sigma_{v c}^{\prime}}{\sigma_{v i}^{\prime}}=\left(\frac{t}{t_{p}}\right)^{\left(C_{\alpha} / C_{c}\right)\left(1-C_{r} / C_{c}\right)}$

where $\sigma^{\prime}{ }_{\mathrm{vc}}=$ preconsolidation pressure from secondary compression

$\sigma^{\prime}{ }_{\mathrm{vi}}=$ consolidation pressure at which secondary compression takes place

$\mathrm{t}=$ total duration of time that the load is maintained

$\mathrm{t}_{\mathrm{p}}=$ time required for completion of primary compression

$\mathrm{C}_{\alpha}=$ coefficient of secondary compression

$\mathrm{C}_{\mathrm{c}}=$ compression index, and $\mathrm{C}_{\mathrm{r}}=$ recompression index.

From equation (6.1), the preconsolidation pressure of LR soil samples developed during curing can be estimated. As described in chapter 4, the soil specimens were cured under a surcharge of $48 \mathrm{kPa}$ for 14 days, and thus $\sigma^{\prime}{ }_{v i}=48 \mathrm{kPa}$ and $\mathrm{t}=14$ days. The average value of $C_{\alpha} / C_{c}$ for organic soils, according to Mesri et al (1997), is about $0.06+/-$ 0.01. As will be discussed later, the value of $C_{r} / C_{c}$ for $L R$ soil is about $1 / 5$. Using these values and equation (6.1), the preconsolidation of LR soil due to secondary compression can be estimated as $60 \mathrm{kPa}\left(\mathrm{C}_{\alpha} / \mathrm{C}_{\mathrm{c}}=0.05, \mathrm{t}_{\mathrm{p}}=60\right.$ minutes $) \sim 70 \mathrm{kPa}\left(\mathrm{C}_{\alpha} / \mathrm{C}_{\mathrm{c}}=0.07, \mathrm{t}_{\mathrm{p}}=\right.$ 20 minutes). It should be noted that since the deformation of soil sample during curing was not monitored with time, it was assumed that the soil sample reached the end of primary consolidation in twenty to sixty minutes. Under these assumptions the preconsolidation pressures of LR soil specimens obtained from the CRS consolidation tests are consistent with the estimated values,.

The compression ratio ( $C R=\Delta \varepsilon_{a} / \Delta \log \left(\sigma^{\prime}{ }_{v}\right)$ ), which is the slope of the virgin compression line, is calculated in the stress range greater than $2 \sigma^{\prime}$. As summarized in Table 6.2, the compression ratios are consistent for all the specimens and increase slightly with vertical effective stress from the average value of 0.305 in 2-4 $\sigma^{\prime}$ p to 0.316 in 4-16 $\sigma_{\mathrm{p}}^{\prime}$. No clear trend of CR was observed with the index properties (OC, LI ). Compared to typical soft soils, the compression ratio of reconstituted LR soil lies 
between the value of a soft clay such as Resedimented Boston Blue Clay (CR=0.150 0.165, Sheahan et al, 1997) and that of highly organic materials such as the fibrous Muskeg from Quebec, Canada (0.358 - 0.646 with organic content $=70-100 \%)$ tested by Lefebvre et al. (1984).

The recompression ratios are scattered at OCR higher than 4 due to the difference of the magnitude of negative excess pore pressure generated during unloading. The recompression ratio of CRS006, which had the highest negative excess pore pressure, is about 0.043 (6-8 OCR) and 0.052 (4-6 OCR) and is almost three times greater than that of CRS008 at the same OCR. However, in the range of OCR smaller than 4, the values are consistent for all specimens. The recompression ratio increases with vertical effective stress from the average value of 0.059 (2-4 OCR) to 0.087 (1-2 OCR). The recompression ratio of $\mathrm{LR}$ soil is approximately $1 / 5$ of the compression ratio, and lies between the value of Resedimented Boston Blue Clay (0.012 - 0.017, Santagata, 1998) and that of the Canadian muskeg (0.030 - 0.148, Lefebvre et al, 1984).

\subsubsection{Constrained Modulus $\left(\mathrm{D}=1 / \mathrm{m}_{\mathrm{v}}\right)$}

Figure 6.3 presents the change of the constrained modulus (D) as a function of vertical effective stress during loading phase of CRS consolidation tests. The constrained modulus is defined as the inverse of the coefficient of volume compressibility $\left(\mathrm{m}_{\mathrm{v}}\right)$, and can be expresses as:

$$
D=\frac{1}{m_{v}}=\frac{\Delta \sigma_{v}^{\prime}}{\Delta \varepsilon_{a}}
$$

As is evident from the concurrent compression curves, the constrained modulus curves are almost identical and show high repeatability. The constrained modulus decreases gradually in the recompression range, and reaches the minimum value near the preconsolidation pressure. According to Janbu et al (1981), the decrease in the constrained modulus before the preconsolidation pressure is reached is related to “structural breakdown”. Degradation of the constrained modulus in the recompression 
range indicates that LR soil exhibits strain-hardening behavior. In the compression range, the constrained modulus increases linearly with vertical effective stress.

The constrained modulus provides another useful method to estimate the preconsolidation pressure, since the change of the constrained modulus takes place near the preconsolidation pressure. The preconsolidation pressure estimated from the constrained modulus plot is about $60 \mathrm{kPa}$ for all the tests and is in good agreement of the values estimated with the strain energy method (Table 6.2).

\subsubsection{Generation of Excess Pore Pressure}

As described in chapter 4, the excess pore pressure is measured as the difference of the cell pressure and the pore pressure measured at the bottom of specimen in CRS consolidation test. Since the coefficient of consolidation $\left(\mathrm{C}_{\mathrm{v}}\right)$ and the hydraulic conductivity $(\mathrm{k})$ are calculated based on the excess pore pressure, it is very critical to measure excess pore pressure accurately.

In the CRS consolidation test, the magnitude of the excess pore pressure depends on the employed strain rate, the stiffness of soil, and the specimen height. According to Force (1998), the excess pore pressure increases linearly with strain rate and the generated excess pore pressure is square function of the specimen height.

The generation of the excess pore pressure during loading and reloading (for CRS008) is shown as a function of vertical effective stress in Figure 6.4. The excess pore pressure is negligible in the recompression range where the stiffness of soil is high. The excess pore pressure at the preconsolidation pressure ranges from $6.9 \mathrm{kPa}$ (CRS007) to $8.7 \mathrm{kPa}$ (CRS006). In the compression range, the excess pore pressure increases more rapidly, due to the continuous decrease in void ratio and in hydraulic conductivity. The change of the hydraulic conductivity with vertical effective stress will be discussed in the following section. The generation of excess pore pressure is consistent in all three CRS consolidation tests. Especially, the generation of excess pore pressure during loading in CRS007 is almost identical to that of CRS008 during reloading.

Figure 6.5 presents the change of the pore pressure ratio, which is the ratio of the excess pore pressure and the total stress at any given time. Similar to the excess pore 
pressure curves, the pore pressure ratio curves of these tests are in good agreement with the pore pressure ratio of CRS006 consistently higher by 2-3\% than the other two tests. The pore pressure ratio remains below $30 \%$ for all the tests in the stress range investigated. Note that a pore pressure of $30 \%$ represents the upper limit according to the ASTM standard.

\subsubsection{Hydraulic conductivity (k)}

The hydraulic conductivity $(\mathrm{k})$ calculated from the CRS data employing the nonlinear theory (Wissa 1971) is shown as a function of axial strain in Figure 6.6. The initial hydraulic conductivity of LR soil is approximately $1.23 \times 10^{-6} \mathrm{~cm} / \mathrm{sec}$. This value is nearly 10-100 times greater than the values reported for clays $\left(\mathrm{k}_{\mathrm{v} 0}=5 \times 10^{-8}-5 \times 10^{-7}\right.$ $\mathrm{cm} / \mathrm{sec}$, Terzhagi et al, 1996), and lies at the lower end of the data typical of peats (for various peat deposits $\mathrm{k}_{\mathrm{v} 0}=6 \cdot \times 10^{-6}-1 \times 10^{-3} \mathrm{~cm} / \mathrm{sec}$, Mesri et al. 1997). Based on this value of hydraulic conductivity the duration of primary consolidation is expected to be relatively shorter compared to soft clays deposits.

The shape of the hydraulic conductivity curve is similar to the compression curve. While the decrease in the hydraulic conductivity is modest in the recompression region, the hydraulic conductivity decreases significantly once the soil becomes normally consolidated. The decrease in the hydraulic conductivity with void ratio in the compression range is expressed in terms of $\mathrm{C}_{\mathrm{k}}$, which is defined as

$$
C_{k}=\frac{\Delta e}{\Delta \log k}
$$

The value of $C_{k}$ for reconstituted LR soil is approximately 1/1.2. The ratio of $C_{k}$ to the initial void ratio of $\mathrm{LR}$ soil $\left(\mathrm{C}_{\mathrm{k}} / \mathrm{e}_{0}\right)$ is about $1 / 4.45$ (Table 6.2), and is close to the average value of $1 / 4$ reported for peats (Mesri et al.1997). The average value of $C_{k} / e_{0}$ for soft clays and silt deposits is close to 1/2. According to Mesri et al. (1997), the low values

of $C_{k} / e_{o}$ and high values of $C_{k}$ for peats as compared to clays and silts suggest that only macropores of peats are serving as flow channels. 
Figure 6.7 presents the change of the hydraulic conductivity as a function of the vertical effective stress. The hydraulic conductivity of LR soil decreases by an order of three from the initial value in the stress range investigated. The inverse of the slope of this curve in the compression range represents the value of $\mathrm{C}_{k} / \mathrm{C}_{c}$, which indicates the change of the log of the hydraulic conductivity for one log cycle of the vertical effective:

$$
\frac{C_{k}}{C_{c}}=\frac{\Delta e / \Delta \log k}{\Delta e / \Delta \log \sigma^{\prime}{ }_{v}}=\frac{\Delta \log \sigma^{\prime}{ }_{v}}{\Delta \log k}
$$

The value of $C_{k} / C_{c}$ for reconstituted LR soil is approximately $1 / 1.77$ (Table 6.2), and lies between the value for many types of clay and slit (near one) and the typical value of peats (1/3 1/2). According to Mesri et al. (1997), the low value of $C_{k} / C_{c}$ compared to many clays and silts indicate that only macropore of peat serves as flow channel, whereas the compression of both micropores and macropores contributes to total volume of flow.

\subsubsection{Coefficient of Consolidation $\left(\mathrm{C}_{\mathrm{v}}\right)$}

Figure 6.8(a) and (b) show the variation of the coefficient of consolidation $\left(\mathrm{C}_{\mathrm{v}}\right)$, derived using non-linear CRS consolidation theory, as a function of the vertical effective stress in semi-log scale and log-log scale, respectively. The coefficient of consolidation is a function of the constrained modulus $\left(\mathrm{D}=1 / \mathrm{m}_{\mathrm{v}}\right)$ and the hydraulic conductivity $(\mathrm{k})$, and can be expressed as:

$$
C_{v}=\frac{k}{m_{v} \gamma_{w}}
$$

where $\gamma_{\mathrm{w}}=$ unit weight of water $\left(9.81 \mathrm{kN} / \mathrm{m}^{3}\right)$

As shown in Figure 6.8 (a), the coefficient of consolidation decreases very rapidly in the recompression range. The rapid decrease of the coefficient of consolidation in the recompression range is related to the decrease of the constrained modulus (Figure 6.3) and the decrease of the hydraulic conductivity (Figure 6.7). While for most soft soils (e.g. Terzaghi et al. 1996, Sheahan et al, 1997, Force, 1998) $C_{v}$ is observed to remain constant or increase moderately in the compression range, the coefficient of consolidation of 
reconstituted LR soil continues to decrease with vertical effective stress albeit less markedly even once the preconsolidation pressure is exceeded. As shown in Figure 6.8 (b), the coefficient of consolidation decreases by approximately one order of magnitude from $4 \times 10^{-4} \mathrm{~cm}^{2} / \mathrm{sec}$ to $3 \times 10^{-5} \mathrm{~cm}^{2} / \mathrm{sec}$ in the stress range investigated. The continuous decrease of the coefficient of consolidation in the compression range is due to the decrease of the hydraulic conductivity. As indicated in Figure 6.3 and 6.6, although the constrained modulus increases with vertical effective stress in the virgin compression range, the decrease of the hydraulic conductivity is sufficiently greater to cause the decrease in the coefficient of consolidation.

\subsubsection{Linear vs. Non-linear theory}

As described in chapter 4, there are two solutions: linear and non-linear solutions, available for analyzing the results of CRS consolidation tests. The main difference of these two solutions lies in the assumption of the stress-strain relationship: constant $\mathrm{m}_{\mathrm{v}}$ in the linear theory and constant $\mathrm{C}_{\mathrm{c}}$ for the non-linear theory.

In addition to the assumption for the stress-strain relationship, the procedure for calculations of the consolidation parameters $\left(\mathrm{C}_{\mathrm{v}}\right.$ and $\left.\mathrm{k}\right)$ is slightly different between these two solutions. In the linear CRS theory, the hydraulic conductivity (k) is directly calculated from the excess pore pressure measured at the base of soil specimen (equation 4.12). The coefficient of consolidation $\left(\mathrm{C}_{\mathrm{v}}\right)$ is, then, calculated as a function of the constrained modulus and the hydraulic conductivity (equation 4.13). In the non-linear CRS theory, on the other hand, the coefficient of consolidation is calculated based on the excess pore pressure (equation 4.18), and the hydraulic conductivity is directly derived from the coefficient of consolidation and the constrained modulus (equation 4.20). In both cases, the hydraulic conductivity and the coefficient of consolidation are related to each other through equation (6.5). Depending on the magnitude of the excess pore pressure, the results obtained using these two solutions can be significantly different: in general, for high excess pore pressure, the linear solution yields higher values of vertical effective stress and of the coefficient of consolidation. 
Wissa et al. (1971) suggest using the linear solution if the time interval between data readings is kept reasonably short and the applied strain rate is slow enough to keep the pore pressure ratio $\left(\Delta \mathrm{u}_{\mathrm{h}} / \sigma_{\mathrm{v}}\right)$ less than $5 \%$. However, based on the pore pressures measured at five points through the specimen depth, Sheahan et al. (1997) showed that the distribution of pore pressure across the specimen in a CRS consolidation test was approximately parabolic. In this case, the vertical effective stress calculated with the nonlinear solution would be more realistic. Therefore, the non-linear solution was selected to analyze the results from CRS consolidation tests performed in this experimental program.

To evaluate the difference of the results obtained using these two solutions, the results from CRS006 was analyzed using both of these solutions. The compression curves, the constrained modulus curves, the hydraulic conductivity curves and the coefficient of consolidation curves of CRS006 obtained from using the linear and nonlinear CRS theories are compared in Figure 6.9 (a)-(d), respectively.

The compression curves and the constrained modulus curves obtained from the two solutions are almost identical and cannot be distinguished in Figures 6.9 (a) and (b). As shown in Figure 6.5, the pore pressure ratio of CRS006 ranges from $11.5 \%$ at $50 \mathrm{kPa}$ to a maximum of $27.5 \%$ at $1450 \mathrm{kPa}$. For this pore pressure ratio range, the maximum difference of the vertical effective stress is approximately 1\% (Figure 4.21).

The hydraulic conductivity curves are almost identical in the stress range investigated. The linear solution yields slightly higher values (about 13\%) of the coefficient of consolidation in the effective stress range $>200 \mathrm{kPa}$. Similar to the deviation of the vertical effective stress, this is related to the pore pressure ratio and is in good agreement with the results shown in Figure 4.22.

The comparison of the results obtained from the linear and non-linear solutions for the CRS consolidation test indicate that for the strain rate employed in the experimental program, the two solutions yield almost identical results. 


\subsection{STRAIN RATE SELECTION AND RATE EFFECTS}

Selection of the appropriate strain rate is a crucial aspect of CRS testing because of the inherent strain rate dependent nature of soil behavior and because this is the factor that ultimately determines testing productivity.

At high rates CRS tests have been shown to cause a shift of the compression curve towards higher stresses, ultimately leading to overestimate the preconsolidation pressure. According to Leroueil et al (1983), the preconsolidation pressure increases by approximately $10 \%$ per log cycle increase in strain rate. This effect, attributed to the soil's "structural viscosity" (Jamiolkowski et al. 1985), is especially significant in the case of sensitive clays such as those present in Canada and Scandinavia.

Excessively low rates, on the other hand, present concerns particularly when testing soils that exhibit significant tendency to creep. If the rate applied is not sufficiently fast to override the deformation of the soil arising from creep, the test leads to underestimate the preconsolidation pressure, and to overestimate the compressibility parameters.

In addition to the above, in a CRS test for a given soil, the magnitude of the excess pore pressure generated at the base of the specimen depends on the applied strain rate. To determine the coefficient of consolidation and the hydraulic conductivity, the strain should be fast enough to generate sufficiently large excess pore pressure so that it can be accurately measured with the available measuring devices (sensor and data acquisition system). However, the strain rate should not be too fast to avoid generation of an excessive hydraulic gradient across the soil specimen, which is not representative of in situ condition.

There are two criteria available for selection of appropriate strain rate for CRS consolidation test, and both of the criteria are based on the absolute value of the pore pressure ratio, which is the ratio of the excess pore pressure and the total stress at any given time $\left(\Delta \mathrm{u}_{\mathrm{h}} / \sigma_{\mathrm{v}}\right)$. Wissa et al (1971) suggest using a strain rate that yields a pore pressure ratio of $2-5 \%$, while the ASTM standard (D 4186-89) recommends to select a strain rate that causes a pore pressure ratio between 3 and $30 \%$. 
In the case of a highly compressible soil such as LR soil, the strain rate selection is further complicated by the fact that the specimen height changes significantly with time. In the CRS consolidation test apparatus employed for this experimental program, the load platen on which the CRS cell rests advances at a constant displacement rate, which causes the soil specimen to strain at a constant rate with respect to the initial specimen height. As a result, the actual strain rate, which is calculated with respect to the current specimen height at any given time, increases continuously as the height of soil specimen decreases. The increase of the actual strain rate during CRS consolidation tests performed on LR soil is illustrated in Figure 6.10. In this test, while the load platen advances at a constant rate of $0.5 \% / h r$, the actual strain rate gradually increased from an initial nominal rate of $0.5 \% / \mathrm{hr}$ to $0.9 \% / \mathrm{hr}$.

To evaluate the effect of the strain rate on the 1-D consolidation behavior of LR soil, additional CRS consolidation tests were performed on reconstituted soil specimens with two strain rates of 025 and $1.0 \% / \mathrm{hr}$. The test information of these tests is summarized in Table 6.3.

\subsubsection{Strain rate effect on the preconsolidation pressure and the compressibility}

Figure 6.11 presents the comparison of the compression curves obtained from CRS consolidation tests performed with three different strain rates. The preconsolidation pressure estimated using the strain energy method and the compression ratio of each test are summarized in Table 6.4.

The preconsolidation pressure increases slightly from $62.8 \mathrm{kPa}(0.25 \% / \mathrm{hr})$ to 67.4 $\mathrm{kPa}(1.0 \% / \mathrm{hr})$ with strain rate. The increase of the preconsolidation pressure with strain rate is in good agreement with the observation by Leroueil et al (1983): 7.3\% increase in the preconsolidation pressure with $\log _{10}(4)$ increase in strain rate. However, the fact that the preconsolidation pressure of the slowest test lies in the range of the three CRS tests performed with the strain rate of $0.5 \% / \mathrm{hr}$ indicates the strain rate effect on the preconsolidation pressure of LR soil is negligible.

The compression curve from the slowest test lies below the other two curves and exhibits slightly higher CR value. No clear relationship is observed between the strain rate and the compressibility of LR soil. 


\subsubsection{Strain rate effect on the generation of the excess pore pressure}

Figure 6.12 presents the effect of strain rate on the generation of excess pore pressure in CRS consolidation test. While the excess pore pressure curve from the fastest test derivates slightly in the recompression range, the magnitude of the excess pore pressure at a given stress level increases approximately linearly with strain rate. This linear relation between the magnitude of the excess pore pressure and the strain rate is evident in Figure 6.13. In this figure, the excess pore pressure normalized by the actual strain rate is plotted as a function of the vertical effective stress. The three curves are almost identical in most of the compression range, which is a clear indication of the linear relationship between the excess pore pressure and the strain rate.

Figure 6.14 presents the effect of strain rate on the pore pressure ratio $\left(\Delta \mathrm{u}_{\mathrm{h}} / \Delta \sigma_{\mathrm{v}}\right)$. The pore pressure ratio curves of the two slower tests exhibit similar shape and show linear relationship with strain rate in the compression range. The results indicate that the strain rates of 0.25 and $0.5 \% / h r$ yield the pore pressure ratio values lower than $30 \%$ in the stress range investigated and thus satisfy the ASTM criteria (D4186-89).

The pore pressure ratio curve of the $1.0 \% / \mathrm{hr}$ test deviates from the curves of the other slower tests especially in the recompression range. One possibility is that the pore pressure distribution with in the soil specimen is no longer parabolic at this strain rate. The pore pressure ratio of this test exceeded the upper limit of the ASTM criterion at the vertical effective stress of $400 \mathrm{kPa}$.

\subsubsection{Strain rate effect on the hydraulic conductivity and the coefficient of consolidation}

The strain rate effects on the hydraulic conductivity and the coefficient of consolidation are shown in Figure 6.15 and 6.16, respectively. The data show some scatter in the recompression range, with no trend with strain rate. The scatter in the recompression range is related to the inconsistent pore pressure response. However, once the soil becomes normally consolidated, the curves collapse on a very narrow band. 
Based on the results obtained from CRS consolidation tests performed with three strain rates in the range of $0.25-1.0 \% / \mathrm{hr}$, it can be concluded that LR soil does not exhibit significant rate dependent behavior in the range investigated. Among the strain rates, the strain rate of $1.0 \% / h r$ yielded pore pressure ratio greater than $30 \%$ and did not satisfy the ASTM criterion. While both of the two slower strain rates satisfy the ASTM criterion, the strain rate of $0.5 \% / h r$ is more advantageous since it will take less time than the strain rate of $0.25 \% / \mathrm{hr}$.

\subsection{EOP-IL CONSOLIDATION TEST ON RECONSTITUTED LR SOIL}

As mentioned in Chapter 4, the CRS consolidation test is suitable for investigation of the primary consolidation behavior of soft soils as it provides a continuous compression curve and values of the coefficient of consolidation and the hydraulic conductivity as a continuous function of the vertical effective stress (or void ratio). This test, on the other hand, provides only very limited information on the creep behavior of the soil.

To investigate this aspect of the behavior of the soils under consideration, additional incremental loading tests were performed. As discussed in more detail in the next chapter, in these tests the soil was allowed to creep extensively at the highest stress level $\left(\sigma^{\prime}=1600 \mathrm{kPa}\right)$. The results of this "long term creep" stage are discussed in Chapter 7.

For all previous increments, application of the incremental load occurred almost immediately after the end of primary of the previous increment, instead of after 24 hours as in conventional IL consolidation test. As a result, these tests are termed end-of-primary (EOP) incremental loading (IL) consolidation tests. Since under these testing conditions

the soil specimens are consolidated following the EOP compression curve (or virgin compression curve) the results can be directly compared to those obtained from the CRS test. 
The results from the EOP-IL consolidation test are compared with two additional CRS consolidation tests performed on reconstituted LR soil in Table 6.5 including test number, soil bin number, test type, strain rate $(\varepsilon)$, organic content (OC), water content $(w)$, specific gravity $\left(G_{s}\right)$ and initial void ratio $\left(e_{o}\right)$. Note that all the soil specimens were prepared following the specimen preparation procedure described in Chapter 4.

\subsubsection{Determination of end-of-primary (EOP) point}

The reliability of the results of an EOP-IL consolidation test depends mainly on the accuracy in determining the EOP point for each load increment. If one load increment is finished before reaching EOP, the compression curve will underestimate the compressibility of the soil. On contrary, if one load increment was applied too long (several orders of $\mathrm{t}_{\mathrm{EOP}}$ ), the soil specimen would go through several orders of secondary compression, yielding overestimation of the compressibility.

In this testing program, EOP-IL consolidation tests were performed employing the same consolidation test apparatus used for the CRS tests. As mentioned in Chapter 4, the CRS cell incorporates cell and pore pressure transducers for continuous measurement of the excess pore pressure (given at any time by the difference between the pore and the cell [i.e. back] pressures). Based on the excess pore pressures measurements, the EOP of each load increment was estimated to the point corresponding to $95 \%$ dissipation of measured peak excess pore pressure, as recommended by Fox et al. (1992).

Figure 6.17 presents the time settlement curve and dissipation of excess pore pressure from one load increment of an EOP-IL consolidation test performed on reconstituted LR soil (CRS037). In this load increment the effective stress was increased from $37.4 \mathrm{kPa}$ to $50.0 \mathrm{kPa}$ with a load increment ratio (LIR) of 0.34 . This small value of LIR was selected to allow more accurate determination of the preconsolidation pressure. The EOP points estimated with excess pore pressures measurements are indicated with open circles on both time settlement and excess pore pressures curves. It should be noted that the slope of the time settlement curve does not decreases after reaching EOP but remains constant. Leonards and Altschaeffl (1964) observed similar results from IL consolidation tests performed on clays with load increment ratios of considerably smaller 
than unity. In this case, measurement of excess pore pressures provides a reliable method for determination of EOP, since Casagrande's graphical method can not be employed.

\subsubsection{Results of EOP-IL consolidation tests performed on reconstituted LR soil}

Table 6.6 summarizes the data of an EOP-IL consolidation test performed on reconstituted LR soil specimen (CRS037), including the applied effective stress, EOP void ratio $\left(\mathrm{e}_{\mathrm{EO}}\right)$, time required for reaching EOP ( $\left.\mathrm{t}_{\mathrm{EOP}}\right)$ estimated from excess pore pressure measurements, maximum excess pore pressure $\left(\Delta \mathrm{u}_{\mathrm{hmax}}\right)$, time for maximum excess pore pressure development $\left(\mathrm{t}_{\Delta} \mathrm{u}_{\mathrm{hmax}}\right)$, duration of each load increment $\left(\mathrm{t}_{\mathrm{f}}\right)$, coefficient of consolidation $\left(\mathrm{C}_{\mathrm{v}}\right)$, hydraulic conductivity $(\mathrm{k})$, compression index $\left(\mathrm{C}_{\mathrm{c}}\right)$ and secondary compression index $\left(\mathrm{C}_{\alpha}\right)$ for each load increment.

Prior to testing, the soil specimen was saturated with back pressure of $312 \mathrm{kPa}$ for 1800 minutes. A vertical effective stress of $2.7 \mathrm{kPa}$ was applied to ensure the contact between piston and soil specimen. The sample height was maintained during the back pressure saturation stage.

During the test, the vertical effective stress was increased with the load increment ratio (LIR) of unity except for the first load increment (LIR $=9$ ). In addition, two load increments were added at $\sigma_{\mathrm{v}}=37.4$ and $74.9 \mathrm{kPa}$ to obtain more data points for better estimation of the preconsolidation pressure.

The EOP compression curve of reconstituted LR soil is plotted in Figure 6.18. As for the CRS consolidation tests, the preconsolidation pressure was estimated employing the strain energy method. Figure 6.19 shows that the preconsolidation pressure of CRS037 is calculated as $56.7 \mathrm{kPa}$, corresponding to the intersection of pre and post yield lines.

The compression curve of reconstituted LR soil is slightly S-shaped with the maximum compression index of 1.421 at $\sigma^{\prime}{ }_{\mathrm{v}}=400-800 \mathrm{kPa}$, which decreases to 1.297 at $\sigma^{\prime}{ }_{\mathrm{v}}=800-1600 \mathrm{kPa}$ (Table 6.7).

As summarized in Table 6.7, the maximum excess pore pressures developed in each load increment are consistently greater than $95 \%$ of actual applied load increments, 
except at the first load increment ( $75 \%)$ and at $\sigma^{\prime}{ }_{\mathrm{v}}=50 \mathrm{kPa}(93.7 \%)$. In the virgin compression range, the time required for the generation of maximum excess pore pressure increases gradually from 0.23 minute at $\sigma^{{ }_{\mathrm{v}}}=74.9 \mathrm{kPa}$ to 2.4 minutes at $\sigma_{\mathrm{v}}{ }_{\mathrm{v}}=$ $1600 \mathrm{kPa}$. These results are consistent with the observations made by Mesri et al. (1997) from consolidation tests performed on Middleton peat with approximately 90 - 95\% organic content. The delay in the generation of maximum pore pressure may be related to significant decrease in the hydraulic conductivity with increasing effective stress of highly organic soils and peats.

The coefficient of consolidation of reconstituted LR soil (CRS037) is plotted in Figure 6.20 as a function of the vertical effective stress. The $C_{v}$ value decreases from 4.53 $\mathrm{x} 10^{-3}$ at $\sigma^{\prime}{ }_{\mathrm{v}}=25.4 \mathrm{kPa}$ to $5.57 \times 10^{-4}$ at $\sigma^{\prime}{ }_{\mathrm{v}}=50.0 \mathrm{kPa}$ in the recompression range. The $\mathrm{C}_{\mathrm{v}}$ continuously decreases in the compression range but less rapidly from $3.19 \times 10^{-4}$ at $\sigma_{\mathrm{v}}=74.9 \mathrm{kPa}$ to $3.63 \times 10^{-5}$ at $\sigma^{\prime}{ }_{\mathrm{v}}=1600.0 \mathrm{kPa}$.

The hydraulic conductivity of reconstituted LR soil (CRS037) is plotted in Figure 6.21 as a function of void ratio. The hydraulic conductivity of reconstituted LR soil decreased by more than three orders of magnitudes from the initial value of $4.31 \times 10^{-7}$ at $\sigma^{\prime}=25.4 \mathrm{kPa}(\mathrm{e}=3.57)$ to $3.71 \times 10^{-10}$ at $\sigma_{\mathrm{v}}=1600.0 \mathrm{kPa}(\mathrm{e}=1.48)$. The value of $\mathrm{C}_{\mathrm{k}}$ ( $=\Delta \mathrm{e} / \Delta \operatorname{logk}$ ) is approximately $\mathrm{e}_{\mathrm{o}} / 3.83$ in the range of $2-16 \sigma_{\mathrm{p}}$, and is close to the average value for peat deposits $\left(\mathrm{e}_{\mathrm{o}} / 4\right)$ (Mesri et al., 1997). The value of $\mathrm{C}_{\mathrm{k}} / \mathrm{Cc}$ ( $=$ $\left.\Delta \sigma_{\mathrm{v}}^{\prime} / \Delta \operatorname{logk}\right)$ is approximately $1 / 1.42$, which indicates that the hydraulic conductivity decreases by a magnitude of 1.42 for one order increase in vertical effective stress. This value lies in between the average values of peats $(1 / 3-1 / 2)$ and clays and silts $(1 / 1)$.

\subsubsection{Comparison with CRS consolidation tests data}

In addition to an EOP-IL consolidation test, two CRS consolidation tests were performed on reconstituted soil using strain rate of $0.1 \% / \mathrm{hr}$ (CRS044) and $1.0 \% / \mathrm{hr}$ (CRS046). The data of soil specimens are summarized in Table 6.5.

The compression curves from the two CRS consolidation tests are plotted in Figure 6.22 and are compared with the compression curve from an EOP-IL consolidation 
test (CRS037). As summarized in Table 6.7, the preconsolidation pressures of CRS044 and CRS046 are 44.26 and $49.27 \mathrm{kPa}$, respectively. While there is a slight variation in the preconsolidation pressure between the three reconstituted LR soil specimens ( $\mathrm{SD} \pm 6.24$ $\mathrm{kPa}$ ) all three compression curves fall in a very narrow band, demonstrating good repeatability in the data. The compression index ranges from 1.28 in 2 - $4 \sigma^{\prime}$, to 1.36 in 4 - $8 \sigma_{\mathrm{p}}^{\prime}$ and 1.36 in $8-16 \sigma_{\mathrm{p}}$.

The hydraulic conductivity of reconstituted LR soil specimens from two CRS and one EOP-IL consolidation tests are plotted and compared in Figure 6.23. Similar to the compression curves, the hydraulic conductivity data show good agreement, with an average $C_{k}$ value of $1 /(4.09 \pm 0.39)$ and average $C_{k} / C_{c}$ value of $1 /(1.47 \pm 0.06)$ in the stress range corresponding to 2 - $16 \sigma_{\mathrm{p}}$.

The data for the coefficient of consolidation of reconstituted LR soil specimens from two CRS and one EOP-IL consolidation tests are plotted and compared in Figure 6.24. The values of the coefficient of consolidation from the two CRS consolidation tests are in good agreement, especially in the normally consolidation region. While the values of $\mathrm{C}_{\mathrm{v}}$ obtained from the EOP-IL test are close to those from CRS046 $(1.0 \% / \mathrm{hr})$ in the over consolidated region, the CRS consolidation tests yield $\mathrm{C}_{\mathrm{v}}$ values two to three times higher than EOP-IL consolidation test in the normally consolidated range. Since the values of hydraulic conductivity and coefficient of consolidation are directly calculated from the excess pore pressure measurements in CRS consolidation tests, these values are more reliable than those from EOP-IL test, and should be used for estimation of degree of consolidation in design stage.

Despite of the difference in loading methods, the results from CRS and EOP-IL consolidation tests appear consistent. This is a further indication that the primary consolidation behavior of reconstituted LR soil does not exhibit significant strain rate sensitivity. 


\subsection{EFFECTS OF TREATMENT WITH PORTLAND CEMENT}

For evaluation of the effects of treatment on the compressibility and the consolidation parameters (e.g. $\mathrm{k}$ and $\mathrm{C}_{\mathrm{v}}$ ) of LR soil, soil specimens were treated with Portland cement. Portland cement was selected as the binder due to the fact that it was the most efficient binder for improvement of the unconfined compressive strength as described in chapter 5 . To investigate the effect of the amount of binder, soil specimens were prepared with different amounts of PC ( $8-103.4 \%$ by dry mass of soil) following the sample preparation procedure described in chapter 4 . For each cement content, two soil samples were prepared: one for CRS and the other for EOP-IL consolidation tests. The specimen data including bin number, test type, amounts of PC added (\%PC), organic contents (OC), water contents (w), specific gravity $\left(\mathrm{G}_{\mathrm{s}}\right)$, and initial void ratio are summarized in Table 6.8. The schedules of the CRS tests performed to evaluate the effects of treatment with PC are summarized in

Table 6.9.

\subsubsection{Effects of treatment with 8.0\% PC}

The effects of treatment with 8.0\% PC on the 1-D consolidation behavior of LR soil were investigated by performing one CRS (CRS049) and one EOP-IL (CRS048) consolidation tests. The data of the EOP-IL consolidation test is summarized in Table 6.10, including: the applied stress at each increment, $\mathrm{e}_{\mathrm{EOP}}$, time required for reaching EOP, maximum excess pore pressures, time taken for generation of maximum excess pore pressures, total duration of load increment, coefficient of consolidation, hydraulic conductivity, and compression index. Both specimens were saturated with back pressure of approximately $560 \mathrm{kPa}$ for one and a half days prior to testing. In the EOP-IL consolidation test, the maximum excess pore pressures are about $80 \%$ of the applied load in the over-consolidated region, and 92\% and higher in the normally consolidated region.

Figure 6.25 presents the compression curves of $8 \%$ PC treated LR soil from both the CRS and the EOP-IL consolidation tests. The representative compression curve of reconstituted LR soil (CRS046) is also plotted for comparison. The results of the CRS 
and EOP-IL consolidation tests performed on 8\% PC treated LR soils are summarized in Table 6.14.

The most apparent effect of treatment on the primary consolidation behavior is the increase in the preconsolidation pressure. The values of the preconsolidation pressures derived from CRS048 and CRS049 are 70.21 and 79.43, respectively, and represent an increase of $49 \%$ from the average preconsolidation pressure of reconstituted LR soil from the same bin $(50.24 \mathrm{kPa})$.

In addition, the compression curve of 8\% PC treated LR soil is shifted to higher effective stresses, indicating the development of structure in the PC treated soil specimens. As a result, the treated soil can sustain higher void ratio than the reconstituted LR soil at the same effective stress.

The development of structure is also indicated by the steeper slope of the compression curves of 8\% PC treated LR soil (Figure 6.25). As summarized in Table 6.14, the compression index of $8 \%$ PC treated LR soil increased to $1.38-1.53$ in the $2-$ $4 \sigma^{\prime}$ p stress range, compared to the average value of reconstituted LR soil (1.28 \pm 0.03$)$. At higher stresses (e.g. in the $8-16 \sigma_{\mathrm{p}}$ stress range) the compression index of 8\% PC treated soil becomes close to the average value of the reconstituted soil.

The hydraulic conductivity curves of 8\% PC treated LR soils are plotted in Figure 6.26. Compared to reconstituted LR soil, the hydraulic conductivity of $8 \%$ PC treated LR soil shows an increase of 1.6-4.6 times at the same effective stress. As mentioned in Chapter 6, the increase in hydraulic conductivity upon treatment may be related to the aggregation of soil particles. The $\mathrm{C}_{\mathrm{k}} / \mathrm{e}_{\mathrm{o}}$ and $\mathrm{C}_{\mathrm{k}} / \mathrm{C}_{\mathrm{c}}$ also increased to average values of $1 / 4.34$ and $1 / 1.58$, respectively.

The coefficient of consolidation curves of 8\% PC treated LR soils are plotted in Figure 6.27. Although the coefficient of consolidation curves of CRS048 and CRS049 show discrepancies, both tests show increase in the coefficient of consolidation by 1.5 8.3 times of the values for reconstituted LR soil at the same effective stress. 


\subsubsection{Effects of treatment with $18.7 \%$ PC}

The compression curves of $18.7 \%$ PC treated LR soil obtained from CRS043 (CRS) and CRS042 (EOP-IL) are plotted in Figure 6.28. A curve for 8\% PC is also shown for comparison purposes. For $18.7 \%$ PC, the preconsolidation pressures increased to 94.40 (CRS042) and $94.83 \mathrm{kPa}$ (CRS043), a 88\% increase compared to the reconstituted LR soil. For $\sigma^{\prime}$, greater than $100 \mathrm{kPa}$ the compression curve for $18.7 \% \mathrm{PC}$ lies above the curve for 8\% PC treated soils, indicating additional development of structure. The compression index values of the $18.7 \%$ PC specimens are lower than the compression index of $8 \%$ PC treated soils and slightly higher than or similar to the average compression index values of reconstituted LR soil (Table 6.14).

As the specimens became stiffer by treating with $18.7 \%$ PC, the maximum excess pore pressures generated in each load increment of the EOP-IL consolidation test were consistently smaller than the applied load increment. As summarized in Table 6.11, the maximum excess pore pressures were less than $80 \%$ of the applied load in the over consolidated region, and were between 85 and $90 \%$ in normally consolidated region. However, this poor generation of excess pore pressure does not have significant effects on the EOP-IL consolidation results as will be discussed later.

While treatment with $18.7 \%$ PC does not change the compression curve significantly compared to $8.0 \%$ PC treated soil, as shown in Figure 6.26, the effect of treatment on the hydraulic conductivity is very marked. Compared to the 8\% PC treated soils, the hydraulic conductivity of $18.7 \%$ PC treated soils is consistently higher by 4.5 6.0 times.

As shown in Figure 6.27, the coefficient of consolidation data of $18.7 \%$ PC treated LR soils show similar trend as for hydraulic conductivity. With $18.7 \%$ PC treatment, the coefficient of consolidation of LR soil increases by 6 times compared to the value measure for the $8.0 \%$ PC treated soil. 


\subsubsection{Effects of treatment with 51.4\% PC}

The effects of treatment of LR soil with $51.4 \%$ PC were evaluated by performing one CRS consolidation test (CRS051) with a strain rate of $0.5 \% / h r$ and one EOP-IL consolidation test (CRS050).

The compression curves from CRS050 and CRS051 are plotted in Figure 6.31, and are compared with the compression curve of 18.7\% PC treated LR soil (CRS043). The compression curves of $51.4 \%$ PC treated LR soils are shifted further to the higher effective stress level, and these soil specimens can sustain 3.67, 490 and $635 \mathrm{kPa}$ higher effective stresses at void ratios of 3.2, 3.0, and 2.8.

The preconsolidation pressures of CRS 050 and CRS051 are 404.4 and $402.6 \mathrm{kPa}$, representing a 700\% increase compared to the reconstituted LR soil. The average value of compression index of $51.4 \%$ PC treated LR soils is $1.70 \pm 0.02$ at $\sigma^{\prime}{ }_{\mathrm{v}}=2-4 \sigma_{\mathrm{p}}$.

The data of the EOP-IL consolidation test (CRS050) are summarized in Table 6.12. In the over consolidated region, the maximum excess pore pressures generated are about $37-71 \%$ of the applied load except at $\sigma^{\prime}{ }_{\mathrm{v}}=49.3 \mathrm{kPa}$ where an excess pore pressure equal to $100 \%$ of the applied load increment was developed. In the normally consolidated region, the maximum excess pore pressures were about $82-86 \%$ of the applied load.

While the compression curves of 51.4\% PC treated soil lie at much higher effective stress level compared to $18.7 \%$ PC treated soil, the hydraulic conductivity curves of $51.5 \%$ PC treated LR soils are almost identical to that of the $18.7 \%$ PC treated soil (Figure 6.32). For 51.4\% PC the values of $C_{k} / e_{o}$ and $C_{k} / C_{c}$ are approximately $1 /(4.16$ $\pm 0.48)$ and $1 /$ (1.93 \pm 0.24$)$, respectively.

Plots of the coefficient of consolidation of $51.4 \%$ PC treated LR soil versus vertical effective stress are shown in Figure 6.33. Compared to 18.7\% PC treated soils, the coefficient of consolidation of $51.4 \%$ PC treated soil increased by 6 - 8 times in the normally consolidated region. 


\subsubsection{Effects of treatment with 103.4\% PC}

To evaluate the effects of treatment with 103.4\% PC, one CRS consolidation test (CRS055) and one EOP-IL consolidation test (CRS054) were performed. For the CRS consolidation tests, a 5000 lbs load cell was used to load the soil specimen up to an effective stress of $4000 \mathrm{kPa}$. In the EOP-IL consolidation test, a $2000 \mathrm{lbs}$ load cell was used and the soil specimen was loaded up to effective stress level of $1387 \mathrm{kPa}$.

The compression curves of $103.4 \%$ PC treated LR soil specimens are plotted in Figure 6.34. Due to the limitation of the load cell employed in the EOP-IL consolidation test, not enough EOP points were obtained to measure the preconsolidation pressure. Therefore, the preconsolidation pressure of the sample was measured from the CRS consolidation test. Using the strain energy method, the preconsolidation pressure of 103.4\% PC treated LR soil specimen is measured as $1249.8 \mathrm{kPa}$. The compression index of the sample is 1.24 at $\sigma_{\mathrm{v}}^{\prime}=2-4 \sigma_{\mathrm{p}}$.

The data of the EOP-IL consolidation test (CRS054) are summarized in Table 6.13. In the highly over consolidated region, especially in the load increments up to $\sigma^{\prime}{ }_{\mathrm{v}}=$ $43.8 \mathrm{kPa}$, the deformation was very small and no excess pore pressures were generated. As a result, EOP points could not be determined and values of coefficient of consolidation and hydraulic conductivity were not calculated. In the load increments between $\sigma^{\prime}{ }_{\mathrm{v}}=86.5 \mathrm{kPa}$ and $\sigma^{\prime}{ }_{\mathrm{v}}=698.1 \mathrm{kPa}$, the values of the maximum excess pore

pressure generated were less than $36 \%$ of the applied load. Even in the normally consolidated region at $\sigma^{\prime}{ }_{\mathrm{v}}=1387.1 \mathrm{kPa}$, the maximum excess pore pressures was only $48 \%$ of the applied load.

Despite the poor generation of excess pore pressures, the coefficient of consolidation and hydraulic conductivity obtained from the EOP-IL consolidation test are close to the values obtained from CRS consolidation test, as shown in Figures 6.35 and 6.36, respectively.

Compared to the values of 51.4\% PC treated specimens, the coefficient of consolidation and hydraulic conductivity increased by approximately four and two times in the normally consolidated region. 


\subsection{SUMMARY OF THE EFFECTS OF TREATMENT ON 1-D CONSOLIDATION BEHAVIOR OF LR SOILS}

6.6.1 Effects of treatment with PC on the preconsolidation pressure and the compressibility of LR soil

The compression curves obtained from CRS consolidation tests performed on reconstituted (CRS037) and PC treated LR soil are shown in Figure 6.37. The most evident effect of treatment with PC is the increase in the preconsolidation pressure. The preconsolidation pressures of all the treated specimens, calculated with the strain energy method, are plotted as a function of the amount of PC added in each specimen in Figure 6.38. The increase in preconsolidation pressure is fairly modest for low dosages of PC with $\sigma_{p}$ increasing from the average value of $50.2 \mathrm{kPa}$ obtained for the reconstituted soil to $74.8 \mathrm{kPa}$ and $94.6 \mathrm{kPa}$ with $8.0 \%$ PC and $18.7 \%$ PC, respectively. Above 20\% PC the increase in $\sigma_{\mathrm{p}}$ is instead very significant: with $51.4 \% \mathrm{PC}$, the preconsolidation pressure increases to $403.3 \mathrm{kPa}$ (8 times the value measured on the reconstituted LR soil). With $103.4 \% \mathrm{PC}$, it further increases to $1249.8 \mathrm{kPa}$ (25 times the value measured on the reconstituted LR soil). In addition, with increasing cement content, the compression curves gradually shifts to higher effective stress level. This indicates that cement treated LR soil specimen can sustain higher effective stress than untreated and reconstituted LR soil at the same void ratio. The compression index in the range of $\sigma^{\prime}{ }_{v}=2-4$ increases with increasing cement content, but becomes similar to the value for reconstituted soil in effective stress range at $8-16 \sigma_{\mathrm{v}}$.

While the preconsolidation pressure increases with treatment, the compression index in the normally consolidated region does not show any significant change. This is illustrated in Figure 6.39 where the compression indices of PC treated LR soil are plotted as a function of the vertical effective stress normalized by the preconsolidation pressure. The compression index of reconstituted LR soil increases up to $4 \sigma_{p}$, then decreases with increasing vertical effective stress, indicating that the compression curve is S-shaped. Although the trend is not clear, the compression indices of LR soil show a slight increase 
with increasing \%PC in $1-2 \sigma_{\mathrm{p}}$, which may be related to the development of structure in the soil specimen. In the stress range above $8 \sigma^{\prime}$, , the compression index of the PC treated LR soil becomes close to that of untreated reconstituted LR soil. The recompression ratios of PC treated LR soil, calculated from the reloading curve, are plotted as a function of OCR in Figure 6.40. The results show that the compression ratio decreases with increasing \%PC throughout the recompression range, evidence of the fact that LR soil becomes much stiffer upon treatment with PC.

Similar observations on the effect of cement treatment on the compressibility of organic soils in the recompressions and virgin compression region are reported by other researchers (Åhnberg 1996, Cortellazzo et al. 1999, Hebib et al. 2003)

6.6.2 Effects of treatment with PC on the hydraulic conductivity and the coefficient of consolidation of LR soil

The values of the hydraulic conductivity of LR soil treated with PC are shown in Figure 6.41. The effect of treatment on hydraulic conductivity is negligible for $8 \%$ PC . With $18.7 \%$ treatment, the hydraulic conductivity of LR soil increases by 6.3 times compared to reconstituted LR soil. The hydraulic conductivity showed an even more marked increase when the amount of cement was increased to 51.4\% and 103.4\% PC (9.7 and 14.4 times the value for the reconstituted soil, respectively).

The increase in the hydraulic conductivity of LR soil with treatment with PC is in good agreement with observations reported by several researchers (Broms and Boman 1979; Brandl 1981; Buensuceso 1990; Townsend and Kylm 1996; Cortellazzo et al. 1999). It is hypothesized that the increase in hydraulic conductivity with treatment is caused by the change of the fabric of soil as a result of the reactions occurring with the cement. Several researchers observed from the results of particle size distribution analysis and scanning electron microscope (SEM) analysis that the soils treated with lime or PC became flocculated and aggregated yielding more open fabric and more granular particles (Tremblay et al 2001; Al-Rawas 2002). As a result, the size of the macropores, which serve as main channels for flow, increase causing the increase in the hydraulic 
conductivity. It should be noted, however, that conflicting results on the effect of treatment with Portland cement or lime on hydraulic conductivity are also reported. For example Terashi et al. (1980), Ghazali et al. (1991), Locat et al. (1996) reported a decrease in the hydraulic conductibity of treated soils. Finally, McCallister and Petry (1992) observed that the hydraulic conductivity increased for addition of lime up to $3 \%$, above which it decreased. Overall this topic seems to require further investigation.

The coefficient of consolidation of treated LR soil is shown as a function of vertical effective stress in Figure 6.42. As shown in this figure, the coefficient of consolidation of the treated soil increases with increasing cement content. This implies that the consolidation process takes place faster in LR soil when treated. Since the coefficient of consolidation changes with vertical effective stress level and with overconsolidation ratio, the values of $C_{v}$ are plotted in Figure 6.43 versus the vertical effective stress normalized by the preconsolidation pressure. This plot clearly highlights the increase of coefficient of consolidation. Compared to the untreated reconstituted LR soil the coefficient of consolidation increases by 1.4 times with $8.0 \%$ PC, 8.1 time with 18.7\% PC, and about 38 times with 51.4\% and 103.4\% PC. Similar results were reported by Kassim et al. (1999), Cortellazzo et al. (1999) and Broms (1999). 
Table 6.1: CRS tests performed on reconstituted LR soil

\begin{tabular}{|c|c|c|c|c|c|c|c|c|c|}
\hline $\begin{array}{c}\text { Test } \\
\text { Number }\end{array}$ & $\mathrm{e}_{\mathrm{o}}$ & $\begin{array}{c}\text { Loading } \\
\dot{\varepsilon} \\
(\% / \mathrm{hr})\end{array}$ & $\begin{array}{c}\text { Target } \\
\text { Stress } \\
(\mathrm{kPa})\end{array}$ & $\begin{array}{c}\text { Creep } \\
\text { time } \\
(\mathrm{hrs})\end{array}$ & $\begin{array}{c}\text { Unloading } \\
\dot{\varepsilon} \\
(\% / \mathrm{hr})\end{array}$ & $\begin{array}{c}\text { Final } \\
\text { Unloading } \\
\text { Stress }(\mathrm{kPa})\end{array}$ & $\begin{array}{c}\text { Creep } \\
\text { time } \\
(\mathrm{hrs})\end{array}$ & $\begin{array}{c}\text { Reloading } \\
\dot{\varepsilon} \\
(\% / \mathrm{hr})\end{array}$ & $\begin{array}{c}\text { Final } \\
\text { Stress } \\
(\mathrm{kPa})\end{array}$ \\
\hline CRS006 & 4.03 & 0.5 & 1591.8 & 48 & 0.5 & 72.7 & 72 & 0.5 & 2006.0 \\
\hline CRS007 & 4.25 & 0.5 & 999.6 & 72 & 0.5 & 89.1 & 72 & 0.5 & 2005.8 \\
\hline CRS008 & 4.25 & 0.5 & 194.0 & 48 & 0.5 & 12.1 & 48 & 0.5 & 2005.1 \\
\hline
\end{tabular}

Table 6.2: Summary of the results from CRS tests performed on reconstituted LR soil

\begin{tabular}{|c|c|c|c|c|c|c|c|c|}
\hline \multirow{2}{*}{ Test Number } & $\sigma_{\mathrm{p}}^{\prime}$ & \multicolumn{2}{|c|}{$\mathrm{CR}$} & \multicolumn{2}{|c|}{$\mathrm{RR}$} & $\mathrm{C}_{\mathrm{k}} / \mathrm{e}_{\mathrm{o}}$ & $\mathrm{C}_{\mathrm{k}} / \mathrm{C}_{\mathrm{c}}$ & \multirow{2}{*}{$\begin{array}{c}\varepsilon_{\mathrm{f}} \\
\end{array}$} \\
\cline { 3 - 9 } & $(\mathrm{kPa})$ & $2-4 \sigma_{\mathrm{p}}{ }^{\prime}$ & $4-16 \sigma_{\mathrm{p}}{ }^{\prime}$ & $2-4$ OCR & $1-2$ OCR & $4-16 \sigma_{\mathrm{p}}{ }^{\prime}$ & $4-16 \sigma_{\mathrm{p}}{ }^{\prime}$ & $(\%)$ \\
\hline CRS006 & 64.0 & 0.305 & 0.319 & 0.067 & 0.090 & $1 / 4.36$ & $1 / 1.77$ & 51.3 \\
\hline CRS007 & 61.5 & 0.288 & 0.312 & 0.057 & 0.081 & $1 / 4.54$ & $1 / 1.77$ & 50.3 \\
\hline CRS008 & 66.8 & 0.321 & - & 0.053 & 0.091 & - & - & 51.8 \\
\hline Average & 64.1 & 0.305 & 0.316 & 0.059 & 0.087 & $1 / 4.45$ & $1 / 1.77$ & - \\
\hline
\end{tabular}

Table 6.3: CRS tests performed to evaluate the strain rate effect

\begin{tabular}{|c|c|c|c|c|c|c|c|c|c|}
\hline $\begin{array}{c}\text { Test } \\
\text { Number }\end{array}$ & $\mathrm{e}_{\mathrm{o}}$ & $\begin{array}{c}\text { Loading } \\
\dot{\varepsilon} \\
(\% / \mathrm{hr})\end{array}$ & $\begin{array}{c}\text { Target } \\
\text { Stress } \\
(\mathrm{kPa})\end{array}$ & $\begin{array}{c}\text { Creep } \\
\text { time } \\
(\mathrm{hrs})\end{array}$ & $\begin{array}{c}\text { Unloading } \\
\dot{\varepsilon} \\
(\% / \mathrm{hr})\end{array}$ & $\begin{array}{c}\text { Final } \\
\text { Unloading } \\
\text { Stress (kPa) }\end{array}$ & $\begin{array}{c}\text { Creep } \\
\text { time } \\
(\mathrm{hrs})\end{array}$ & $\begin{array}{c}\text { Reloading } \\
\dot{\varepsilon} \\
(\% / \mathrm{hr})\end{array}$ & $\begin{array}{c}\text { Final } \\
\text { Stress } \\
(\mathrm{kPa})\end{array}$ \\
\hline CRS016 & 4.32 & 0.25 & 992.1 & 48 & 0.25 & 26.9 & 48 & 0.25 & 2005.3 \\
\hline CRS017 & 4.45 & 1.0 & 994.3 & 72 & 1.0 & 6.76 & 96 & 1.0 & 1534.6 \\
\hline CRS030 & 4.20 & 1.0 & 1233.7 & 72 & 0.1 & 56.1 & 72 & 1.0 & 2008.2 \\
\hline
\end{tabular}


Table 6.4: Summary of the results from CRS tests performed to evaluate the strain rate effect

\begin{tabular}{|c|c|c|c|c|c|c|}
\hline \multirow{2}{*}{ Test Number } & \multirow{2}{*}{$\begin{array}{l}\text { Loading } \\
\dot{\varepsilon}(\% / \mathrm{hr})\end{array}$} & \multirow{2}{*}{$\begin{array}{c}\sigma_{\mathrm{p}}^{\prime} \\
(\mathrm{kPa})\end{array}$} & \multicolumn{2}{|c|}{ CR } & $\mathrm{C}_{\mathrm{k}} / \mathrm{e}_{\mathrm{o}}$ & $\mathrm{C}_{\mathrm{k}} / \mathrm{C}_{\mathrm{c}}$ \\
\hline & & & $2-4 \sigma_{p}{ }^{\prime}$ & $4-16 \sigma_{p}{ }^{\prime}$ & $4-16 \sigma_{p}{ }^{\prime}$ & $4-16 \sigma_{p}^{\prime}$ \\
\hline CRS016 & 0.25 & 62.8 & 0.330 & 0.330 & $1 /$ & $1 / 1.82$ \\
\hline $\begin{array}{c}\text { Average of } \\
\text { CRS006-008 }\end{array}$ & 0.5 & 64.1 & 0.305 & 0.316 & $1 / 4.45$ & $1 / 1.77$ \\
\hline CRS017 & 1.0 & 67.4 & 0.336 & 0.321 & & $1 / 1.67$ \\
\hline
\end{tabular}

Table 6.5: Summary of reconstituted LR soil specimen data used for CRS and IL consolidation tests

\begin{tabular}{|c|c|c|c|c|c|c|c|}
\hline Test No. & Bin\# & Test type & $\dot{\varepsilon}(\% / \mathrm{hr})$ & OC (\%) & $\mathrm{w}(\%)$ & $\mathrm{G}_{\mathrm{s}}$ & $\mathrm{e}_{\mathrm{o}}$ \\
\hline CRS037 & LR-B8 & IL & - & 55.97 & 176.55 & 1.99 & 3.67 \\
\hline CRS044 & LR-B9 & CRS & 0.1 & 36.57 & 156.27 & 2.19 & 3.80 \\
\hline CRS046 & LR-B9 & CRS & 1.0 & 37.47 & 155.42 & 2.18 & 3.64 \\
\hline
\end{tabular}


Table 6.6: Summary of data from EOP-IL consolidation test performed on reconstituted LR soil (CRS037)

\begin{tabular}{|c|c|c|c|c|c|c|c|c|c|}
\hline Steps & $\begin{array}{c}\sigma_{\mathrm{v}}^{\prime} \\
(\mathrm{kPa})\end{array}$ & $\mathrm{e}_{\mathrm{EOP}}$ & $\begin{array}{c}\mathrm{t}_{\mathrm{EOP}} \\
(\mathrm{min})\end{array}$ & $\begin{array}{c}\Delta \mathrm{u}_{\mathrm{hmax}} \\
(\mathrm{kPa})\end{array}$ & $\begin{array}{c}\mathrm{t}_{\text {पuhmax }} \\
(\mathrm{min})\end{array}$ & $\begin{array}{c}\mathrm{t}_{\mathrm{f}} \\
(\mathrm{min})\end{array}$ & $\begin{array}{c}\mathrm{C}_{\mathrm{v}} \\
\left(\mathrm{cm}^{2} / \mathrm{sec}\right)\end{array}$ & $\begin{array}{c}\mathrm{k} \\
(\mathrm{cm} / \mathrm{sec})\end{array}$ & $\mathrm{C}_{\mathrm{c}}$ \\
\hline $\begin{array}{c}\text { BP } \\
\text { Saturation }\end{array}$ & 2.71 & 3.67 & & & & & & & \\
\hline Step1 & 25.4 & 3.57 & 41.3 & 17.1 & 0.18 & 63.2 & $4.53 \mathrm{E}-03$ & $4.31 \mathrm{E}-07$ & 0.106 \\
\hline Step2 & 37.4 & 3.48 & 111.4 & 11.4 & 0.22 & 200.5 & $1.17 \mathrm{E}-03$ & $1.77 \mathrm{E}-07$ & 0.514 \\
\hline Step3 & 50.0 & 3.40 & 178.4 & 11.8 & 0.30 & 343.6 & $5.57 \mathrm{E}-04$ & $7.87 \mathrm{E}-08$ & 0.673 \\
\hline Step4 & 74.9 & 3.24 & 338.2 & 24.4 & 0.23 & 510.4 & $3.19 \mathrm{E}-04$ & $4.26 \mathrm{E}-08$ & 0.902 \\
\hline Step5 & 100 & 3.10 & 429.7 & 24.8 & 0.23 & 1000.0 & $1.92 \mathrm{E}-04$ & $2.29 \mathrm{E}-08$ & 1.133 \\
\hline Step6 & 200.5 & 2.72 & 712.7 & 98.4 & 0.43 & 1001.0 & $1.65 \mathrm{E}-04$ & $1.31 \mathrm{E}-08$ & 1.253 \\
\hline Step7 & 399.6 & 2.30 & 909.2 & 191.8 & 0.75 & 1440.1 & $9.96 \mathrm{E}-05$ & $4.37 \mathrm{E}-09$ & 1.391 \\
\hline Step8 & 800.7 & 1.87 & 1204.4 & 382.8 & 1.4 & 1440.1 & $5.94 \mathrm{E}-05$ & $1.33 \mathrm{E}-09$ & 1.421 \\
\hline Step9 & 1600.0 & 1.48 & 1529.7 & 760.2 & 2.4 & 50065.2 & $3.63 \mathrm{E}-05$ & $3.71 \mathrm{E}-10$ & 1.297 \\
\hline
\end{tabular}

Table 6.7: Summary of results from CRS and IL consolidation tests performed on reconstituted LR soil

\begin{tabular}{|c|c|c|c|c|c|c|c|c|}
\hline \multirow{2}{*}{ Test No. } & \multirow{2}{*}{$\begin{array}{l}\text { Test } \\
\text { type }\end{array}$} & \multirow{2}{*}{$\begin{array}{c}\dot{\varepsilon} \\
(\% / \mathrm{hr})\end{array}$} & \multirow{2}{*}{$\begin{array}{c}\sigma_{\mathrm{p}}^{\prime} \\
(\mathrm{kPa})\end{array}$} & \multicolumn{3}{|c|}{$\mathrm{C}_{\mathrm{c}}$} & \multirow{2}{*}{$\frac{\mathrm{C}_{\mathrm{k}} / \mathrm{e}_{\mathrm{o}}}{2-16 \sigma_{\mathrm{p}}^{\prime}}$} & \multirow{2}{*}{$\frac{\mathrm{C}_{\mathrm{k}} / \mathrm{C}_{\mathrm{c}}}{2-16 \sigma_{\mathrm{p}}^{\prime}}$} \\
\hline & & & & $2-4 \sigma_{p}^{\prime}$ & $4-8 \sigma_{p}^{\prime}$ & $8-16 \sigma_{p}^{\prime}$ & & \\
\hline CRS037 & IL & - & 56.70 & 1.25 & 1.39 & 1.42 & $1 / 3.83$ & $1 / 1.42$ \\
\hline CRS044 & CRS & 0.1 & 44.26 & 1.28 & 1.30 & 1.28 & $1 / 4.54$ & $1 / 1.54$ \\
\hline CRS046 & CRS & 1.0 & 49.72 & 1.30 & 1.39 & 1.38 & $1 / 3.91$ & $1 / 1.46$ \\
\hline \multicolumn{3}{|c|}{ Average } & 50.23 & 1.28 & 1.36 & 1.36 & $1 /(4.09$ & $1 /(1.47$ \\
\hline \multicolumn{3}{|c|}{ \pm standard deviation } & \pm 6.24 & \pm 0.03 & \pm 0.05 & \pm 0.07 & $\pm 0.39)$ & $\pm 0.06)$ \\
\hline
\end{tabular}


Table 6.8: Summary of PC treated LR soil specimen data used for CRS and IL Consolidation tests

\begin{tabular}{|c|c|c|c|c|c|c|c|}
\hline Test No. & Bin\# & Test type & $\%$ PC & OC (\%) & $\mathrm{w}(\%)$ & $\mathrm{G}_{\mathrm{s}}$ & $\mathrm{e}_{\mathrm{o}}$ \\
\hline CRS042 & LR-B9 & IL & 18.7 & 40.67 & 166.85 & 2.15 & 3.74 \\
\hline CRS043 & LR-B9 & CRS & 18.7 & 40.89 & 169.01 & 2.15 & 3.65 \\
\hline CRS048 & LR-B10 & IL & 8.0 & 42.36 & 180.17 & 2.13 & 3.95 \\
\hline CRS049 & LR-B10 & CRS & 8.0 & 43.12 & 174.44 & 2.12 & 3.75 \\
\hline CRS050 & LR-B10 & IL & 51.4 & 44.18 & 167.98 & 2.11 & 3.66 \\
\hline CRS051 & LR-B10 & CRS & 51.4 & 43.87 & 167.58 & 2.11 & 3.57 \\
\hline CRS054 & LR-B10 & IL & 103.4 & 44.71 & 125.97 & 2.10 & 2.65 \\
\hline CRS055 & LR-B10 & CRS & 103.4 & 43.34 & 125.20 & 2.12 & 2.57 \\
\hline
\end{tabular}

Table 6.9: CRS tests performed to evaluate the effects of treatment with PC

\begin{tabular}{|c|c|c|c|c|c|c|c|c|}
\hline $\begin{array}{c}\text { Test } \\
\text { Number }\end{array}$ & $\begin{array}{c}\text { Loading } \\
\dot{\varepsilon}(\% / h r)\end{array}$ & $\begin{array}{c}\text { Target } \\
\text { Stress } \\
(\mathrm{kPa})\end{array}$ & $\begin{array}{c}\text { Creep } \\
\text { time } \\
(\mathrm{hrs})\end{array}$ & $\begin{array}{c}\text { Unloading } \\
\dot{\varepsilon} \\
(\% / \mathrm{hr})\end{array}$ & $\begin{array}{c}\text { Final Unloading } \\
\text { Stress (kPa) }\end{array}$ & $\begin{array}{c}\text { Creep } \\
\text { time } \\
(\mathrm{hrs})\end{array}$ & $\begin{array}{c}\text { Reloading } \\
\dot{\varepsilon} \\
(\% / \mathrm{hr})\end{array}$ & $\begin{array}{c}\text { Final } \\
\text { Stress } \\
(\mathrm{kPa})\end{array}$ \\
\hline CRS043 & 0.5 & 1016.3 & 72 & 0.05 & 99.2 & 72 & 0.5 & 1598.5 \\
\hline CRS049 & 0.5 & 1061.8 & 72 & 0.05 & 103.0 & 72 & 0.5 & 2021.6 \\
\hline CRS051 & 0.5 & 1005.9 & 72 & 0.05 & 102.6 & 72 & 0.5 & 2014.6 \\
\hline CRS055 & 0.5 & 4015.3 & 72 & 0.05 & 406.1 & - & 0.5 & 6322.4 \\
\hline
\end{tabular}


Table 6.10: Summary of data from EOP-IL consolidation test performed on 8\% PC treated LR soil (CRS048)

\begin{tabular}{|c|c|c|c|c|c|c|c|c|c|}
\hline Steps & $\begin{array}{c}\sigma_{\mathrm{v}}^{\prime} \\
(\mathrm{kPa})\end{array}$ & $\mathrm{e}_{\mathrm{EOP}}$ & $\begin{array}{c}\mathrm{t}_{\mathrm{EOP}} \\
(\mathrm{min})\end{array}$ & $\begin{array}{c}\Delta \mathrm{u}_{\mathrm{hmax}} \\
(\mathrm{kPa})\end{array}$ & $\begin{array}{c}\mathrm{t}_{\Delta \mathrm{uhmax}} \\
(\mathrm{min})\end{array}$ & $\begin{array}{c}\mathrm{t}_{\mathrm{f}} \\
(\mathrm{min})\end{array}$ & $\begin{array}{c}\mathrm{C}_{\mathrm{v}} \\
\left(\mathrm{cm}^{2} / \mathrm{sec}\right)\end{array}$ & $\begin{array}{c}\mathrm{k} \\
(\mathrm{cm} / \mathrm{sec})\end{array}$ & $\mathrm{C}_{\mathrm{c}}$ \\
\hline $\begin{array}{c}\text { BP } \\
\text { Saturation }\end{array}$ & 1.55 & 3.95 & & & & & & & \\
\hline Step1 & 6.32 & 3.93 & 6.8 & 3.7 & 0.18 & 9.0 & $3.08 \mathrm{E}-02$ & - & - \\
\hline Step2 & 12.1 & 3.91 & 22.0 & 4.6 & 0.23 & 27.0 & $4.12 \mathrm{E}-03$ & - & 0.045 \\
\hline Step3 & 23.9 & 3.87 & 30.3 & 9.9 & 0.1 & 35.1 & $4.58 \mathrm{E}-03$ & $3.65 \mathrm{E}-07$ & 0.160 \\
\hline Step4 & 48.6 & 3.66 & 254.6 & 19.7 & 0.08 & 260.4 & $7.53 \mathrm{E}-04$ & $1.25 \mathrm{E}-07$ & 0.672 \\
\hline Step5 & 98.3 & 3.30 & 355.7 & 49.6 & 0.23 & 380.8 & $3.30 \mathrm{E}-04$ & $4.70 \mathrm{E}-08$ & 1.170 \\
\hline Step6 & 196.1 & 2.89 & 383.0 & 94.6 & 0.57 & 500.6 & $2.51 \mathrm{E}-04$ & $2.09 \mathrm{E}-08$ & 1.368 \\
\hline Step7 & 393.5 & 2.47 & 437.7 & 188.4 & 0.4 & 480.6 & $1.86 \mathrm{E}-04$ & $7.91 \mathrm{E}-09$ & 1.400 \\
\hline Step8 & 786.9 & 2.04 & 542.1 & 377.6 & 1.0 & 600.6 & $1.21 \mathrm{E}-04$ & $2.58 \mathrm{E}-09$ & 1.407 \\
\hline Step9 & 1572.7 & 1.67 & 605.1 & 719.6 & 1.3 & 23625.7 & $8.43 \mathrm{E}-05$ & $8.00 \mathrm{E}-10$ & 1.251 \\
\hline
\end{tabular}

Table 6.11: Summary of data from EOP-IL consolidation test performed on 18.7\% PC treated LR soil (CRS042)

\begin{tabular}{|c|c|c|c|c|c|c|c|c|c|}
\hline Steps & $\begin{array}{c}\sigma_{\mathrm{v}}^{\prime} \\
(\mathrm{kPa})\end{array}$ & $\mathrm{e}_{\text {EOP }}$ & $\begin{array}{c}\mathrm{t}_{\mathrm{EOP}} \\
(\mathrm{min})\end{array}$ & $\begin{array}{c}\Delta \mathrm{u}_{\mathrm{hmax}} \\
(\mathrm{kPa})\end{array}$ & $\begin{array}{c}\mathrm{t}_{\Delta \text { uhmax }} \\
(\mathrm{min})\end{array}$ & $\begin{array}{c}\mathrm{t}_{\mathrm{f}} \\
(\mathrm{min})\end{array}$ & $\begin{array}{c}\mathrm{C}_{\mathrm{v}} \\
\left(\mathrm{cm}^{2} / \mathrm{sec}\right)\end{array}$ & $\begin{array}{c}\mathrm{k} \\
(\mathrm{cm} / \mathrm{sec})\end{array}$ & $\mathrm{C}_{\mathrm{c}}$ \\
\hline $\begin{array}{c}\text { BP } \\
\text { Saturation }\end{array}$ & 0.2 & 3.74 & & & & & & & \\
\hline Step1 & 5.87 & 3.68 & 14.2 & 5.67 & 0.28 & 20.1 & $1.02 \mathrm{E}-02$ & - & - \\
\hline Step2 & 11.9 & 3.67 & 6.4 & 4.19 & 0.20 & 17.0 & $1.80 \mathrm{E}-02$ & $9.24 \mathrm{E}-07$ & 0.049 \\
\hline Step3 & 24.1 & 3.64 & 10.9 & 8.98 & 0.15 & 17.0 & $1.48 \mathrm{E}-02$ & $6.86 \mathrm{E}-07$ & 0.089 \\
\hline Step4 & 48.9 & 3.58 & 22.2 & 19.6 & 0.27 & 41.1 & $7.33 \mathrm{E}-03$ & $3.87 \mathrm{E}-07$ & 0.205 \\
\hline Step5 & 98.3 & 3.33 & 78.0 & 42.2 & 0.17 & 90.2 & $2.02 \mathrm{E}-03$ & $2.13 \mathrm{E}-07$ & 0.831 \\
\hline Step6 & 196.4 & 2.99 & 79.4 & 88.4 & 0.25 & 100.2 & $1.60 \mathrm{E}-03$ & $1.14 \mathrm{E}-07$ & 1.125 \\
\hline Step7 & 394.1 & 2.59 & 102.2 & 176.4 & 0.08 & 110.2 & $1.07 \mathrm{E}-03$ & $4.50 \mathrm{E}-08$ & 1.325 \\
\hline Step8 & 785.2 & 2.19 & 109.1 & 352.4 & 0.21 & 140.4 & $7.45 \mathrm{E}-04$ & $1.56 \mathrm{E}-08$ & 1.323 \\
\hline Step9 & 1557.8 & 1.80 & 153.1 & 694.8 & 0.38 & 26808 & $4.47 \mathrm{E}-04$ & $4.49 \mathrm{E}-09$ & 1.271 \\
\hline
\end{tabular}


Table 6.12: Summary of data from EOP-IL consolidation test performed on 51.4\% PC treated LR soil (CRS050)

\begin{tabular}{|c|c|c|c|c|c|c|c|c|c|}
\hline Steps & $\begin{array}{c}\sigma_{\mathrm{v}}^{\prime} \\
(\mathrm{kPa})\end{array}$ & $\mathrm{e}_{\mathrm{EOP}}$ & $\begin{array}{c}\mathrm{t}_{\mathrm{EOP}} \\
(\mathrm{min})\end{array}$ & $\begin{array}{c}\Delta \mathrm{u}_{\mathrm{hmax}} \\
(\mathrm{kPa})\end{array}$ & $\begin{array}{c}\mathrm{t}_{\Delta \mathrm{uhmax}} \\
(\mathrm{min})\end{array}$ & $\begin{array}{c}\mathrm{t}_{\mathrm{f}} \\
(\mathrm{min})\end{array}$ & $\begin{array}{c}\mathrm{C}_{\mathrm{v}} \\
\left(\mathrm{cm}^{2} / \mathrm{sec}\right)\end{array}$ & $\begin{array}{c}\mathrm{k} \\
(\mathrm{cm} / \mathrm{sec})\end{array}$ & $\mathrm{C}_{\mathrm{c}}$ \\
\hline $\begin{array}{c}\text { BP } \\
\text { Saturation }\end{array}$ & 1.28 & 3.66 & & & & & & & \\
\hline Step1 & 5.62 & 3.65 & 0.20 & 1.62 & 0.08 & 5.0 & $3.31 \mathrm{E}-01$ & - & - \\
\hline Step2 & 12.4 & 3.65 & 0.52 & 2.00 & 0.10 & 6.0 & $1.98 \mathrm{E}-01$ & - & - \\
\hline Step3 & 24.6 & 3.65 & 1.43 & 8.01 & 0.08 & 5.0 & $1.15 \mathrm{E}-01$ & $6.43 \mathrm{E}-07$ & 0.011 \\
\hline Step4 & 49.3 & 3.64 & 1.47 & 25.4 & 0.08 & 5.0 & $1.10 \mathrm{E}-01$ & $6.51 \mathrm{E}-07$ & 0.027 \\
\hline Step5 & 97.7 & 3.63 & 0.48 & 34.6 & 0.07 & 4.0 & $2.72 \mathrm{E}-01$ & $5.71 \mathrm{E}-07$ & 0.030 \\
\hline Step6 & 199.1 & 3.62 & 0.50 & 38.1 & 0.05 & 6.0 & $2.84 \mathrm{E}-01$ & $6.68 \mathrm{E}-07$ & 0.055 \\
\hline Step7 & 395.8 & 3.44 & 17.52 & 109.0 & 0.22 & 19.0 & $9.17 \mathrm{E}-03$ & $2.75 \mathrm{E}-08$ & 0.601 \\
\hline Step8 & 788.9 & 3.01 & 28.68 & 322.8 & 0.12 & 30.0 & $4.87 \mathrm{E}-03$ & $1.24 \mathrm{E}-08$ & 1.435 \\
\hline Step9 & 1574.2 & 2.49 & 38.72 & 675.4 & 0.12 & 31701 & $2.73 \mathrm{E}-03$ & $3.87 \mathrm{E}-08$ & 1.719 \\
\hline
\end{tabular}

Table 6.13: Summary of data from EOP-IL consolidation test performed on 103.4\% PC treated LR soil (CRS054)

\begin{tabular}{|c|c|c|c|c|c|c|c|c|c|}
\hline Steps & $\begin{array}{c}\sigma_{\mathrm{v}}^{\prime} \\
(\mathrm{kPa})\end{array}$ & $\mathrm{e}_{\mathrm{EOP}}$ & $\begin{array}{c}\mathrm{t}_{\mathrm{EOP}} \\
(\mathrm{min})\end{array}$ & $\begin{array}{c}\Delta \mathrm{u}_{\mathrm{hmax}} \\
(\mathrm{kPa})\end{array}$ & $\begin{array}{c}\mathrm{t}_{\Delta \mathrm{uhmax}} \\
(\mathrm{min})\end{array}$ & $\begin{array}{c}\mathrm{t}_{\mathrm{f}} \\
(\mathrm{min})\end{array}$ & $\begin{array}{c}\mathrm{C}_{\mathrm{v}} \\
\left(\mathrm{cm}^{2} / \mathrm{sec}\right)\end{array}$ & $\begin{array}{c}\mathrm{k} \\
(\mathrm{cm} / \mathrm{sec})\end{array}$ & $\mathrm{C}_{\mathrm{c}}$ \\
\hline $\begin{array}{c}\text { BP } \\
\text { Saturation }\end{array}$ & 3.5 & 2.65 & & & & & & & \\
\hline Step1 & 5.6 & - & - & - & - & 4.0 & - & - & - \\
\hline Step2 & 10.6 & - & - & - & - & 5.0 & - & - & - \\
\hline Step3 & 22.2 & - & - & - & - & 6.0 & - & - & - \\
\hline Step4 & 43.8 & - & - & - & - & 4.0 & - & - & - \\
\hline Step5 & 86.5 & 2.64 & 0.20 & 1.8 & 0.07 & 9.0 & $2.14 \mathrm{E}-01$ & $1.05 \mathrm{E}-06$ & - \\
\hline Step6 & 174.2 & 2.62 & 0.27 & 32.1 & 0.05 & 6.0 & $4.26 \mathrm{E}-01$ & $2.19 \mathrm{E}-06$ & 0.055 \\
\hline Step7 & 348.6 & 2.61 & 0.35 & 33.5 & 0.05 & 5.0 & $4.23 \mathrm{E}-01$ & $7.42 \mathrm{E}-07$ & 0.038 \\
\hline Step8 & 698.1 & 2.58 & 2.43 & 65.0 & 0.07 & 6.0 & $1.40 \mathrm{E}-01$ & $2.58 \mathrm{E}-07$ & 0.080 \\
\hline Step9 & 1387.1 & 2.42 & 24.5 & 332.7 & 0.5 & 22942 & $1.22 \mathrm{E}-02$ & $7.71 \mathrm{E}-08$ & 0.546 \\
\hline
\end{tabular}


Table 6.14: Summary of the results from CRS and IL consolidation tests performed to evaluate the effects of treatment with PC

\begin{tabular}{|c|c|c|c|c|c|c|c|c|c|}
\hline \multirow{2}{*}{ Test No. } & \multirow{2}{*}{$\%$ PC } & \multirow{2}{*}{$\begin{array}{c}\text { Test } \\
\text { type }\end{array}$} & $\begin{array}{c}\sigma_{\mathrm{p}}^{\prime} \\
(\mathrm{kPa})\end{array}$ & \multicolumn{3}{|c|}{$\mathrm{C}_{\mathrm{c}}$} & $\mathrm{Cr}$ & $\mathrm{C}_{\mathrm{k}} / \mathrm{e}_{\mathrm{o}}$ & $\mathrm{C}_{\mathrm{k}} / \mathrm{C}_{\mathrm{c}}$ \\
\cline { 5 - 11 } & & & $2-4 \sigma_{\mathrm{p}}^{\prime}$ & $4-8 \sigma_{\mathrm{p}}^{\prime}$ & $2-16 \sigma_{\mathrm{p}}^{\prime}$ & $2-4$ OCR & $2-16 \sigma_{\mathrm{p}}^{\prime}$ & $2-16 \sigma_{\mathrm{p}}^{\prime}$ \\
\hline CRS037, & \multirow{2}{*}{ RC } & & $\begin{array}{c}50.23 \\
\pm 6.24\end{array}$ & $\begin{array}{c}1.28 \\
\pm 0.03\end{array}$ & $\begin{array}{c}1.36 \\
\pm 0.05\end{array}$ & $\begin{array}{c}1 /(4.09 \\
\pm 0.39)\end{array}$ & $\begin{array}{c}0.11 \\
\pm 0.06\end{array}$ & $\begin{array}{c}1 /(4.09 \\
\pm 0.39)\end{array}$ & $\begin{array}{c}1 /(1.47 \\
\pm 0.06)\end{array}$ \\
\hline CRS042 & 18.7 & IL & 94.40 & 1.33 & 1.32 & $1 / 4.45$ & 0.01 & $1 / 4.45$ & $1 / 1.56$ \\
\hline CRS043 & 18.7 & CRS & 94.83 & 1.33 & 1.34 & $1 / 4.30$ & 0.01 & $1 / 4.30$ & $1 / 1.57$ \\
\hline CRS048 & 8.0 & IL & 70.21 & \multicolumn{7}{|c|}{1.38} & & 0.02 & $1 / 4.58$ & $1 / 1.57$ \\
\hline CRS049 & 8.0 & CRS & 79.43 & 1.53 & 1.46 & $1 / 4.10$ & 0.02 & $1 / 4.10$ & $1 / 1.58$ \\
\hline CRS050 & 51.4 & IL & 404.44 & 1.72 & & $1 / 3.68$ & 0.006 & $1 / 3.68$ & $1 / 1.69$ \\
\hline CRS051 & 51.4 & CRS & 402.06 & 1.68 & & $1 / 4.64$ & 0.006 & $1 / 4.64$ & $1 / 2.17$ \\
\hline CRS054 & 103.4 & IL & & & & & - & & \\
\hline CRS055 & 103.4 & CRS & 1249.83 & 1.24 & & $1 / 4.06$ & - & $1 / 4.06$ & $1 / 1.96$ \\
\hline
\end{tabular}




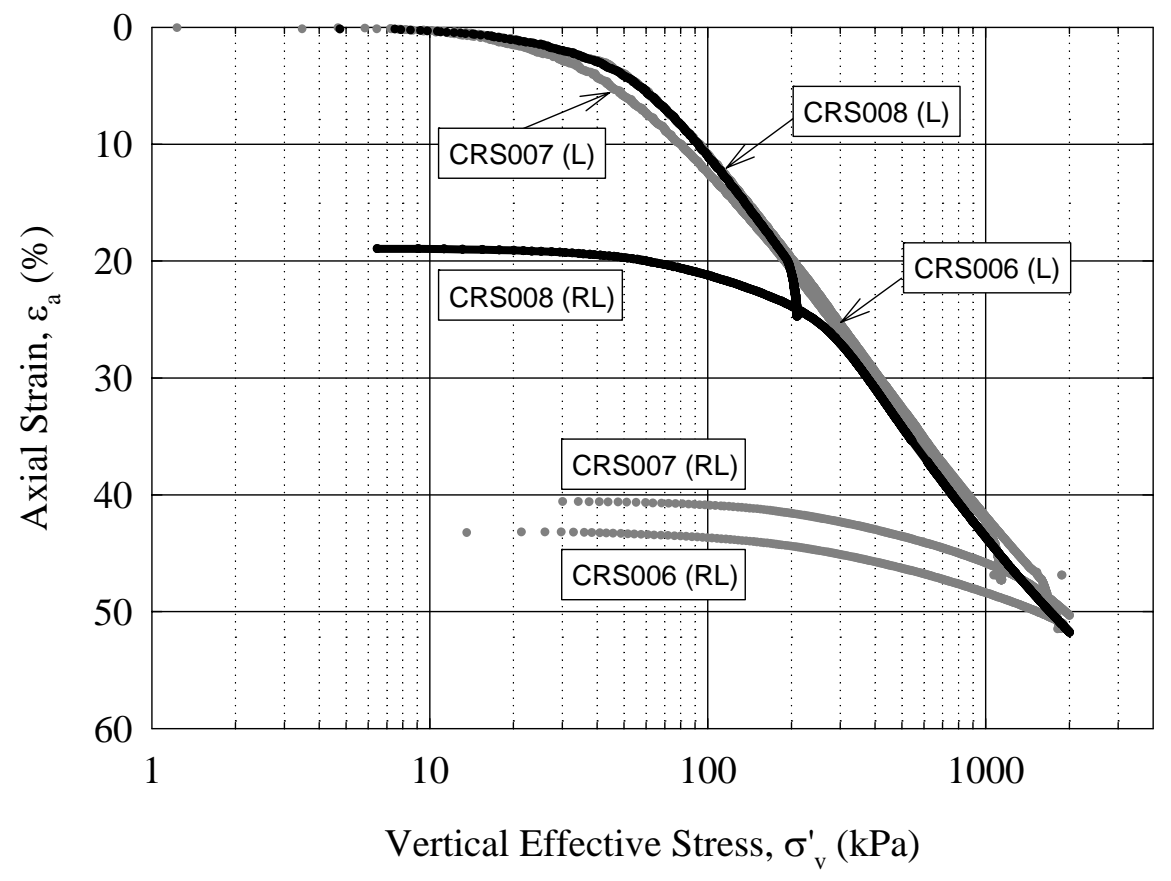

Figure 6.1: Compression curves from three CRS consolidation tests performed on reconstituted LR soil

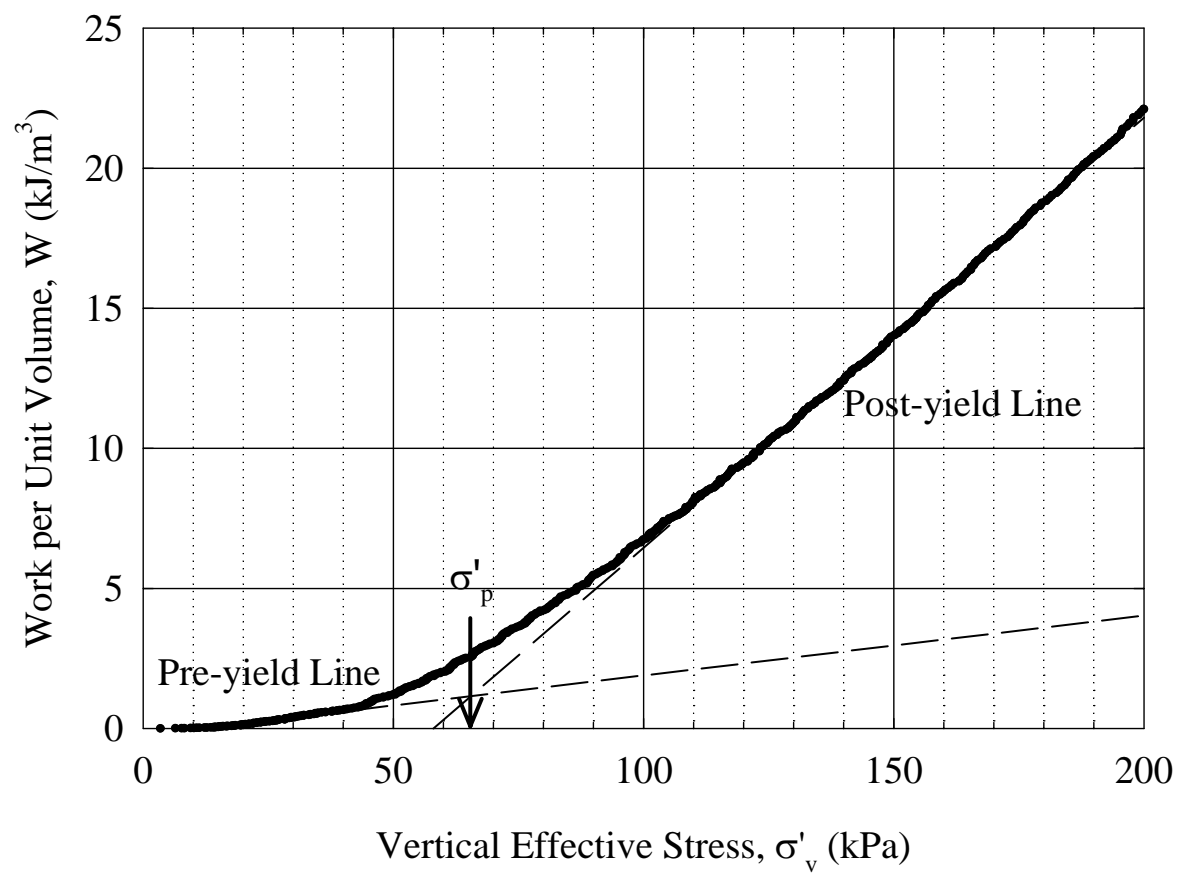

Figure 6.2: Determination of preconsolidation pressure with the strain energy method (CRS006) 


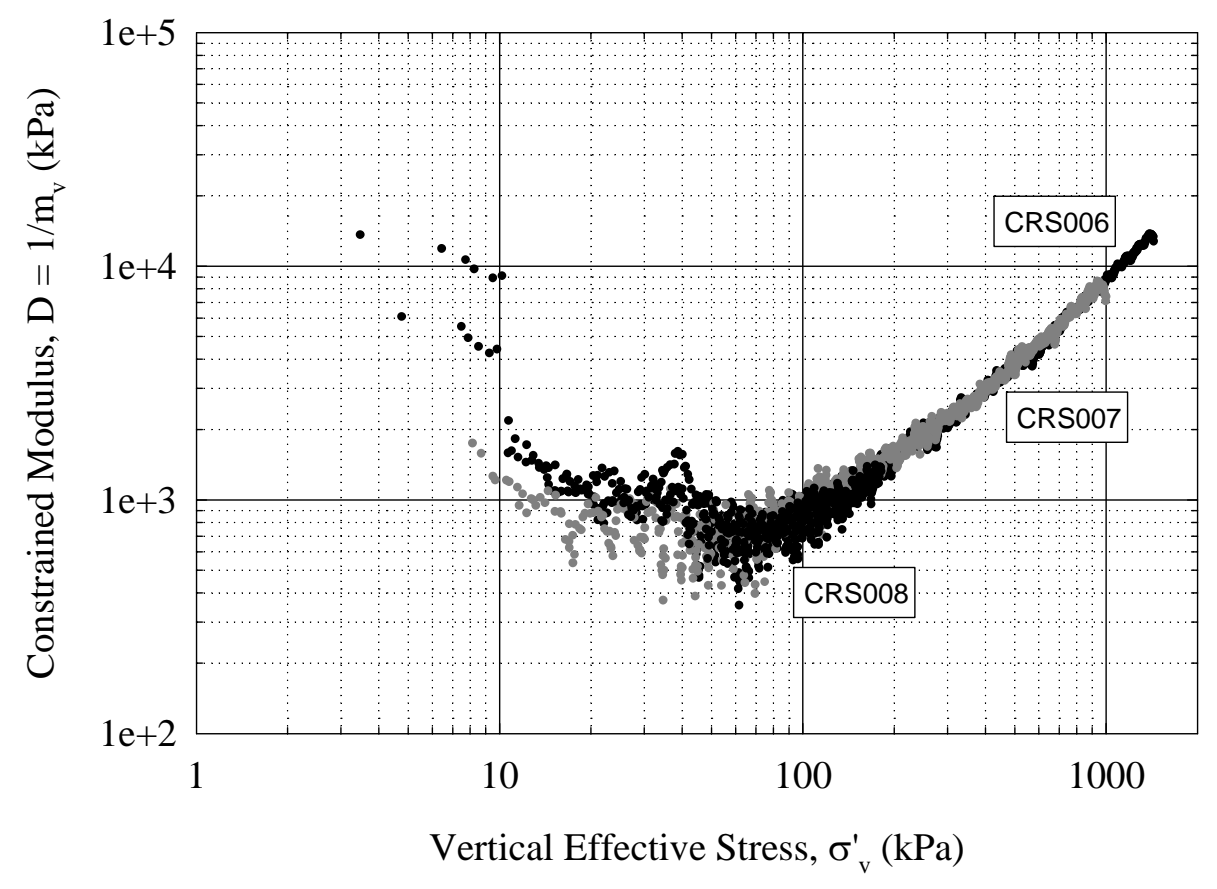

Figure 6.3: Change of the constrained modulus $\left(\mathrm{D}=1 / \mathrm{m}_{\mathrm{v}}\right)$ during loading

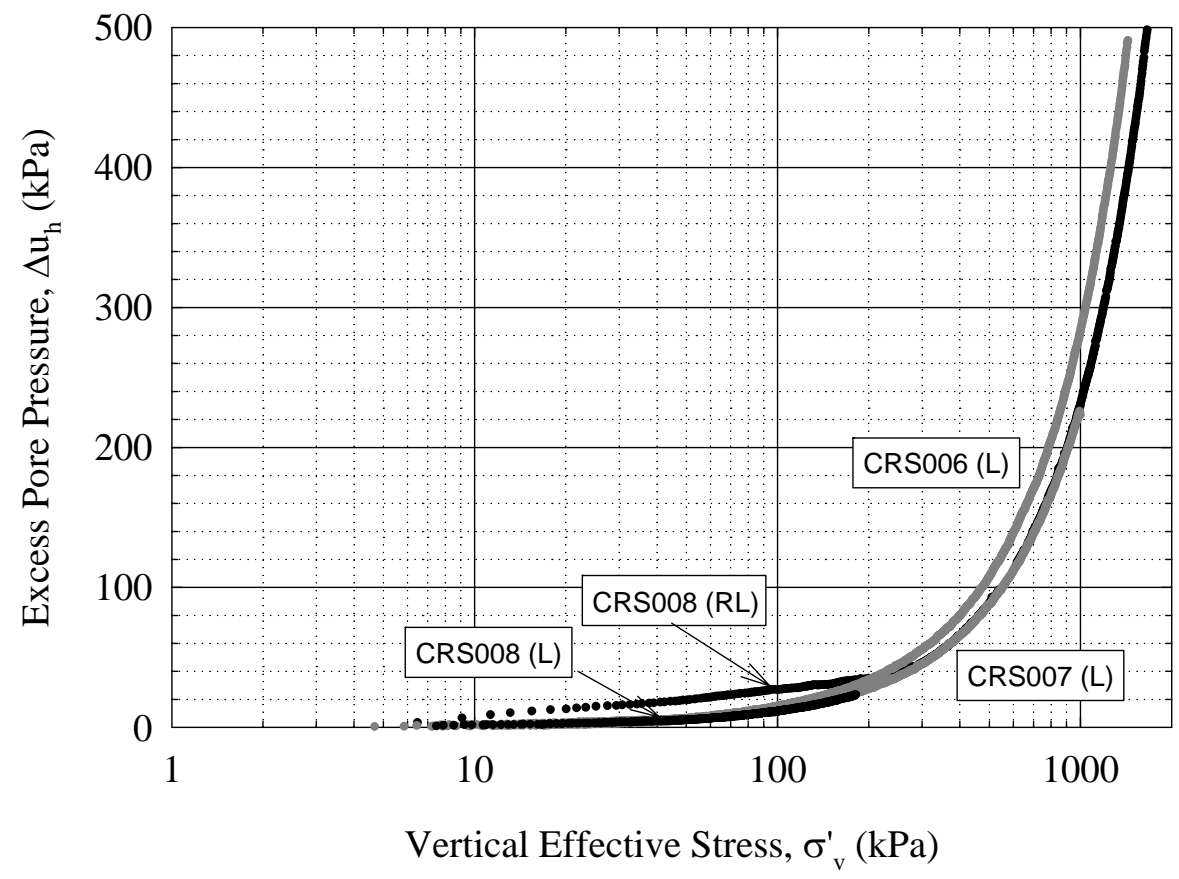

Figure 6.4: Generation of excess pore pressure $\left(\Delta \mathrm{u}_{\mathrm{h}}\right)$ during loading and reloading 


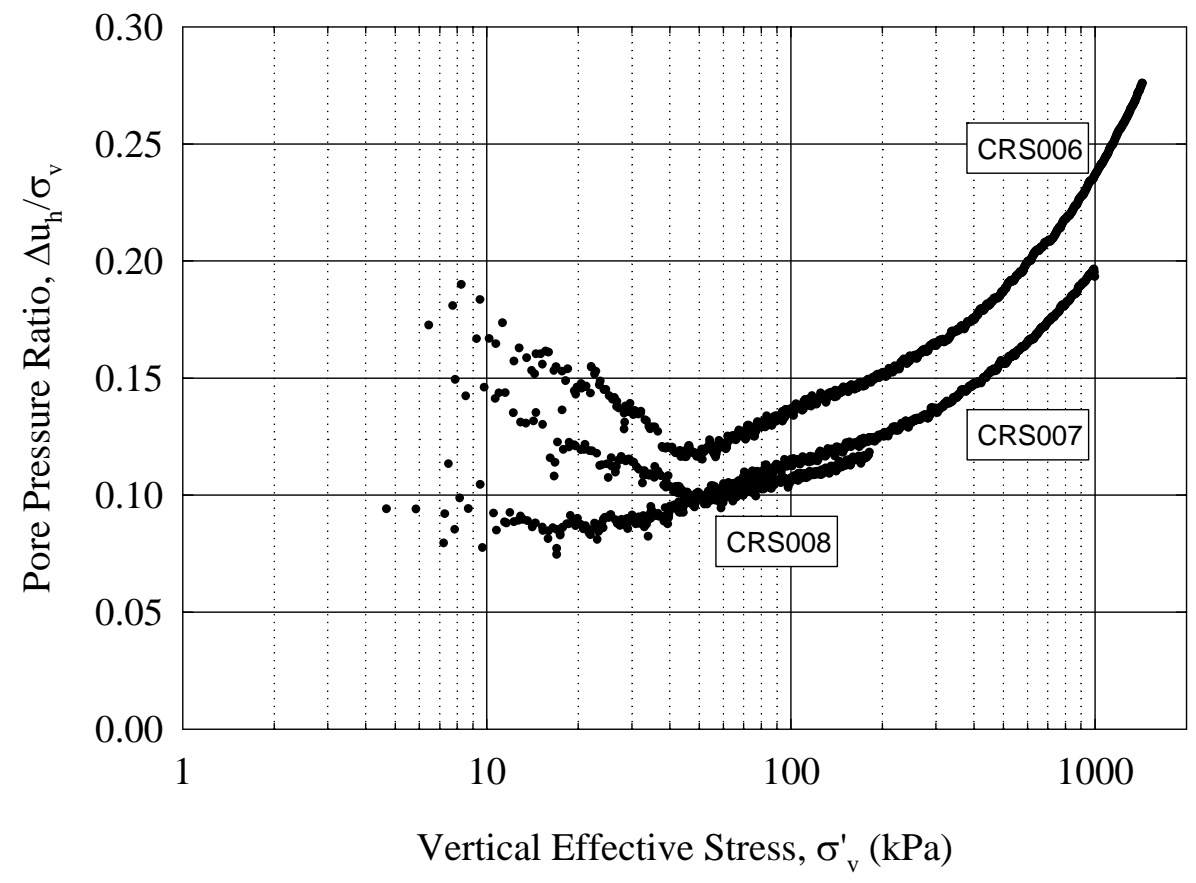

Figure 6.5: Plot of the pore pressure ratio vs. vertical effective stress

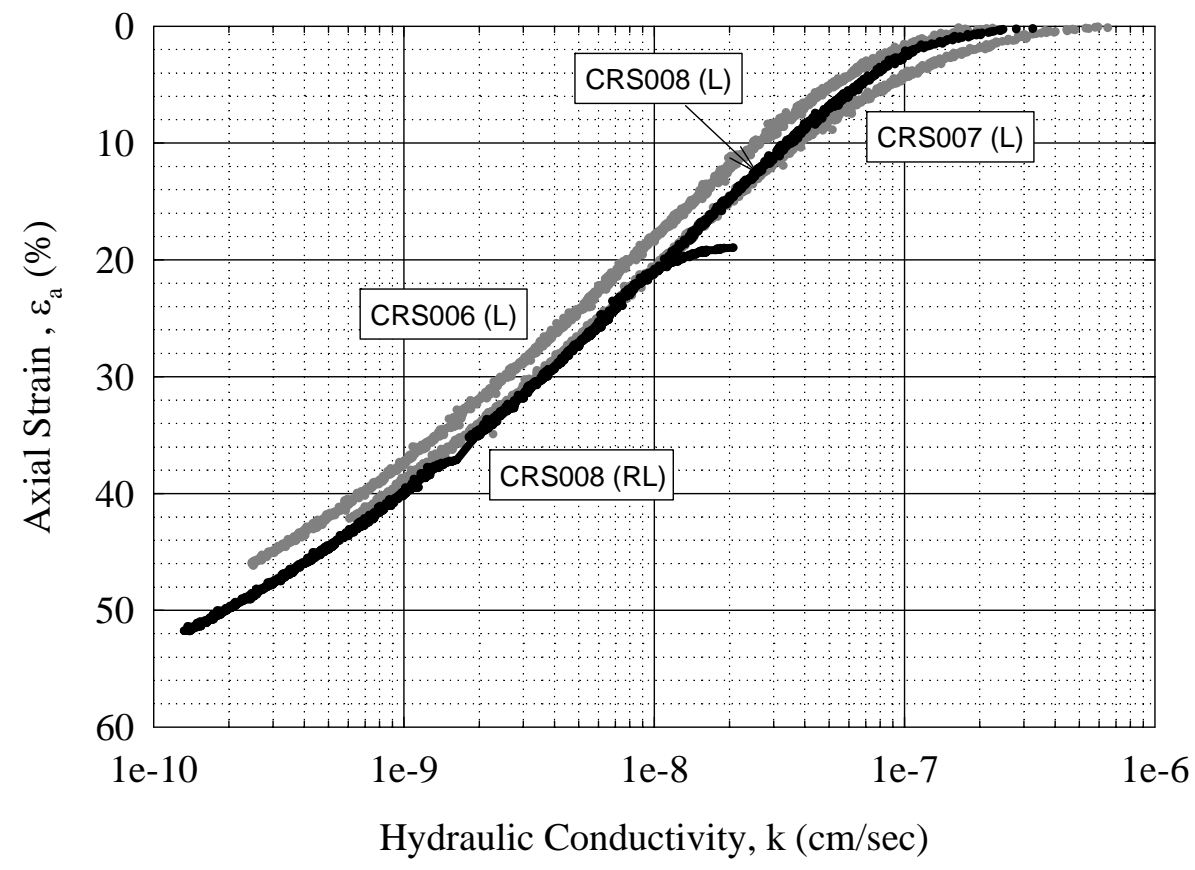

Figure 6.6: Plot of the hydraulic conductivity (k) vs. axial strain 


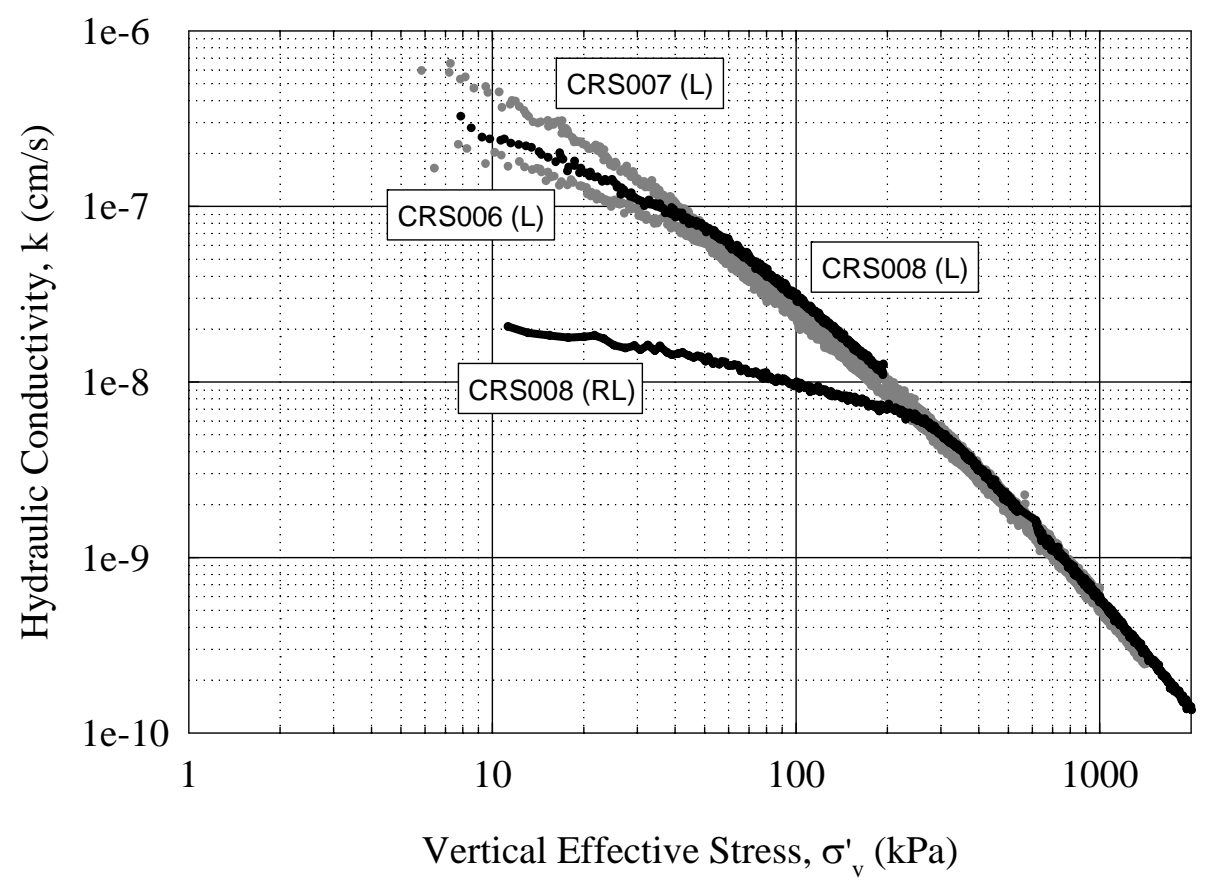

Figure 6.7: Plot of the hydraulic conductivity (k) vs. vertical effective stress 

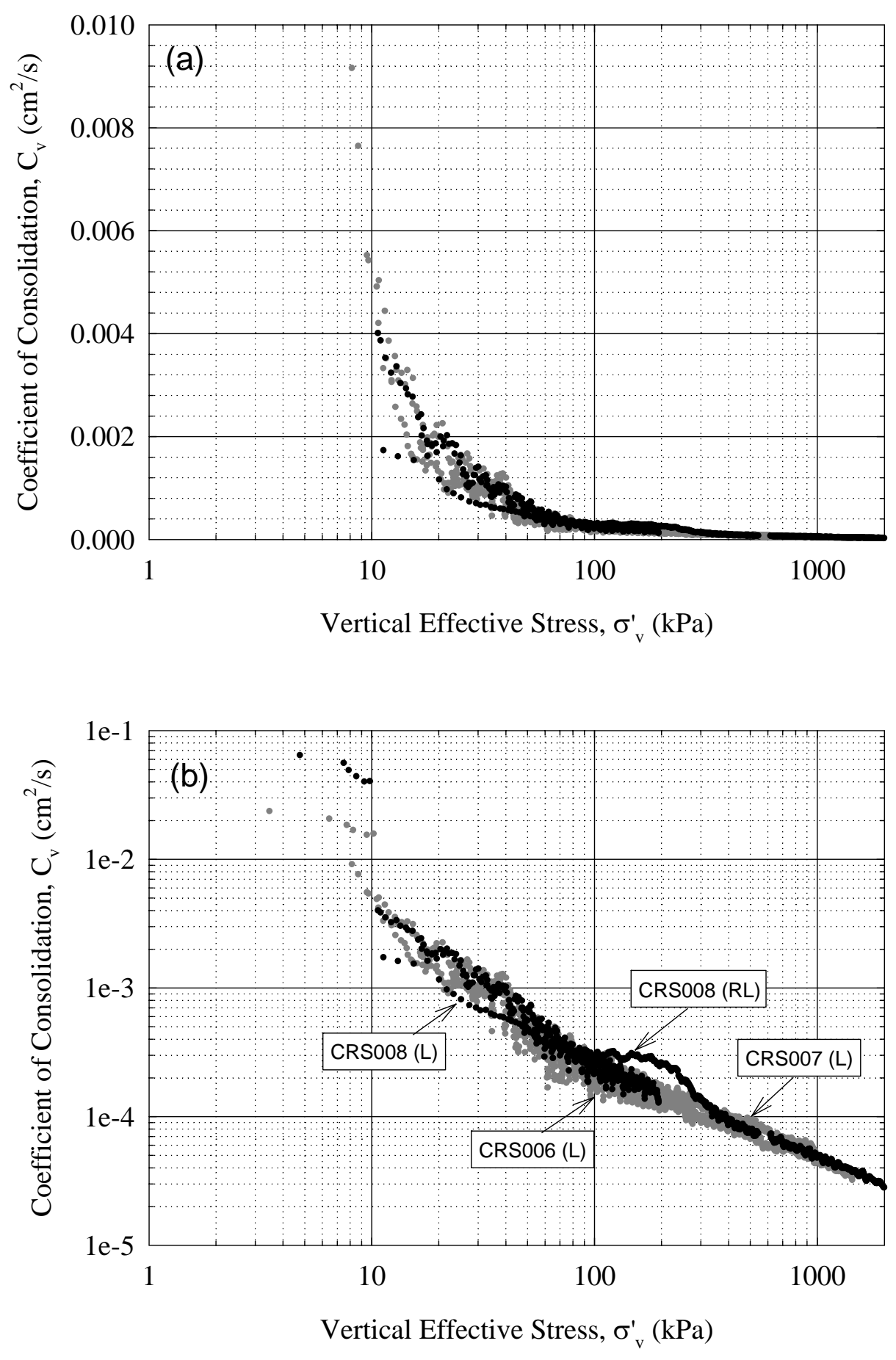

Figure 6.8: Coefficient of consolidation $\left(\mathrm{C}_{\mathrm{v}}\right)$ as a function of the vertical effective stress: (a) semi-log scale and (b) log-log scale 

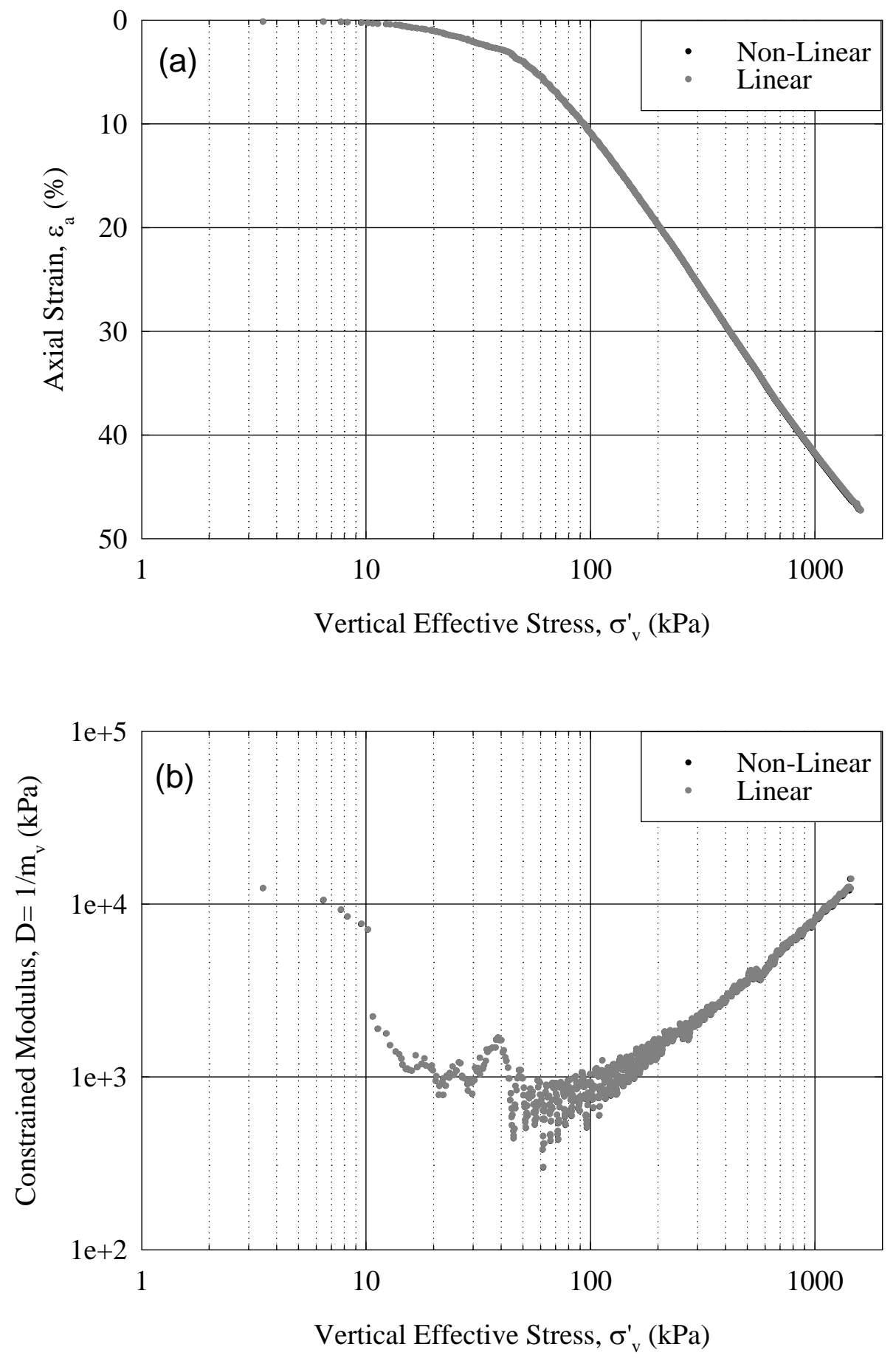

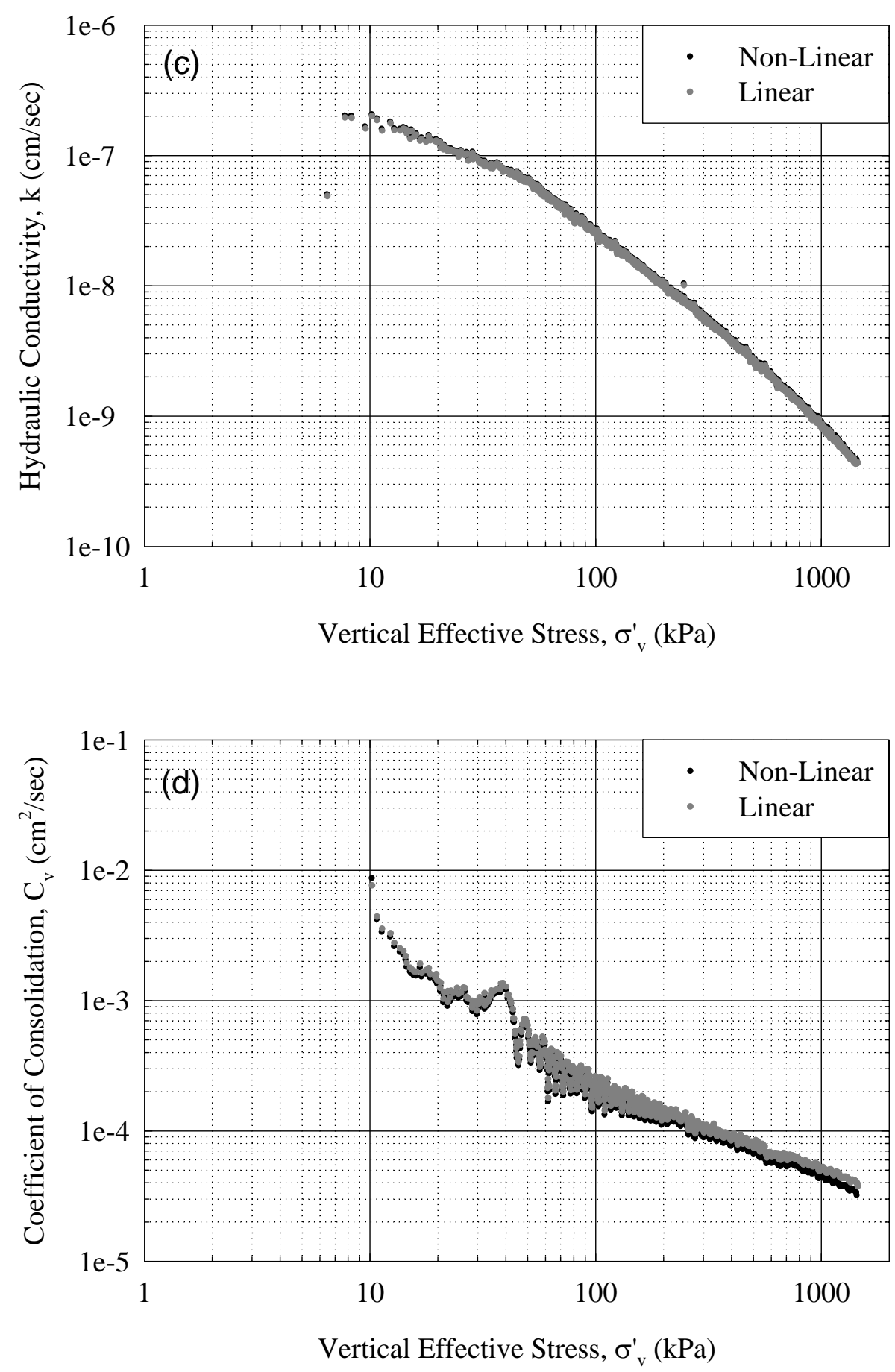

Figure 6.9: Comparison of linear vs. non-linear theory (CRS006): (a) compression curve, (b) the constrained modulus, (c) the hydraulic conductivity and (d) the coefficient of consolidation 


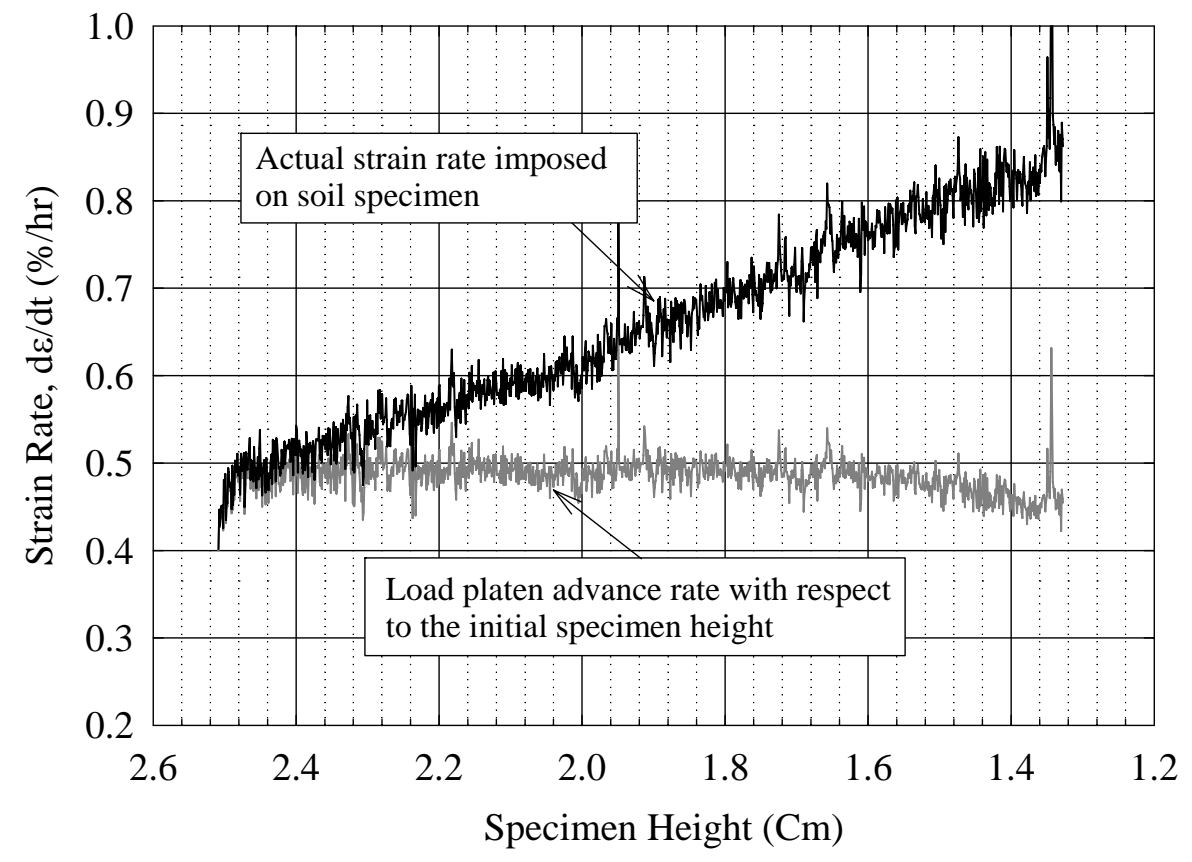

Figure 6.10: Increase of the actual strain rate during CRS consolidation test (CRS006)

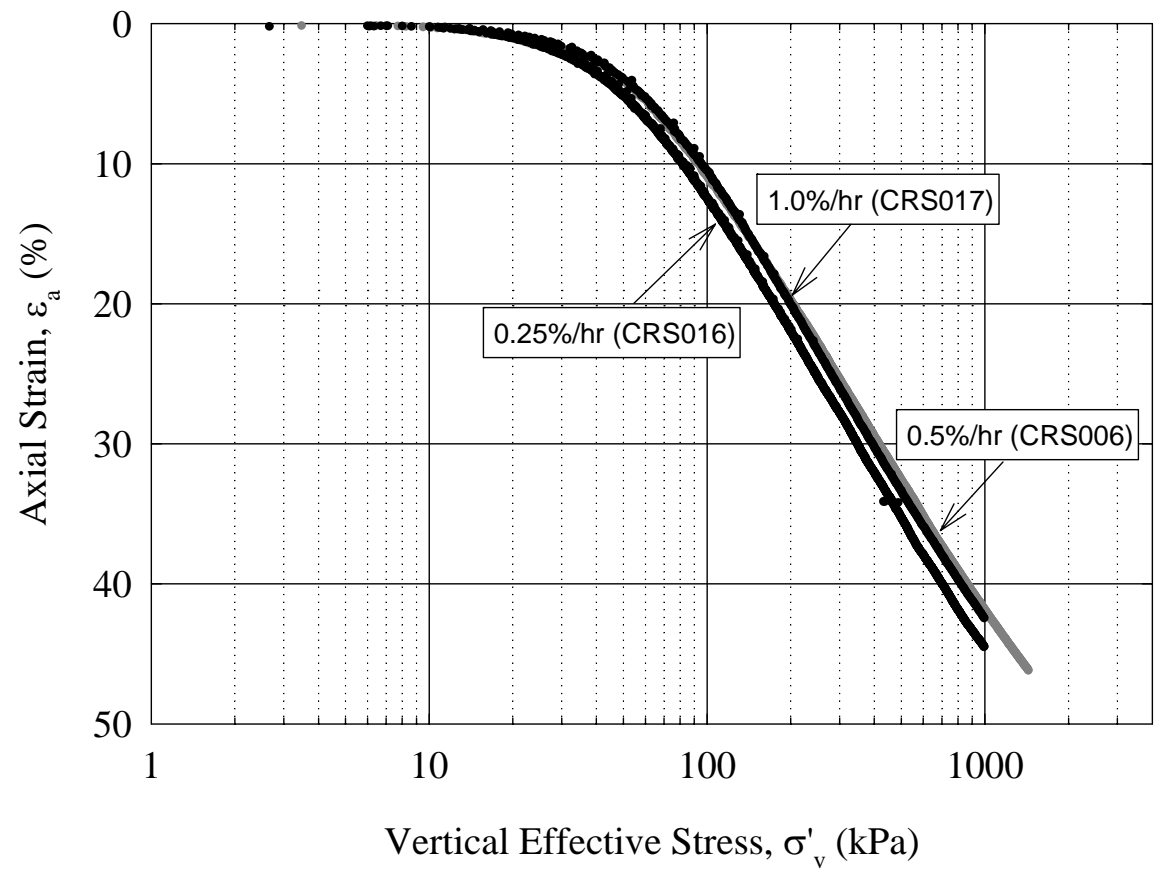

Figure 6.11: Strain rate effect on the compression curve 


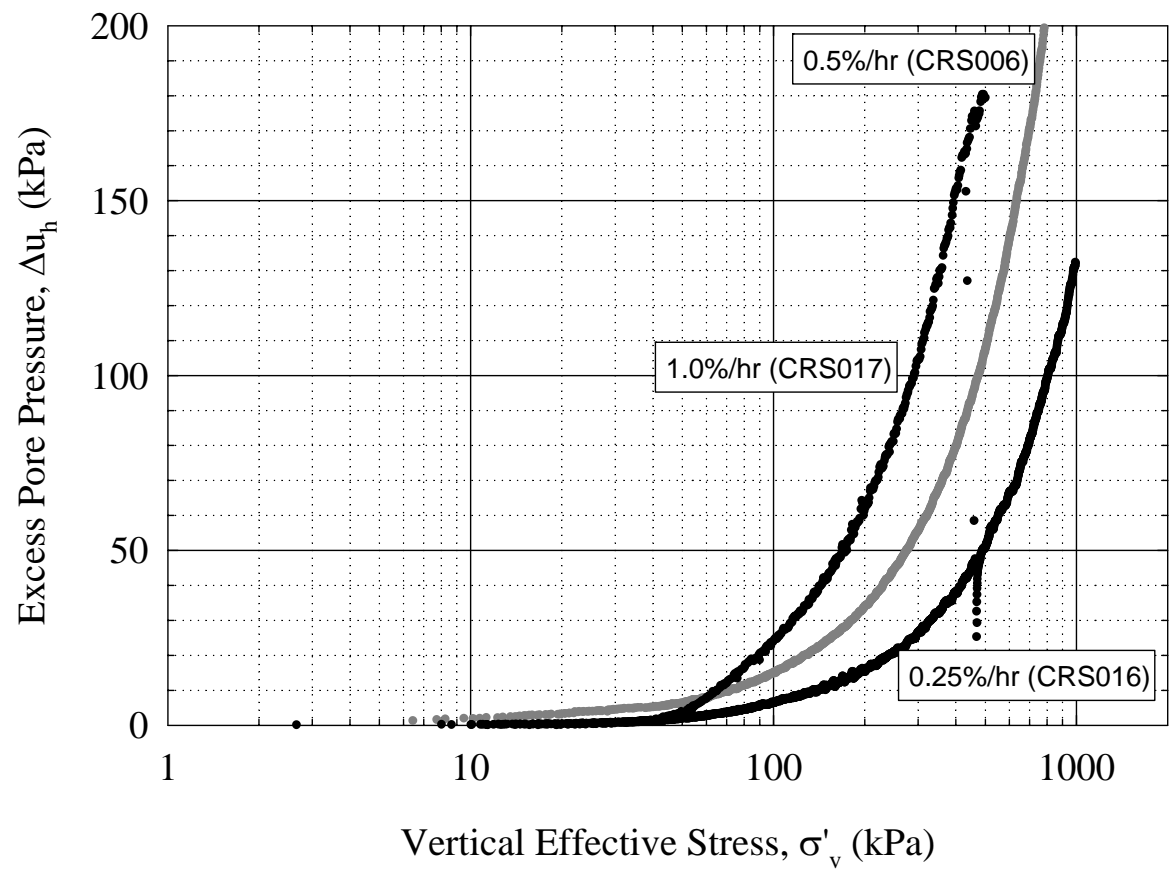

Figure 6.12: Strain rate effect on generation of excess pore pressure

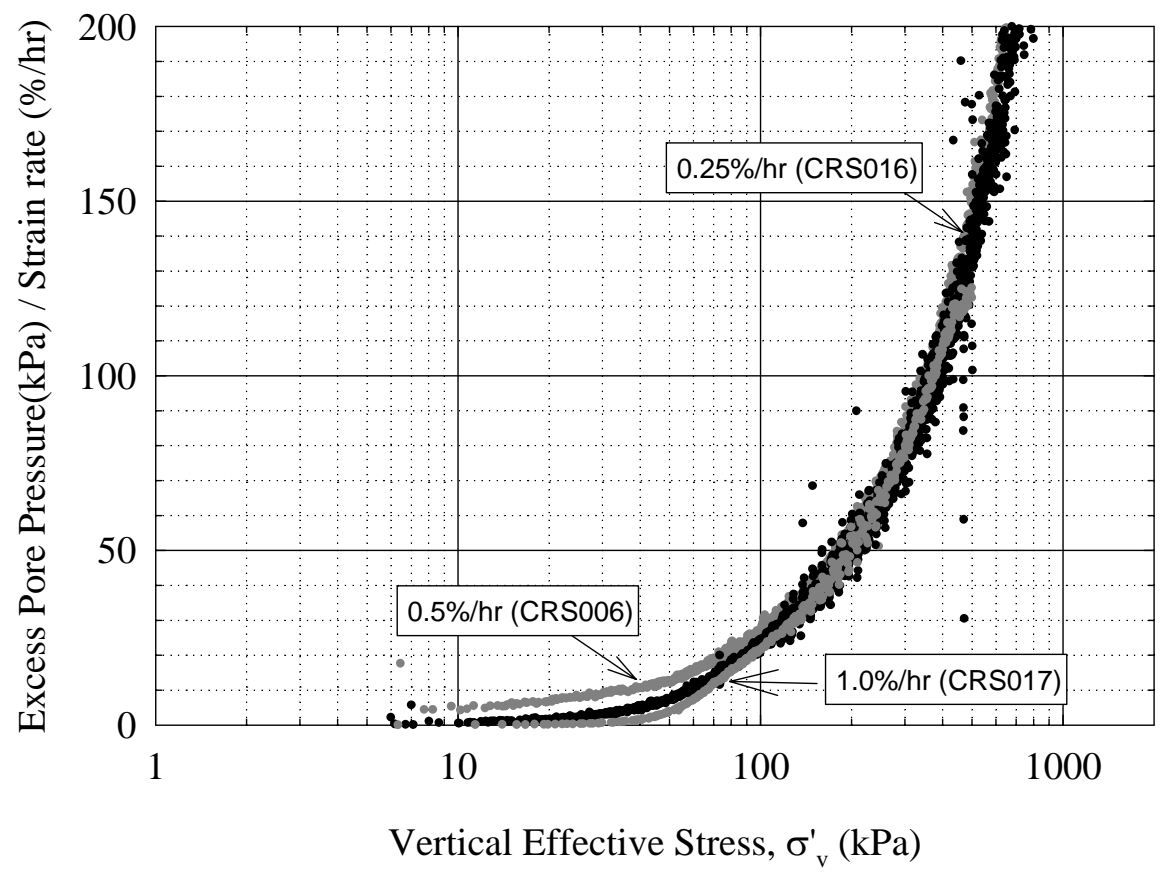

Figure 6.13: Normalization of excess pore pressure with actual strain rate 


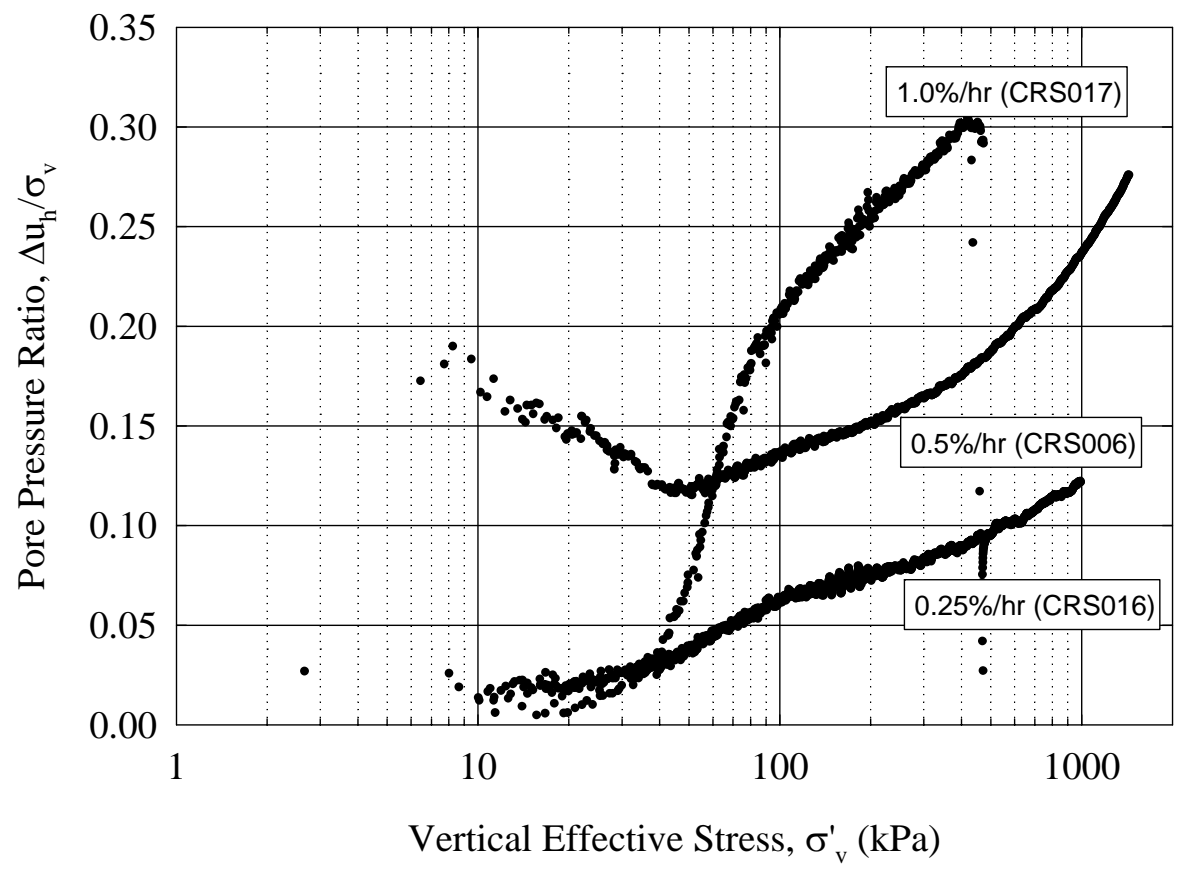

Figure 6.14: Strain rate effect on pore pressure ratio

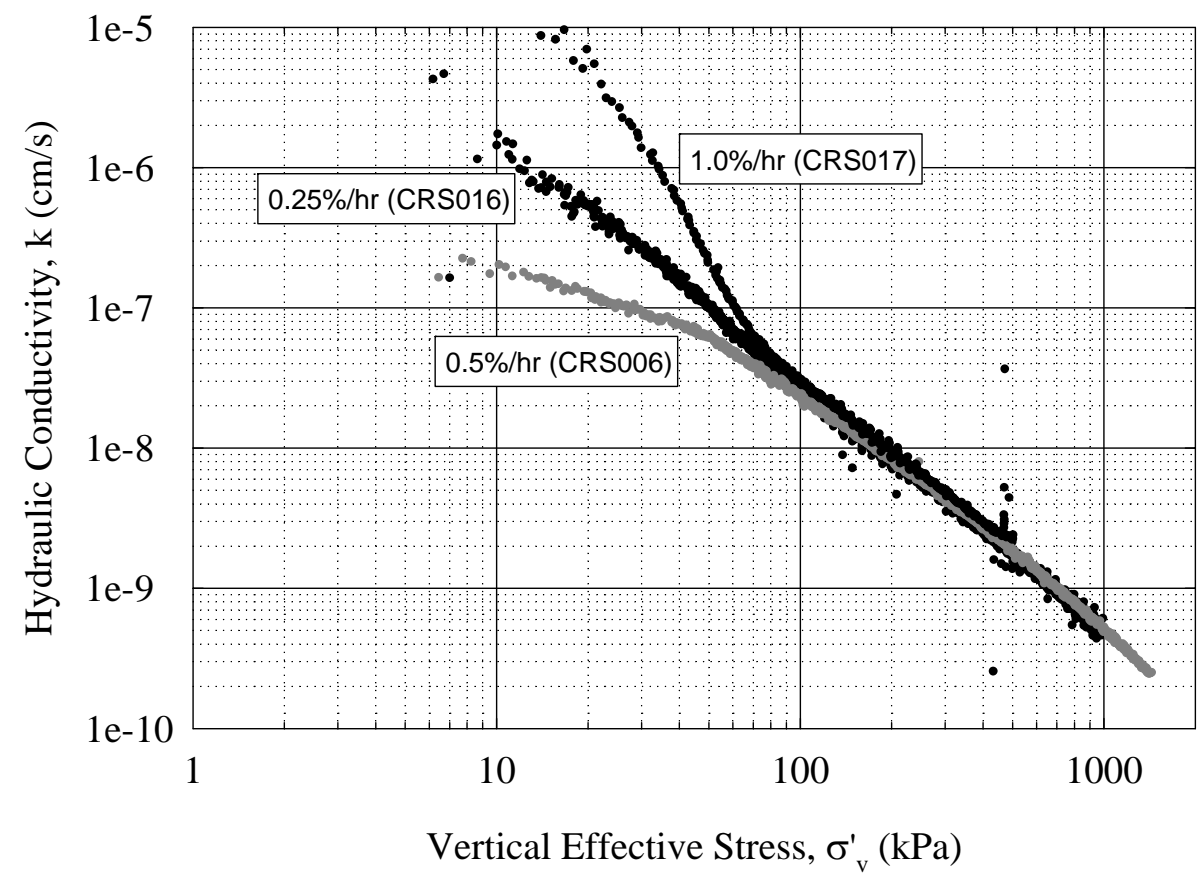

Figure 6.15: Strain rate effect of the hydraulic conductivity 


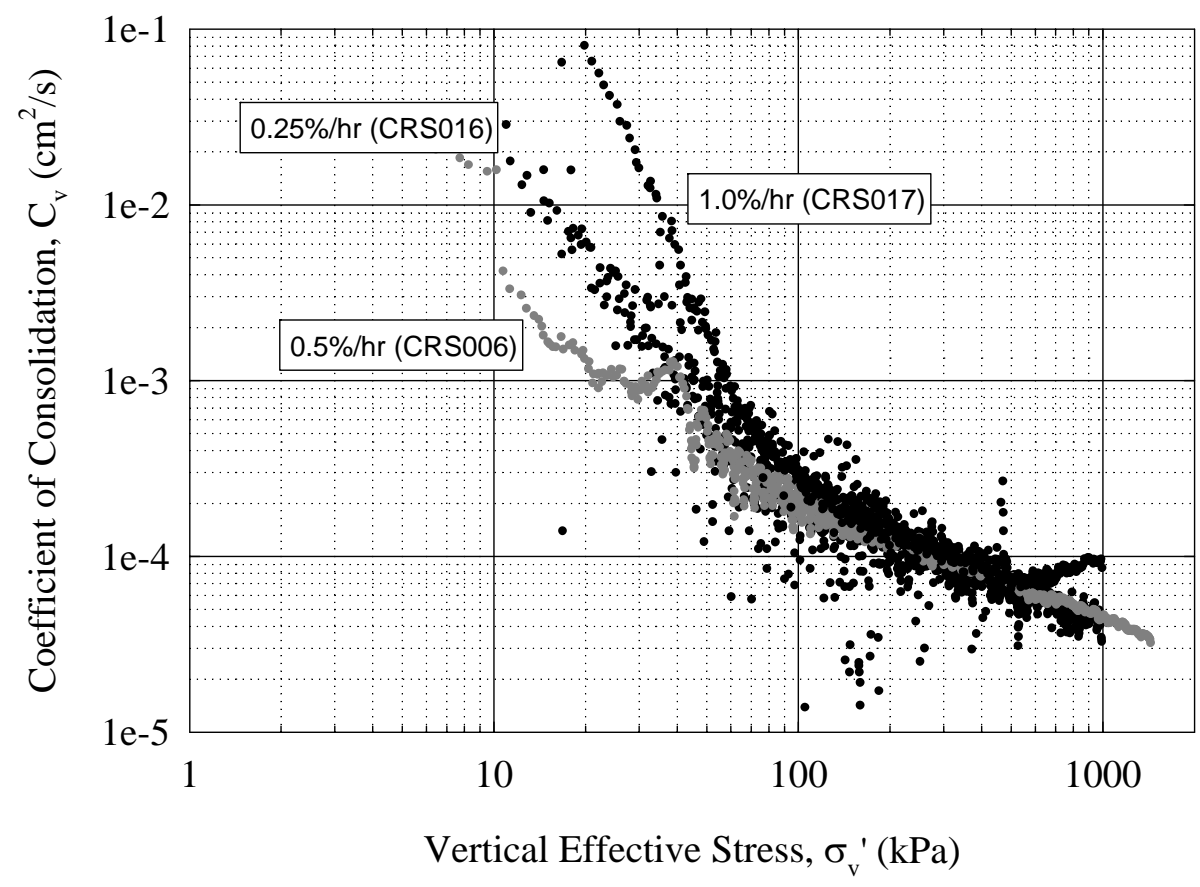

Figure 6.16: Strain rate effect of the coefficient of consolidation

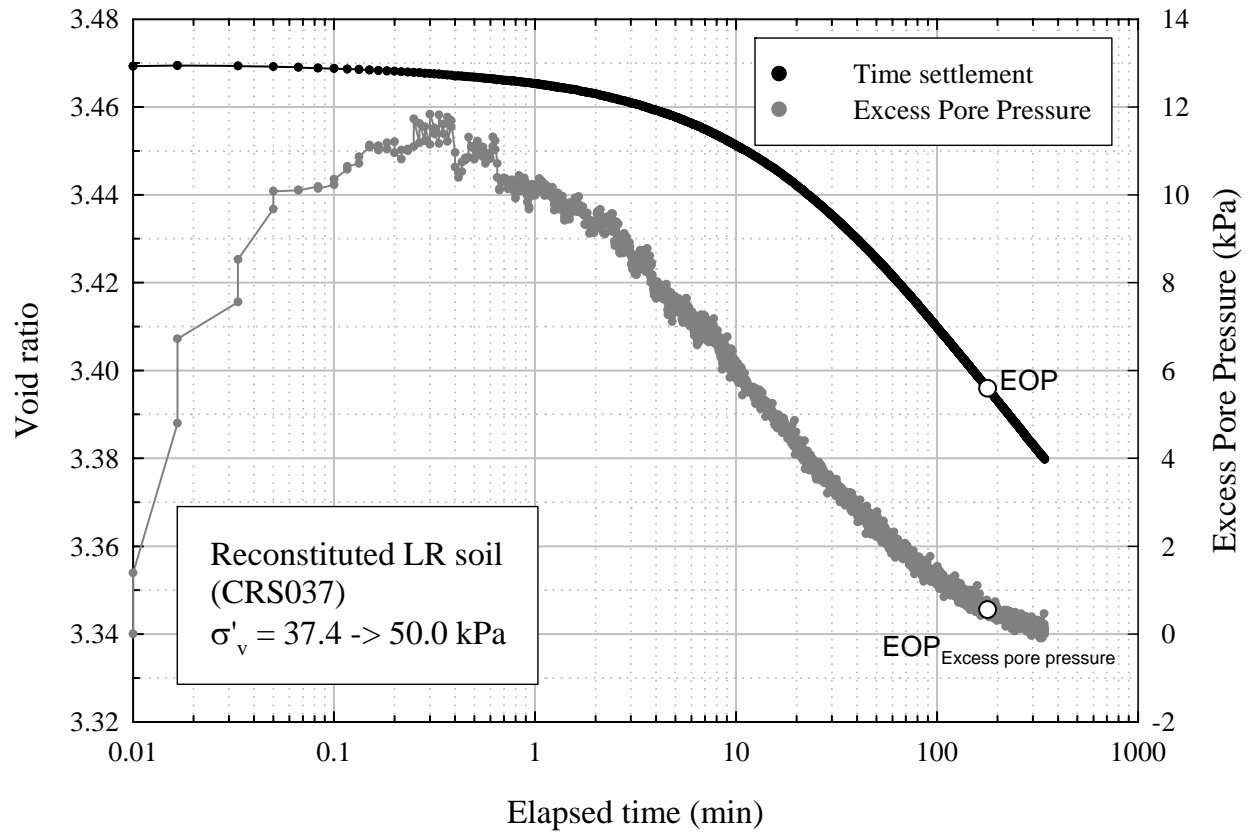

Figure 6.17: Determination of End-of-primary point based on measurements of excess pore pressures 


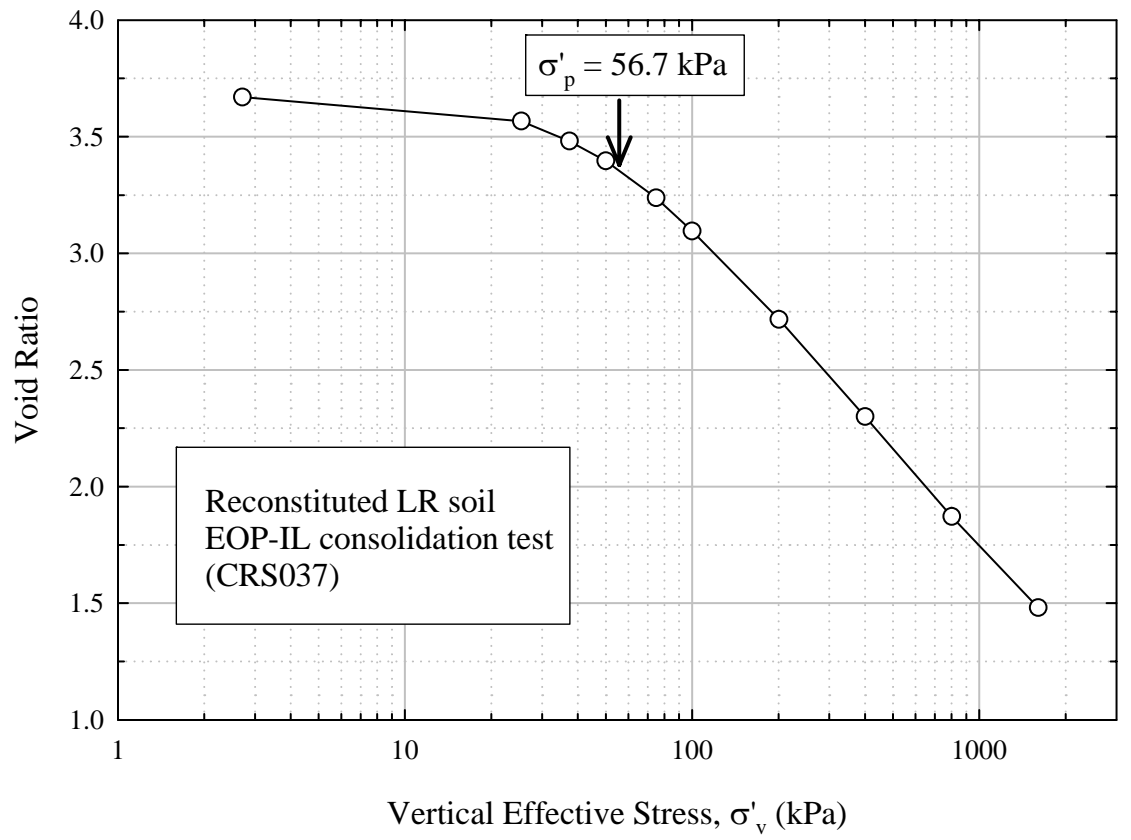

Figure 6.18: EOP compression curve of reconstituted LR soil (CRS037)

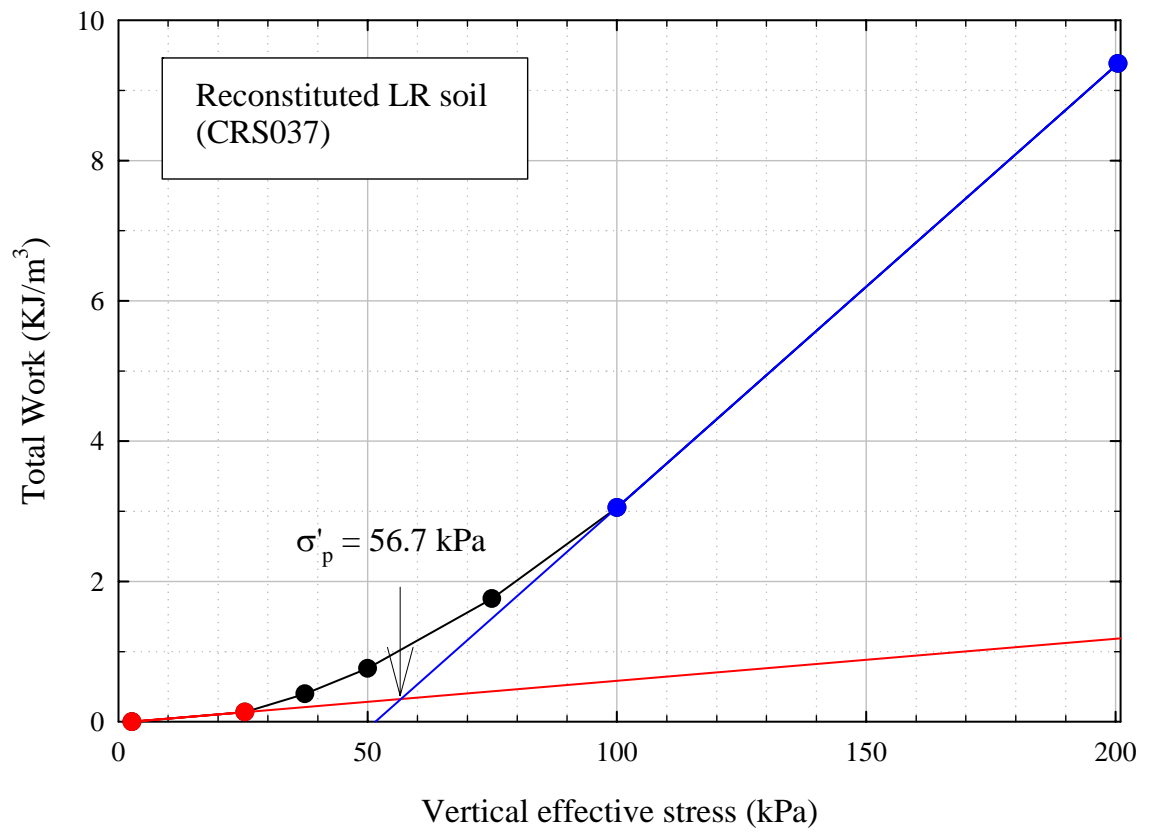

Figure 6.19: Determination of preconsolidation pressure of reconstituted LR soil (CRS037) employing strain energy method 


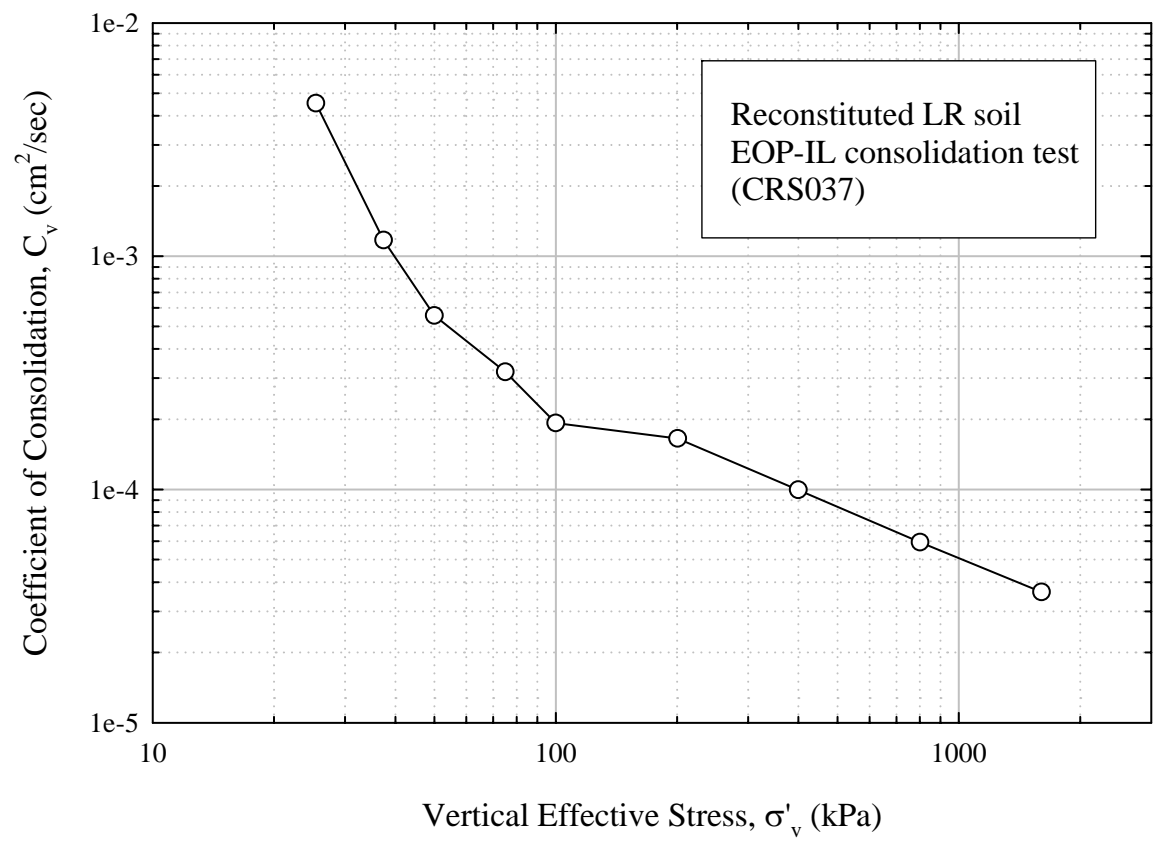

Figure 6.20: Coefficient of consolidation of reconstituted LR soil (CRS037)

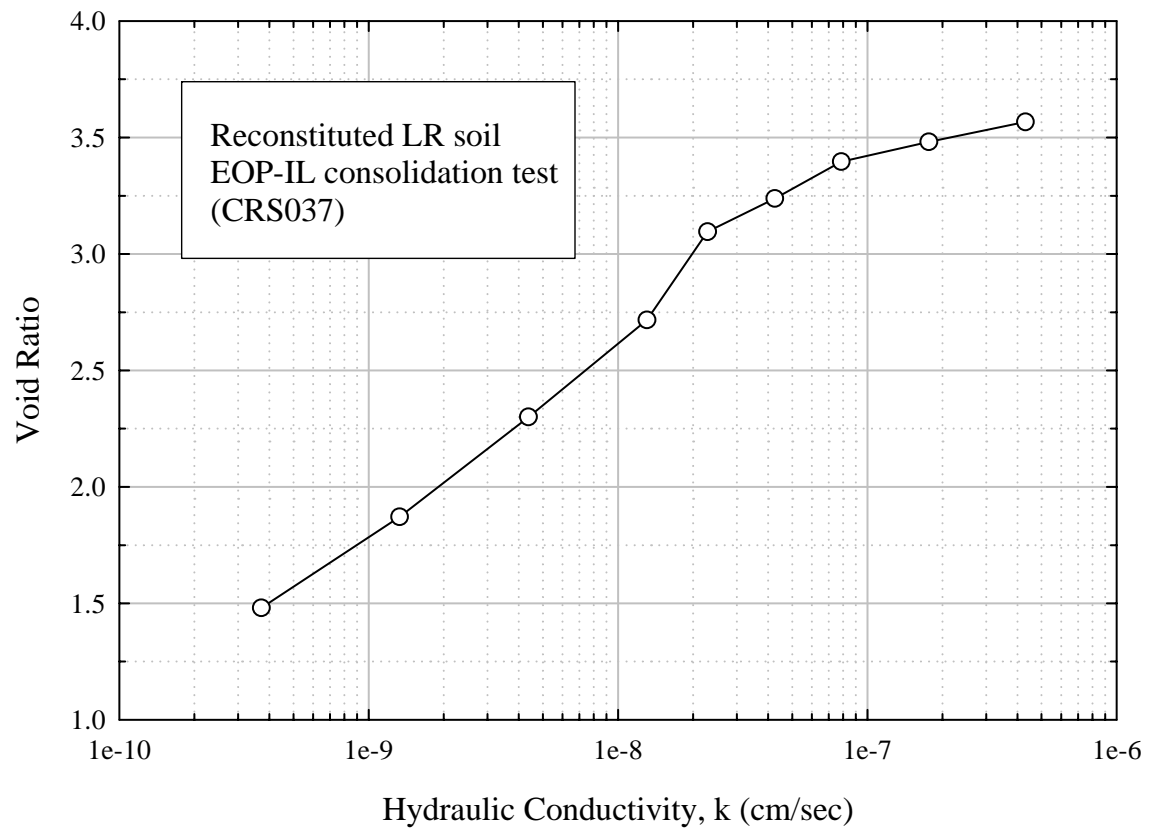

Figure 6.21: Hydraulic conductivity of reconstituted LR soil (CRS037) 


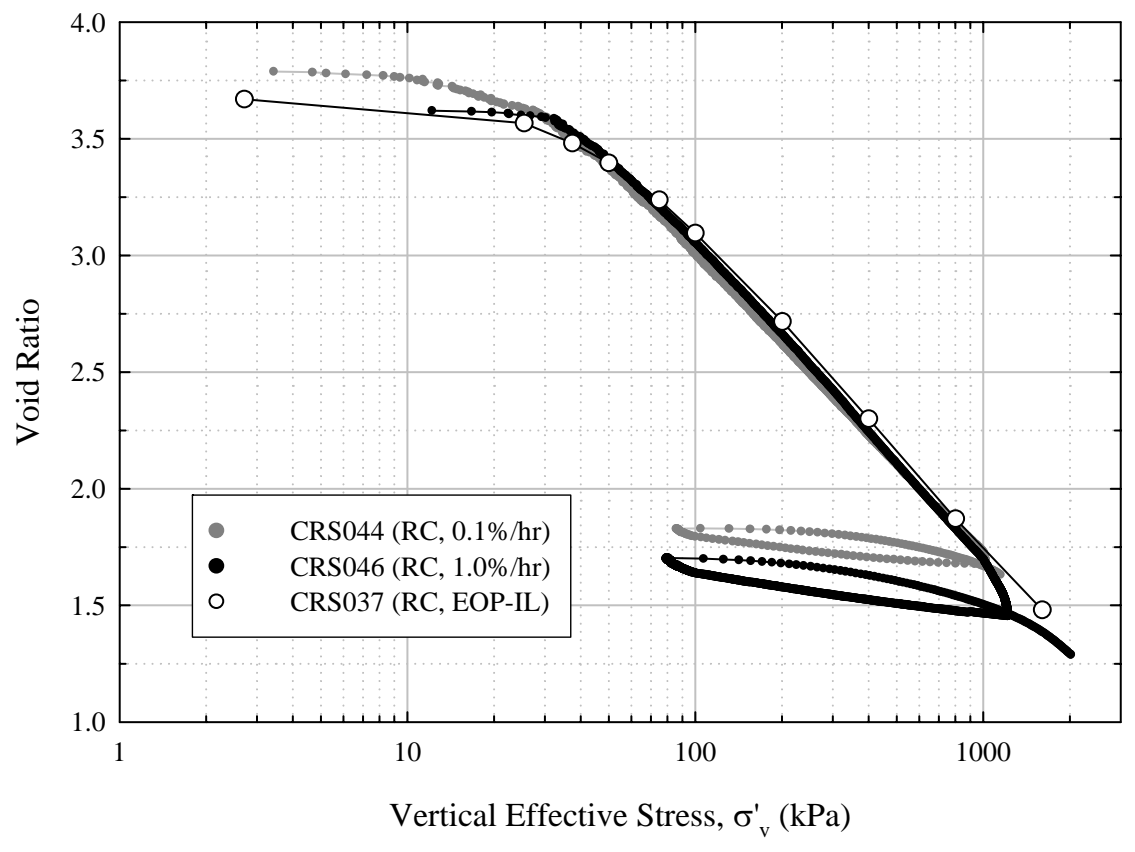

Figure 6.22: Comparison of EOP compression curves of reconstituted LR soil obtained from CRS and EOP-IL consolidation tests 


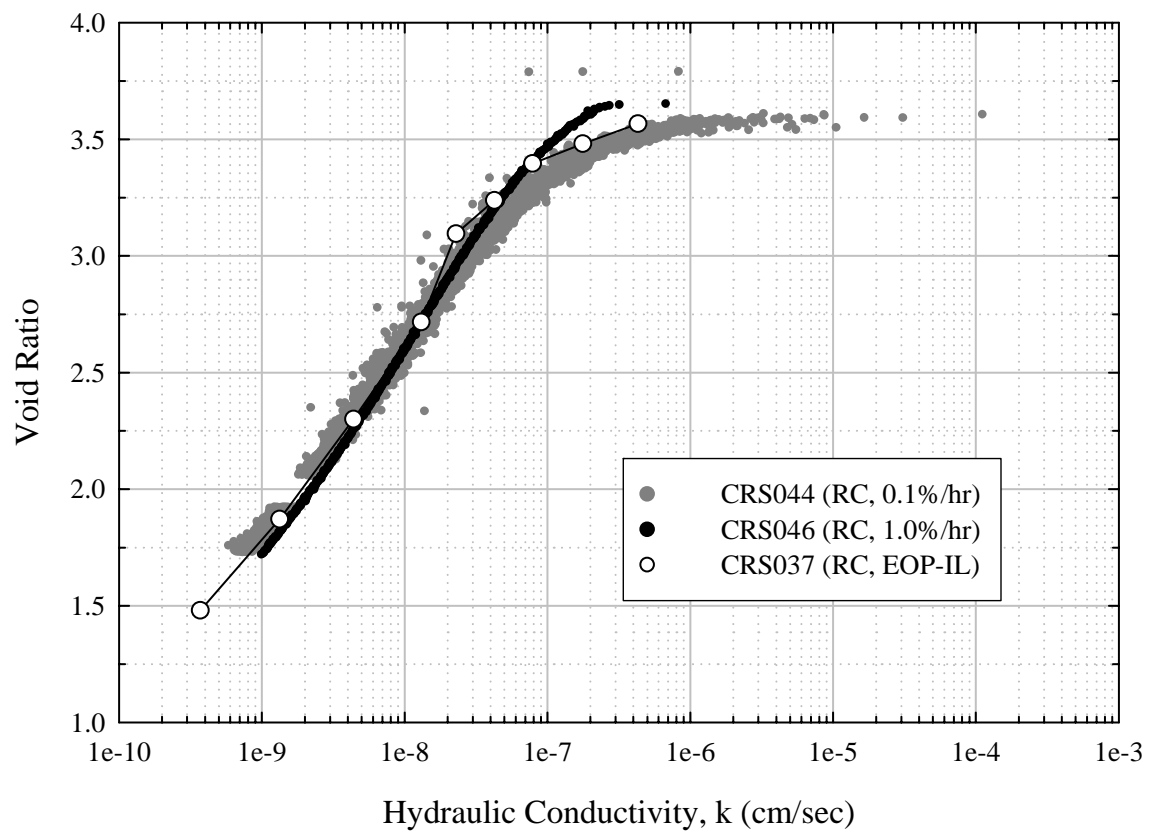

Figure 6.23: Comparison of hydraulic conductivity of reconstituted LR soil obtained from CRS and EOP-IL consolidation tests

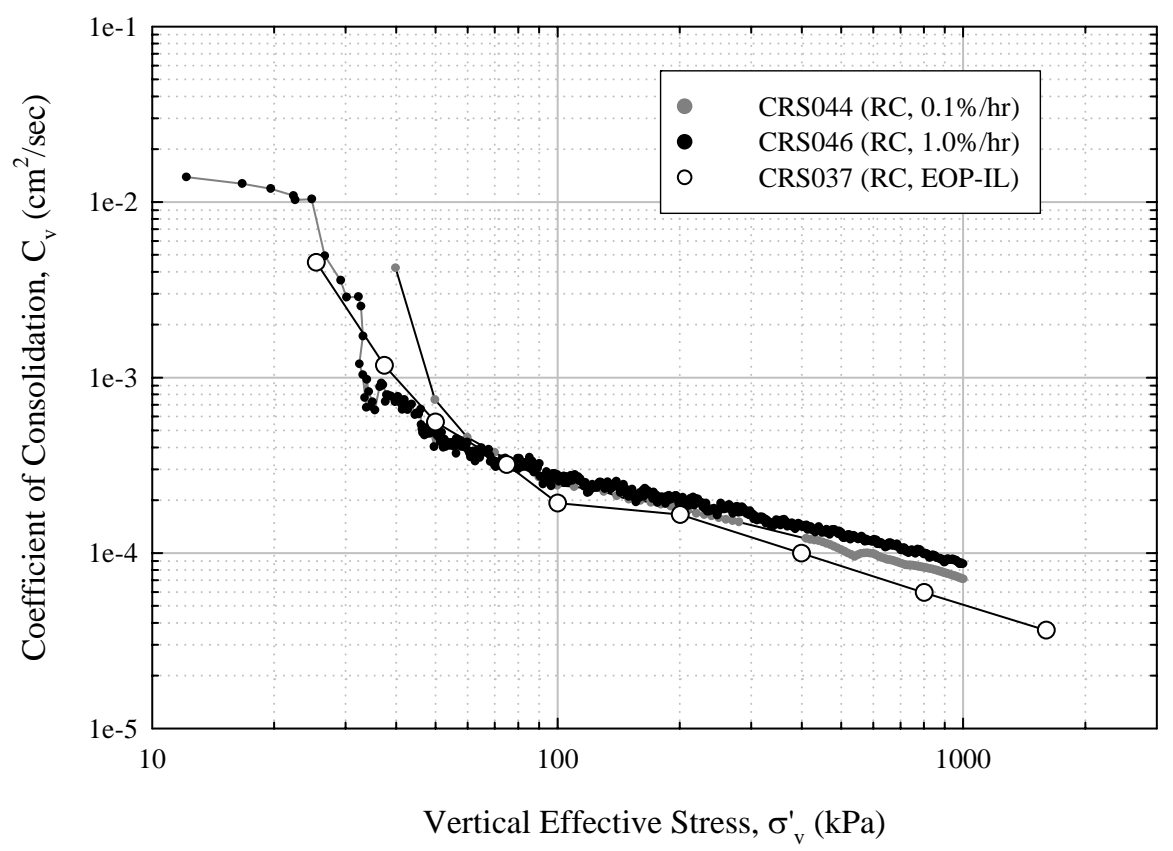

Figure 6.24: Comparison of coefficient of consolidation of reconstituted LR soil obtained from CRS and EOP-IL consolidation tests 


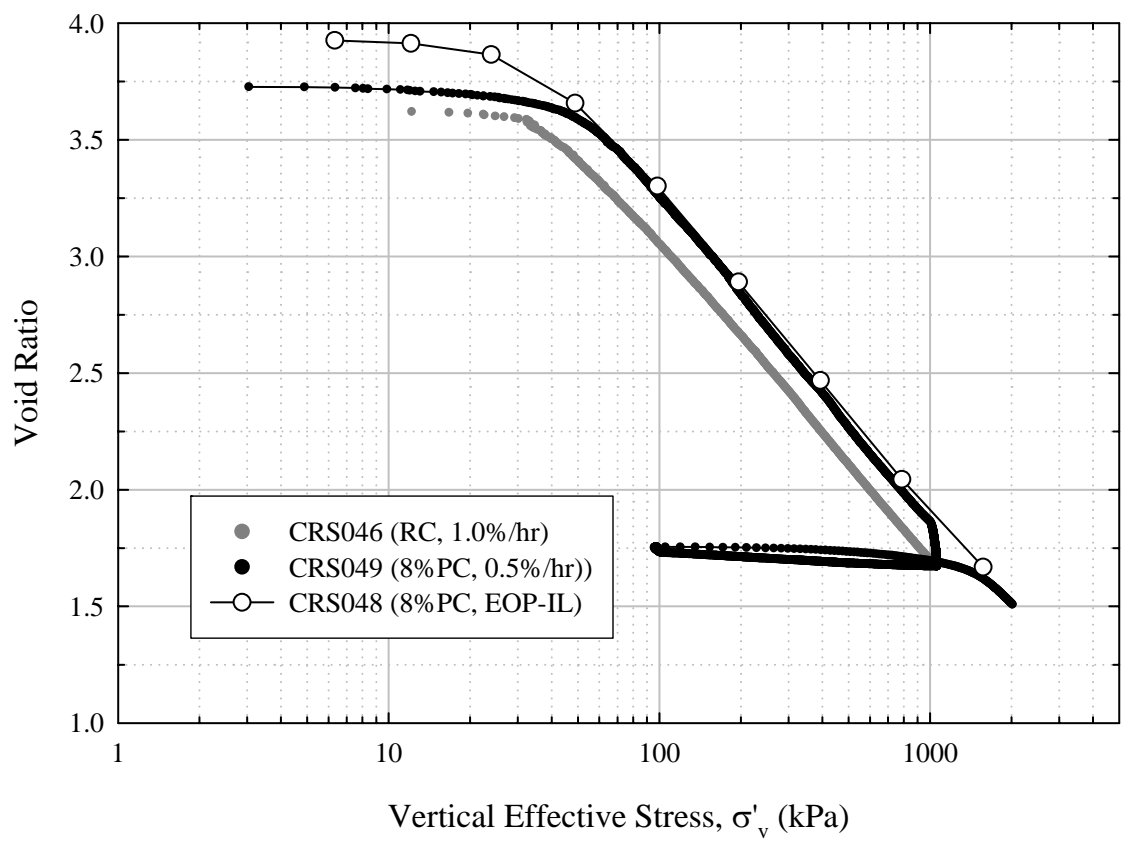

Figure 6.25: EOP compression curves of 8\% PC treated LR soil obtained from CRS and EOP-IL consolidation tests

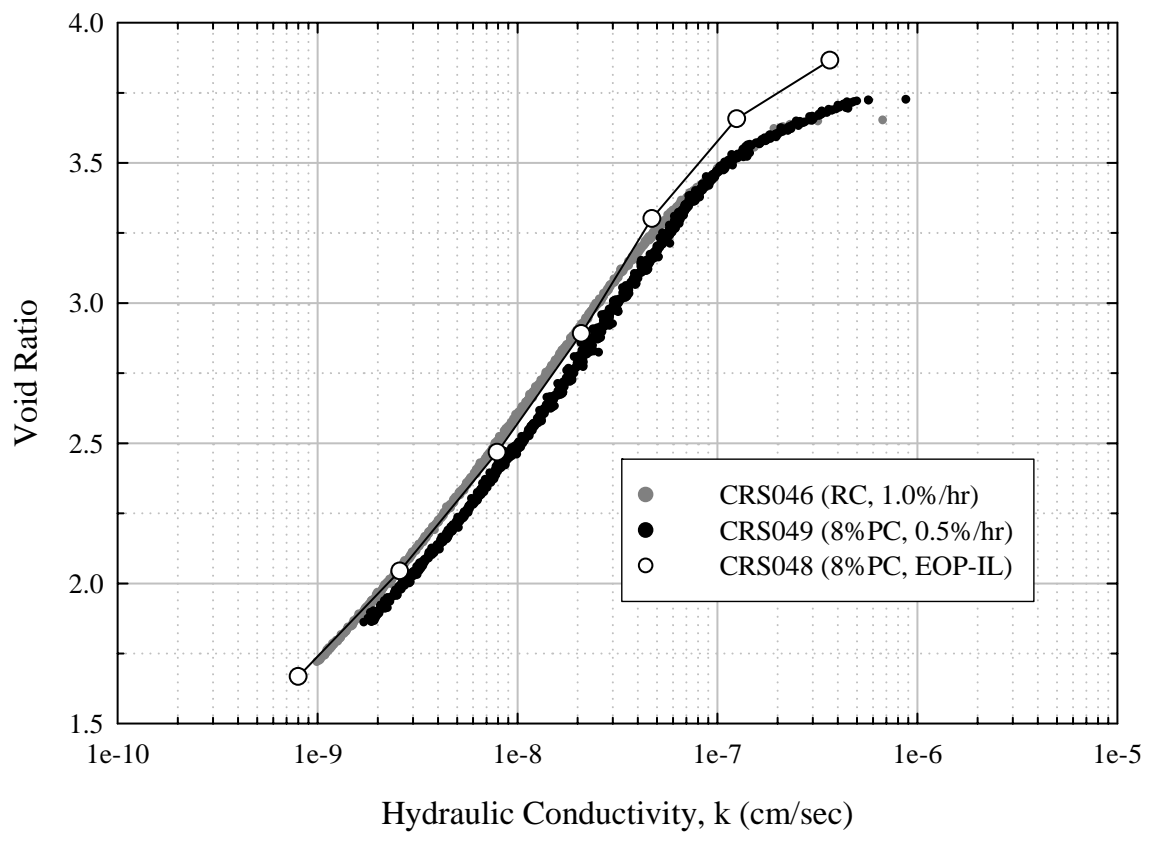

Figure 6.26: Hydraulic conductivity of 8\% PC treated LR soil obtained from CRS and EOP-IL consolidation tests 


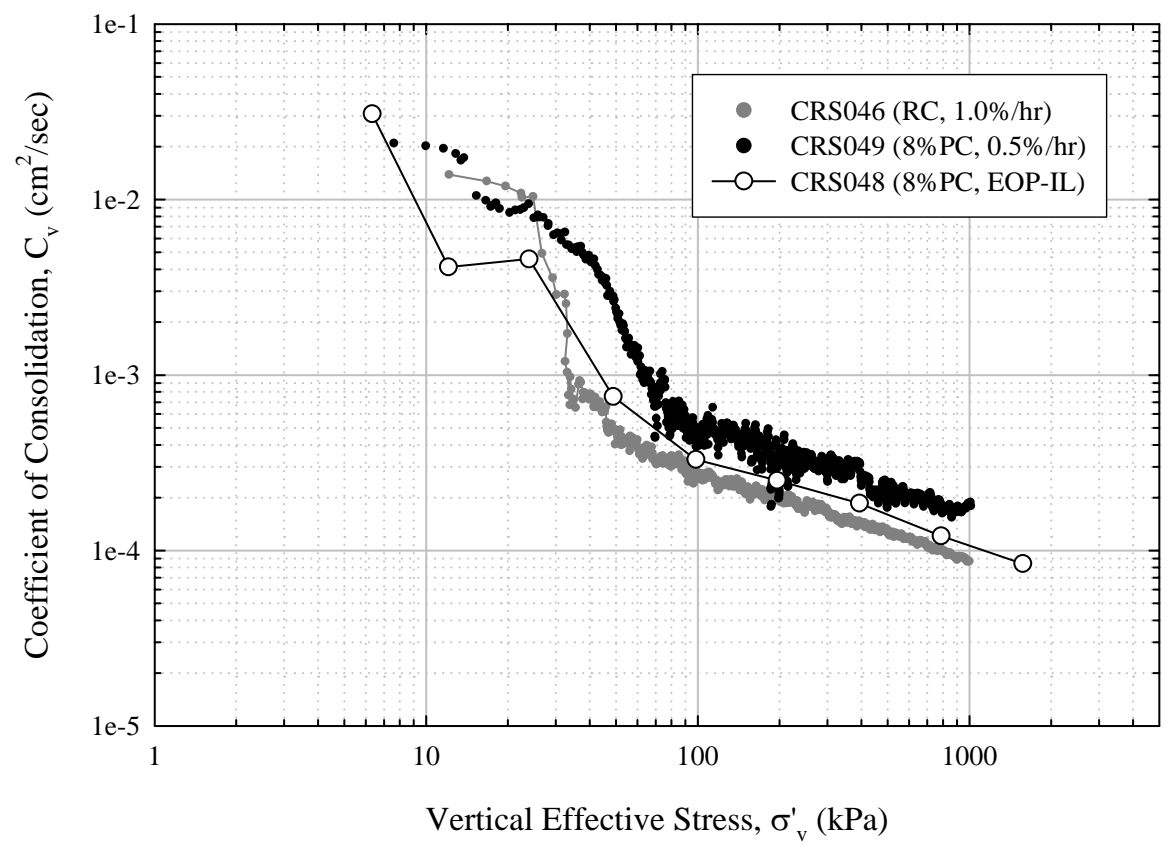

Figure 6.27: Coefficient of consolidation of 8\% PC treated LR soil obtained from CRS and EOP-IL consolidation tests

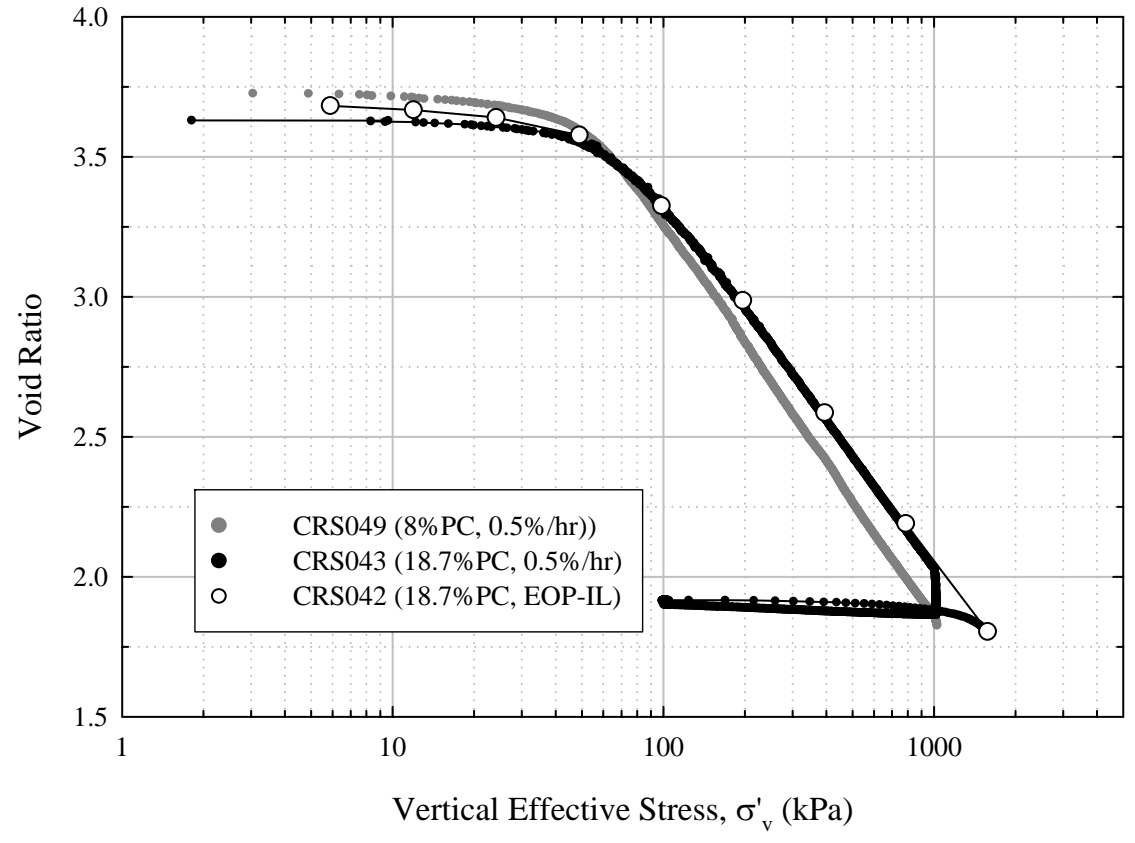

Figure 6.28: EOP compression curve of 18.7\% PC treated LR soil obtained from CRS and EOP-IL consolidation tests 


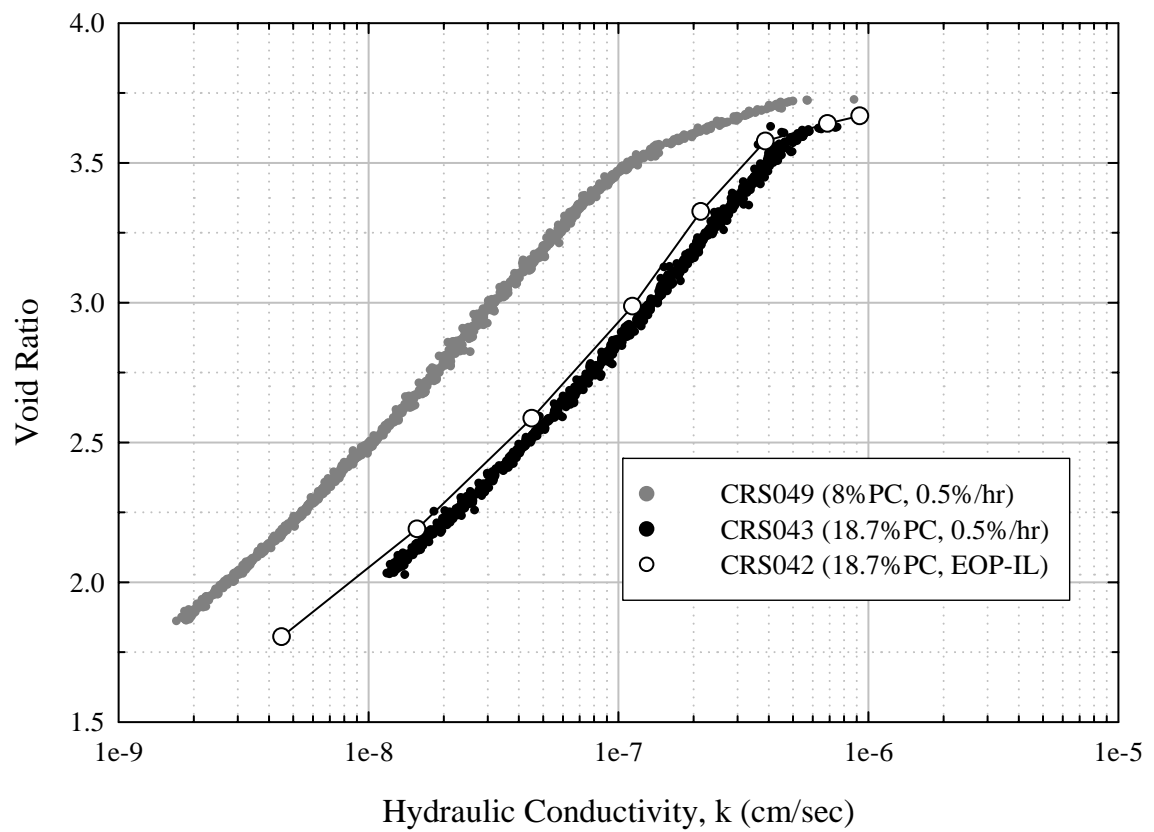

Figure 6.29: Hydraulic conductivity of 18.7\% PC treated LR soil from CRS and EOP-IL consolidation tests

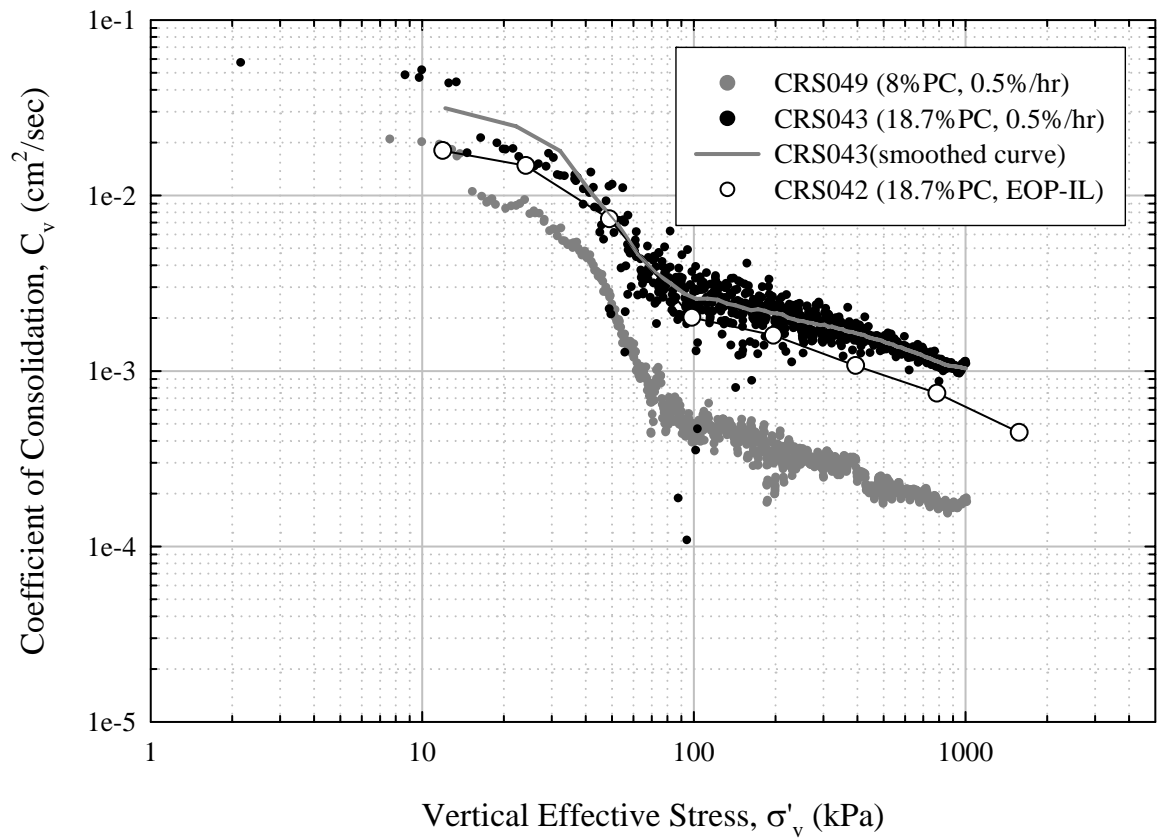

Figure 6.30: Coefficient of consolidation of 18.7\% PC treated LR soil from CRS and EOP-IL consolidation tests 


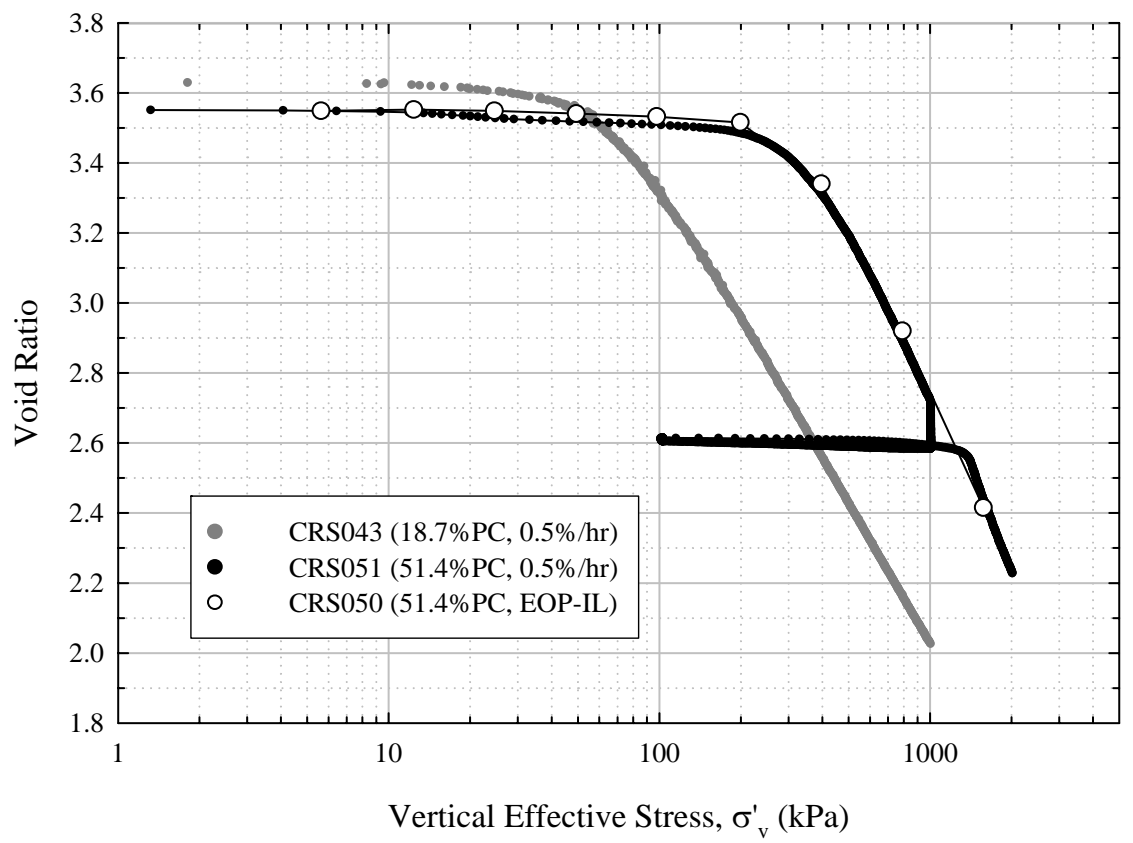

Figure 6.31: EOP compression curve of 51.4\% PC treated LR soil obtained from CRS and EOP-IL consolidation tests

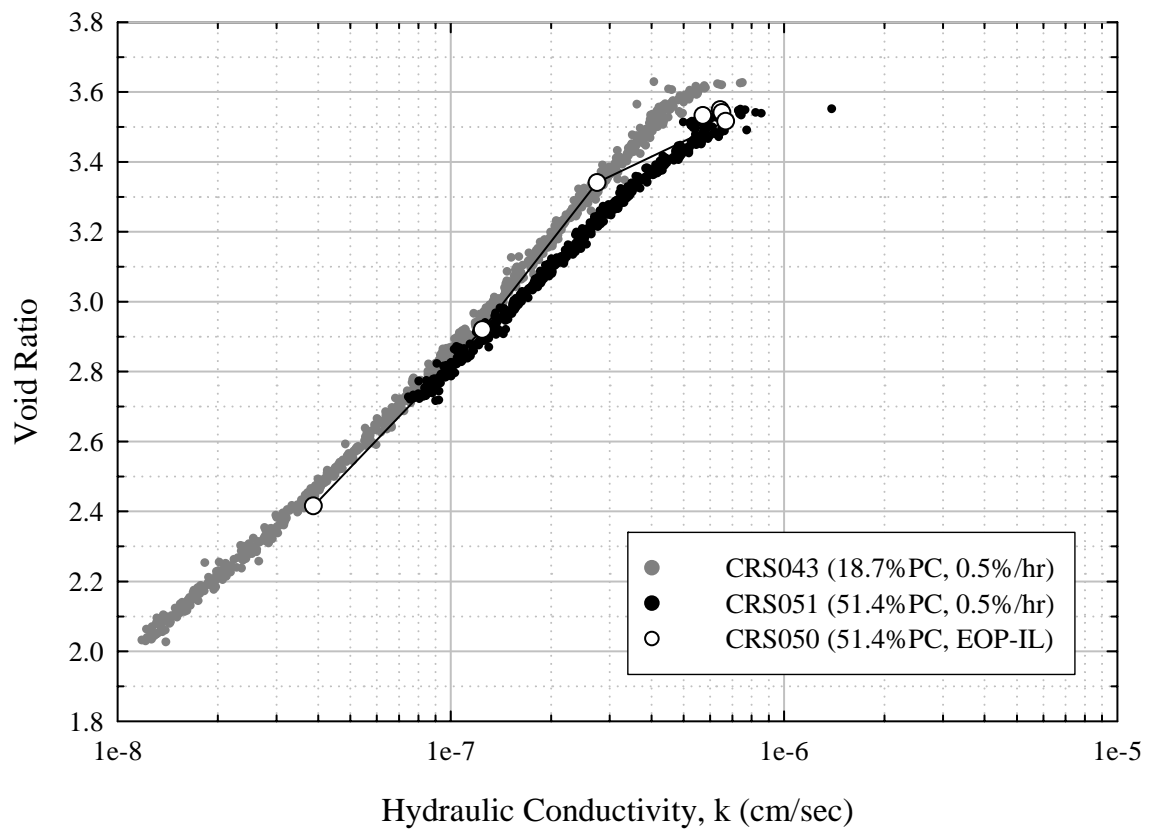

Figure 6.32: Hydraulic conductivity of 51.4\% PC treated LR soil obtained from CRS and EOP-IL consolidation tests 


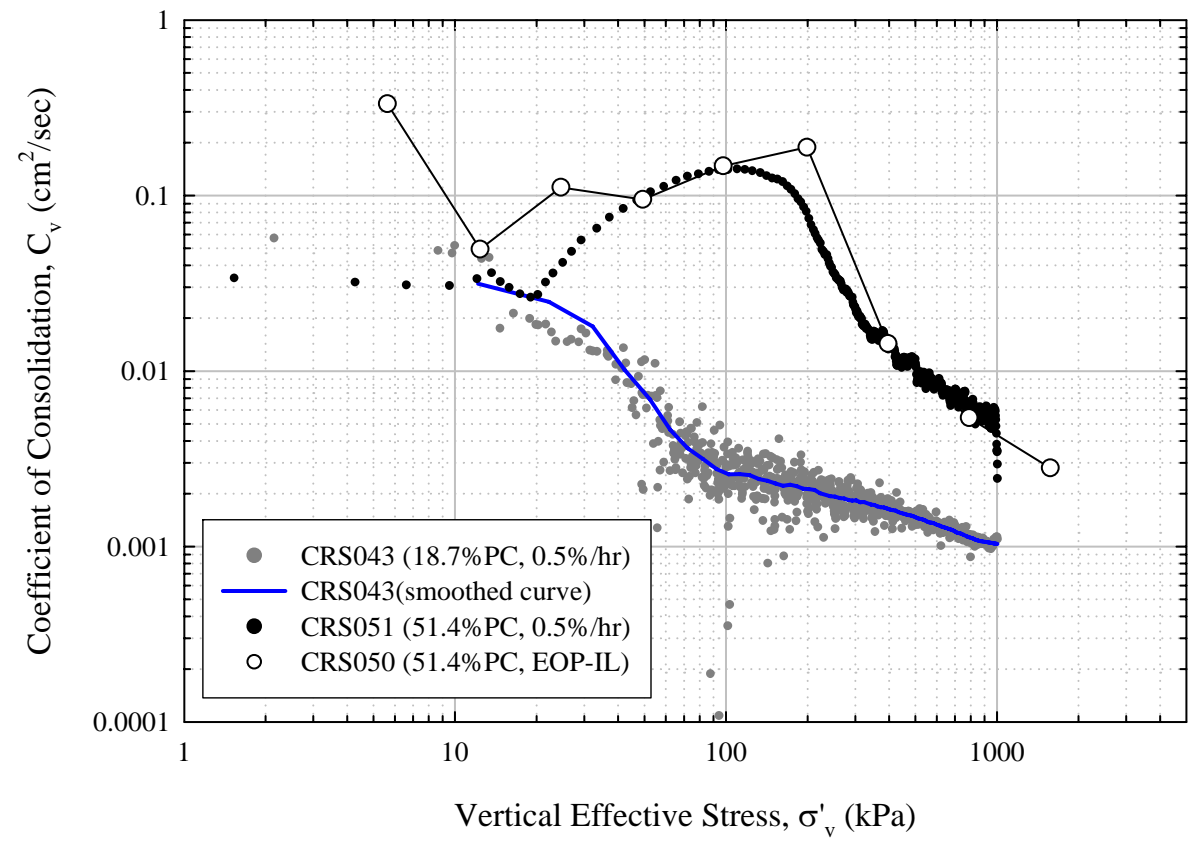

Figure 6.33: Coefficient of consolidation of 51.4\% PC treated LR soil obtained from CRS and EOP-IL consolidation tests

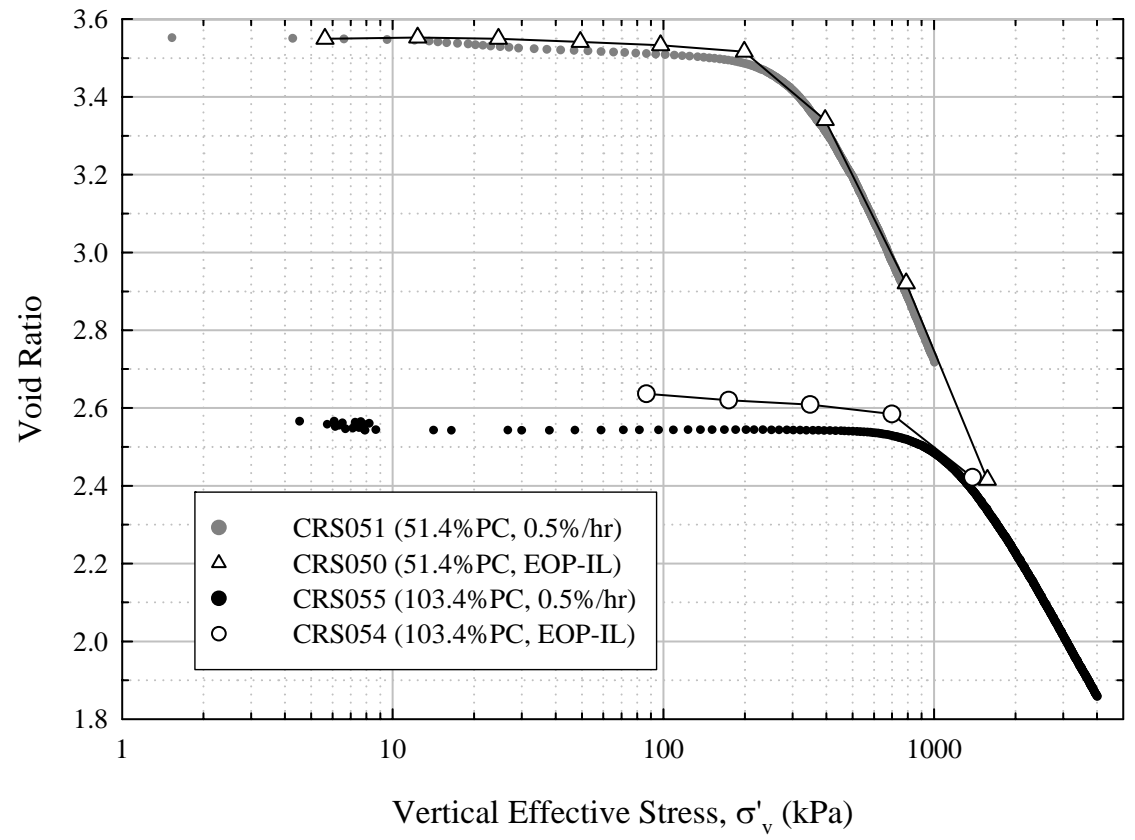

Figure 6.34: EOP compression curve of 103.4\% PC treated LR soil obtained from CRS and EOP-IL consolidation tests 


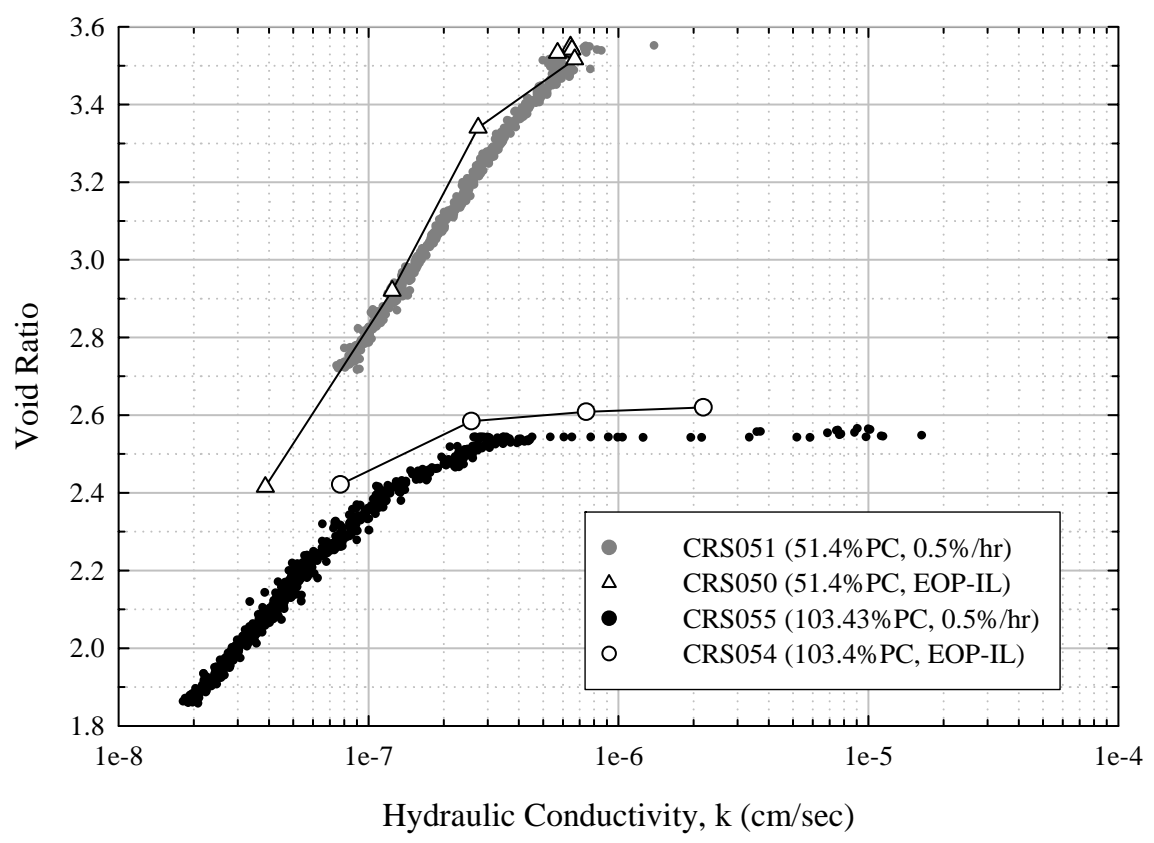

Figure 6.35: Hydraulic conductivity of 103.4\% PC treated LR soil obtained from CRS and EOP-IL consolidation tests

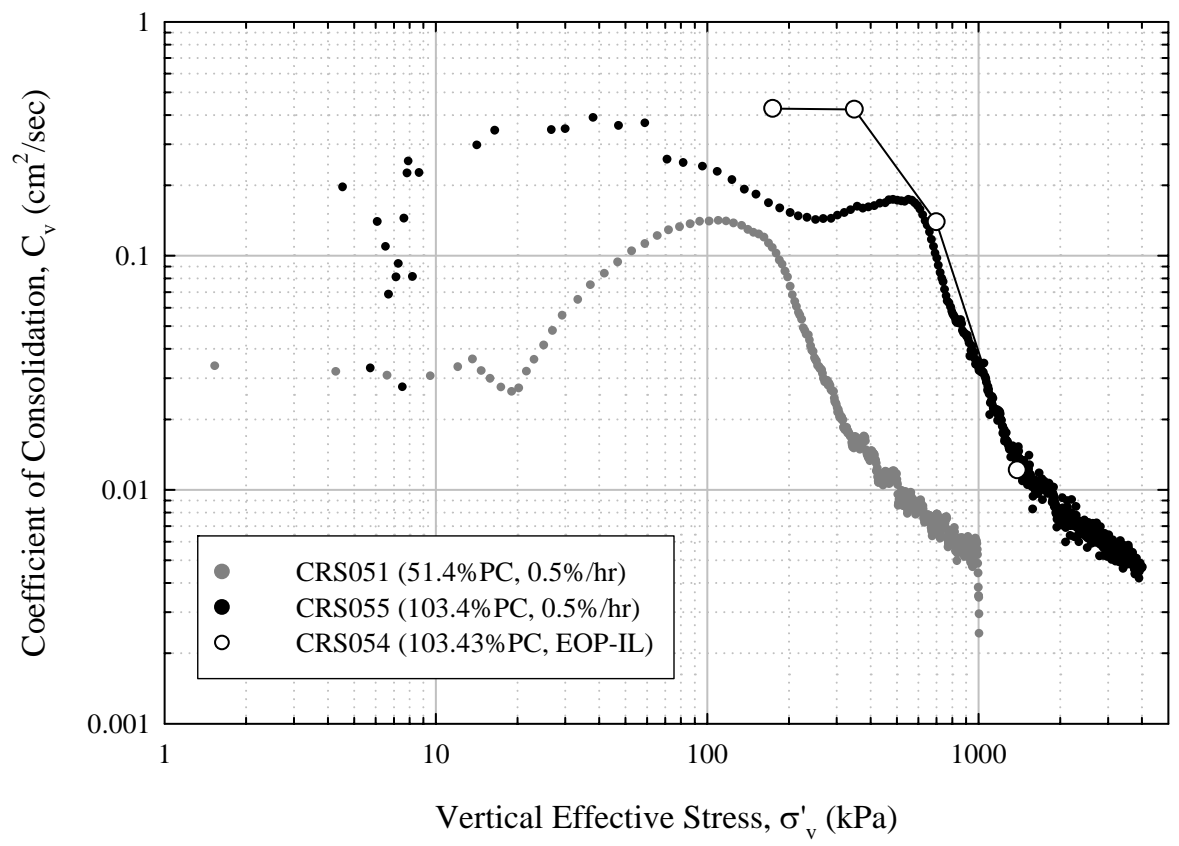

Figure 6.36: Coefficient of consolidation of $103.4 \%$ PC treated LR soil obtained from CRS 


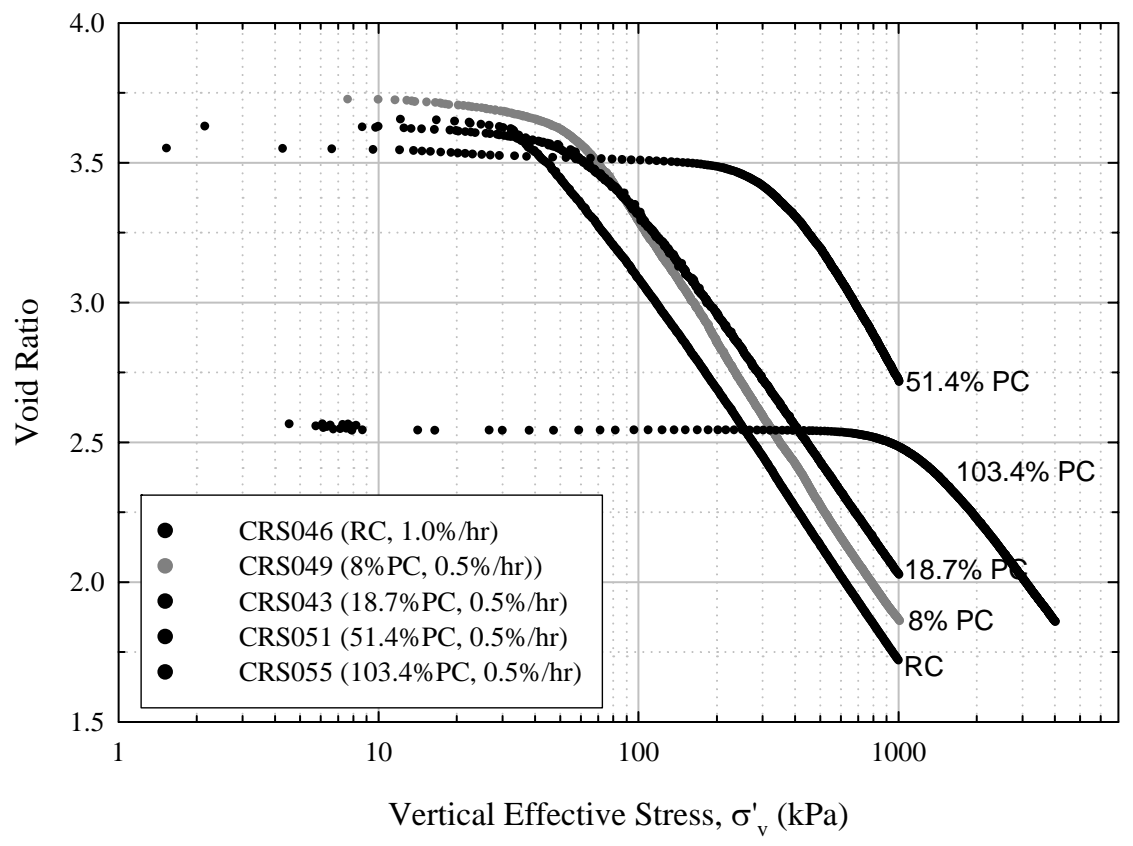

Figure 6.37: Comparison of the compression curves of LR soil treated with different amounts of PC

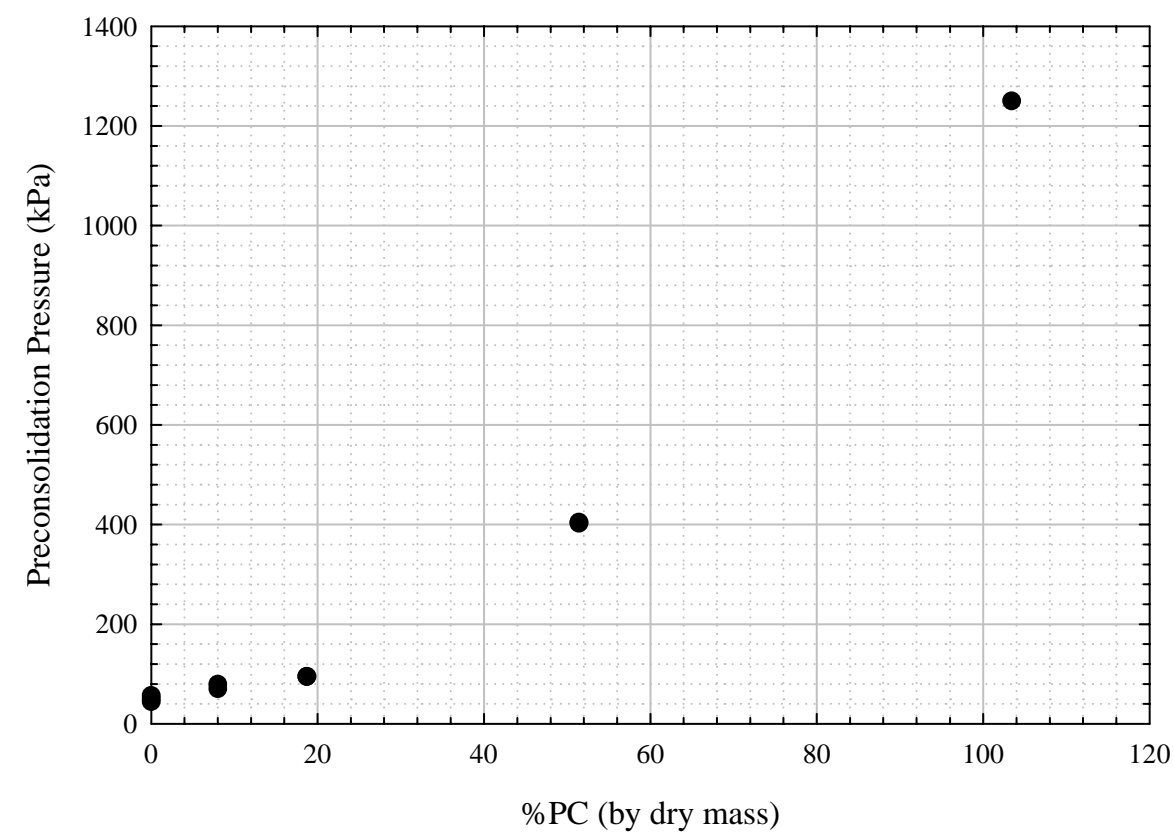

Figure 6.38: Increase of the preconsolidation pressure with increase in the amount of PC 


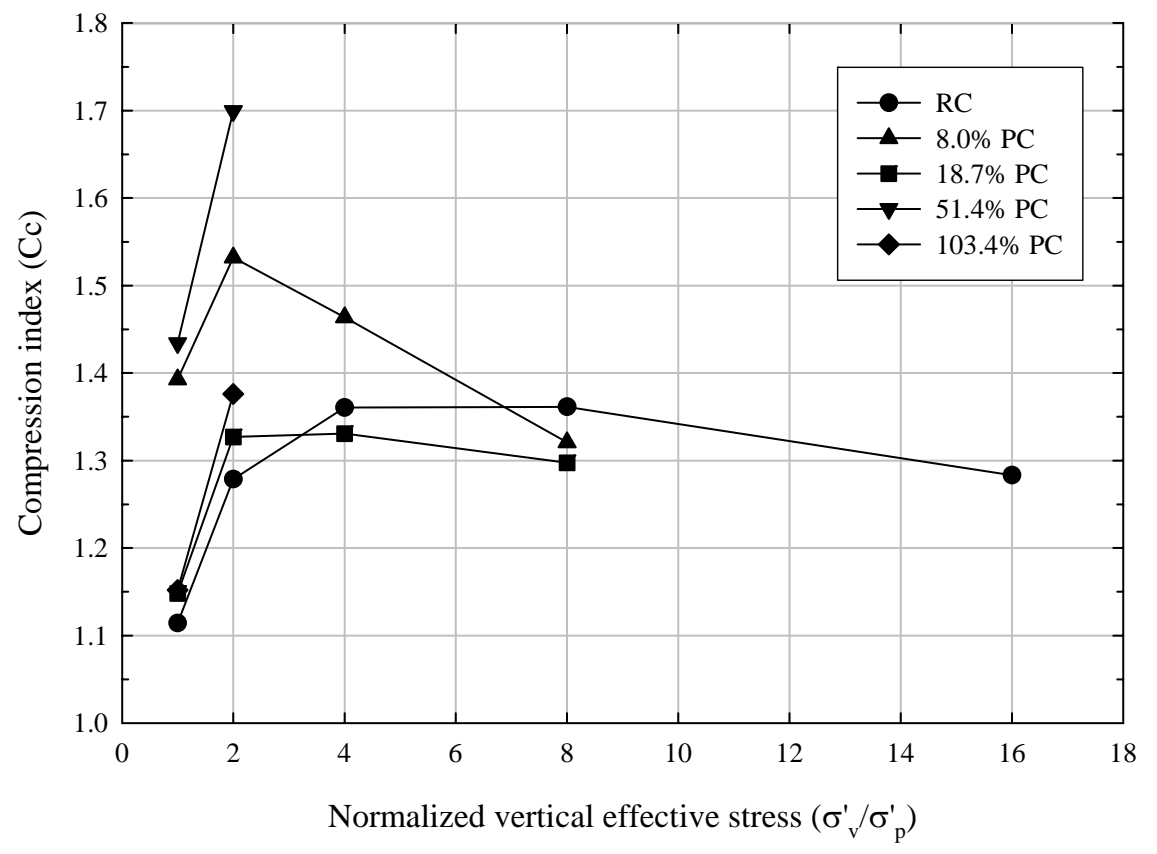

Figure 6.39: Effect of treatment with PC on the compression index of LR soil

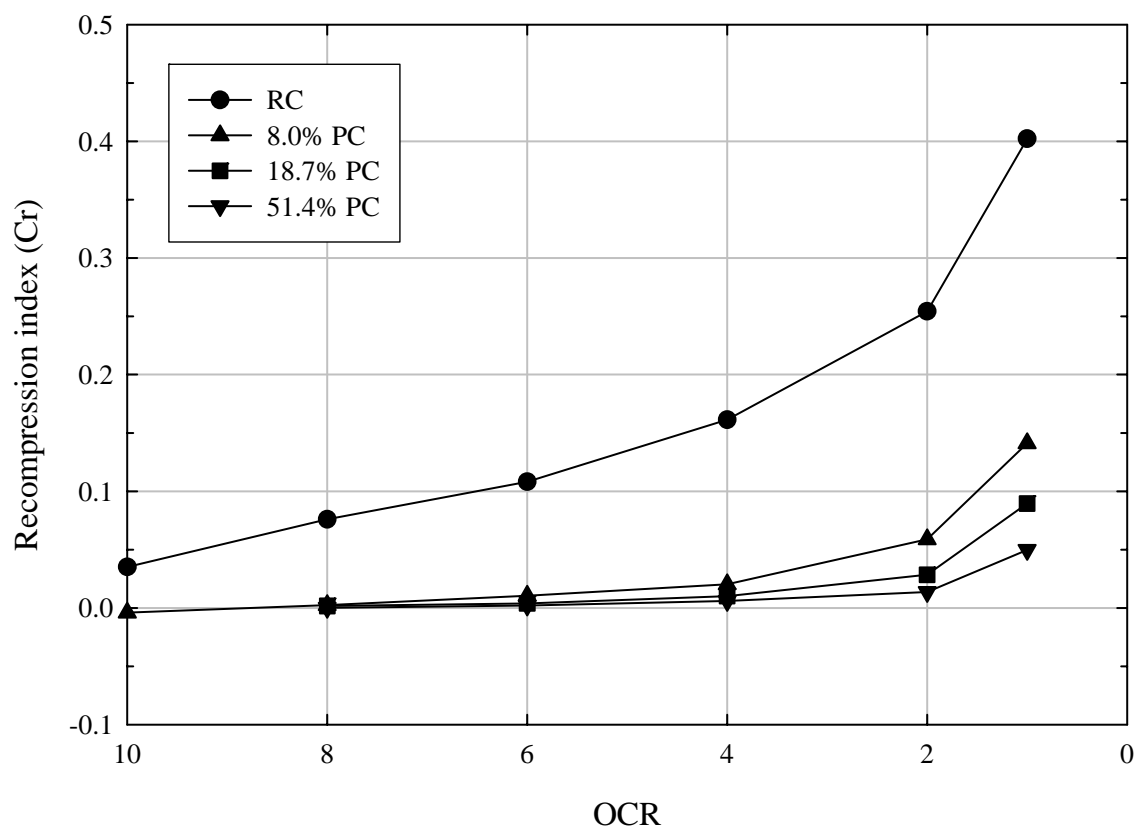

Figure 6.40: Effect of treatment with PC on the recompression index of LR soil 


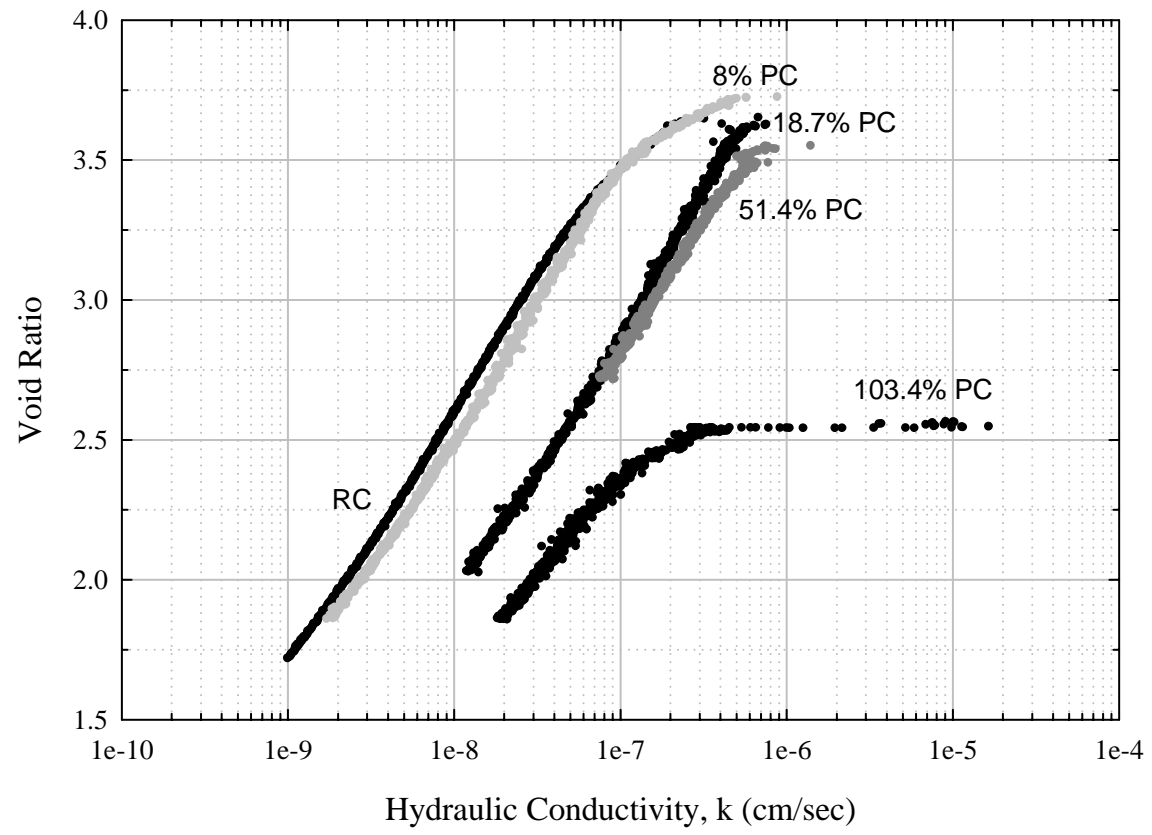

Figure 6.41: Comparison of the hydraulic conductivity of LR soil treated with different amounts of PC

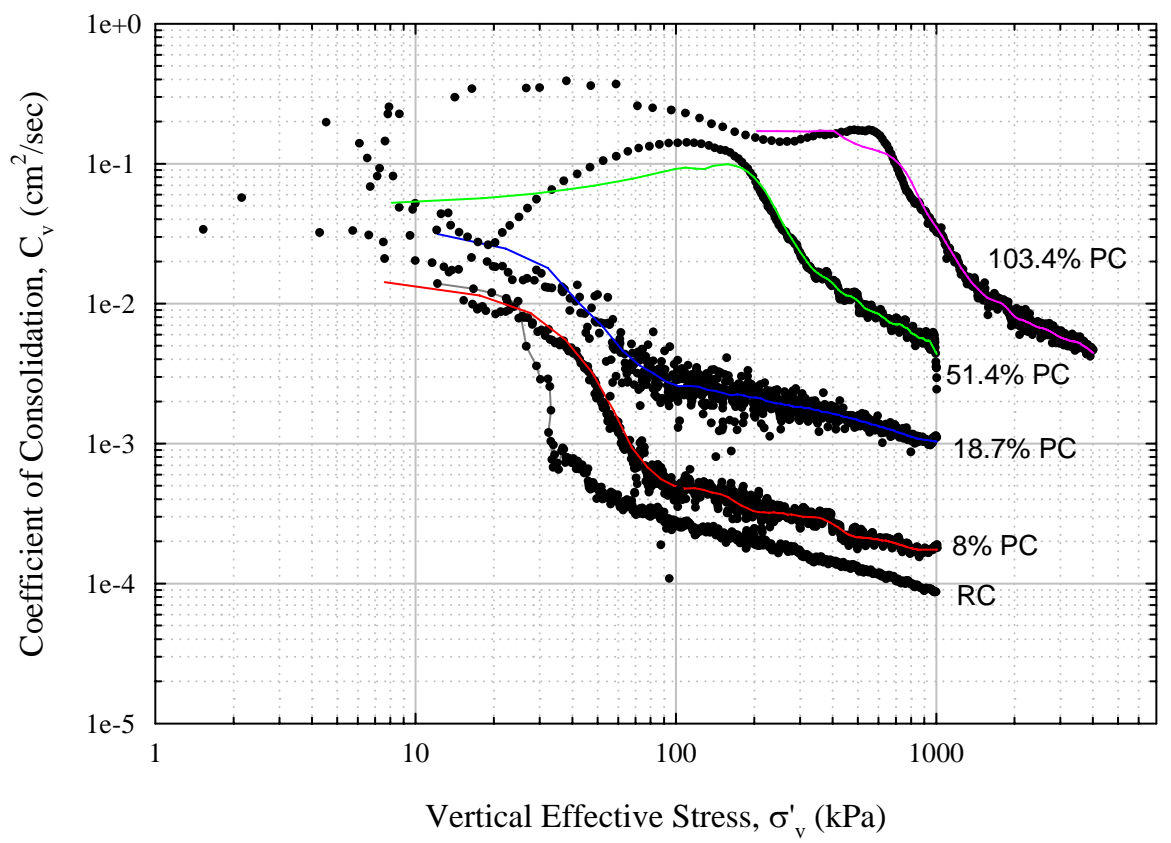

Figure 6.42: Comparison of the coefficient of consolidation of untreated and PC treated LR soil 


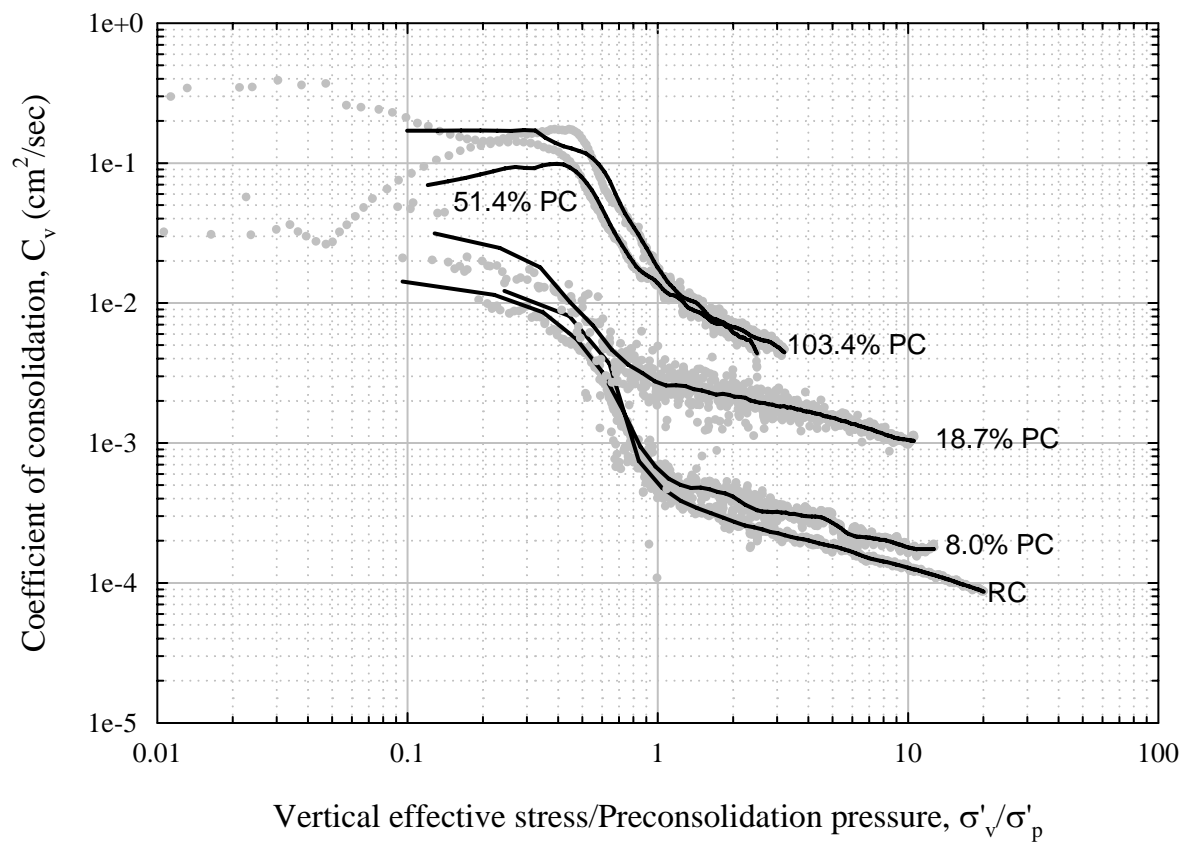

Figure 6.43: Normalized vertical effective stress vs. coefficient of consolidation 


\section{CHAPTER 7: SECONDARY COMPRESSION: CREEP TESTS}

\subsection{INTRODUCTION}

The objects of this chapter are (1) to present the results of the incremental loading (IL) consolidation tests and long term creep tests performed on untreated and PC treated LR soils and (2) to discuss the effects of treatment on the secondary compression of PC treated LR soils.

Section 7.2 presents the results of EOP-IL consolidation and long term creep test performed on untreated and reconstituted LR soil.

Section 7.3 presents the results of EOP-IL and long term creep tests performed on LR soils treated with $8-103.4 \%$ PC. The effects of treatment are evaluated by comparing the results from these tests with the results for reconstituted LR soils.

Section 7.4 summarizes the effects of treatment on the creep behavior of PC treated LR soils.

\subsection{TESTING APPROACH}

One of the most serious engineering problems associated with highly organic soils and peats is their tendencies for significant secondary compression (creep). In geotechnical engineering, creep is defined as the deformation under constant effective stress. In general, the creep behavior of soils is investigated by performing IL consolidation test. In conventional IL consolidation tests, the load is applied for 24 hours and the load is increased by a load increment ratio of unity. At select increments (typically the maximum vertical stress) the soil is allowed to creep for additional $24 \mathrm{hrs}$. From each load increment data, the time settlement curve is obtained, and the secondary compression index $\left(C_{\alpha}=\Delta e / \Delta \log t\right)$ is obtained as the slope of the time settlement curve after the end-of-primary (EOP). Identification of the end-of-primary typically relies on Casagrande's procedure, requiring that the soil undergoes some secondary compression.

In this research, a slightly different testing approach was employed. The IL consolidation tests were performed by applying each load increment soon after the EOP for the previous increment had been reached. This was possible because the end of 
primary was determined based on the base excess pore pressure measurements. As discussed in Chapter 6 such tests are referred to as EOP IL consolidation tests. The only exception in this loading scheme was represented by the load increment corresponding to the maximum vertical stress at which the soil was allowed to creep for an extended period (a month or more). In addition to the value of the secondary compression index derived from this creep phase, additional values of $\mathrm{C}_{\alpha}$ could be derived for most of other stress increments given that while the test is referred to as an EOP IL test, there was some delay in the application of the load increment after reaching the EOP. Albeit short, in most cases this delay was sufficient to allow determination of $\mathrm{C}_{\alpha}$.

The decision to perform EOP IL tests was motivated primarily by the desire to compare the results of incremental tests to those of CRS tests (see Chapter 6). With regard to characterizing the creep behavior, this testing procedure has a second advantage in that for each increment the $C_{\alpha} / C_{c}$ ratio can be obtained directly from compression index at any vertical effective stress level (in both over-consolidated and normally consolidated regions) and the secondary compression index obtained at the corresponding vertical effective stress. The following sections discuss the evaluation of $\mathrm{C}_{\alpha}$ and of the $\mathrm{C}_{\alpha} / \mathrm{C}_{\mathrm{c}}$ ratio from the EOP IL test results.

\subsection{CREEP BEHAVIOR OF RECONSTIUTUED LR SOIL}

Data for the reconstituted LR soil specimen (CRS037) used for the EOP-IL consolidation test is summarized in Table 7.1 including test number, soil bin number, organic content (OC), initial water content $\left(\mathrm{w}_{\mathrm{o}}\right)$, specific gravity $\left(\mathrm{G}_{\mathrm{s}}\right)$ and initial void ratio $\left(\mathrm{e}_{\mathrm{o}}\right)$. Table 7.2 summarizes the data for the EOP-IL consolidation test (CRS037) including the applied effective stress, EOP void ratio $\left(\mathrm{e}_{\mathrm{EOP}}\right)$, time required for reaching EOP ( $\left.\mathrm{t}_{\mathrm{EOP}}\right)$ estimated from excess pore pressures measurements, maximum excess pore pressures $\left(\Delta \mathrm{u}_{\mathrm{hmax}}\right)$, the ratio of maximum excess pore pressures to applied load increment $\left(\Delta \mathrm{u}_{\mathrm{hmax}} / \Delta \sigma^{\prime}{ }_{\mathrm{v}}\right)$, time for maximum excess pore pressures development $\left(\mathrm{t}_{\Delta} \mathrm{u}_{\mathrm{hmax}}\right)$, duration of each load increment $\left(\mathrm{t}_{\mathrm{f}}\right)$, the ratio of duration of each load increment to the time required 
for EOP $\left(\mathrm{t}_{\mathrm{f}} / \mathrm{t}_{\mathrm{EOP}}\right)$, compression index $\left(\mathrm{c}_{\mathrm{c}}\right)$ and secondary compression index $\left(\mathrm{c}_{\alpha}\right)$ of each load increment.

Prior to testing, the soil specimen was saturated with back pressure of $312 \mathrm{kPa}$ for 1800 minutes. Vertical effective stress of $2.7 \mathrm{kPa}$ was applied to ensure the contact between piston and soil specimen. The sample height was maintained during the back pressure saturation stage.

During the test, the vertical effective stress was increased with the load increment ratio (LIR) of unity except for the first load increment (LIR $=9$ ). In addition, two load increments were added at $\sigma_{\mathrm{v}}=37.4$ and $74.9 \mathrm{kPa}$ to obtain more data points for better estimation of the preconsolidation pressure.

The EOP compression curve of reconstituted LR soil is plotted and compared with curves from two CRS consolidation tests in Figure 7.1. As mentioned in Chapter 6, the results from EOP-IL and CRS consolidation tests are in good agreement and show high repeatability in the compressibility parameters as well as in the coefficient of consolidation and the hydraulic conductivity.

As summarized in Table 7.2, the maximum excess pore pressures developed in each load increment are consistently greater than $95 \%$ of actual load increments, except at the first load increment ( $75 \%$ ) and at $\sigma_{\mathrm{v}}^{\prime}=50 \mathrm{kPa}$ ( 93.7\%). In the normally consolidated region $\left(\sigma^{\prime}{ }_{\mathrm{v}}>56.7 \mathrm{kPa}\right)$, the time required for the generation of maximum excess pore pressure increases gradually from 0.23 minute at $\sigma^{\prime}{ }_{\mathrm{v}}=74.9 \mathrm{kPa}$ to 2.4 minutes at $\sigma_{\mathrm{v}}=1600 \mathrm{kPa}$. These results are consistent with the observation made by Mesri et al. (1997) from consolidation tests performed on Middleton peat with approximately 90 - 95\% of organic content. The delay of the generation of maximum pore pressure may be related to significant decrease of the hydraulic conductivity with increase in effective stress of highly organic soils and peats.

The secondary compression index of reconstituted LR soil was obtained from the long term creep test performed at $\sigma^{\prime}{ }_{\mathrm{v}}=1600 \mathrm{kPa}$ in EOP-IL consolidation test (CRS037). As summarized in Table 7.2, each load increment except the one corresponding to $\sigma^{\prime}{ }_{\mathrm{v}}=$ $1600 \mathrm{kPa}$ was applied for between $1.2\left(\sigma^{\prime}{ }_{\mathrm{v}}=800 \mathrm{kPa}\right)$ and $2.3\left(\sigma^{\prime}{ }_{\mathrm{v}}=100 \mathrm{kPa}\right)$ times the time required for reaching the end of primary $\left(\mathrm{t}_{\mathrm{EOP}}\right)$. 
During the long term creep test performed at $\sigma^{{ }_{\mathrm{v}}}=1600 \mathrm{kPa}$, the soil specimen was allowed to creep for 50065.2 minutes ( 34.8 days), or 1.5 cycles of secondary compression. The time settlement curve and dissipation of excess pore pressures are plotted in Figure 7.2. The time settlement curve of LR soil exhibits the characteristics of time settlement curve derived from Terzaghi's consolidation theory: a linear portion in the primary consolidation stage where excess pore pressures are dissipating, a second linear portion in the secondary compression stage where all the excess pore pressure has dissipated, and gradual change of slope between two linear portions.

In this load increment, the EOP point could be obtained using both Casagrande's method and excess pore pressure measurements. As indicated with open circles in Figure 7.2, the EOP point obtained with Casagrande's method corresponds to $\mathrm{e}_{\mathrm{EOP}}=1.503$ and $t_{\mathrm{EOP}}=1104.4$ minutes, where the EOP from excess pore pressures data corresponds to $\mathrm{e}_{\mathrm{EOP}}=1.481$ and $\mathrm{t}_{\mathrm{EOP}}=1529.7$ minutes. The variations in $\mathrm{e}_{\mathrm{EOP}}$ and $\mathrm{t}_{\mathrm{EOP}}$ between these two methods would change the values of coefficient of consolidation and hydraulic conductivity as follows

$$
\begin{aligned}
& \mathrm{Cv} \text { Casagrande }=4.13 \mathrm{E}-05\left(\mathrm{~cm}^{2} / \mathrm{sec}\right) \text { and } \mathrm{k}_{\text {Casagrande }}=4.22 \mathrm{E}-10(\mathrm{~cm} / \mathrm{sec}) \\
& \mathrm{Cv} \text { excess pore pressure }=3.63 \mathrm{E}-05\left(\mathrm{~cm}^{2} / \mathrm{sec}\right) \text { and } \mathrm{k}_{\text {excess pore pressure }}=3.71 \mathrm{E}-10(\mathrm{~cm} / \mathrm{sec})
\end{aligned}
$$

The values of coefficient of consolidation and hydraulic conductivity calculated based on Casagrande method are about 12\% greater than the values from excess pore pressures measurements. This may be one of the reasons that the coefficient of consolidation values from the EOP-IL consolidation test were less than the values from CRS consolidation tests, as discussed in Chapter 6. Yet, the measurements of excess pore pressure provide a reliable method for determining the EOP without allowing significant secondary compression of the soil.

The secondary compression index $\left(\mathrm{C}_{\alpha}\right)$ of reconstituted LR soil calculated from $\mathrm{t}$ $=1529.7$ to $t=50045.5$ minutes is 0.123 . As indicated as linear line of time settlement curve during secondary compression stage in Figure 7.2, the secondary compression index remains constant during the time range investigated. 
Although the secondary compression index is used to describe the creep behavior of soils, it must be emphasized that its magnitude as that of many other soil parameters (e.g. $\mathrm{C}_{\mathrm{c}}, \mathrm{C}_{\mathrm{v}}$ and $\mathrm{k}$ ) changes depending on the effective stress level, as well as on the overconosolidation ratio. This is well illustrated in Figure 7.3, where the time settlement curves for three different stress increments $\left(\sigma^{\prime}{ }_{v}=50.0,200.5\right.$, and $\left.1600 \mathrm{kPa}\right)$ from CRS037 are plotted together. As indicated in this figure, the secondary compression index of reconstituted LR soil changes from $0.056\left(\sigma^{\prime}{ }_{\mathrm{v}}=50.0 \mathrm{kPa}\right)$ to $0.145\left(\sigma_{\mathrm{v}}{ }_{\mathrm{v}}=200.5\right.$ $\mathrm{kPa})$ and to $0.123\left(\sigma_{\mathrm{v}}=1600.0 \mathrm{kPa}\right)$.

Another parameter for describing the susceptibility of a soil to creep is the $C_{\alpha} / C_{c}$ ratio. According to Mesri et al. (1997), the change of $C_{\alpha}$ with time is directly related to the change of $C_{c}$ with vertical effective stress, and thus as $C_{c}$ remains constant, decreases, or increases with vertical effective stress, $\mathrm{C}_{\alpha}$ also remains constant, decreases, or increases with time, respectively. It should be noted that the $C_{\alpha} / C_{c}$ concept can be applied to in both over consolidated and normally consolidated regions, so $\mathrm{C}_{\mathrm{c}}$ represents the slope of compression curve in both regions. Using the $\mathrm{C}_{\alpha} / \mathrm{C}_{\mathrm{c}}$ concept, the change of $\mathrm{C}_{\alpha}$ of reconstituted LR soil with vertical effective stress illustrated in Figure 7.3 can be explained in the following manner: $\mathrm{C}_{\alpha}$ increases from $\sigma^{\prime}{ }_{\mathrm{v}}=50.0 \mathrm{kPa}$ to $\sigma^{{ }_{\mathrm{v}}}=200.5 \mathrm{kPa}$ because the compression index increased as the soil specimen is consolidated from the over consolidated region to normally consolidated region. The decrease of $\mathrm{C}_{\alpha}$ from $\sigma^{{ }_{\mathrm{v}}}=$ $200.5 \mathrm{kPa}$ to $\sigma_{\mathrm{v}}=1600.0 \mathrm{kPa}$ is related to the fact that the compression curve of reconstituted LR soil is slightly S-shaped so that the compression index decreases with vertical effective stress in this range.

The secondary compression indices and the corresponding compression indices at the same vertical effective stress are plotted in Figure 7.4. From this curve, the ratio of $\mathrm{C}_{\alpha} / \mathrm{C}_{\mathrm{c}}$ for reconstituted LR soil can be obtained as the slope of the linear best-fitting curve that goes through the origin. The result shows that the value of $\mathrm{C}_{\alpha} / \mathrm{C}_{\mathrm{c}}$ for reconstituted LR soil is approximately 0.103 . It should be also noted that the $C_{\alpha} / C_{c}$ for reconstituted LR soil calculated with a single value of secondary compression index 
obtained at $\sigma^{\prime}{ }_{\mathrm{v}}=1600 \mathrm{kPa}$ and the corresponding compression index yields a value of 0.094, only $8 \%$ less than the average $C_{\alpha} / C_{c}$ value obtained from the linear regression.

The values of $\mathrm{C}_{\alpha} / \mathrm{C}_{\mathrm{c}}$ for various geotechnical materials are summarized in Table 7.3. Compared to the average value of $C_{\alpha} / C_{c}$ for peat and muskeg $(0.06 \pm 0.01)$, reconstituted LR soil has much higher value of $\mathrm{C}_{\alpha} / \mathrm{C}_{\mathrm{c}}$. However, as indicated in Table 7.4, the $\mathrm{C}_{\alpha} / \mathrm{C}_{\mathrm{c}}$ for peat deposits reported in literatures ranges from 0.035 to 0.10 , and the $\mathrm{C}_{\alpha} / \mathrm{C}_{\mathrm{c}}$ for reconstituted LR soil falls at the upper bound of this range.

\subsection{EFFECTS OF TREATMENT WITH PORTLAND CEMENT}

The effects of treatment with Portland cement on the creep behavior of LR soil were investigated by performing EOP-IL consolidation tests and long term creep tests on LR soil treated with 8.0, 18.7, 51.4 and $103.4 \%$ PC by dry mass of soil following the specimen preparation procedure described in Chapter 4.

The specimen data for these tests are summarized in Table 7.1 including test number, soil bin number, the amounts of PC added (\%PC), organic content (OC), initial water content $\left(\mathrm{w}_{\mathrm{o}}\right)$, specific gravity $\left(\mathrm{G}_{\mathrm{s}}\right)$ and initial void ratio $\left(\mathrm{e}_{\mathrm{o}}\right)$. For each cement contents, two soil specimens were prepared from the same batch, and one CRS and one EOP-IL consolidation tests were performed to check the repeatability and consistency of the results. The results of the compression parameters of these tests are reported in Chapter 6. In this section, only the creep data are discussed.

\subsubsection{Effects of treatment with 8.0\% PC}

The data of the EOP-IL consolidation test performed on 8\% PC treated LR soil (CRS0048) is summarized in Table 7.5, including the applied effective stress, EOP void ratio $\left(\mathrm{e}_{\mathrm{EOP}}\right)$, time required for reaching $\mathrm{EOP}\left(\mathrm{t}_{\mathrm{EOP}}\right)$ estimated from excess pore pressures measurements, maximum excess pore pressures $\left(\Delta \mathrm{u}_{\mathrm{hmax}}\right)$, the ratio of maximum excess pore pressures to applied load increment $\left(\Delta \mathrm{u}_{\mathrm{hmax}} / \Delta \sigma^{\prime}{ }_{\mathrm{v}}\right)$, time for maximum excess pore pressures development $\left(\mathrm{t}_{\Delta} \mathrm{u}_{\mathrm{hmax}}\right)$, duration of each load increment $\left(\mathrm{t}_{\mathrm{f}}\right)$, the ratio of duration 
of each load increment to the time required for EOP $\left(\mathrm{t}_{\mathrm{f}} / \mathrm{t}_{\mathrm{EOP}}\right)$, compression index $\left(\mathrm{c}_{\mathrm{c}}\right)$ and secondary compression index $\left(\mathrm{c}_{\alpha}\right)$ of each load increment. All the PC treated LR soil specimens were saturated with back pressure of approximately $560 \mathrm{kPa}$ for one and a half to two days prior to testing. In this test, the maximum excess pore pressures are about $80 \%$ of the applied load in over consolidated region, and are about $92 \%$ and higher in normally consolidated region.

The specimen was loaded from $\sigma^{\prime}{ }_{\mathrm{v}}=6.3 \mathrm{kPa}$ to $\sigma^{\prime}{ }_{\mathrm{v}}=1573 \mathrm{kPa}$ with a load increment ratio of unity. Each load increment was applied for $1.02\left(\sigma^{\prime}{ }_{\mathrm{v}}=48.6 \mathrm{kPa}\right)$ to $1.32\left(\sigma^{\prime}{ }_{\mathrm{v}}=6.32 \mathrm{kPa}\right)$ times of $\mathrm{t}_{\mathrm{EOP}}$, until reaching $\sigma_{{ }_{\mathrm{v}}}=1573 \mathrm{kPa}$ at which long term creep test was performed. Given the very short creep period at the lower effective stresses the secondary compression index was determined only at $1573 \mathrm{kPa}$.

The EOP compression curve obtained from this test is plotted and compared with the results from CRS048 in Figure 7.5. Despite the variation in initial void ratios, the two compression curves show good repeatability in normally consolidated region.

The time settlement curve and dissipation of excess pore pressures obtained from the long term creep test performed at $\sigma^{\prime}{ }_{\mathrm{v}}=1573 \mathrm{kPa}$ are plotted in Figure 7.6. The load was applied for 23626 minutes and the soil specimen was allowed to creep for 39.0 times of $\mathrm{t}_{\mathrm{EOP}}$ or 1.6 cycles of secondary compression. Similar to the reconstituted LR soil, the slope of the time settlement curve in the secondary compression stage remains constant, with the secondary compression index being 0.106. Based on the data for the stress increment at $\sigma^{\prime}{ }_{\mathrm{v}}=1573 \mathrm{kPa}$ the $\mathrm{C}_{\alpha} / \mathrm{C}_{\mathrm{c}}$ ratio for $8 \% \mathrm{PC}$ treated $\mathrm{LR}$ soil is 0.085 .

\subsubsection{Effects of treatment with 18.7\% PC}

The data of the EOP-IL consolidation test performed on 18.7\% PC treated LR soil (CRS0042) is summarized in Table 7.6. In this test, the maximum excess pore pressures generated in each load increment were consistently smaller than the applied load increment: less than $80 \%$ of the applied load in the overconsolidated region $\left(\sigma^{\prime}{ }_{\mathrm{v}}<94.4\right.$ $\mathrm{kPa}$ ), and between 85 and $90 \%$ in the normally consolidated region. 
In this test, the soil specimen was loaded from $\sigma^{{ }_{\mathrm{v}}}=5.9 \mathrm{kPa}$ to $\sigma^{{ }_{\mathrm{v}}}=1558 \mathrm{kPa}$ with a load increment ratio of unity. Each load increment was applied for $1.08\left(\sigma^{\prime}{ }_{\mathrm{v}}=\right.$ $394.1 \mathrm{kPa})$ to $2.66\left(\sigma^{\prime}{ }_{\mathrm{v}}=11.9 \mathrm{kPa}\right)$ times of $\mathrm{t}_{\mathrm{EOP}}$, except at $\sigma^{{ }_{\mathrm{v}}}=1558 \mathrm{kPa}$ where a long term creep test was performed. The EOP compression curve from this test is also in good agreement with the compression curve obtained from CRS consolidation test (CRS043) as shown in Figure 7.7.

The time settlement and dissipation of excess pore pressures of 18.7\% PC treated specimen are plotted in Figure 7.8. The EOP was reached in 153 minutes, and the soil specimen was allowed to creep for 26808 minutes or 2.2 cycles of secondary compression. Similar to the previous results, the slope of the time settlement curve during secondary compression stage remained constant, with the secondary compression index of 0.08 . The $\mathrm{C}_{\alpha} / \mathrm{C}_{\mathrm{c}}$ of $18.7 \%$ PC treated LR soil is calculated as 0.063 using the compression index from the EOP-IL consolidation test at $\sigma^{\prime}{ }_{\mathrm{v}}=1600 \mathrm{kPa}$.

\subsubsection{Effects of treatment with 51.4\% PC}

Table 7.7 summarizes the data of the EOP-IL consolidation test performed on LR soil treated with 51.4\% PC (CRS050). In the over consolidated region $\left(\sigma^{\prime}{ }_{\mathrm{v}}<404.4 \mathrm{kPa}\right)$, the maximum excess pore pressures generated were about $37-71 \%$ of the applied load except at $\sigma_{\mathrm{v}}=49.3 \mathrm{kPa}$ where $100 \%$ of excess pore pressures were developed. In the normally consolidated region, the excess pore pressures response improved with the maximum values of about $82-86 \%$ of the applied load.

As shown in Figure 7.9, the EOP compression curve from this test follows the same curve obtained from CRS consolidation test (CRS051).

Figure 7.10 presents the time settlement curve and dissipation of excess pore pressure of $51.4 \%$ PC treated soil specimen at $\sigma^{\prime}{ }_{\mathrm{v}}=1574 \mathrm{kPa}$. The EOP was reached after 38.7 minutes, and the specimen was allowed to creep for 31701 minutes or 2.9 cycles of secondary compression. The secondary compression index of 51.4\% PC treated LR soil was measured as 0.068 , and the ratio of $\mathrm{C}_{\alpha} / \mathrm{C}_{\mathrm{c}}$ calculated with the Cc at $\sigma^{\prime}{ }_{\mathrm{v}}=$ $1574 \mathrm{kPa}$ was 0.040 . 
Due to poor response of excess pore pressures, in the overconsolidated region load increments were applied for significantly longer periods than in the previous two cases, and the secondary compression indices were obtained at $\sigma^{{ }_{\mathrm{v}}}=49.3 \mathrm{kPa}, 97.7 \mathrm{kPa}$ and $199.1 \mathrm{kPa}$. The secondary compression indices and the compression indices measured at these vertical effective stress levels are plotted in Figure 7.11. The $C_{\alpha} / C_{c}$ calculated as the slope of the linear best-fitting curve is 0.040 and shows very good correlation with $\mathrm{R}^{2}$ value of 0.9924 . The result indicates that $C_{\alpha} / C_{c}$ value obtained at single effective stress level well represents the average value of $C_{\alpha} / C_{c}$ for PC treated LR soil.

\subsubsection{Effects of treatment with $103.4 \%$ PC}

The data of the EOP-IL consolidation test (CRS054) performed on LR soil treated with $103.4 \%$ PC are summarized in

Table 7.8. In the highly over consolidated region, up to $\sigma^{{ }_{v}}=43.8 \mathrm{kPa}$, the deformation was very small and no excess pore pressures were generated so the EOP points could not be determined. In the load increments between $\sigma^{\prime}{ }_{\mathrm{v}}=86.5 \mathrm{kPa}$ and $\sigma_{\mathrm{v}}{ }_{\mathrm{v}}=$ $698.1 \mathrm{kPa}$, the maximum excess pore pressures generated were less than $36 \%$ of the applied load. Even in the normally consolidated region $\left(\sigma^{\prime}{ }_{v}>1249.8 \mathrm{kPa}\right)$, the maximum excess pore pressures was only $48 \%$ of the applied load.

Due to the limitation of the sensors, only one EOP point was obtained in this test. However, the comparison of compression curve with the one obtained from the CRS consolidation test (CRS055), where the specimen was loaded up to $\sigma_{\mathrm{v}}=6322 \mathrm{kPa}$, shows that the compression curves follow the same trend in normally consolidated region (Figure 7.12)

The time settlement and dissipation of excess pore pressure of 103.4\% PC treated LR soil specimens obtained at $\sigma_{\mathrm{v}}{ }^{\prime}=1387 \mathrm{kPa}$ are shown in Figure 7.13. The EOP was reached in 24.5 minutes, and the soil specimen was allowed to creep for 22942 minutes or 3.0 cycles of secondary compression. The measured secondary compression index of 
the specimen is 0.030. Using the compression index obtained from CRS consolidation test at $\sigma^{{ }_{\mathrm{v}}}=1387 \mathrm{kPa}$, the value of $\mathrm{C}_{\alpha} / \mathrm{C}_{\mathrm{c}}$ of $103.4 \% \mathrm{PC}$ treated soil is measured as 0.027 .

\subsection{SUMMARY OF THE EFFECTS OF TREATMENT ON CREEP BEHAVIOR OF PC TREATED LR SOILS}

The effects of treatment with Portland cement on the creep behavior of LR soil were evaluated by performing EOP-IL consolidation tests involving one prolonged creep stage at the highest effective stress level $(\sim 1600 \mathrm{kPa})$. The secondary compression indices of untreated and PC treated LR soils were measured during this creep stage and at select load increments where the load was applied long enough to obtain a linear time settlement curve in secondary compression stage. The values of secondary compression index, effective stress at which long term creep tests were performed, and the values of $\mathrm{C}_{\alpha} / \mathrm{C}_{\mathrm{c}}$ for these soils are summarized in Table 7.9.

The time settlement curves from the long prolonged creep stages all lasting a month or more are compared in Figure 7.14. It should be noted that the relative vertical effective stress levels with respect to the preconsolidation pressures $\left(\sigma^{\prime}{ }_{v} / \sigma_{p}{ }_{p}\right)$ are different for these tests. However, the figure indicates that the secondary compression index of LR soil decreases gradually with increasing \%PC from the value of 0.123 for untreated LR soil to 0.106 (8\%PC), 0.080 (18.7\%PC), 0.068 (51.4\%PC), and 0.030 (103.4\%PC).

For reconstituted and 51.4\% PC treated LR soil, average value of $\mathrm{C}_{\alpha} / \mathrm{C}_{\mathrm{c}}$ were obtained from the long term creep tests and from additional select load increments. For 8.0, 18.7 and $103.4 \%$ PC treated LR soil, the $\mathrm{C}_{\alpha} / \mathrm{C}_{\mathrm{c}}$ values were obtained at one vertical effective stress level.

The $\mathrm{C}_{\alpha} / \mathrm{C}_{\mathrm{c}}$ values for untreated and PC treated LR soil are plotted against \%PC in Figure 7.15. The corresponding values of cement factors ( $\mathrm{kg}$ of cement in unit volume of treated soil) are shown in the upper $\mathrm{x}$-axis. The $\mathrm{C}_{\alpha} / \mathrm{C}_{\mathrm{c}}$ for untreated and reconstituted LR soil is 0.103 and falls at the upper range of values reported for peats. With $8.0 \%$ and $18.7 \% \mathrm{PC}, \mathrm{C}_{\alpha} / \mathrm{C}_{\mathrm{c}}$ decreases to 0.085 and 0.063 , but remains still higher than the average value for peats and muskeg $(0.06 \pm 0.01)$. With $51.4 \% \mathrm{PC}, \mathrm{C}_{\alpha} / \mathrm{C}_{\mathrm{c}}$ decreases to 0.04 and 
falls in the range of average value for inorganic clays and silts $(0.04 \pm 0.01)$. With $103.4 \% \mathrm{PC}, \mathrm{C}_{\alpha} / \mathrm{C}_{\mathrm{c}}$ further decreases to 0.024 and falls in the range of average values for granular soils $(0.02 \pm 0.01)$. 
Table 7.1: Summary of soil specimen data used in EOP-IL consolidation tests

\begin{tabular}{|c|c|c|c|c|c|c|}
\hline Test No. & Bin\# & \%PC & OC (\%) & $\mathrm{w}_{\mathrm{o}}(\%)$ & $\mathrm{G}_{\mathrm{s}}$ & $\mathrm{e}_{\mathrm{o}}$ \\
\hline CRS037 & LR-B8 & 0.0 & 55.97 & 176.55 & 1.99 & 3.67 \\
\hline CRS042 & LR-B9 & 18.7 & 40.67 & 166.85 & 2.15 & 3.74 \\
\hline CRS048 & LR-B10 & 8.0 & 42.36 & 180.17 & 2.13 & 3.95 \\
\hline CRS050 & LR-B10 & 51.4 & 44.18 & 167.98 & 2.11 & 3.66 \\
\hline CRS054 & LR-B10 & 103.4 & 44.71 & 125.97 & 2.10 & 2.65 \\
\hline
\end{tabular}

Table 7.2: Summary of data from EOP-IL consolidation test performed on reconstituted LR soil (CRS037)

\begin{tabular}{|c|c|c|c|c|c|c|c|c|c|c|}
\hline Steps & $\begin{array}{c}\sigma_{\mathrm{v}}^{\prime} \\
(\mathrm{kPa})\end{array}$ & $\mathrm{e}_{\mathrm{EOP}}$ & $\begin{array}{c}\mathrm{t}_{\mathrm{EOP}} \\
(\mathrm{min})\end{array}$ & $\begin{array}{c}\Delta \mathrm{u}_{\text {hmax }} \\
(\mathrm{kPa})\end{array}$ & $\begin{array}{c}\Delta \mathrm{u}_{\text {hmax }} / \Delta \sigma_{\mathrm{v}}^{\prime} \\
(\%)\end{array}$ & $\begin{array}{c}\mathrm{t}_{\text {पuhmax }} \\
(\mathrm{min})\end{array}$ & $\begin{array}{c}\mathrm{t}_{\mathrm{f}} \\
(\mathrm{min})\end{array}$ & $\mathrm{t}_{\mathrm{f}} / \mathrm{t}_{\text {EOP }}$ & $\mathrm{C}_{\mathrm{c}}$ & $\mathrm{C}_{\alpha}$ \\
\hline $\begin{array}{c}\text { BP } \\
\text { Saturation }\end{array}$ & 2.71 & 3.67 & & & & & & & & \\
\hline Step1 & 25.4 & 3.57 & 41.3 & 17.1 & 75.4 & 0.18 & 63.2 & 1.53 & 0.106 & 0.037 \\
\hline Step2 & 37.4 & 3.48 & 111.4 & 11.4 & 95.0 & 0.22 & 200.5 & 1.80 & 0.514 & 0.047 \\
\hline Step3 & 50.0 & 3.40 & 178.4 & 11.8 & 93.7 & 0.30 & 343.6 & 1.93 & 0.673 & 0.056 \\
\hline Step4 & 74.9 & 3.24 & 338.2 & 24.4 & 98.0 & 0.23 & 510.4 & 1.51 & 0.902 & 0.093 \\
\hline Step5 & 100 & 3.10 & 429.7 & 24.8 & 98.8 & 0.23 & 1000.0 & 2.33 & 1.133 & 0.098 \\
\hline Step6 & 200.5 & 2.72 & 712.7 & 98.4 & 97.9 & 0.43 & 1001.0 & 1.40 & 1.253 & 0.145 \\
\hline Step7 & 399.6 & 2.30 & 909.2 & 191.8 & 96.3 & 0.75 & 1440.1 & 1.58 & 1.391 & 0.159 \\
\hline Step8 & 800.7 & 1.87 & 1204.4 & 382.8 & 95.4 & 1.4 & 1440.1 & 1.20 & 1.421 & 0.154 \\
\hline Step9 & 1600.0 & 1.48 & 1529.7 & 760.2 & 95.1 & 2.4 & 50065.2 & 32.7 & 1.297 & 0.123 \\
\hline
\end{tabular}


Table 7.3: Values of $C_{\alpha} / C_{c}$ for various geotechnical materials (Terzaghi et al, 1996)

\begin{tabular}{|c|c|}
\hline Material & $\mathrm{C}_{\alpha} / \mathrm{C}_{\mathrm{c}}$ \\
\hline Granular soils including rock fill & $0.02 \pm 0.01$ \\
\hline Shale and mudstone & $0.03 \pm 0.01$ \\
\hline Inorganic clays and silts & $0.04 \pm 0.01$ \\
\hline Organic clays and silts & $0.05 \pm 0.01$ \\
\hline Peat and muskeg & $0.06 \pm 0.01$ \\
\hline
\end{tabular}

Table 7.4: Values of natural water content $\left(\mathrm{w}_{0}\right)$, and $\mathrm{C}_{\alpha} / \mathrm{C}_{\mathrm{c}}$ for peat deposits (Mesri et al., 1997)

\begin{tabular}{|l|c|c|l|}
\hline \multicolumn{1}{|c|}{ Peat } & $\mathrm{w}_{\mathrm{o}}(\%)$ & $\mathrm{C}_{\alpha} / \mathrm{C}_{\mathrm{c}}$ & \multicolumn{1}{|c|}{ Reference } \\
\hline Fibrous peat & 850 & $0.06-0.10$ & Hanrahan (1954) \\
\hline Peat & 520 & $0.061-0.078$ & Lewis (1956) \\
\hline Amorphous and fibrous peat & $500-1500$ & $0.035-0.083$ & Lea and Brawner (1963) \\
\hline Canadian muskeg & $200-600$ & $0.09-0.10$ & Adams (1965) \\
\hline Amorphous to fibrous peat & 705 & $0.073-0.091$ & Keene and Zawodniak (1968) \\
\hline Peat & $400-750$ & $0.075-0.085$ & Weber (1969) \\
\hline Fibrous peat & $605-1290$ & $0.052-0.072$ & Samson and LaRochell (1972) \\
\hline Fibrous peat & $613-886$ & $0.06-0.085$ & Berry and Vickers (1975) \\
\hline Amorphous to fibrous peat & 600 & $0.042-0.083$ & Dhowian and Edil (1981) \\
\hline Fibrous peat & $660-1590$ & 0.06 & Lefebvre et al. (1984) \\
\hline Dutch peat & 370 & 0.06 & Den Haan (1994) \\
\hline Fibrous peat & $610-850$ & 0.052 & Mesri et al. (1997) \\
\hline
\end{tabular}


Table 7.5: Summary of data from EOP-IL consolidation test performed on 8\% PC treated LR soil (CRS048)

\begin{tabular}{|c|c|c|c|c|c|c|c|c|c|c|}
\hline Steps & $\begin{array}{c}\sigma_{\mathrm{v}}^{\prime} \\
(\mathrm{kPa})\end{array}$ & $\mathrm{e}_{\mathrm{EOP}}$ & $\begin{array}{c}\mathrm{t}_{\mathrm{EOP}} \\
(\mathrm{min})\end{array}$ & $\begin{array}{c}\Delta \mathrm{u}_{\mathrm{hmax}} \\
(\mathrm{kPa})\end{array}$ & $\begin{array}{c}\Delta \mathrm{u}_{\mathrm{hmax}} / \Delta \sigma_{\mathrm{v}}^{\prime} \\
(\%)\end{array}$ & $\begin{array}{c}\mathrm{t}_{\Delta \mathrm{uhmax}} \\
(\mathrm{min})\end{array}$ & $\begin{array}{c}\mathrm{t}_{\mathrm{f}} \\
(\mathrm{min})\end{array}$ & $\mathrm{t}_{\mathrm{f}} / \mathrm{t}_{\mathrm{EOP}}$ & $\mathrm{C}_{\mathrm{c}}$ & $\mathrm{C}_{\alpha}$ \\
\hline $\begin{array}{c}\text { BP } \\
\text { Saturation }\end{array}$ & 1.55 & 3.95 & & & & & & & & \\
\hline Step1 & 6.32 & 3.93 & 6.8 & 3.7 & 77.6 & 0.18 & 9.0 & 1.32 & - & - \\
\hline Step2 & 12.1 & 3.91 & 22.0 & 4.6 & 79.6 & 0.23 & 27.0 & 1.23 & 0.045 & - \\
\hline Step3 & 23.9 & 3.87 & 30.3 & 9.9 & 83.9 & 0.1 & 35.1 & 1.16 & 0.160 & - \\
\hline Step4 & 48.6 & 3.66 & 254.6 & 19.7 & 79.8 & 0.08 & 260.4 & 1.02 & 0.672 & - \\
\hline Step5 & 98.3 & 3.30 & 355.7 & 49.6 & 99.8 & 0.23 & 380.8 & 1.07 & 1.170 & - \\
\hline Step6 & 196.1 & 2.89 & 383.0 & 94.6 & 96.7 & 0.57 & 500.6 & 1.31 & 1.368 & - \\
\hline Step7 & 393.5 & 2.47 & 437.7 & 188.4 & 95.4 & 0.4 & 480.6 & 1.10 & 1.400 & - \\
\hline Step8 & 786.9 & 2.04 & 542.1 & 377.6 & 96.0 & 1.0 & 600.6 & 1.11 & 1.407 & - \\
\hline Step9 & 1572.7 & 1.67 & 605.1 & 719.6 & 91.6 & 1.3 & 23625.7 & 39.0 & 1.251 & 0.106 \\
\hline
\end{tabular}

Table 7.6: Summary of data from EOP-IL consolidation test performed on $18.7 \%$ PC treated LR soil (CRS042)

\begin{tabular}{|c|c|c|c|c|c|c|c|c|c|c|}
\hline Steps & $\begin{array}{c}\sigma_{\mathrm{v}}^{\prime} \\
(\mathrm{kPa})\end{array}$ & $\mathrm{e}_{\mathrm{EOP}}$ & $\begin{array}{l}t_{\mathrm{EOP}} \\
(\mathrm{min})\end{array}$ & $\begin{array}{c}\Delta \mathrm{u}_{\mathrm{hmax}} \\
(\mathrm{kPa})\end{array}$ & $\begin{array}{c}\Delta \mathrm{u}_{\mathrm{hmax}} / \Delta \sigma_{\mathrm{v}}^{\prime} \\
(\%)\end{array}$ & $\begin{array}{l}\mathrm{t}_{\Delta \text { uhmax }} \\
\text { (min) }\end{array}$ & $\begin{array}{c}\mathrm{t}_{\mathrm{f}} \\
(\mathrm{min})\end{array}$ & $\mathrm{t}_{\mathrm{f}} / \mathrm{t}_{\mathrm{EOP}}$ & $\mathrm{C}_{\mathrm{c}}$ & $\mathrm{C}_{\alpha}$ \\
\hline $\begin{array}{c}\text { BP } \\
\text { Saturation }\end{array}$ & 0.2 & 3.74 & & & & & & & & \\
\hline Step1 & 5.87 & 3.68 & 14.2 & 5.67 & 100.0 & 0.28 & 20.1 & 1.42 & - & - \\
\hline Step2 & 11.9 & 3.67 & 6.4 & 4.19 & 69.5 & 0.20 & 17.0 & 2.66 & 0.049 & - \\
\hline Step3 & 24.1 & 3.64 & 10.9 & 8.98 & 73.6 & 0.15 & 17.0 & 1.56 & 0.089 & - \\
\hline Step4 & 48.9 & 3.58 & 22.2 & 19.6 & 79.0 & 0.27 & 41.1 & 1.85 & 0.205 & - \\
\hline Step5 & 98.3 & 3.33 & 78.0 & 42.2 & 85.4 & 0.17 & 90.2 & 1.16 & 0.831 & - \\
\hline Step6 & 196.4 & 2.99 & 79.4 & 88.4 & 90.1 & 0.25 & 100.2 & 1.26 & 1.125 & - \\
\hline Step7 & 394.1 & 2.59 & 102.2 & 176.4 & 89.2 & 0.08 & 110.2 & 1.08 & 1.325 & - \\
\hline Step8 & 785.2 & 2.19 & 109.1 & 352.4 & 90.1 & 0.21 & 140.4 & 1.29 & 1.323 & - \\
\hline Step9 & 1557.8 & 1.80 & 153.1 & 694.8 & 89.9 & 0.38 & 26808 & 175.1 & 1.271 & 0.080 \\
\hline
\end{tabular}


Table 7.7: Summary of data from EOP-IL consolidation test performed on 51.4\% PC treated LR soil (CRS050)

\begin{tabular}{|c|c|c|c|c|c|c|c|c|c|c|}
\hline Steps & $\begin{array}{c}\sigma_{\mathrm{v}}^{\prime} \\
(\mathrm{kPa})\end{array}$ & $\mathrm{e}_{\mathrm{EOP}}$ & $\begin{array}{c}\mathrm{t}_{\mathrm{EOP}} \\
(\mathrm{min})\end{array}$ & $\begin{array}{c}\Delta \mathrm{u}_{\mathrm{hmax}} \\
(\mathrm{kPa})\end{array}$ & $\begin{array}{c}\Delta \mathrm{u}_{\mathrm{hmax}} / \Delta \sigma_{\mathrm{v}}^{\prime} \\
(\%)\end{array}$ & $\begin{array}{c}\mathrm{t}_{\Delta \mathrm{uhmax}} \\
(\mathrm{min})\end{array}$ & $\begin{array}{c}\mathrm{t}_{\mathrm{f}} \\
(\mathrm{min})\end{array}$ & $\mathrm{t}_{\mathrm{f}} / \mathrm{t}_{\mathrm{EOP}}$ & $\mathrm{C}_{\mathrm{c}}$ & $\mathrm{C}_{\alpha}$ \\
\hline $\begin{array}{c}\text { BP } \\
\text { Saturation }\end{array}$ & 1.28 & 3.66 & & & & & & & & \\
\hline Step1 & 5.62 & 3.65 & 0.20 & 1.62 & 37.3 & 0.08 & 5.0 & 25.0 & - & - \\
\hline Step2 & 12.4 & 3.65 & 0.52 & 2.00 & 29.5 & 0.10 & 6.0 & 11.5 & - & - \\
\hline Step3 & 24.6 & 3.65 & 1.43 & 8.01 & 65.7 & 0.08 & 5.0 & 3.50 & 0.011 & - \\
\hline Step4 & 49.3 & 3.64 & 1.47 & 25.4 & 100.0 & 0.08 & 5.0 & 3.40 & 0.027 & 0.001 \\
\hline Step5 & 97.7 & 3.63 & 0.48 & 34.6 & 71.5 & 0.07 & 4.0 & 8.33 & 0.030 & 0.002 \\
\hline Step6 & 199.1 & 3.62 & 0.50 & 38.1 & 37.6 & 0.05 & 6.0 & 12.0 & 0.055 & 0.007 \\
\hline Step7 & 395.8 & 3.44 & 17.52 & 109.0 & 55.4 & 0.22 & 19.0 & 1.08 & 0.601 & - \\
\hline Step8 & 788.9 & 3.01 & 28.68 & 322.8 & 82.1 & 0.12 & 30.0 & 1.05 & 1.435 & - \\
\hline Step9 & 1574.2 & 2.49 & 38.72 & 675.4 & 86.0 & 0.12 & 31701 & 818.7 & 1.719 & 0.068 \\
\hline
\end{tabular}

Table 7.8: Summary of data from EOP-IL consolidation test performed on 103.4\% PC treated LR soil (CRS054)

\begin{tabular}{|c|c|c|c|c|c|c|c|c|c|c|}
\hline Steps & $\begin{array}{c}\sigma_{\mathrm{v}}^{\prime} \\
(\mathrm{kPa})\end{array}$ & $\mathrm{e}_{\mathrm{EOP}}$ & $\begin{array}{l}\mathrm{t}_{\mathrm{EOP}} \\
(\min )\end{array}$ & $\begin{array}{r}\Delta \mathrm{u}_{\mathrm{hmax}} \\
(\mathrm{kPa})\end{array}$ & $\begin{array}{c}\Delta \mathrm{u}_{\mathrm{hmax}} / \Delta \sigma_{\mathrm{v}}^{\prime} \\
(\%)\end{array}$ & $\begin{array}{l}\mathrm{t}_{\Delta \mathrm{uhmax}} \\
(\min )\end{array}$ & $\begin{array}{c}\mathrm{t}_{\mathrm{f}} \\
(\mathrm{min})\end{array}$ & $\mathrm{t}_{\mathrm{f}} / \mathrm{t}_{\mathrm{EOP}}$ & $\mathrm{C}_{\mathrm{c}}$ & $\mathrm{C}_{\alpha}$ \\
\hline $\begin{array}{c}\text { BP } \\
\text { Saturation }\end{array}$ & 3.5 & 2.65 & & & & & & & & \\
\hline Step1 & 5.6 & - & - & - & - & - & 4.0 & - & - & - \\
\hline Step2 & 10.6 & - & - & - & - & - & 5.0 & - & - & - \\
\hline Step3 & 22.2 & - & - & - & - & - & 6.0 & - & - & - \\
\hline Step4 & 43.8 & - & - & - & - & - & 4.0 & - & - & - \\
\hline Step5 & 86.5 & 2.64 & 0.20 & 1.8 & 4.2 & 0.07 & 9.0 & 45.0 & - & - \\
\hline Step6 & 174.2 & 2.62 & 0.27 & 32.1 & 36.6 & 0.05 & 6.0 & 22.2 & 0.055 & - \\
\hline Step7 & 348.6 & 2.61 & 0.35 & 33.5 & 19.2 & 0.05 & 5.0 & 14.3 & 0.038 & - \\
\hline Step8 & 698.1 & 2.58 & 2.43 & 65.0 & 18.6 & 0.07 & 6.0 & 2.47 & 0.080 & - \\
\hline Step9 & 1387.1 & 2.42 & 24.5 & 332.7 & 48.3 & 0.5 & 22942 & 936.4 & 0.546 & 0.030 \\
\hline
\end{tabular}


Table 7.9: Summary of the secondary compression index and the ratio of $C_{\alpha} / C_{c}$ for untreated (reconstituted) and PC treated LR soils

\begin{tabular}{|c|c|c|c|}
\hline Test No. & \%PC & $\mathrm{C}_{\alpha}$ & $\mathrm{C}_{\alpha} / \mathrm{C}_{\mathrm{c}}$ \\
\hline CRS037 & 0.0 & 0.123 (at $1600.0 \mathrm{kPa})$ & 0.103 \\
\hline CRS048 & 8.0 & $0.106($ at $1572.7 \mathrm{kPa})$ & 0.085 \\
\hline CRS042 & 18.7 & $0.080($ at $1557.8 \mathrm{kPa})$ & 0.063 \\
\hline CRS050 & 51.4 & $0.068($ at $1574.2 \mathrm{kPa})$ & 0.040 \\
\hline CRS054 & 103.4 & 0.030 (at $1387.1 \mathrm{kPa})$ & 0.024 \\
\hline
\end{tabular}




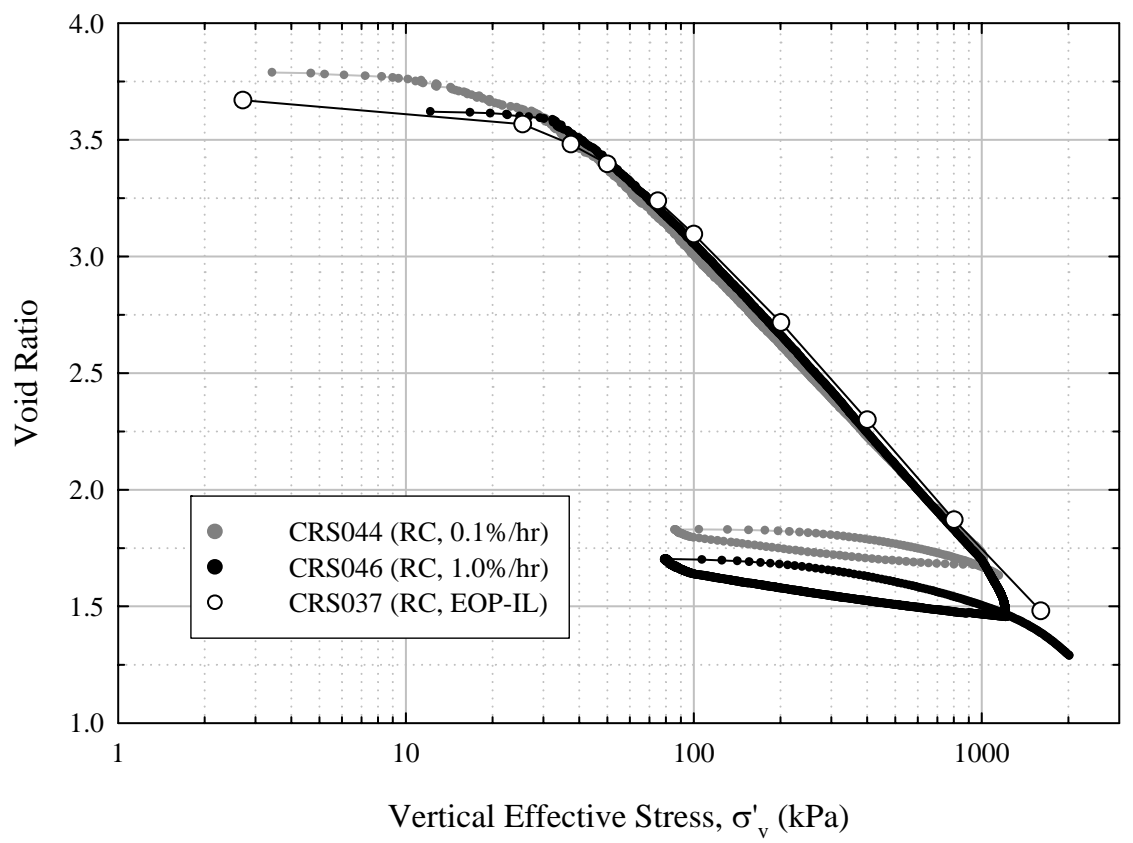

Figure 7.1: EOP compression curve of reconstituted LR soil obtained form two CRS and one EOP-IL consolidation tests

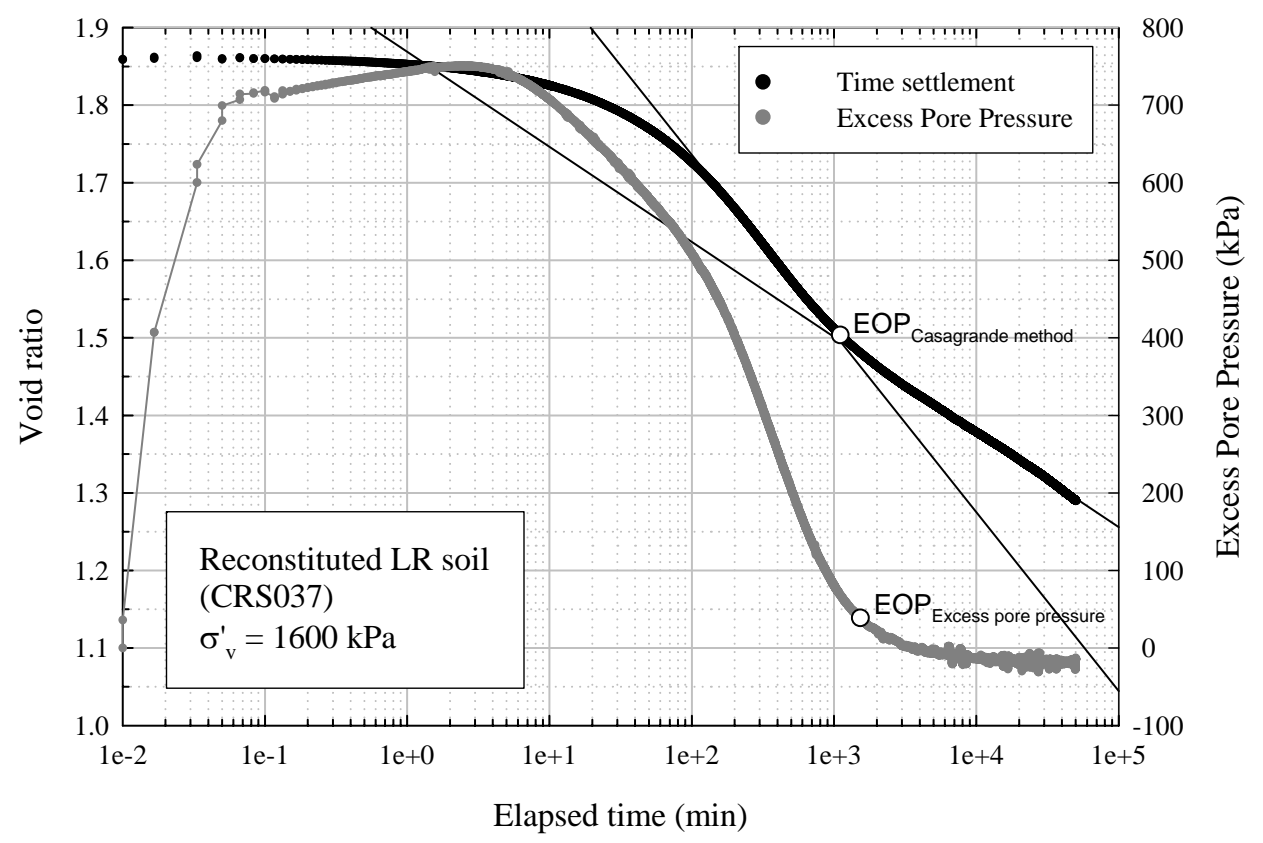

Figure 7.2: Long term creep test for determination of secondary compression index $\left(\mathrm{C}_{\alpha}\right)$ of reconstituted LR soil 


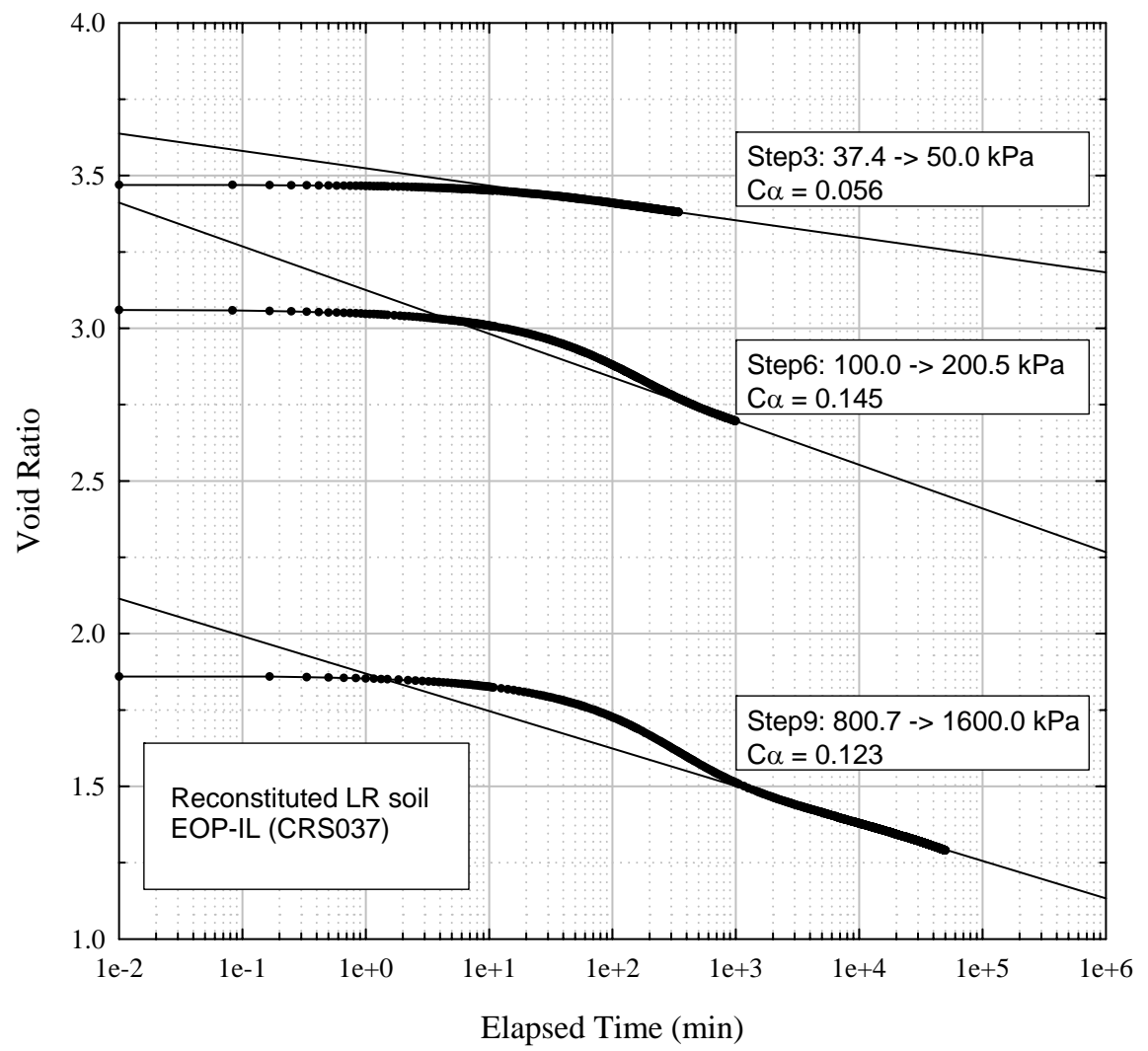

Figure 7.3: Comparison of the secondary compression index of reconstituted LR soil at different vertical effective stress levels 


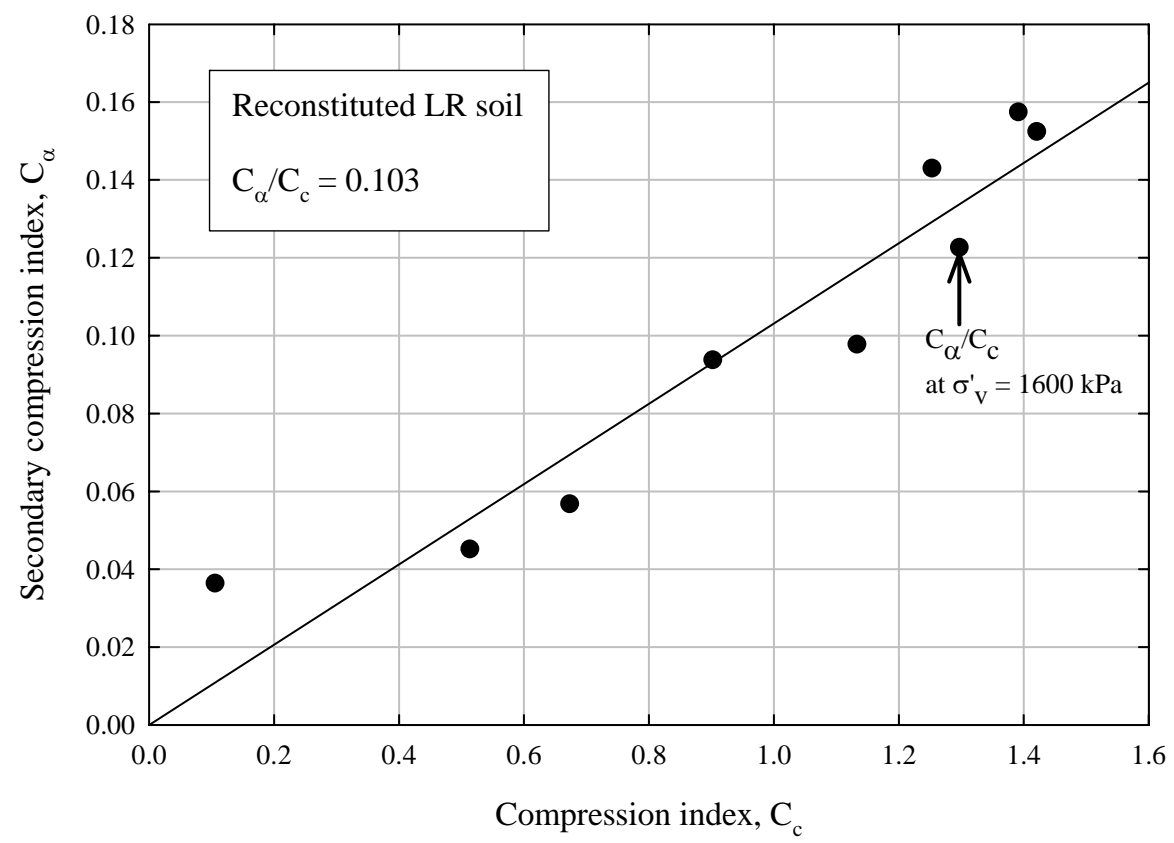

Figure 7.4: Calculation of $\mathrm{C}_{\alpha} / \mathrm{C}_{\mathrm{c}}$ for reconstituted LR soil (CRS037)

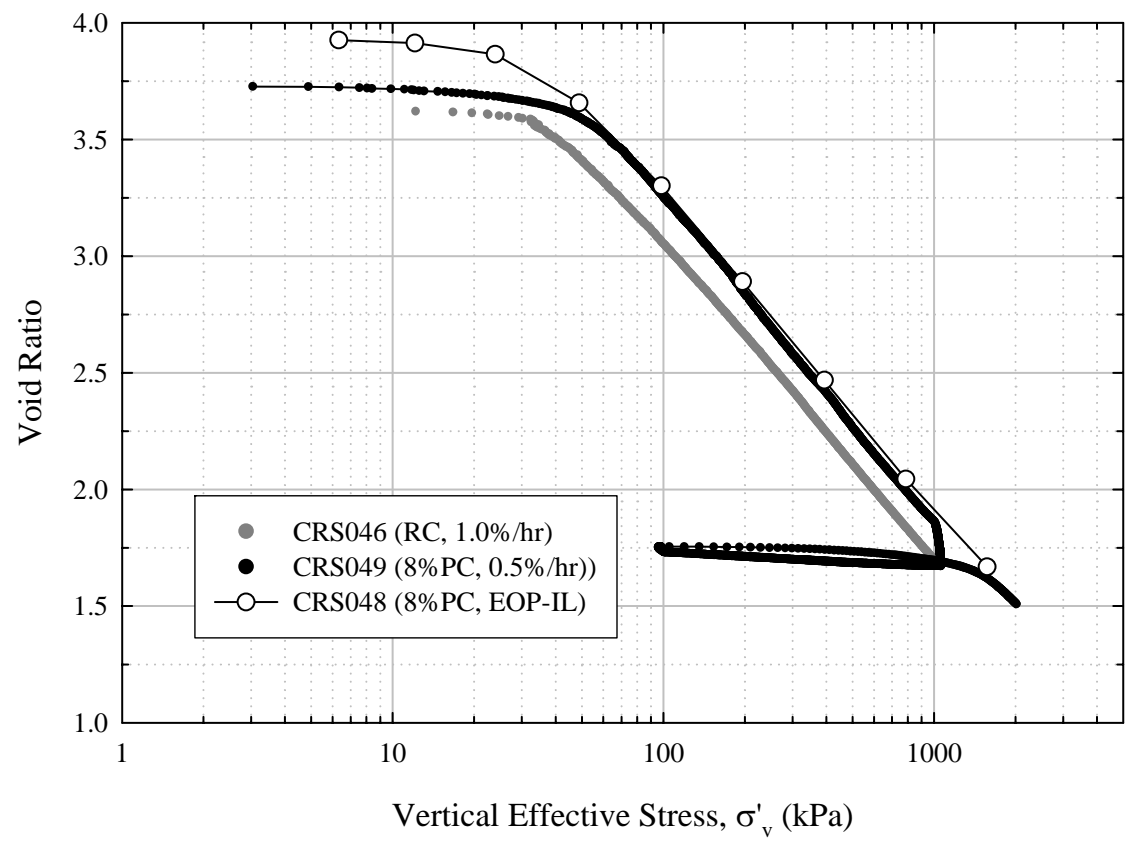

Figure 7.5: EOP compression curves of 8\% PC treated LR soil obtained from CRS and EOP-IL consolidation tests 


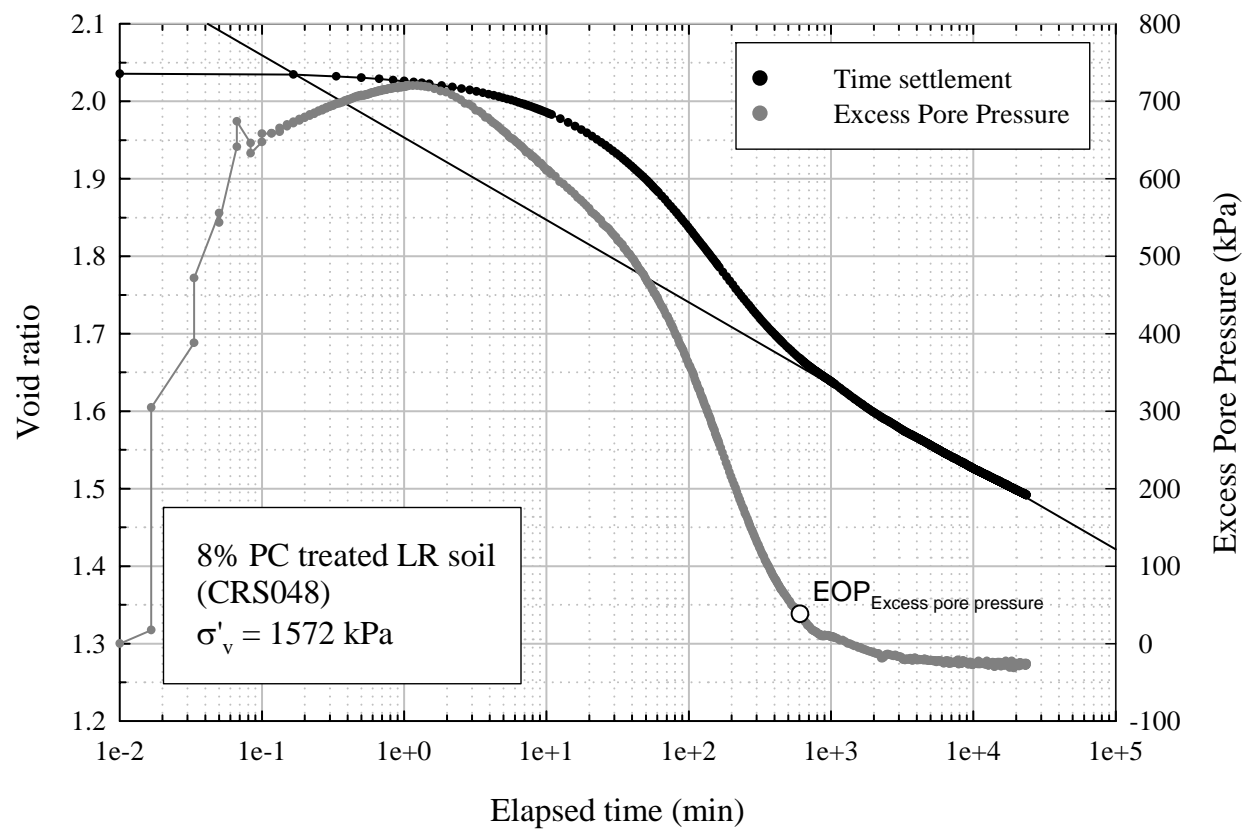

Figure 7.6: Long term creep test for determination of secondary compression index $\left(\mathrm{C}_{\alpha}\right)$ of $8 \%$ PC treated LR soil

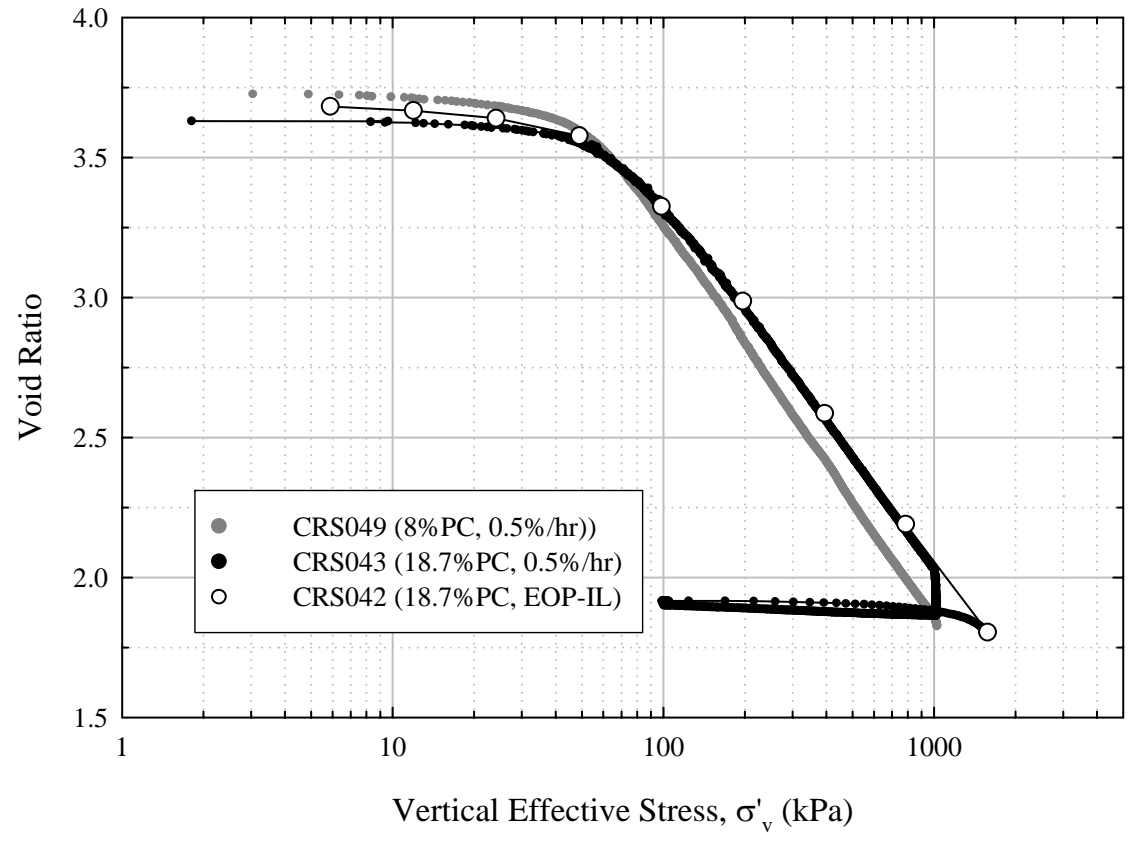

Figure 7.7: EOP compression curve of 18.7\% PC treated LR soil obtained from CRS and EOP-IL consolidation tests 


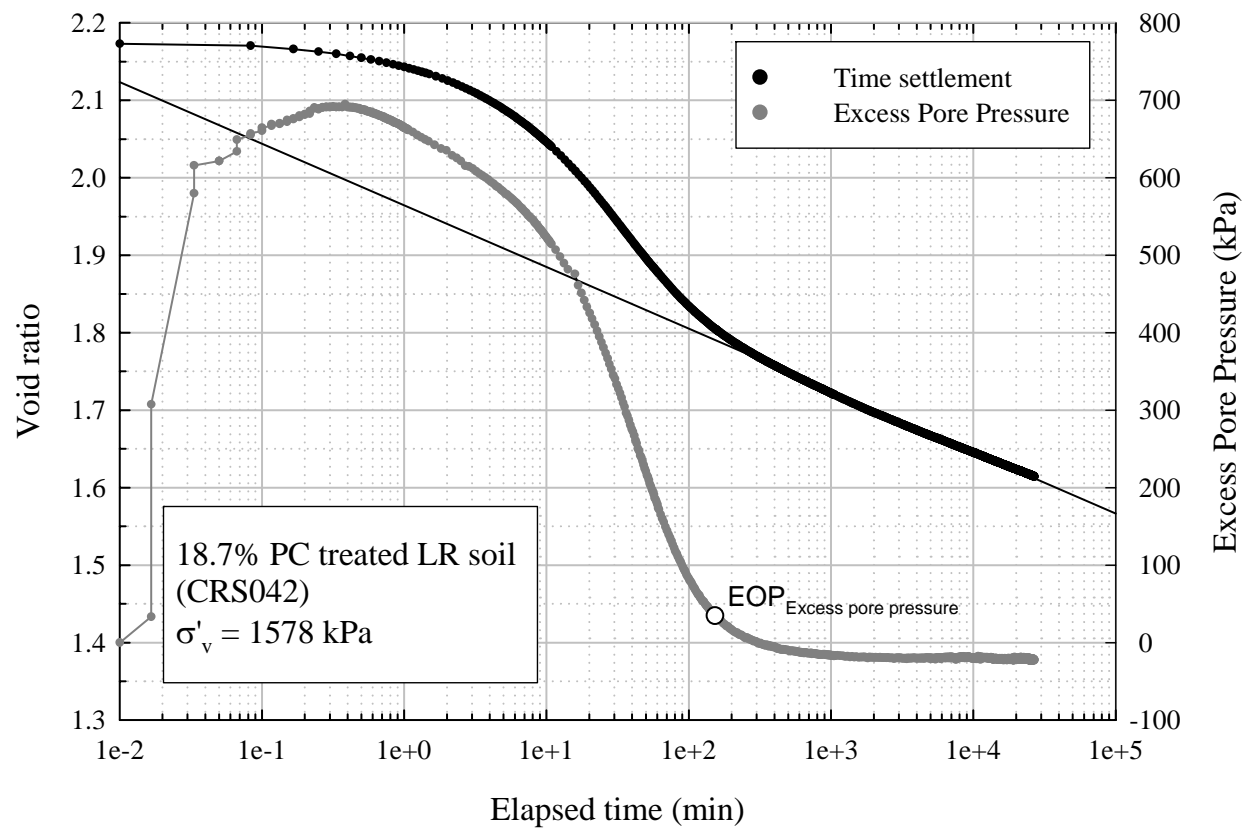

Figure 7.8: Long term creep test for determination of secondary compression index $\left(\mathrm{C}_{\alpha}\right)$ of $18.7 \%$ PC treated LR soil

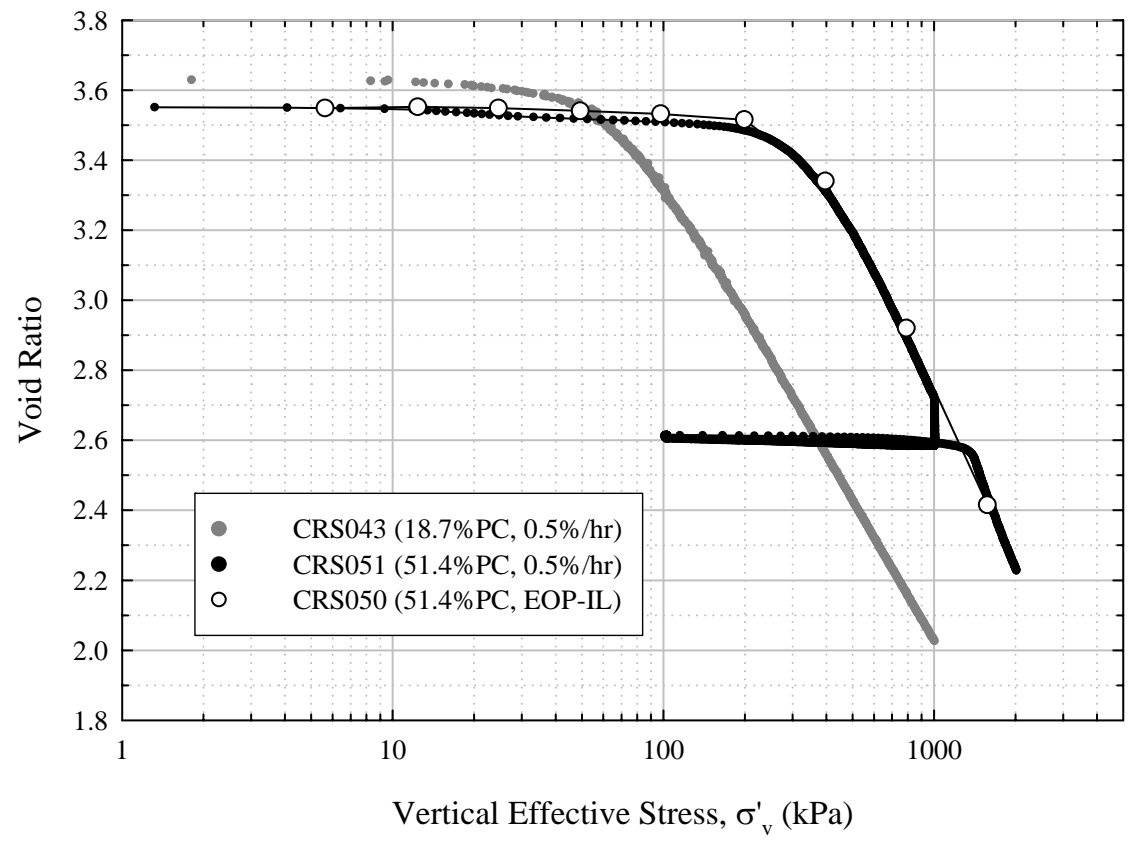

Figure 7.9: EOP compression curve of 51.4\% PC treated LR soil obtained from CRS and EOP-IL consolidation tests 


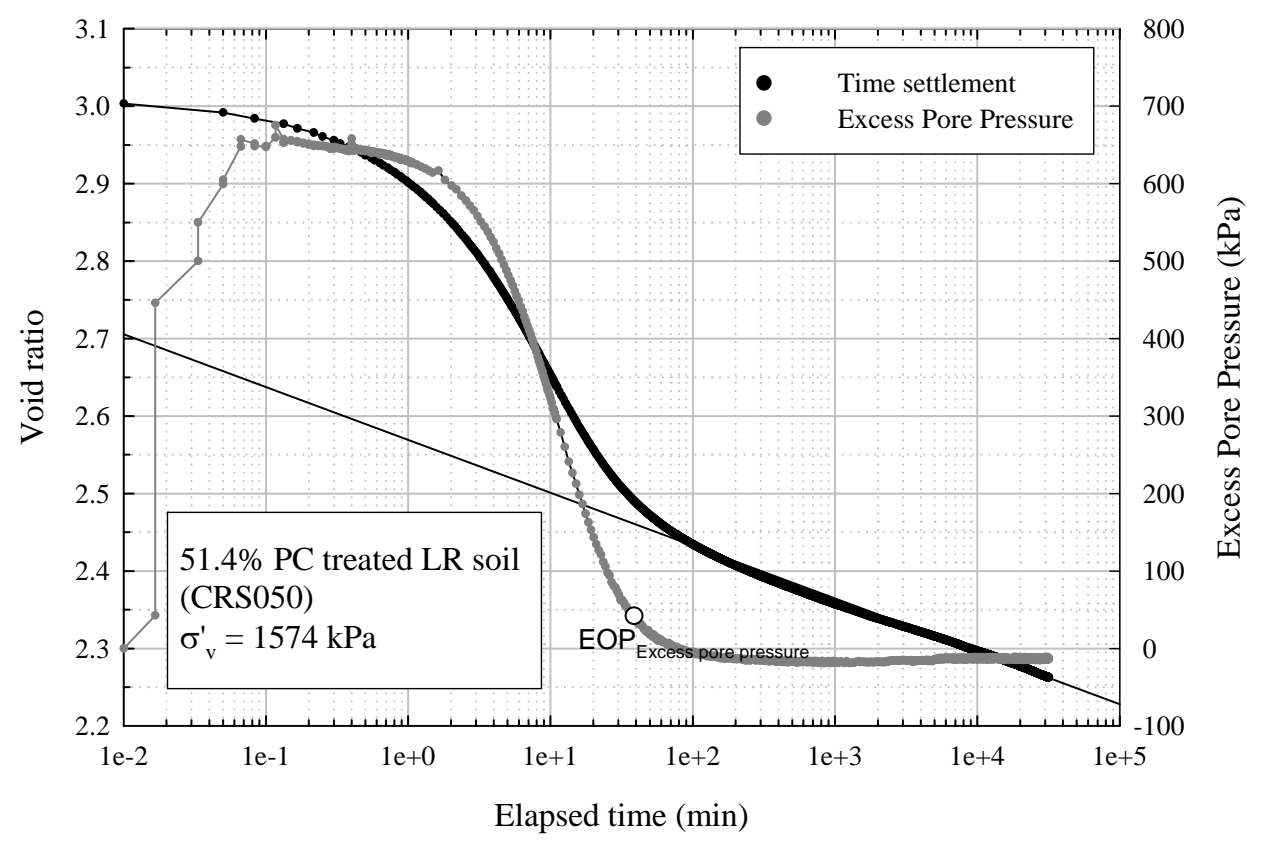

Figure 7.10: Long term creep test for determination of secondary compression index $\left(\mathrm{C}_{\alpha}\right)$ of 51.4\% PC treated LR soil

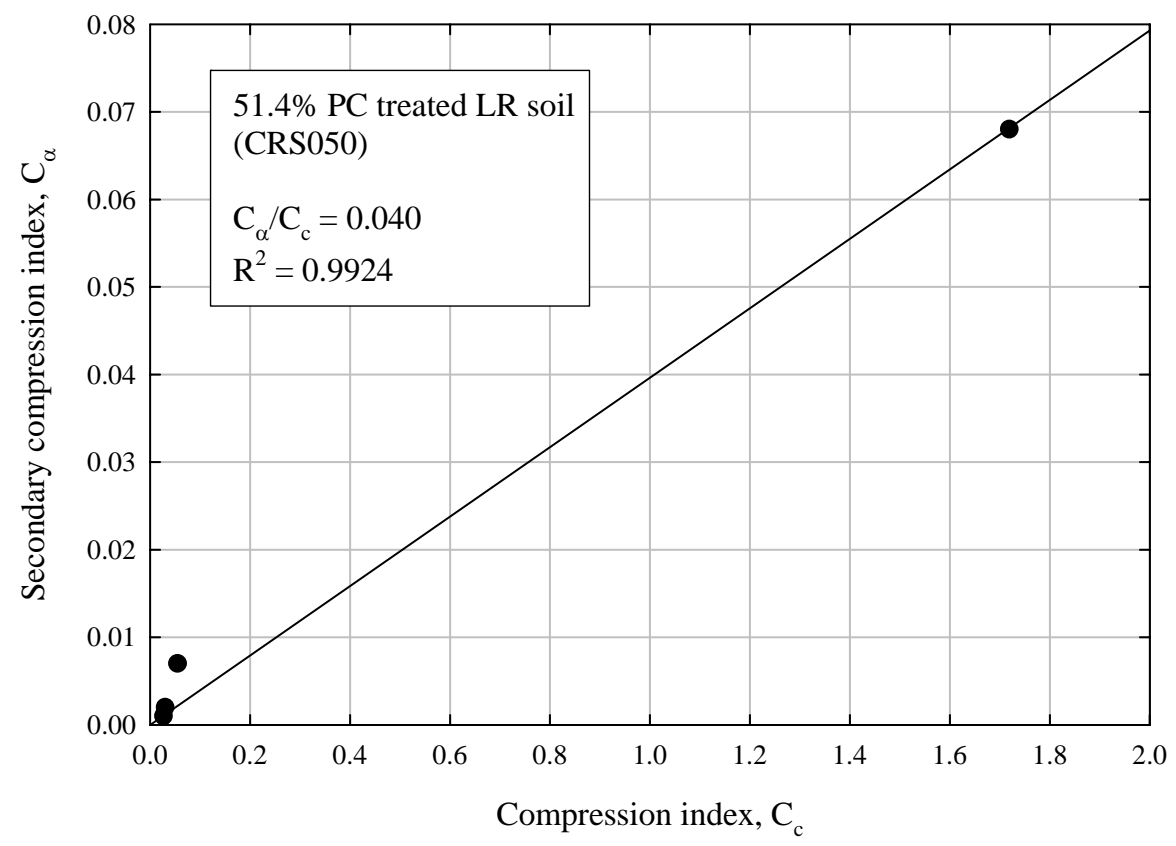

Figure 7.11: Determination of $\mathrm{C}_{\alpha} / \mathrm{C}_{\mathrm{c}}$ for $51.4 \%$ PC treated LR soil (CRS050) 


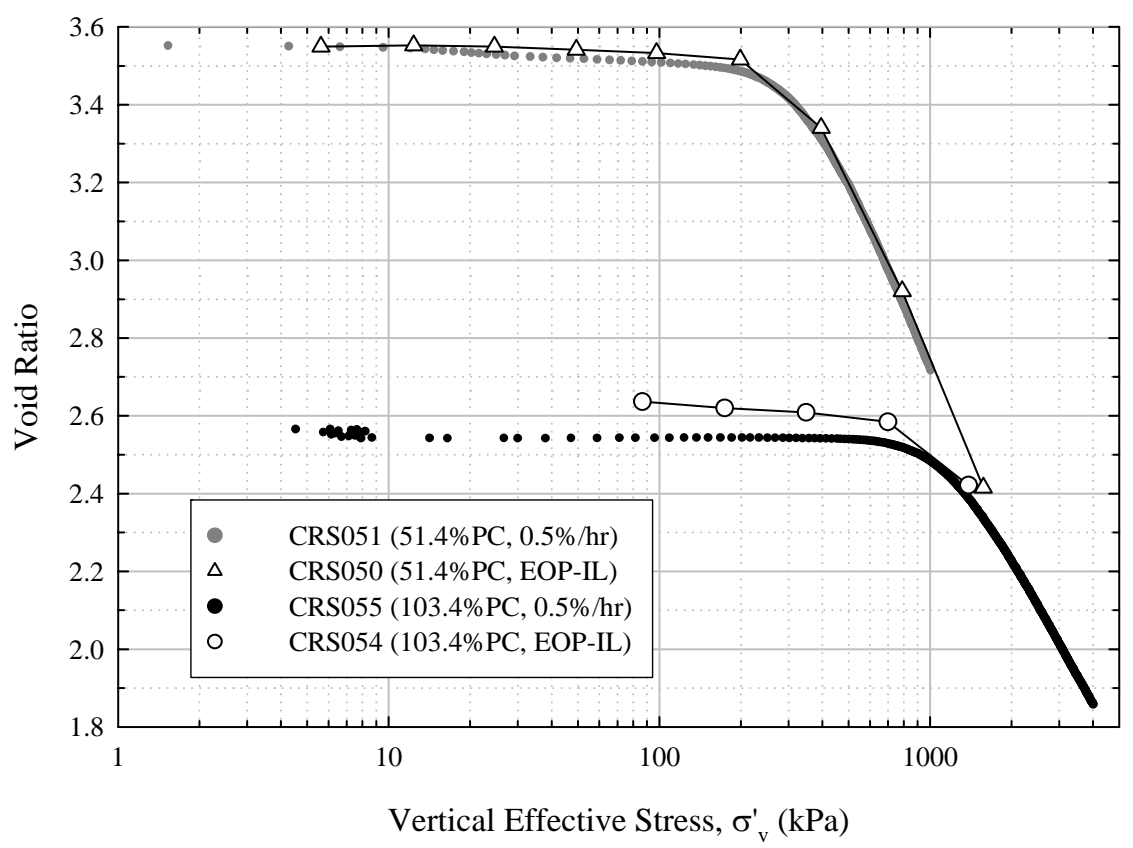

Figure 7.12: EOP compression curve of 103.4\% PC treated LR soil obtained from CRS and EOP-IL consolidation tests

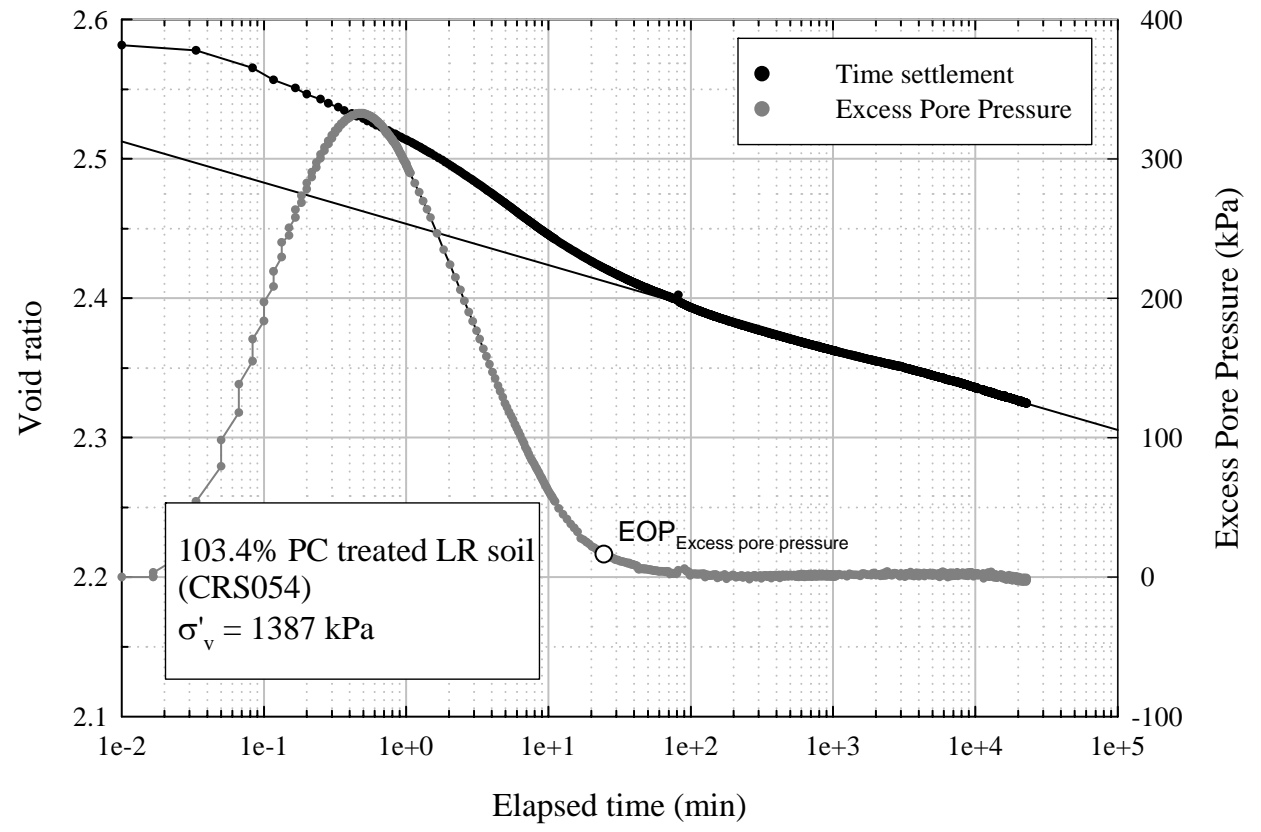

Figure 7.13: Long term creep test for determination of secondary compression index $\left(\mathrm{C}_{\alpha}\right)$ of $103.4 \%$ PC treated LR soil 


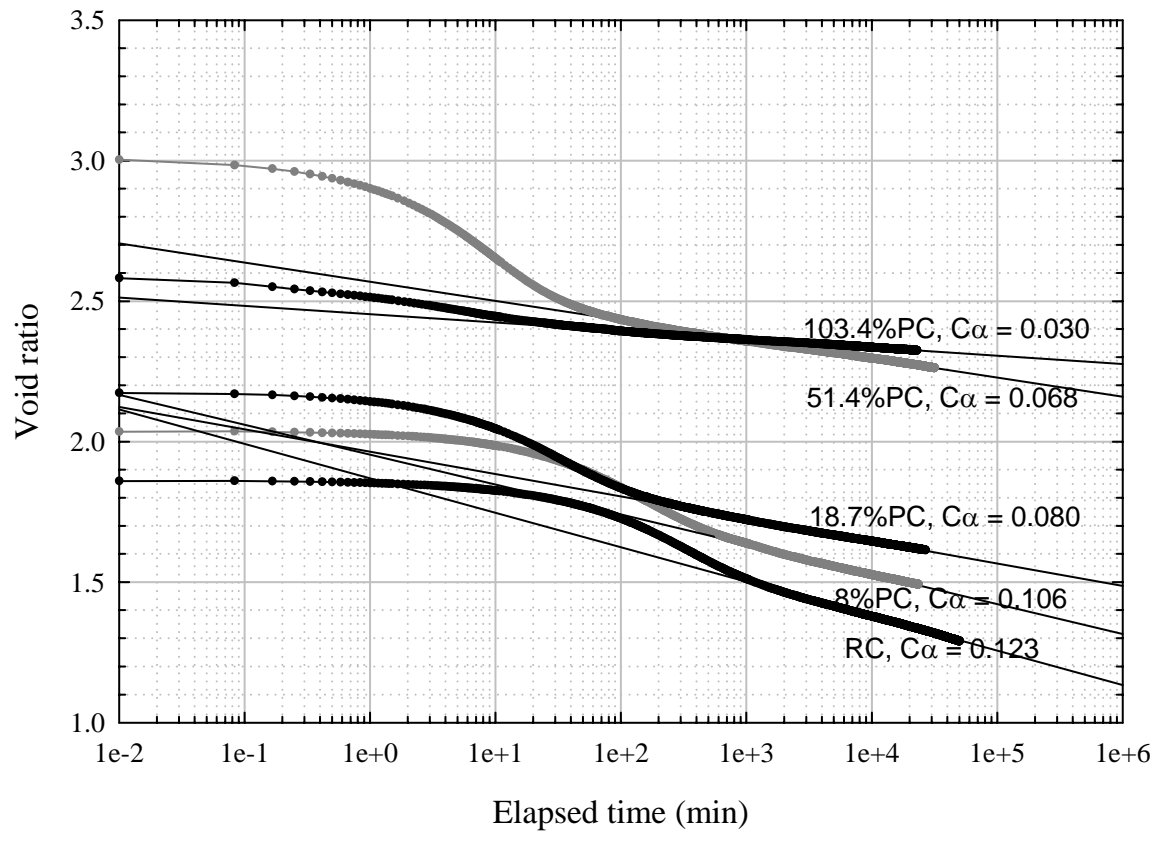

Figure 7.14: Comparison of time settlement curves from long term creep tests

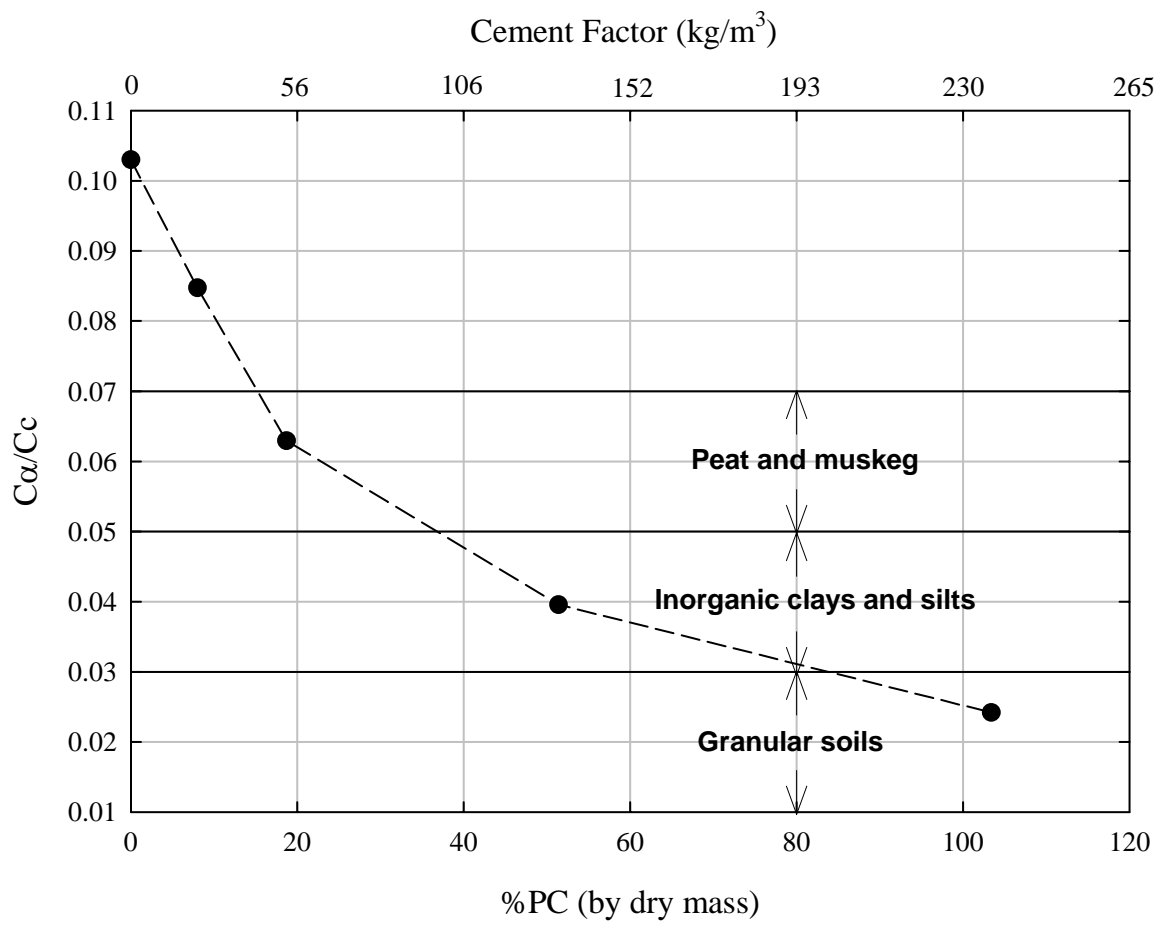

Figure 7.15: Change of the ratio of $\mathrm{Ca} / \mathrm{Cc}$ for $\mathrm{PC}$ treated $\mathrm{LR}$ soil 


\section{CHAPTER 8: CONCLUSIONS AND RECOMMENDATIONS}

\subsection{Conclusions}

This section is a summary of the most important conclusions reached with this research. It is divided in two sub-sections which address the effects of treatment on strength and stiffness, respectively.

\subsubsection{Effects of treatment on strength}

The effects of treatment on the strength of organic soils were investigated by performing Unconfined Compression (UC) tests. The effects of the following variables on the strength of the treated organic soils were evaluated:

(1) Curing time : 1,7 , and 28 days

(2) Curing surcharge : 14.5, 48, and $96 \mathrm{kPa}$

(3) Types of binder : Portland cement, calcium oxide (CaO), High Calcium Flue Dust (HC), Marblehead Buffington Dust (MD), and bentonite

(4) Amount of binder

(5) Organic content: 9.5\% (Soil Mixture I), 20\% (Soil Mixture II), and 50\% (Lindberg road soil)

The results of the UC tests indicate that the strength and stiffness of the treated and untreated soils increase with increasing curing time. This increase in strength with time may be attributed to the reduction of the void ratio in the soil during consolidation and aging of the specimen as new bonds are created between particles.

The strength and stiffness of the treated and untreated soil exhibit an essentially linear increase with surcharge. The increase of strength and stiffness with surcharge is more significant in cement treated soils. This may be due to the combined effects of the reduction in soil water content and the hydration of the cement which leads to the creation of bonds between the soil particles.

The introduction of Portland cement results in an increase of the strength and stiffness for cement contents up to $8 \%$. No significant differences are observed for PC 
additions between $8 \%$ and $20 \%$. For $50 \%$ PC the increase in strength is instead very marked.

Treatment with calcium oxide increases the strength and stiffness up to a calcium oxide content of approximately 8\%. Increasing calcium content above $8 \%$ produces a negligible increase in strength and stiffness. Since treatment with $\mathrm{CaO}$ is thought to contribute to the reduction of the water content of the soil, its improvement capabilities appear to be limited.

The use of $2-12 \%$ calcium oxide in combination with $8 \%$ Portland cement results in strength values lower than those measured on the soil treated with $8 \%$ Portland cement alone.

High Calcium Flue Dust (HC) and Marblehead Buffington Dust (MD) are not very effective in increasing the strength of organic soils. The addition of High Calcium Flue dust and Marblehead Buffington Dust results in a decrease of the moisture content of the soil. Soil treated with these additives does not consistently have a higher compressive strength as more additive is used. The addition of bentonite decreases the compressive strength of organic soil.

Unlike the results for the PC treated organic soil (LR soil, 50\% LOI), the compressive strength of Mixture I (9.5\% LOI) increases as the percentage of cement is increased, at least within the range investigated (0 - 30\% PC). The increase of compressive strength with PC depends on the organic content of the soil treated. For example, UC tests on LR soil treated with 50\% PC and on Mixture I treated with 20\% PC show that the compressive strength of Mixture I is substantially higher than LR soil.

Several general observations can be made from the results of UC tests. First, among the binders investigated, both cement and $\mathrm{CaO}$ increase the strength and stiffness of a soil mixture, while the improvements obtained with the two commercial limes, HC and $\mathrm{MB}$, and with the bentonite are small or none. Second, cement provides a much more pronounced effect, especially when considering the increase in strength with curing time. Portland cement alone provides much greater strengthening than $\mathrm{CaO}$ or the combination of $\mathrm{CaO}$ and cement, although the addition of $\mathrm{CaO}$ to a cement treated soil appears to increase the stiffness of the treated soil. Third, the surcharge has a significant effect on 
the strength and stiffness of the soil mixture. The compressive strength is directly proportional to the surcharge that is applied. The surcharge provides a greater strengthening effect on cement treated soil than on a soil with no binder. Finally, the treatment with PC is more effective for soils with lower organic content.

\subsubsection{Effects of treatment on stiffness and creep behavior}

The 1-D consolidation behavior of LR soil was investigated by performing constant rate of strain (CRS) and incremental loading (IL) consolidation tests. The strain rate effects were evaluated from the results of CRS consolidation tests data performed on reconstituted LR soil with strain rates of $0.1,0.25,0.5$ and $1.0 \% / \mathrm{hr}$. The results indicate that the behavior (compression curve, coefficient of consolidation $\left(\mathrm{C}_{\mathrm{v}}\right)$ and hydraulic conductivity (k)) of LR soil does not show significant strain rate dependency in the range investigated,. The excess pore pressures generated with the strain rate of $0.5 \% / \mathrm{hr}$ were high enough to be measured accurately with the pressure transducers employed and the resulting pore pressure ratio satisfied the ASTM recommendation. Based on these results, the strain rate of $0.5 \% / h r$ was selected for all further CRS tests.

The most evident effect of treatment with PC is the increase in the preconsolidation pressure. The increase in preconsolidation pressure is fairly modest for low dosages of PC with $\sigma_{\mathrm{p}}$ increasing from the average value of $50.2 \mathrm{kPa}$ obtained for the reconstituted soil to $74.8 \mathrm{kPa}$ and $94.6 \mathrm{kPa}$ with $8.0 \% \mathrm{PC}$ and $18.7 \% \mathrm{PC}$, respectively. Above $20 \%$ PC the increase in $\sigma_{p}^{\prime}$ is instead very significant: with $51.4 \%$ PC the preconsolidation pressure increases to $403.3 \mathrm{kPa}$ (8 times the value measured on the reconstituted LR soil). With 103.4 \%PC it further increases to $1249.8 \mathrm{kPa}$ (25 times the value measured on the reconstituted LR soil). In addition, with increasing cement content, the compression curves gradually shifts to higher effective stress level. This indicates that cement treated LR soil specimen can sustain higher effective stress than untreated reconstituted LR soil at the same void ratio.

While the preconsolidation pressure increases with treatment, the compression index $\left(\mathrm{C}_{\mathrm{c}}\right)$ in the normally consolidated region does not show any significant change 
Although the trend is not clear, the compression indices of LR soil show a slight increase with increasing \% PC in the $1-2 \sigma^{\prime}{ }_{p}$ effective stress range which may be related to the development of structure in the soil specimen. In the stress range above $8 \sigma_{p}{ }_{p}$, the compression index of the PC treated LR soil becomes close to that of the untreated reconstituted LR soil. The recompression ratios of PC treated LR soil, calculated from the reloading curve, show that the compression ratio decreases with increasing \%PC throughout the recompression range, evidence of the fact that LR soil becomes much stiffer upon treatment with PC.

The hydraulic conductivity and coefficient of consolidation of the treated soil increase with increasing cement content. It is hypothesized that the increase in hydraulic conductivity with treatment is caused by the change of the fabric of soil as a result of the reactions occurring with the cement. As a result, the size of the macropores, which serve as main channels for flow, increase causing the increase in the hydraulic conductivity. The increase in coefficient of consolidation is associated with both the increase in $\mathrm{k}$ and the increase in stiffness. The increase in coefficient of consolidation implies that the consolidation process takes place faster in LR soil when treated. Compared to the untreated reconstituted LR soil the coefficient of consolidation increases by 1.4 times with $8.0 \%$ PC, 8.1 times with $18.7 \%$ PC, and about 38 times with $51.4 \%$ and $103.4 \%$ PC.

The effects of treatment on creep behavior of LR soil were investigated by performing long term creep tests. The $\mathrm{C}_{\alpha} / \mathrm{C}_{\mathrm{c}}$ for untreated and reconstituted LR soil is 0.103 and falls at the upper range of values reported for peats. With $8.0 \%$ and $18.7 \%$ PC, $\mathrm{C}_{\alpha} / \mathrm{C}_{\mathrm{c}}$ decreases to 0.085 and 0.063 , but remains still higher than the average value for peats and muskeg $(0.06 \pm 0.01)$. With $51.4 \% \mathrm{PC}, \mathrm{C}_{\alpha} / \mathrm{C}_{\mathrm{c}}$ decreases to 0.04 and falls in the range of average value for inorganic clays and silts $(0.04 \pm 0.01)$. With $103.4 \%$ PC, $\mathrm{C}_{\alpha} / \mathrm{C}_{\mathrm{c}}$ further decreases to 0.024 and falls in the range of average values for granular soils $(0.02 \pm 0.01)$. 


\subsection{Comparison to other improvement methods and recommendations}

There are definite advantages in treating organic soils with PC over other methods such as preloading, sand drains or excavation. To illustrate the advantages of soil treatment, a settlement analysis is performed for an example case where a $3 \mathrm{~m}$ high embankment is constructed at a site where an organic soil deposit is encountered.

The subsoil conditions at this example site are shown in Figure 8.1. A 3 m thick sand layer is found immediately below the surface. The dry unit weight $\left(\gamma_{d}\right)$ of the sand is $18 \mathrm{kN} / \mathrm{m}^{3}$. The sand layer is underlain by a $4 \mathrm{~m}$ thick organic soil layer. The organic soil contains about $50 \%$ organic matter and has the same properties as the reconstituted LR soil: natural water content $(\mathrm{w})=289 \%$, liquidity index $(\mathrm{LI})=0.77$, and compression ratio $(C R)=0.305$. The groundwater table is located at a depth of $3 \mathrm{~m}$. The saturated unit weight $\left(\gamma_{\text {sat }}\right)$ of the organic soil is $15 \mathrm{kN} / \mathrm{m}^{3}$. The organic soil layer is underlain by a clay layer and limestone. The hydraulic conductivity of the clay is assumed to be significantly lower than that of the organic soil.

The most commonly used methods for soil stabilization are preloading, sand drains, and deep mixing. These methods are considered here for the organic layer only. For each method, the primary consolidation settlement, the time required for $95 \%$ of consolidation, and the secondary settlement (creep) are calculated. The settlement associated with consolidation of the clay layer is not considered.

To simplify the calculations, the following assumptions are made:

(1) the organic soil is normally consolidated

(2) the embankment is placed in a very short period of time

(3) drainage takes places only at the top of the organic soil layer

(4) the preconsolidation pressures and the compressibility parameters (CR and RR) of PC treated organic soils are the same as those of PC treated LR soil

(5) the organic soil has the same hydraulic conductivity and coefficient of consolidation as the LR soil.

(6) the hydraulic conductivity and the coefficient of consolidation are the same in vertical and horizontal directions: $\mathrm{k}_{\mathrm{v}}=\mathrm{k}_{\mathrm{h}}$ and $\mathrm{C}_{\mathrm{v}}=\mathrm{C}_{\mathrm{h}}$ 
(7) the coefficient of consolidation does not vary during consolidation despite the increase in vertical effective stress

(8) secondary consolidation takes place after the end of primary consolidation

(9) the ratio of $C_{\alpha} / C_{c}$ of the organic soil is 0.10

Finally note that stability issues are not addressed in this example which focuses exclusively on settlement calculation.

8.2.1 Construction of embankment in 1 stage (similar results apply for preloading)

The average vertical effective stress $\left(\mathrm{p}_{\mathrm{o}}\right)$ at the middle of the organic soil layer is

$$
p_{o}=3 \gamma_{d(\text { sand })}+2\left(\gamma_{\text {sat(organic soil })}-\gamma_{w}\right)=3 \times 18+2 \times(15-9.81)=64.38 \mathrm{kPa}
$$

After placing a 3m thick fill on the surface, a surcharge $(\Delta \mathrm{p})$ of $60 \mathrm{kPa}$ is applied. Then, the total settlement (S) of the organic soil layer caused by primary consolidation is:

$$
S=C R \cdot H_{c} \cdot \log \left[\frac{p_{o}+\Delta p_{(p)}}{p_{o}}\right]=0.305 \times 4 \times \log \left(\frac{64.38+60}{64.38}\right)=34.9 \mathrm{~cm}
$$

where $\mathrm{H}_{\mathrm{c}}=$ thickness of the organic soil layer (m)

The coefficient of consolidation of untreated and reconstituted LR soil in this stress range is approximately $0.873 \mathrm{~m}^{2} / \mathrm{yr}$. With this value, the time required for $95 \%$ of consolidation can be calculated as:

$$
t=\frac{T_{v} \cdot H_{d r}^{2}}{C_{v}}=\frac{1.129 \times(4)^{2}}{0.873}=20.7 \mathrm{yrs}
$$

where $\mathrm{H}_{\mathrm{dr}}=$ drainage height $(\mathrm{m})$

Using the ratio of $\mathrm{C}_{\alpha} / \mathrm{C}_{\mathrm{c}}$ and $\mathrm{CR}$ for reconstituted LR soil, the secondary consolidations $\left(\mathrm{S}_{\mathrm{s}}\right)$ of the organic soil 50 and 100 years after construction of embankment are calculated as: 


$$
\begin{aligned}
& S_{\text {s50years }}=C R \cdot \frac{C_{\alpha}}{C_{c}} H_{c} \cdot \log \left[\frac{t}{t_{p}}\right]=0.305 \times 0.10 \times(4-0.349) \times \log \left(\frac{50}{20.7}\right)=4.3 \mathrm{~cm} \\
& S_{\text {s100years }}=C R \cdot \frac{C_{\alpha}}{C_{c}} H_{c} \cdot \log \left[\frac{t}{t_{p}}\right]=0.305 \times 0.10 \times(4-0.349) \times \log \left(\frac{100}{20.7}\right)=7.6 \mathrm{~cm}
\end{aligned}
$$

The calculations above also illustrate the very long times associated with application of the preloading method.

\subsubsection{Sand drains}

The layout of the sand drains is shown in Figure 8.2. It is assumed that the sand drains have a radius $\left(\mathrm{r}_{\mathrm{w}}\right)=0.2 \mathrm{~m}$, and that the radius of the effective zone of drainage $\left(\mathrm{r}_{\mathrm{e}}=\mathrm{d}_{\mathrm{e}} / 2\right)=1 \mathrm{~m}$. It is also assumed that during the installation of the sand drains, the organic soils around the drains are not smeared, and the hydraulic conductivity does not change. Note that this is an important assumption and there are many cases reported in the literature where this problem rendered the sand drains ineffective.

The total settlement due to primary consolidation of the organic soil layer is the same as calculated above. However, since the drainage length is reduced due to the installation of the sand drains, the time required for $95 \%$ of settlement is reduced. The time for $95 \%$ of settlement is:

$$
t=\frac{T_{r} \cdot d_{e}{ }^{2}}{C_{v r}}=\frac{0.3507 \times(2)^{2}}{0.873}=1.6 \mathrm{yrs}
$$

where $T_{\mathrm{r}}=$ non-dimensional time factor for radial drainage only

$\mathrm{d}_{\mathrm{e}}=$ diameter of the effective zone of drainage $(\mathrm{m})$

$\mathrm{C}_{\mathrm{vr}}=$ coefficient of consolidation for radial drainage $\left(\mathrm{m}^{2} / \mathrm{yrs}\right)$

The secondary consolidations $\left(\mathrm{S}_{\mathrm{s}}\right)$ of the organic soil 50 and 100 years after construction of embankment are:

$$
\begin{aligned}
& S_{\text {s50years }}=C R \cdot \frac{C_{\alpha}}{C_{c}} H_{c} \cdot \log \left[\frac{t}{t_{p}}\right]=0.305 \times 0.10 \times(4-0.349) \times \log \left(\frac{50}{1.6}\right)=16.6 \mathrm{~cm} \\
& S_{\text {s100 years }}=C R \cdot \frac{C_{\alpha}}{C_{c}} H_{c} \cdot \log \left[\frac{t}{t_{p}}\right]=0.305 \times 0.10 \times(4-0.349) \times \log \left(\frac{100}{1.6}\right)=20.0 \mathrm{~cm}
\end{aligned}
$$




\subsubsection{Treatment with PC (deep mixing)}

For deep mixing, the following cases are considered: treatment of the organic soil with 8, 20 and 50\% PC. In terms of cement factor, the amounts of PC used in 1 cubic meter of treated soil are 25.6, 64.0 and $160.0 \mathrm{~kg}$, respectively.

\section{(1) Treatment with $8 \%$ PC}

The preconsolidation pressure of the treated organic soil will be increased to 75.0 $\mathrm{kPa}$. Under these conditions the treated soil will become overconsolidated with an OCR 1.2. For this OCR range, the recompression ratio (RR) of the organic soil treated with $8 \%$ PC is 0.060 . After the fill is placed, the treated soil will be loaded into the normally consolidated region. As a result of this loading the total primary consolidation settlement is:

$$
\begin{aligned}
S & =R R \cdot H_{c} \cdot \log \left[\frac{p_{p}}{p_{o}}\right]+C R \cdot H_{c} \cdot \log \left[\frac{p_{o}+\Delta p_{(p)}}{p_{p}}\right] \\
& =0.060 \times 4 \times \log \left(\frac{75.0}{64.38}\right)+0.305 \times 4 \times \log \left(\frac{64.38+60}{75.0}\right)=28.4 \mathrm{~cm}
\end{aligned}
$$

The coefficient of consolidation of the organic soil treated with 8\% PC is approximately $1.15 \mathrm{~m}^{2} / \mathrm{yr}$. The time required for $95 \%$ of settlement is calculated as:

$$
t=\frac{T_{v} \cdot H_{d r}{ }^{2}}{C_{v}}=\frac{1.129 \times(4)^{2}}{1.15}=15.8 \mathrm{yrs}
$$

With $8.0 \%$ PC treatment, the $\mathrm{C}_{\alpha} / \mathrm{C}_{\mathrm{c}}$ for the soil will decrease to 0.085 . The secondary consolidations $\left(\mathrm{S}_{\mathrm{s}}\right)$ of the soil 50 and 100 years after construction of embankment will be: 


$$
\begin{aligned}
& S_{s 50 \text { years }}=C R \cdot \frac{C_{\alpha}}{C_{c}} H_{c} \cdot \log \left[\frac{t}{t_{p}}\right]=0.305 \times 0.085 \times(4-0.284) \times \log \left(\frac{50}{15.8}\right)=4.8 \mathrm{~cm} \\
& S_{\text {s100 years }}=C R \cdot \frac{C_{\alpha}}{C_{c}} H_{c} \cdot \log \left[\frac{t}{t_{p}}\right]=0.305 \times 0.085 \times(4-0.284) \times \log \left(\frac{100}{15.8}\right)=7.7 \mathrm{~cm}
\end{aligned}
$$

(2) Treatment with $20 \%$ PC

The preconsolidation pressure of the organic soil treated with 20\% PC will increase to $95 \mathrm{kPa}$. Similar to the previous case, the treated soil will become overconsolidated with an OCR $\sim 1.5$ and with a recompression ratio of 0.049 . The total settlement due to primary consolidation is calculated as:

$$
\begin{aligned}
S & =R R \cdot H_{c} \cdot \log \left[\frac{p_{p}}{p_{o}}\right]+C R \cdot H_{c} \cdot \log \left[\frac{p_{o}+\Delta p_{(p)}}{p_{p}}\right] \\
& =0.049 \times 4 \times \log \left(\frac{95.0}{64.38}\right)+0.305 \times 4 \times \log \left(\frac{64.38+60}{95.0}\right)=17.6 \mathrm{~cm}
\end{aligned}
$$

The coefficient of consolidation of the $20 \%$ PC treated soil is approximately 9.10 $\mathrm{m}^{2} / \mathrm{yr}$. The time required for $95 \%$ of settlement is:

$$
t=\frac{T_{v} \cdot H_{d r}{ }^{2}}{C_{v}}=\frac{1.129 \times(4)^{2}}{9.10}=2.0 \mathrm{yrs}
$$

With $20.0 \%$ PC treatment, the $\mathrm{C}_{\alpha} / \mathrm{C}_{\mathrm{c}}$ for the soil will decrease to 0.063 . The secondary consolidations $\left(\mathrm{S}_{\mathrm{s}}\right)$ of the soil 50 and 100 years after construction of embankment will be:

$$
\begin{aligned}
& S_{s 50 \text { years }}=C R \cdot \frac{C_{\alpha}}{C_{c}} H_{c} \cdot \log \left[\frac{t}{t_{p}}\right]=0.305 \times 0.063 \times(4-0.176) \times \log \left(\frac{50}{2.0}\right)=10.3 \mathrm{~cm} \\
& S_{\text {s100 years }}=C R \cdot \frac{C_{\alpha}}{C_{c}} H_{c} \cdot \log \left[\frac{t}{t_{p}}\right]=0.305 \times 0.063 \times(4-0.176) \times \log \left(\frac{100}{2.0}\right)=12.5 \mathrm{~cm}
\end{aligned}
$$

(3) Treatment with 50\% PC 
The preconsolidation pressure will increase to $400 \mathrm{kPa}$. In this case, the soil remains in the overconsolidated state even once the full effective stress increase associated with placing the fill has occurred. With the recompression ratio of 0.032, the settlement of the soil is:

$$
S=R R \cdot H_{c} \cdot \log \left[\frac{p_{p}}{p_{o}}\right]=0.032 \times 4 \times \log \left(\frac{64.38+60}{64.38}\right)=3.6 \mathrm{~cm}
$$

The coefficient of consolidation of the 50\% PC treated soil is approximately $52.60 \mathrm{~m}^{2} / \mathrm{yr}$. The time required for $95 \%$ of settlement is:

$$
t=\frac{T_{v} \cdot H_{d r}{ }^{2}}{C_{v}}=\frac{1.129 \times(4)^{2}}{52.60}=0.35 \mathrm{yrs}
$$

With $50 \% \mathrm{PC}$ treatment, the $\mathrm{C}_{\alpha} / \mathrm{C}_{\mathrm{c}}$ for the soil will decrease to 0.040 . The secondary consolidations $\left(\mathrm{S}_{\mathrm{s}}\right)$ of the soil 50 and 100 years after construction of embankment will be:

$$
\begin{aligned}
& S_{s 50 \text { years }}=C R \cdot \frac{C_{\alpha}}{C_{c}} H_{c} \cdot \log \left[\frac{t}{t_{p}}\right]=0.032 \times 0.040 \times(4-0.036) \times \log \left(\frac{50}{0.35}\right)=1.1 \mathrm{~cm} \\
& S_{\text {s10 years }}=C R \cdot \frac{C_{\alpha}}{C_{c}} H_{c} \cdot \log \left[\frac{t}{t_{p}}\right]=0.032 \times 0.040 \times(4-0.036) \times \log \left(\frac{100}{0.35}\right)=1.2 \mathrm{~cm}
\end{aligned}
$$

The results of the settlement analyses for the three methods considered are summarized in Table 8.1 and Figure 8.4. The total settlements due to primary consolidation of the organic soil when using preloading or sand drains are the same. However, the time required for completion of primary consolidation can be significantly reduced with the sand drains. When deep mixing is used, the total settlement due to primary consolidation and the time required for $95 \%$ of consolidation are significantly reduced.

Each stabilization method has advantages and disadvantages. Preloading has been used for a long time and there is a large knowledge from experience; in addition it is inexpensive. However, the time required for settlement completion may render the solution infeasible. Theoretically the time for consolidation is reduced with sand (wick) 
drains, although in practice an effective installation of the drains may be problematic and large soil deformations may render the drains inoperative. It is important to mention that neither preloading nor drainage decreases deformation due to creep (secondary consolidation).

In this particular case excavation of the organic may not have been possible due to the depth and volume of the soil.

Excavation of the organic soil is perhaps the best solution when the organic soil is close to the surface, it is not very thick, and there are no environmental concerns.

Deep mixing offers the advantages of rapid implementation, large reduction of primary consolidation due to the increase of the preconsolidation pressure of the soil and reduced creep. The disadvantages are that it requires a specialty contractor and the method may result in higher construction costs (note that the higher construction costs may be compensated with smaller maintenance costs).

\subsection{Recommendations}

Soils with low to moderate ( $15 \%$ to $50 \%$ Loss of Ignition, LOI) to high organic content (more than 50\% LOI) are characterized by high water content, high compressibility, both primary and creep, and low strength. There are three methods that have been traditionally used in projects where such soils are encountered: removal, preloading, and improvement. Removal consists of the excavation of the problem soils and replacement with an engineered fill that satisfies the project requirements, usually in terms of strength and stiffness. Preloading requires the temporary addition of a load (usually a fill) on top of the soil for a given period of time; the load may be larger than the final load that the soils will sustain. The process can be accelerated with the addition of vertical drains (sand or stone columns, or wick drains). The end result, after some degree of consolidation, is a soil with a higher preconsolidation pressure. Soil improvement usually requires the addition of a chemical agent (usually a binding agent) to the soil and mixture of the soil with the agent. The result is a different soil with improved strength and stiffness characteristics.

While there is no single decision that can be applied to all projects, some trends and practical recommendations can be made based on the research conducted. Each 
project is different; the soils and soil properties are different, the project requirements are also different, and the construction constrains change, even during the project. In the end the best solution is the one that provides the results required and is the most cost effective.

The following are general recommendations based on the thickness of the organic soil and the LOI of the soil.

Shallow organic soil. The problem soil is very close to the surface and its thickness is moderate. As a reference, this case would include organic soils that extend from the surface to about 6-10 ft. In this case an option that could be advantageous is removal of the problem soil and replacement by an engineered fill with the required specifications. Due to the small volume of soil, preloading, including vertical drains, and soil improvement, even though technically feasible, may not be as cost-effective as excavation. If the problem soil affects only the first 3-4 ft, an alternative option could be to mix the soil in-situ with either lime or cement. Both additives improve the engineering properties of the soil. The results, for the same quantity of additive, improve as the organic content decreases; thus soil treatment may be an attractive solution for shallow low organic soil deposit. In particular for those cases were the primary objective is the reduction of the water content of the soil.

Deep organic soil deposit. The problem soil is found beyond 6-10 ft below the surface. Preloading with or without vertical drainage may be successful only in soils with low organic content (less than 15\%). The smaller compressibility of these soils compared to high organic soils will induce smaller settlements due to consolidation and creep. In addition the shear strength of low organic soils may be large enough to allow for significant preloading thus increasing preconsolidation pressure. For soils with high organic content, preloading may not be technically or economically feasible. The time required to induce a significant change in preconsolidation pressure may be too long. On the one hand the magnitude of the preload may be limited by the low shear strength of the soil and, on the other hand, vertical drains may not be effective due to the large settlements induced in the soils even with a small load; in particular it is well documented that wick drains may be inoperative when large ground deformations occur, which is 
generally the case in organic soils. Furthermore, preloading does not change the rate of creep in soils with high organic content, and thus long-term settlements may be still too large. For such soils treatment with deep cement mixing is recommended. The treatment increases the preconsolidation pressure of the soil and decreases settlements due to creep. Deep mixing requires advance planning and a specialty contractor. Even though the solution is more costly than other methods such as preloading, it may be the only one technically feasible. The use of the technology is rapidly increasing in the country and abroad, and there is already and extensive experience with successful treatment with organic soils. All this brings confidence in the method and in its results. 
Table 8.1: Comparisons of settlement due to primary consolidation, time for 95\% consolidation and secondary consolidation

\begin{tabular}{|c|c|c|c|c|c|c|}
\hline \multirow{2}{*}{\multicolumn{2}{|c|}{ Stabilization methods }} & \multirow{2}{*}{ Preloading } & \multirow{2}{*}{ Sand drains } & \multicolumn{3}{|c|}{ Deep mixing } \\
\hline & & & & $8 \%$ PC & $20 \%$ PC & $50 \% \mathrm{PC}$ \\
\hline \multicolumn{2}{|c|}{$\begin{array}{l}\text { Settlement due to primary } \\
\text { consolidation }(\mathrm{cm})\end{array}$} & 34.9 & 34.9 & 28.4 & 17.6 & 3.6 \\
\hline \multicolumn{2}{|c|}{$\begin{array}{c}\text { Time for 95\% settlement } \\
\text { (yrs) }\end{array}$} & 20.7 & 1.6 & 15.8 & 2.0 & 0.35 \\
\hline \multirow{2}{*}{$\begin{array}{c}\text { Secondary } \\
\text { consolidation } \\
\text { (cm) }\end{array}$} & $\begin{array}{l}\text { after } 50 \\
\text { years }\end{array}$ & 4.3 & 16.6 & 4.8 & 10.3 & 1.1 \\
\hline & $\begin{array}{c}\text { after } 100 \\
\text { years }\end{array}$ & 7.6 & 20.0 & 7.7 & 12.5 & 1.2 \\
\hline
\end{tabular}




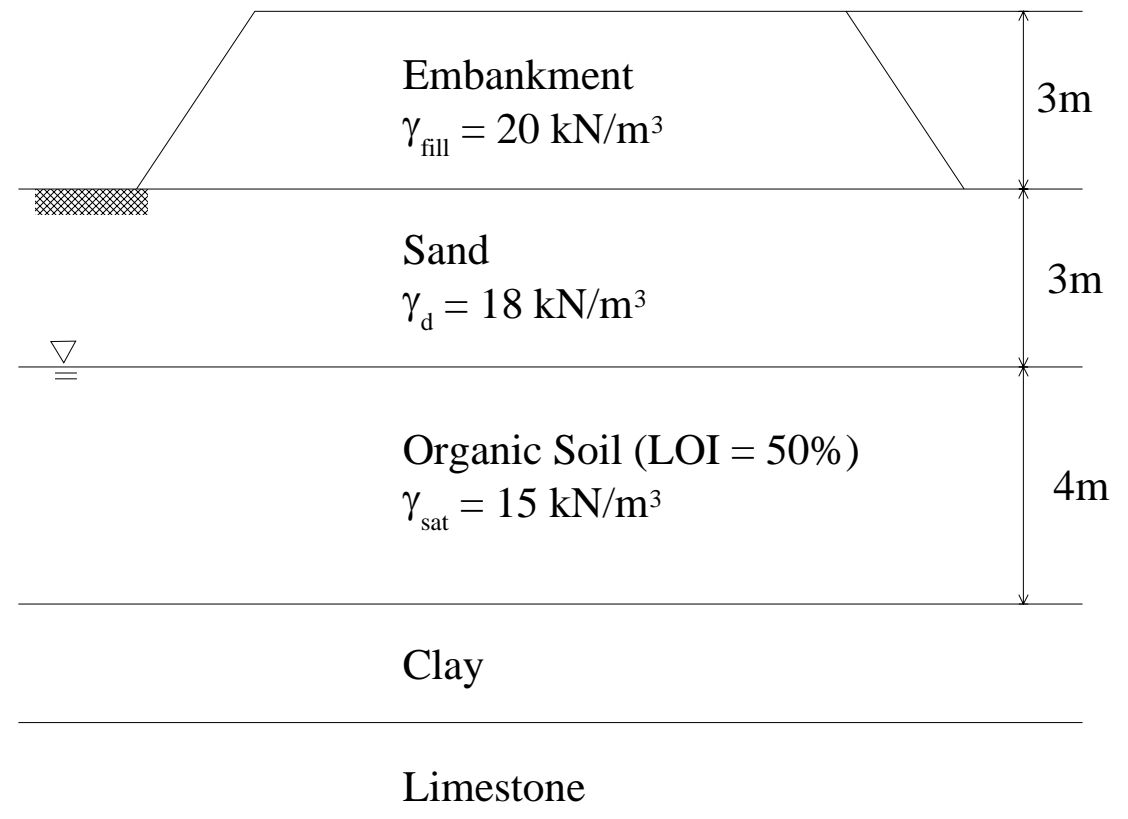

Figure 8.1: Soil profile under the embankment

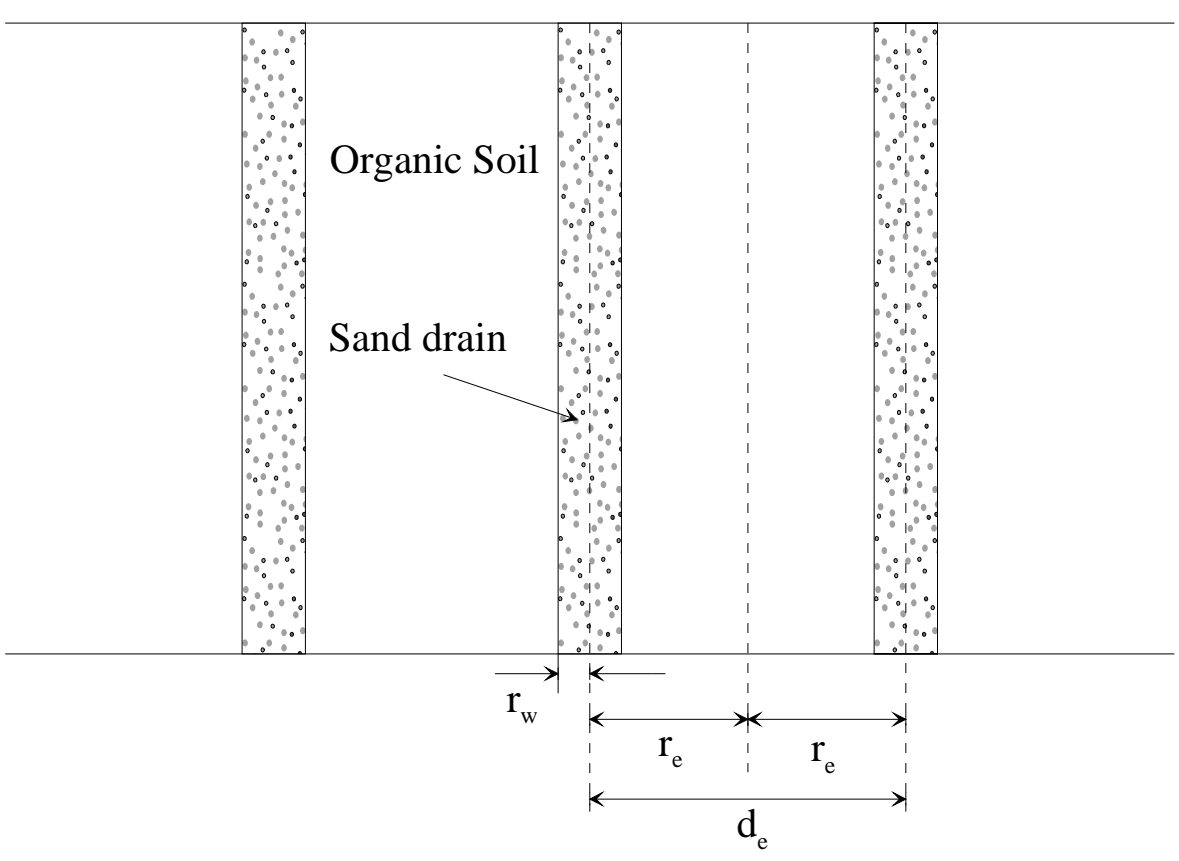

Figure 8.2: Diagram of sand drains 

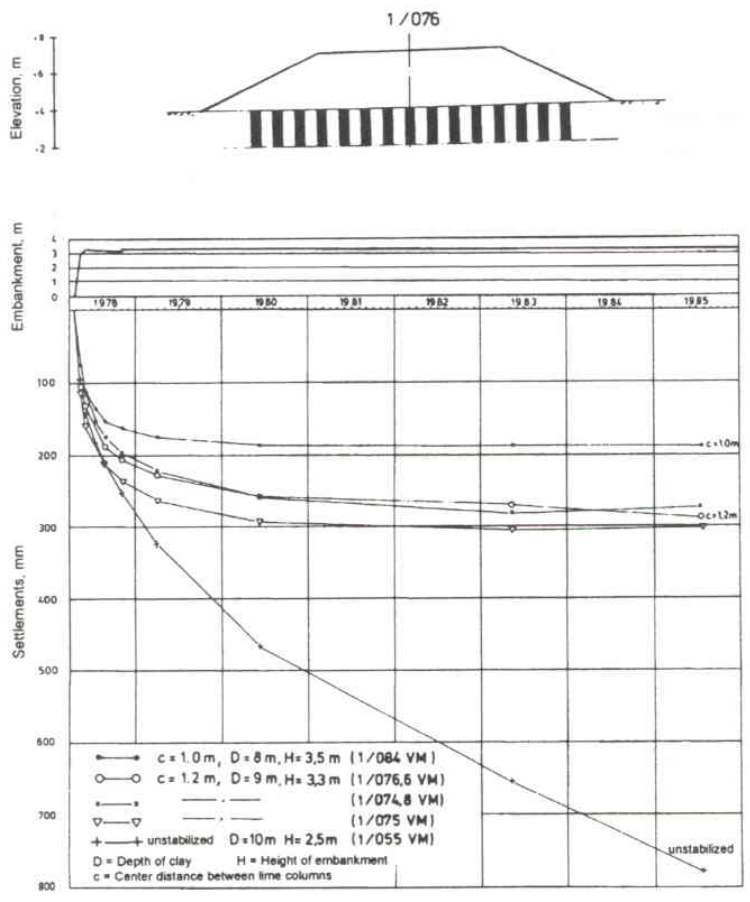

Figure 8.3: Long term behavior of a road embankment stabilized with deep mixing method (Holm, 2003)

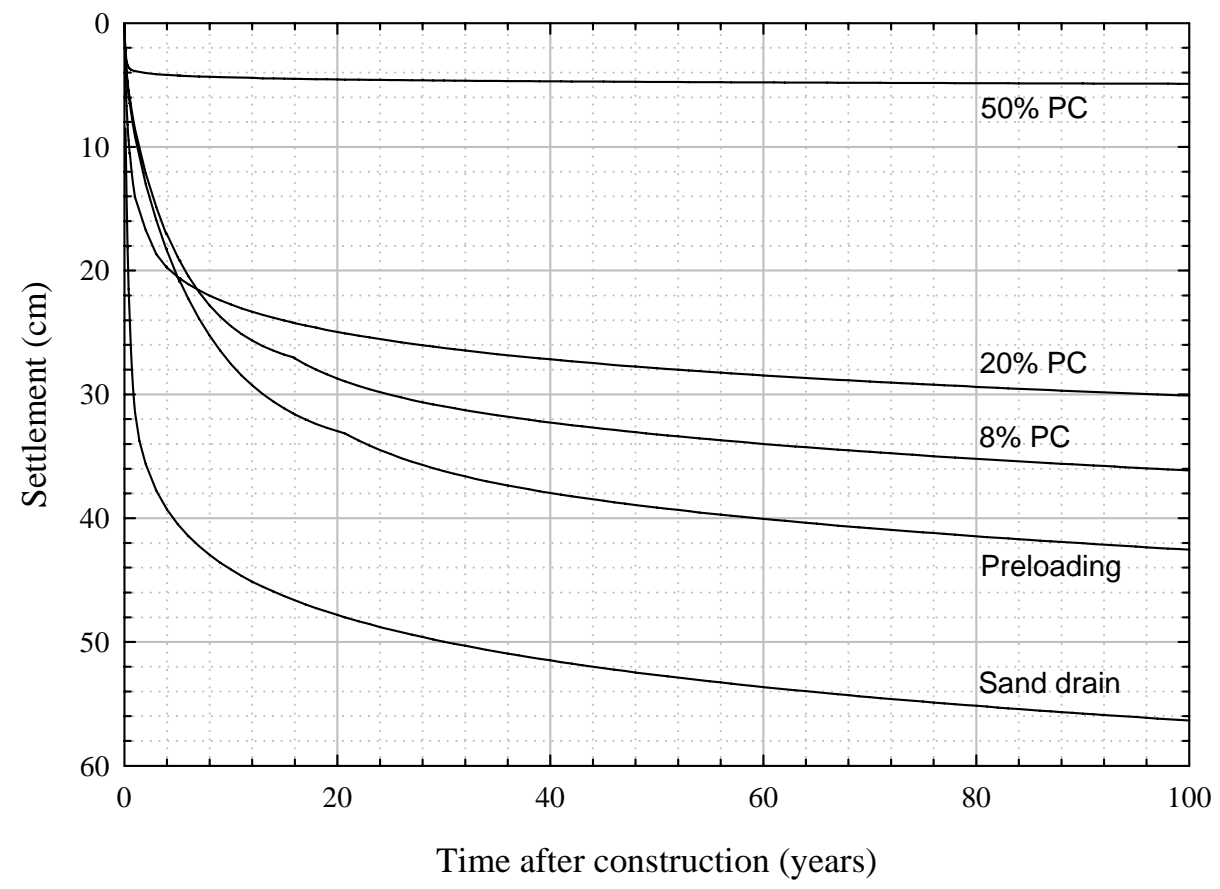

Figure 8.4: Comparison of the time-settlement curves 


\section{LIST OF REFERENCES}

Åhnberg, H. (1996). "Stress dependent parameters of cement and lime stabilized soils.” Grouting and Deep Mixing, Proceedings of IS-Tokyo'96/ The second International Conference on Ground Improvement Geosystems, Tokyo, 14-17 May 1996, 387-392.

Åhnberg, H., and G. Holm. (1999). "Stabilization of some Swedish organic soils with different types of binder.” Dry Mix Methods for Deep Soil Stabilization. Proc. Of international conference on dry mix methods for deep soil stabilization., 13-15 Oct. 1999, Stockholm. Ed. Håkan Bredenberg, Göran Holm, and Bengt B. Broms. Rotterdam: A.A. Balkema, 101-108.

American Society for Testing and Materials. (1999). 1999 Annual Book of ASTM Standards. ASTM.

Al-Rawas, A.A. (2002). "Microgabric and mineralogical studies on the stabilization of an expansive soil using cement bypass dust and some types of slags”, Can. Geotech. J., 39 (5), 1150-1167.

Aziz, M.A. (1984). “A New Method of Stabilization of Soft Soils.” International Conference on Case Histories in Geotechnical Engineering. Proceeding of International Conference on Case Histories in Geotechnical Engineering. 6-11 May 1984, St. Louis. Ed. Shamsher Prakash. Rolla: University of Missouri-Rolla, 1215-1219.

Becker, D.E., Crooks, J.H.A., Been, K. and Jefferies, M.G. (1987). "Work as a criterion for determining in situ and yield stresses in clays”, Can. Geotech. J., 24 (4), 549-564.

Brandl, H. (1981), “Alteration of soil parameters by stabilisation with lime”, Proceedings, 10th International Conference on Soil Mechanics and Foundation Engineering, Stockholm, Sweden, 587-594. 
Broms, B.B (1999). “Design of lime, lime/cement and cement columns”, Dry Mix Methods for Deep Soil Stabilization, Proceedings of the International Conference on Dry Mix Methods for Deep Soil Stabilization, Stockholm, Sweden, 13-15 Oct, 1999, 125-153.

Broms, B. and Boman, P. (1977). “Stabilization of soil with lime columns”, Royal Institute of Technology, Department of Soils and Rock Mechanics, Sweden, Design Handbook.

Bruce, D.A. and Bruce, E.C. (2003). “The practitioner's guide to deep mixing”, Geotechnical Special Publication No. 120, ASCE, 474-488.

Buensuceso, B.R. (1990). "Engineering behavior of lime treated soft Bangkok Clay", Ph.D. thesis, Asian Institute of Technology, Bangkok.

Casagrande, A. (1948). “Classification and Identification of soils”, Transactions of the American Society of Civil Engineers, 113, 901-930.

Colleselli, Francesco, Ciampaolo Cortellazzo, and Simonetta Cola (2000). "Laboratory Testing of Italian Peaty Soils.” Geotechnics of High Water Content Materials: ASTM Special Technical Publication 1374. Ed. Tuncer B. Edil and Patrick Fox. West Conshohocken: ASTM, 226-240.

Cortellazzo, G. and Cola, S. (1999). “Geotechnical characteristics of two Italian peats stabilized with binders”, Dry Mix Methods for Deep Soil Stabilization, Proceddings of the International Conderence on Dry Mix Methods for Deep Soil Stbilization, Stockholm, Sweden, 13-15 Oct. 1999, 93-100.

Dhowian, A.B., and Edil, E.B. (1980). “Consolidation Behavior of Peats”, Geotech. Testing J., 3 (3), 105-114. 
Earth Exploration, INC. (1993). "Preliminary geotechnical evaluation: Proposed improvements to Lindberg road in West Lafayette, Tippecanoe County, Indiana”

Edil, Tuncer B. and Xiaodong Wang. (2000). "Shear Strength and Ko of Peats and Organic Soils.” Geotechnics of High Water Content Materials: ASTM Special Technical Publication 1374. Ed. Tuncer B. Edil and Patrick Fox. West Conshohocken: ASTM, 209225.

Esrig, M.I. (1999). “Keynote lecture: Properties of binders and stabilized soils.” Dry Mix Methods for Deep Soil Stabilization. Proc. of international conference on dry mix methods for deep soil stabilization., 13-15 Oct. 1999, Stockholm. Ed. Håkan Bredenberg, Göran Holm, and Bengt B. Broms. Rotterdam: A.A. Balkema, 67-72.

Esrig, M.I., Mac Kenna, P.E., and Forte, E.P. (2003) “Ground stabilization in the United States by the Scandinavian lime cement dry mix process”, Geotechnical Special Publication No. 120, ASCE, 501-514.

Force, E.A. (1998). "Factors controlling pore pressure generation during Ko consolidation of laboratory tests” MS Thesis, MIT Dept. of Civil and Env. Eng., Cambridge, MA

Fox, Patrick J., and Tuncer B. Edil. (1996). "Effects of stress and temperature on secondary compression of peat.” Can. Geotech. J., 33, 405-415.

Fox, Patrick J., Nina Roy-Chowdhury, and Tuncer B. Edil. (1999). "Secondary Compression of Peat With or Without Surcharging.” J. Geotech. and Geoenviron. Engrg., 125 (2), 160-162.

Ghazali, F.M., Baghdadi, Z.A., and Khan, A.M. (1991). “Overconsolidated behavior of phosphoric acid and lime-stabilized kaolin clay”, Transportation Research Record 1295, 62-69. 
Hebib, S., and E.R. Farrell. (1999). "Some experience of stabilizing Irish organic soils.” Dry Mix Methods for Deep Soil Stabilization. Proc. of international conference on dry mix methods for deep soil stabilization., 13-15 Oct. 1999, Stockholm. Ed. Håkan Bredenberg, Göran Holm, and Bengt B. Broms. Rotterdam: A.A. Balkema, 81-84.

Hebib, S. and Farrel, E.R. (2003). "Some experiences on the stabilization of Irish peats”, Can. Geotech. J., $\mathbf{4 0}$ (1), 107-120.

Huttunen, E. and K. Kujala. (1996). “On the stabilization of organic soils.” Grouting and Deep Mixing. Proc. of IS-Tokyo '96/ The second international conference on ground improvement geosystems., 14-17 May 1996, Tokyo. Ed. Ryozo Yonekura, Masaaki Terashi, and Mitsuhiro Shibazaki. Rotterdam: A.A. Balkema., 411-414.

Huttunen, E., K. Kujala, and H. Vesa. (1996). “Assesment of the quality of stabilized peat and clay.” Grouting and Deep Mixing. Proc. of IS-Tokyo '96/ The second international conference on ground improvement geosystems., 14-17 May 1996, Tokyo. Ed. Ryozo Yonekura, Masaaki Terashi, and Mitsuhiro Shibazaki. Rotterdam: A.A. Balkema., 607612.

Indiana Department of Transportation. (1999). Division of Contracts and Construction Standards Section. 1999 Standards. Indianapolis: Indiana.

Janbu, N., Tokheim, O. and Semmeset, K. (1981). “Consolidation tests with continuous loading.” 10th Int. Conf. Soil Mechanics \& foundation Eng. Stockholm, Vol. 1. 645-654.

Jamiolkowski, M., Ladd, C.C., Germaine, J.T. and Lancellotta, R. (1985). "New developments in field and laboratory testing of soils" Proc. 11th Int. Conference on Soil Mech. and Found. Engrg., San Francisco, USA, 1, 57-153. 
Kassim, K.A. and Clarke, B.G. (1999). “Constant rate of strain consolidation equipment and procedure for stabilized soils”, Geotech. Testing J., 22, 13-21.

Koda, E., Wolski, W. (1994). "The influence of strip drains on the consolidation performance of organic soils.” Advances in Understanding and Modelling the Mechanical Behavior of Peat. Ed. Evert den Haan, Ruud Termaat \& Tuncer B. Edil. A.A. Balkema: Rotterdam, 347-359.

Kirov, B. (1994). “Experience of peat preloading in the Varna West Harbour.” Advances in Understanding and Modelling the Mechanical Behavior of Peat. Ed. Evert den Haan, Ruud Termaat \& Tuncer B. Edil. A.A. Balkema: Rotterdam, 341-345.

Kujala, K., M. Mäkikyrö, and O. Lehto. (1996). "Effect of humus on the binding reaction in stabilized soil.” Grouting and Deep Mixing. Proc. of IS-Tokyo '96/ The second international conference on ground improvement geosystems., 14-17 May 1996, Tokyo. Ed. Ryozo Yonekura, Masaaki Terashi, and Mitsuhiro Shibazaki. Rotterdam: A.A. Balkema., 415-420.

Lahtinen, P., H. Jyrävä, and K. Kuusipuro. (1999). "Development of binders for organic soils.” Dry Mix Methods for Deep Soil Stabilization. Proc. of international conference on dry mix methods for deep soil stabilization., 13-15 Oct. 1999, Stockholm. Ed. Håkan Bredenberg, Göran Holm, and Bengt B. Broms. Rotterdam: A.A. Balkema, 109-114.

Lambe, William T., and Robert V. Whitman. (1969). “Soil Mechanics”. New York: John Wiley \& Sons.

Lambrechts, J.R., Ganse, M.A, and Layhee, C.A. (2003). "Soil mixing to stabilize organic clay forl-95 Widening, Alexandria, VA”. Geotechnical Special Publication No. 120, ASCE, 575-585. 
Landva, Arvid O., Peter E. Pheeney, and Donald E. Mersereau. (1983). "Undisturbed Sampling of Peat.” Testing of Peats and Organic Soils: ASTM Special Technical Publication 820. Proc. of a symposium sponsored by ASTM committee D-18 on Soil and Rock., 23 June 1982, Toronto. Ed. P.M. Jarrett. Philadelphia: ASTM, 141-156.

Landva, A.O., and P.E. Pheeney. (1980). "Peat Fabric and Structure.” Can. Geotech. J., 17, 416-435

Lefebvre, G., Langlois, P. and Lupien, V. (1984). "Laboratory Testing and in situ Behavior of Peat as Embankment Foundation”, Can. Geotech. J., 21 (2), 322-337.

Leroueil S., Tavenas F., Samson L. and Morin P. (1983). "Preconsolidation pressure of Champlain clays. Part II: Laboratory determination”, Can Geotech. J., 20 (4), 803-816.

Locat, J. Tremblay, H. and Leroueil, S. (1996). "Mechanical and hydraulic behavior of a soft inorganic clay treated with lime”, Can Geotech. J., 33 (4), 654-669.

McCallister, L.D., and Petry, T.M. (1992). “Leach tests on lime-treated clays”, Geotech. Testing J., 15, 106-114.

Mesri, G. and Casto, A. (1987). “Ca/Cc concept and Ko during secondary compression”, J. Geotech.. Engrg., 113 (3), 230-247.

Mesri, G., Stark, M.D, Ajlouni, M.A. and Chen, C.S. (1997). "Secondary Compression of Peat with or Without Surcharging”, J. Geotech. and Geoenviron. Engrg., 123 (5), 411421.

Nalbantoglu, Z. and Tuncer E. R. (2001). “Compressibility and hydraulic conductivity of a chemically treated expansive clay”, Can Geotech. J., 38 (1), 154-160. 
Ng, Samuel Y., and James C. Rudd. (1984). "Embankments Built Over Swamps.” International Conference on Case Histories in Geotechnical Engineering. Proceeding of International Conference on Case Histories in Geotechnical Engineering. 6-11 May 1984, St. Louis. Ed. Shamsher Prakash. Rolla: University of Missouri-Rolla, 609-618.

Pousette, K., J Mácsik, A. Jacobson, R. Andersson, and P. Lahtinen. (1999). "Peat soil samples stabilized in laboratory - Experiences from manufacturing and testing.” Dry Mix Methods for Deep Soil Stabilization. Proc. of international conference on dry mix methods for deep soil stabilization., 13-15 Oct. 1999, Stockholm. Ed. Håkan Bredenberg, Göran Holm, and Bengt B. Broms. Rotterdam: A.A. Balkema, 85-92.

Rao, S.N. and Rajasekaran, G. (1996) "Reaction products formed in lime-stabilized marine clays”, J. Geotech. Engrg., 122 (5), 329-336.

Radforth, N.W. (1969). “Classification of Muskeg.” Muskeg Engineering Handbook. Ed. Ivan C. MacFarlane. Toronto, University of Toronto, 31-39.

Santangata, M.C. (1994). "Investigation of Sample Disturbance in Soft Clays Using Triaxial Element Tests”, S.M. Thesis, Department of Civil and Environmental Engineering, MIT, Cambridge, MA, 289p.

Sasaki, Harumi. (1985). "Effectiveness and applicability of the methods of foundation improvement for embankments over peat deposits.” Recent Developments in Ground Improvement Techniques. Proc. of Symposium on Recent Developments in Ground Improvement Techniques ., 29 Nov. - 3 Dec. 1982, Bangkok. Ed. A.S. Balasubramaniam. Boston: A.A. Balkema, 543-561.

Sheahan, T.C. and Watters, P.J. (1997) "Experimental Verification of CRS Consolidation Theory”, J. Geotech. and Geoenviron. Engrg., 123 (5), 430 - 437. 
Terashi, M. Tanaka, H. Niidome, Y., and Sakanoi, H. (1980). "Permeability of treated soils”, Proceedings 15th Japan Conference on Soil mechanics and Foundation Engineering, 773-776.

Terzaghi, K., Peck, R.B. and Mesri, G. (1996). "Soil Mechanics in Engineering Practice”, 3rd edition, John Wiley \& Sons, Inc.

Townsend, D.C. and Klym, T.W. (1996). “Durability of lime-stabilized soils”, Highway Research Board Bulletin 139, 25-41.

Tremblay, H, Leroueil, S. and Locat, J. (2001). “Mechanical improvement and vertical yield stress prediction of clayey soils from eastern Canada treated with lime or cement”, Can Geotech. J., 38, 567-579.

Venema, Thomas P., C.R. Waletzko, and Frank J. Swekosky. (1990). "Ground Modification for a 14-story Structure over a Deep Peat Deposit.” Hayward Baker. Ground Modification Case Histories. Odenton: Hayward Baker.

Wissa, A E. Z., Christian, J. T., Davis, E.H. and Heiberg, S. (1971). “Consolidation at Constant Rate of Strain”, J. Soil Mech. and Found Div., ASCE, 97 (10), 1393-1413. 